University of South Florida

DIGITAL COMMONS

Digital Commons @ University of

@ UNIVERSITY OF SOUTH FLORIDA

South Florida

USF Tampa Graduate Theses and Dissertations

USF Graduate Theses and Dissertations

$11-10-2016$

\title{
Locomotion in Virtual Reality for Room Scale Tracked Areas
}

\author{
Evren Bozgeyikli \\ University of South Florida, evrenbozgeyikli@gmail.com
}

Follow this and additional works at: https://digitalcommons.usf.edu/etd

Part of the Computer Sciences Commons

\section{Scholar Commons Citation}

Bozgeyikli, Evren, "Locomotion in Virtual Reality for Room Scale Tracked Areas" (2016). USF Tampa Graduate Theses and Dissertations.

https://digitalcommons.usf.edu/etd/6470

This Dissertation is brought to you for free and open access by the USF Graduate Theses and Dissertations at Digital Commons @ University of South Florida. It has been accepted for inclusion in USF Tampa Graduate Theses and Dissertations by an authorized administrator of Digital Commons @ University of South Florida. For more information, please contact digitalcommons@usf.edu. 
Locomotion in Virtual Reality for Room Scale Tracked Areas

by

Evren C. Bozgeyikli

A dissertation submitted in partial fulfillment of the requirements for the degree of

Doctor of Philosophy

Department of Computer Science and Engineering

College of Engineering University of South Florida

\author{
Co-Major Professor: Srinivas Katkoori, Ph.D. \\ Co-Major Professor: Andrew Raij, Ph.D. \\ Rajiv Dubey, Ph.D. \\ Paul Rosen, Ph.D. \\ Sriram Chellappan, Ph.D. \\ Eleazar Vasquez, Ph.D.
}

Date of Approval:

October 24, 2016

Keywords: Redirected Walking, Point and Teleport, Controllers, Walk in Place, Head Mounted Display

Copyright @ 2016, Evren C. Bozgeyikli 


\section{DEDICATION}

To my brilliant and beloved wife, Gamze. 


\section{ACKNOWLEDGMENTS}

I would like to thank my co-advisor Dr. Raij, who believed in me and started my PhD journey. He also helped me throughout all those years without any doubt. Without his guidance and generous help, this dissertation would not have been possible.

I would also like to thank my co-advisor Dr. Katkoori, who helped a lot in solving the hardest situations during my dissertation. His support cleared the obstacles during difficult times.

I would like to express the deepest appreciation to my supervisor Dr. Alqasemi, who has been one of the closest mentors to me. He supported me and my studies without any hesitation. Without his infinite help, my dissertation would not have been completed.

I would like to thank my committee members, Dr. Dubey, Dr. Chellappan, and Dr. Rosen, for their valuable feedback and constructive criticism. I also would like to thank Dr. Vasquez for accepting to be in my committee and sharing his valuable expertise to guide me during my dissertation. I would also like to acknowledge Associate Director of CARRT, Stephen, for his help and support.

I would like to thank the Florida Department of Education's Division of Vocational Rehabilitation, the Center for Rehabilitation Engineering and Technology (CARRT), and the Powerful Interactive Experiences (PIE) Lab for their funding and support. 
I would like to thank the Computer Science and Engineering staff, Gabriela, Shanie, and Kim, and Academic Program Specialist Catherine for their help. I would like to also thank my lab mates Andoni, Luis, Tylar, Juan, and Dimitri for their companionship.

I would also like to thank my former advisor and life-long mentor Dr. Isler, who made my first steps in this area possible.

Last but not least, I would like to thank my family members, Oya, Halil, Mihriban, Haluk, Ozlem, Arda, Huseyin, for their unconditional support. And my beautiful wife Gamze, without her love and understanding, none of these could have been possible. 


\section{TABLE OF CONTENTS}

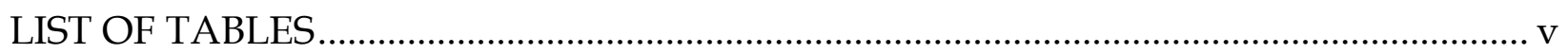

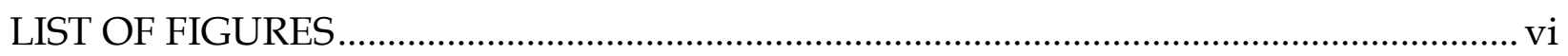

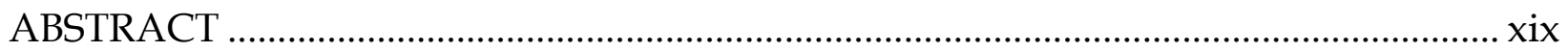

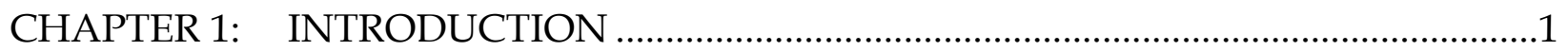

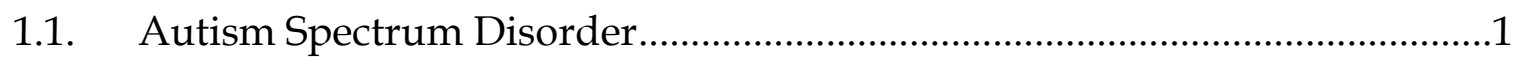

1.2. Virtual Reality Applications .......................................................................

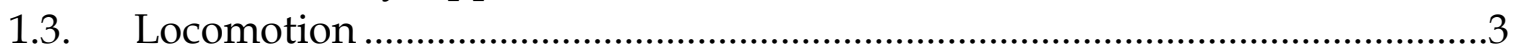

1.4. Motivation and Contributions of the Dissertation............................................

1.5. Organization of the Dissertation......................................................................8

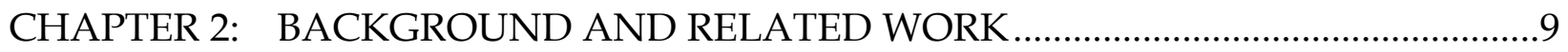

2.1. Terminology and Definitions on Virtual Reality, Interaction and

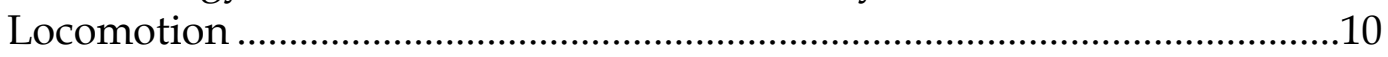

2.1.1. Interaction Techniques ..................................................................12

2.1.2. Locomotion Techniques .........................................................................15

2.2. Algorithm Based Locomotion Techniques .....................................................16

2.2.1. Real Walking.................................................................................16

2.2.1.1. Redirected Walking..................................................................17

2.2.1.2. Environment Change ……........................................................20

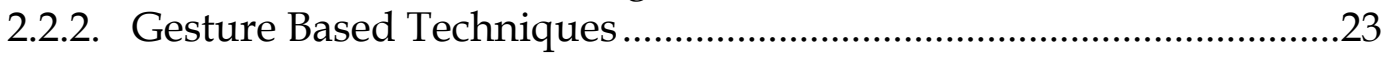

2.2.2.1. Walking in Place ....................................................................23

2.2.2.2. Flying/Leaning .......................................................................27

2.3. Tool Based Locomotion Techniques.............................................................30

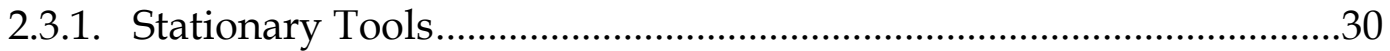

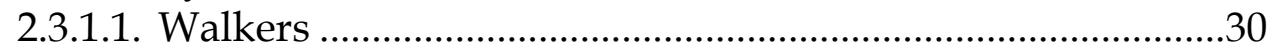

2.3.1.2. Standard Controllers.....................................................................35

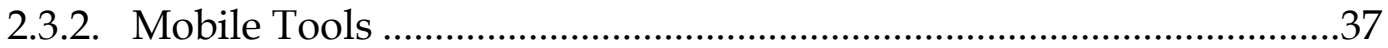

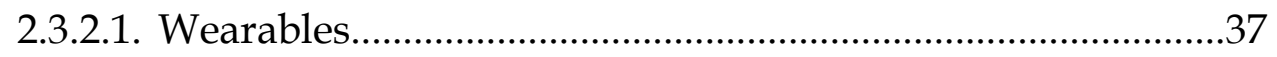

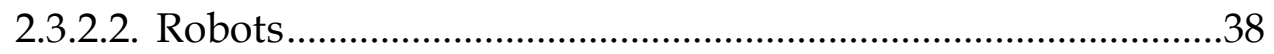

2.4. Cognitive Comparison Studies ....................................................................39

2.5. Virtual Reality Locomotion in Applications for Autism ................................41

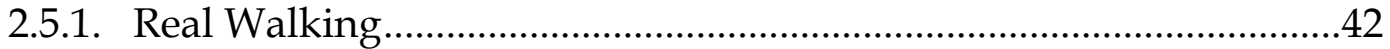

2.5.2. Standard Controller ...........................................................................4 
CHAPTER 3: VIRTUAL REALITY FOR VOCATIONAL REHABILITATION OF INDIVIDUALS WITH SEVERE DISABILITIES SYSTEM:

LOCOMOTION, INTERACTION AND DISPLAYS

3.1. Note to Reader

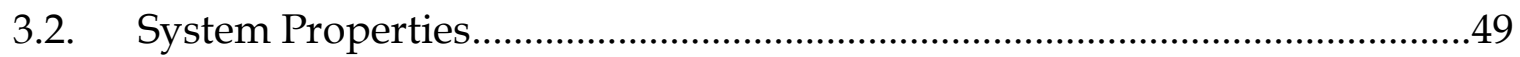

3.3. Locomotion Techniques in the VR4VR System ............................................51

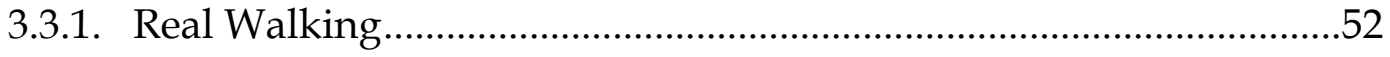

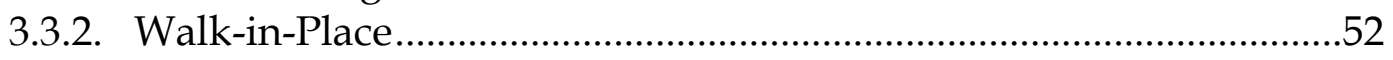

3.4. Object Selection and Manipulation Interaction ............................................55

3.4.1. Tangible Object Manipulation..............................................................56

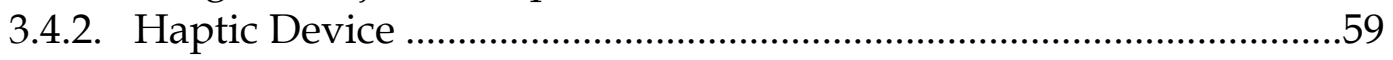

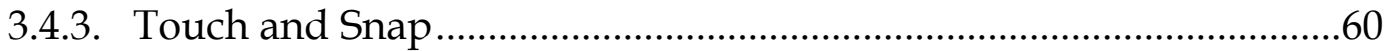

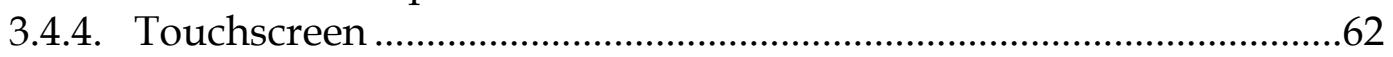

3.5. Display Methods in the VR4VR System …………........................................63

3.5.1. Head Mounted Display …………………............................................64

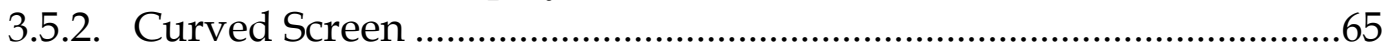

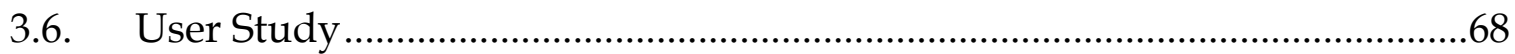

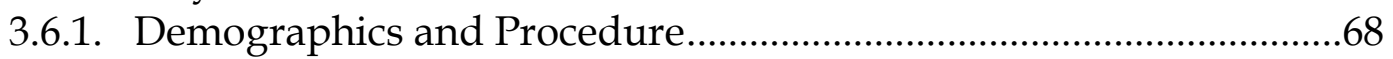

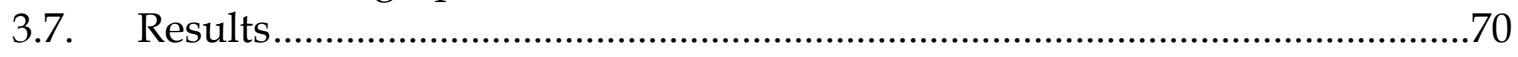

3.7.1. Locomotion Techniques .....................................................................

3.7.2. Object Selection and Manipulation Techniques ..................................73

3.7.3. Display Methods ...............................................................................77

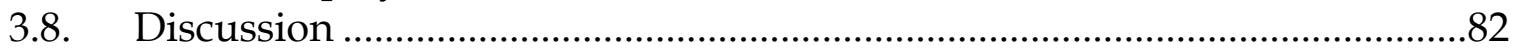

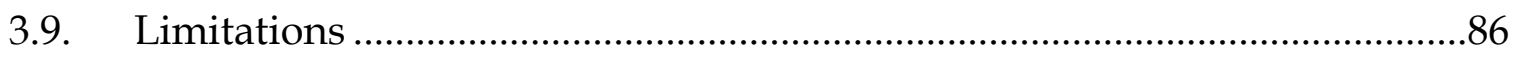

3.10. Summary and Conclusion ..........................................................................86

CHAPTER 4: IMPLEMENTATION OF THE LOCOMOTION TECHNIQUES...............88

4.1. System Hardware and Software ...................................................................8

4.2. General Implementation ......................................................................

4.2.1. Real User ....................................................................................94

4.2.2. Virtual Character..............................................................................98

4.2.3. Position Warnings..............................................................................102

4.2.4. Data Recording..............................................................................102

4.2.5. Virtual Cameras ................................................................................105

4.3. Redirected Walking ………………………..............................................106

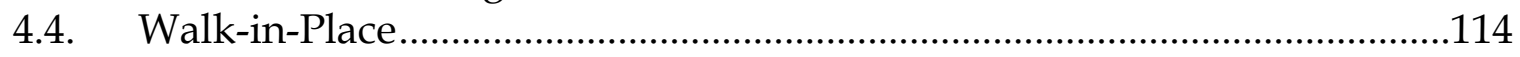

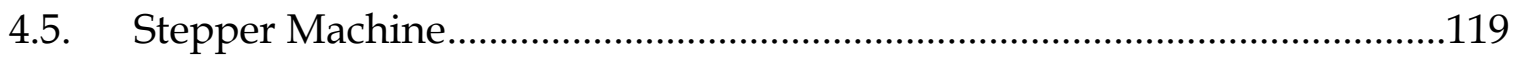

4.6. Point \& Teleport .......................................................................................124

4.6.1. Point \& Teleport with Direction Specification .....................................131

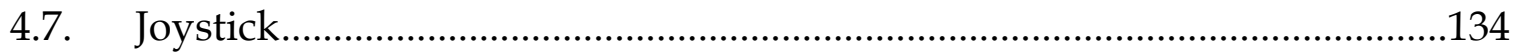

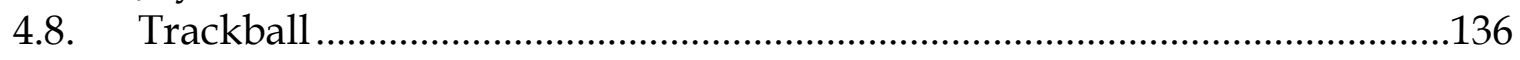

4.9. Hand Flapping...................................................................................138

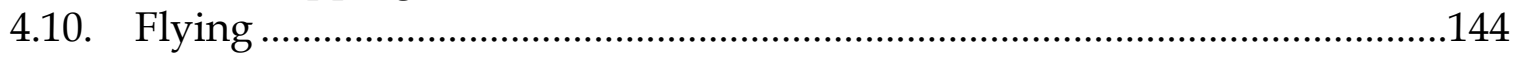




\section{CHAPTER 5: COMPARISON OF EIGHT LOCOMOTION TECHNIQUES}

IN ROOM SCALE TRACKED VIRTUAL REALITY ................................148

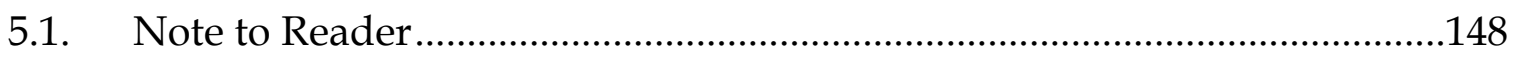

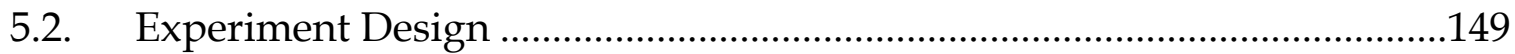

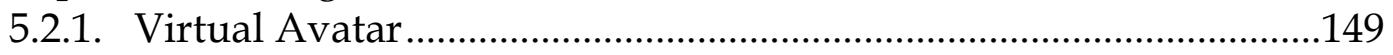

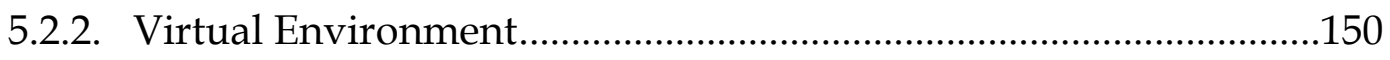

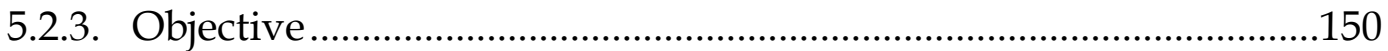

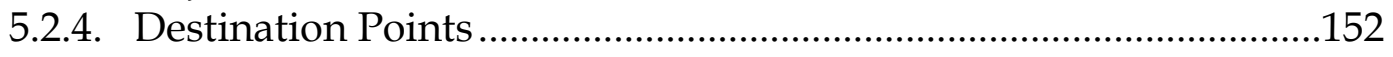

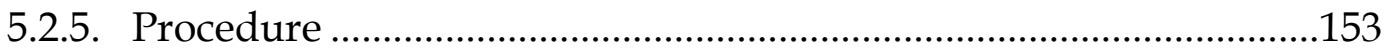

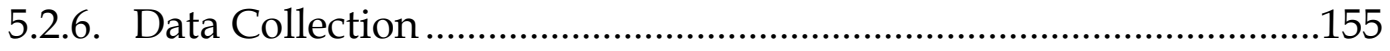

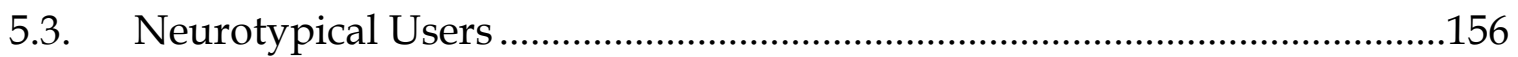

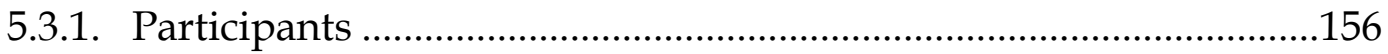

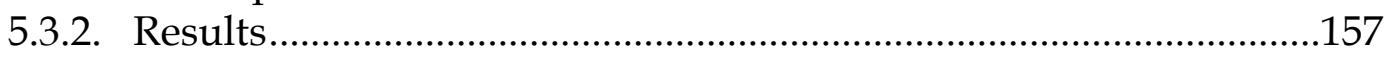

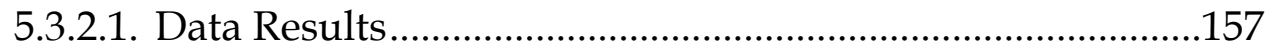

5.3.2.2. Survey Results...................................................................159

5.3.2.3. Participant Comments ...............................................................173

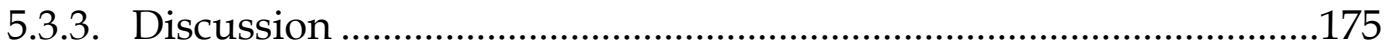

5.3.3.1. Point \& Teleport..................................................................175

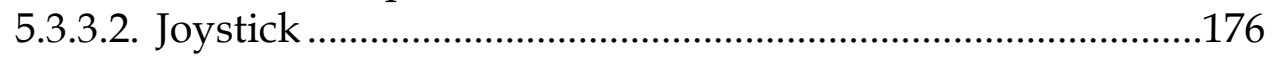

5.3.3.3. Redirected Walking.................................................................177

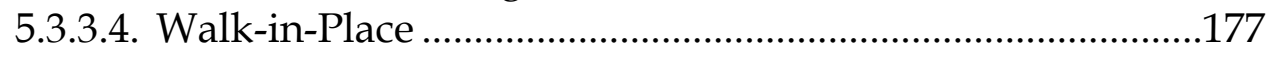

5.3.3.5. The Other Techniques............................................................178

5.3.3.6. Time to Reach the Destination Points and Number

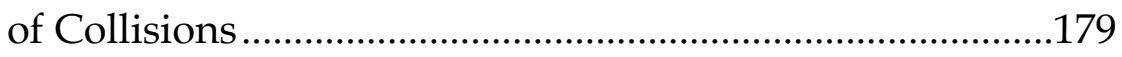

5.3.3.7. Survey Metrics .....................................................................180

5.3.4. Summary, Conclusion and Future Work ..........................................181

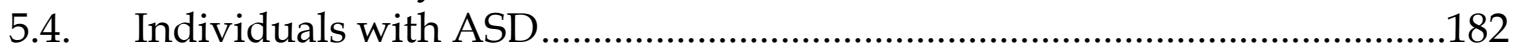

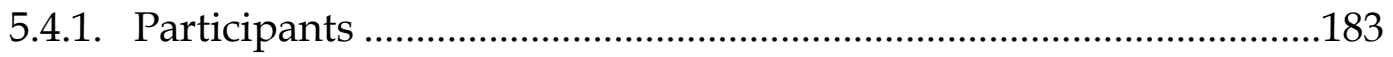

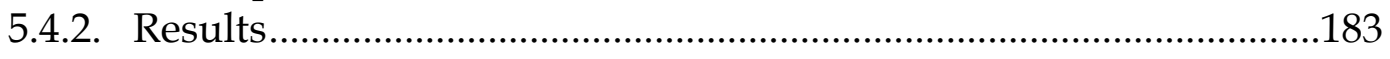

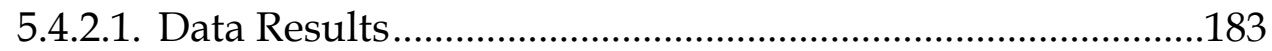

5.4.2.2. Survey Results........................................................................185

5.4.2.3. Participant Comments .........................................................199

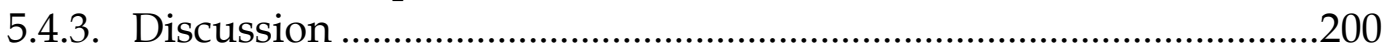

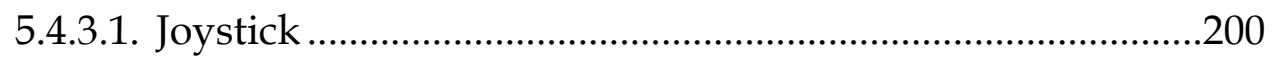

5.4.3.2. Point \& Teleport....................................................................201

5.4.3.3. Redirected Walking.............................................................202

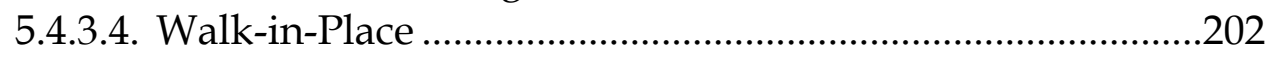

5.4.3.5. The Other Techniques............................................................203

5.4.3.6. Time to Reach the Destination Points and Number

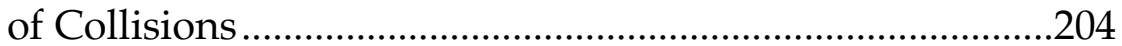

5.4.3.7. Survey Metrics ........................................................................205

5.4.4. Summary, Conclusion and Future Work .......................................206

5.5. Comparison of Neurotypical Individuals and Individuals with ASD......206 
5.6. Limitations .210

CHAPTER 6: DIRECTION SPECIFICATION MODIFICATION OF

THE POINT \& TELEPORT LOCOMOTION TECHNIQUE .212

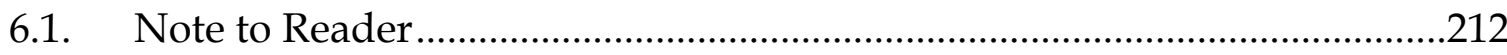

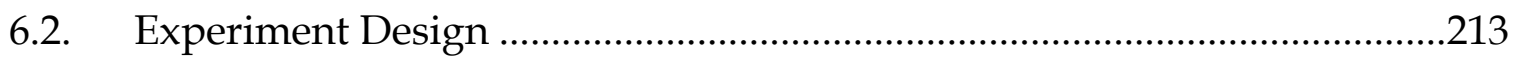

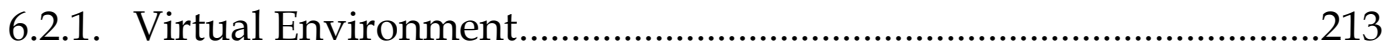

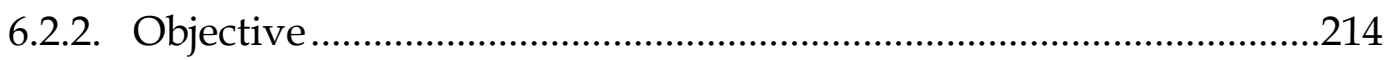

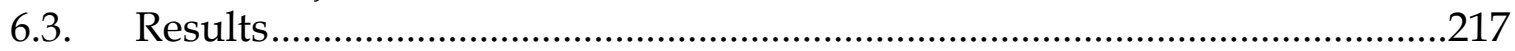

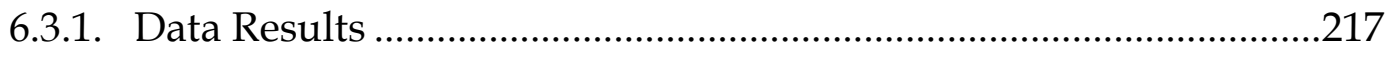

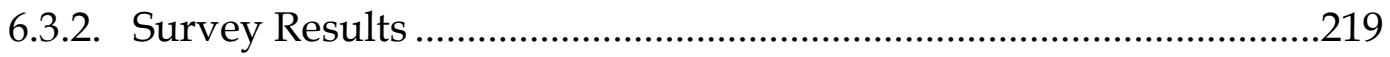

6.3.3. Participant Comments......................................................................222

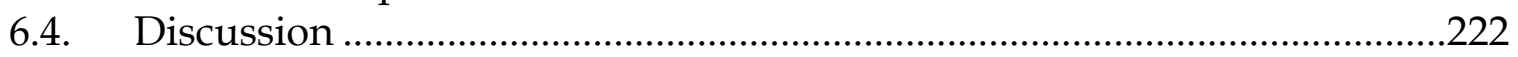

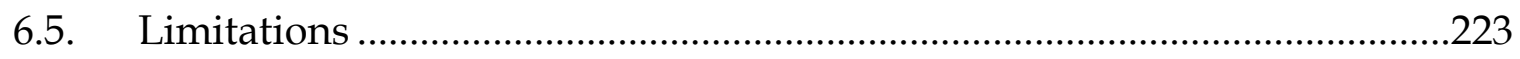

6.6. Summary, Conclusion and Future Work ………………………………....224

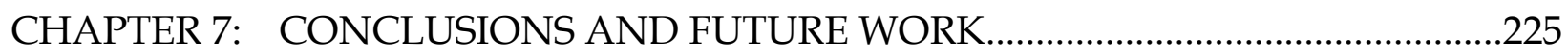

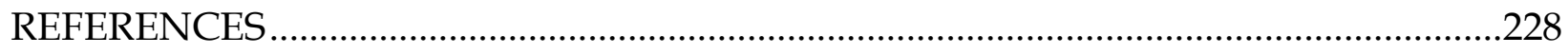

APPENDIX A: SUMMARY OF THE STATE-OF-THE-ART PREVIOUS STUDIES.........242

APPENDIX B: SUMMARY OF THE VIRTUAL REALITY APPLICATIONS FOR INDIVIDUALS WITH AUTISM ........................................................24

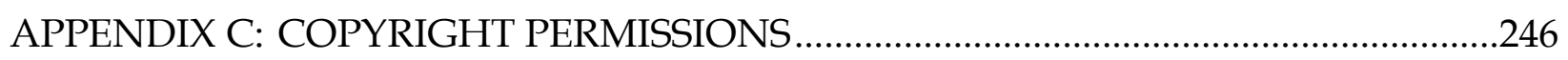

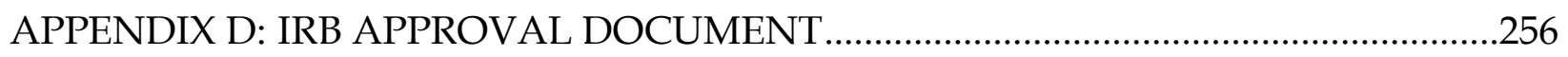

APPENDIX E: DATA ANALYSIS OF THE VR4VR EXPERIMENT .................................258

APPENDIX F: SURVEYS THAT WERE USED IN THE USER STUDIES .........................273

APPENDIX G: DATA ANALYSIS OF THE LOCOMOTION TECHNIQUES EXPERIMENT FOR NEUROTYPICAL INDIVIDUALS AND INDIVIDUALS WITH ASD.

APPENDIX H: DETAILED COMPARISON ANALYSIS BETWEEN NEUROTYPICAL INDIVIDUALS AND INDIVIDUALS WITH ASD FOR THE LOCOMOTION TECHNIQUES EXPERIMENT .336

APPENDIX I: DATA ANALYSIS OF THE POINT \& TELEPORT DIRECTION SPECIFICATION EXPERIMENT. 


\section{LIST OF TABLES}

Table 3-1: The interaction and locomotion techniques and the displays in the VR4VR system

Table 4-1: $\quad$ Real and virtual position and forward direction variables.

Table 5-1: $\quad$ Paired t-test results for the largest and the smallest significant mean differences for average destination clearance times for the neurotypical users

Table 5-2: One-way ANOVA results for the user experience survey for the neurotypical users

Table 5-3: $\quad$ Paired t-test results for the largest and the smallest significant mean differences for the user experience survey for the neurotypical users.

Table 5-4: $\quad$ Paired t-test results for the largest and the smallest significant mean differences for the presence results for the neurotypical users

Table 5-5: One-way ANOVA results the user experience survey for the users with ASD.

Table 5-6: $\quad$ Paired t-test results for the largest and the smallest significant mean differences for the user experience survey for the users with ASD

Table 5-7: Locomotion technique recommendations for neurotypical individuals and individuals with ASD

Table 6-1: T-test results for the survey data of the direction specification modification of the point \& teleport experiment

Table A-1: A summary of the key studies with generalized properties .242

Table B-1: A summary of virtual reality applications for individuals with autism, with properties regarding locomotion. 


\section{LIST OF FIGURES}

Figure 2-1: Taxonomy for locomotion technique studies ..............................................16

Figure 3-1: VR4VR system's sketch ............................................................................

Figure 3-2: Picture of the real walking locomotion technique in the VR4VR system

Figure 3-3: Walk-in-place algorithm's flowchart

Figure 3-4: Pictures of the walk-in-place locomotion technique in the VR4VR system .55

Figure 3-5: Tangible box manipulation in the VR4VR system .58

Figure 3-6: Tangible stick manipulation in the VR4VR system .59

Figure 3-7: The haptic device interaction in the VR4VR system.....................................60

Figure 3-8: The touch and snap interaction in the VR4VR system ................................61

Figure 3-9: The touchscreen interaction in the VR4VR system .......................................63

Figure 3-10: Head mounted display in the VR4VR system ................................................65

Figure 3-11: Curtain display in the VR4VR system ......................................................66

Figure 3-12: Survey results for the locomotion techniques in the VR4VR system for neurotypical individuals .71

Figure 3-13: Survey results for the locomotion techniques in the VR4VR system for individuals with ASD .72

Figure 3-14: Performance scores for different locomotion techniques in the VR4VR system for both population groups.

Figure 3-15: Survey results for the selection and manipulation techniques in the VR4VR system for neurotypical individuals. 
Figure 3-16: Survey results for the selection and manipulation techniques in the VR4VR system for individuals with ASD.

Figure 3-17: Performance scores for different selection and manipulation techniques in the VR4VR system for both population groups. .75

Figure 3-18: Preference of the users on the selection and manipulation techniques in the VR4VR system .76

Figure 3-19: Survey results for the display methods in the VR4VR system for neurotypical individuals .78

Figure 3-20: Survey results for the display methods in the VR4VR system for individuals with ASD.

Figure 3-21: Performance scores for the different display methods in the VR4VR system for both populations

Figure 3-22: Preference of the users on the display methods in the VR4VR system

Figure 4-1: An infrared camera used in the motion tracking system

Figure 4-2: Head mounted display that was used in the user studies .90

Figure 4-3: Hand and foot markers that were used in the user studies...........................90

Figure 4-4: Fictional area for the real user and the total virtual environment...... .91

Figure 4-5: Real user position readings and the estimated reference positions and direction .95

Figure 4-6: Code excerpt from the real user implementation .97

Figure 4-7: Virtual hands and feet in the virtual world

Figure 4-8: Code excerpt from the virtual character implementation .100

Figure 4-9: Real and calculated virtual character positions. .101

Figure 4-10: Real vs. virtual character movements .101

Figure 4-11: The virtual position warning. .102

Figure 4-12: A sample output of the program .104 
Figure 4-13: A sample position data.

Figure 4-14: Marker positions for the redirected walking locomotion technique

Figure 4-15: Vectors to calculate the translational gain....................................................108

Figure 4-16: Vectors to calculate the rotational gain.......................................................110

Figure 4-17: Code excerpt from the redirected walking locomotion technique implementation

Figure 4-18: Virtual obstacle for redirecting the user

Figure 4-19: Photo of a testing session with the redirected walking locomotion technique

Figure 4-20: Redirected walking locomotion technique descriptive reference icon

Figure 4-21: Marker positions for the walk-in-place locomotion technique .114

Figure 4-22: Walk-in-place locomotion technique implementation flowchart .115

Figure 4-23: Code excerpt from the walk-in-place locomotion technique implementation

Figure 4-24: Photo of a testing session with the walk-in-place locomotion technique .118

Figure 4-25: Walk-in-place locomotion technique descriptive reference icon .118

Figure 4-26: Marker positions for the stepper machine locomotion technique

Figure 4-27: The stepper machine, the marker attachment and the holding stage

Figure 4-28: Head rotation vs. auto virtual rotation speed chart.

Figure 4-29: Code excerpt from the stepper machine locomotion technique implementation

Figure 4-30: Photo of a testing session with the stepper machine locomotion technique...... 
Figure 4-31: Stepper machine locomotion technique descriptive reference icon

Figure 4-32: Marker positions for the point \& teleport technique

Figure 4-33: Illustration of the (a) point \& teleport and (b) point \& teleport with direction specification locomotion techniques

Figure 4-34: Code excerpt from the point \& teleport locomotion technique implementation

Figure 4-35: Photo of a testing session with the point \& teleport locomotion technique

Figure 4-36: Point \& teleport locomotion technique descriptive reference icon

Figure 4-37: Vectors to control the direction of the arrow in the point \& teleport with direction specification

Figure 4-38: Hand roll vs. virtual arrow rotation graph for point \& teleport with direction specification

Figure 4-39: Additional code excerpt from the point \& teleport with direction specification locomotion technique implementation

Figure 4-40: Marker positions for the joystick locomotion technique.

Figure 4-41: Photo of a testing session with the joystick locomotion technique

Figure 4-42: Joystick locomotion technique descriptive reference icon .135

Figure 4-43: Marker positions for the trackball locomotion technique...... .136

Figure 4-44: Photo of a testing session with the trackball locomotion technique .137

Figure 4-45: Trackball locomotion technique descriptive reference icon .138

Figure 4-46: Marker positions for the hand flapping locomotion technique

Figure 4-47: Vectors forming a single flap for the hand flapping locomotion technique 
Figure 4-48: Consecutive flaps forming a flapping motion

Figure 4-49: Code excerpt from the hand flapping locomotion technique implementation

Figure 4-50: Photo of a testing session with the hand flapping locomotion technique

Figure 4-51: Hand flapping locomotion technique descriptive reference icon

Figure 4-52: Marker positions for the flying technique.

Figure 4-53: Code excerpt from the flying locomotion technique implementation

Figure 4-54: Photo of a testing session with the flying locomotion technique

Figure 4-55: Flying locomotion technique descriptive reference icon 147

Figure 5-1: Cylindrical roman pillars as static obstacles

Figure 5-2: Destination point in virtual environment

Figure 5-3: Block diagram for the experiment of the comparison of the eight locomotion techniques

Figure 5-4: Sorting chart for the participants with ASD

Figure 5-5: Average time to reach to the destination points without and with obstacles for the neurotypical users with the eight locomotion techniques

Figure 5-6: Average number of collisions with static obstacles for the neurotypical users.

Figure 5-7: Survey results for the eight locomotion techniques for the neurotypical users

Figure 5-8: Average motion sickness and presence scores for the neurotypical users

Figure 5-9: Weighted averages of the preference ranking scores for the neurotypical users 
Figure 5-10: Redirected walking locomotion technique projected paths for the neurotypical users

Figure 5-11: Walk-in-place locomotion technique projected paths for the neurotypical users.

Figure 5-12: Stepper machine locomotion technique projected paths for the neurotypical users

Figure 5-13: Point \& teleport locomotion technique projected paths for the neurotypical users.

Figure 5-14: Joystick locomotion technique projected paths for the neurotypical users.

Figure 5-15: Trackball locomotion technique projected paths for the neurotypical users

Figure 5-16: Hand flapping locomotion technique projected paths for the neurotypical users

Figure 5-17: Flying locomotion technique projected paths for the neurotypical users

Figure 5-18: Average time to reach to the destination points without and with obstacles for the users with ASD with the eight locomotion techniques

Figure 5-19: Average number of collisions with static obstacles for the users with ASD

Figure 5-20: Survey results for the eight locomotion techniques for the users with ASD

Figure 5-21: Average motion sickness and presence scores for the users with ASD

Figure 5-22: Weighted averages of the preference ranking data for all locomotion techniques

Figure 5-23: Redirected walking locomotion technique projected paths for the users with ASD

Figure 5-24: Walk-in-place locomotion technique projected paths for the users with ASD. 
Figure 5-25: Stepper machine locomotion technique projected paths for the users with ASD.

Figure 5-26: Point \& teleport locomotion technique projected paths for the users with ASD.

Figure 5-27: Joystick locomotion technique projected paths for the users with ASD

Figure 5-28: Trackball locomotion technique projected paths for the users with ASD

Figure 5-29: Hand flapping locomotion technique projected paths for the users with ASD.

Figure 5-30: Flying locomotion technique projected paths for the users with ASD

Figure 6-1: The virtual maze environment

Figure 6-2: Block design of the direction specification modification of the point \& teleport experiment

Figure 6-3: Photo of a testing session with the point \& teleport locomotion technique in the virtual maze.

Figure 6-4: Photo of a testing session with the point \& teleport with direction specification locomotion technique in the virtual maze. .216

Figure 6-5: Average time to reach the destination points in the direction specification modification of the point \& teleport experiment

Figure 6-6: Average number of collisions with maze walls in the direction specification modification of the point \& teleport experiment

Figure 6-7: Survey results for point \& teleport and point \& teleport with direction specification locomotion techniques

Figure 6-8: Average user preference score results for point \& teleport and point \& teleport with direction specification locomotion techniques

Figure 6-9: Point \& teleport technique's projected virtual (left) and real (right) user paths for the direction specification modification of the point \& teleport experiment. 
Figure 6-10: Point \& teleport with direction specification technique projected virtual (left) and real (right) user paths for the direction specification modification of the point \& teleport experiment

Figure E-1: Paired samples t-test results of VR4VR locomotion techniques for neurotypical individuals .258

Figure E-2: Paired samples t-test results of VR4VR locomotion techniques for individuals with ASD. .258

Figure E-3: Pairwise comparison factors for the data analysis of selection and manipulation techniques .258

Figure E-4: Ease of interaction detailed data analysis for VR4VR's object selection and manipulation techniques for neurotypical individuals

Figure E-5: Ease of interaction detailed data analysis for VR4VR's object selection and manipulation techniques for individuals with ASD

Figure E-6: Enjoyment detailed data analysis for VR4VR's object selection and manipulation techniques for neurotypical individuals

Figure E-7: Enjoyment detailed data analysis for VR4VR's object selection and manipulation techniques for individuals with ASD

Figure E-8: Frustration detailed data analysis for VR4VR's object selection and manipulation techniques for neurotypical individuals .263

Figure E-9: Frustration detailed data analysis for VR4VR's object selection and manipulation techniques for individuals with ASD

Figure E-10: Tiredness detailed data analysis for VR4VR's object selection and manipulation techniques for neurotypical individuals.

Figure E-11: Tiredness detailed data analysis for VR4VR's object selection and manipulation techniques for individuals with ASD

Figure E-12: Score detailed data analysis for VR4VR's object selection and manipulation techniques for neurotypical individuals

Figure E-13: Score detailed data analysis for VR4VR's object selection and manipulation techniques for individuals with ASD 
Figure E-14: Presence detailed data analysis for VR4VR's object selection and manipulation techniques for neurotypical individuals.

Figure E-15: Presence detailed data analysis for VR4VR's object selection and manipulation techniques for individuals with ASD.

Figure E-16: Detailed data analysis of VR4VR's display methods for neurotypical individuals

Figure E-17: Detailed data analysis of VR4VR's display methods for individuals with ASD

Figure E-18: Detailed score analysis between neurotypical individuals and individuals with ASD

Figure F-1: A sample survey that was used in the user studies about a locomotion technique for neurotypical individuals

Figure F-2: A sample survey that was used in the user studies about the stepper machine locomotion technique for individuals with ASD

Figure G-1: Pairwise comparison factors for the data analysis of the eight virtual reality locomotion techniques

Figure G-2: Detailed data analysis for the completion time without obstacles for neurotypical individuals

Figure G-3: Detailed data analysis for the completion time without obstacles for individuals with ASD

Figure G-4: Detailed data analysis for the completion time with obstacles for neurotypical individuals

Figure G-5: Detailed data analysis for the completion time with obstacles for individuals with ASD

Figure G-6: Detailed data analysis for the number of collisions for neurotypical individuals

Figure G-7: Detailed data analysis for the number of collisions for individuals with ASD

Figure G-8: Detailed data analysis for the difficulty in understanding for neurotypical individuals 
Figure G-9: Detailed data analysis for the difficulty in understanding for individuals with ASD

Figure G-10: Detailed data analysis for the difficulty in operating for neurotypical individuals

Figure G-11: Detailed data analysis for the difficulty in operating for individuals with ASD .298

Figure G-12: Detailed data analysis for the feeling of being in control for neurotypical individuals

Figure G-13: Detailed data analysis for the feeling of being in control for individuals with ASD

Figure G-14: Detailed data analysis for the enjoyment for neurotypical individuals

Figure G-15: Detailed data analysis for the enjoyment for individuals with ASD .306

Figure G-16: Detailed data analysis for the required effort for neurotypical individuals .308

Figure G-17: Detailed data analysis for the required effort for individuals with ASD

Figure G-18: Detailed data analysis for the tiredness for neurotypical individuals

Figure G-19: Detailed data analysis for the tiredness for individuals with ASD

Figure G-20: Detailed data analysis for the overwhelmedness for neurotypical individuals

Figure G-21: Detailed data analysis for the overwhelmedness for individuals with ASD

Figure G-22: Detailed data analysis for the frustration for neurotypical individuals

Figure G-23: Detailed data analysis for the frustration for individuals with ASD 
Figure G-24: Detailed data analysis for the motion sickness for neurotypical individuals

Figure G-25: Detailed data analysis for the motion sickness for individuals with ASD

Figure G-26: Detailed data analysis for the presence for neurotypical individuals .328

Figure G-27: Detailed data analysis for the presence for individuals with ASD .330

Figure G-28: Detailed data analysis for the preference ranking scores for neurotypical individuals

Figure G-29: Detailed data analysis for the preference ranking scores for individuals with ASD

Figure $\mathrm{H}-1$ : Detailed comparison analysis for the completion time without obstacles for neurotypical individuals and individuals with ASD

Figure H-2: Detailed comparison analysis for the completion time with obstacles for neurotypical individuals and individuals with ASD .338

Figure H-3: Detailed comparison analysis for the number of collisions for neurotypical individuals and individuals with ASD

Figure $\mathrm{H}-4$ : Detailed comparison analysis for the difficulty in understanding for neurotypical individuals and individuals with ASD .340

Figure $\mathrm{H}-5$ : Detailed comparison analysis for the difficulty in operating for neurotypical individuals and individuals with ASD

Figure H-6: Detailed comparison analysis for the feeling of being in control for neurotypical individuals and individuals with ASD

Figure H-7: Detailed comparison analysis for the enjoyment for neurotypical individuals and individuals with ASD.

Figure H-8: Detailed comparison analysis for the required effort for neurotypical individuals and individuals with ASD

Figure H-9: Detailed comparison analysis for the tiredness for neurotypical individuals and individuals with ASD 
Figure H-10: Detailed comparison analysis for the overwhelmedness for neurotypical individuals and individuals with ASD

Figure H-11: Detailed comparison analysis for the frustration for neurotypical individuals and individuals with ASD

Figure H-12: Detailed comparison analysis for the motion sickness for neurotypical individuals and individuals with ASD

Figure H-13: Detailed comparison analysis for the presence for neurotypical individuals and individuals with ASD

Figure H-14: Detailed comparison analysis for the preference ranking scores for neurotypical individuals and individuals with ASD

Figure I-1: Paired samples t-test for the completion time for neurotypical individuals for the point \& teleport direction specification experiment

Figure I-2: Paired samples t-test for the number of collision with maze walls for neurotypical individuals for the point \& teleport direction specification experiment

Figure I-3: Paired samples t-test for the difficulty in understanding for neurotypical individuals for the point \& teleport direction specification experiment

Figure I-4: Paired samples t-test for the difficulty in operating for neurotypical individuals for the point \& teleport direction specification experiment

Figure I-5: Paired samples t-test for the feeling of being in control for neurotypical individuals for the point \& teleport direction specification experiment

Figure I-6: Paired samples t-test for the enjoyment for neurotypical individuals for the point \& teleport direction specification experiment

Figure I-7: Paired samples t-test for the required effort for neurotypical individuals for the point \& teleport direction specification experiment 
Figure I-8: Paired samples t-test for the tiredness for neurotypical individuals for the point \& teleport direction specification experiment

Figure I-9: Paired samples t-test for the overwhelmedness for neurotypical individuals for the point \& teleport direction specification experiment

Figure I-10: Paired samples t-test for the frustration for neurotypical individuals for the point \& teleport direction specification experiment

Figure I-11: Paired samples t-test for the motion sickness for neurotypical individuals for the point \& teleport direction specification experiment

Figure I-12: Paired samples t-test for the presence for neurotypical individuals for the point \& teleport direction specification experiment

Figure I-13: Paired samples t-test for the preference ranking scores for neurotypical individuals for the point \& teleport direction specification experiment 


\begin{abstract}
In the recent years, virtual reality has been used as an effective tool for a wide range of areas such as training, rehabilitation, education and games. The affordability of the new generation headsets helped this medium to become more widespread. However, in order for virtual reality to become mainstream, more content that is specifically designed for this medium is needed. Since virtual reality is a different technology than the computer systems, different design principles may be required for these content for better user experience. One of the crucial components of virtual reality applications is locomotion, since the viewpoint of the user is very important in immersing the users into virtual reality and locomotion is used for moving the viewpoint of user in virtual environments. Locomotion in virtual reality is expected to have a direct effect on user experience in terms of many elements such as effort, enjoyment, frustration, motion sickness and presence. Up to date, many locomotion techniques for virtual reality have been studied in the literature. However, many of these techniques were evaluated in large tracked areas. Although professional motion tracking systems can track large areas, today's new generation affordable commercial virtual reality systems can only track room scale environments. This dissertation aims at evaluating different locomotion techniques in room scale tracked areas for neurotypical individuals and individuals with ASD. Several previous studies concurred that virtual reality is an effective medium for
\end{abstract}


the training and rehabilitation of individuals with ASD. However, no previous study evaluated locomotion in virtual reality for this specific population. Thus, this dissertation aims at finding out the suitable virtual reality locomotion techniques for individuals with ASD.

With these motivations, in this dissertation, locomotion techniques for room scale virtual reality were evaluated under three experiments: virtual reality for vocational rehabilitation system, evaluation of eight virtual reality locomotion techniques, and point \& teleport direction specification experiment.

In the first experiment of virtual reality for vocational rehabilitation system, locomotion, interaction, and display components in an immersive virtual reality system for vocational rehabilitation was evaluated by 10 neurotypical individuals and 9 individuals with high functioning ASD. The results indicated that neurotypical individuals favored real walking over walk-in-place; tangible interaction over haptic device, touch \& snap and touch screen; and head mounted display over curtain screen. For the participants with high functioning ASD, real walking was favored over walk-inplace; touch screen was favored over haptic device, tangible interaction and touch \& snap; and curtain screen was favored over head mounted display.

In the second experiment, eight virtual reality locomotion techniques were evaluated in a room scale tracked area $(2 \mathrm{~m}$ by $2 \mathrm{~m})$. These eight locomotion techniques were: redirected walking, walk-in-place, stepper machine, point \& teleport, joystick, trackball, hand flapping and flying. Among these locomotion techniques, the three were commonly used in virtual reality (redirected walking, walk-in-place and joystick), the 
two were unexplored -explored previously only by a few related studies (stepper machine and point \& teleport), and the three were selected and/or modified for individuals with ASD based on their common characteristics (trackball, hand flapping and flying). These eight techniques were evaluated in an immersive virtual reality test environment. A user study was performed with 16 neurotypical participants and 15 participants with high functioning ASD. The results indicated that for neurotypical individuals, point \& teleport, joystick and redirected walking were suitable virtual reality locomotion techniques for room scale tracked areas whereas hand flapping and flying were not suitable. For individuals with high functioning ASD, point \& teleport, joystick and walk-in-place were suitable virtual reality locomotion techniques for room scale tracked areas whereas hand flapping and flying were not suitable.

Locomotion techniques that are similar to point \& teleport have been starting to be used in commercial video games, however were not evaluated in the literature. For this reason, a separate experiment was performed as the third experiment to investigate the effects of an additional direction specification component of point \& teleport. Since this direction specification component exerted an additional cognitive load into the use of the same technique, which was recommended to be avoided for individuals with ASD in the literature, it was only evaluated by neurotypical individuals. An immersive virtual maze environment was developed and a user study was performed with 16 neurotypical users. The results indicated that the additional direction specification feature did not improve the user experience. 


\section{CHAPTER 1: INTRODUCTION}

Virtual reality has become more affordable and widely used in the recent years than ever. Besides the various applications for neurotypical individuals such as video games and educational experiences; virtual reality is considered as a promising area for individuals with autism since it offers realistic experiences that can be used for training and rehabilitation. Virtual reality applications have many aspects such as hardware, interaction and locomotion. Locomotion is an important component of video games since it can have a strong influence on user experience. A good virtual reality experience needs to be designed carefully, considering the needs of the targeted audience. Individuals with autism are described to have deficits and strengths attributed to their condition. This chapter presents general information about autism spectrum disorder, virtual reality applications, locomotion, motivation and contributions, and the organization of this dissertation.

\subsection{Autism Spectrum Disorder}

Autism spectrum disorder (ASD) is described as a neurodevelopmental disorder with persistent deficits of social skills, communication, cognitive processing, languagerelated abilities, executive functioning, adapting to changes, and increased repetitive behaviors or constant interests [1]. Autism is reported to be prevalent in $1 \%$ of the world population. Since autism is a disorder that is spread on a spectrum, individuals with ASD 
cannot be characterized by strict characteristics. There is no currently known treatment to autism; however supportive training and intervention programs are known to provide improvement in the skills of individuals with ASD that are needed to live more typical lives [2].

\subsection{Virtual Reality Applications}

Although virtual reality (VR) has been present for decades, in the recent years it has become more accessible and popular for many uses. Entertainment and training are the two main areas in which virtual reality applications are used. Many immersive virtual environments combine head mounted displays and motion tracking devices to immerse users in visual experiences that change consistently with their body motion [3]. Head mounted displays improved significantly -both in capability and cost- since they were first introduced in 1968 [4]. Similar advances in the tracking technology have also emerged. Now, relatively inexpensive, commodity tools, like the Oculus Rift [5] and Microsoft Kinect [6], allow the general public to experience and use immersive virtual reality in their homes. With virtual reality systems, users can experience different places and scenarios than their actual physical environment. This has many advantages such as; time and expense savings because of the reduced transportation costs to different work places, and safety that comes with the training in a controlled environment. Furthermore, virtual reality systems can even help people experience situations that would be difficult or impossible to access otherwise, such as war zones, underwater deep spaces, and the center of a tornado. These capabilities enable VR systems to enhance training, architecture, entertainment, and many other areas. [7]. Among these training areas, using 
virtual reality for training individuals with autism has been getting attention recently [8$11]$.

Individuals with autism are known to have strong interests in computer technologies $[12,13]$. In particular, virtual reality training systems have several properties that resonates with the characteristics of individuals with autism. These properties include: interactivity; real time feedback; practicing potentially dangerous situations in safe, simulated encounters; the ability to layer on more complexity; the predictability of the virtual environments; customization; and being primarily visual.

\subsection{Locomotion}

Effective virtual reality systems often require several characteristics that are designed harmoniously, such as interaction, locomotion, audio, visual and task design. Each of these components is important in themselves for a good virtual reality experience. Specifically, locomotion is among the most common and important tasks within 3D virtual reality systems [14]. Locomotion is defined as travel that is restricted to selfpropulsion [15]. Locomotion is required to move to an aimed location in virtual world. In immersive virtual environments with head-tracking, the virtual viewpoint can be adjusted via moving and rotating the head while walking on the tracked area. Immersive virtual environments that use head-mounted displays are usually suitable to be explored on foot. But in virtual reality, it is usually difficult to maintain spatial knowledge in the virtual environment [16]. Furthermore, locomotion methods likely contributed to user motion sickness, since they directly control viewpoint. 
In a virtual environment, the walkable space may have infinite size and the user should be able to walk and explore that space freely. However, in real physical places, users have limited space. If the virtual space and the real space have similar sizes, then a 1:1 mapping can be used for navigation. But if the virtual area is larger than the actual area, users may eventually go outside the real tracking space. This interrupts the tracking and makes a negative impact on the presence and user experience. To solve this problem, numerous different locomotion techniques have been proposed by the academic community so far.

Locomotion usually is not the main goal of most immersive virtual reality systems. These systems usually have the aim of training, giving therapies or entertaining the users while the users move around in the virtual environment. Nonetheless, a wrong selection of locomotion technique or a poorly implemented one may distract the user, which may result in less benefits gained from the system. The users may not even want to continue using the system due to motion sickness or frustration.

\subsection{Motivation and Contributions of the Dissertation}

As with many other components of virtual reality, locomotion has several attributes that need to be selected carefully according to the context, goals, and audience of the system. For neurotypical users, a general input system is recommended to be hands-free and eyes free in order to let the user perform main tasks easily [17]. Similarly, a good locomotion technique is recommended not use the hands or visual focus of the user unless the main purpose of the system is navigation. Techniques taking advantage of proprioception are favored since they are suggested to provide more presence. 
Proprioception is defined as a person's sense of where their body parts are. As an example, a driver can change the gears of a car without looking, because of proprioception [15]. However, most of the previous studies in the literature investigated locomotion in large tracked areas. Today's commercial virtual reality systems can only track room scale environments and no extensive literature exists on the implications of virtual reality locomotion techniques for room scale tracked areas. On the other hand, all of the previous studies were performed with neurotypical populations, not with individuals with autism. Although there has been extensive research on locomotion techniques for neurotypical populations, no research on VR locomotion has been done for individuals with autism. Even in virtual reality studies that involved individuals with autism, no study looked into how these techniques were performed or what the preferences of the users were. Most of these studies for training individuals with ASD employed standard controllers such as keyboard and joystick. Studies using CAVE - like projection based immersive environments allowed for real walking of the users only in a limited area and did not provide virtual navigation beyond this physical limit. The common tendencies and deficits of people with autism may not be compatible with the commonly used locomotion techniques for typical individuals. Thus, it is important to study the understanding and the experience of this specific population with the existing commonly used locomotion techniques. New locomotion techniques exploiting the strengths and deficits of people with autism may also be developed and evaluated. 
With these motivations, our research questions were as follows:

- What are the implications of the state of the art VR locomotion techniques for room scale tracked environments?

- Which locomotion technique would provide the best user experience for high functioning individuals with ASD in VR?

- What are the implications of the increasingly utilized and recently popular VR point \& teleport technique?

- Can point \& teleport technique be improved with an additional direction specification feature?

Based on all these motivations and research questions, the contributions of this dissertation can be summarized as follows:

- An extensive literature review on the state-of-the-art virtual reality locomotion techniques.

- Implementation of locomotion and interaction techniques and display preferences in an advanced immersive virtual reality tool for vocational rehabilitation of individuals with disabilities, and evaluation with a user study of 10 neurotypical individuals and 9 individuals with high functioning ASD.

- Implications of the user study results for locomotion, interaction and displays in room scale virtual reality systems for neurotypical individuals and individuals with high functioning ASD. 
- Design and implementation of eight locomotion techniques in a room scale tracked immersive virtual reality experiment: three commonly used techniques (redirected walking, walk-in-place and joystick); two unexplored techniques (stepper machine and point \& teleport); and three ASD aimed techniques (trackball controller, hand flapping and flying), and evaluation with a user study of 16 neurotypical individuals and 15 individuals with high functioning ASD.

- Implications of the user study results on the comparison of eight locomotion techniques in room scale virtual reality systems for neurotypical individuals and individuals with high functioning ASD.

- Design and implementation of a modified version of point \& teleport with direction specification in an immersive room scale tracked virtual maze environment, and evaluation with a user study of 16 neurotypical individuals.

- Implications of the results for the use of point \& teleport -a technique that is being used commercial virtual reality games but has not been explored in the literature- in room scale virtual reality systems for neurotypical individuals.

- Detailed information about the implementation of the eight virtual reality locomotion techniques and the direction specification modification of point \& teleport. 


\subsection{Organization of the Dissertation}

The remainder of this dissertation is organized as described as mentioned below: Chapter 2 presents previous studies about the virtual reality locomotion techniques and systems for neurotypical individuals and individuals with ASD. Chapter 3 discusses the first experiment of virtual reality for vocational rehabilitation of individuals with disabilities system, with a focus on locomotion, interaction and display preferences in a user study with both neurotypical individuals and individuals with high functioning ASD. Chapter 4 presents information about the implementation of the locomotion techniques in this dissertation. Chapter 5 includes the second experiment on the evaluation of eight virtual reality locomotion techniques: redirected walking, walk-inplace, stepper machine, point \& teleport, joystick, trackball, hand flapping and flying with a user study with both neurotypical individuals and individuals with high functioning ASD. Chapter 6 discusses the third experiment on evaluating the direction specification feature of the point \& teleport locomotion technique in a user study with neurotypical individuals. Chapter 7 draws conclusions from the results of the user studies in this dissertation and specifies directions for future work. 


\section{CHAPTER 2: BACKGROUND AND RELATED WORK}

Several studies show that the advances in technology have been assisting individuals with ASD for a long time. Goldsmith et al. collected and published the early examples of the technologies that were used for children with ASD [18]. In general, the developed systems used an interaction technique that seemed to be the most suitable by the authors for the application and the selection of that technique was rarely justified since the main goal of these studies was to explore the effectiveness of virtual reality in training individuals with ASD. Interaction techniques among the uppermost important elements of virtual reality since they are directly related to the user's experience with the system. Existing systems support a variety of interaction techniques for different platforms and input devices, from conventional devices such as mice or joysticks, to modern devices such as touch gestures, speech-recognition devices, and digitally augmented environments. Locomotion is an important aspect of virtual reality that can affect user experience significantly in terms of presence, enjoyment, frustration, and tiredness.

In this chapter, a new taxonomy was proposed and used in an extensive survey of state-of-the-art virtual reality locomotion techniques. Virtual reality applications for individuals with autism were shared with a focus on identifying appropriate locomotion 
techniques for these individuals. Major issues and future research considerations were discussed.

\subsection{Terminology and Definitions on Virtual Reality, Interaction and Locomotion}

The important terms that are used in this dissertation are shared in this subsection with their definitions.

- Avatar: a virtual and interactive representation of a person in virtual reality applications [15].

- CAVE: a physical environment with projections on the walls, ceiling and floor [15].

- Desktop virtual systems: virtual reality applications which are displayed on a 2D desktop computer screen so that a user can see through the eyes of the virtual character, but the experience would not be 3D [15].

- Gesture: a meaningful motion that can be categorized as a sign, signal, or symbol, usually made by the hand, foot or body [15].

- Haptic device: an interaction device that enables the users to feel the force feedback when touched a virtual object.

- Head-mounted display (HMD): a visual display that covers the eyes of the user so that the virtual world could be rendered based on the head position and orientation [15].

- Immersion: the experience of being physically within a virtual world that a virtual reality application provides to the users [15]. 
- Locomotion: means of travel that is restricted to the user's self-propulsion [15].

- Motion sickness: disturbances such as nausea, disorientation and tired eyes that are caused by a virtual experience [15].

- Optical motion tracking: A motion tracking system that uses surrounding cameras to track the reflective markers via infrared light.

- Point \& teleport: a locomotion technique in which the user points to where they would like to go and teleported there instantaneously.

- Presence: the feeling of being part of a virtual environment. The level of immersiveness of a virtual reality application is likely to increase the presence [15].

- Proprioceptive: the internal sense of body position and movement even when not seeing [15].

- Real time: actions that take place with no delay [15].

- Redirected Walking: a locomotion technique in which the view of the user is altered based on their movements [19].

- Tactile: sensory information that arises from making contact with objects [15].

- Virtual reality: a model of reality with which users can interact and get sensory information such as sight, sound and touch, may be used interchangeably with virtual environments [15]. 
- Virtual world: the entire virtual environment in a virtual reality application [15].

- Walk-in-place: a locomotion technique in which the user marches in place and is moved in the virtual world according to their head or body directions [20].

- Wayfinding: navigation with cognitive effort [15].

\subsubsection{Interaction Techniques}

Most of the assistive training applications were implemented using a single interaction technique. The recent applications usually used touchscreen devices since they are easy to use, affordable and available. Furthermore, one of the recent studies showed that the tablet applications with multi touch interactions could make children with ASD more verbally and physically engaged as compared to the traditionally performed similar activities [21]. In a study conducted by Madsen et al., the researchers developed touch screen applications for teaching children with ASD to recognize facial expressions [22]. In this study, lessons learned about the software and hardware design of touch screen applications for this specific population were shared very briefly. In a study on developing an expression recognition game for individuals with ASD using touch enabled mobile devices, the research team has studied the previously existing popular ASD games and tried to consolidate some good design practices regarding designing user interfaces for an audience of children with ASD [23].

Another popular approach in designing applications for individuals with ASD is using touchless interactions. The availability of the depth sensors, such as Microsoft 
Kinect and their usage for skeleton tracking made this technique easily usable and popular. Moreover, some researchers suggest not to use wearable sensors since some individuals with ASD may not prefer to wear any sensors on them [24]. A study made on five children with ASD showed that games with touchless interaction helped in improving the attention skills for children with autism. However, the authors stated that the interaction technique was not tested on being appropriate or not for this special user group [25]. Another recent study for individuals with ASD was aiming at improving their motor skills [26]. With this goal, the researchers developed a motion based touchless application and tested the results. This study focused on the importance of physical activity, but did not justify why the authors chose to use this interaction technique while developing the application.

There were also some applications that used more than one interaction technique simultaneously. One study focused on full body interaction techniques for low functioning children with ASD [27]. An environment similar to a virtual reality cave was developed with surrounding projectors, cameras and sensors. Some touchless interaction techniques as well as touch based interaction techniques were implemented, and the children's acceptance of the system was discussed. Most of the children accepted the system and used it effectively.

With the emerging technology of virtual reality, some researchers have been integrating virtual reality interaction techniques into training applications for people with ASD. In a study, researchers utilized a VR system to train children with ASD on street crossing [28]. The results showed that training in virtual reality improved the 
related skills of those children. In another study, a virtual reality driving training system was developed [29]. In this system, gaze tracking was implemented to track where the users looked during the training sessions since individuals with ASDs' gaze positions were reported to be different from neurotypical individuals. The users were trained to look at the important regions such as traffic lights, traffic signs and pedestrians. The results showed that effective training was achieved using the developed virtual reality system with the incorporation of gaze positions.

Although many studies focused on using only one interaction technique per application, there have been some studies in the literature that used two different interaction techniques in the same application or in different applications that were developed for the same purpose for individuals with ASD. One example was a study that aimed at increasing the social engagement of children with ASD [30]. Two different games were used with two different interaction techniques. One was using multiple mice while the other was using a Diamond touch surface. The study did not test the differences observed while using these interaction techniques and did not make any suggestions for researchers. There was a detailed study on a training tool for children with ASD [31]. In the study, a tangible user interface design was compared with the traditional mousebased approach. The results of the study showed more learning progress using the tangible user interface. Another recent study showed observations on the usability of basic 3D interactions such as translation and rotation for the adolescents with ASD [32]. The authors aimed at finding the differences in the usage of 3D user interaction techniques between neurotypical individuals and individuals with autism. The results 
showed that the deficits in hand-eye coordination of individuals with ASD caused some difficulties in using the 3D interaction techniques. The authors suggested that developers should add some assistive cues to aid individuals with ASD with the hand-eye coordination.

\subsubsection{Locomotion Techniques}

To discuss the existing commonly used virtual reality locomotion techniques in the literature, we propose a new taxonomy that is focused on the virtual reality system components. Our taxonomy has three levels. We first divide the previous studies into two as algorithm based locomotion techniques and tool based locomotion techniques. We then divide these two groups into two as real walking and gesture based locomotion techniques, and stationary and mobile tools. Finally, we divide these groups into two according to the technique as redirected walking and environment change, walking in place and flying/leaning, walkers and standard controllers, and wearables and robots. A summary of this new taxonomy that is proposed in this dissertation can be seen in Figure $2-1$.

Obviously, the tool based techniques also involve some algorithms that drive their implementation. The distinction we make here emphasizes whether the algorithm can be implemented across different types of hardware (e.g., marker based optical or magnetic motion tracking systems, or markerless depth sensors), or it was developed for use with a specific device, such as an omnidirectional treadmill. 


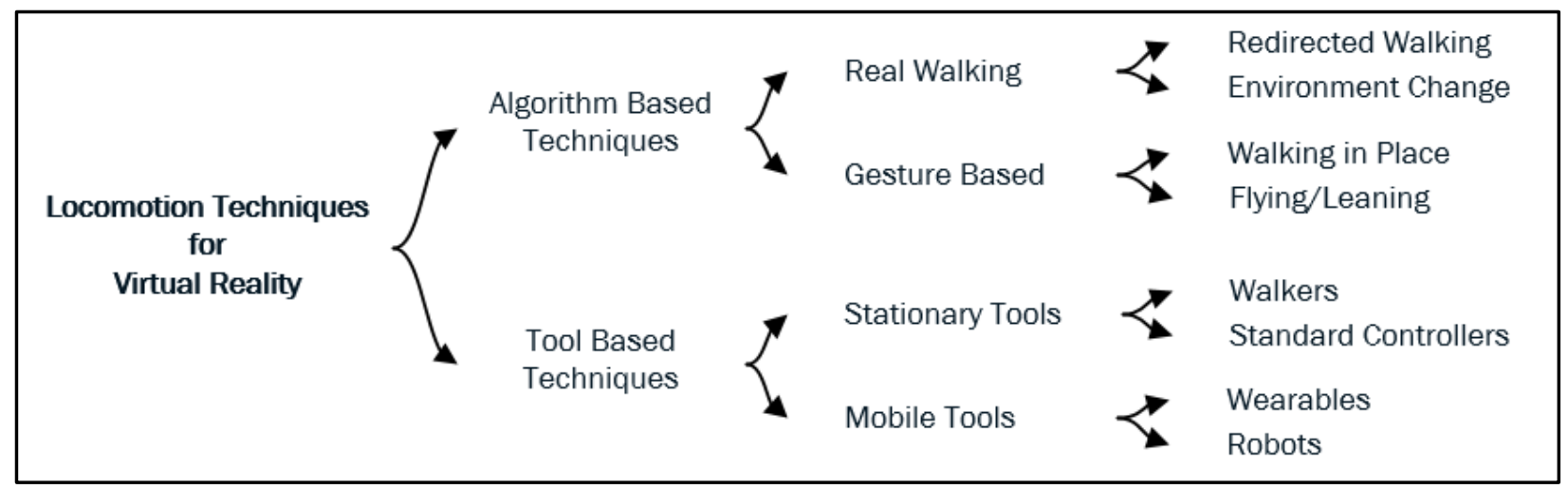

Figure 2-1: Taxonomy for locomotion technique studies.

\subsection{Algorithm Based Locomotion Techniques}

Some locomotion techniques that are used in immersive virtual environments are heavily based on algorithms. Although the tool based techniques also use some advanced algorithms, they depend on the hardware they are using. In this subsection, the locomotion techniques that use general virtual reality hardware that are not specifically designed for tracking or helping locomotion in virtual environments are discussed. The main groups of this taxonomy level are: real walking and gesture based techniques. Both have some advantages and disadvantages and both are used in immersive virtual environments extensively.

\subsubsection{Real Walking}

Real walking is considered to be the most intuitive locomotion type in immersive VR. It was also found to be more presence-enhancing as compared to the other navigation techniques [33]. Furthermore, it was asserted to be superior over other the techniques across the navigational tasks [34], cognitive map buildings [35], and cognitive demands [36]. But there are strong limitations for this technique as well, and sometimes it is 
impossible to use real walking in immersive VR because of the motion tracking constraints [37]. The main limitation is size of the tracked area. If the area of the virtual environment is bigger than the physical area, individuals may eventually walk outside the physical space while trying to reach far spaces in the virtual environment. To overcome this limitation, some techniques have been implemented to help the users in exploring larger virtual environments with real walking. These techniques are discussed in the following sub-subsections.

\subsubsection{Redirected Walking}

It was found out that, when only visual input was supplied, people could successfully estimate the amount of change in the direction but not the path they followed [38]. This makes it possible to manipulate the visual cues to keep the users in the tracking area without being noticed. Various experiments have been performed to analyze the user's perception on virtual and real worlds. It was found that the perceived egocentric distances were often underestimated in VR as compared to actual distances [39], unless the virtual environment started as a replica of the real environment [40]. Similarly, travelled distances in virtual worlds were underestimated [41]. Likewise, virtual walking speeds were often underestimated by the users as compared to the real walking speeds [42].

Redirection is a way of manipulating the user's visual cues to keep them in the tracking area [19]. With this technique, larger virtual environments can be explored within a smaller tracking area. There are some variations of redirected walking techniques, and different taxonomies have been proposed in the literature. Steinicke et 
al. proposed a classification based on the types of gains applied; translation, rotation or curvature [43]. On the other hand, Suma et al. proposed a different classification based on the geometric flexibility, the detectability of the technique and the continuity [44]. In this taxonomy, the repositioning and reorientation techniques can either be overt or subtle according to the detectability, and either continuous or discrete according to the gain application time.

In their study, Steinicke et al. reported the positive and negative limits of the gains that could be applied without getting noticed by the users [43], [45], [46]. Reported limits were listed as $-14 \%$ and $26 \%$ for translation, $-20 \%$ and $49 \%$ for rotation, and 22 meters for curvature radius. For the rotational gains, limits were also examined in detail for the body rotations [47]. For small body rotations like 10 degrees, the limits became $-14 \%$ and $102 \%$. The sensitivity for scene motion was further examined for head yaws [48]. The scene could be rotated $5 \%$ against the head rotation and $11 \%$ with the head rotation. Using gain values outside these limit values were suggested by the researchers to increase the cognitive load and decrease the task performance [49].

Some sophisticated redirected walking techniques use dynamic curvature gains according to the speed of the user [50]. However, no significant difference was found between changing the rotational gain gradually and instantaneously during a full rotation [51]. Another technique called "Seven League Boots" predicted the aimed travel direction by combining eye direction and former displacement data, and applied translational gains on that direction [52]. The displacements in the perpendicular directions were not scaled so that unintentional vertical and sideways head movements 
were not exaggerated. For traveling in long and straight hallways, this method was preferred by the users. Another recent study suggested calculating the redirection parameters based on the architectural layout of the virtual environment [53]. If the users were asked to focus on a cognitive task, these limits could be expanded and no negative impact was noted in spatial memory [54]. Bruder et al. examined the limits of the gains for individuals using an electric wheelchair controlled by joystick [55]. The possible range for the gain values was found to be larger for such redirected driving.

Even if redirection techniques are used, in some cases, users may still reach the edge of the tracked area. For those situations, some additional methods were developed and used alongside the redirection technique. Williams at al. proposed "Freeze-Backup", "Freeze-Turn", and "2:1-Turn" methods as alternatives [56]. In the Freeze-Backup method, when the user approaches to the border of the actual area, the virtual environment positions stay frozen, and the user can take a few steps backwards. In the Freeze-Turn method, again the virtual environment stays frozen and the user can make a 180 degree turn to continue, walking back toward the center of the tracked area. Finally, in the 2:1 Turn method, the user makes a 180 degree turn and the virtual world makes a simultaneous 360 degree turn. The 360 degree turn better masks the unusual 180 degree turn the user is taking.

These methods can be effective but they also inject interruptions into the user experience that have a negative effect on the presence and the quality of the experience [57]. As an alternative, Kohli et al. [58] and Peck et al. [59] integrated virtual distracters into the experience to make the users rotate and face towards the center of the tracked 
area without explicitly interrupting the experience to prompt the user to turn. The aim was to avoid the explicit interruptions that caused breaks in the presence. Using distracters was found to be more natural as compared to mentioned explicit breaks. With the distracter based methods, the redirection was also less noticeable. Furthermore, addition of these distracters did not degrade wayfinding and navigation as compared to basic redirected walking [59]. A recent study suggested using a context-sensitive approach by spawning some events in the virtual world that are related to the narrative, so that the user's orientation is changed with the help of redirection gains without being noticed [60].

Redirection algorithms can also be altered to involve passive haptic feedback objects as well [45], [61]. A proxy object in the real environment representing virtual objects with similar size, shape and surface structure can support passive haptic feedback to the users. Although more difficult to utilize, these passive haptic feedbacks was reported to improve the virtual reality experience significantly [62]. Another redirection technique for exploring architectural 3D models scales the virtual room to fit into the real room, so that users can feel the real walls when they reach to the virtual walls [63]. In this study, to let the users go through a virtual door in a virtual wall, an intense redirection was used so that they did not hit the real walls.

\subsubsection{Environment Change}

Since real walking is the most intuitive locomotion technique, the researchers also looked for other alternatives to utilize redirected walking in virtual reality experiences. A few creative solutions have been suggested and tested. Some of them proposed 
changing the architecture of the virtual environment; others proposed teleporting the users in the virtual world via portal like doors to let the users walk in a larger virtual environment without going out of the physical tracked area.

Usually the techniques involving change of the architecture of the virtual environment are only suitable for interior environments. Suma et al. proposed changing the orientation of the doors in an indoor environment while the user was looking elsewhere, so that users stayed in the tracking area [64]. Although it seems to be an obvious change, only one out of 77 users were reported to notice the change in the study. Despite the changes in the environment during exploration, the users drew consistent sketches of the environment structure and they maintained their spatial orientation in the virtual world. Although this technique was effective, it is limited to indoor environments where the architecture can be manipulated.

Another study showed promising results in maximizing the virtual environment space to be explored [65]. In this technique called "Impossible Spaces", the architecture layout was self-overlapping so that it fitted a large virtual environment into a smaller physical tracked area. It was found out that until 56\% overlap, users could not detect these overlapping spaces. The perceived distances to the objects in different overlapping rooms were not affected by the impossible space approach and remained as if it was not overlapped. Even if the users identified the impossibility of the space, their judgement of distance was not affected. Using this technique alone is again suitable for only interior environments and the success of the technique is highly dependent on the design of the architecture, which imposes a limitation on the application design. A similar study 
expanded this idea with a technique that was called "Flexible Spaces" [66]. With this technique, the environment layouts were generated dynamically to enable infinite real walking in virtual environments. The technique generated many corridors and turns between the rooms to keep the users inside the tracked area, but unfortunately this technique also led to user discomfort. The "Flexible Spaces" approach seemed most suitable for applications in which content is more important than spatial layout, such as virtual museums and virtual sightseeing experiences.

Some researchers used doors that are similar to portals to make larger virtual environments more suitable for exploration. In a study called "Arch-Explore", a virtual down sized architectural model was placed inside a virtual world and the user chose the rooms to explore with a handheld controller [63]. Then a portal like virtual door appeared and the user could walk through that door to walk into the selected room. After a few seconds, the portal vanished. To be able to return to the previous room, the user must have pushed a button on the controller and the portal appeared again. With this method, users can experience a large number of places in a limited tracked area, however it comes with the cost of the reduced realism and presence. Another study used portals to reorient the users in a CAVE-like projection based environment [67]. As individuals reached the border of the actual area, a barrier tape visual feedback appeared. In that case, the user selected a destination point in the virtual environment with a handheld controller by pointing. After the destination was selected, a portal appeared in the center of the actual tracking area. The individual walked near the portal to be teleported to the destination, effectively moving to the center of the tracked area. This technique introduced no 
additional simulator sickness, likely because the viewpoint movement was not displayed to the users.

\subsubsection{Gesture Based Techniques}

Another main technique that is used for locomotion in immersive virtual environments is utilizing body gestures. The most natural gesture was found to be the walking in place gesture, since it was the closest gesture to real walking [68]. Still, other gestures such as leaning or bending could effectively be used in immersive virtual environments. Another commonly used technique is flying. Although it was not reported to be the best technique in terms of reality and presence [33], it was stated to be easy to implement and easy to use.

\subsubsection{Walking in Place}

In this technique, users make body gestures similar to the real world walking, without actually moving in the actual area. This way, users can walk virtually and explore a larger virtual world. Important advantages of the walking in place technique can be listed as cost effectiveness [20], naturalness, stronger feeling of presence and being easy to learn as compared to other approaches [69], and proprioceptive feedback similar to real walking [70].

One of the first scientific implementations of the walking in place technique was published in 1995 [71], [69]. In the implementation, the head movements were analyzed while performing walk-in-place gesture, and the virtual walking was triggered by the movement of the head. The latencies were large; the system required four steps in place to start the virtual walking, since false-positive steps (moving viewpoint when the user 
was not walking in place) were considered more confusing than a late start. Similarly, the system looked for no steps for two cycles to stop the virtual walking.

Since then, different aspects of the walking in place technique have been examined, such as step detections, start and stop latency [20]; and smooth speed control [72]. Smooth speed control was important because of the phenomenon of visual cues making the users feel like they are running with a slower speed than the actual treadmill speed [73], [74]. A detailed study showed that the recommended range for the visual gain was between 1.65 and 2.44 for the walking in place technique [75]. The same study showed that the gain values varied across different field of views; gain values increasing as the field of view decreasing.

One of the lowest latency walking in place technique was proposed by Feasel et al. [20]. In their study, they used a series of filters and numeric differentiations to obtain heel speed, and then they calculated the virtual locomotion speed after some signal processing operations. Their technique not only had low latency for both start and stop, but also had smooth movement, speed control during stepping and high turning responsiveness. They used magnetic sensors on the feet and the knees for tracking, but the same technique could work with more common optical motion tracking systems as well. A similar study was performed by Wendt et al. [76]. The proposed system used a biomechanical state machine to control the virtual walking, and found more consistent output speeds as compared to the study of Feasel et al. A similar recent study used two smart phones that were attached to the ankles of the user to track leg movement using the inertial sensors that were built into the phones. It triggered the walking in place 
technique after analyzing the acceleration data collected by the smart phones in real time [77].

Usually, the walking in place gesture looks like alternately pulling one's knees up, similar to soldiers marching in place. A recent study proposed two different gestures as alternatives to the original walking in place gesture [78]. The first one was called the "Wiping Gesture" in which the user alternately bended each leg backwards whereas kept the upper leg almost still, instead of pulling the knees up as in the original walk-in-place gesture. The second one was called the "Tapping Gesture", where individuals alternately lifted their heels without lifting their toes. The study showed that the Tapping Gesture was perceived as the most natural gesture as compared to the other two gestures. Furthermore, the perceived required physical effort for the Tapping Gesture was closer to real walking. In another study, some of the same authors examined two more input gestures; hip movement and arm swinging [79]. The user study results showed that arm swinging was perceived by the users as natural as the original walk-in-place technique, in terms of the perceived energy required, arm swinging was perceived as closest to real walking.

Different tracking techniques have been proposed for the locomotion techniques in this category. Some applications used knee positions to detect the walking in place gesture [80], and some techniques tracked shins for lower latency [20], [76]. Other techniques tracked the contact points of the feet with the ground. One study used an inexpensive commercial product, the Nintendo Wii Fit Balance Board, for this purpose [81]. They could successfully detect the walking gesture and found similar results in 
turning errors and mean latency, as compared to real walking. Another study used a camera under the ground plane of a six-sided CAVE-like projection system for processing the feet shadows when they were in contact with the ground [82]. The technique was called "Shadow Walking," and could identify different feet gestures to be used for a broader range of movements, such as sidesteps. Shadow Walking was easy to install and suggested as an inexpensive solution to detect walking in place in six-sided CAVE systems. It also did not require an attachment on the body of the user.

For applications that require walking in three-sided CAVE-like systems, the user is likely to walk into the missing fourth wall. For this purpose, a technique called "Redirected Walking in Place" [83] combines the previously discussed redirected walking and walking in place techniques to reduce the frequency with which the user sees the missing wall. In a study, this technique did not lower the mean value of the fraction of time the users saw the missing wall, but it reduced the variance value.

Lastly, Terziman et al. studied a variation of the walking in place technique which required explicit head gestures [84]. For different actions, different head gestures were defined and a simple web camera checked these gestures in real time. These gestures were; lateral head motion for walking, head roll motion for turning, and vertical head motion for jumping and crawling. This technique worked in both sitting and standing configurations. While easy to implement and inexpensive, head gestures for walking was unintuitive and required training and practice before comfortable use. 


\subsubsection{Flying/Leaning}

Flying is a simple locomotion technique alternative for immersive virtual environments [85], [86]. The technique uses some form of input from the user to move the viewpoint automatically in the virtual environment. Inputs can be taken from the user in different ways. It can be a simple joystick input, or a leaning body gesture input. The input may require continuous action to keep walking such as pushing the joystick forward or a single action to start and stop the movement such as pushing a button. Although locomotion by pushing the joystick forward continuously can also be considered flying, in this dissertation, the flying technique was defined as the automatic movement of the user by triggering the start and stop of the locomotion.

The difference of flying by leaning as compared to using a joystick is that in leaning, the movement direction is defined by the direction of a tracked body part; head, hand or torso. The travel direction is usually chosen to be the gaze direction since it was stated to be more natural, easier to learn and provide easier traveling in a straight line [87]. On the other hand, using an additional pointing gesture may also provide comfortable travel since the users could then look around while moving in a constant direction.

Although it is straightforward to implement and use, the flying technique was reported to be less realistic and the sense of presence was reported to be lower compared to other techniques like redirected walking and walking in place [33]. The lack of speed control, which is usually done via a separate controller or a hand/body gesture, reduced the naturalness of the technique. 
A more complicated locomotion technique is leaning. With the leaning technique, users can control both the speed and the rotation at the same time. By using the center of gravity on the 2D ground plane as a directional vector, users can accelerate or decelerate, and rotate the viewpoint clockwise or counter-clockwise. One study used a Nintendo Wii Balance Board to detect the shifts in the body weight [88]. They proposed a human transporter (i.e. Segway PT) metaphor to control the speed and steering. A similar study called "Human Joystick" compared the leaning gesture with joystick and walking in place methods [89]. According to the study, leaning techniques had lower latency and turning error as compared to the joystick. Performance on spatial orientation was similar to the walking in place technique, but in overall the users preferred the leaning technique. The leaning technique in general is easy to use and implement. It gives the user more control as compared to flying by using only one gesture to trigger start and stop of the locomotion. It must also be noted that this technique demands more balance and body control skills compared to the other ones.

A hybrid technique called "Magic Barrier Tape" [90] was suggested. This technique also enabled the users to explore infinite virtual environments with a combination of real walking and flying. The users walked freely in a limited tracked area. When they reached the edge of the tracked area, a virtual barrier tape appeared to show that they could not walk in that direction anymore. In that case, a hand gesture such as pushing the tape triggered flying in the gesture direction. Instead of the gesture, a triggering device such as joystick can also be used to trigger flying. This technique was claimed to be intuitive and easy to use. 
To control flying in virtual environments, any triggering method can be used. Due to the increased availability of skeleton tracking, using body gestures for this purpose has been becoming more popular. One recent study proposed a navigation model called "LazyNav", which explored ways to control the locomotion without any inputs from hands, arms, eyes and local head orientation [91]. The purpose was to let the user do other required actions with their hands and head, and control the locomotion with other possible tracked body parts. The study examined different body motions based on the ease of control, accuracy, required effort and social acceptance. The shoulder rotation and the bust rotation turned out to be the favorite gestures to control the rotation and speed respectively in the pilot study.

Instant locomotion techniques can also be considered under this classification. One of the most important examples of this approach is teleportation. Although teleportation is a simple yet powerful possible alternative to the previous locomotion techniques, it has not quite been explored yet. One example developed for CAVE-like environments used teleportation in the virtual world thorough portals [67]. This technique utilized redirected walking and when a user created a portal by using a controller, a conjugate portal gate appeared in the center of tracked space, so that users were kept inside. Another study used teleportation approach to help the users walk long distances in the virtual environment [92]. The locomotion was done by real walking, and when the user made a jumping gesture, teleportation was triggered in the head direction. No other studies that we are aware of studied teleportation for locomotion in virtual reality. 


\subsection{Tool Based Locomotion Techniques}

Many locomotion techniques are often built on top of optical or magnetic tracking devices. These devices can give the position and rotation of rigid bodies in real-time at a reasonable latency. However, they have some weaknesses as well, such as including occlusion, and high dependency on the environmental conditions (such as poor lighting or nearby metal materials). The techniques described in this subsection avoid these limitations by relying on mechanical hardware designed and developed specifically for tracking the locomotion.

\subsubsection{Stationary Tools}

Stationary systems are usually heavy tools that are attached to the ground. These systems help users walk in virtual environments while wearing a head mounted display (HMD) or standing on an area within projected surfaces such as CAVE-like environments. Stationary systems either work with physical effort as in real walking, or with a triggering mechanism such as a joystick or mouse. The main purpose of these systems is to keep the user in the very same physical space while allowing virtual walking, which allows for more safety. The main groups of stationary tools are walker machines and standard joystick-like controllers.

\subsubsection{Walkers}

Treadmills, bicycle machines, and similar exercise equipment are also well-suited to enabling virtual reality locomotion. For example, in a previous study, a traditional treadmill was used for locomotion [93]. The treadmill supported one directional 
movement, and the user could rotate by using a steering bar similar to a bicycle. The onedirectional imposition was limiting but allowed for locomotion via natural walking.

The U.S. Army's Dismounted Infantry Training Program performed an extensive research on locomoation devices [94]. Three generations of locomotion devices were developed and tested. The first generation used a unicycle as a locomotion interface. Using the unicycle did not feel natural and it did not allow for making sidesteps. The second generation system was a standard unidirectional treadmill with a constraining mechanism that was attached to the user's waist. This was better than the first generation in that it allowed realistic walking, but was still limited to one direction of movement. The third generation system was an "Omni-Directional Treadmill" which enabled users to walk freely towards any direction. The system was composed of two dimensional rotary motors that moved the treadmill belts in order to keep the user in the same place. The study showed that accurate user tracking and precise control over the speed of the belts were critical for the usability of the system. Otherwise, the users experienced uncomfortable sudden movements. A similar system was developed in later studies and compared to a three degrees of freedom motion platform with controller based locomotion [95], [96]. The mean error distance for real walking on the motion platform was statistically significantly lower than that was with the controller. In the more recent studies, an improved Omni-directional treadmill was compared with real walking [97], [98]. The new treadmill, which was called "CyberWalk", was based on a torus design. The system applied imperceptible accelerations on the motion platform to keep the user at the center. They system allowed for a more continuous walking surface, with lower 
vibration and noise, and was compatible with other virtual reality devices. Although the system was found to be effective in locomotion, it was expensive and may not be suitable for daily use.

Another approach for developing a locomotion device was using a low friction surface. The user could walk naturally on the surface but stayed at the same place since the surface was low friction. This could either be achieved by using ball bearings or by covering the surface with low friction material and using suitable shoes. But keeping the surface low friction was not sufficient. Another complimentary technique also needed to keep the user at the center of the system. This could be achieved by using a curved surface to utilize gravity, using a stationary belt to physically keep the user at the center, or using motor systems. The "Omni-directional Ball-bearing Disc Platform" used a custom made disc with ball-bearing sensors to track the walking of the user [99]. No skeletal tracking was required other than the head tracking for rendering. The user was kept at the center by the concave shape of the surface. Another system used a similar surface with ball bearings [100], but the bearings were standard bearings without sensors. A camera system was used for capturing the walking of the user real-time. Another approach was called "CyberCarpet" [101]. The proposed approach combined the bearing system with a belt system. The user walked on a series of ball bearings under which there was a belt system. The belt system was rotated according to the user's direction of walking via a turntable. The belt rotated the bearings which kept the user at the center. This system did not restrict the user with a stationary belt, and the surface was not concave which made the walking more natural. 
Some similar works in this area have recently been emerging as commercial products to be released in the near future [102], [103]. Both works use low friction surfaces and special shoes to reduce surface friction and keep the user at the center of the device. Once these products will be available commercially, everyday users will be able to use these devices in their homes and experience intuitive walking in immersive virtual environments.

So far, the mentioned locomotion devices were designed to allow for real walking movement. There have been also some devices that were designed to work with some gestures such as leaning and walking in place. For example, "Joyman" [104] was similar to a human sized joystick. Users leaned on a board to control the locomotion. A spring system introduced a repelling force to balance the user. Inertial sensors measured the inclination of the board with which the virtual locomotion speed was calculated. Sideway leans were used to rotate the viewpoint in the virtual environment. Another device, called "Walking-Pad," was designed to track the walking in place gesture [105]. The system was a platform with a grid of switch sensors which could detect pressure (a predecessor of Wii Balance Board). The platform could detect walking speed and direction as well as jumping.

Some other studies employed everyday objects as locomotion devices. A recent study used a "Swopper Chair" with an orientation sensor to get input from the user and could also provide vibration feedback [106]. Users could lean forward in the chair with the help of chair's unique design to control the navigation. The head rotation was tracked by internal sensors of the head mounted display. Another study used a stepper machine 
for locomotion in virtual environments [107]. The authors used an Arduino microcontroller with wind and vibration sensors to translate movement on the stepper machine into steps for virtual locomotion. In a user study, the stepper machine received the highest scores for joy and immersion compared to a Wii Balance Board and joystick. However, within the same research, users also reported that the stepper machine was difficult to use. On a similar note, Nilsson et al. compared the stepper machine, Wii Balance Board, keyboard and mouse for movement in a virtual skiing game [108]. The stepper machine was found out to be the best in terms of enjoyment and the second best in terms of ease of use.

Finally, there are some atypical approaches to locomotion in this category. One of these studies was called "Cybersphere". The authors used a large sphere in which the user could walk, run, jump or crawl freely in any direction to explore an infinite virtual environment. Another similar product, which was commercialized, is called "VirtuSphere" [109]. The VirtuSphere was designed to work with head mounted displays. It was reported to be difficult to start and stop walking in the sphere due to its size and large mass. Once the user started to run, the momentum of the ball made it hard to stop or make fine adjustments. The system was suggested to be more useful for low velocity applications. Another atypical device was called "String Walker" [110]. In this system, each foot was attached to four motor pulleys with strings. Once a forward motion was detected, the strings pulled the user to the center. The tension was only applied when the foot was on the ground. This information was gathered with a touch sensor that was placed on each foot. The turning motion was performed with a turntable that was placed 
under the pulley-motor mechanism. Walking with this device seemed to be neither easy nor natural for beginners.

\subsubsection{Standard Controllers}

Standard controllers such as joysticks, joypads, touchpads and keyboards are the most commonly used locomotion devices [111], [112]. These controllers are usually low priced, easy to install, familiar to users and easy to use. There have been many studies that compared different locomotion techniques with standard controllers. Keyboards are binary controllers and joypads may have limited sensitivity compared to joysticks. Thus, a previous study found that joysticks were slightly better than joypads and keyboards in maintaining longer continuous control of velocity [113]. Locomotion trajectory with joysticks also had a higher conformity to real world trajectories. In another recent study, a joystick controller was compared with real walking using both a CAVE-like environment and head mounted display [114]. In the study, users performed perceptualmotor coordination tasks with different locomotion techniques. The results showed that the different velocity controls of each locomotion technique affected the timing and success rate of actions. In real walking, the speed could be controlled easily whereas with a joystick an almost constant speed was provided. Another study by Peck et al. compared joysticks with other locomotion techniques in a virtual maze environment $[115,116]$. Participants who used joystick-based navigation performed significantly worse than the participants who used redirected walking; in terms of navigating, pathfinding, unnecessary walking distances, wrong turns, and recalling and pointing targets. In the same study, joystick and walking in place techniques performed similarly on the above 
metrics. In another study, joystick locomotion did not perform well as compared to natural human walking [37].

On the other hand, one study found that joystick performed better than natural and semi-natural locomotion interfaces [117]. In this study, joystick was compared to a natural technique (real walking) and a semi-natural technique (VirtuSphere). Despite being a non-natural locomotion technique, joystick got better results than VirtuSphere in terms of fatigue, ease of learning and walking, and accuracy. The authors concluded that well designed locomotion techniques of lower fidelity levels such as joysticks tended to yield improved results as compared to moderate interaction fidelity like VirtuSphere.

Another study compared real walking and joystick locomotion with an additional alternative of real rotation with joystick walking [118]. The task was to visit different positions in a virtual environment to find target objects. Combining real rotation with joystick-based walking produced similar task performance scores as real walking. Both techniques outperformed joystick locomotion. The results showed that large tracked areas were not required for reasonable navigation performance in virtual reality. Finally, joystick and keyboard like devices were inferior for controlling spatial orientation as compared to the physical locomotion techniques such as redirected walking [119].

In light of these previous works, we can summarize that most users are familiar with standard controllers and do not need any prior training to use them, on a positive note. However, applications using these standard controllers may lack some degree of realism in terms of interaction and sense of presence. 


\subsubsection{Mobile Tools}

Some locomotion devices are designed to be mobile and are usually worn on the body of the users. The main problem they try to solve is similar to the tool based systems. These tracking area independent tools can sense gestures, transmit them to the system to make the necessary updates on the viewport, and keep the user in a safe physical place, if required. So far, mobile tools were primarily examined in experimental studies and they are not usually utilized in virtual reality applications. In this sub-subsection, these systems are discussed under two categories: wearables and robots.

\subsubsection{Wearables}

For spatial tasks with low accuracy requirements, the foot can be used for interaction [120]. One of the first examples of wearable virtual reality locomotion devices was called "Cyber Boots" [121]. It had four pressure sensors for each foot: one under the heel, one under the toes and two in the middle of the foot. The proposed system could identify walking and leaning gestures. Another wearable locomotion device was called "Waraji" [122], [123]. Waraji had three rotary sensors attached to a sandal, from which the ankle motion was detected and used for locomotion in virtual environments. However, the system needed separate calibration for each different user. Recently, the researchers have developed another shoe prototype with six different sensors placed on the highest pressure points of the feet [124]. It could track walking in place to trigger the locomotion. Furthermore, it could track some other gestures such as shifting pressure or jumping. One of the strongest properties of this system is its ability to give feedback to the users. The system gives tactile feedback as well as temperature feedback. Besides 
these, there are some other applications that can track the feet and understand the feet gestures, but these systems were not primarily developed for virtual reality applications [125], [126].

Another wearable locomotion device was called "Powered Shoes" [127]. It was a pair of roller skates that were driven by flexible shaft motors. It had a lightweight design that could be worn comfortably by the users. The motion of the user was tracked by optical sensors and the motors rotated the roller skates to keep the user at the same place. It was a good system to explore large virtual spaces without a need to have a large real tracked space. The walking was similar to real walking so it did not involve learning and is natural. The drawback of this system was that it needed accurate low-latency tracking to stop the movement of the user. Otherwise, sudden movements and disturbances in the user's presence were observed. Furthermore, the Powered Shoes system required powerful motors to be able to move the weight of the user. The motor and the batteries were carried separately in a backpack that was worn by the user. The system relied on the motor abilities of the user.

\subsubsection{Robots}

An interesting approach to locomotion in virtual reality was proposed with the name "CirculaFloor" [128], a set of robotic, movable tiles that the user could freely walk on. The tiles kept the user at the same physical place even though they kept walking in the same direction in virtual world. The system tracked knees and assumed the midpoint of the knees showed the center of the body on the ground. Once the midpoint left the dead zone, which was placed at the center of the physical area, the tiles started moving 
to keep the user in the dead zone. There were four tiles in the system. In every cycle, two of the tiles pulled the walking user to the center, and the rest positioned themselves. The tile rotation did not change so it did not give a sense of rotation to the user while providing a sense of walking with proprioceptive feedback. Each tile had 568mm width and depth and they supported a maximum walking velocity of $330 \mathrm{~mm} / \mathrm{s}$. A further improvement made to the system was to give a feeling of walking on staircases by controlling the height of the tiles. The prototype was not stable and the users needed to walk slowly in small steps on them. This system also relied on motor abilities and balancing of the user.

\subsection{Cognitive Comparison Studies}

Some studies compared various locomotion techniques based on cognitive criteria. A study investigated real walking and flying locomotion techniques in terms of information gathering and cognition [129]. The authors used joystick for triggering the flying technique. In their first experiment, the authors found that real walking and flying in the gaze direction could maintain higher immersion as compared to flying in the pointed direction. Furthermore, no significant difference was found for recalling the objects and drawing the map of the environment. In their second experiment, the authors compared virtual maze results with real maze results. Virtual locomotion techniques turned out to be an acceptable alternative for real walking in complex virtual mazes for learning and reasoning tasks. On the other hand, for tasks that required speed and navigation efficiency, real walking was found to be better than flying locomotion with

joystick. Another study compared different locomotion techniques based on cognition in 
three categories: knowledge, understanding, and mental processing [130], [131]. In the study, three techniques of real walking, flying with joystick and joystick controlled locomotion were compared. The results showed that real walking technique was significantly better than flying and joystick techniques in terms of understanding and mental processing. The same study also looked at the map sketching scores for these three techniques. Real walking results were significantly better compared to the joystick controlled locomotion. Finally, for presence, real walking and flying techniques which were implemented with a head mounted display got significantly higher scores than when viewed on a monitor and used the joystick controlled locomotion. A few other studies likewise showed that real walking provided more presence than other techniques such as walking in place or flying [69], [33], [130].

A more recent study performed experiments to investigate the cognitive costs of various locomotion interfaces [36]. It was shown that locomotion techniques required spatial working memory resources. Unnatural locomotion techniques were stated to affect performance in cognitive tasks negatively. Furthermore, virtual reality applications with low visual feedback such as low field of view displays required more general attention resources.

As mentioned, there are several approaches to locomotion in virtual environments. A summary of the key studies that have been discussed in this literature review is given in Appendix A, with their generalized properties. Each technique has its own strengths and drawbacks. All studies considered, real walking seems to be the best choice for virtual reality applications in which realism and presence are desired. If there 
is a limitation in the size of the tracked area and real walking cannot be utilized, walk-inplace technique can also be used without much sacrifice in these aspects. Standard controllers seem to be the poorest choice in terms of realism, presence and enjoyment. On the other hand, these controllers may provide ease of use and less cognitive effort. However, it should be considered that these studies targeted neurotypical individuals. These suggestions may not apply to individuals with autism. We believe that characteristics of the targeted population is among the most important factors in selecting or designing a locomotion technique for a virtual reality application. Hence, locomotion techniques should be evaluated according to the characteristics of the targeted audience as well.

\subsection{Virtual Reality Locomotion in Applications for Autism}

Although locomotion techniques have not been evaluated for virtual reality applications for individuals with autism, many previous virtual reality studies used simple forms of locomotion, such as keyboard and joystick. Movement was performed with pressing keyboard buttons or maneuvering the joystick. Some studies taking place in CAVE projection environments allowed for limited real walking within a small area. This subsection presents previous virtual reality studies for individuals with autism with respect to their locomotion techniques. Brief descriptions of the studies are also presented to give a better understanding of the contexts in which the locomotion techniques were used. These previous works usually used one of the following locomotion approaches: real walking or standard controller. 


\subsubsection{Real Walking}

Virtual reality applications for individuals with autism did not utilize real walking with the aim of navigation in larger virtual environments. However, some studies utilized real walking to allow for movement of the users in a small tracked real world area.

Finkelstein et al. studied implications of an exercise game on motivation of individuals with ASD [132]. The system tracked the body motion of users. The users could control the game with their physical movements. The tasks did not require navigation but the users were free to walk within the small CAVE-like environment. In a follow-up research, the authors found out that the system provided high levels of enjoyment and motivation for exercise [8]. Cai et al. designed and developed a virtual dolphin lagoon for the therapy of children with autism [133]. The system used electromagnetic markers for the position and orientation tracking. The users were free to move in the CAVE-like environment. They needed to perform different hand gestures such as raising hands and waving, to play the role of a dolphin trainer. Pilot user study results indicated the engagement of the participants with autism with the system. However, some participants had trouble in understanding and performing the hand gestures. Bartoli et al. looked into the potential benefits of the motion based games for children with autism [25]. The researchers utilized Microsoft Kinect for motion tracking. The children experimented with a set of commercial Microsoft Kinect games, and the user study results showed improvement in their attention skills. Although no usability data was shared, the researchers reported that the users interacted with the games without 
any difficulties. Garzotto et al. also explored the use of motion games for children with autism [26]. The authors developed a motion based touchless game that mainly encouraged the social interactions and imaginative thinking. Children were required to match the postures that were shown on the screen with their own bodies. User study results demonstrated development in the self-awareness and imitation skills of children with autism.

Pares et al. developed a full body interaction application that included several virtual reality games [27]. The primary goal of this study was developing a fun tool targeting users with autism. The users controlled the games with several body movements. The games did not have concrete goals but provided the users with environments that responded to their actions with visual stimuli such as flying leaves and traces. User studies revealed that children with autism were able to use body movement based interaction. Users engaged with the games, showed positive reactions and no signs of discomfort. Lorenzo et al. investigated the use of virtual reality as an educational intervention for children with autism [9]. The users fulfilled several tasks in virtual environments, including a classroom, home and playground. Some example tasks included talking with virtual characters and collecting the books that were needed for the next school day. The results favored the use of this virtual reality based tool as an intervention for children with ASD. Social skills of the children were improved after using the system. 


\subsubsection{Standard Controller}

Controller based locomotion does not utilize full body movements of the users. Users mainly use their hands to control an input controller such as keyboard, joystick or gamepad. Controller based interaction have been utilized by some previous studies with the aim of navigation in the virtual world.

In their study, Bernardes et al. examined whether virtual reality could be used as an assistive tool to improve the capabilities of individuals with autism [10]. The tasks were centered on travelling. The users were required to move to the predefined destinations such as a bus stop. A gamepad was used for walking in the virtual world. Preliminary user study results indicated the acceptance of the system by users with autism. Self et al. studied the benefits of virtual reality as compared to traditional teaching of safety skills to children with autism [134]. The users had to navigate inside a building to find the exit during an emergency situation. Results showed that virtual reality offered faster learning of the emergency strategies. Strickland et al. developed a similar safety training system for street crossing [135] that was controlled with a joystick. Some problems with using the joystick were reported for children with autism. In a later study on teaching fire safety with a non-immersive computer based application, the authors observed difficulties in using the joystick again, and restricted the degrees of freedom to moving forward, backward and turning left and right. This simplification resulted in better acceptance of the joystick-based locomotion by the children with autism, although some children kept running into corners of objects in the virtual world. As a second simplification, the authors tried mouse and keyboard alternatives. The mouse was found 
to be confusing. Children had difficulty understanding that they needed to bring the mouse back to the center to keep moving in the virtual world. The authors observed that the keyboard interaction appeared to be the most comfortable option among the other alternatives, although no alternative locomotion methods were evaluated in the study. Along similar lines, Josman et al. examined VR for training children with autism on street crossing [28]. The users crossed a virtual street using three keyboard keys. Children with autism learned using the VR tool and their real life street crossing skills were also improved.

Mitchell et al. used a virtual café environment for providing training on social skills to children with ASD [136]. The users were required to move in the virtual world and also have interaction with some virtual objects. User study results showed improvement in the real life social skills of children with autism who used the system. Participants learned using the VR system and got more comfortable with training. Kandalaft et al. investigated the feasibility of a VR tool for social skills training of individuals with ASD [11]. The tasks were mainly communicating with virtual characters. Keyboard arrow keys were used for walking in the virtual environment. User study results indicated improvement in the real life social skills of the participants with autism. Participants were reported to learn how to navigate in the virtual world easily.

Fornasari et al. studied the behavioral differences between children with autism and neurotypically developed children in navigation [137]. For this purpose, the authors utilized a computer based virtual environment in which the users navigated using mouse. There were no differences between children with autism and neurotypically 
developed children for the exploration with a goal task. On the other hand, it was found out that children with autism spent less time in the virtual environment for the free exploration task.

A summary of the studies for training individuals with autism using virtual reality can be seen in Appendix B with a focus on the locomotion techniques. The mentioned studies were limited to basic locomotion techniques. Intuitive locomotion techniques like redirected walking and walking in place were not employed or evaluated in virtual reality systems that aimed for effective training of individuals with autism. Findings on the understanding and use of locomotion techniques by individuals with autism were not explored and shared in these studies. For desktop virtual systems that use computers instead of motion tracking areas, only a few studies shared their observations on users with autism having no difficulties in using keyboard. However, these observations were limited to the alternative locomotion methods that were available in the study and were not based on statistical comparisons. Thus, we believe that more comparative studies are needed to understand the user experience of individuals with autism with virtual reality locomotion methods. This dissertation aims to contribute to remedying this deficiency. 


\section{CHAPTER 3: VIRTUAL REALITY FOR VOCATIONAL REHABILITATION OF INDIVIDUALS WITH SEVERE DISABILITIES SYSTEM: LOCOMOTION, INTERACTION AND DISPLAYS}

Autism spectrum disorder (ASD) is a lifelong development related disability which may impact people's understanding of their environment. It can result in difficulties in social relationships and behavior [138]. The latest data from the U.S. Department of Health and Human Services [139] shows that today, about 1 in 68 children is identified with ASD. Attention to this specific group and applications for them has increased recently because of an increase in the awareness of prevalence of ASD. According to the Centers for Disease Control and Prevention, National Center for Health Statistics, prevalence of autism has increased by $289.5 \%$ from 1997 to 2008 [140]. The three most significant impairments that are associated with autism are listed as; social interaction, communication and behavior [141]. Because of these impairments, individuals with autism often have difficulty in their daily lives, especially while interacting with other individuals. Because of the limiting properties of ASD, it is usually harder for individuals with autism to find jobs and succeed in them without proper training.

There are several advantages of using virtual reality over traditional training methods, such as active participation in accurately represented real-life like situations, 
opportunities for repetitive practice on simulators, unique training experiences with suitable and customizable difficulty levels, consistent and real time feedback, and opportunity for users to train and correct errors without severe consequences [142]. Due to these positive properties, virtual reality has been used in many different training applications for neurotypical individuals, such as training law enforcement agents on interrogation [143] and physicians on intervention [144]. Virtual reality is found to be especially useful for populations with ASD, since virtual reality training offers several aspects that resonate with their characteristics, such as the predictability and the ability to repeat the exercises with adjusted difficulty levels until the user feels ready for the task to be performed [145].

There have been many scientific studies for training of individuals with ASD using virtual reality. These studies showed that virtual reality is an effective tool in training individuals with ASD. However, there is little work to understand which virtual reality locomotion and interaction techniques are useful for individuals with ASD. This dissertation aims to contribute to this area. This chapter presents an advanced virtual reality system for vocational training of individuals with severe disabilities, with a focus on locomotion, interaction and display preferences. First, system properties are presented, them locomotion techniques, interaction techniques and the display methods are discussed. Following, user study design and results are reported. Finally, results are discussed and conclusions are drawn. 


\subsection{Note to Reader}

Portions of this chapter were published in HCI International 2016 (Bozgeyikli, E., et al. Virtual Reality Interaction Techniques for Individuals with Autism Spectrum Disorder: Design Considerations and Preliminary Results. International Conference on HumanComputer Interaction, Springer International Publishing, 2016.). Permission is included in Appendix C.

\subsection{System Properties}

The training applications for individuals with ASD that were implemented with virtual reality were reported to usually have positive effects [28, 29] but the systems showed different results in terms of the effectiveness and the acceptance by the users. Among the main reasons for these differences could be the locomotion and interaction techniques that were used in these systems. The literature has not yet extensively examined different virtual reality locomotion and interaction techniques for individuals with ASD. Although there are many possible advantages of using virtual reality systems for job training, the effective locomotion and interaction techniques must be implemented for the users to truly benefit from the advantages of virtual reality. Since the perception and behaviors of the individuals with ASD are different from neurotypical individuals, using the same interaction techniques that work well for neurotypical individuals may not be a good practice for individuals with ASD. In this study, we examined and evaluated different virtual reality interaction techniques for individuals with ASD. For this purpose, several different interaction techniques for object selection and manipulation (tangible object manipulation, haptic device interaction, touch and snap 
technique and touchscreen interaction) and locomotion (real walking and walk-in-place technique) were implemented in different modules of the virtual reality for vocational rehabilitation of individuals with severe disabilities (VR4VR) System. The VR4VR system is a highly immersive virtual reality system that aims to train individuals with severe disabilities on transferrable job skills. This dissertation focuses on the autism population and the locomotion, interaction and display components of the VR4VR system. The locomotion and interaction techniques along with two different display methods (head mounted display and curtain screen) were evaluated based on different aspects such as the ease of interaction, level of enjoyment, frustration, dizziness, nauseousness, tiredness, and user statements.

In VR4VR, there are six modules that are developed for the training of six different transferrable vocational skills: shelving, cleaning, environmental awareness, loading the back of a truck, money management, and social skills. In each different skill, the most convenient interaction technique to be tested was decided by research and discussions with the professional job trainers of the individuals with ASD. These job trainers have been training the individuals with ASD professionally for vocational rehabilitation for a long time and are highly experienced in this area. In this user study, the real walking and walk-in-place locomotion techniques, the selection and manipulation interaction techniques, and the head mounted display and curtain display methods were explored. To implement these locomotion and interaction techniques, the Unity game engine [146] and MiddleVR software [147] were used. The implemented software was run on a desktop computer with the following specifications: AMD FX-8150 3.61Ghz Eight-Core 
CPU, AMD FirePro W600 GPU and 16GB RAM. For motion tracking, the OptiTrack [148] V100R2 FLEX optical motion tracking system with 12 cameras was used in a $2 \mathrm{~m} \times 2 \mathrm{~m}$ tracked area (Figure 3-1).

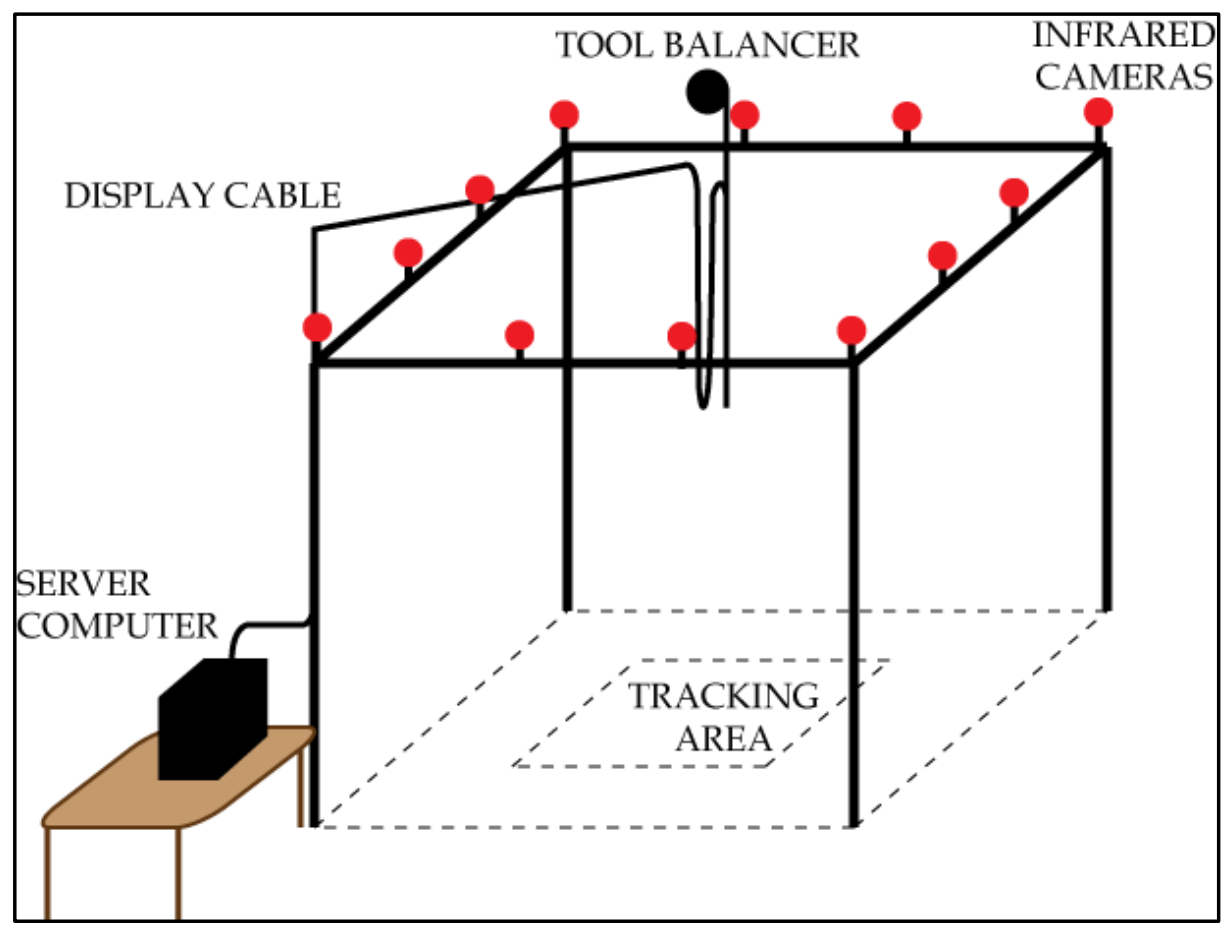

Figure 3-1: VR4VR system's sketch.

\subsection{Locomotion Techniques in the VR4VR System}

Locomotion techniques are used to move the viewpoint (and the avatar, if used) of the user in the virtual world. There are many different techniques of locomotion in virtual reality. In this user study, two locomotion techniques were implemented and evaluated: real walking and walk-in-place. This subsection presents information about these two locomotion techniques in the VR4VR system. 


\subsubsection{Real Walking}

To move the avatar in the virtual world, the user really walks in the tracked area as they would do in real life. Although this is a very intuitive method, there is a significant restriction of the limited tracked area. The user was equipped with reflective markers on their hands and head so that the real position of the user was approximated by these tracked position values and transferred into the virtual world. The virtual world was viewed inside from a virtual camera that was attached to the position of the virtual head and this view was rendered to the HMD. The movement and the rotation of the real head affected the virtual camera's position and rotation so that a realistic view of the virtual world could be displayed in the HMD.

In this project, real walking interaction technique was evaluated in a virtual warehouse environment with the shelving skills. Since this technique is restricted by a limited tracking area, the user was surrounded by two physical shelves and one desk. All the tasks were designed so that they could be performed inside that limited tracked area (see Figure 3-2). The design of the application allowed for the use of a limited tracking area and real walking as the locomotion method.

\subsubsection{Walk-in-Place}

If the real tracked area is smaller than the virtual world, then real walking technique becomes hard to use due to the restriction. To overcome this limitation, walkin-place technique is commonly used in VR implementations. In this technique, the user marches in the same place while the virtual avatar walks in the virtual environment in the direction the user faces. This way, the limitation of the physical tracking area can 
easily be overcome. But this comes with the additional gesture of walking in place instead of real walking. In the VR4VR system, the implementation of this technique included different walking speeds, depending on the speed of walking in place gesture, so that the user could adjust the virtual speed of the avatar by modifying their real marching speed. The walking direction of the virtual avatar was controlled by the head direction of the user. We assumed that the head of the user was aligned with the user's body orientation and the neck of the user was not rotated. If the user turned their head to the left while their body was front facing and marched in place, they would have moved towards the left in the virtual world, where their head was facing.

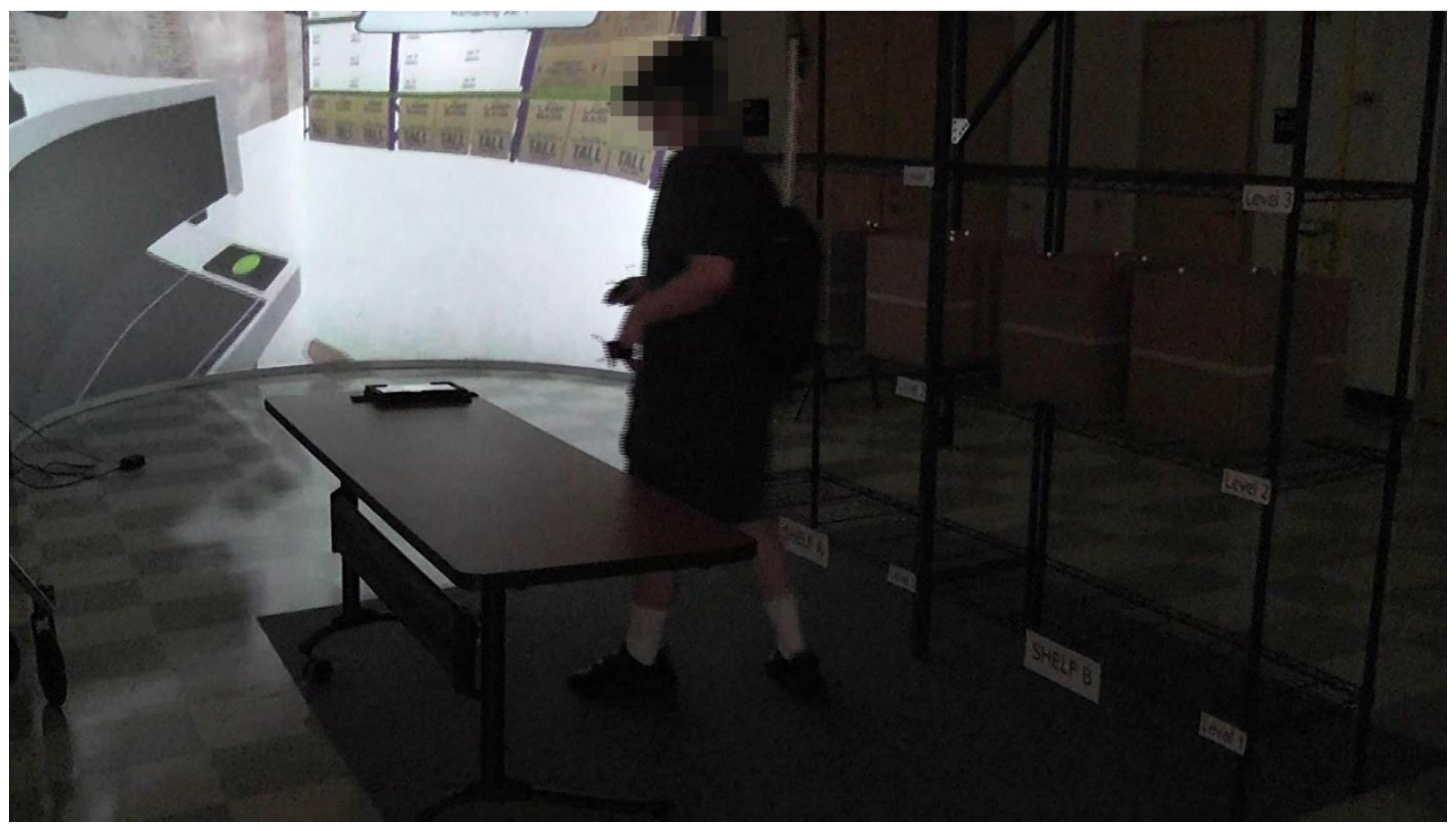

Figure 3-2: Picture of the real walking locomotion technique in the VR4VR system. The user walks inside the tracked area in the shelving module. 
To be able to detect the walk-in-place gesture, a marker set was attached to the same position on user's both feet. The difference of the heights $\left(\mathrm{h}_{0}\right)$ of the left and the right foot markers $\left(h_{0}=h_{r}-h_{l}\right)$ was calculated in each cycle of the program. If the difference of the heights of the foot markers became higher than a threshold $\left(h_{t}\right)$, the system got ready for a possible walking action. In a specific time interval $(\Delta t)$, if the difference of the heights of the foot markers $\left(h_{1}\right)$ became higher than a threshold again but this time in the opposite direction, this triggered the walking action. The walking speed was calculated by collecting the time between the two triggers, and dividing the average one step length to the collected time. After each trigger, system looked for another trigger in a specific time interval. If another trigger happened, the walking speed got updated and walking proceeded. If no trigger was initiated in that time interval, the walking ended (see Figure 3-3 for a flowchart of the walk-in-place algorithm).

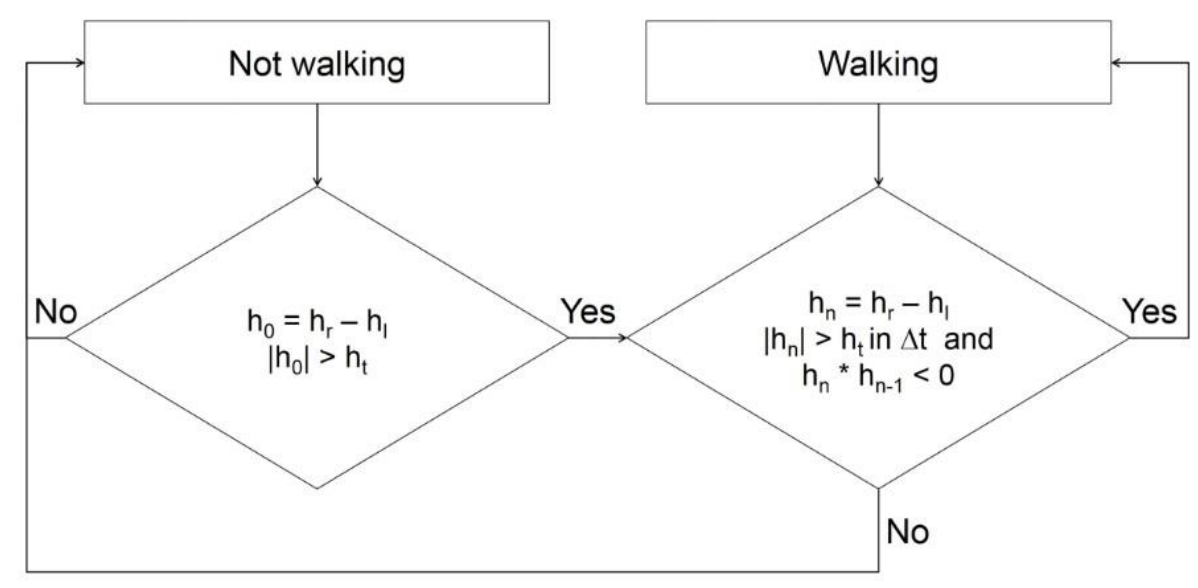

Figure 3-3: Walk-in-place algorithm's flowchart.

To evaluate this technique, two skill modules of the VR4VR system were used. The first one was the cleaning module in which the user was required to go near the dirty 
areas to clean them or go near the litter to collect them (see Figure 3-4). The locomotion was performed by the walk-in-place technique. The other module that was used to evaluate this interaction technique was the environmental awareness module. In this module, the users were required to walk to the specified check points in the parking lot of a virtual shopping mall environment. This module also used only the walk-in-place technique for locomotion.

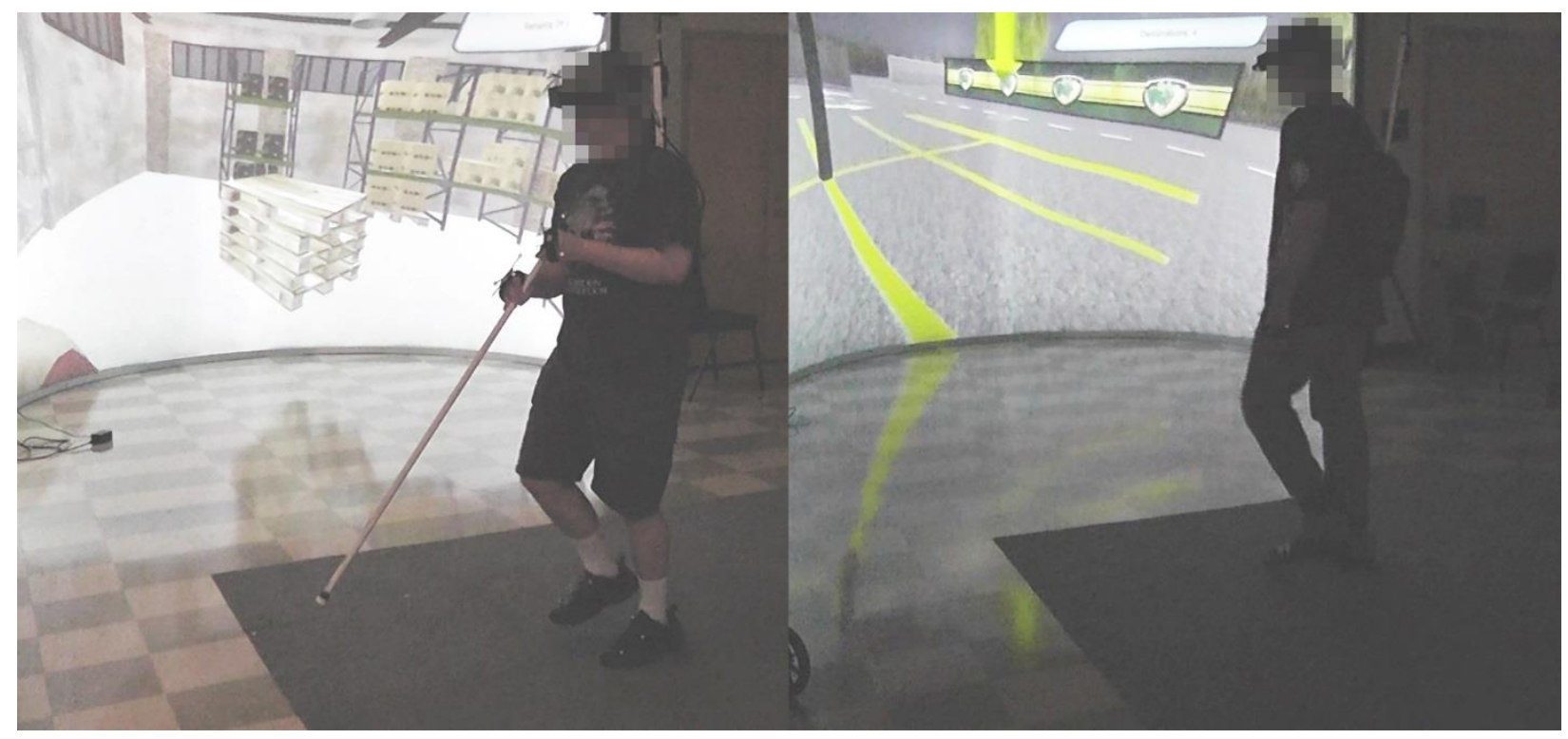

Figure 3-4: Pictures of the walk-in-place locomotion technique in the VR4VR system. The user navigates in the virtual environment by walking in place in the actual world. Cleaning module (left), environmental awareness module (right).

\subsection{Object Selection and Manipulation Interaction}

In the VR4VR system, different object selection and manipulation techniques were implemented and used in different skill modules. The selection of the interaction techniques was performed according to the requirements of the task and inputs received from the job trainers. 
For object selection and manipulation, four different interaction techniques were implemented and tested: tangible object manipulation, haptic device, touch and snap, and touchscreen. These were used in different skill modules in order to interact with the virtual world. These four interaction techniques are presented in the following subsubsections.

\subsubsection{Tangible Object Manipulation}

In this interaction technique, two types of real tangible objects were tracked and represented in the virtual world: (1) identical looking real boxes that were shown in the virtual world with different textures or labels, and (2) a broomstick handle that was represented as a vacuum cleaner or a mop that the user used for cleaning the virtual environments.

For the evaluation of these interaction techniques, some tasks were assigned to the user to be performed in a virtual warehouse environment. In the shelving skill module, there were two physical shelves and one physical table in the real world environment. The virtual conjugates of those objects were created and placed at the same positions in the virtual world. Furthermore, there were four real boxes that were identical in appearance with reflective markers placed on top of each (for infrared camera tracking). The virtual conjugates of the boxes were created and placed at the same positions in the virtual world with different virtual textures projected on them. The first task was to rotate the boxes on the shelves such that the front sides faced the user. In the second task, the user was asked to put the required boxes on the table and then place the boxes on the correct levels of the correct shelves according to their projected label textures. In the third 
task, instead of the label textures, labels with different code numbers were projected on the boxes. These code numbers indicated the shelf/level the boxes belonged to. The user was again asked to put the required boxes on the table and then place the boxes on the correct levels of the correct shelves according to the code numbers.

An immersive tangible object manipulation technique was implemented and tested. With this technique, the users could move and rotate the real tangible boxes in the tracked area (Figure 3-5). This enabled a tactile feedback during the interaction, which was expected to increase the presence for the users. Head mounted display (HMD) was used along with hand bands with reflective markers on them. This enabled real time head and hand tracking. The user was able to see two virtual hands in the virtual world approximately at the same position and orientation with their real hands. We used virtual hand models representing the real hands of the users since it was reported to increase the realism and the immersiveness in virtual reality applications [149] and help the users to better understand the virtual distances. The required actions to be performed in this module were to rotate the real boxes and to move the boxes from the shelves to the table and vice versa.

The tangible object manipulation interaction was also used in the cleaning skill module. In this module, there were two tasks that involved vacuuming and mopping in which the user used a tangible broomstick handle to interact with the virtual world. The real broomstick handle was replaced with a virtual vacuum cleaner or a virtual mop in different tasks (see Figure 3-6). To be able to track the real stick by the optical cameras in real-time, three pieces of reflector marker tape were attached around the cylinder. Since 
the cylinder was symmetric along its longitudinal axis, we used software calculations to visualize the cleaning head (nozzle or mop) according to the angle between the cylinder and the ground. This time, in addition to HMD and hand bands, feet bands with reflective markers were also worn by the user. This enabled real time head, hand and feet tracking. The user was able to see two virtual hands and feet in the virtual world. Required actions included moving and carrying the broomstick and pointing it to the required areas. The objectives of these tasks were to clean the virtual warehouse by using the real broomstick handle to vacuum clean the dry dirt piles and to mop the wet dirt piles.

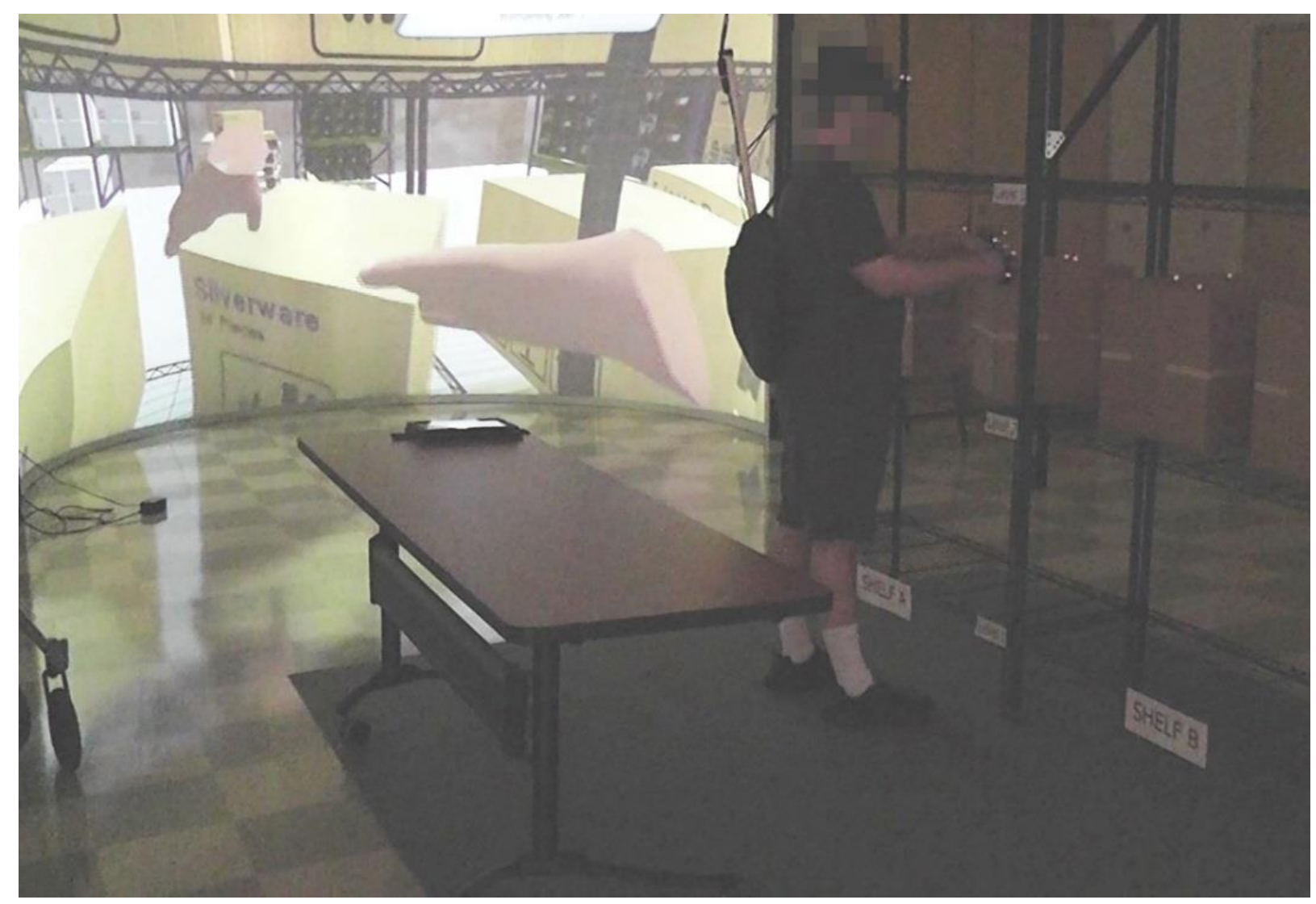

Figure 3-5: Tangible box manipulation in the VR4VR system. The user is rotating a real box. The curtain screen displays the user's view through the HMD. 


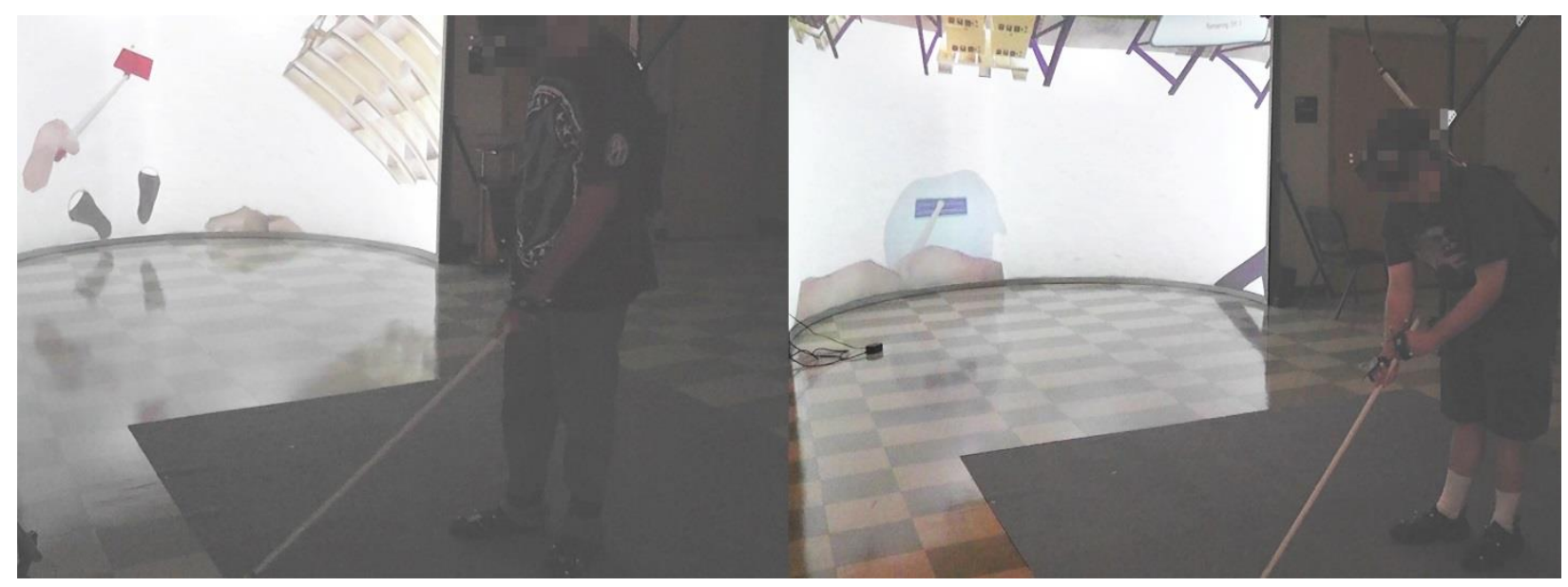

Figure 3-6: Tangible stick manipulation in the VR4VR system. Real broomstick handle with virtual representations of a vacuum cleaner (left) and a mop (right).

\subsubsection{Haptic Device}

Haptic device interaction was evaluated in the loading the back of a truck module. Haptic devices utilize force feedback to create a tactile sense of touch. In this module, Phantom Omni ${ }^{\circledR}$ haptic device by SensAble Technologies [150] was utilized for interacting with virtual world. Phantom Omni® haptic device created a sense of weight for the users so that they could feel if they were interacting with a light or heavy object. This was expected to help in increasing the immersion.

In the module that was utilized to evaluate this interaction technique, the user was expected to fill up an empty truck's back with different sized 2D boxes in a virtual environment. The boxes were presented to the user one by one instead of making all of the boxes visible at the beginning. The boxes had different properties such as weight, fragility and directional arrows. The working area of the haptic device was restricted to a planar surface that was parallel to the display area. This helped the users to relate the 
haptic device input to the visual output easily and also removed the ambiguity coming from the extra degree of freedom for the sake of this task.

The buttons on the haptic device handle were assigned for specific commands (see Figure 3-7). One of the buttons was used to hold the boxes similar to the vastly used mouse gesture for drag and drop. The other button was used to rotate the boxes by 90 degrees counterclockwise. The aim of this module was to fit all of the boxes into a limited area by moving and rotating the boxes.

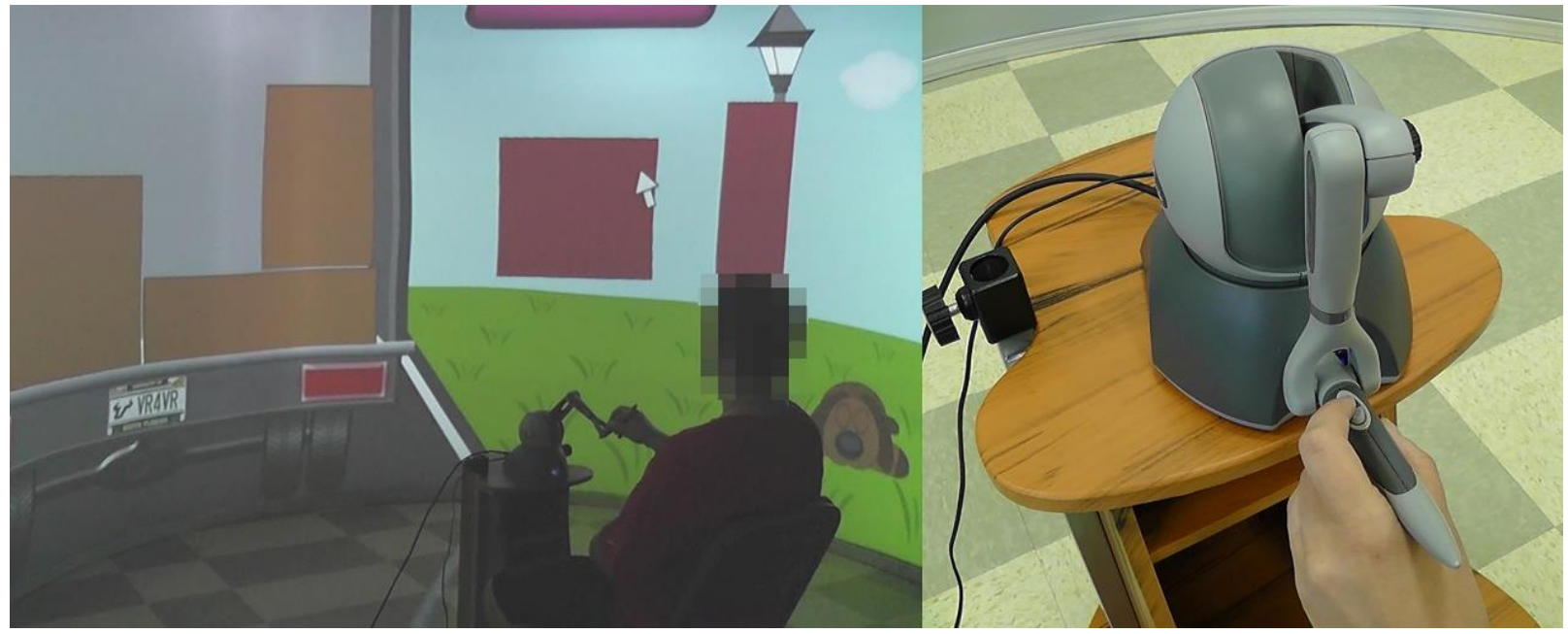

Figure 3-7: The haptic device interaction in the VR4VR system. The user controls the curser in the virtual world by using the haptic device (left). Haptic device with two buttons on the handle (right).

\subsubsection{Touch and Snap}

Touch and snap interaction technique is often used in the existing virtual reality applications. In this technique, a virtual object is snapped to a moving object which usually is selected to be the virtual hand of the user. To trigger the release of the snapped 
object, different techniques can be used such as time triggering, position triggering or gesture triggering.

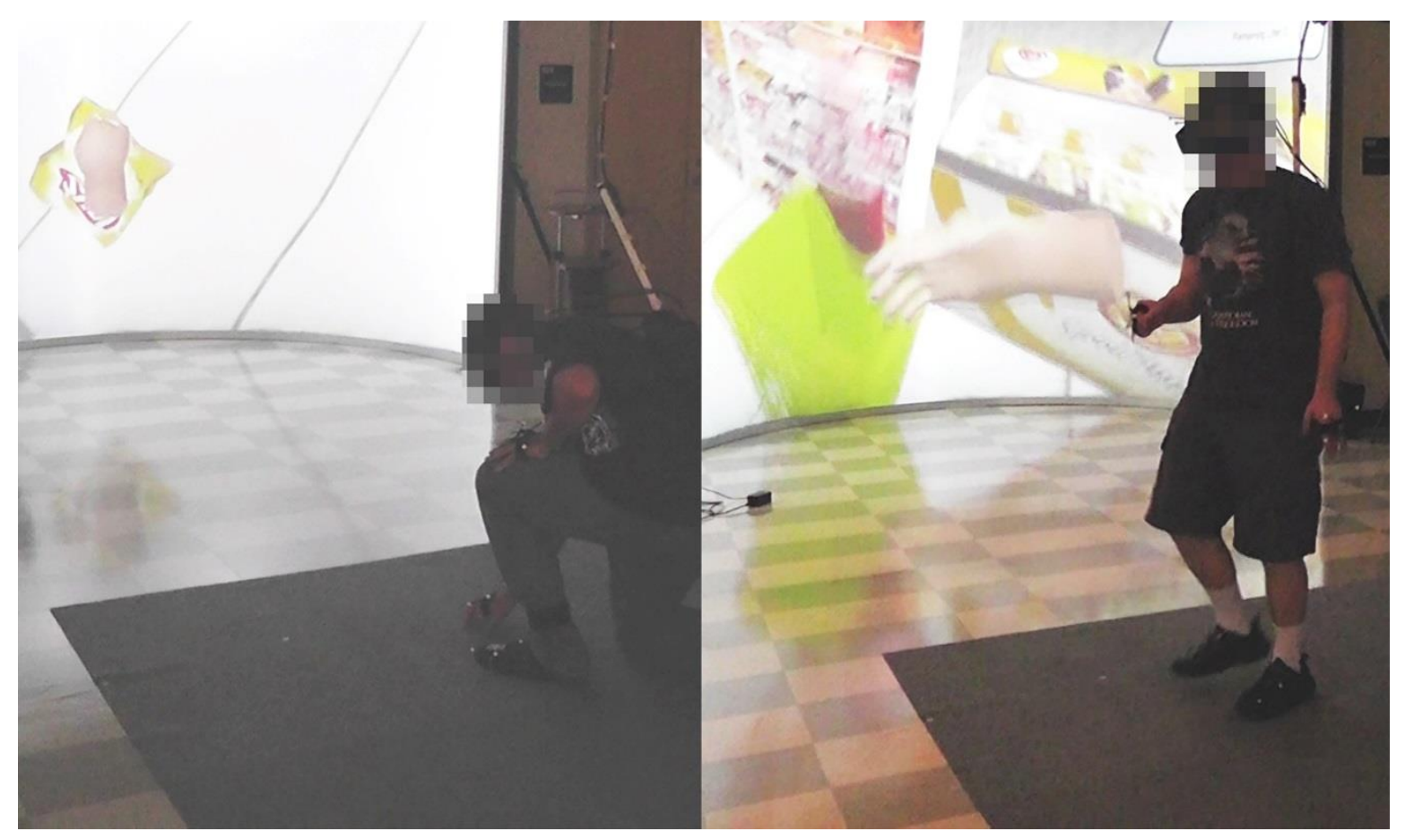

Figure 3-8: The touch and snap interaction in the VR4VR system. The user is grabbing a virtual litter (left) and releasing their hand to throw the litter into a green virtual trash bin (right).

To evaluate this interaction technique, the litter collection task that took place in a virtual grocery store was used. In this task, the users were asked to collect randomly distributed litter objects from the ground and throw them into the trash bins that were located around. User's hands were equipped with passive reflective markers that were tracked real time via the optical tracking cameras. Those positions were used to place the virtual hands into the virtual world. Virtual litter object was snapped to the user's virtual hands when the user bended and their hands came close to the litter. Users carried the litter objects in the virtual world and once the litter arrived in the vicinity of a trash bin, 
it disengaged from the hand and fell into the trash bin. Required actions in this task were moving the hands close to the litter in the virtual world by bending and reaching out in the real word and then bringing the held litter close to a virtual trash bin to release it by extending the arm in the real world (see Figure 3-8).

\subsubsection{Touchscreen}

With the increasing number of mobile devices such as cell phones and tablet computers, touch interaction became one of the most popular and prevalent interaction techniques in daily lives of the users. Even some personal computers, televisions and projectors are currently using this technique for interaction. Since the visual output and the touch input are aligned perfectly, this interaction method is very intuitive and easy to use for the users.

In the VR4VR project, a touchscreen ASUS T100 10 inch display tablet computer was used as another interaction method. Touchscreen interaction was utilized in a module that was related to the cash register skills. Three modules were utilized for this interaction: (1) recognizing money, (2) counting money, and (3) giving change. Currently, most of the digital cash registers are using touchscreens to get the input from the cashier, so this technique was decided to be implemented in the money management skill module to increase the immersiveness of the system (see Figure 3-9).

In this module, only the single touch static touching technique was used instead of the more complicated dynamic or multi touch interactions. The tasks required the user: (1) to identify the given bill or coin amounts by touching on the corresponding value among the given options, (2) to type the sum of the presented bill and coin amounts by 
touching the numbers on the touchscreen keypad, and (3) touching the bill and coin visuals on the cash register to fetch the required change to be given to a customer based on the shopping simulation. A touchscreen keypad similar to the real cash register keypads was presented to the user. The only possible interaction technique in this module was the touch interaction.

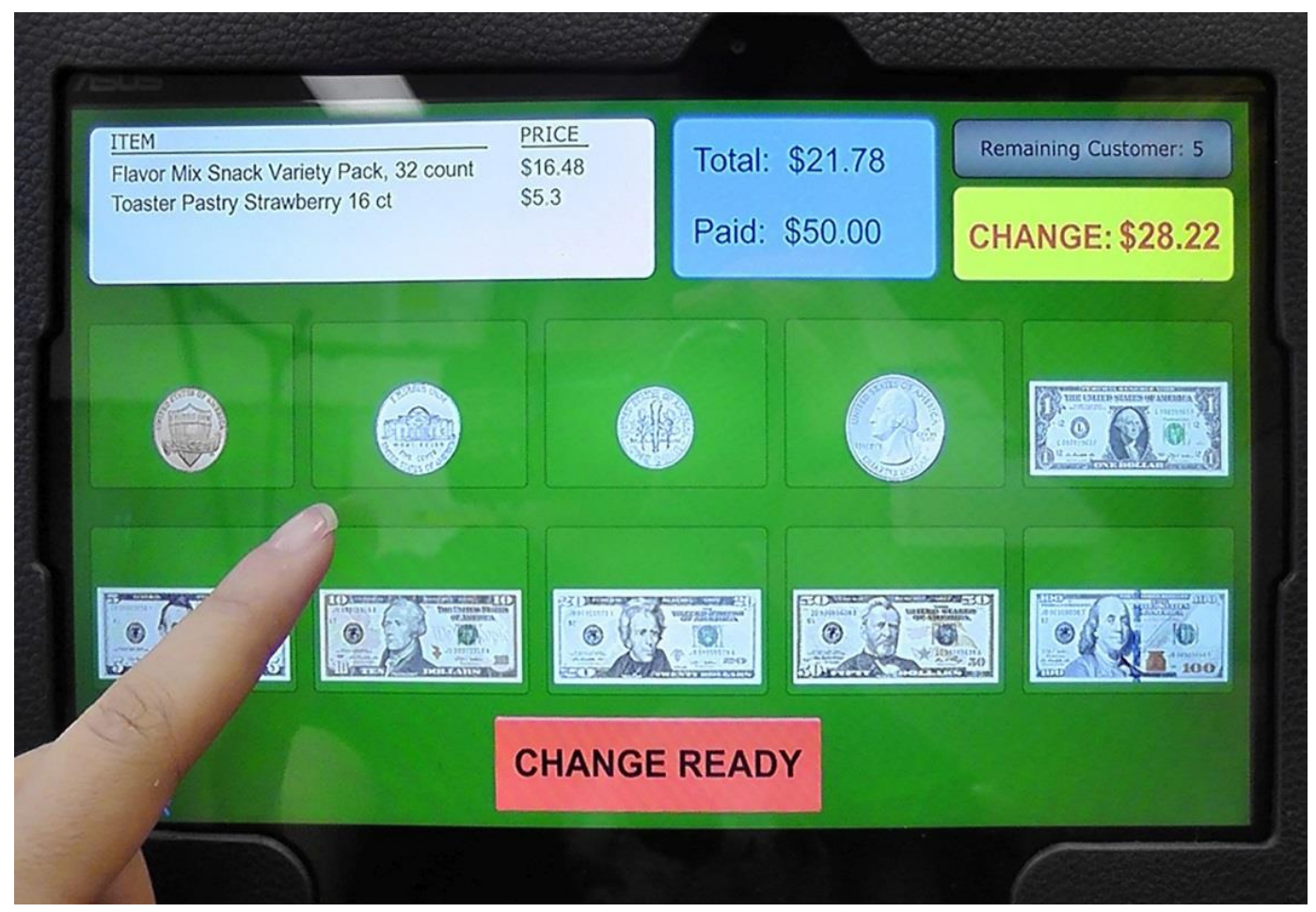

Figure 3-9: The touchscreen interaction in the VR4VR system. The digital cash register interface implemented on a touchscreen tablet computer.

\subsection{Display Methods in the VR4VR System}

Different locomotion and interaction techniques were implemented in different modules of the VR4VR system. Each implemented locomotion and interaction technique was more suitable for a specific display method. In this project, head mounted display and a 180 degree curved screen were used as the available display methods. In the study, 
the most suitable display method was selected for each locomotion interaction technique and the user preference on the display methods were evaluated.

\subsubsection{Head Mounted Display}

Using head mounted display is an immersive way of displaying the virtual world to the user. A pair of digital displays is placed near the eyes so that the user sees the virtual world through them (see Figure 3-10). VR2200 head mounted display with high resolution XGA (1024x768) was used. The main reason for selecting this HMD was to provide individuals with ASD with empty space around their heads since the professional job trainers for ASD stated that covering all of their view with the HMD could create a sense of feeling trapped and disconnected from the real world in individuals with ASD. The job trainers also stated that having open space in the HMD will make sure that they still have some connection with the real world and provide a more comfortable training experience for individuals with ASD. Hence, instead of using a highly immersive HMD that surrounds all of the user's vision, we preferred to use a more open spaced HMD that could be flipped up when not in use.

The modules of the VR4VR system in which the HMD was used were: shelving, cleaning and environmental awareness. The locomotion of real walking and walk-inplace were implemented for the head mounted display since those techniques required the tracking of the rotation of the user's head, to which a virtual camera was aligned. Real rotation of the head was used for rotating the virtual camera in the virtual world instead of using an additional rotating gesture. This approach decreased the gesture learning burden for the users by applying a more intuitive interaction technique. In these modules 
the "tangible object manipulation" and the "touch and snap interactions" were tested as well.

\subsubsection{Curved Screen}

For the interaction techniques that were implemented from a stationary point of view -money management, loading the back of a truck and social skills- a 180 degree large curved curtain screen with two projectors were employed (see Figure 3-11). The curved screen had $3.5 \mathrm{~m}$ diameter and $2.4 \mathrm{~m}$ height and its surface was white fabric so that the projections of the virtual world would be easily visible. This way, the discomfort of the users while using HMDs was eliminated and the two different display methods could be evaluated.

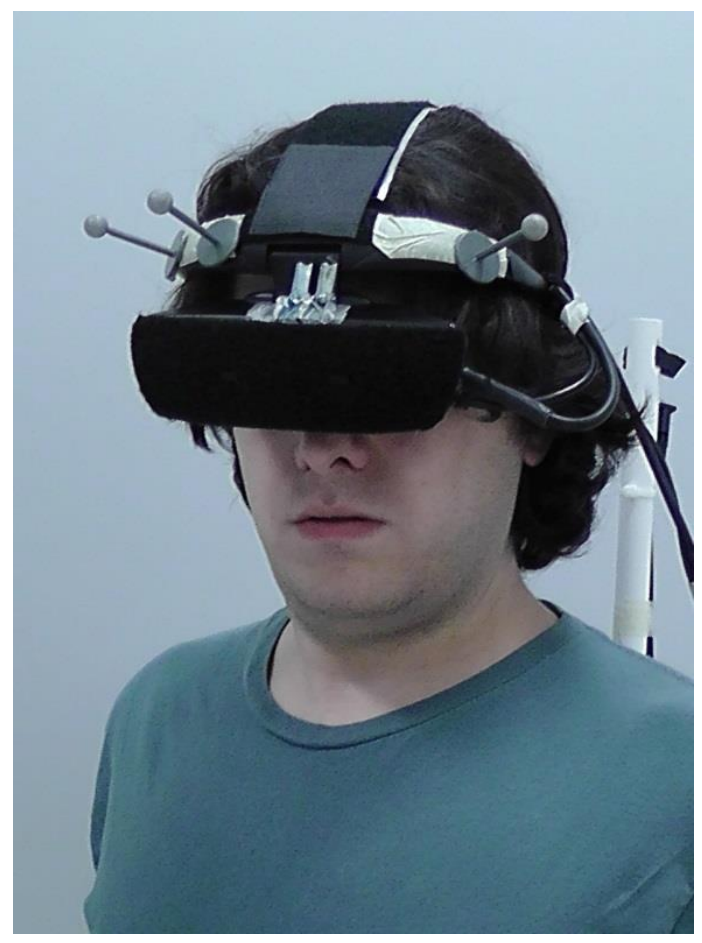

Figure 3-10: Head mounted display in the VR4VR system. The user is wearing the head mounted display with reflective markers on top. 
The modules of the VR4VR system in which the curtain screen was used were: loading, cash register skills and social skills. Interaction techniques that were tested with the curtain screen display method were haptic device and touchscreen. Curtain screen was also used for the social skills in which the user was expected to talk with virtual people. All of the interaction techniques with the skill modules they were tested in the VR4VR system and the relevant interaction tasks are presented in Table 3-1.

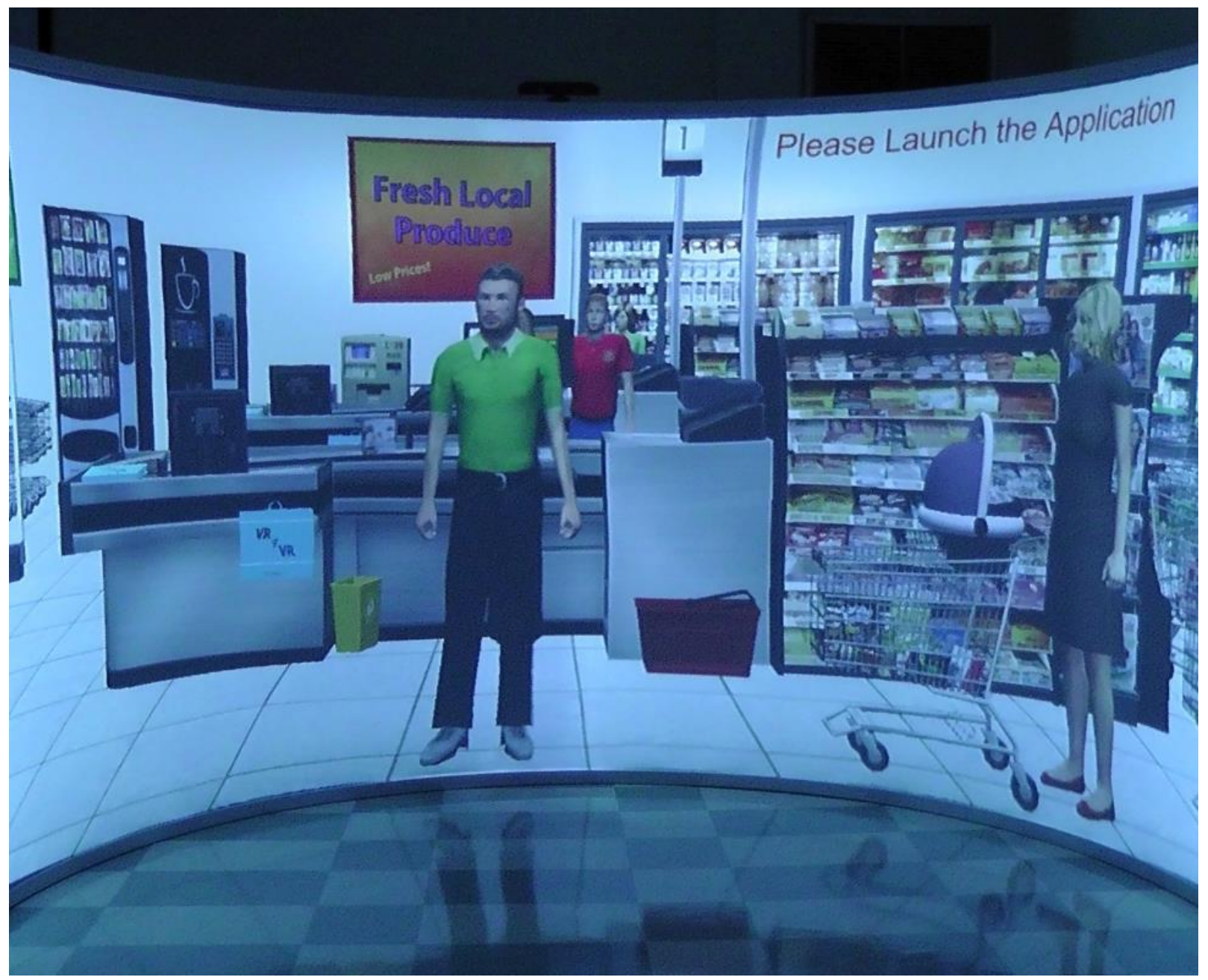

Figure 3-11: Curtain display in the VR4VR system. The virtual grocery store was projected onto the 180 degrees curtain display. 
Table 3-1: The interaction and locomotion techniques and the displays in the VR4VR system. The skill modules they were implemented in and the tasks that were used within these skill modules.

\begin{tabular}{|c|c|c|c|}
\hline Category & Interaction Technique & Skill Module & Interaction Tasks \\
\hline \multirow{5}{*}{$\begin{array}{l}\text { Object Selection } \\
\text { and } \\
\text { Manipulation }\end{array}$} & $\begin{array}{l}\text { Tangible Object } \\
\text { Manipulation (Real Boxes) }\end{array}$ & Shelving & $\begin{array}{l}\text { - Rotating the Boxes } \\
\text { - Placing the Boxes }\end{array}$ \\
\hline & $\begin{array}{l}\text { Tangible Object } \\
\text { Manipulation } \\
\text { (Real Broomstick) }\end{array}$ & Cleaning & $\begin{array}{l}\text { - Vacuuming } \\
\text { - Mopping }\end{array}$ \\
\hline & Haptic Device & Loading & $\begin{array}{l}\text { - Moving the Boxes Inside the } \\
\text { Back of a Truck }\end{array}$ \\
\hline & Touch and Snap & Cleaning & - Litter Collection \\
\hline & Touchscreen & Cash Register & $\begin{array}{l}\text { - Selection on a Touchscreen } \\
\text { Tablet Computer }\end{array}$ \\
\hline \multirow{3}{*}{ Locomotion } & Real Walking & Shelving & $\begin{array}{l}\text { - Rotating the Boxes } \\
\text { - Placing the Boxes }\end{array}$ \\
\hline & Walk-in-place & Cleaning & $\begin{array}{l}\text { - Vacuuming } \\
\text { - Mopping } \\
\text { - Litter collection }\end{array}$ \\
\hline & Walk-in-place & $\begin{array}{l}\text { Environmental } \\
\text { Awareness }\end{array}$ & $\begin{array}{l}\text { - Walking to Destination } \\
\text { Points }\end{array}$ \\
\hline \multirow{6}{*}{$\begin{array}{l}\text { Display } \\
\text { Methods }\end{array}$} & Head Mounted Display & Shelving & $\begin{array}{l}\text { - Rotating the Boxes } \\
\text { - Placing the Boxes }\end{array}$ \\
\hline & Head Mounted Display & Cleaning & $\begin{array}{l}\text { - Vacuuming } \\
\text { - Mopping } \\
\text { - Litter Collection }\end{array}$ \\
\hline & Head Mounted Display & $\begin{array}{l}\text { Environmental } \\
\text { Awareness }\end{array}$ & $\begin{array}{l}\text { - Walking to Destination } \\
\text { Points }\end{array}$ \\
\hline & Curtain Screen & Loading & $\begin{array}{l}\text { - Moving the Boxes Inside the } \\
\text { Back of a Truck }\end{array}$ \\
\hline & Curtain Screen & Cash Register & $\begin{array}{l}\text { - Selection on a Touchscreen } \\
\text { Tablet Computer }\end{array}$ \\
\hline & Curtain Screen & Social & - Talking with Virtual People \\
\hline
\end{tabular}




\subsection{User Study}

A user study was performed to evaluate the experience of neurotypical individuals and individuals with autism with the mentioned locomotion and interaction techniques and the display methods. In this section, demographics, procedure and results of the user study are reported.

\subsubsection{Demographics and Procedure}

Ten neurotypical individuals (10 males, aged between 21 and 50) and nine individuals with high functioning ASD (7 males and 2 females, aged between 20 and 41) participated in the user study. The participants came to our research facility to try all six modules of the VR4VR system with their accompanying professional job trainers. Three different professional job trainers accompanied the nine users with autism throughout the user study. The users completed the skill modules in two different sessions that were scheduled on two different days. Each session was approximately two hours long and there were at least three days between the consecutive sessions. After each skill module, participants filled out a survey about their experience using the VR4VR system. After completing the final skill module of the system, the users filled out a general survey including questions about preferences on the different components of the system. The job trainers were also presented with questionnaires asking for their opinions about the VR4VR system. Other than these methods of data collection, we also asked the users' and the job trainers' opinions about the interaction techniques during the breaks between the consecutive sessions in the form of interviews. The user study was performed under the 
IRB with the number Ame7_Pro00013008, the IRB approval letter can be seen in Appendix D.

The questions that were asked after each skill module were about the users' experience on the locomotion and interaction techniques and the display methods. The questions were about how easy it was to interact with the system, how much they enjoyed, got frustrated or tired while interacting with the system. We used the answers to these questions to evaluate the used locomotion and interaction techniques since they were one of the major differences between the six modules besides the tasks. The users were asked to choose from five available answer choices based on a five-level Likert scale [151]. The users were also asked if they felt dizzy or nauseous, during and/or after the virtual reality training. In addition, the users were asked to select their preferred interaction technique and their preferred display method after completing all of the modules. The users and the job trainers were also asked to state their own opinions about these evaluated aspects.

Other than the surveys, the system also has collected automated data about the performance of the users. Those performance variables were, success counts, fail counts, and the count of additional pictograph helps that were displayed for the user. An overall score was calculated after the session was ended and was stored for all users. For every failure during the session, 30 points were deducted from a total 100 points. For every pictograph help, additional 20 points were deducted from the score. The overall score could not go below 0 . 


\subsection{Results}

The results obtained from the participants and the job trainers are presented in this subsection to provide a general idea on the preference of a cohort of users with autism on several virtual reality locomotion and interaction techniques. However, since the system was not designed with the aim of comparing virtual reality locomotion and interaction techniques, these results are only expected to give a general understanding instead of generalizable powerful conclusions.

The user study results are presented in three categories; locomotion techniques, selection and manipulation techniques, and display methods.

\subsubsection{Locomotion Techniques}

The results for the two locomotion techniques (real walking and walk-in-place) are shown in Figure 3-12 and Figure 3-13 for neurotypical individuals and individuals with high functioning ASD respectively. Real walking received higher scores for ease of interaction, enjoyment and immersion when compared to walk-in-place technique for both populations. The results of the two locomotion techniques were quite similar for tiredness. The users found the walk-in-place locomotion technique more frustrating as compared to the real walking. Detailed data analysis showed that walk-in-place locomotion method was significantly harder and introduced significantly more frustration on the neurotypical users as compared to the real walking. Average scores for each locomotion technique is shown in Figure 3-14. Analysis did not indicate significant difference between the scores of the locomotion techniques for both groups of individuals. Detailed statistical analysis results can be seen in Appendix E. 
During the breaks, some of the users with ASD complained about the difficulty of trying to walk-in-place while trying to concentrate on tasks to do and they said that they liked the real walking much better. The job trainers stated that they found real walking very intuitive and easy to use for the users with autism. In contrast, they said that they found the walk-in-place locomotion technique difficult to learn and perform for individuals with ASD. They observed that most of the users walked forward unintentionally while marching and stepped outside the tracking area, hence got frustrated. They stated that the users with ASD were too focused on performing the walking gesture so that they had difficulty in concentrating practicing the tasks. Thus, the job trainers advised us to look for alternatives for the walk-in-place technique since they did not find it very reasonable to use for individuals with ASD, after their observations during the user study sessions.

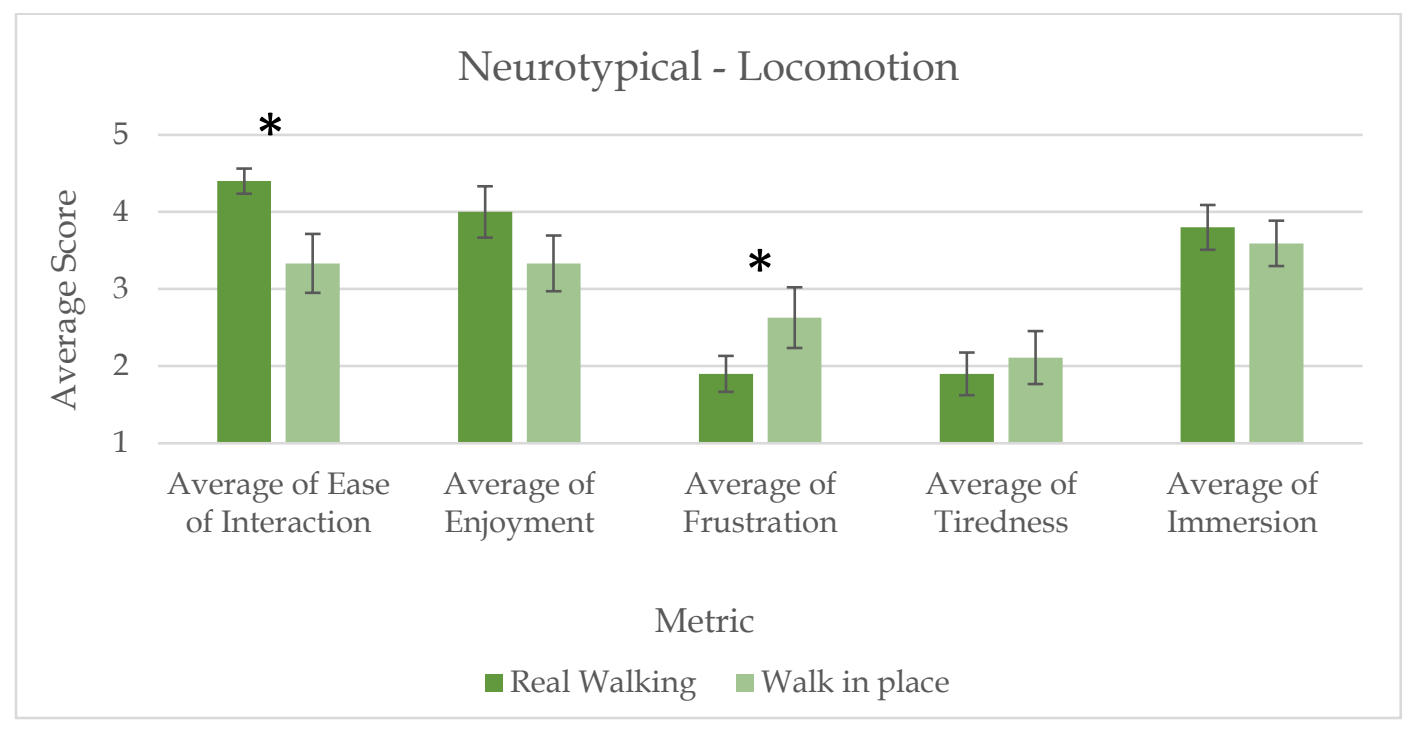

Figure 3-12: Survey results for the locomotion techniques in the VR4VR system for neurotypical individuals. 


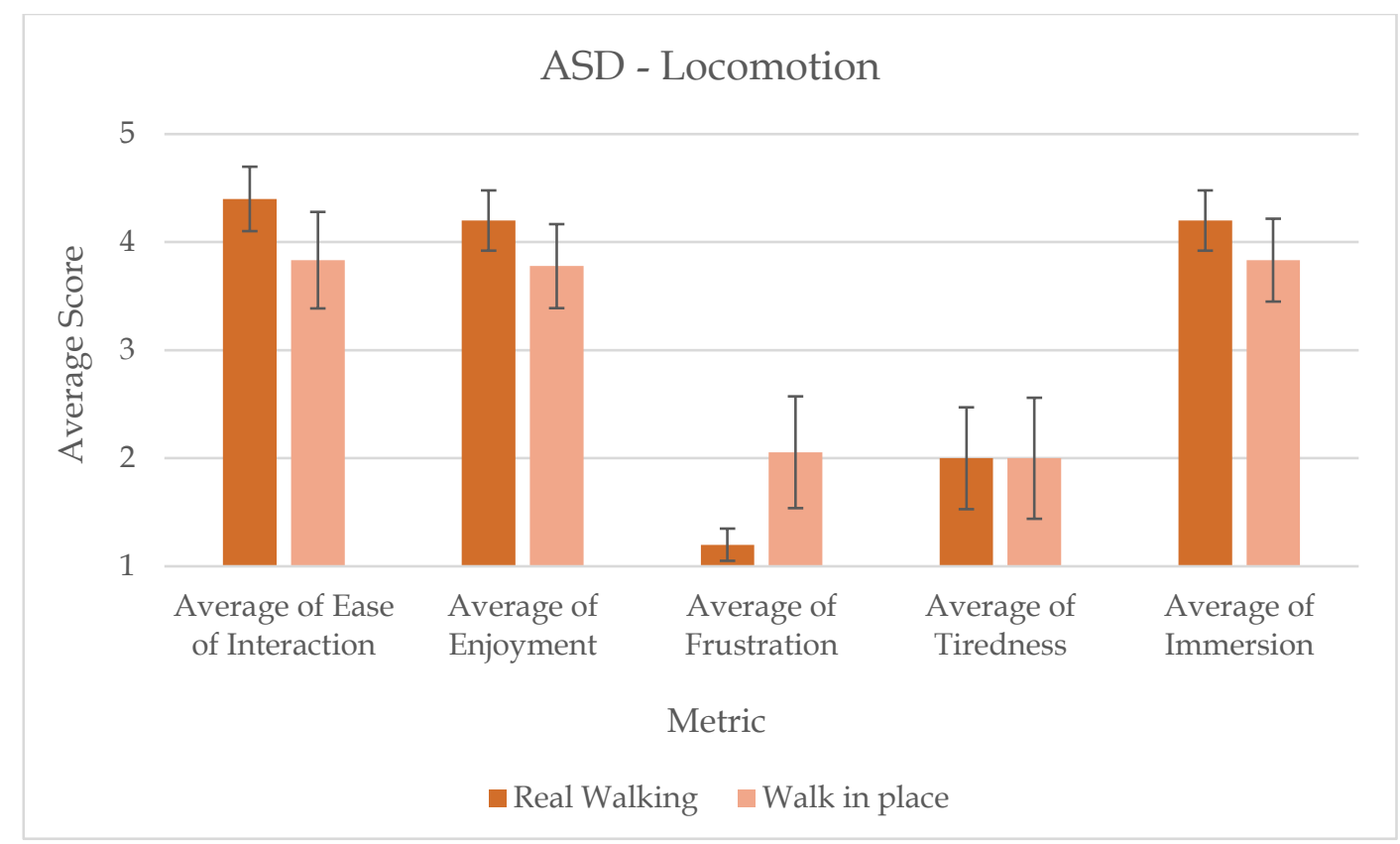

Figure 3-13: Survey results for the locomotion techniques in the VR4VR system for individuals with ASD.

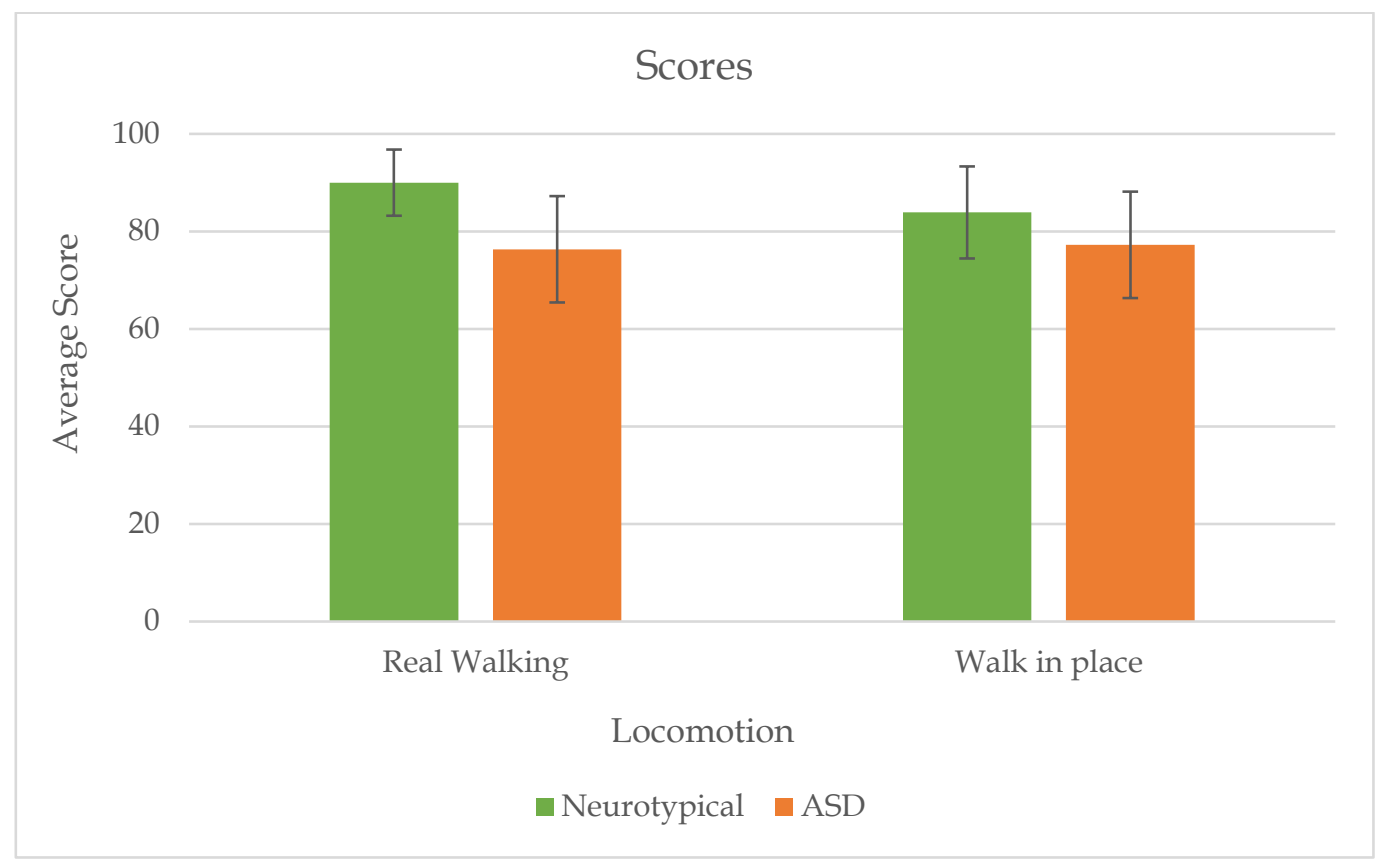

Figure 3-14: Performance scores for different locomotion techniques in the VR4VR system for both population groups. 


\subsubsection{Object Selection and Manipulation Techniques}

The results obtained from the users for the four different selection and manipulation techniques -tangible object manipulation, haptic device, touch and snap, and touchscreen- are presented in this sub-subsection. Figure 3-15 shows the average scores for the neurotypical users, whereas Figure 3-16 shows the average scores for individuals with ASD. A score of 1 represents very little while a score of 5 represents very much of the related aspect. Users in general, felt little tired and frustrated with all interaction techniques. The averages were between "Not tired at all" and "Not tired" for tiredness and between "Not frustrated at all" and "Neutral" for frustration. On the other hand, for ease of interaction, enjoyment and immersion; the averages were above 3.0. The users with ASD found the haptic device hard to interact as compared to the other interaction techniques. Touchscreen interaction received the best results for the ease of interaction, enjoyment, and immersion aspects as compared to the other three interaction techniques.

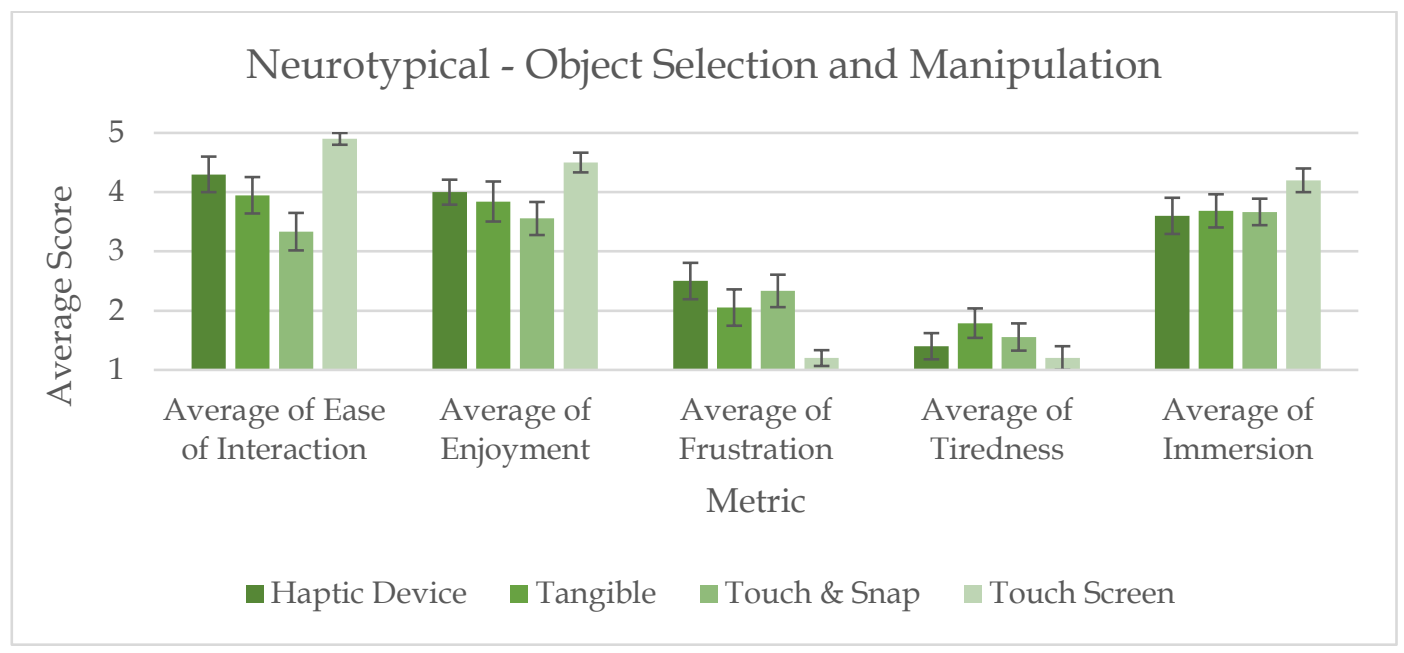

Figure 3-15: Survey results for the selection and manipulation techniques in the VR4VR system for neurotypical individuals. 


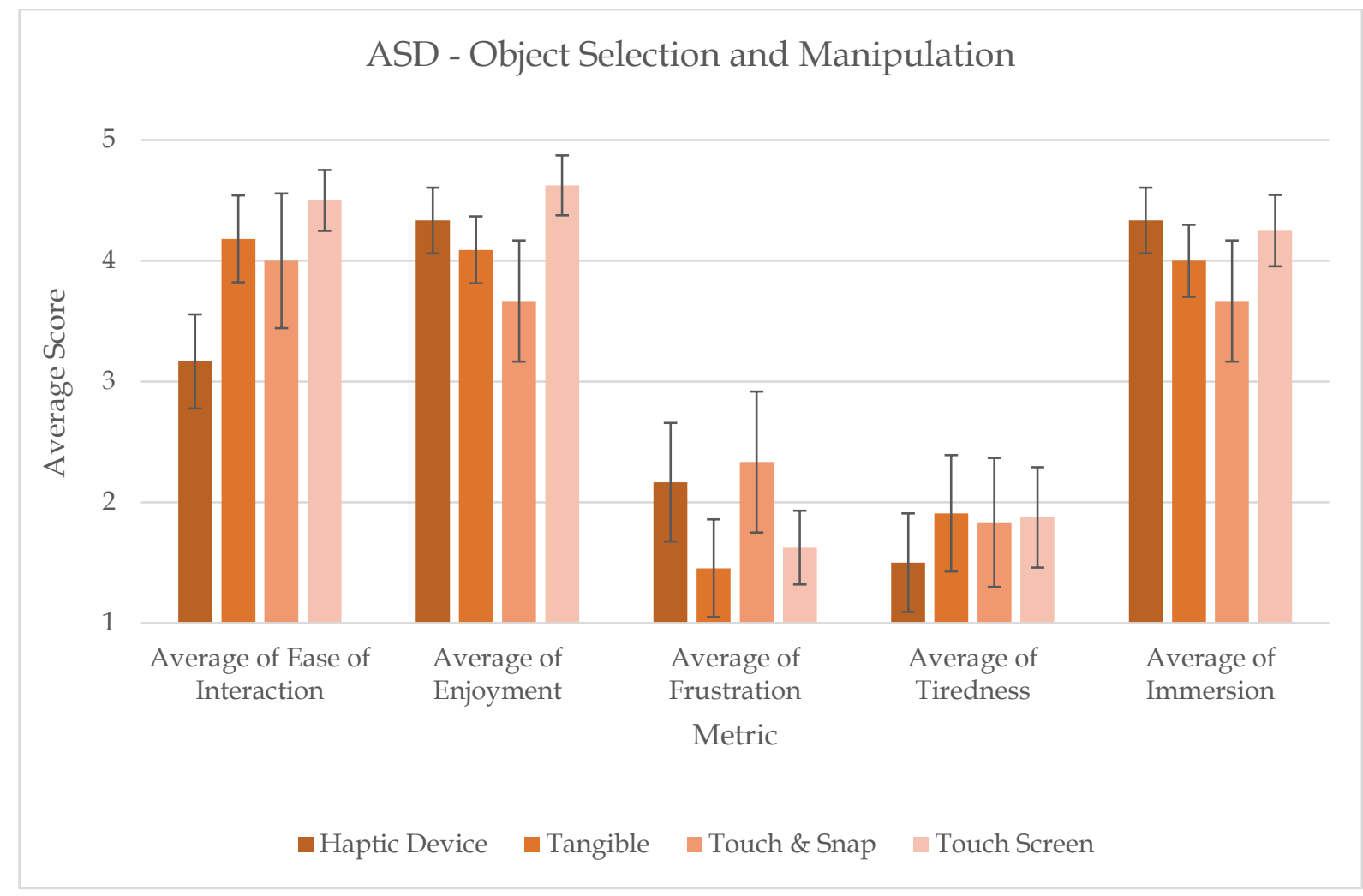

Figure 3-16: Survey results for the selection and manipulation techniques in the VR4VR system for individuals with ASD.

A detailed data analysis was performed on the collected data. The results showed that there were significant differences for the ease of interaction for both population groups. For the neurotypical users, there were also significant difference for frustration and tiredness aspects. The detailed results of the statistical analysis can be found in Appendix E. Further analysis showed that the touchscreen interaction led to a significantly lower frustration than all other techniques for the neurotypical users. Tangible interaction made the neuroty pical users tired as compared to the touch and snap and touchscreen interactions, but the overall results were very low. For the users with 
ASD, the haptic device was found to be significantly hard to interact as compared with the touchscreen.

A chart of the scores are also shown in Figure 3-17 for both neurotypical users and individuals with ASD. Detailed statistical analysis showed that the scores were significantly different for both population groups. The neurotypical users received significantly lower scores with the haptic interaction as compared to all the other interaction techniques. On the other hand, individuals with ASD scored significantly higher with the touch and snap interaction as compared to the other interaction techniques. Detailed analysis results for individuals with ASD can also be seen in Appendix E.

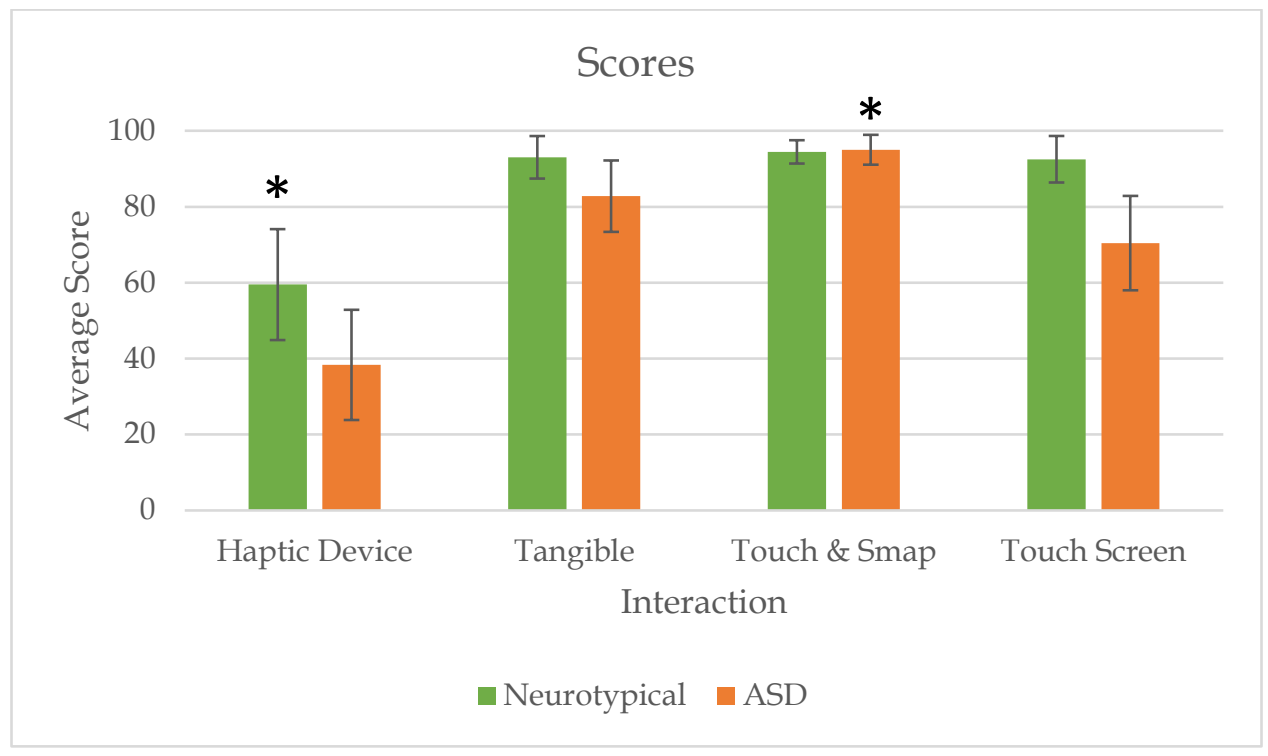

Figure 3-17: Performance scores for different selection and manipulation techniques in the VR4VR system for both population groups.

As the users were presented with all of the object selection and manipulation techniques they have used and asked to select one as their most preferred one, most of 
the users with ASD stated preference for the touchscreen interaction and some users with ASD stated preference for the tangible object manipulation. On the other hand, most of the neurotypical users preferred tangible interaction. None of the users stated preference over haptic device or touch and snap interaction. A chart of the results is presented in Figure 3-18.

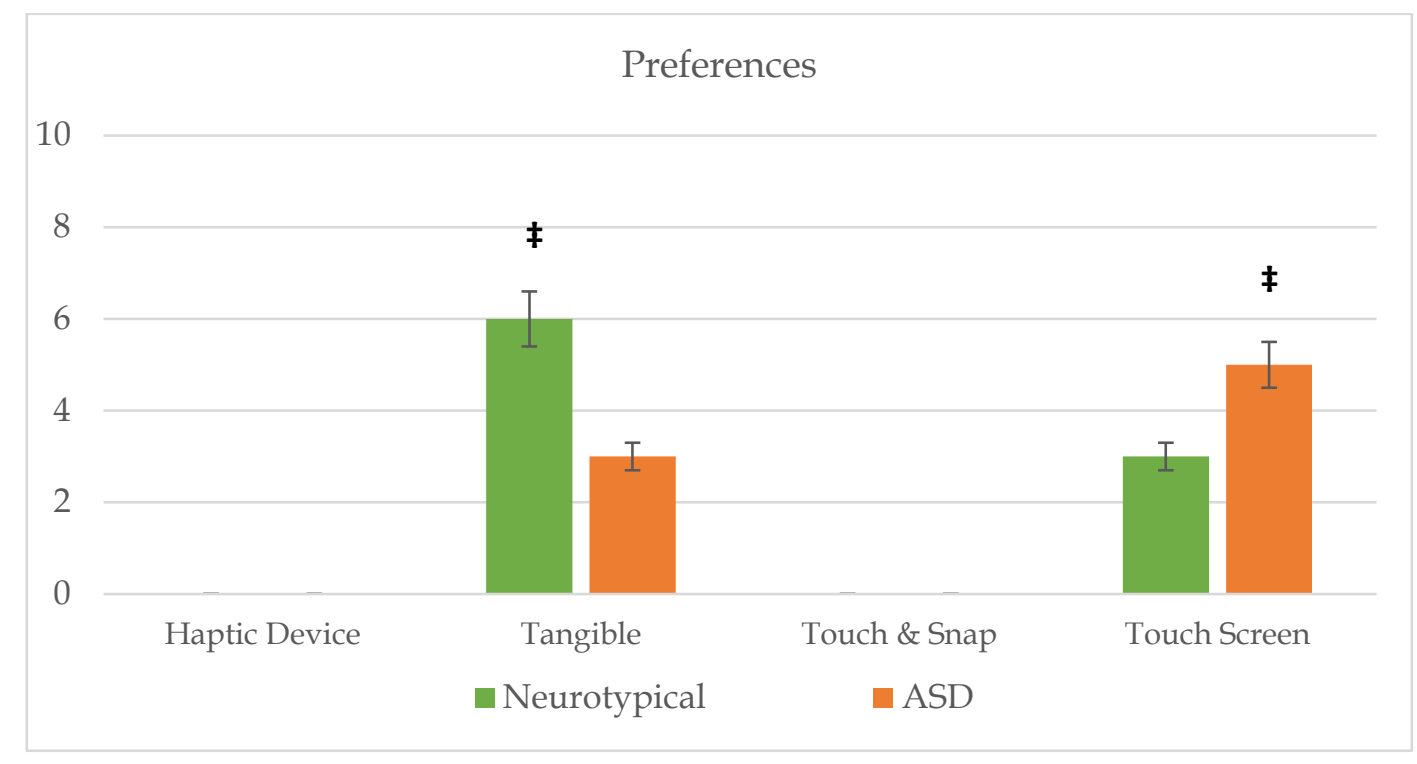

Figure 3-18: Preference of the users on the selection and manipulation techniques in the VR4VR system.

About the selection and manipulation techniques, in the conversations during the breaks, some users stated that they found shelving simplest and easiest to do and manage and some users stated that they thought that the tablet computing cashier was the most fun among the six skill modules.

The job trainers stated that they found all of the object selection and manipulation techniques reasonable to use for the individuals with ASD. They stated that they liked the tangible interaction the most as compared to the other techniques for individuals with 
ASD. They found the tangible interaction very useful for the transition of the training to real life jobs and creating a sense of presence in the users via the tactile feedback. They also said that the touchscreen was very intuitive since it was just like using a smart phone, with which all of our participants with ASD were already familiar. The job trainers finally said that they observed some confusion in some of the users with ASD while using the haptic device but still they did not find it not reasonable for the use of individuals with ASD. They stated that they found the sense of weight applied by the haptic device while carrying objects in the virtual world very useful for contributing to the users' sense of presence. As we offered to switch to the commonly used mouse interaction instead of the haptic device due to the relative low scores in ease of interaction, the job coaches stated that they preferred that we continued using the haptic device for a more effective training for individuals with ASD.

\subsubsection{Display Methods}

The average survey results for the modules with different display methods are presented in this sub-subsection. Figure 3-19 shows the average scores for the neurotypical users and Figure 3-20 shows the average scores for individuals with ASD. Curtain display had higher scores for the ease of interaction for both population groups. The tiredness values were lower for the curtain display for neurotypical individuals as well as individuals with ASD. This was a predicted result since the tasks performed with curtain display required low or no effort. The detailed statistical analysis revealed that for neurotypical users there were significant differences for the ease of interaction, enjoyment, frustrating and tiredness aspects. The performance scores for both population 
groups can be seen in Figure 3-21. Although there was no significant difference between these scores, individuals from both population groups received higher scores in the modules with the HMD as compared to the modules with the curtain display. For the details of the statistical analysis, please see Appendix E.

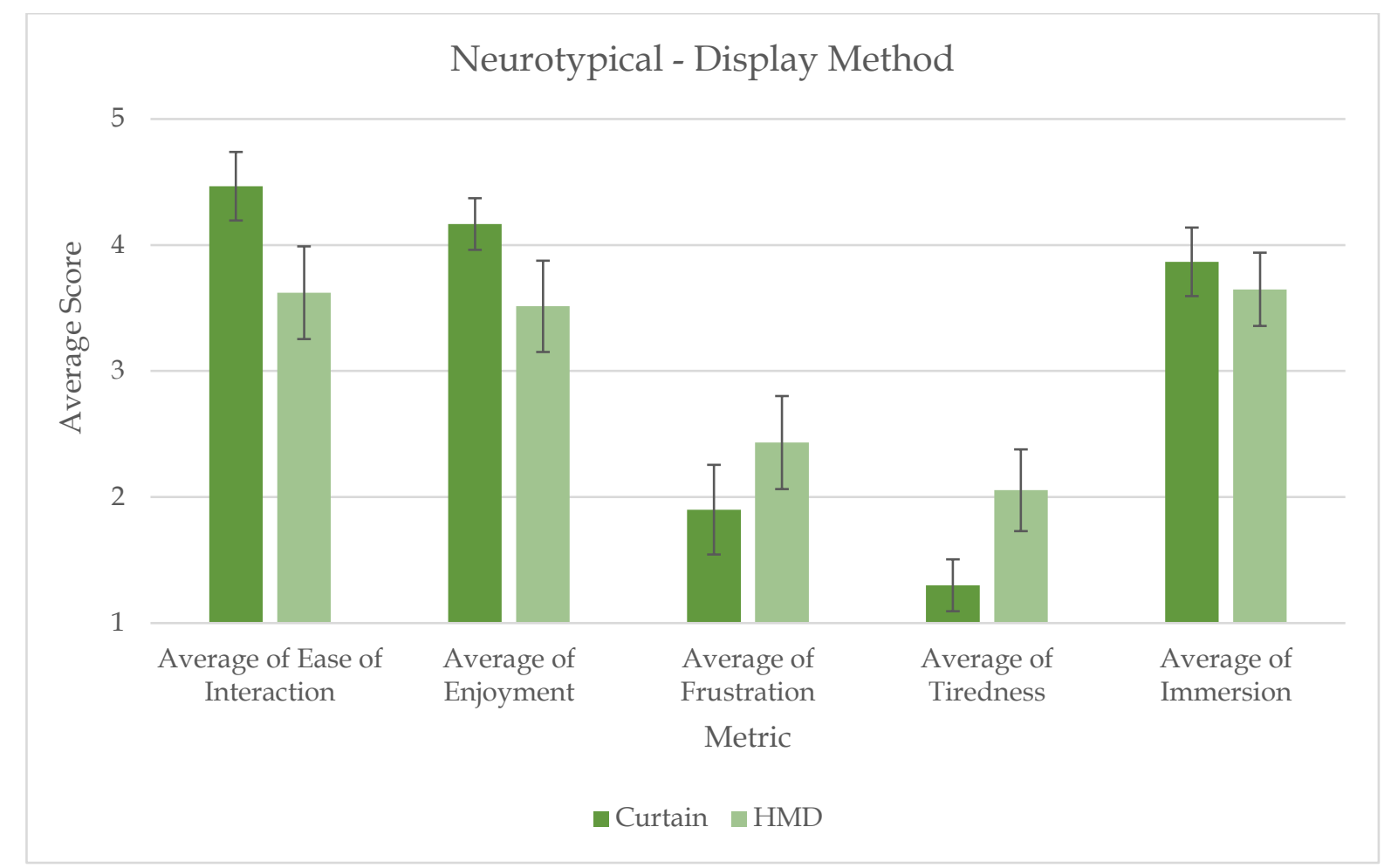

Figure 3-19: Survey results for the display methods in the VR4VR system for neurotypical individuals.

After training, users were inquired about their preference of the display methods: head mounted display and curtain screen. These preference results are presented in Figure 3-22. Most of the participants with ASD stated preference for the curtain display while less participants with ASD stated preference for the head-mounted display. On the other hand, for the neurotypical users, the preference results indicated the opposite. 


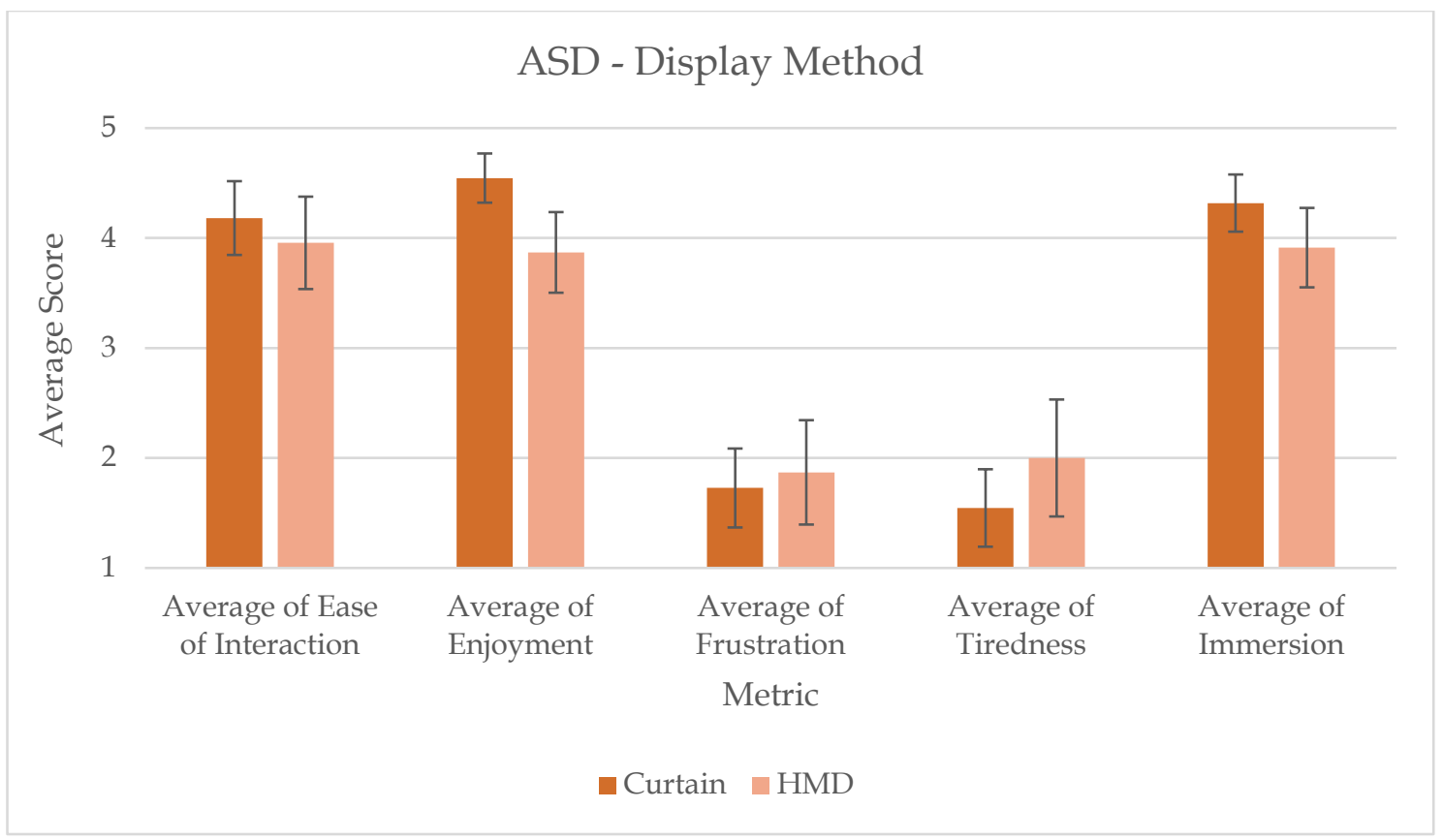

Figure 3-20: Survey results for the display methods in the VR4VR system for individuals with ASD.

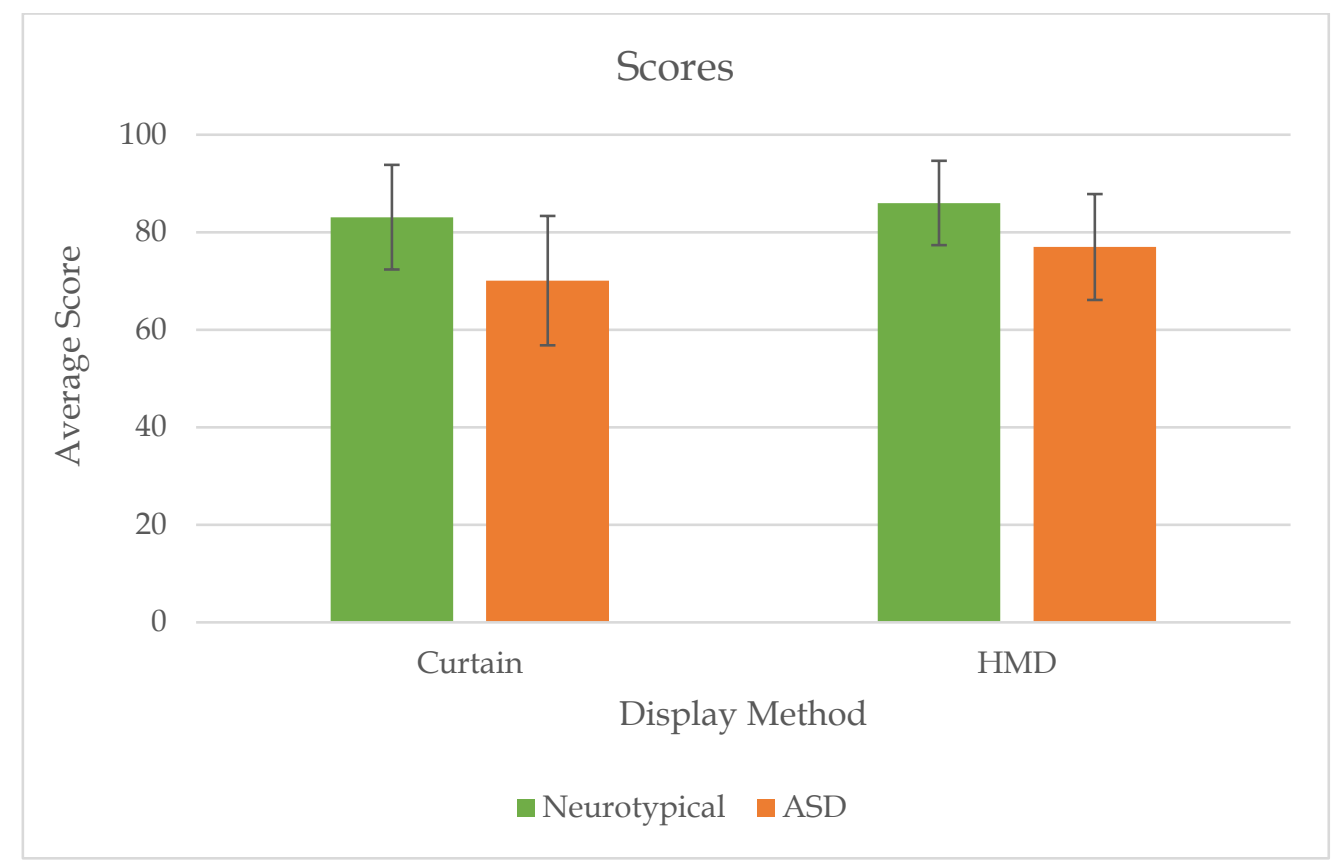

Figure 3-21: Performance scores for the different display methods in the VR4VR system for both populations 
Three out of the nine participants with ASD declared that they felt dizzy and nauseous during and/or after the testing while using the head mounted display. Similarly, four of the ten neurotypical individuals declared that they felt dizzy and nauseous during and/or after the testing while using the head mounted display. Only one participant from each population stated feeling dizzy and/or nauseous while using the curtain screen.

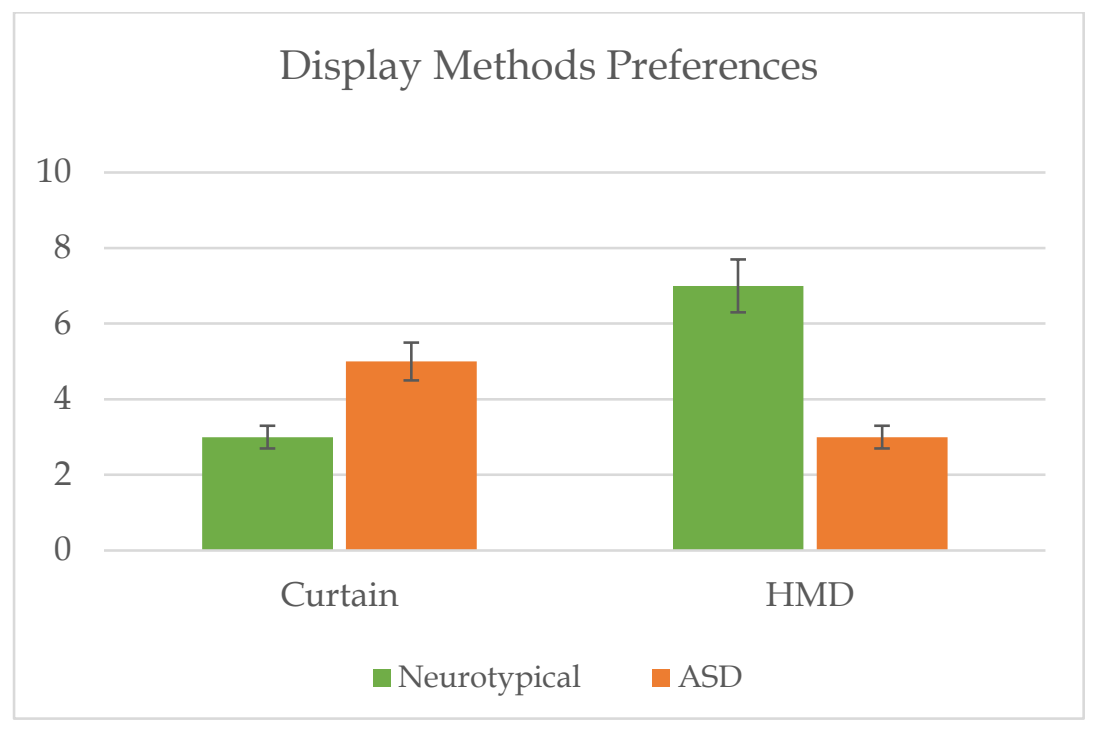

Figure 3-22: Preference of the users on the display methods in the VR4VR system.

As the individuals with ASD were asked about their opinions on the HMD display, only one of them stated that they did not like the concept of the HMD due to its resemblance to a television that was placed very near. The user also stated that their nearsightedness might be the cause of them finding it a bit annoying to use the HMD. Other than that, no users with ASD stated any negative comments about the HMD. We did not observe any problems in the individuals with autism's acceptance of the HMD. All users with ASD made positive comments about the curtain display method. 
Although they were a little bit anxious about the HMD acceptance of individuals with autism, the job trainers did not observe any negative effects of the HMD on users as well. They stated that it was reasonable to continue using HMD as a display for individuals with autism in the VR4VR system, since the users were very quick to adapt to using the HMD although none of them had any prior experience of using it. The job trainers stated positive comments about the curtain display as well.

Below, the user comments stated in the surveys for the locomotion, interaction and display aspects of the VR4VR system are shared for the neurotypical users. Individuals with ASD did not fill out these sections of the surveys and thus were interviewed about these aspects afterwards.

Question: "Have you ever felt limited/restricted while using any of the display/interaction methods? If so, please describe the situation and what made you feel limited."

- UserID 4: "The walk steps made me feel limited and tired."

- UserID 6: "I can't walk very well."

- UserID 8: “Head mounted display, there was some kind of inconvenience not able to move quickly and properly and confidently as well."

- UserID 10: "I felt limited while I was trying to walk in the parking lot."

- UserID 11: “Field of view on head mounted device too narrow. 3D mouse felt strange for 2D application."

- UserID 12: "Yes, walking was a little slow, maybe make more adjustable speeds." 
- UserID 13: “When loading the back of the truck, some of the finer motions were difficult to perform."

Question: "Please describe the most positive experience you had while using the VR4VR system."

- UserID 6: “The tangible boxes were pretty nice. I felt this will help people a lot. I really enjoyed it."

- UserID 10: “The most positive experience was the interaction between the real world and the virtual world."

- UserID 12: "The interactions with the head mounted display."

- UserID 13: "Tangible boxes, the tracking is very good.”

Question: "Please describe the most negative experience you had while using the VR4VR system."

- UserID 12: "Walking."

\subsection{Discussion}

For the locomotion techniques, the most significant difference between real walking and walk-in-place techniques were observed on the frustration scores of the users. Users got more frustrated while they were walking-in-place than real walking. It was hard for the users with ASD to comprehend the walk-in-place gesture and keep doing that locomotion technique without really walking forward in the real world. They gave better scores to real walking for the ease of interaction, enjoyment, and immersion aspects. The users preferred the real walking technique and did not like the walk-in-place technique. These results aligned with $[33,69,130]$. The job trainers found the walk-in- 
place technique difficult to learn and perform for individuals with ASD. Hence, we interpret these results and views of the users and the job trainers as walk-in-place being a questionable locomotion technique to be used for individuals with ASD in virtual reality applications. On the other hand, the real walking locomotion technique was found very comfortable by the job coaches for the use of individuals with ASD as well. We interpret this as real walking being a very suitable method for virtual reality implementations for individuals with autism. Of course this is not easy to achieve due to the limitation imposed by the motion tracking cameras but as a solution, the tasks in the virtual world would be designed such that the users do not need to go outside the physical tracking area in the application.

Among the selection and manipulation techniques, touchscreen received the best result from the users with autism for the ease of interaction and haptic device received the worst score. This may be caused by the users' previous experiences. None of the users with ASD were familiar with the haptic device whereas all of them stated that they used touch enabled devices regularly on a daily basis. We observed that most of our participants with ASD were interacting with their touchscreen phones during the breaks. The same fact may also be the reason why touchscreen also received the best results for the enjoyment, frustration, and presence aspects. Since we were not expecting the touchscreen to get high presence scores, we asked the users with ASD why they gave better results for the presence of touchscreen. The participants expressed the reason as the touchscreen did not require any extra thinking or effort for them to use it. Tangible object manipulation and touch and snap interaction techniques were found to be the most 
tiring interaction techniques. Those results were expected, since they required more physical activities such as carrying the boxes and bending. The users stated positive comments about the tangible object manipulation technique. Some of the users stated that it was much easier for them to interact with the tangible objects in virtual reality than the virtual ones. Some users also stated that the tangible boxes gave them physical cues and it made the tasks easier to perform for them.

None of the users stated preference over the haptic device or touch and snap interaction. We did not observe any difficulties in using the touch and snap interaction. The users with ASD found the haptic device difficult to interact as compared to the other interaction techniques. We observed some difficulty in some users with ASD in using the haptic device. It took longer for the individuals with ASD to get comfortable with using this interaction technique as compared to the other interaction techniques. The job trainers also found the haptic device a bit challenging for the use of individuals with ASD but still thought that the challenging aspect was a positive contribution for their training and the haptic device was a suitable interaction method for them. The job trainers especially liked the sense of weight provided by the haptic device as they saw it as a significant positive contribution to the training of individuals with ASD.

Finally, further data analysis was performed to compare the performance scores between neurotypical individuals and individuals with ASD. The results of this analysis was only shared as supplementary information since the main purpose of this study was not to show the differences between those populations. The results showed significant differences on the performance scores between these populations for the tangible object 
selection and manipulation, touch screen interaction and real walking locomotion techniques. The detailed analysis results can be seen in Appendix E.

In overall, most of the users with ASD preferred the touchscreen over all of the other interaction techniques tested. Although the job trainers stated that they found all of the selection and manipulation techniques suitable for use for individuals with autism, they stated that they found the tangible interaction the most suitable due to the tactile feedback and the similarity to real world interactions. They also found touchscreen interaction familiar and easy to use for individuals with ASD.

We interpret these results as it is better to implement selection and manipulation techniques that utilize commonly used real life interactions such as tangible and touchscreen interaction as much as possible for the effective use of individuals with autism in virtual reality applications.

As the display methods were evaluated, most of the users with ASD preferred the curtain screen over the head mounted display. But as they were interviewed, only one user with ASD stated negative comments about the view through the HMD, mostly about the tired eyes. The job trainers also stated that they found both the curtain display and the HMD suitable to use for individuals with autism. There weren't any acceptance or adjustment time problems, thus we interpret that both the curtain display and the HMD might be used for virtual reality viewing tools for users with ASD. The neurotypical users stated preference for the HMD over the curtain screen. The results were in alignment with $[130,131]$. 


\subsection{Limitations}

The user study was performed with nine individuals with high functioning autism. Thus, it should be avoided to generalize these results. The study aimed at providing general understanding in the use of locomotion, interaction and display methods in virtual reality by individuals with ASD. Another factor to keep in mind is that these interaction techniques were not examined in isolation, but within a larger scope tasks, hence might have been affected by other factors such as the task design and different virtual environments. Even still, the long hours of testing sessions with the participants gave us and the job trainers the opportunity to observe, discuss and have an initial idea on the suitability of the several virtual reality locomotion, interaction and display methods for the use of individuals with autism.

\subsection{Summary and Conclusion}

Locomotion and interaction techniques constitute a crucial part of the user experience in virtual reality applications. While a good design of locomotion and interaction techniques may enhance the experience and make it seamless for the users, a bad design may have serious negative effects on the user experience. Individuals with autism have their own characteristics and preferences that are related to their information processing. This makes the effectiveness of the previously proven virtual reality interaction techniques for neurotypical individuals speculative when it comes to individuals with autism.

This study aims at evaluating different virtual reality interaction techniques for individuals with autism within the VR4VR system. Several techniques of locomotion, 
object selection and manipulation interaction, and display methods were implemented and tested in different contexts. User experience with these interaction techniques were explored with a user study of nine individuals with high functioning ASD with accompanying professional job trainers. For the object selection and manipulation, touchscreen and tangible interaction methods were preferred by the individuals with ASD. The walk-in-place locomotion technique were found frustrating and difficult to perform by the individuals with ASD. Curtain display method received higher preference scored from individuals with ASD although they accepted the HMD as well. The results and the opinions of the users and the job trainers contributed to the inference that users with autism prefer the most realistic and real life linkable locomotion and interaction techniques in virtual reality while having some difficulties with the gesture based and more abstract ones. 


\section{CHAPTER 4: IMPLEMENTATION OF THE LOCOMOTION TECHNIQUES}

This chapter presents the implementation of the locomotion techniques that were evaluated with user studies in this dissertation. The technical implementation details are included with code excerpts and descriptive visualizations. The following topics are discussed: system hardware and software, general implementation information, redirected walking, walk-in-place, stepper machine, point \& teleport, joystick, trackball, hand flapping and flying.

\subsection{System Hardware and Software}

The tracking system that was used for the experiments was an optical motion tracking system with 12 cameras (see Figure 4-1) and passive reflective markers for head, hands and feet tracking (see Figure 4-3). A head mounted display (Figure 4-2) was used for display purposes. The tracking area was a $2 \mathrm{~m}$ by $2 \mathrm{~m}$ square area where the user could freely move.

Specifications of the motion tracking cameras were as follows:

- OptiTrack V100R2:FLEX

- $640 \times 480$ resolution

- 100FPS

- Sub-millimeter accuracy

- Latency: $10 \mathrm{~ms}$ 


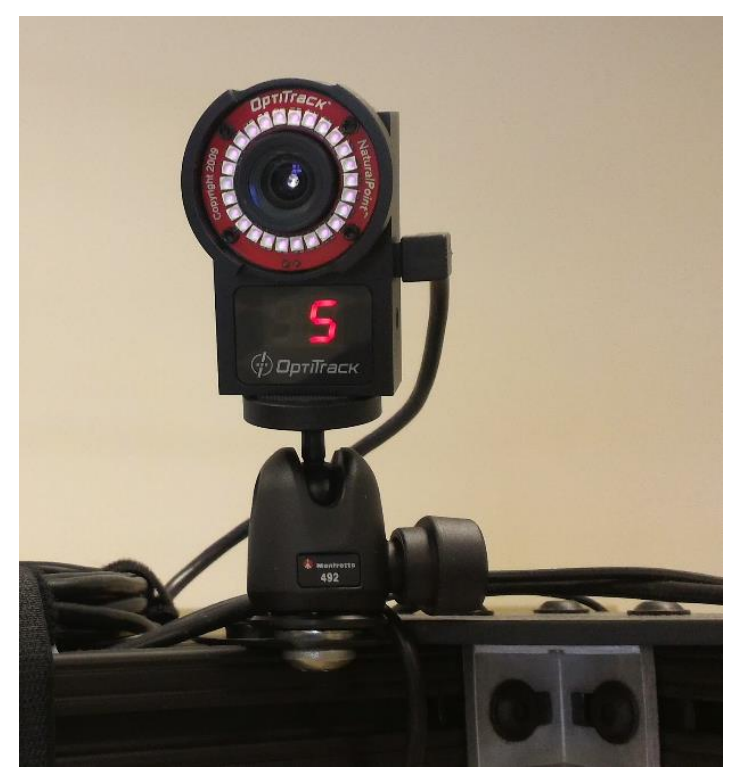

Figure 4-1: An infrared camera used in the motion tracking system.

Specifications of the head mounted display (HMD) were as follows:

- Virtual Realities LLC. VR2200

- Resolution: $1024 \times 768$ XGA

- Field of View: 45 Degrees Diagonal

- Input Signal: VGA, DVI and RCA Composite - NTSC/PAL

The main program was implemented with the Unity game engine. All of the locomotion techniques and the virtual world was developed using C\# in Visual Studio with the Unity game engine integration. For the tracking software, Motive was used which triangulated the marker positions and sent the 3D world coordinates of the marker positions. The calibration process was done by a standard marker set which was captured by the surrounding infrared cameras. The relative positions of the cameras with respect to each other was calculated. After the calculation, another standard marker set was used 
to mark the origin position. The origin position was marked as the ground at the center of the tracking area.

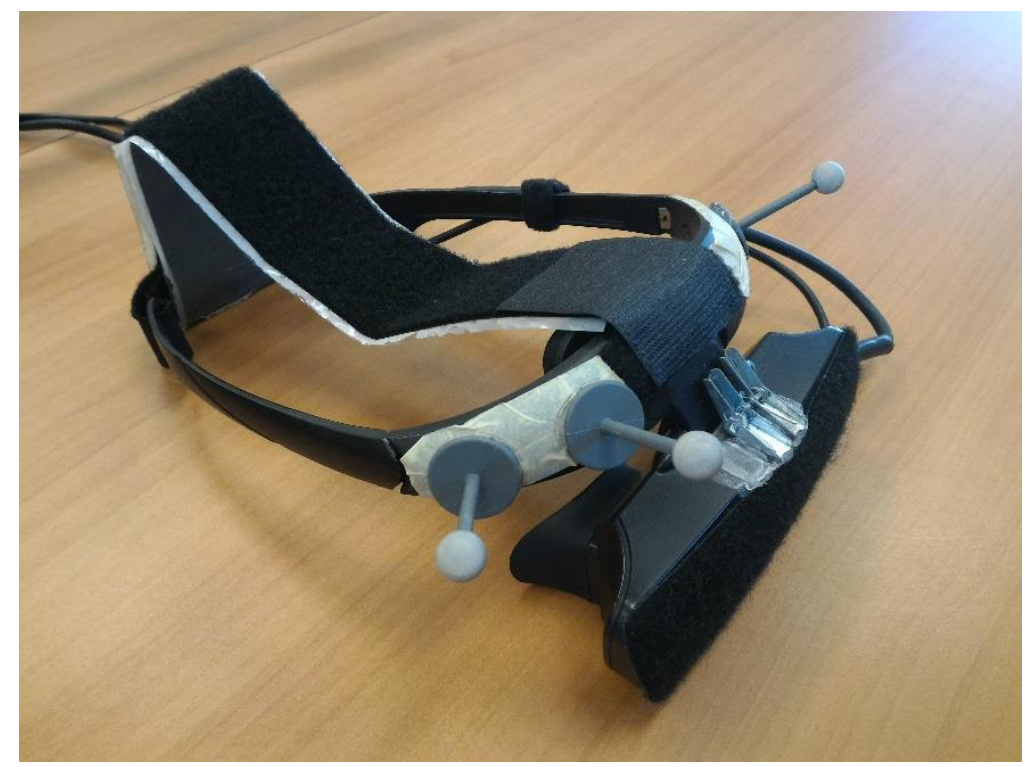

Figure 4-2: Head mounted display that was used in the user studies.

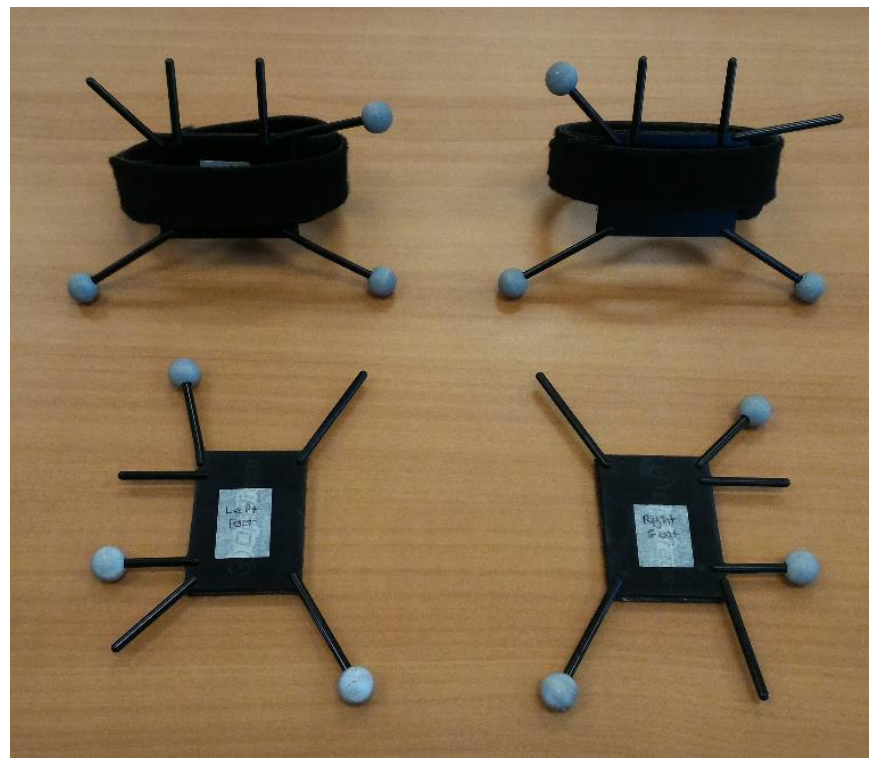

Figure 4-3: Hand and foot markers that were used in the user studies. 


\subsection{General Implementation}

In the game engine, the virtual and real character positions of the user were placed in different areas. The reason was, in some techniques, the displacement of the virtual character was different than the real user. It was also helpful for the virtual environments with physical objects around. With this technique the virtual character could be kept away from penetrating into the solid objects. The white circle with $1 \mathrm{~m}$ radius was the fictional area for the real user and the square area $(16 \mathrm{~m} \times 16 \mathrm{~m})$ was the total virtual environment for the virtual character to explore (see Figure 4-4).

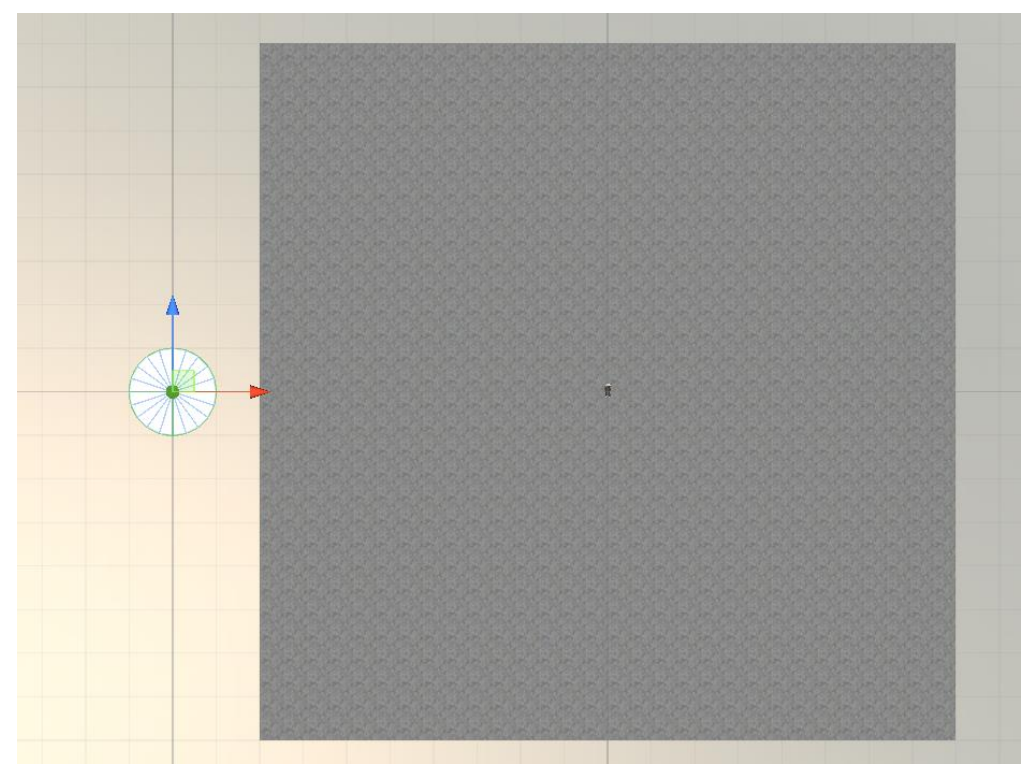

Figure 4-4: Fictional area for the real user and the total virtual environment.

The obvious size difference between the real tracked area and the virtual world could be overcome by utilizing different locomotion techniques. Without special locomotion techniques, the users could only explore a small portion of the large virtual 
world. The implementation of these locomotion techniques will be explained in the following subsections.

The required position and rotation data was captured by optical motion tracking system. Due to the limitations imposed by the tracking system, we could not track all the joints of the user. Instead, five important points were equipped with markers and captured by the system. These five points were:

- Head

- Right Hand

- Left Hand

- Right Foot

- Left Foot

The variables that were related to the user's tracked position and virtual position are listed in Table 4-1.

Table 4-1: Real and virtual position and forward direction variables.

Po Origin position of the tracking system $(0,0,0)$ and the origin of the fictional area for the real user.

$\mathrm{P}_{\mathrm{H}-\mathrm{O}} \quad$ Real position of the head with respect to the $\mathrm{P}_{\mathrm{O}}$

$\mathrm{P}_{\mathrm{RH}-\mathrm{O}} \quad$ Real position of the right hand with respect to the $\mathrm{P}_{\mathrm{O}}$

$\mathrm{P}_{\mathrm{LH}-\mathrm{O}} \quad$ Real position of the left hand with respect to the $\mathrm{P}_{\mathrm{O}}$

PRF-O Real position of the right foot with respect to the $P_{O}$

$\mathrm{P}_{\mathrm{LF}-\mathrm{O}} \quad$ Real position of the left foot with respect to the $\mathrm{P}_{\mathrm{O}}$

$P_{R} \quad$ Reference position of the real user with respect to the $P_{O}$. 
Table 4-1 (Continued)

$\mathrm{P}_{\mathrm{H}} \quad$ Real position of the head with respect to the $\mathrm{P}_{\mathrm{R}}$.

$P_{R H} \quad$ Real position of the right hand with respect to the $P_{R}$.

$\mathrm{P}_{\mathrm{LH}} \quad$ Real position of the left hand with respect to the $P_{\mathrm{R}}$.

$\mathrm{P}_{\mathrm{RF}} \quad$ Real position of the right foot with respect to the $\mathrm{P}_{\mathrm{R}}$.

$P_{L F} \quad$ Real position of the left foot with respect to the $P_{R}$.

po Origin position of the virtual environment.

$\mathrm{p}_{\mathrm{R}} \quad$ Reference position of the virtual character with respect to the $\mathrm{p}_{\mathrm{O}}$.

$\mathrm{p}_{H} \quad$ Virtual position of the head with respect to the $\mathrm{p}_{\mathrm{R}}$.

$\mathrm{p}_{\mathrm{RH}} \quad$ Virtual position of the right hand with respect to the $\mathrm{p}_{\mathrm{R}}$.

$\mathrm{p}_{\mathrm{LH}} \quad$ Virtual position of the left hand with respect to the $\mathrm{p}_{\mathrm{R}}$.

$\mathrm{p}_{\mathrm{RF}} \quad$ Virtual position of the right foot with respect to the $\mathrm{p}_{\mathrm{R}}$.

$\mathrm{p}_{\mathrm{LF}} \quad$ Virtual position of the left foot with respect to the $\mathrm{p}_{\mathrm{R}}$

$\mathrm{D}_{\mathrm{H}-\mathrm{O}} \quad$ Real forward direction of the head with respect to the $\mathrm{P}_{\mathrm{O}}$

DRH-O Real forward direction of the right hand with respect to the PO.

D LH-O Real forward direction of the left hand with respect to the Po.

DRF-O Real forward direction of the right foot with respect to the $P_{\mathrm{O}}$.

DLf-O Real forward direction of the left foot with respect to the PO.

$D_{R} \quad$ Reference forward direction of the real user with respect to the $P_{O}$.

$D_{H} \quad$ Real forward direction of the head with respect to the $P_{R}$.

$D_{R H} \quad$ Real forward direction of the right hand with respect to the $P_{R}$.

$D_{\mathrm{LH}} \quad$ Real forward direction of the left hand with respect to the $P_{R}$.

$D_{\mathrm{RF}} \quad$ Real forward direction of the right foot with respect to the $P_{\mathrm{R}}$. 


\section{Table 4-1 (Continued)}

$D_{\mathrm{LF}} \quad$ Real forward direction of the left foot with respect to the $P_{\mathrm{R}}$.

$\mathrm{d}_{\mathrm{H}} \quad$ Virtual forward direction of the head with respect to the $\mathrm{p}_{\mathrm{R}}$

$\mathrm{d}_{\mathrm{RH}} \quad$ Virtual forward direction of the right hand with respect to the $\mathrm{p}_{\mathrm{R}}$.

$\mathrm{d}_{\mathrm{LH}} \quad$ Virtual forward direction of the left hand with respect to the $\mathrm{p}_{\mathrm{R}}$.

$d_{R F} \quad$ Virtual forward direction of the right foot with respect to the $p_{R}$.

$d_{L F} \quad$ Virtual forward direction of the left foot with respect to the $p_{R}$.

The real position data was captured with respect to the origin point of the motion tracking system. The origin point was set to the ground at the center of the tracking area during the calibration process. The center of the tracking area was also assumed to be the center of the fictional area for the real user. Since the ground had the same height for both cases $(0 \mathrm{~m})$, the height of the real positions (y-axis) of the points were the same with the height of the virtual objects. But the other axes ( $\mathrm{x}$ - axis and $\mathrm{z}$ - axis) were changed for the virtual character. Similarly, all the rotations were changed according the technique implementation.

\subsubsection{Real User}

The real user was a fictional character which was composed of five position data. The reference position of the real user was estimated by using the five position readings. These readings were relative to the origin point (see Figure 4-5 for an explanative illustration). 


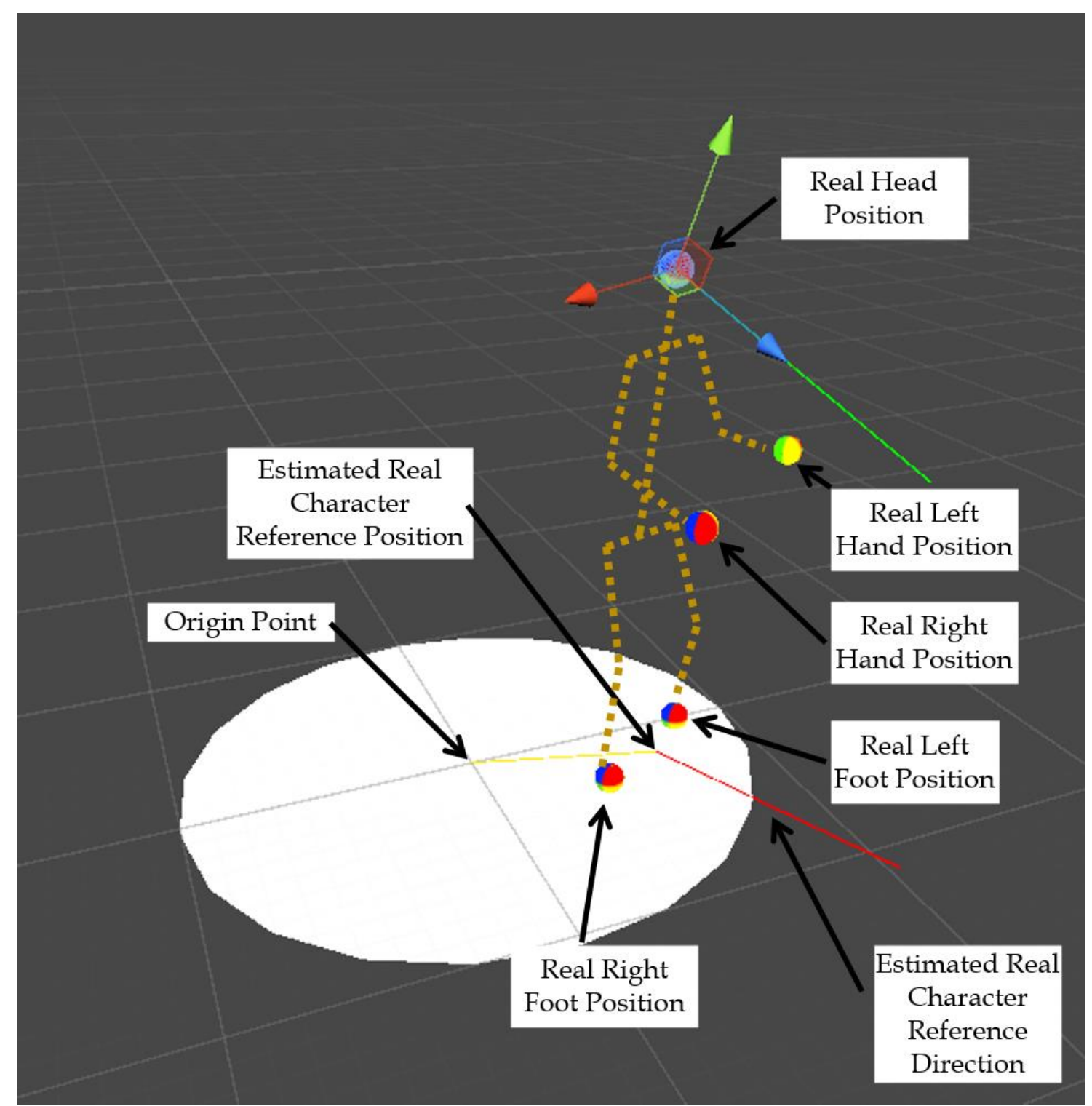

Figure 4-5: Real user position readings and the estimated reference positions and direction.

The reference position and the reference direction of the real user was calculated by taking the average of the five positions with different weights for different points.

$$
P_{R}=\frac{\sum P_{i-O} * W_{i}}{\sum W_{i}}=\frac{P_{H-O} * W_{H}+P_{R H-O} * W_{R H}+P_{L H-O} * W_{L H}+P_{R F-O} * W_{R F}+P_{L F-O} * W_{L F}}{W_{H}+W_{R H}+W_{L H}+W_{R F}+W_{L F}}
$$




$$
\begin{aligned}
& D_{R}=\frac{\sum D_{i-O} * W R_{i}}{\sum W R_{i}} \\
& =\frac{P_{H-O} * W R_{H}+P_{R H-O} * W R_{R H-O}+P_{L H} * W R_{L H-O}+P_{R F} * W R_{R F-O}+P_{L F} * W R_{L F-O}}{W R_{H}+W R_{R H}+W R_{L H}+W R_{R F}+W R_{L F}}
\end{aligned}
$$

The weights that were used were as follows: $\mathrm{W}_{\mathrm{H}}=5.0, \mathrm{~W}_{\mathrm{RF}}=\mathrm{W}_{\mathrm{LF}} 1.0, \mathrm{~W}_{\mathrm{RH}}=\mathrm{W}_{\mathrm{LH}}$ $=0.0, \mathrm{WR}_{\mathrm{H}}=1.0, \mathrm{WR}_{\mathrm{RF}}=\mathrm{WR}_{\mathrm{LF}} 0.0$, and $\mathrm{WR}_{\mathrm{RH}}=\mathrm{WR}_{\mathrm{LH}}=0.0$.

These weights were decided after in-house testing sessions. For the position weights, the head position was chosen to be larger than the foot weights, so that the reference position of the user could be largely determined by the position of the head instead of the feet. This approach made the reference position more stable while the user was moving in the system. Similarly, no weights were given to the hands. This also helped with the stability when the user reached somewhere or while walking with their hands swinging.

For the rotation weights, only the head weight was used after in-house testing sessions. The forward direction was used for moving direction in most of the locomotion techniques. In those cases, the head direction was more intuitive for the users to feel the walking direction. Although they could not move in a different direction than their looking direction, with this approach, they could see anything on their ways. The direction was projected to the $\mathrm{x}-\mathrm{z}$ plane and normalized. The code excerpt can be seen in Figure 4-6. 


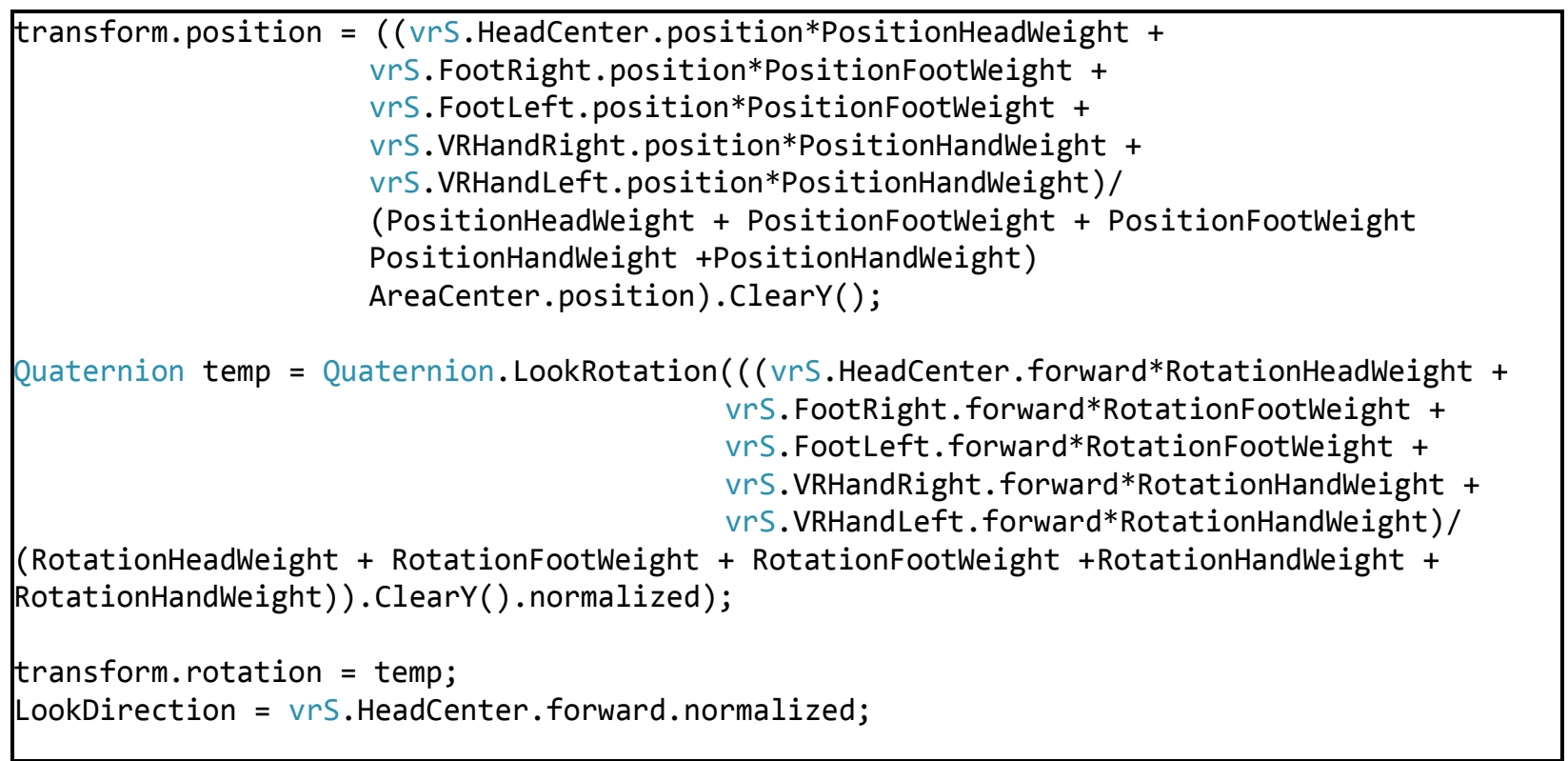

Figure 4-6: Code excerpt from the real user implementation.

After calculating the reference points and the reference direction of the real user, each position of the tracked points was recalculated with respect to the reference point.

$$
\begin{gathered}
P_{H}=P_{H-O}-P_{R} \\
P_{R H}=P_{R H-O}-P_{R} \\
P_{L H}=P_{L H-O}-P_{R} \\
P_{R F}=P_{R F-O}-P_{R} \\
P_{L F}=P_{L F-O}-P_{R}
\end{gathered}
$$

Similarly, the forward directions were recalculated with respect to the reference forward direction.

$$
\begin{gathered}
D_{H}=D_{H-O}-D_{R} \\
D_{R H}=D_{R H-O}-D_{R} \\
D_{L H}=D_{L H-O}-D_{R}
\end{gathered}
$$




$$
\begin{aligned}
& D_{R F}=D_{R F-O}-D_{R} \\
& D_{L F}=D_{L F-O}-D_{R}
\end{aligned}
$$

\subsubsection{Virtual Character}

For the virtual position of the user and the five tracked objects, the following position and rotation values were used.

$$
\begin{gathered}
p_{H}=P_{H} \\
p_{R H}=P_{R H} \\
p_{L H}=P_{L H} \\
p_{R F}=P_{R F} \\
p_{L F}=P_{L F} \\
d_{H}=D_{H} \\
d_{R H}=D_{R H} \\
d_{L H}=D_{L H} \\
d_{R F}=D_{R F} \\
d_{L F}=D_{L F}
\end{gathered}
$$

The virtual position and the rotation of objects with respect to virtual reference point were kept equal to the real position and rotation of the objects with respect to the real reference point.

3D models of the hands and the feet were placed to the corresponding virtual positions to provide the users with a view of their virtual hands and feet at any time for a more realistic and immersive experience (see Figure 4-7). 


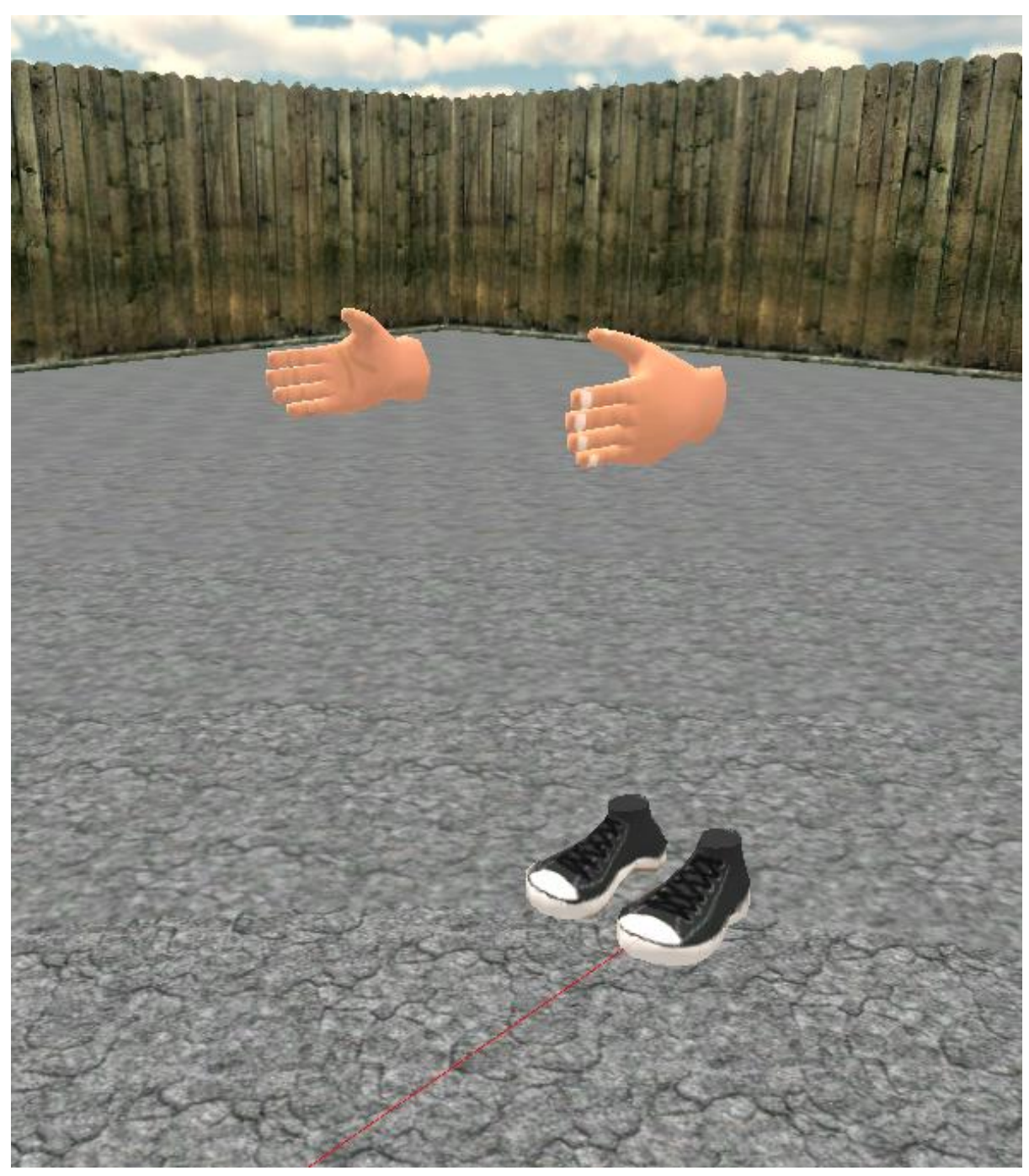

Figure 4-7: Virtual hands and feet in the virtual world.

The code excerpt for the virtual character parameter calculations can be seen in Figure 4-8. The real and calculated vectors for the virtual character positions can be seen in Figure 4-9.

With this approach, the position of the virtual character was simplified from the combination of five positions and rotations to a single position and rotation while keeping the distances between each tracked object unchanged. Another advantage of this 
approach was that it helped resolving the collisions with the virtual character and the virtual objects in the virtual world. If there was a virtual object in front of the virtual character, even if the real user walked forward, the virtual character was blocked by the virtual object. This phenomenon is illustrated in the Figure 4-10.

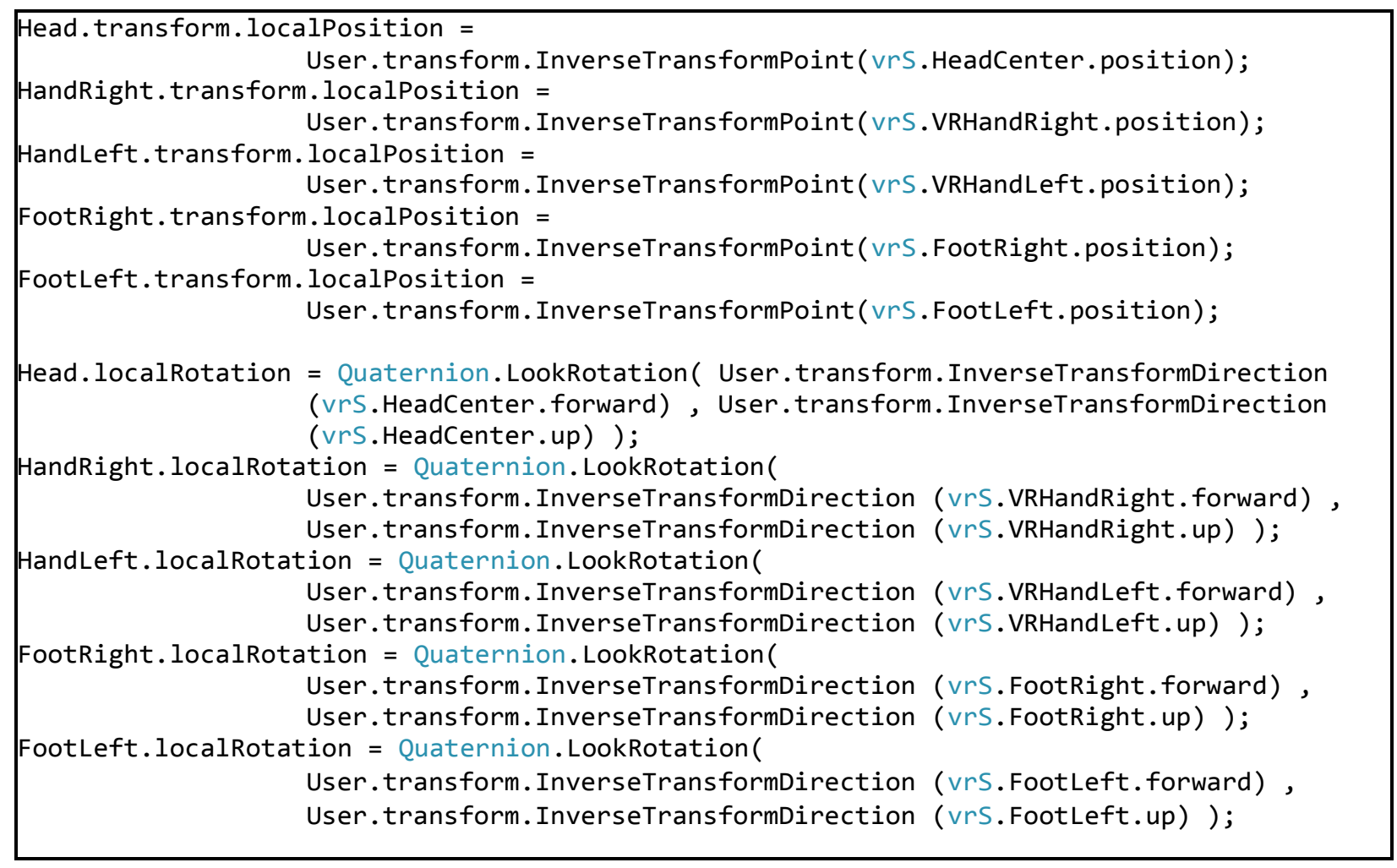

Figure 4-8: Code excerpt from the virtual character implementation.

The virtual character was equipped with a character collider. The required displacements and the rotations were performed on this character collider. Furthermore, the virtual collisions were checked between the virtual colliders on the virtual objects and the character collider. 


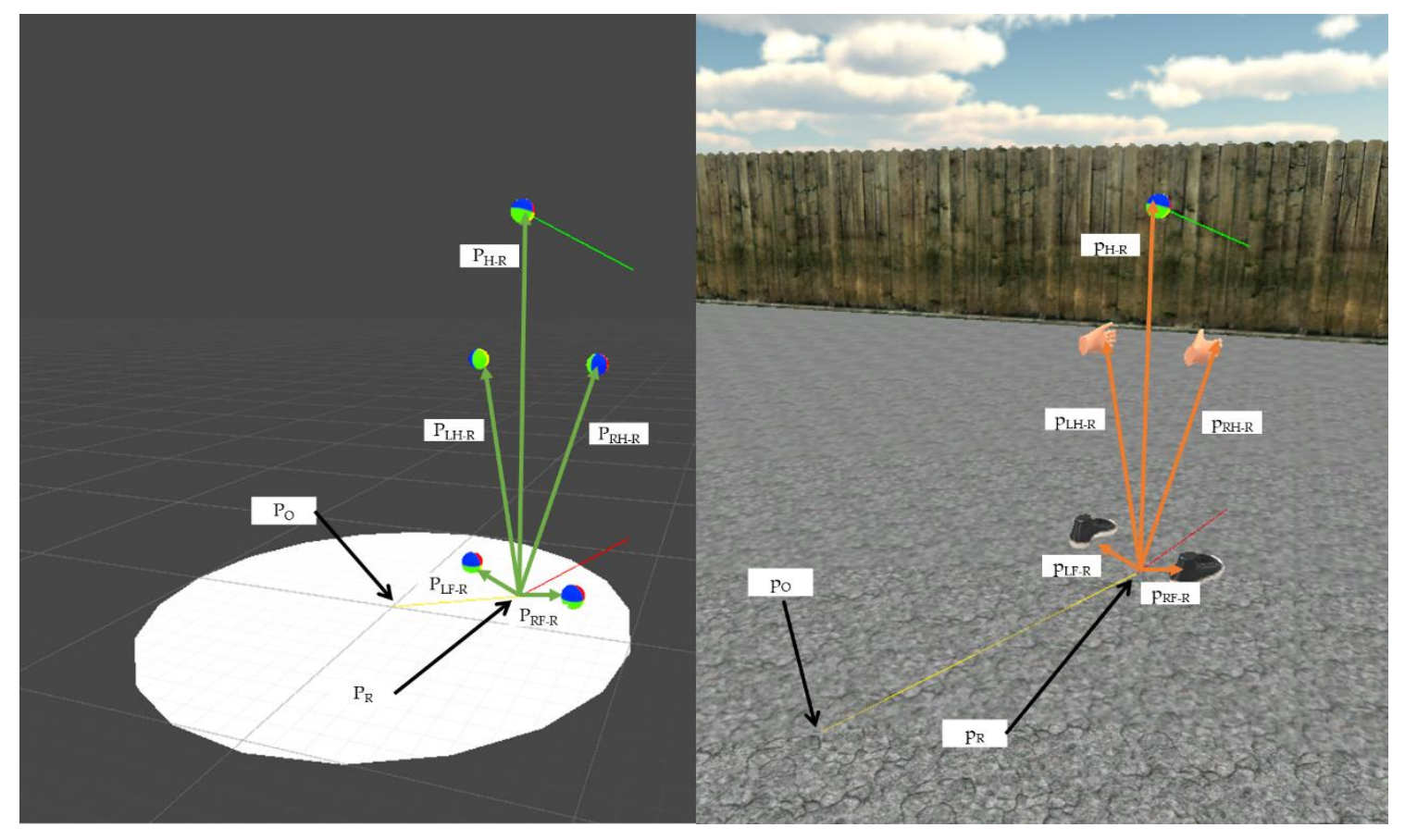

Figure 4-9: Real and calculated virtual character positions.
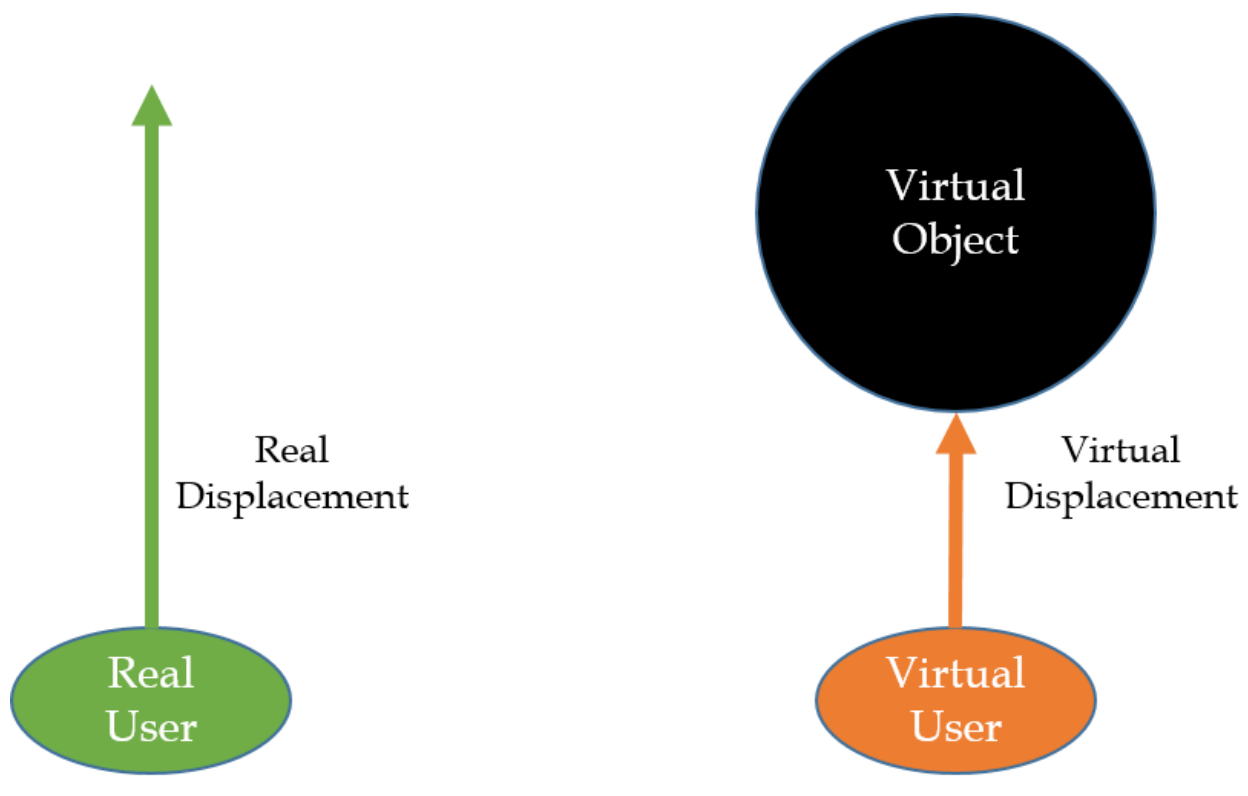

Figure 4-10: Real vs. virtual character movements. 


\subsubsection{Position Warnings}

The reference position of the real user $\left(\mathrm{P}_{\mathrm{R}}\right)$ was used for estimating real position of user on actual tracking area. In every frame, the magnitude of the $P_{R}$ was calculated and if it was greater than $0.8 \mathrm{~m}$, a warning sign was displayed on the HMD to warn the user and encourage them to move to the center of the tracking area (see Figure 4-11). The warning sign was kept on the display until the value of the $P_{R}$ was smaller than $0.3 \mathrm{~m}$.

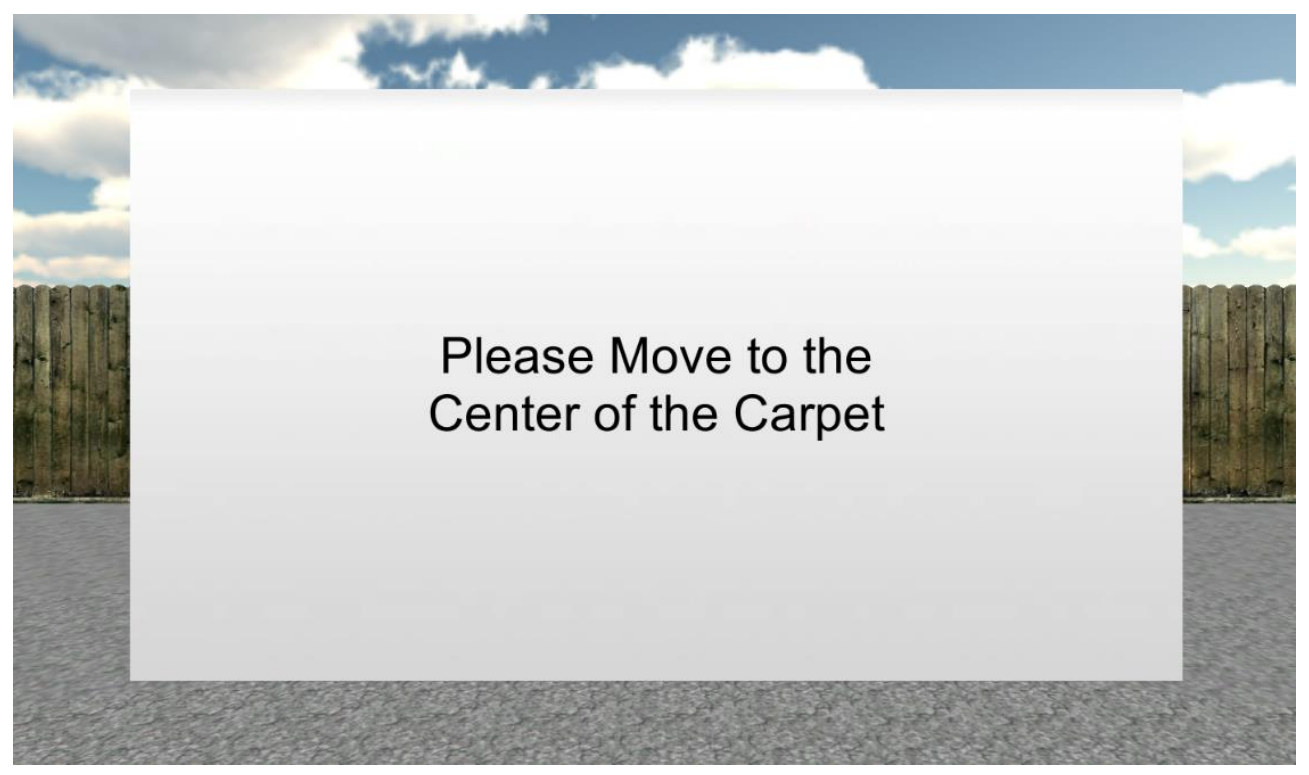

Figure 4-11: The virtual position warning.

\subsubsection{Data Recording}

The real and the virtual reference positions were recorded during all testing sessions. Each time a new session with a new locomotion technique was started, a new text document with a time stamp was created. The positions were written in every $0.1 \mathrm{sec}$ and were saved after the session. These data were used to sketch the walking paths in the real tracked area and virtual environment. 
A sample output of the program can be seen in Figure 4-12. The presented portion was taken from a redirected walking locomotion technique session. First, the file name, which was the date and time combination, and the positions of all the destination points were listed. Then, the real time timestamps and the actions were listed. The actions that were included in the output file were as follows:

- Destination point appearance

- Destination point disappearance

- Users entering the destination area

- Users exiting the destination area

- Hit with border walls

- Hit with static obstacles

- Hit with dynamic obstacle (Available in redirected walking)

- Hit with maze walls (Available in maze experiment)

- Position warning

- Start of a session

- End of a session

In addition to these collected data, in a separate file, the position data for the real user and virtual avatar was stored (for a sample portion of these files, see Figure 4-13). For each technique session, a separate file was created. The file contained the $\mathrm{x}$ and $\mathrm{y}$ coordinates of both real and virtual character in the real and virtual environment. The position data was collected roughly in every $0.1 \mathrm{sec}$, and the timestamps were saved as 
well. This data was combined with the overall data and the walking paths of the users were generated.

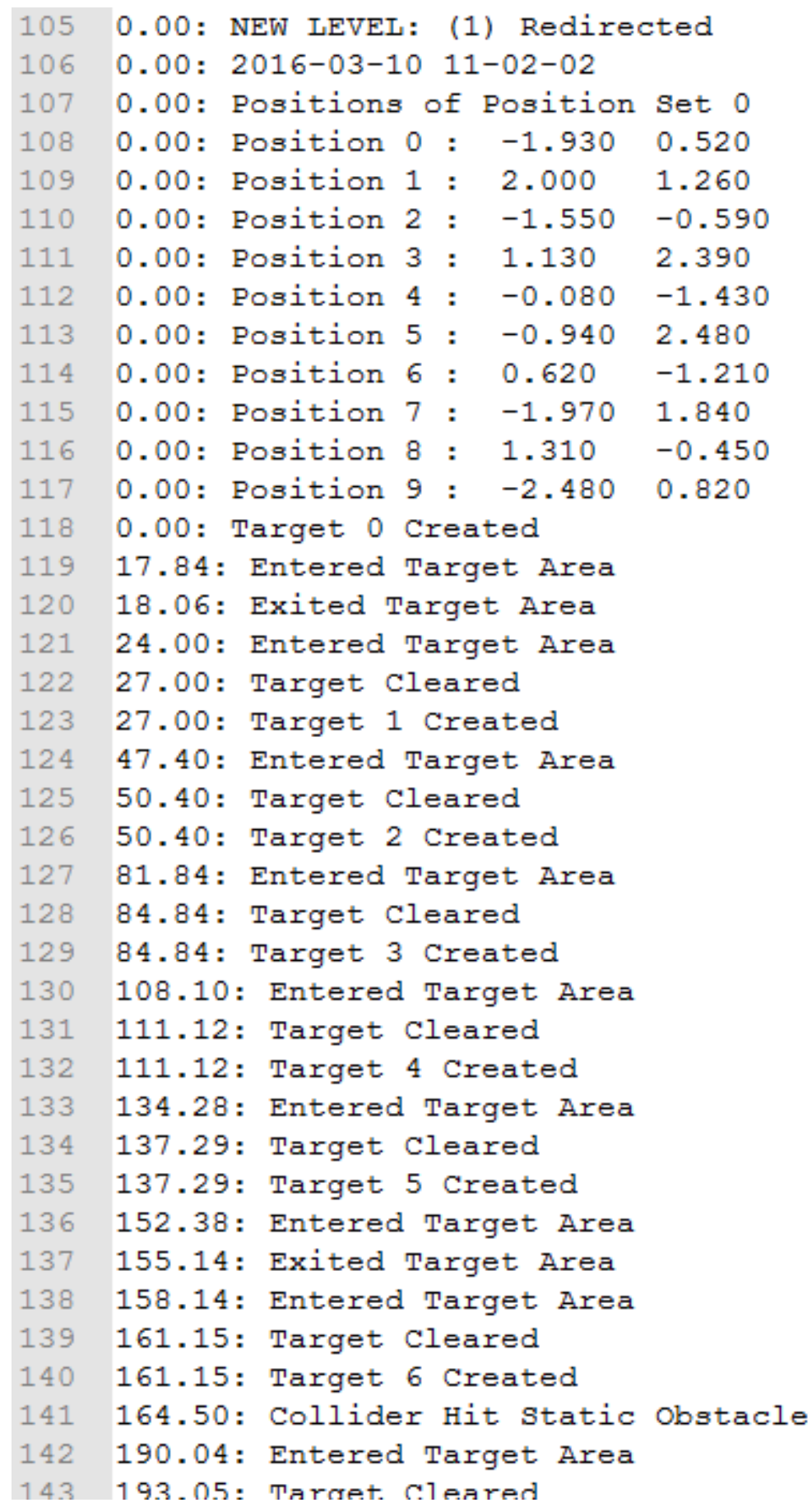

Figure 4-12: A sample output of the program. 


\begin{tabular}{rlllll}
1 & \multicolumn{5}{l}{ Positions } \\
2 & Time & User Positions & Avatar & Position \\
3 & 0.00 & 0.000 & 0.000 & 0.000 & 0.000 \\
4 & 0.10 & -0.087 & -0.206 & -0.456 & -0.772 \\
5 & 0.21 & -0.087 & -0.206 & -0.456 & -0.770 \\
6 & 0.31 & -0.087 & -0.205 & -0.455 & -0.770 \\
7 & 0.41 & -0.086 & -0.207 & -0.453 & -0.776 \\
8 & 0.52 & -0.084 & -0.208 & -0.449 & -0.780 \\
9 & 0.63 & -0.082 & -0.206 & -0.445 & -0.778 \\
10 & 0.74 & -0.081 & -0.205 & -0.441 & -0.776 \\
11 & 0.85 & -0.080 & -0.203 & -0.437 & -0.773 \\
12 & 0.95 & -0.080 & -0.201 & -0.437 & -0.769 \\
13 & 1.06 & -0.082 & -0.199 & -0.439 & -0.763 \\
14 & 1.17 & -0.084 & -0.197 & -0.444 & -0.757 \\
15 & 1.27 & -0.086 & -0.195 & -0.447 & -0.750 \\
16 & 1.38 & -0.087 & -0.193 & -0.449 & -0.744 \\
17 & 1.48 & -0.088 & -0.191 & -0.450 & -0.740 \\
18 & 1.58 & -0.088 & -0.191 & -0.451 & -0.739 \\
19 & 1.69 & -0.088 & -0.190 & -0.450 & -0.738 \\
20 & 1.79 & -0.087 & -0.191 & -0.448 & -0.742 \\
21 & 1.90 & -0.087 & -0.191 & -0.448 & -0.742 \\
22 & 2.01 & -0.089 & -0.187 & -0.450 & -0.731 \\
23 & 2.13 & -0.092 & -0.183 & -0.457 & -0.721 \\
24 & 2.24 & -0.095 & -0.181 & -0.464 & -0.714 \\
25 & 2.35 & -0.098 & -0.180 & -0.472 & -0.710 \\
26 & 2.46 & -0.100 & -0.179 & -0.479 & -0.708 \\
27 & 2.57 & -0.101 & -0.179 & -0.484 & -0.706 \\
28 & 2.68 & -0.103 & -0.178 & -0.487 & -0.704 \\
29 & 2.79 & -0.104 & -0.176 & -0.492 & -0.701 \\
30 & 2.89 & -0.106 & -0.174 & -0.500 & -0.697 \\
31 & 3.00 & -0.103 & -0.171 & -0.496 & -0.689 \\
22 & 2.10 & -0.096 & -0164 & -0.187 & -0.671
\end{tabular}

Figure 4-13: A sample position data.

\subsubsection{Virtual Cameras}

The perspective cameras were positioned at the head node of the virtual character where the eyes should be placed. The positions of these cameras were updated every 
frame and were kept at the head node. All cameras were rendered and a stereoscopic view of the virtual world was displayed for the user through the head mounted display.

\subsection{Redirected Walking}

Redirected walking is a locomotion technique which tries to manipulate the perception of the users in order to keep them in the physical tracking area. This manipulation can be done on either the virtual environment or the virtual character. With the first alternative, it is easier to implement, but this technique translates and rotates all the virtual elements other than the user, which makes it unusable for most of the cases. For example, if there are physical objects in the environment that can interact with each other in the virtual world, moving them with high velocities (rotations at the far places induce high velocity) disturbs the physics calculations and unexpected results become likely to occur.

The second alternative changes the linear and angular displacement of the user. Some factors (gains) are introduced to the displacement of the virtual character to help them to stay in the tracked area. These gains can increase or decrease the displacement of the user to keep them in the tracking area. In this dissertation, the second technique was implemented.

The reflective motion tracking markers were placed at their regular places on the user; head, right hand, left hand, right foot and left foot (see Figure 4-14 for a descriptive sketch). 


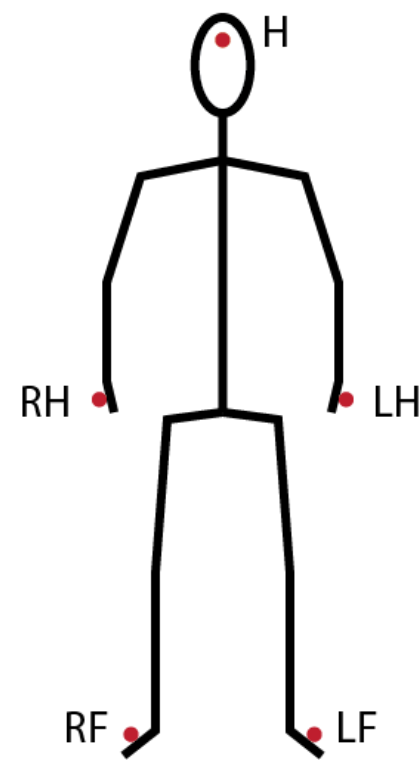

Figure 4-14: Marker positions for the redirected walking locomotion technique.

Three distinctive gains were used in the implementation. These gains were as follows:

- Translational gain

- Rotational gain

- Curvature gain

Translational gain increased the linear displacement depending on the direction of the displacement. First, the displacement of the real user was found for each frame.

$$
\text { Displacement }=P_{R}(n)-P_{R}(n-1)
$$

To calculate the dynamic gain value, a dot product was taken with normalized $P_{R}(n)$ and normalized Displacement vector. The dot product was actually the projection of the Displacement vector onto the $P_{R}(n)$ (see Figure 4-15).

$$
M_{T}(n)=P_{R}(n) \cdot\left(P_{R}(n)-P_{R}(n-1)\right)
$$




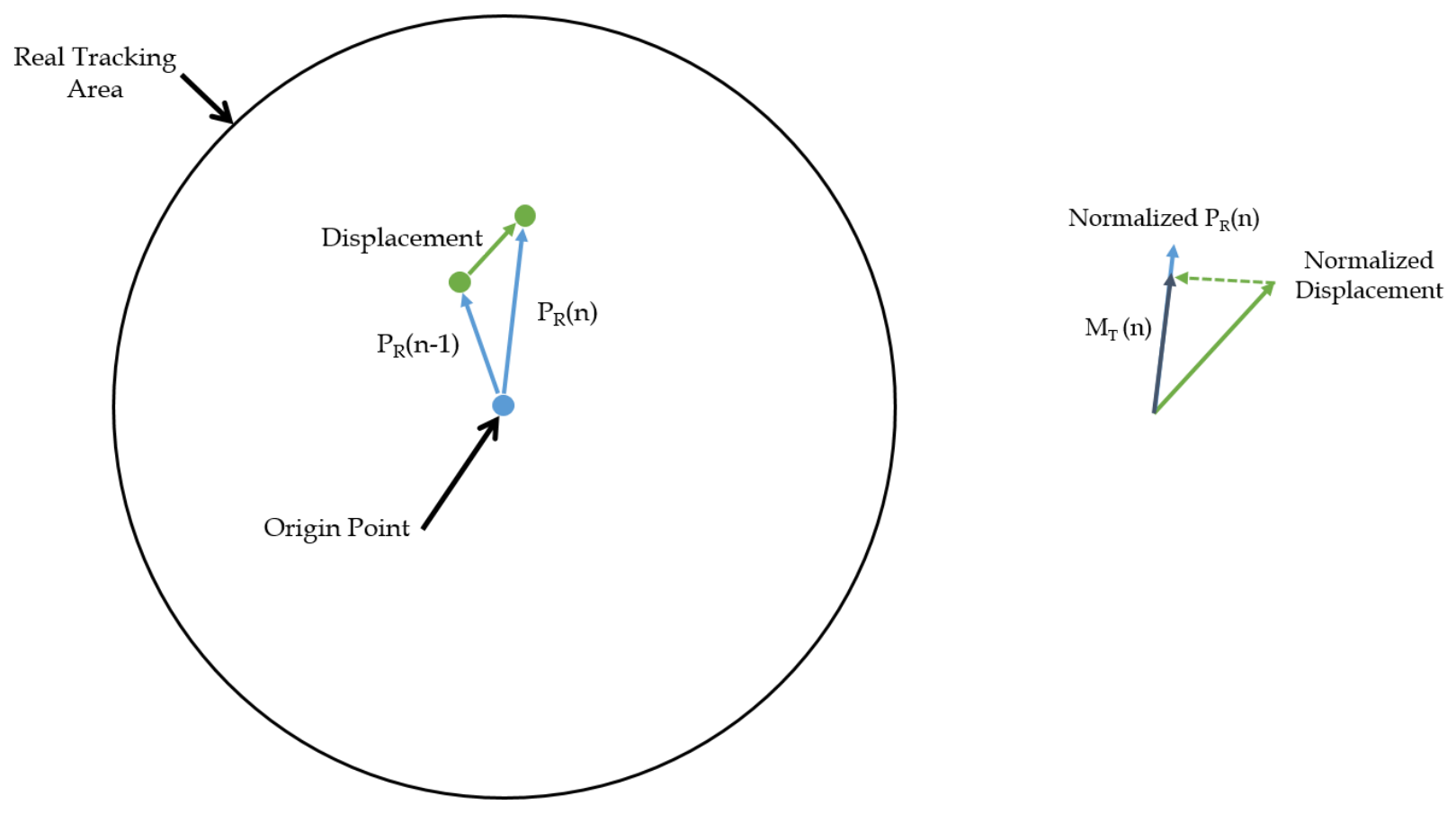

Figure 4-15: Vectors to calculate the translational gain.

The value of the dot product is between -1 and 1 . If two vectors are parallel it gives 1 , if they are opposite it gives -1 . This value shows if the user is walking towards the center of the tracking area or walking away from it. The gain values were kept low if the user was walking towards the center, which makes the user walk relatively more towards the center. On the other hand, if the user was walking away from the center, the larger gain value made the user walk relatively less, trying to prevent the user from going outside of the tracking area.

The dot product result was modified so that it was from minimum translational gain $\left(\mathrm{G}_{\mathrm{Tmin}}\right)$ to the maximum translational gain $\left(\mathrm{G}_{\mathrm{Tmax}}\right)$ value. Those values were decided to be 2 and 4 respectively, after in-house testing sessions.

$$
G_{T}=\frac{\left(M_{T}+1\right)\left(G_{T \max }-G_{T \min }\right)}{2}+G_{T \min }
$$


To apply the final result to the virtual character, the resulting displacement vector was transformed to the local coordinates and applied to the virtual character in its own local coordinates.

For the rotational gain, this time, the change of the forward vector was examined. The angle forming between the two consecutive forward vectors was calculated by taking the cross product of those two vectors. The sinus inverse gives the angle in radians (see Figure 4-16).

$$
\beta=\sin ^{-1}\left(D_{R}(n-1) \times D_{R}(n)\right)
$$

The multiplier was found by taking a dot product with the normalized $D_{R}(n)$ and minus normalized $P_{R}(n)$. The dot product is actually the projection of $D_{R}(n)$ onto the $\mathrm{P}_{\mathrm{R}(\mathrm{n}) .}$

$$
\begin{gathered}
M_{R}(n)=D_{R}(n) \cdot-P_{R}(n) \\
M 2_{R}(n)=D_{R}(n) \cdot-P_{R}(n) * \varphi(n-1)
\end{gathered}
$$

where $\varphi$ is a constant either 1 or -1 depending on the change of forward vector.

$$
\varphi(n)=\left\{\begin{array}{lr}
1 & \text { if } M_{R}(n-1)>M_{R}(n) \\
-1 & \text { otherwise }
\end{array}\right.
$$

The final value is between -1 and 1 . This value shows if the individual is turning towards the center of tracked space or turning away from it. The gain values were kept low if the user was turning towards the center, which makes the user turn relatively more though the center. On the other hand, if the user was turning away from the center, the larger gain value made the user turn relatively less, trying to prevent the user turn through the outside of the tracking area. 


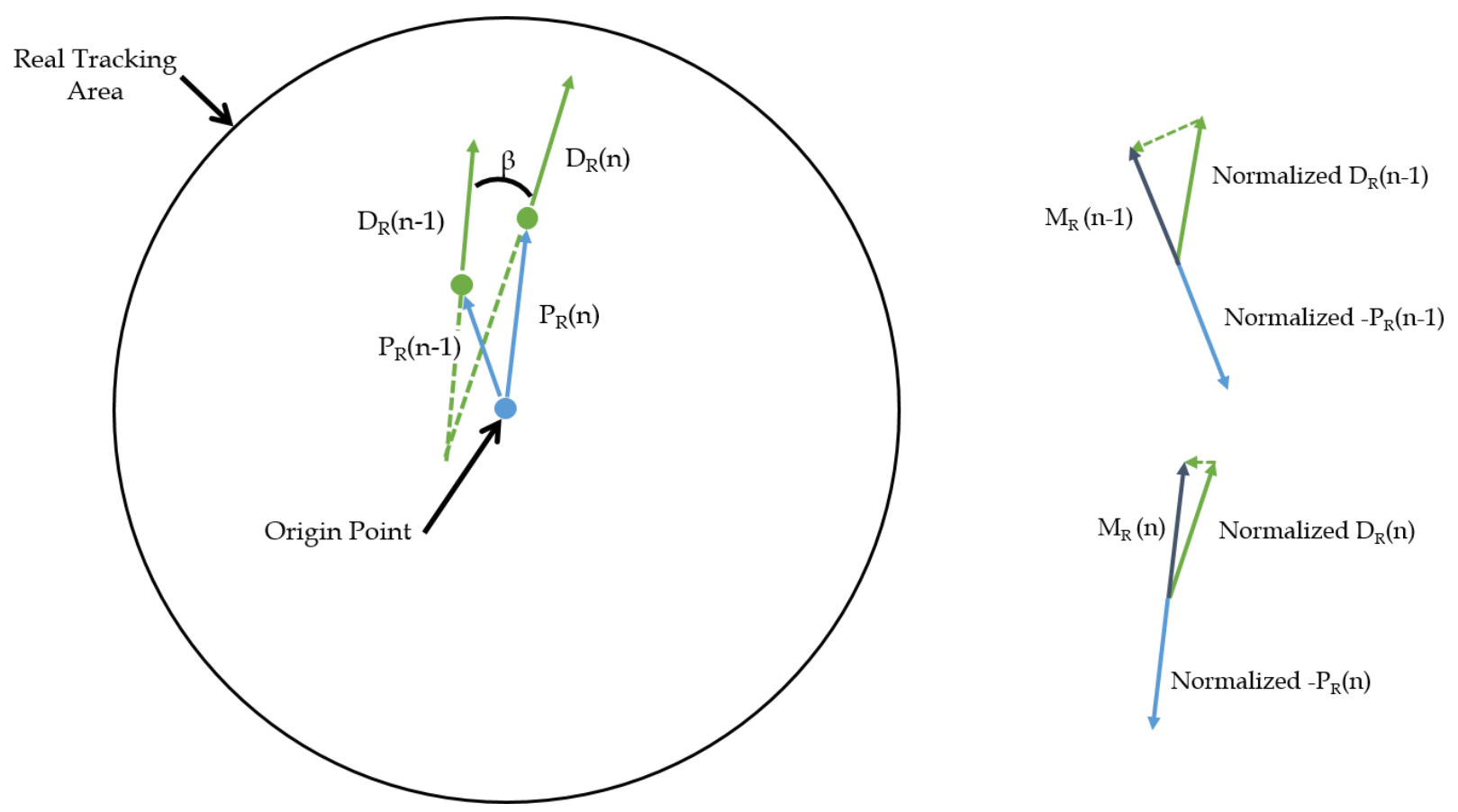

Figure 4-16: Vectors to calculate the rotational gain.

The dot product result was modified so that it was from the minimum rotational gain $\left(G_{R \min }\right)$ to the maximum translational gain $\left(G_{R \max }\right)$ value. Those values were decided to be 0.2 and 2.5 respectively, after in-house testing sessions.

$$
G_{R}=\frac{\left(M 2_{R}+1\right)\left(G_{R \max }-G_{R \min }\right)}{2}+G_{R \min }
$$

Only the modified rotation was applied to the virtual character. Note that it can be higher or lower than the real rotation.

Finally, the curvature gain was also implemented and applied to the virtual character. It takes values of 1 or -1 depending on the displacement vector. This gain makes the users walk in circles while they think they are walking straight. To calculate the curvature gain, first the rotation direction was found by the cross product of was taken 
with normalized $\mathrm{P}_{\mathrm{R}}(\mathrm{n})$ and normalized Displacement vector. And depending on the result of the product in the y-axis, the curvature gain is determined.

$$
G_{R}=\left\{\begin{array}{lr}
1 & \text { if }\left(P_{R}(n) \times\left(P_{R}(n)-P_{R}(n-1)\right)\right) \cdot y<0 \\
-1 & \text { othervise }
\end{array}\right.
$$

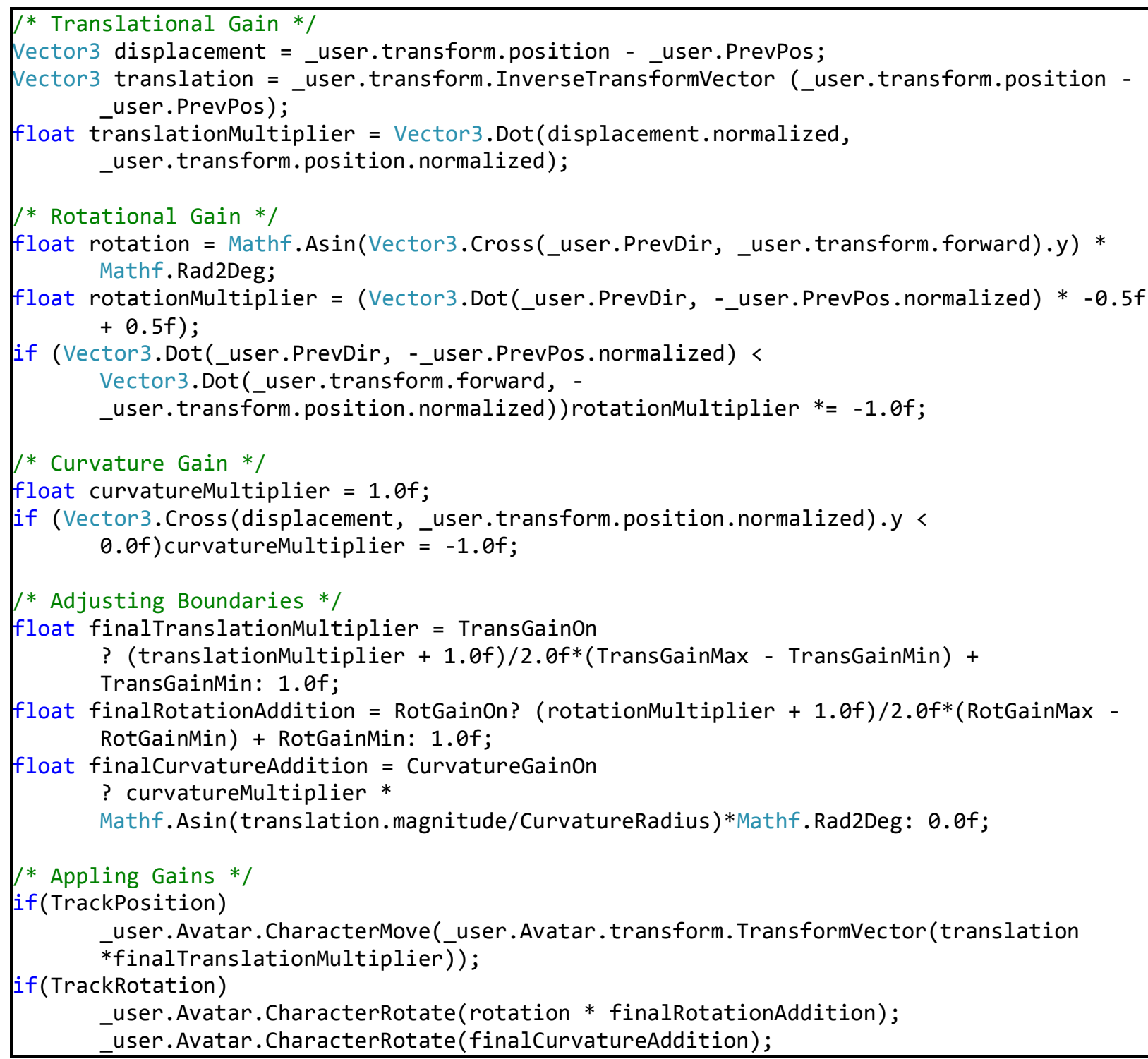

Figure 4-17: Code excerpt from the redirected walking locomotion technique implementation. 
This time an additional rotation was introduced to the virtual character to keep the user inside the tracking area. Since our tracking area was $2 \mathrm{~m}$ by $2 \mathrm{~m}$, the curvature radius was chosen as $0.9 \mathrm{~m}$. The code excerpt can be seen in Figure 4-17.

During the sessions even if the gains were applied successfully, there could be times that the user came to the edge of the tracked area. In these cases, a separate redirection was needed to be applied to keep the user inside the tracking area. For this purpose, a dynamic obstacle in the form of a concave wall was introduced if the user approached to the border of the tracked space (Figure 4-18). This was checked by looking the magnitude of the position of the real user $\left(\mathrm{P}_{\mathrm{R}}\right)$. If it exceeded a threshold value $(0.8 \mathrm{~m})$, the wall popped up from the ground. The position of the wall was placed in the virtual world, in the direction of the approached edge, concave face looking through the user.

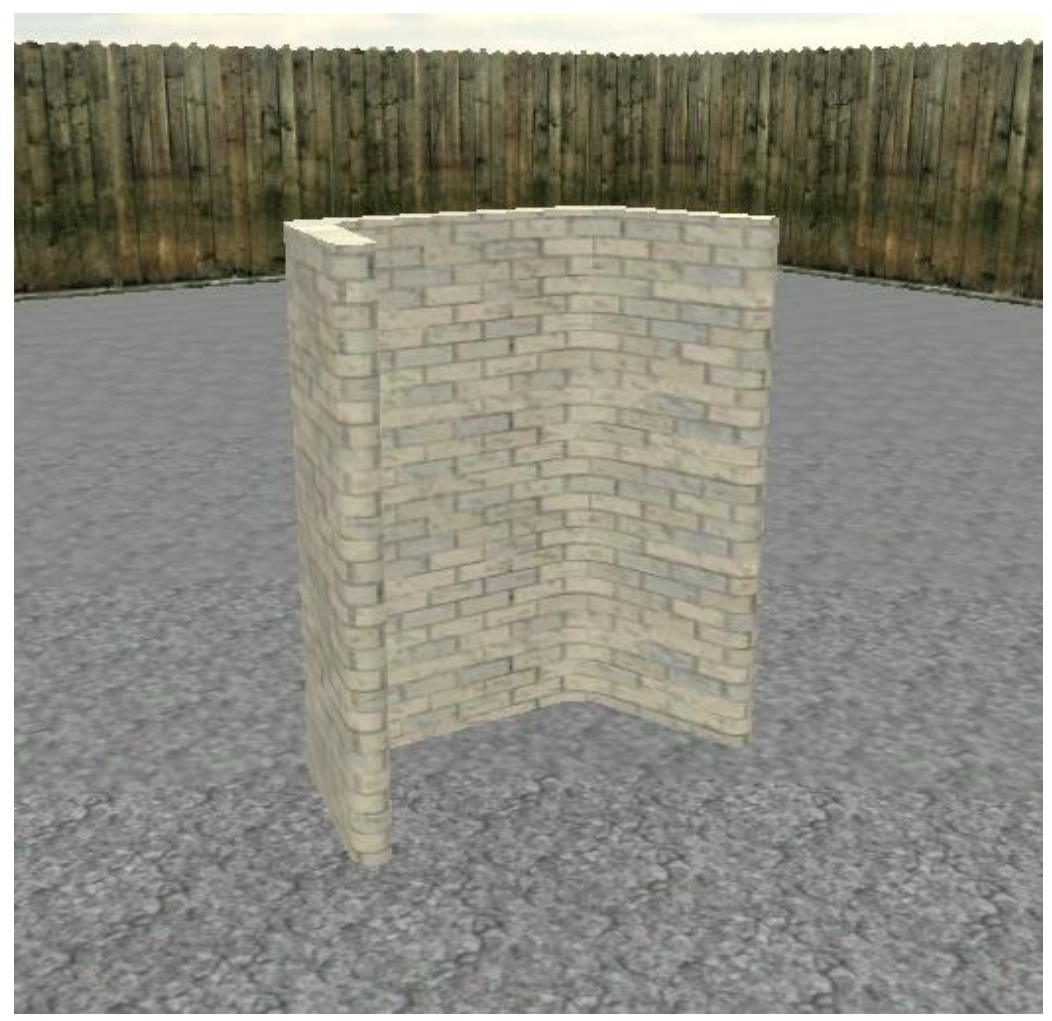

Figure 4-18: Virtual obstacle for redirecting the user. 


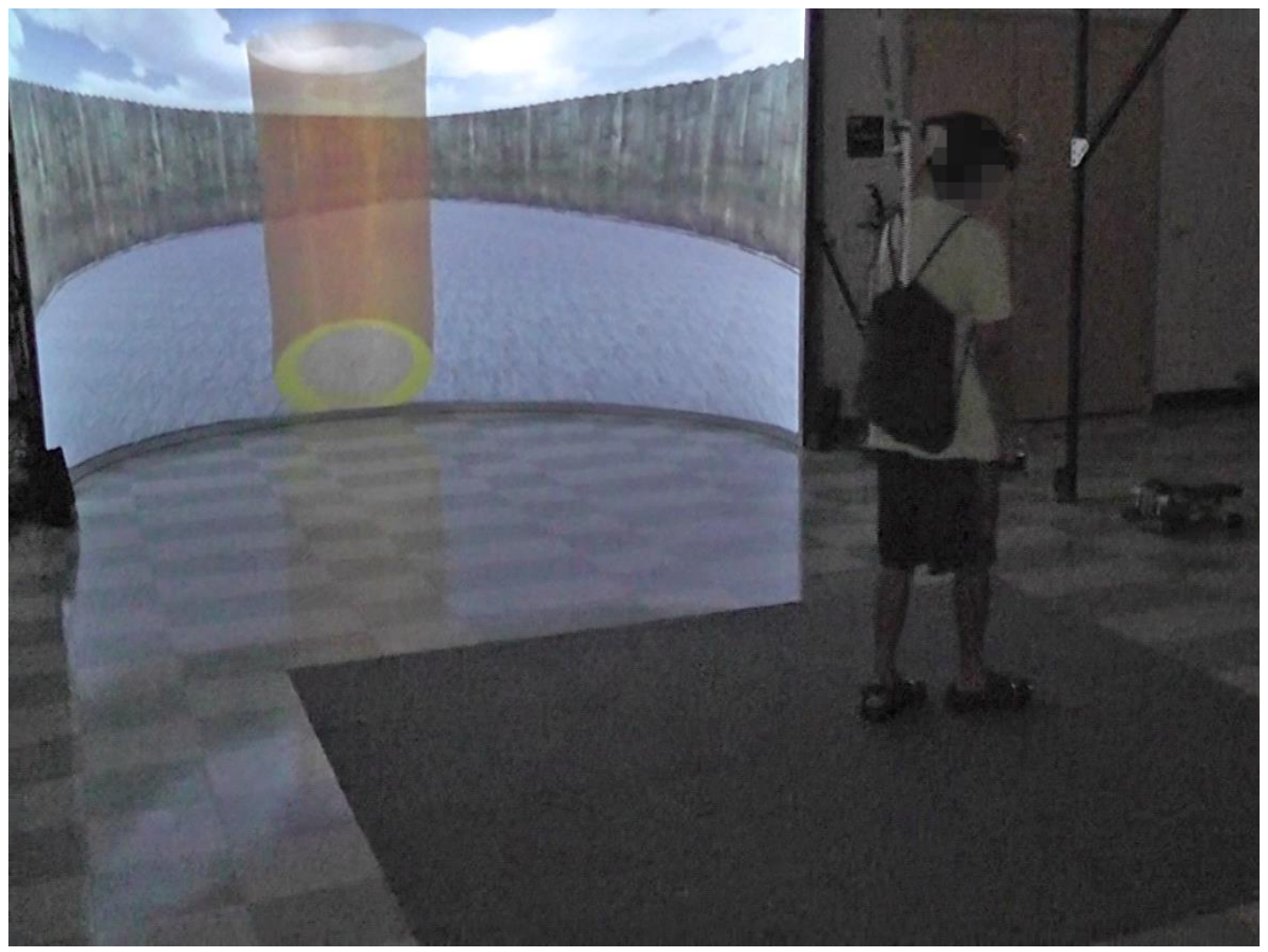

Figure 4-19: Photo of a testing session with the redirected walking locomotion technique.

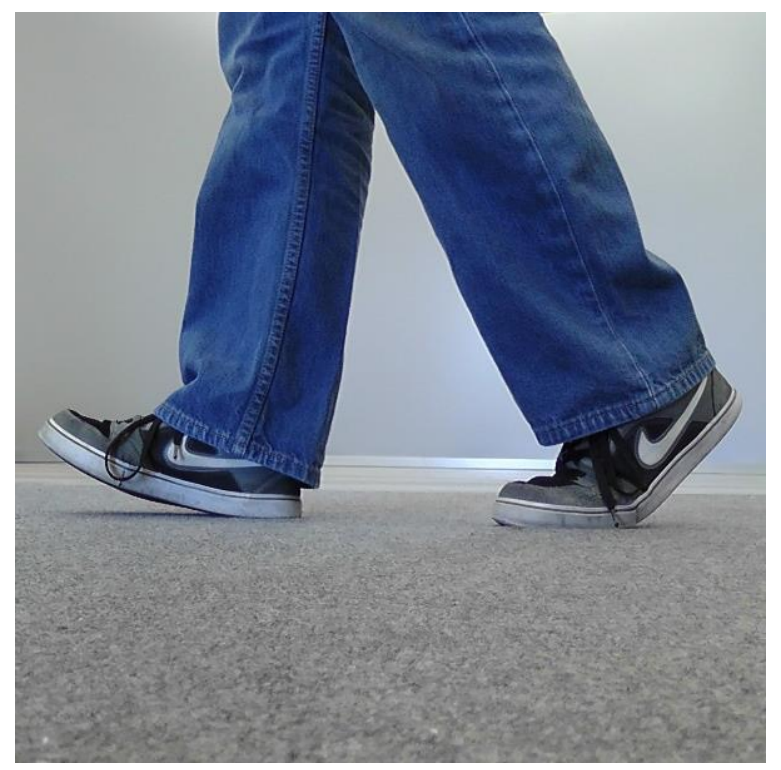

Figure 4-20: Redirected walking locomotion technique descriptive reference icon. 
A photo taken during a testing session with the redirected walking locomotion technique can be seen in Figure 4-19. The descriptive icon that was used to refer to the redirected walking locomotion technique can be seen in Figure 4-20.

\subsection{Walk-in-Place}

In the walk-in-place locomotion technique implementation, the foot positions were tracked to trigger the locomotion. The markers were placed at their regular places on the user; the head, right hand, left hand, right foot and left foot (see Figure 4-21).

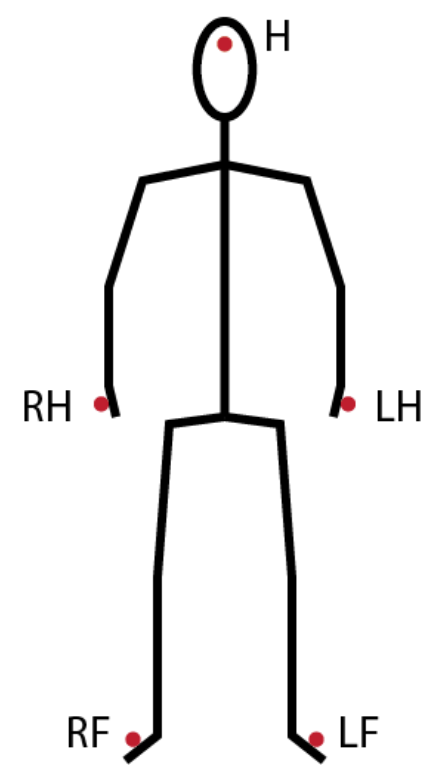

Figure 4-21: Marker positions for the walk-in-place locomotion technique.

At each frame, the position data of the feet markers ( $\mathrm{P}_{\mathrm{RF}}$ and $\left.\mathrm{P}_{\mathrm{LF}}\right)$ were examined. The height of each foot was assumed to be the difference of the readings in the y-axis. This assumption was made because the foot markers were attached on top of the shoes of the users, and different types and different sizes of shoes had different heights. To overcome this problem without the need of calibration, the differences were used. 


$$
\begin{gathered}
h_{R F}=P_{R F} \cdot y-P_{L F} \cdot y \\
h_{L F}=-h_{R F}=P_{L F} \cdot y-P_{R F} \cdot y
\end{gathered}
$$

A simple two stage walking cycle was implemented. The flowchart of the walk-inplace locomotion technique implementation is presented in Figure 4-22.

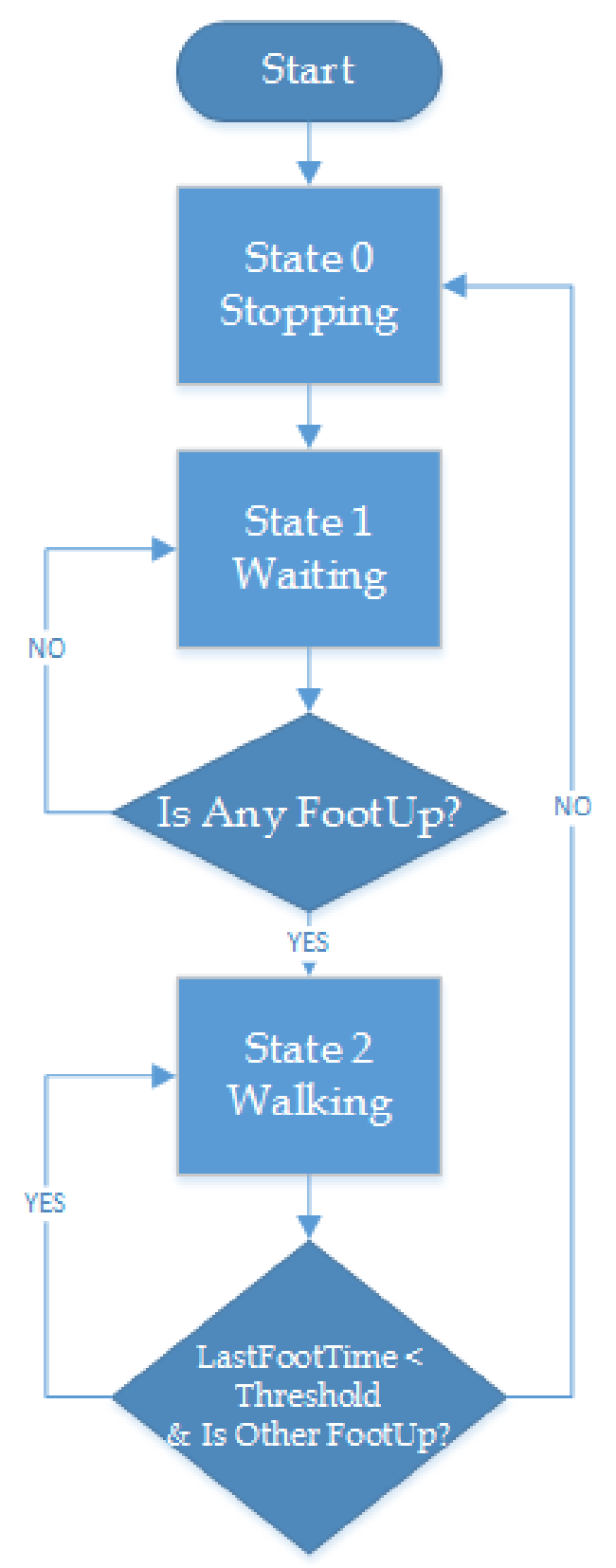

Figure 4-22: Walk-in-place locomotion technique implementation flowchart. 
After the program started, it immediately went into the state 0 . State 0 set the maximum velocity to zero and incremented the state variable.

At state 1, the program started looking for marching. Once one foot was sensed to be active (the height difference was larger than the threshold $(0.1 \mathrm{~m})$ ), the activation time and the side of the active foot (right or left) was stored and the state was incremented.

At state 2 , the program waited for a specific time $(0.7 \mathrm{sec})$ and looked for the other foot to be activated. If the other foot was not activated within that timeframe, the state was set to 0 to stop. If the other foot was activated in that specific time, the activation time and the side of the active foot was updated.

The virtual walking speed was determined by the speed of walking-in-place. The length of one step was assumed to be $0.5 \mathrm{~m}$. With each step, the maximum velocity was updated.

$$
V \max =\frac{0.5 m}{\Delta t}
$$

Until the next step, the $V_{\max }$ value was kept constant, whilst the instantaneous velocity might have been different. During the velocity change, a constant acceleration was used. It was chosen to be $5 \mathrm{~m} / \mathrm{s}^{2}$ after the in-house testing sessions. This caused a smooth transition between different velocities that could be obtained by walking-in-place faster or slower. The state machine code excerpt for the walk-in-place implementation is presented in Figure 4-23. 


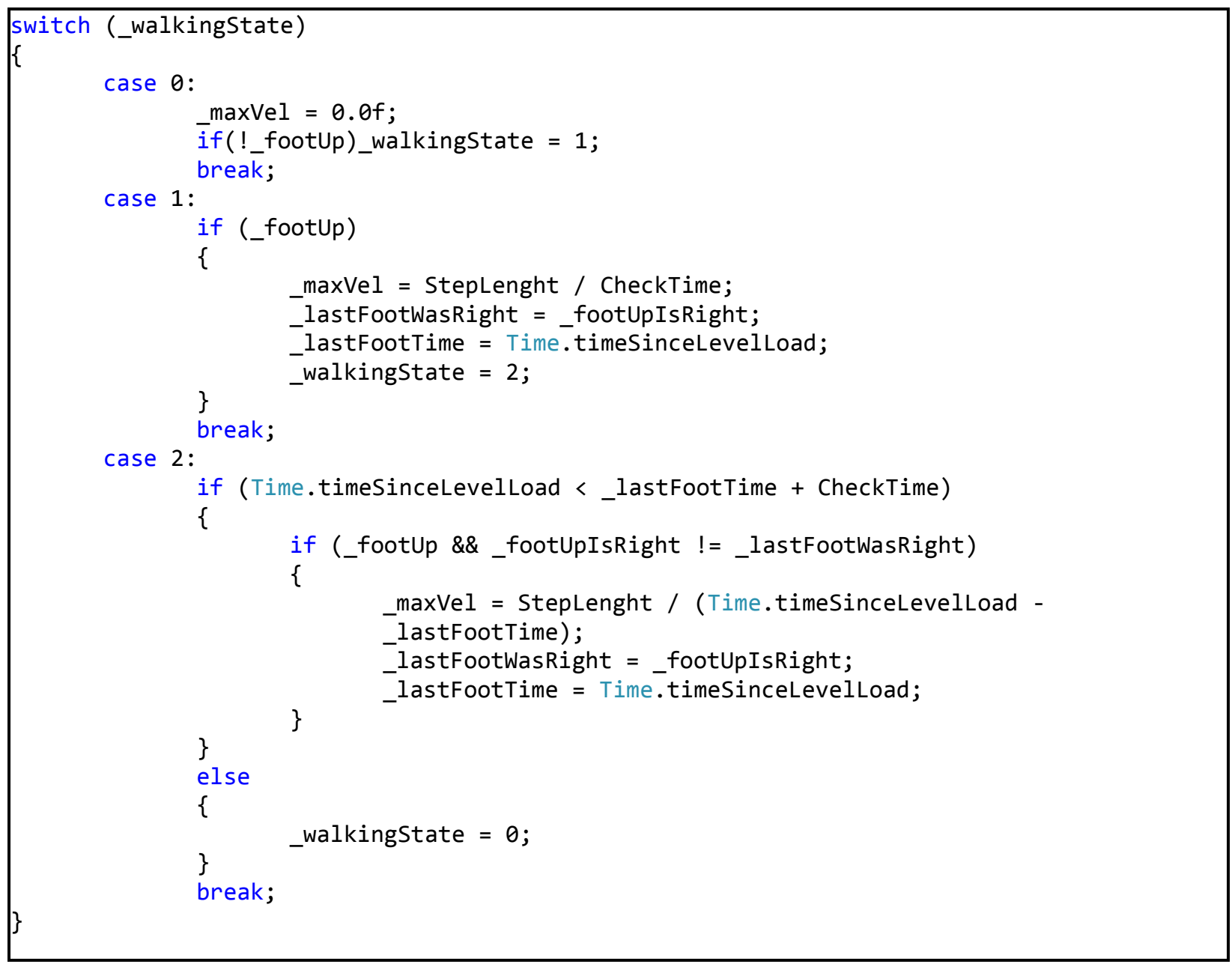

Figure 4-23: Code excerpt from the walk-in-place locomotion technique implementation.

The walking direction was chosen to be the head forward direction. This data was coming from the real head position and transformed into the virtual space. With this approach, the users could change the walking direction by rotating their heads. A photo taken during a testing session with the walk-in-place locomotion technique can be seen in Figure 4-24. The descriptive icon that was used to refer to the walk-in-place locomotion technique is presented in Figure 4-25. 


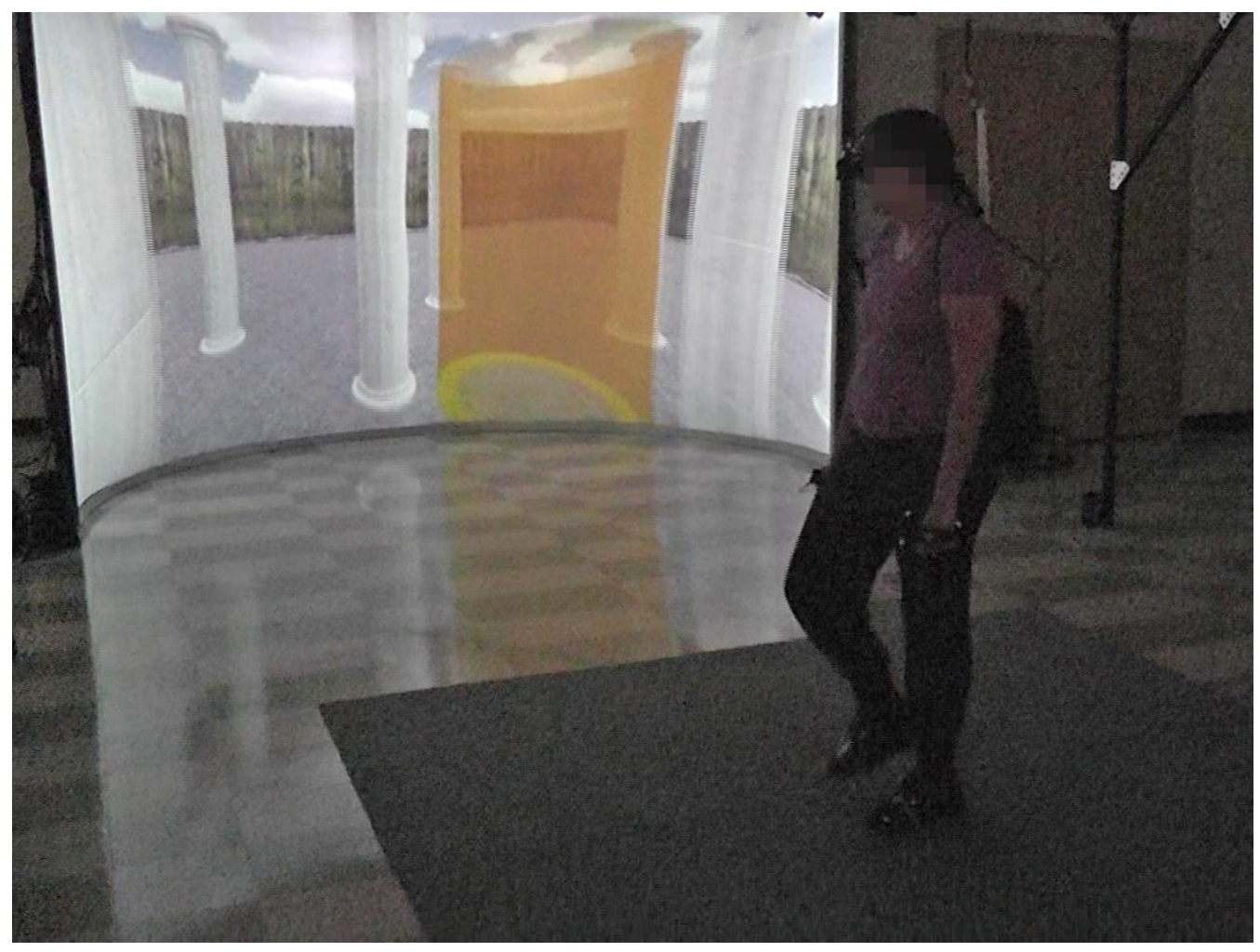

Figure 4-24: Photo of a testing session with the walk-in-place locomotion technique.

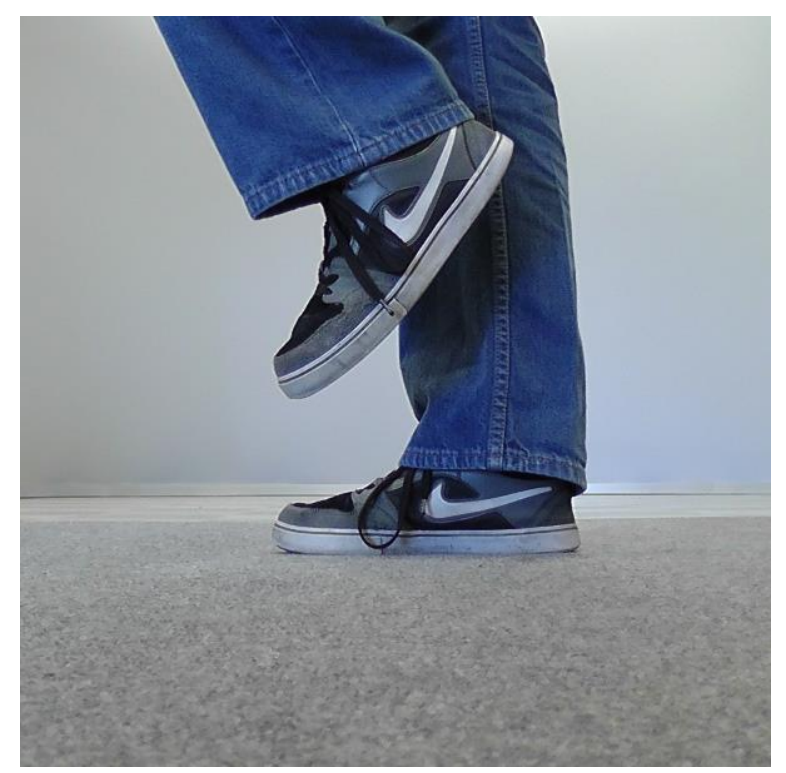

Figure 4-25: Walk-in-place locomotion technique descriptive reference icon. 


\subsection{Stepper Machine}

In this locomotion technique, a simple commercial stepper machine was used. It was placed at the center of the tracking area with a marker attached on the right pedal of it. The marker set that were used in this technique was shown in the descriptive sketch in Figure 4-26.

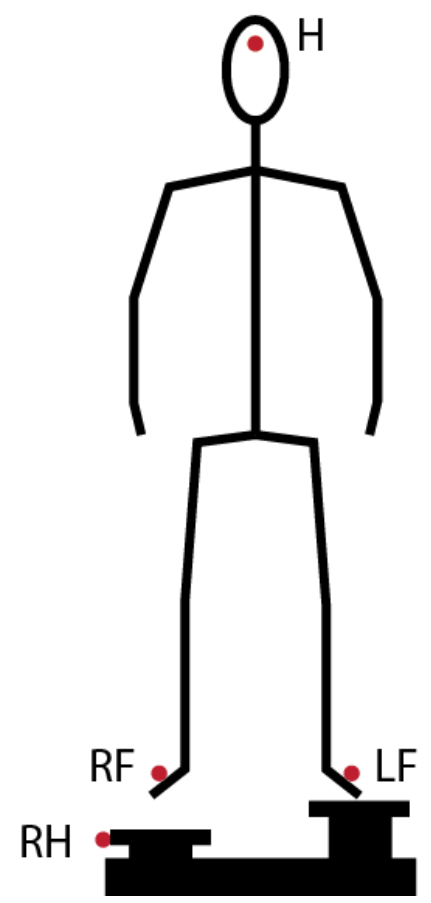

Figure 4-26: Marker positions for the stepper machine locomotion technique.

The right hand marker was attached to the right pedal of the stepper machine. The reason behind using this technique was, due to the nature of the stepper motion, the tip of the feet which we attached the markers did not move enough to have accurate position and orientation readings. The right hand marker was attached to a specially added protrusion on the side of the right pedal of the stepper (see Figure 4-27). 


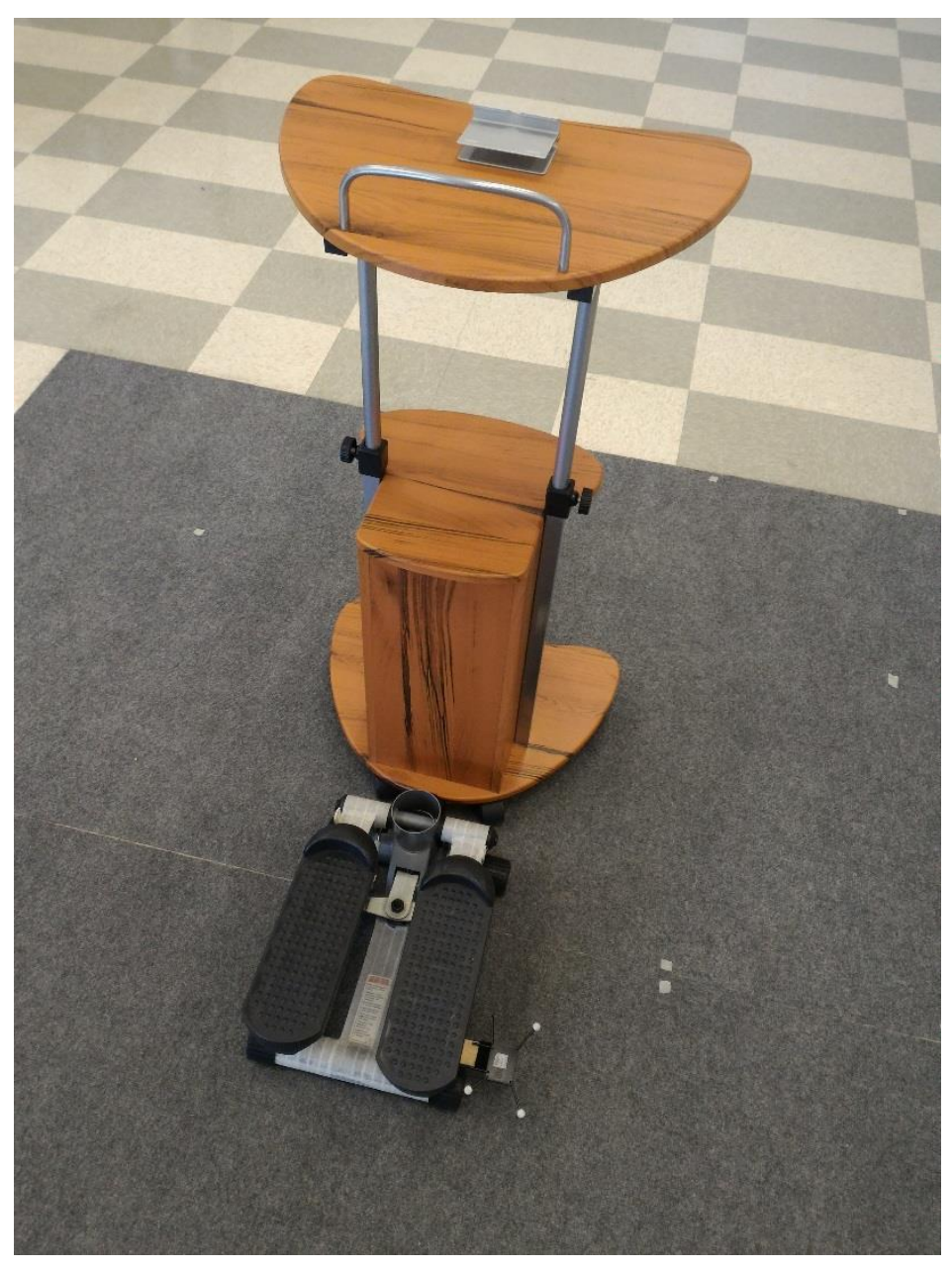

Figure 4-27: The stepper machine, the marker attachment and the holding stage.

We wanted to keep the feet markers on the feet of the users, in order to help the users about their exact place in the virtual environment.

During the in-house testing sessions, it was found out that locomotion with stepper machine and HMD could be quite dangerous due to the balance issues. That's why we introduced a holding stage in front of the stepper machine. The users held the bars while using the stepper machine locomotion technique. With this addition, no more issues on the balance have been observed. 
In this technique, the users were on the stepper machine and they could not rotate their bodies during the sessions due to the nature of the stepper machine. That's why an alternative rotation technique was implemented. The rotation was performed by the head rotation in the y-axis. But frequently the users had to rotate their heads back, which could be difficult or even impossible for large angles. In this technique, if the rotation of the head was more than a threshold value ( \pm 35 degrees), the virtual viewpoint was started to rotate automatically until the rotation of the head became lower than the threshold. The automatic rotation speed was implemented to be proportional to the amount of the head rotation. A maximum head rotation threshold ( \pm 90 degrees) was also introduced to limit the head rotation of the users. Once the users understood that the rotation does not speed up after that threshold, they did not rotate their heads more.

$$
\text { HeadRotation }=\sin ^{-1}\left(D_{H}(n) \times\{0,0,1\}\right)
$$

A linear correlation was used to determine the resulting rotation speed (see Figure $4-28)$.

To control the translation in the virtual world, the positon changes of the right hand marker was observed. The amount of change was multiplied by a constant and it gave the linear locomotion distance in the head direction.

$$
\text { Distance }=\left|P_{R H}(n)-P_{R H}(n-1)\right| * c
$$

The constant $\mathrm{c}$ was chosen to have a natural walking speed which was comparable with the other techniques. After our in-house testing sessions, the constant was decided to be 400 . The code excerpt for the stepper machine locomotion implementation can be seen in Figure 4-29. 


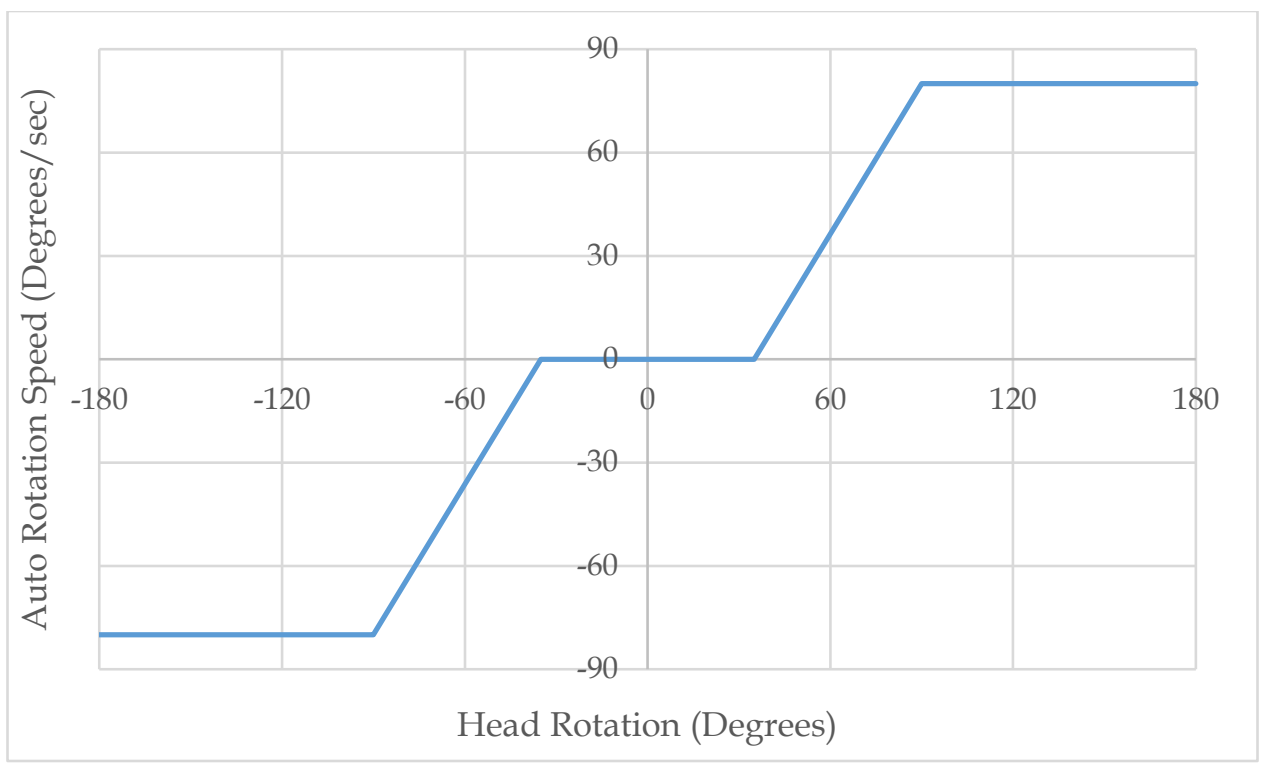

Figure 4-28: Head rotation vs. auto virtual rotation speed chart.

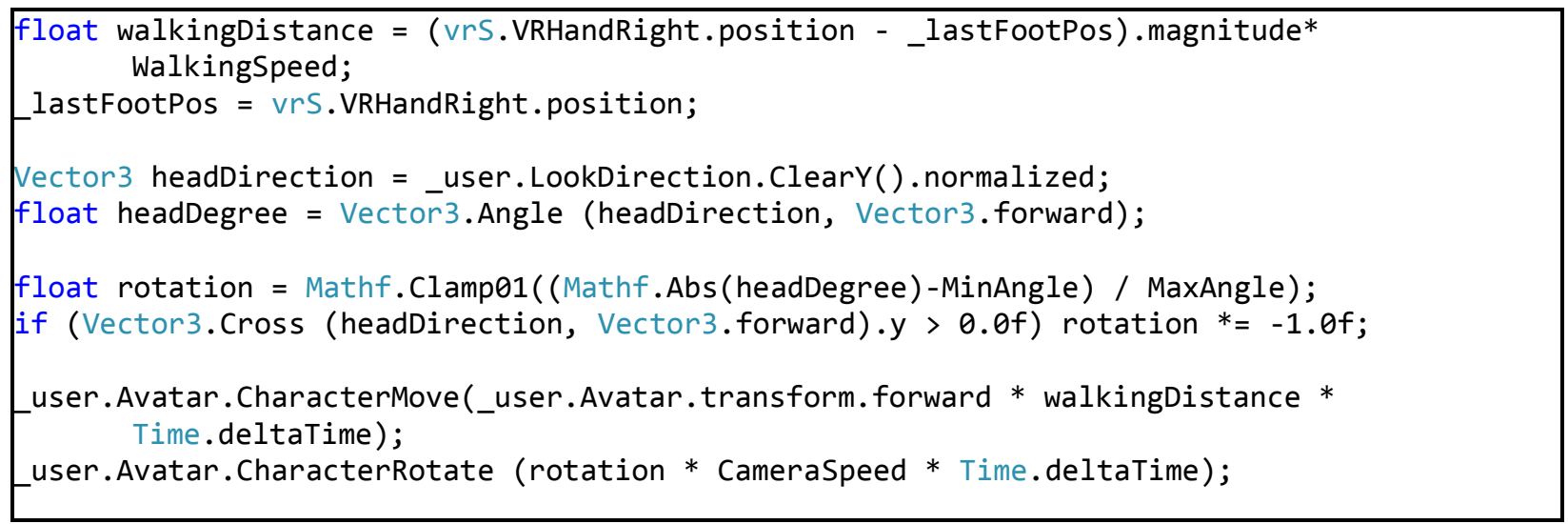

Figure 4-29: Code excerpt from the stepper machine locomotion technique implementation.

A photo taken during a testing session with the stepper machine locomotion technique can be seen in Figure 4-30. The descriptive icon that was used to refer to the technique is presented in Figure 4-31. 


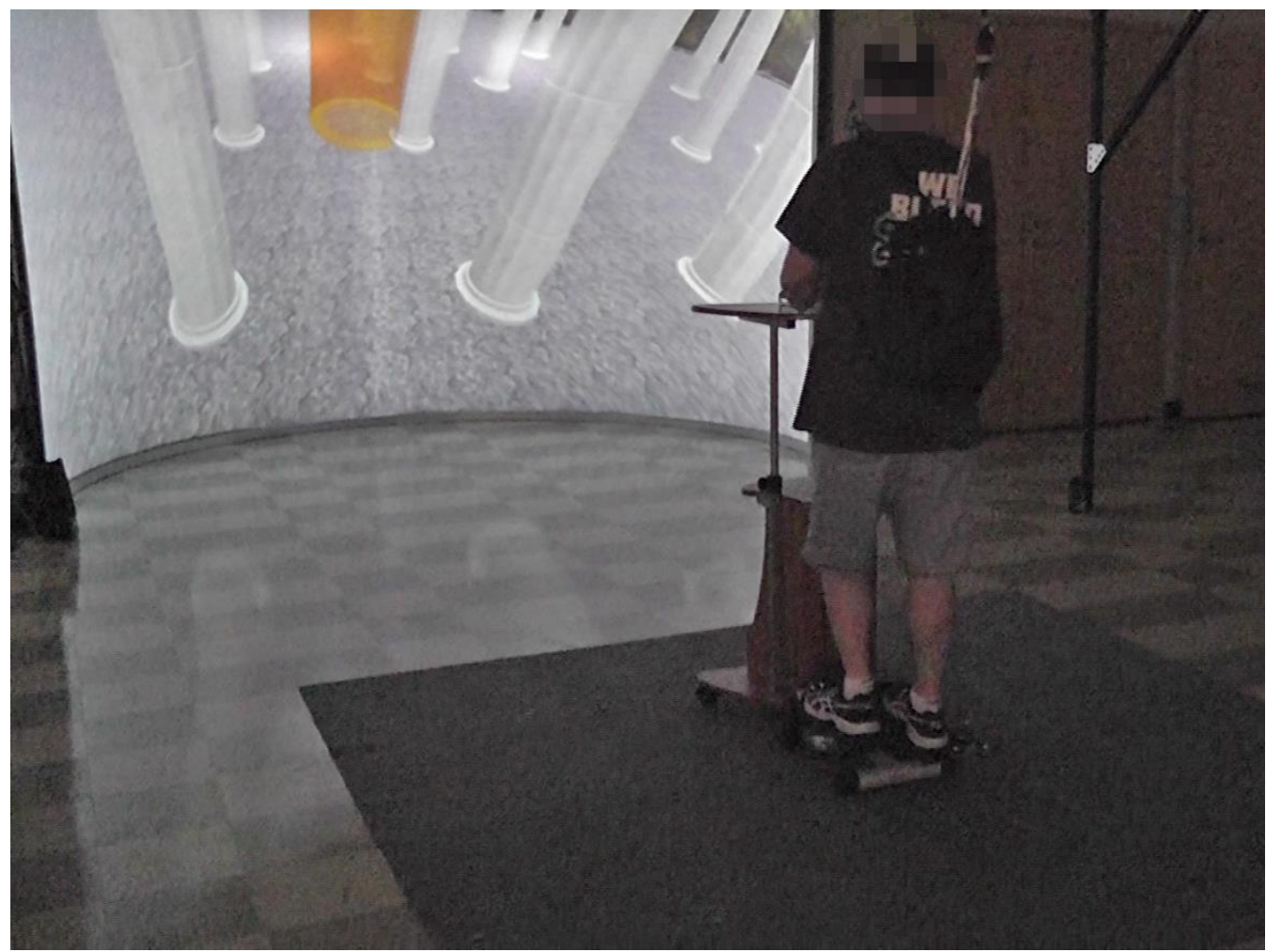

Figure 4-30: Photo of a testing session with the stepper machine locomotion technique.

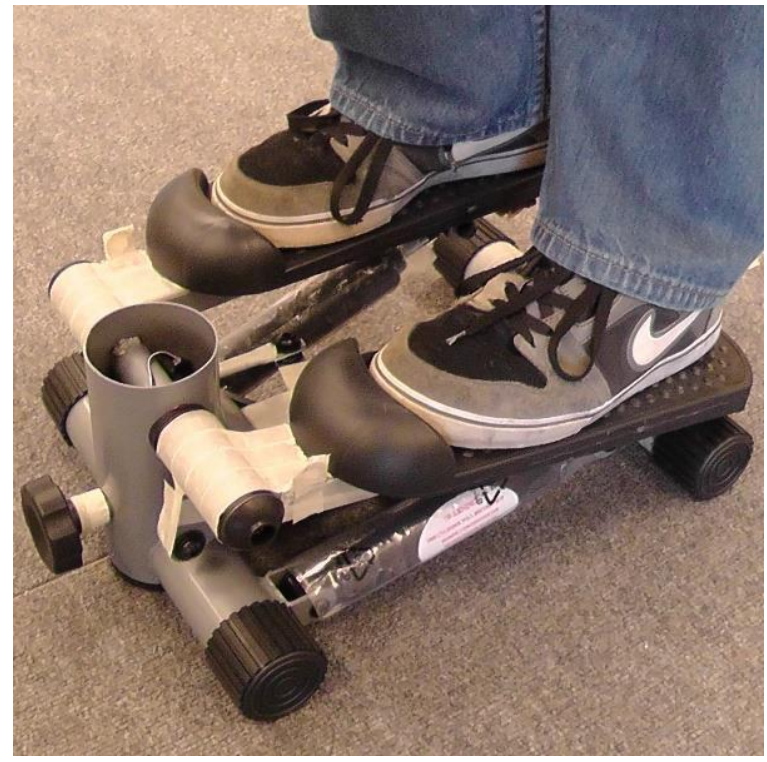

Figure 4-31: Stepper machine locomotion technique descriptive reference icon. 


\subsection{Point \& Teleport}

For the point \& teleport technique, the following marker positions were used depending on the user's dominant hand. The algorithm worked the same using different marker positions for different dominant hand configurations. The non-dominant hand marker was used on the shoulder of the user's dominant side (see Figure 4-32).

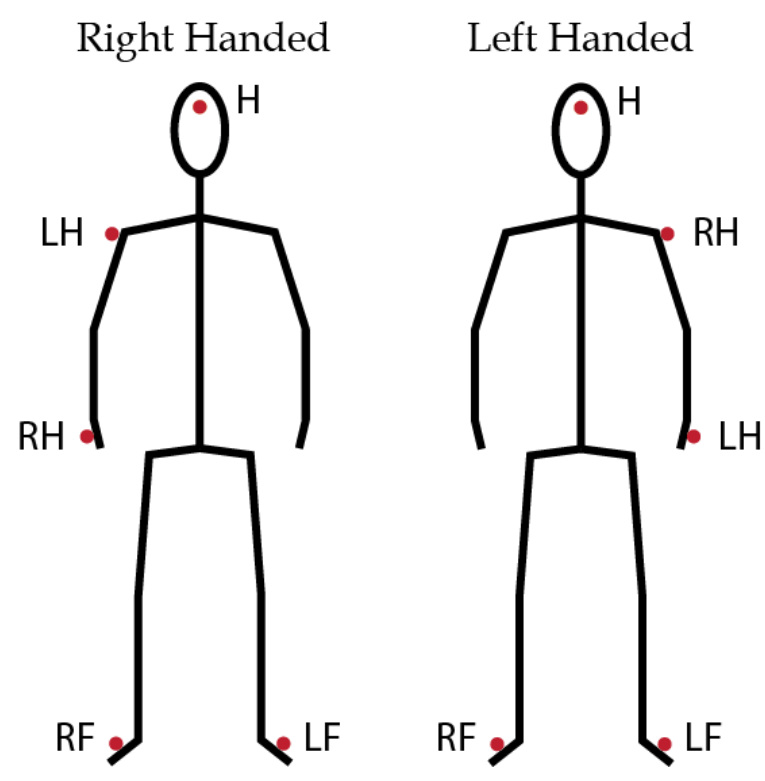

Figure 4-32: Marker positions for the point \& teleport technique.

To use the point \& teleport technique, the users should point to wherever they wanted to be in the virtual world and the virtual viewpoint would be teleported to that position. In our design, to trigger the teleportation, the users should point to the same place or a close vicinity for two seconds. After that, the teleportation was triggered and the virtual avatar was instantaneously moved to that position. An illustration of the point \& teleport technique can be seen in Figure 4-33. 


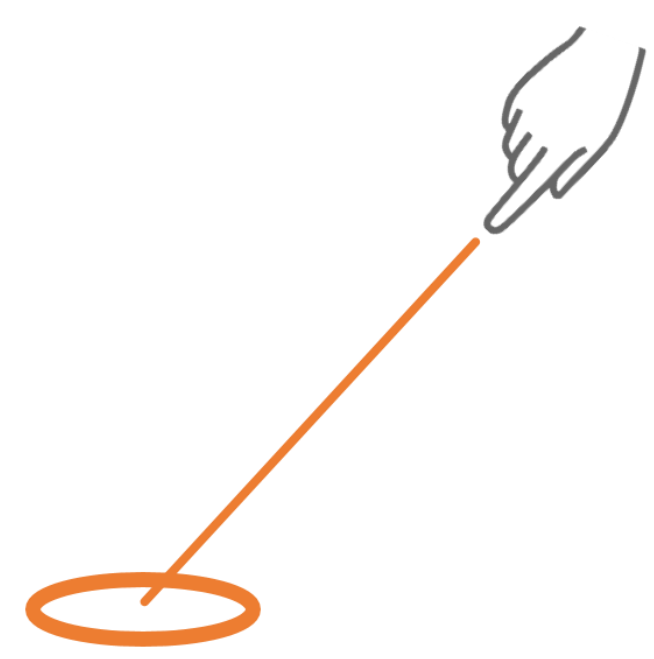

(a)

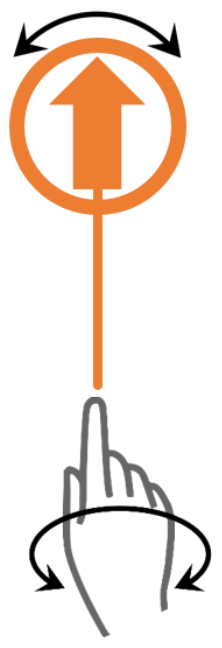

(b)

Figure 4-33: Illustration of the (a) point \& teleport and (b) point \& teleport with direction specification locomotion techniques.

The pointing direction was determined as the vector from the virtual shoulder position data to the virtual hand position data. In this approach, the wrist was assumed to be straight. Another alternative approach to determine the pointing direction could have been using only the hand position and the orientation data. According to our inhouse testing sessions, with the latter approach it was harder to track the user's pointing direction accurately. Small unintentional hand movements or errors in the motion tracking of the hand orientation could cause large displacements at the pointed position. This made the technique harder to control, which might introduce frustration. When both the hand and the shoulder were tracked as in the first approach, the aiming was more accurate and easier to control. Furthermore, the virtual viewpoint was usually close to the vector formed from the shoulder to the hand, which made the aiming easier for the users. 
The pointed position in the virtual world was calculated with ray casting approach. The ray origin was the virtual hand position and the ray direction was the pointing direction calculated by subtracting the virtual position of shoulder from virtual position of user's hand. The collision detection was performed between the ray and the possible teleportation surfaces. In our case, the only possible surface was the ground of the virtual environment. Once a collision was detected, the position of the collision point was stored. In the upcoming frames, if the distance between new collision position value and stored value was smaller than a threshold, it was assumed that the user was pointing to the same point constantly, and the timer was increased by the frame length. If the distance was larger than the threshold, the stored collision position was updated with the new collision position and the timer was reset. If no collision was detected, the stored collision position was cleared until another collision was detected. Balancing the threshold value was important since the larger the threshold was, the larger the tolerance was to the unintentional hand movements. But this came with the cost of lowered precision when the vicinity of the target was pointed. After in-house testing sessions, we found out that a threshold value similar to the virtual character's bounding capsule diameter worked well, since it would occupy the same virtual space after the teleportation.

In our design, to exclude interfering effects of any additional components, we did not include any controllers to trigger the teleportation. The users needed to point to the same place or a close vicinity for two seconds. Two seconds was decided by in-house 
testing sessions to be just long enough to eliminate the unintentional teleportation instances, yet to be short enough not to cause tiredness in the users.

The technique was always active unless the tracked arm was lowered by the user's sides. Since we did not use any handheld controller in our implementation to activate or deactivate the teleportation, we utilized the lowered arm posture to make the teleportation inactive. With this implementation, the users could wait at a constant position in a relaxed posture with their arms lowered. Before checking for collision, the angle between the pointing vector and the down vector was calculated and if the angle was smaller than a threshold, the teleportation was deactivated. This was important for the users to be able to stay in the same position without getting constantly teleported unintentionally.

To make the locomotion more user-friendly, in our implementation, an orange ring overlay was placed on the pointed position in the virtual world if the pointed position was on a possible teleportation surface. This way, the users could easily see where they were pointing at and where they were going to be teleported. The color of this ring was gradually turned into green as long as the user pointed at the vicinity of the initial position. The color feedback was helpful for the users in understanding if they were pointing the same position and how much longer they needed to point. Furthermore, a virtual laser beam was displayed that originated from the user's virtual hand and extended towards the ring, parallel to the pointing direction. In our in-house testing sessions, this laser beam helped with the sense of being in control and the distance estimation. Without the laser beam, it was difficult to see the ring if the user pointed to a 
position that was far away from their viewpoint. The color of the laser beam was kept the same with the ring color if the ring was active; otherwise the laser beam's color was kept red, indicating that no possible teleportation surface was pointed currently.

Once the teleportation was triggered by the user, the virtual character and the virtual viewpoint was moved to the pointed position in the virtual world instantaneously. The orientation of the user was kept the same during the teleportation. In our implementation, the user rotated in virtual environment via rotating their bodies in actual world. So users were able to adjust their orientations after the teleportation. Different approaches were tested in-house before designing the experiment. One approach was to move the virtual character in the virtual world until it reached to the destination point. The speed could be adjusted as an average walking speed or a faster speed. In both these conditions, the approach has introduced motion sickness to the testers. Motion sickness with this approach was not unexpected since the users saw themselves moving in the virtual world while they were standing still in the real world. Another approach was to make the teleportation with a fade-out and fade-in effect in order to help the users cope with the instantly changing virtual world. This approach was found to break the presence and turned out to be unnecessary since the users did not get overwhelmed by the changing virtual world during the teleportations. In addition, the fading introduced wasted time for each teleportation which caused impatience in the testers. The users could expect what to see once they were teleported because they already saw where they were going to be teleported. The code excerpt for the implementation of the point \& teleport locomotion technique can be seen in Figure 4-34. 


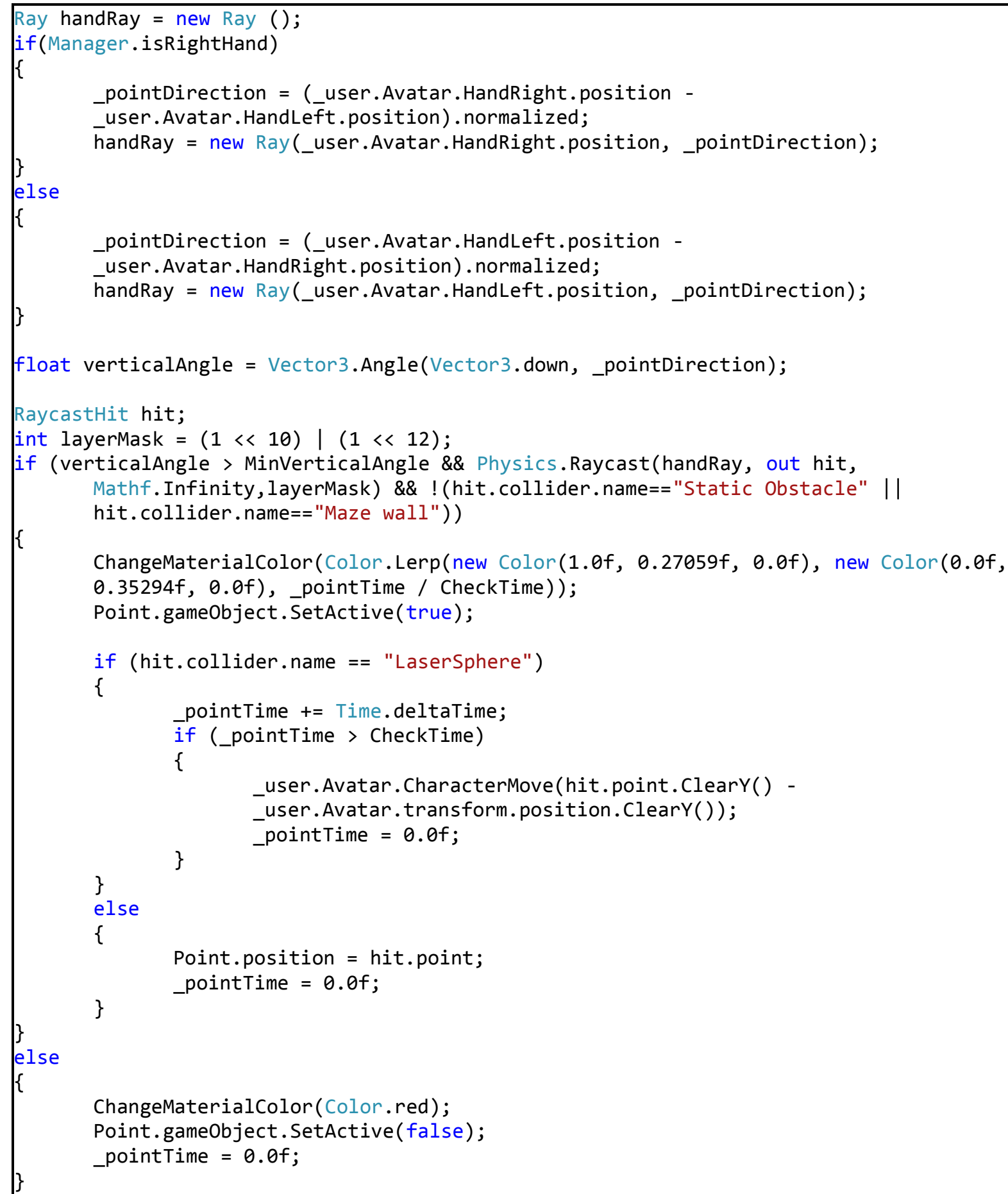

Figure 4-34: Code excerpt from the point \& teleport locomotion technique implementation. 


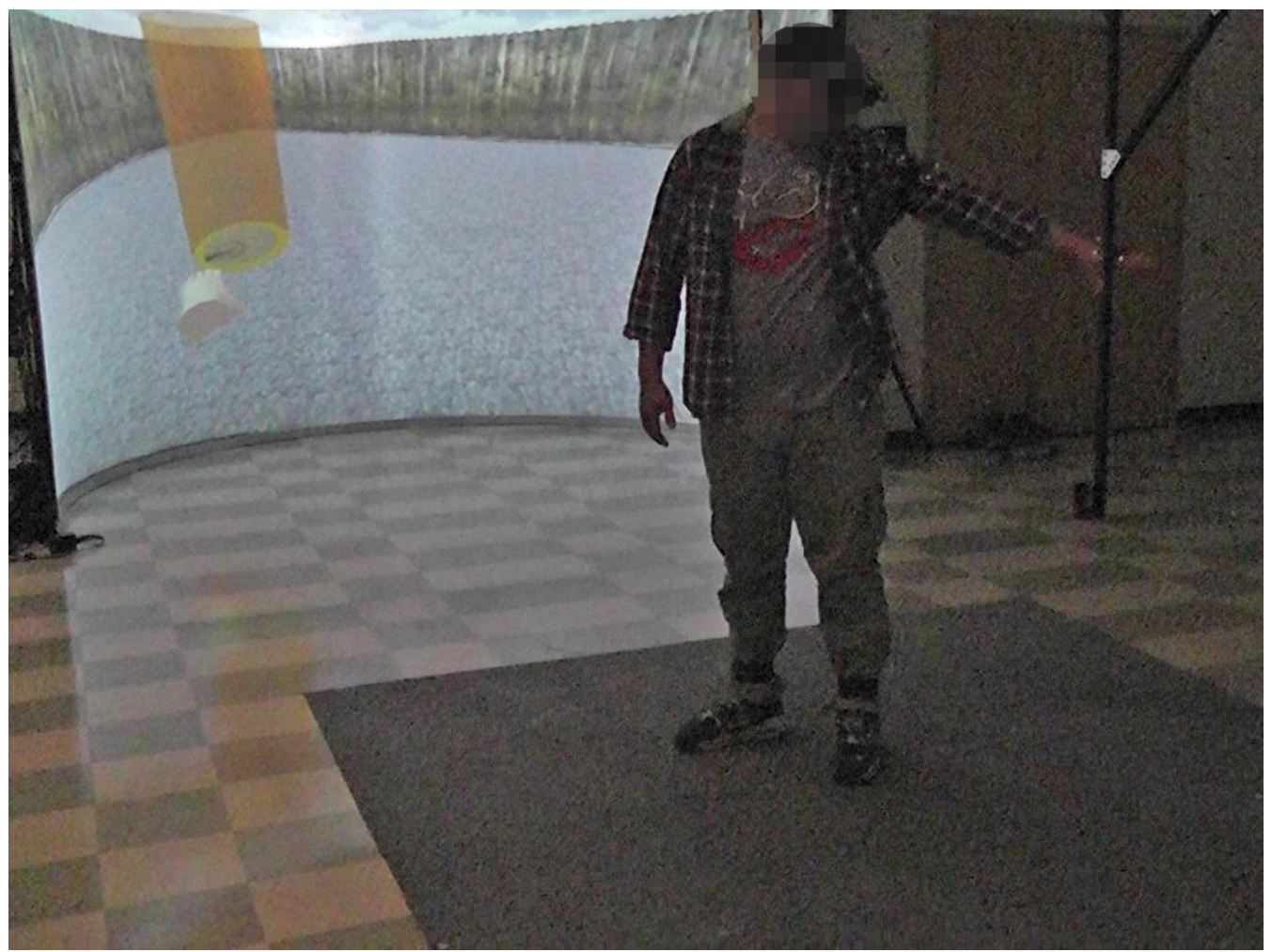

Figure 4-35: Photo of a testing session with the point \& teleport locomotion technique.

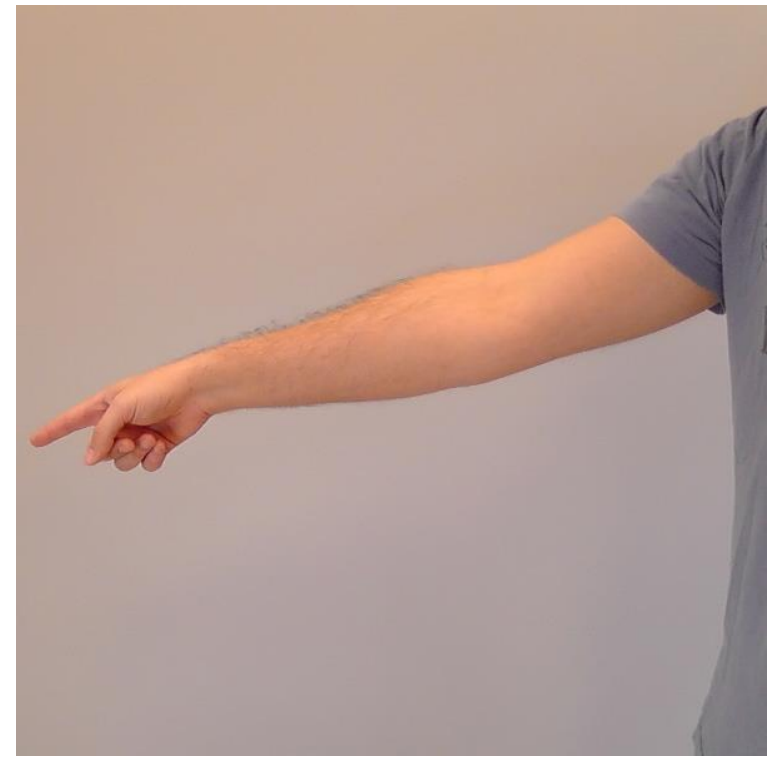

Figure 4-36: Point \& teleport locomotion technique descriptive reference icon. 
A photo taken during a testing session with the point \& teleport locomotion technique can be seen in Figure 4-35. The descriptive icon that was used to refer to the technique is presented in Figure 4-36.

\subsubsection{Point \& Teleport with Direction Specification}

In the explained implementation of the point \& teleport locomotion technique so far, the teleportation was performed without any change in the orientation before and after the teleportation. To be able to turn in the virtual world, the users were required to make a real turn after the teleportation. We wondered if we could improve the point \& teleport technique by adding a direction feature. Hence, a variation of this technique was implemented with a direction specification. With this modified technique, while the users pointed to a position in the virtual world, they could also specify which direction they wanted to be facing after the teleportation. For this purpose, in our implementation, a 3D arrow was placed above the ring. The arrow was restricted to $x-z$ plane and the rotation in the y-axis was determined by the rolling axis of the pointing hand (see Figure 4-33). This way, the users could both point to the destination position and specify the direction they would be facing when they were teleported, by only using their one arm. After our initial in-house testing sessions, the rolling axis was found to be the easiest for the rotation of the hand to specify the direction among other alternatives in terms of understanding and operating the gesture.

The virtual arm vector was formed between the virtual shoulder position $\left(\mathrm{p}_{\mathrm{RS}}(\mathrm{n})\right)$ and the virtual hand position $\left(\mathrm{p}_{\mathrm{H}}(\mathrm{n})\right)$. The right handed configuration is assumed in the descriptions in this sub-subsection. The right shoulder position was actually taken from 
the left hand marker set, which was attached on the right shoulder of the user for this technique.

The angle between the virtual right hand's normal direction and the normal direction for the arm vector gave the rolling angle $(\beta)$. The user could control the angle between the forward vector for the user and the direction of the arrow on the ground (a) by changing the angle $\beta$ (see Figure 4-37).

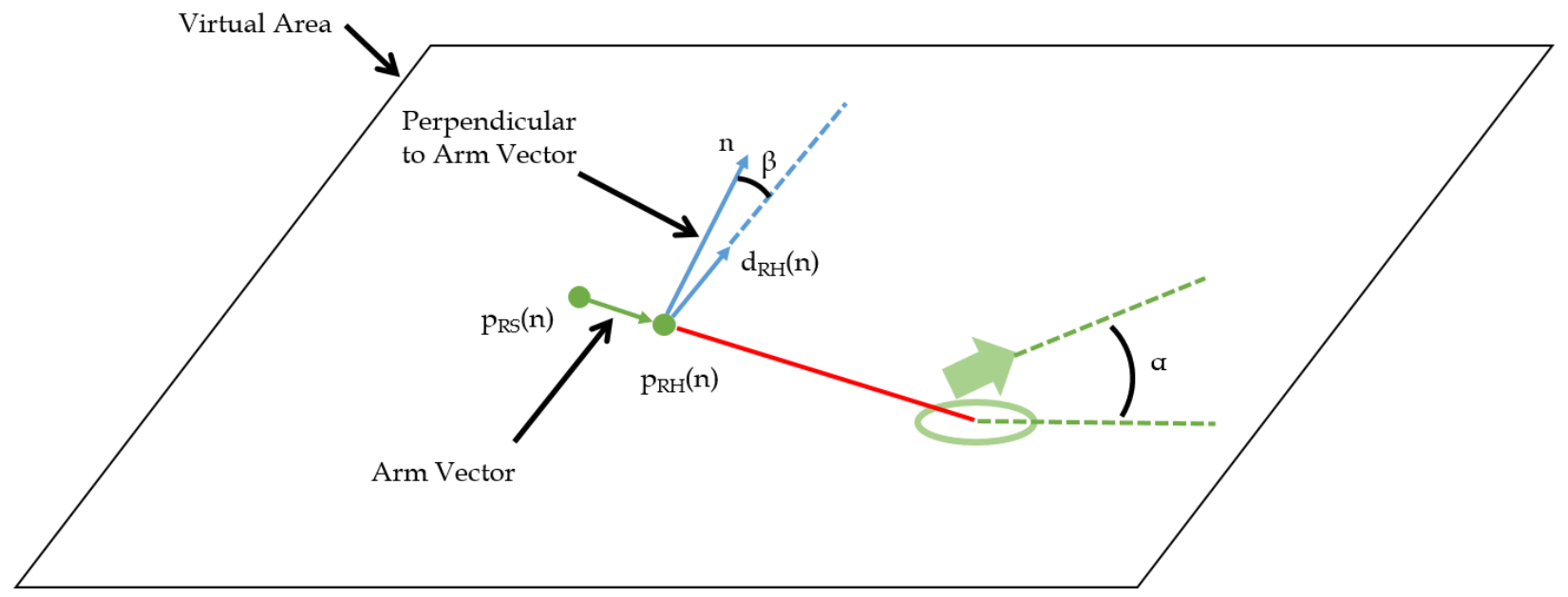

Figure 4-37: Vectors to control the direction of the arrow in the point \& teleport with direction specification.

There was a linear relation between $\beta$ and $a$. Since the markers were attached to the top of the hands and the cameras were located on the top of the tracked area, rotating the hand more than 90 degrees could cause self-blocking of the markers. That's why a constant multiplier (3.0) was introduced to keep the maximum hand rotation as 60 degrees. The explanatory chart can be seen in Figure 4-38. The additional codes that were required for the direction specification and the details of the implementation can be seen in the code excerpt presented in Figure 4-39. 


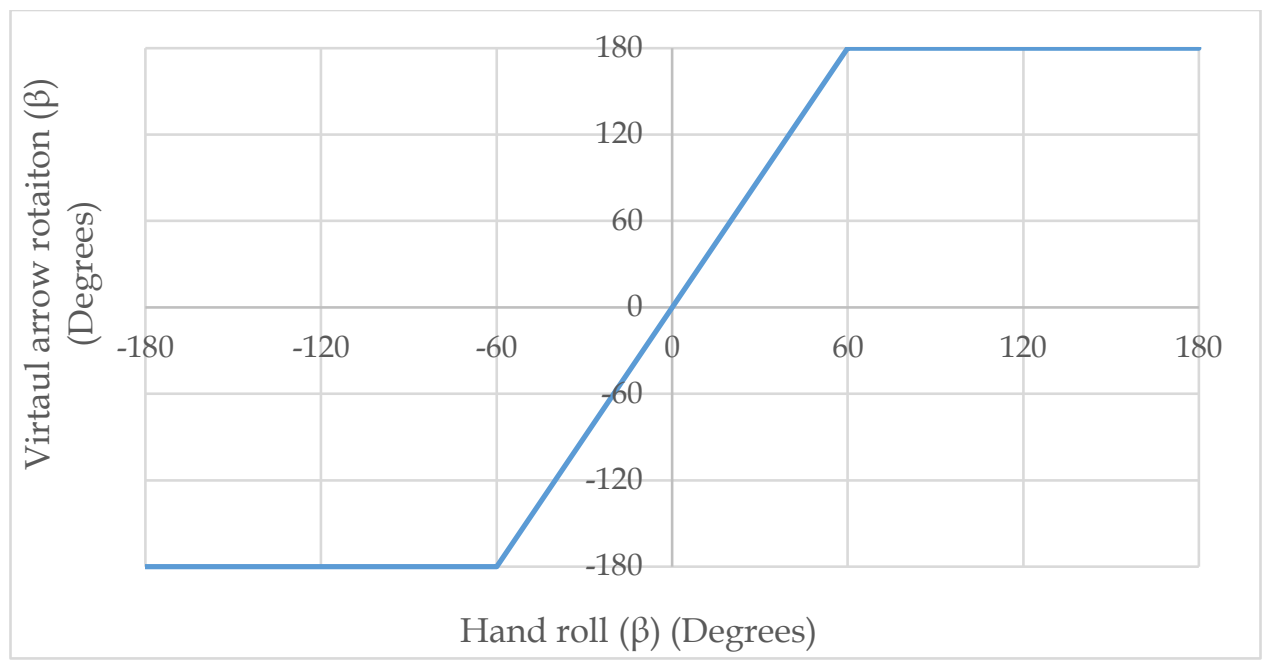

Figure 4-38: Hand roll vs. virtual arrow rotation graph for point \& teleport with direction specification.

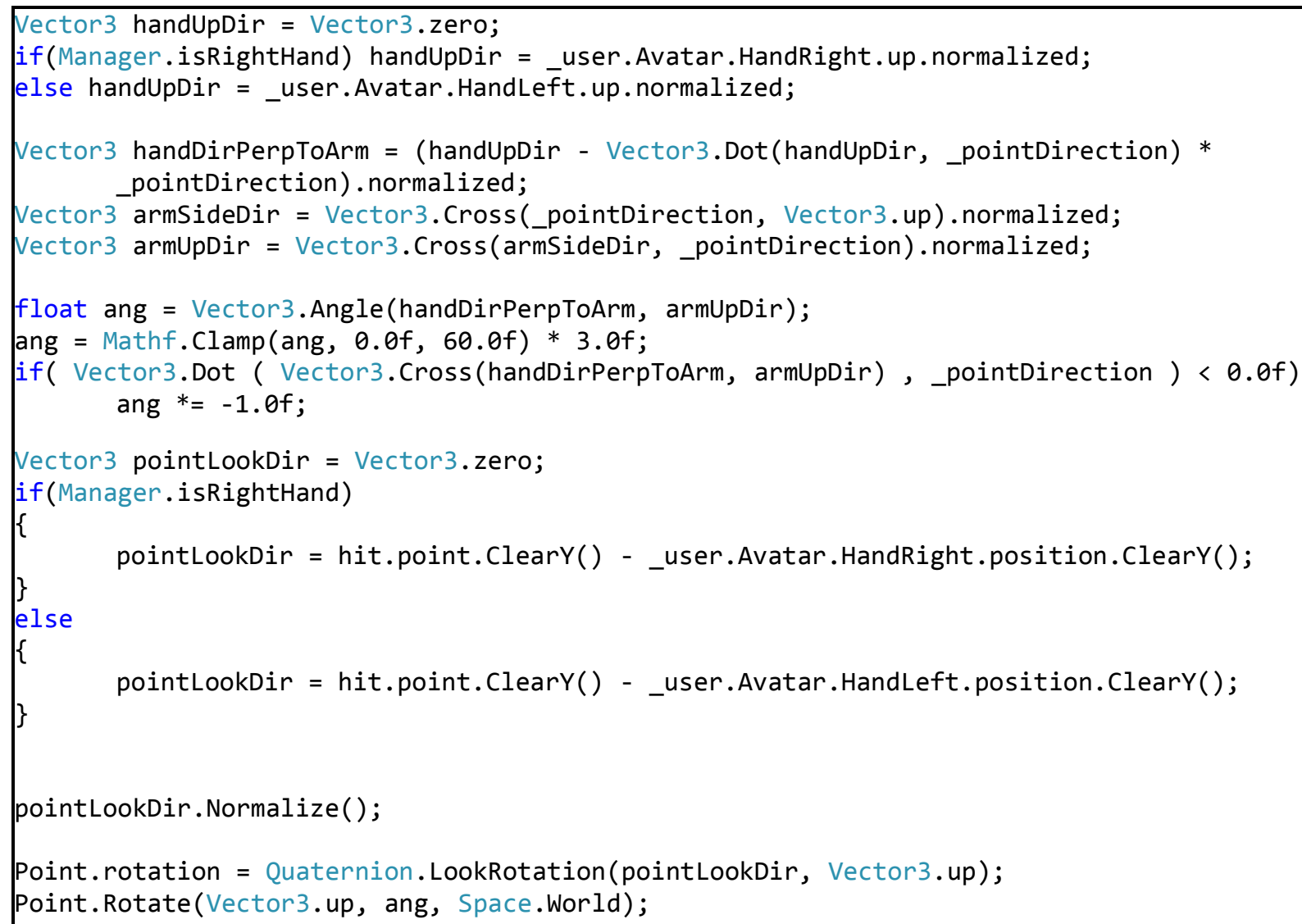

Figure 4-39: Additional code excerpt from the point \& teleport with direction specification locomotion technique implementation. 


\subsection{Joystick}

This technique was used as a reference for the other locomotion techniques. The marker positions were as shown in Figure 4-40. The users were standing in front of a high table with a joystick (Logitech Extreme 3D Pro Joystick [152]). The joystick was used by the dominant hand of the user. To go forward in the looking direction, the user should push the joystick forward. To turn, the user could either push the joystick in the turning direction, or rotate their head accordingly.

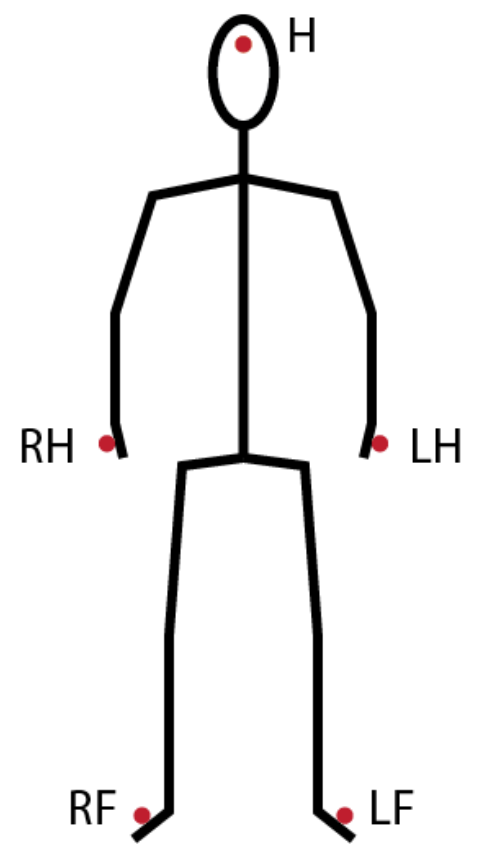

Figure 4-40: Marker positions for the joystick locomotion technique.

A photo taken during a testing session with the joystick technique can be seen in

Figure 4-41. The descriptive icon that was used to refer to the technique is presented in Figure 4-42. 


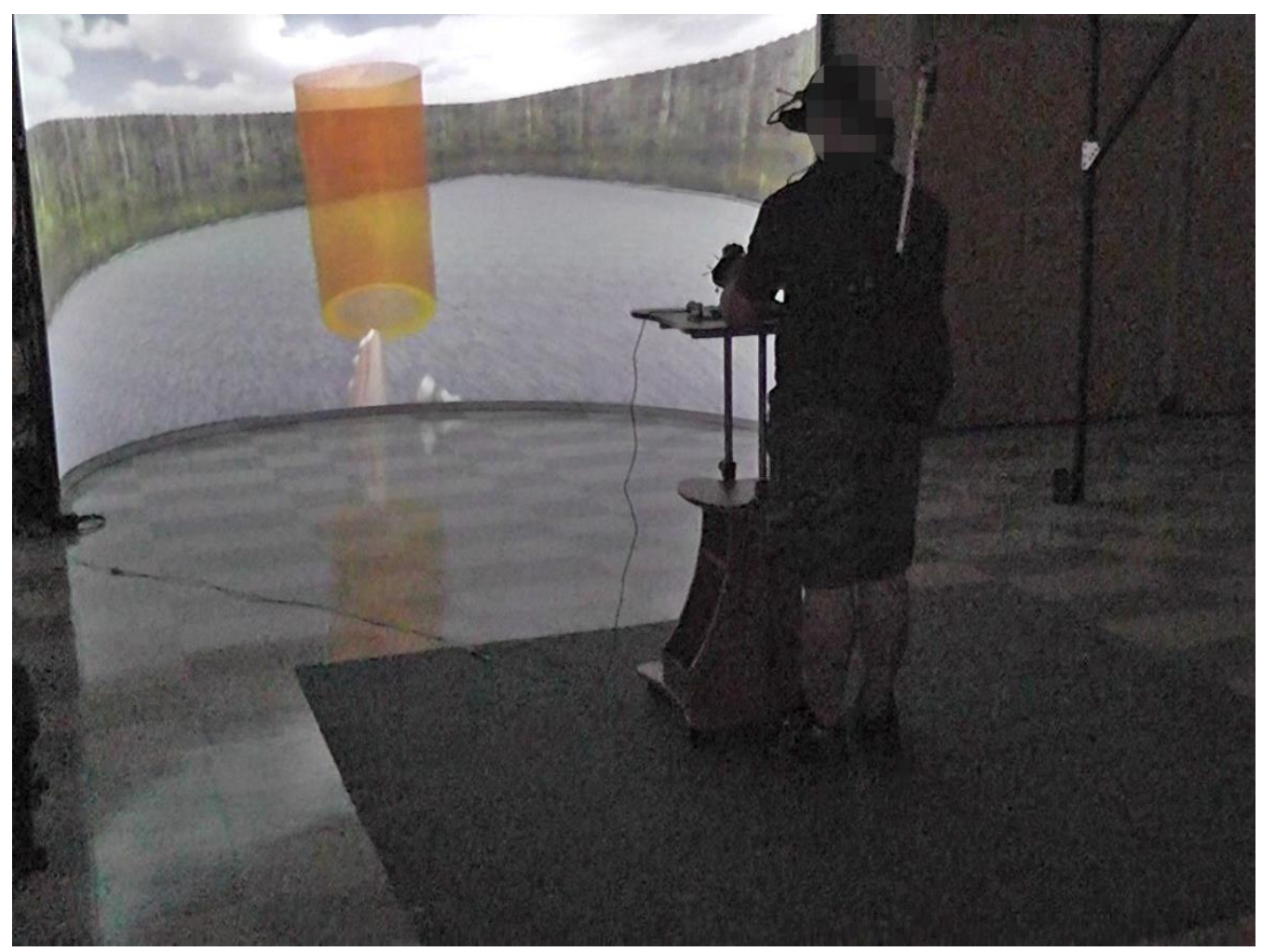

Figure 4-41: Photo of a testing session with the joystick locomotion technique.

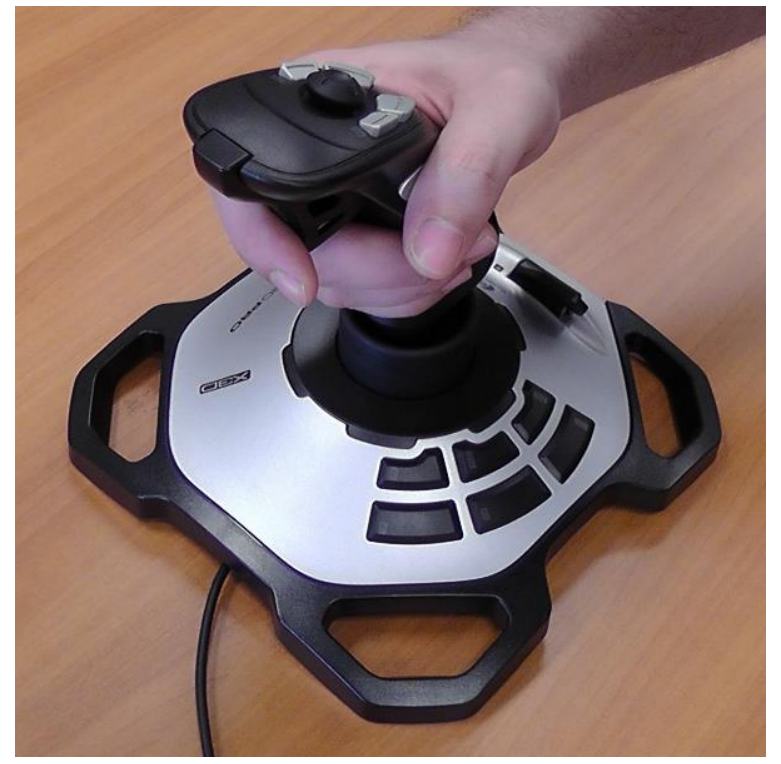

Figure 4-42: Joystick locomotion technique descriptive reference icon. 


\subsection{Trackball}

Individuals with autism are commonly characterized to be fascinated by or obsessed with spinning objects, such as wheels of toys or washing machines [153]. For that reason, a new locomotion alternative was designed in this dissertation with a trackball (Kensington Expert Trackball [154]). This technique implementation was quite similar to the joystick locomotion technique. The marker positions were as shown in Figure 4-43.

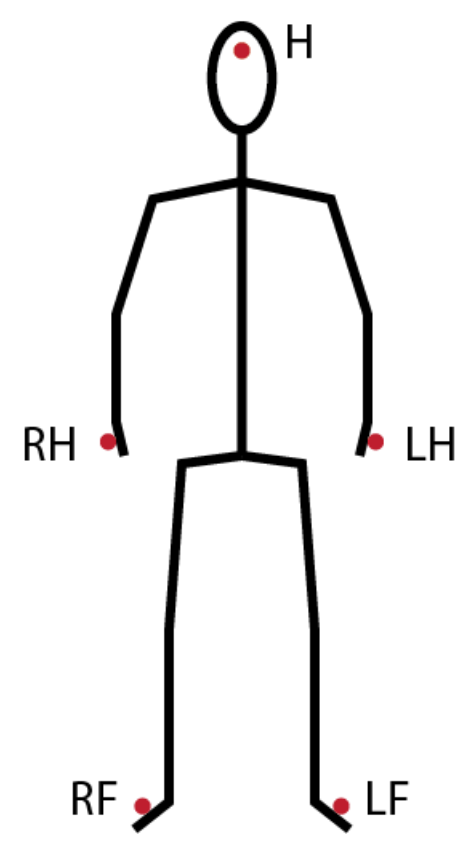

Figure 4-43: Marker positions for the trackball locomotion technique.

The users were standing in front of a high table with a trackball controller on it. The trackball was used by the dominant hand of the user and was placed accordingly on the table. This controller had a smooth surfaced large ball that could be span in any direction. The spinning of the ball controlled the locomotion in the virtual environment. 
Forward spinning resulted in forward movement and side spinning controlled the rotation.

The users needed to keep spinning the ball to move the virtual world viewpoint. One rotation of the ball provided a movement of one step in the virtual world. The user could spin the ball fast or slow, resulting in more or less rotation, and more or less movement in the virtual world respectively. A photo taken during a testing session with the trackball locomotion technique can be seen in Figure 4-44. The descriptive icon that was used to refer to the technique can be seen in Figure 4-45.

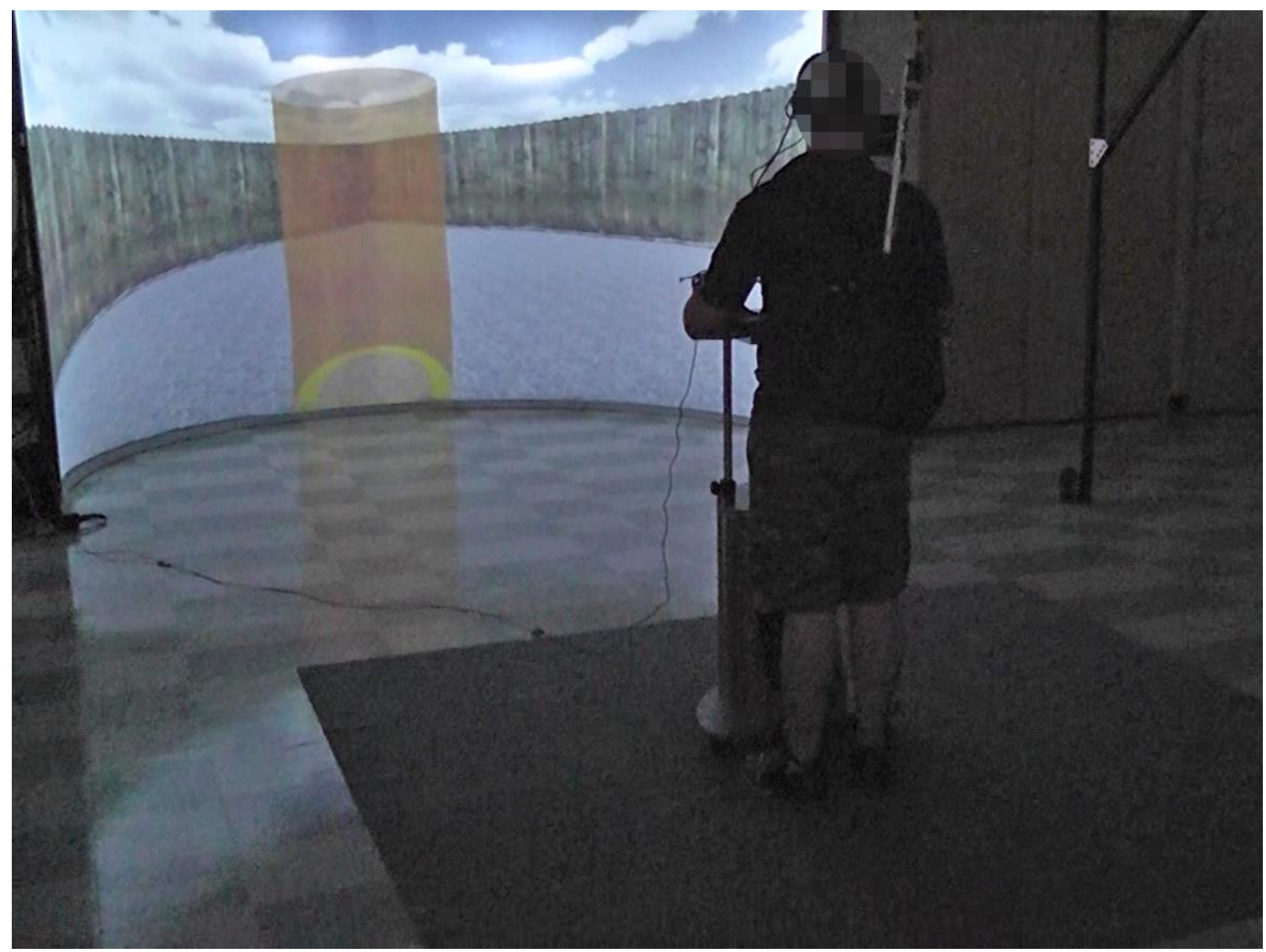

Figure 4-44: Photo of a testing session with the trackball locomotion technique. 


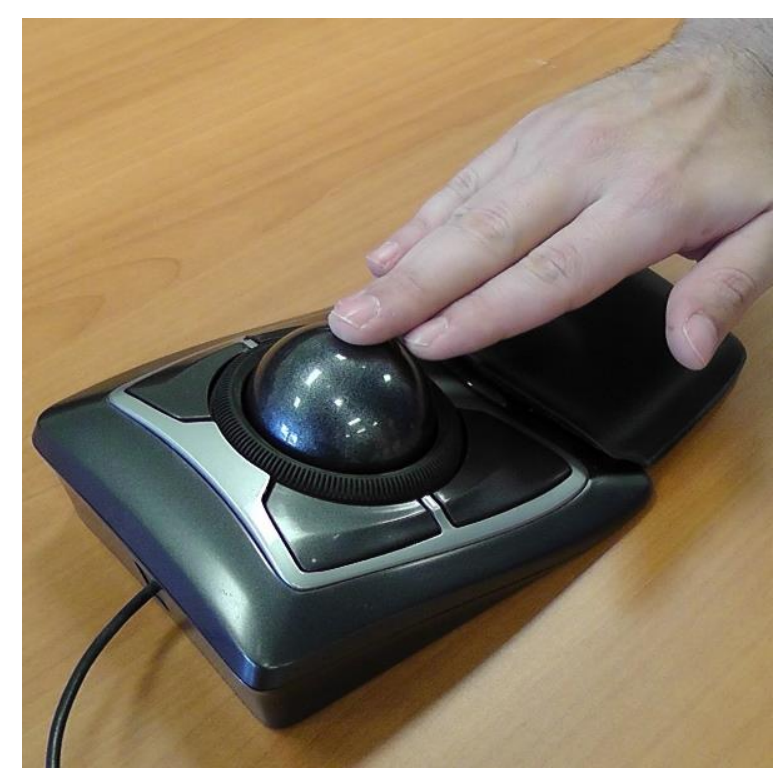

Figure 4-45: Trackball locomotion technique descriptive reference icon.

\subsection{Hand Flapping}

Individuals with autism commonly engage in self-stimulating (stimming) behaviors such as flapping arms and hands or rocking [153]. These movements are observed to provide soothing for them. Hence, the hand flapping technique was designed in this dissertation, in which the hand flapping movement was used for the locomotion. This technique could be described as the hand equivalent of the walk-in-place locomotion technique. The flapping motion was kept independent from the position of the user's hand. It could be performed wherever was more comfortable for the user such as near the hips, near the shoulder or in front of the torso. As long as the user flipped their hand, the viewpoint in the virtual environment was moved continuously. This technique was thought to provide the users with ASD soothing and help in practicing controlling the unintentional stimming behaviors since the user needed to stop the stimming to stop 
moving in the virtual world. For the hand flapping technique, the marker set positions that is shown in Figure 4-46 were used.

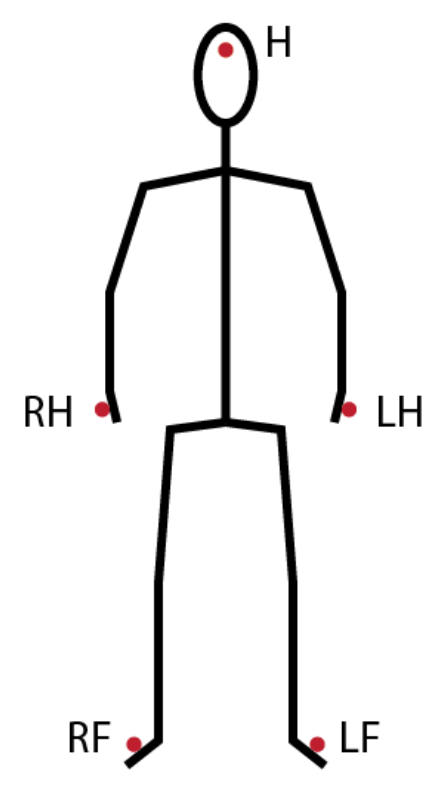

Figure 4-46: Marker positions for the hand flapping locomotion technique.

For the implementation of this locomotion technique, a structure called 'flap' was defined with two world coordinates. First one represented the starting point of a flap and the second one represented the end point.

The controlling hand was assumed to be making a flapping motion all the time. Each flap must have a specific property: all the displacements in a single flap must point to a similar direction. Once this property was rejected, the flap was assumed to be ended, and the next flap was started.

To check this property, the displacement vector was taken from the controlling hand. In the formulas in this sub-subsection, the controlling hand was assumed to be the right hand. 


$$
D(n)=P_{R H}(n)-P_{R H}(n-1)
$$

After calculating the displacement, consecutive vectors were checked for the similar direction. For this purpose, a dot product operation was used and compared a threshold value $(\mathrm{t})$ which was set to 0.98 . This threshold value corresponded to a maximum 11.4-degree difference between two consecutive displacement vectors.

$$
D(n-1) \cdot D(n)>t
$$

If this equation was violated, the flap was assumed to be finished and the new one began (see Figure 4-47).

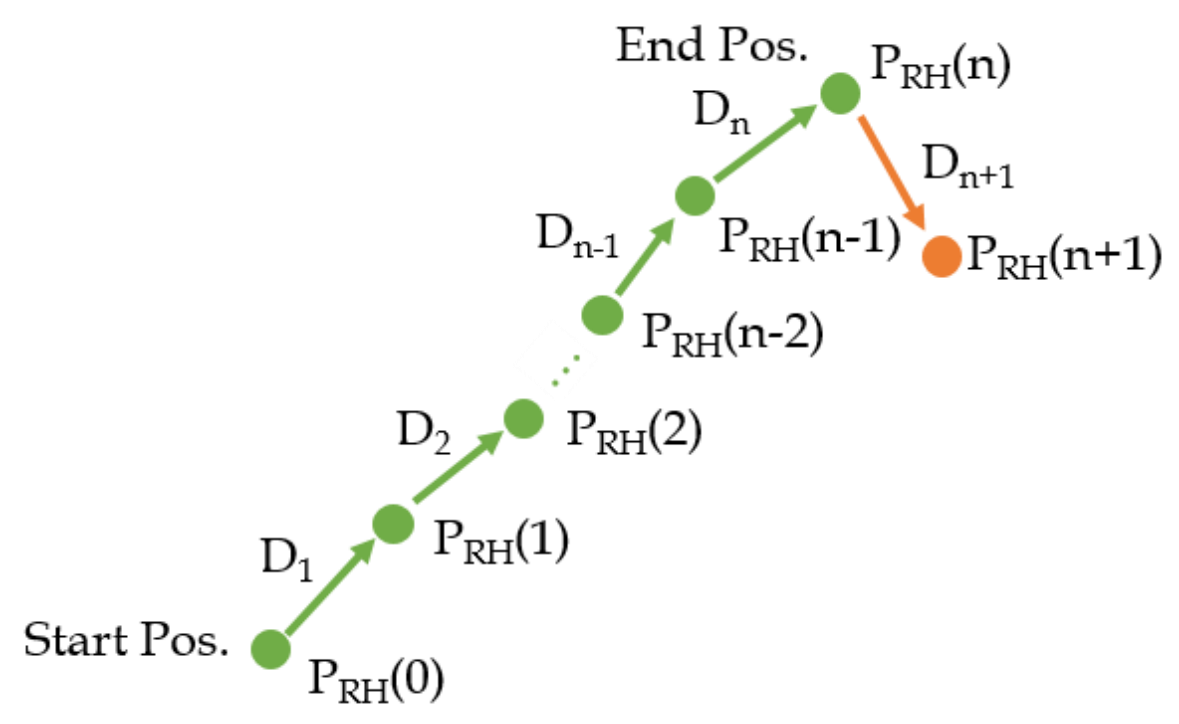

Figure 4-47: Vectors forming a single flap for the hand flapping locomotion technique.

After two flaps were completed, we compared the properties with respect to the threshold values and decided if the user was flapping or not (see Figure 4-48 for a descriptive illustration). A successful flapping action composed of two flaps must have the following properties: 
- Each flap should have a minimum total length of $\mathrm{L}_{\min }=0.05 \mathrm{~m}$.

- The distance between the start position of the first flap and the end position of the second flap should be smaller than $\Delta \mathrm{r}=0.02 \mathrm{~m}$.

- Each flap must be completed in a certain time period $\Delta \mathrm{t}=0.5 \mathrm{sec}$.

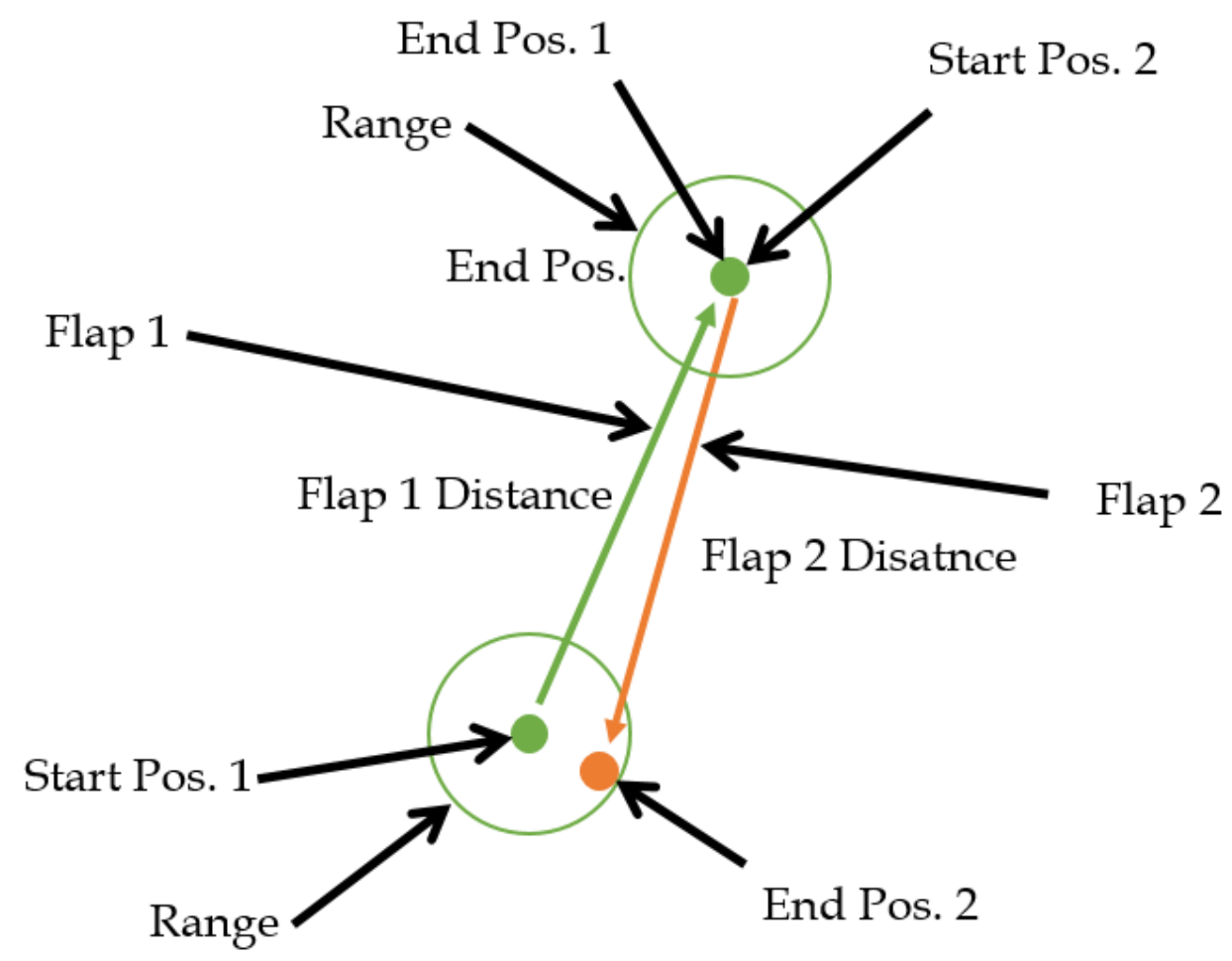

Figure 4-48: Consecutive flaps forming a flapping motion.

These conditions were checked for all consecutive flaps, and if all were met, the locomotion was started. The code excerpt for the hand flapping locomotion technique can be seen in Figure 4-49. A photo taken during a testing session with the hand flapping locomotion technique is presented in Figure 4-50. The descriptive icon that was used to refer to the technique can be seen in Figure 4-51. 


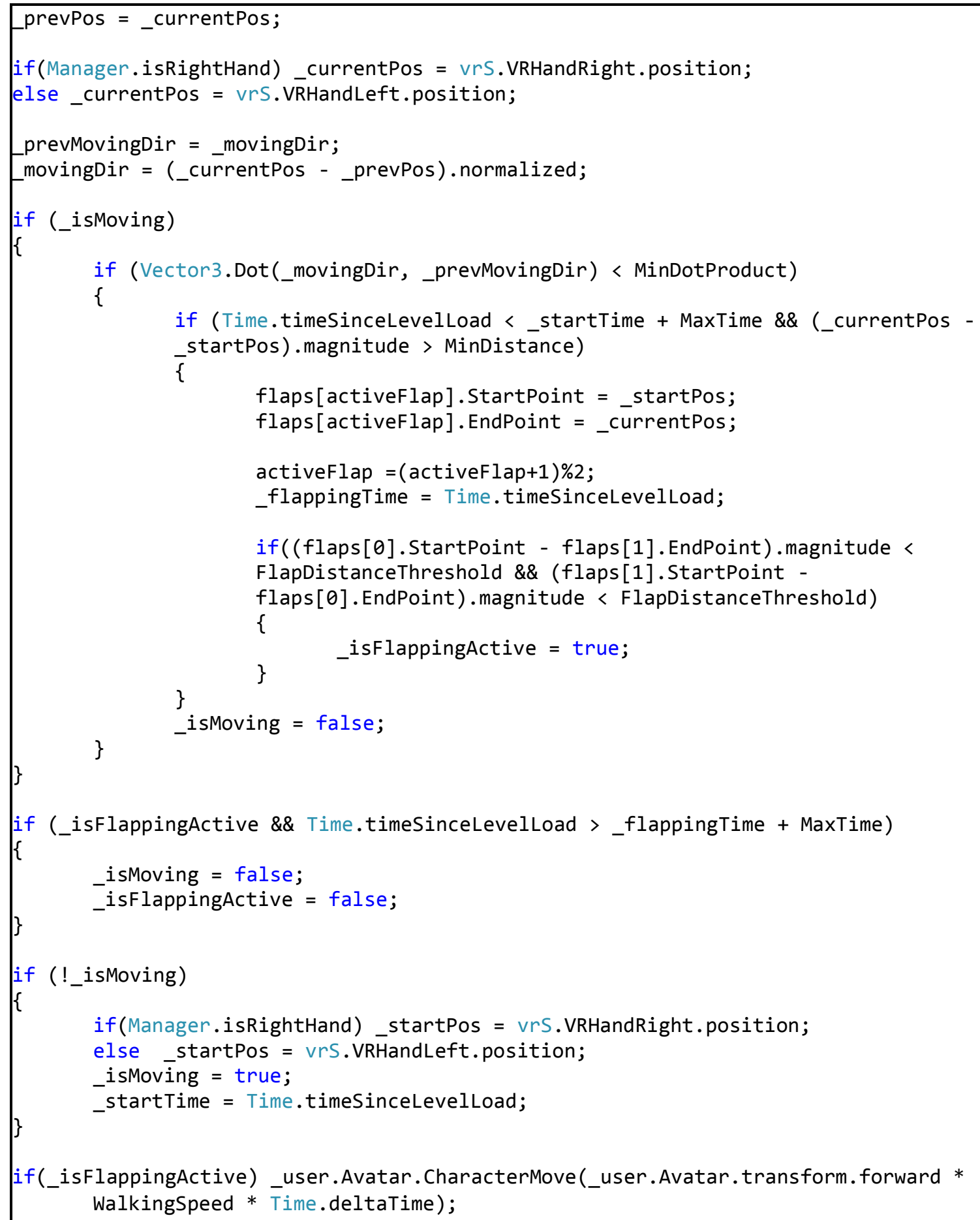

Figure 4-49: Code excerpt from the hand flapping locomotion technique implementation. 


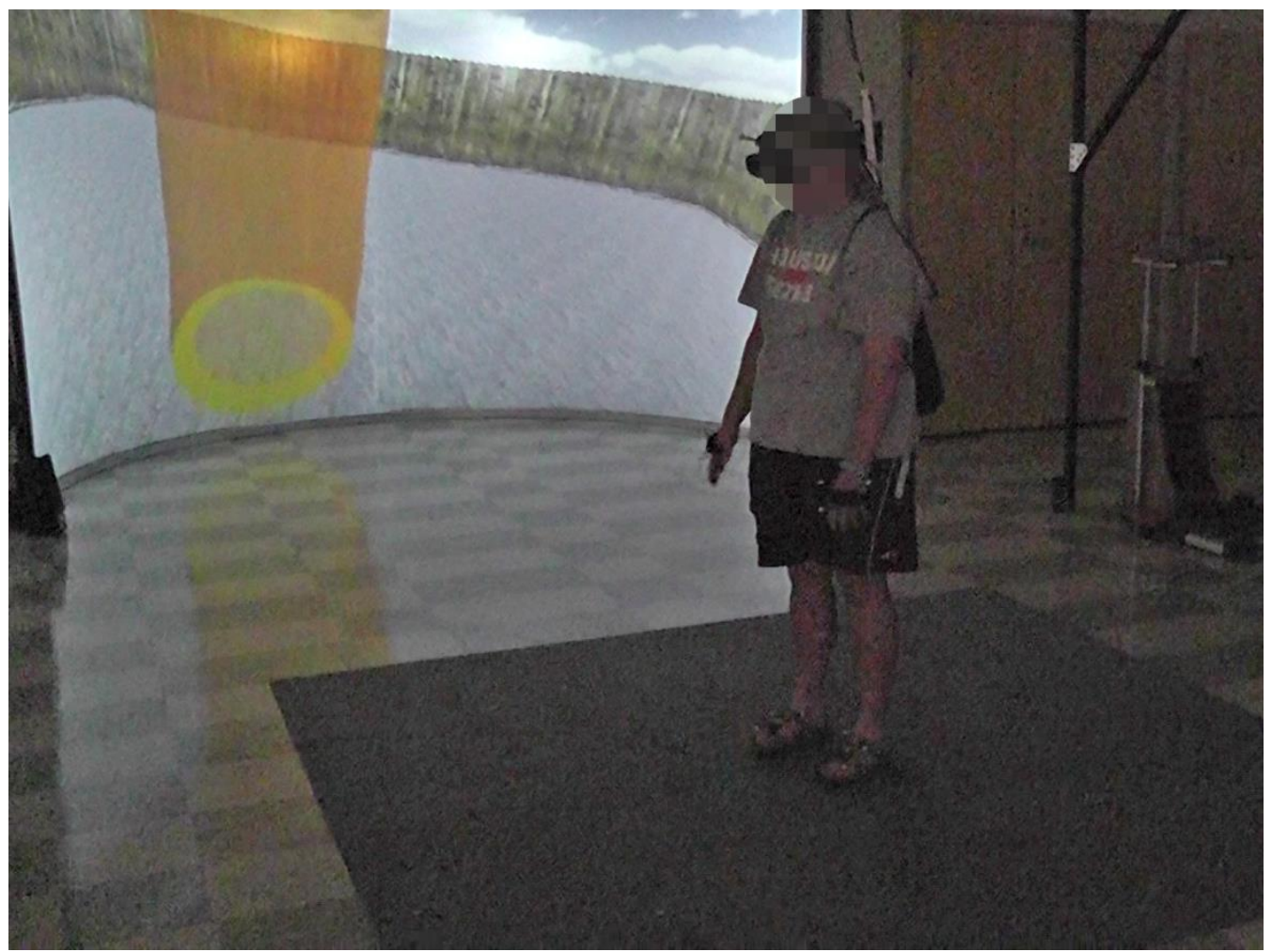

Figure 4-50: Photo of a testing session with the hand flapping locomotion technique.

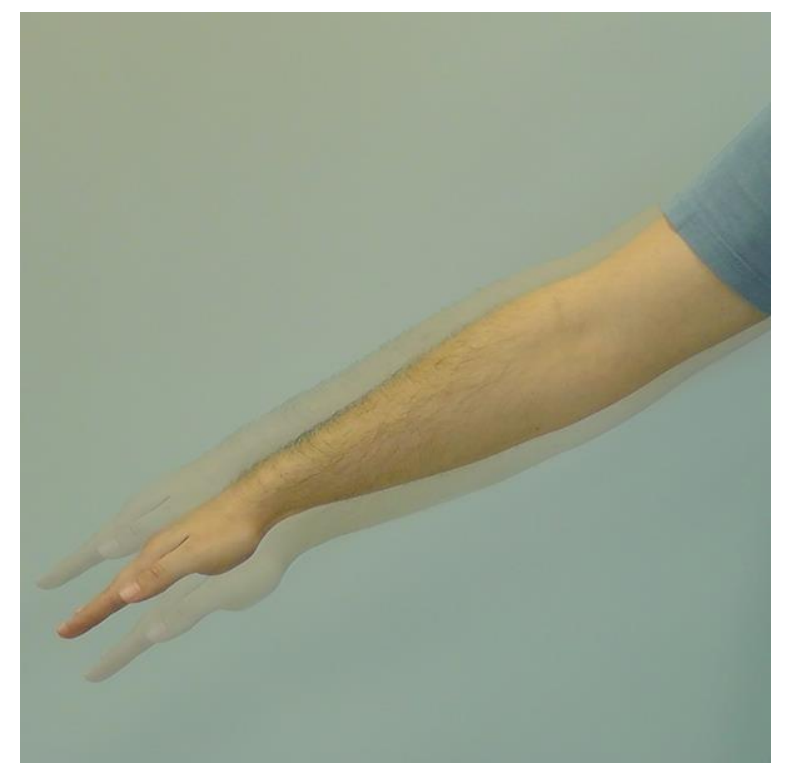

Figure 4-51: Hand flapping locomotion technique descriptive reference icon. 


\subsection{Flying}

Individuals with ASD may not handle extensive cognitive load well, especially when paired with other tasks to perform. Hence, flying was selected as a locomotion technique to be suitable for individuals with autism in this dissertation. Flying is usually characterized to be one of the simplest locomotion techniques. It gets an input from the user to move viewpoint of virtual world [86]. Input can be either continuous to keep moving or instantaneous to start or stop the movement. For the input, a controller button or a body gesture can be used.

In our study, in order to avoid the cognitive load of an additional controller, a hand raising gesture was selected for triggering the automatic locomotion in the virtual environment. The same gesture was used to stop the locomotion as well. To reduce the physical load on the users, raising the hand up to the shoulder level was defined as the trigger threshold. Hand raising gesture was selected because of its common use for showing that an action is needed to be done. The controlling hand was chosen as the dominant hand of the user. This gesture was selected because it does not introduce constant strain on the arm, either during the locomotion or standing still. The marker set positions for this technique was as shown in Figure 4-52.

This time the height value of the dominant hand was checked at every cycle. In the formulas in this sub-subsection, the dominant hand was assumed to be the right hand of the user.

$$
\left(P_{R H}(n)\right) \cdot y>\left(P_{H}(n)\right) \cdot y-c
$$


If this equation was approved, it meant that the user has triggered the locomotion. The constant $\mathrm{c}$ is the difference between the required hand raising height and the head marker height. It was chosen to be $0.4 \mathrm{~m}$, which is about the average distance between the top of the head and the shoulders.

This approach gave a different required height for the users with different heights without initial calibration of the system for each user. The code excerpt for the implementation of the flying locomotion technique can be seen in Figure 4-53.

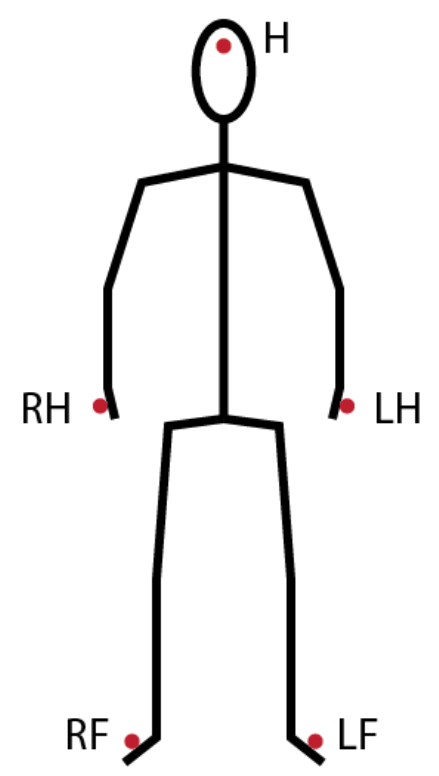

Figure 4-52: Marker positions for the flying technique.

The walking speed was chosen to be constant $(0.8 \mathrm{~m} / \mathrm{s})$, a reasonable walking speed in the virtual environment. A photo taken during a testing session with the flying locomotion technique can be seen in Figure 4-54. The descriptive icon that was used to refer to the technique is presented in Figure 4-55. 


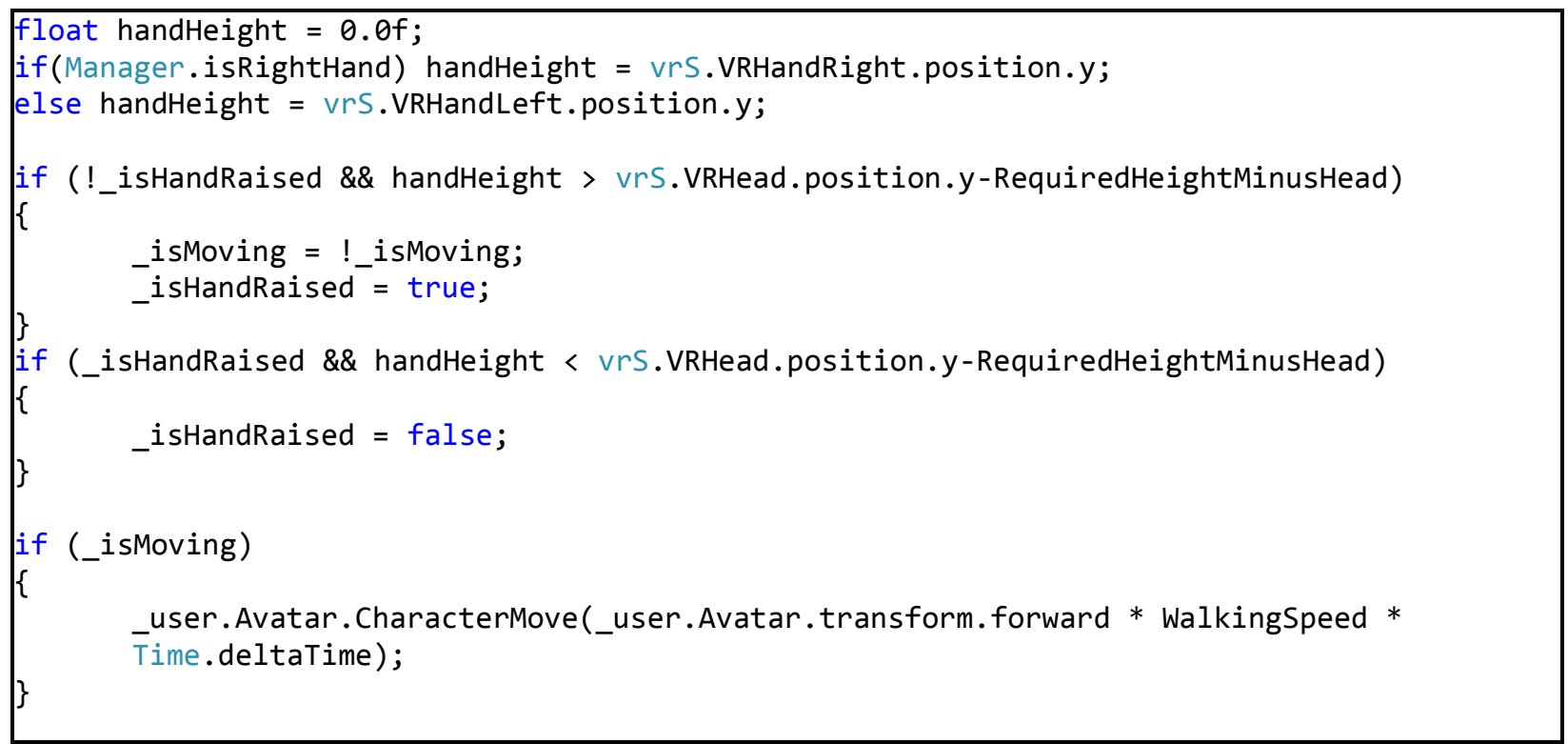

Figure 4-53: Code excerpt from the flying locomotion technique implementation.

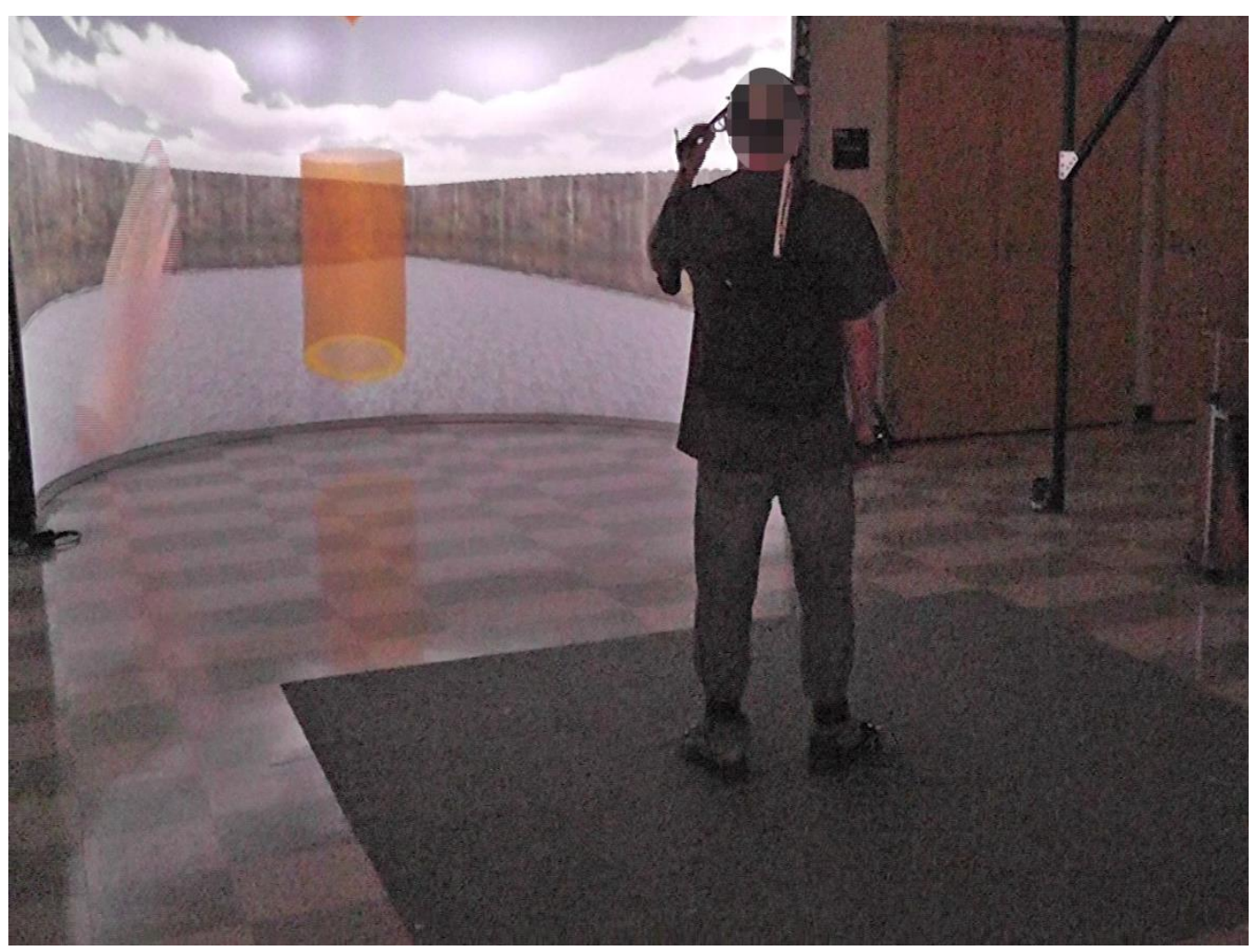

Figure 4-54: Photo of a testing session with the flying locomotion technique. 


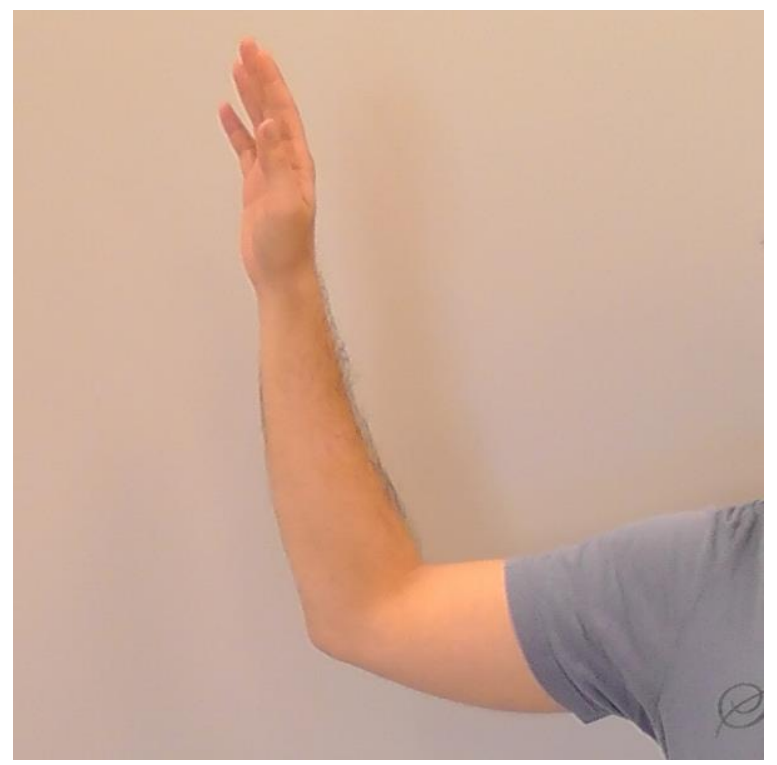

Figure 4-55: Flying locomotion technique descriptive reference icon. 


\section{CHAPTER 5: COMPARISON OF EIGHT LOCOMOTION TECHNIQUES IN ROOM SCALE TRACKED VIRTUAL REALITY}

This chapter presents the evaluation of eight locomotion techniques in room scale tracked virtual reality. An immersive virtual reality experiment was designed and implemented to evaluate the eight different locomotion techniques with both neurotypical individuals and individuals with high functioning ASD. The eight locomotion techniques that were evaluated are: redirected walking, walk-in-place, stepper machine, point \& teleport, joystick, trackball, hand flapping and flying. In this chapter, the experiment design, results for neurotypical users, results for high functioning individuals with autism, and comparison of results of these two populations were discussed.

\subsection{Note to Reader}

Portions of this chapter were published in CHI PLAY 2016 (Bozgeyikli, E., Raij, A., Katkoori, S., and Dubey, R. Point \& Teleport Locomotion Technique for Virtual Reality. In Proceedings of the 2016 Annual Symposium on Computer-Human Interaction in Play (CHI PLAY '16). ACM, New York, NY, USA.) and in ACM Symposium on Spatial User Interaction 2016 (Bozgeyikli, E., Raij, A., Katkoori, S., and Dubey, R. Locomotion in Virtual Reality for Individuals with Autism Spectrum Disorder. In Proceedings of the $4^{\text {th }}$ 
ACM Symposium on Spatial User Interaction (SUI'16). ACM, New York, NY, USA.) Permissions are included in Appendix C.

\subsection{Experiment Design}

A within-subjects experiment was designed with the independent variable of the locomotion technique having eight levels: redirected walking, walk-in-place, stepper machine, point \& teleport, joystick, trackball, hand flapping and flying. All of these eight locomotion techniques were tried by all users with a randomly assigned order. Counterbalancing was applied to have a distribution close to equal for all combinations of ordering. For each technique, the locomotion direction was defined as the head direction and the head rotation could be used for rotating the virtual viewpoint. For the controller based techniques, the virtual rotation could be controlled via the controller and/or the head rotation. All applicable techniques (point \& teleport, hand flapping and flying) were implemented to work with either left hand or right hand to cater for both right handed and left handed users. A different set of destination points were used for each technique, to eliminate any learning effect and possible memorization.

\subsubsection{Virtual Avatar}

The user was represented with two hand and two feet models in the virtual environment. The users were able to see these virtual hands and feet in the virtual environment, which were moved according to their real hand and foot movement. The reason for this was to give feedback on their position and orientation. For an accurate representation, marker sets were attached to the hands and the feet of the user. The

physical space that user's body occupied in the virtual environment was defined as a 
vertical capsule with a $0.5 \mathrm{~m}$ diameter that was placed at the weighted center of the user's two feet and the head.

\subsubsection{Virtual Environment}

To evaluate the eight locomotion techniques, a simple yet realistic looking outdoor virtual environment with $16 \mathrm{~m}$ by $16 \mathrm{~m}$ dimensions was designed. The reason behind the virtual environment's being produced as realistic looking was the previous studies stating that locomotion was more efficient when the virtual environment looked visually more realistic $[36,155]$. The virtual environment was restricted on all sides with virtual walls of $2.2 \mathrm{~m}$ height. The users initially appeared in the center of the virtual environment. They were free to move inside the virtual environment but they could not go beyond the virtual walls. The virtual environment was designed plain to avoid exerting additional cognitive load to the users, overwhelming or distracting them. A basic ambient light was used along with low intensity directional lights to create a good visibility from all sides of the virtual environment. A simple, relaxing outdoor sound was played in low volume to increase the immersion.

\subsubsection{Objective}

The users were asked to go to ten destination points with each locomotion technique. Once arrived to a destination point, the users needed to wait inside until the marking objects around them disappeared. The disappearance of the marking objects was done after three continuous seconds that was started when the user stepped inside the destination point area. The clearance of the destination points was not designed to be instantaneous since the users' control on stopping the locomotion technique was also 
desired to be observed and evaluated. The objective of the experiment was kept as simple as possible to be able to evaluate the user experience on the locomotion techniques without the additional effects that may come from different factors.

After the sixth destination point, 21 obstacles in the form of cylindrical roman pillars appeared in the virtual world (see Figure 5-1). Each obstacle was of $0.4 \mathrm{~m}$ diameter and $2.4 \mathrm{~m}$ height and was $1.77 \mathrm{~m}$ away from the neighboring obstacle, which gave enough space to the user to move around them.

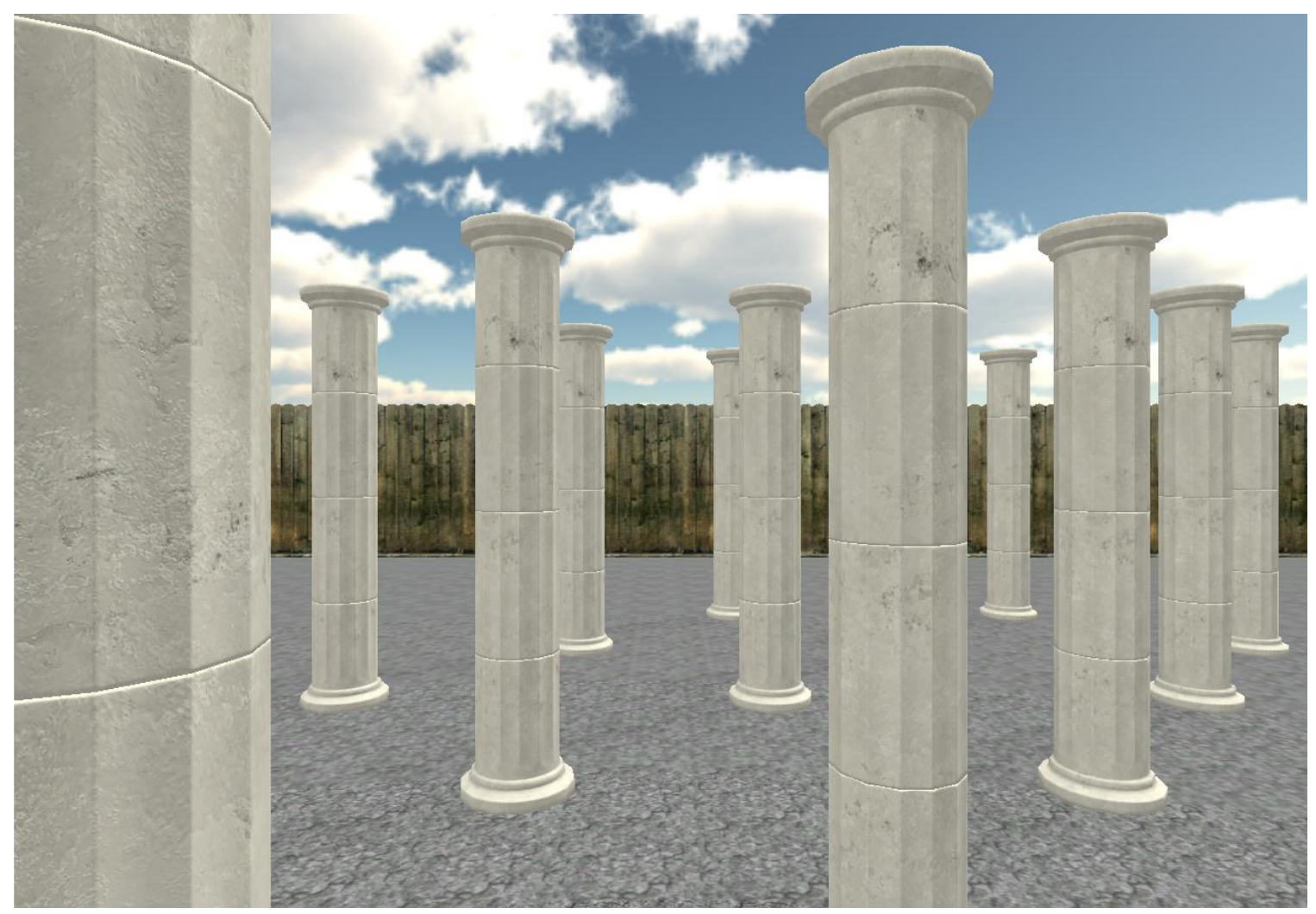

Figure 5-1: Cylindrical roman pillars as static obstacles.

The reason behind these obstacles was to observe and evaluate the users' control on making turns with the locomotion techniques. The users were supposed to go to the destination points without colliding with the obstacles. Without the obstacles, the users 
could reach to the destination points with movements that were close to straight lines. The placement of the obstacles and the destination points were designed so that the users required to make turns to avoid collision with the obstacles.

\subsubsection{Destination Points}

The destination points were marked with three objects to ensure a clear visibility; a circle on the ground having $1.2 \mathrm{~m}$ diameter, a semi-transparent cylinder with $2 \mathrm{~m}$ height and $1.2 \mathrm{~m}$ diameter, and a 3D arrow above the cylinder oscillating in the y-axis to point to the destination point (see Figure 5-2). All these objects were designed to be easily seen and identified even from the longest distance in the virtual environment. The marker objects were designed in orange to be easily visible.

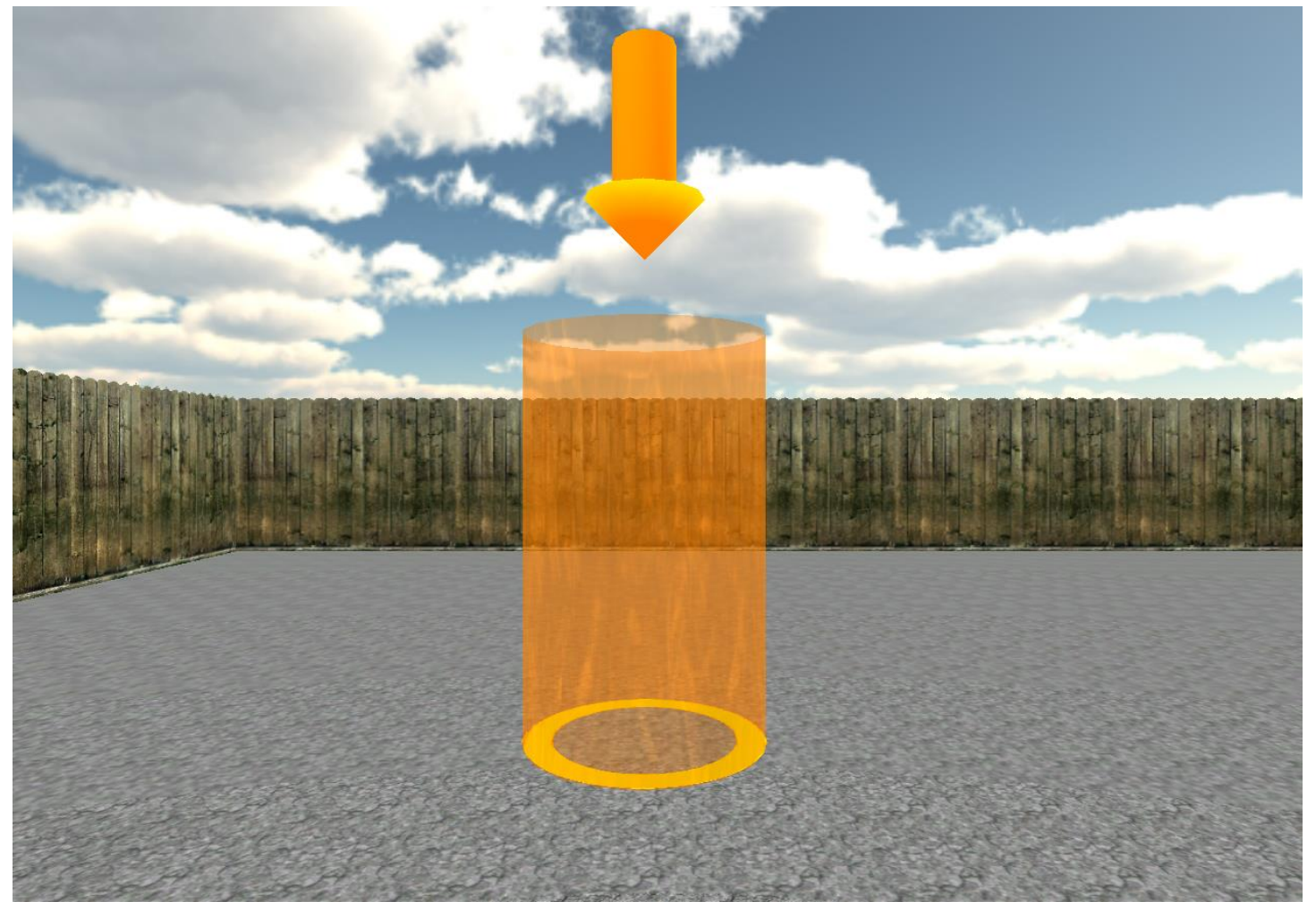

Figure 5-2: Destination point in virtual environment. 
Once the user stepped inside the destination point area, the color of the objects immediately turned to green to give real time feedback to the users. As the user stayed inside these marker objects, the color gradually turned to blue in 3 seconds. If the user stepped outside of the destination point before 3 seconds, the cycle was reset and the color of the marker objects was turned back to orange. For each technique, a different set of destination points were used to eliminate any possible learning effect that might be caused by memorization. The first destination point appeared 2 meters away from the user. After the first one, each new destination point appeared 4 meters away from the previous one. Furthermore, each destination point required $180^{\circ} \pm 30^{\circ}$ turns to be reached after the previous one.

\subsubsection{Procedure}

The participants arrived at the research laboratory. They first read then signed the IRB consent form and filled out a demographics related survey. Then, the research staff explained the VR system and their objective in the experiment to the participants. The destination points, color changing dynamics of the marker objects and the obstacles that appear after the sixth destination point were explained to the participants. They were requested to try not hitting the obstacles. Then, the research staff helped the participants to wear the HMD, and hand and feet marker bands for motion tracking. After answering any possible questions of the participants, the experiment began. The participants tried one of the randomly assigned eight locomotion techniques. When they completed all 10 destination points with the assigned locomotion technique, a user experience survey was given to the participants for evaluation of the tried locomotion technique. After all of the 
locomotion techniques were tried, an overall survey was given to the participants that requested them to rank the techniques according to their preference. The surveys can be seen in Appendix F. Block diagram for the experiment can be seen in Figure 5-3.

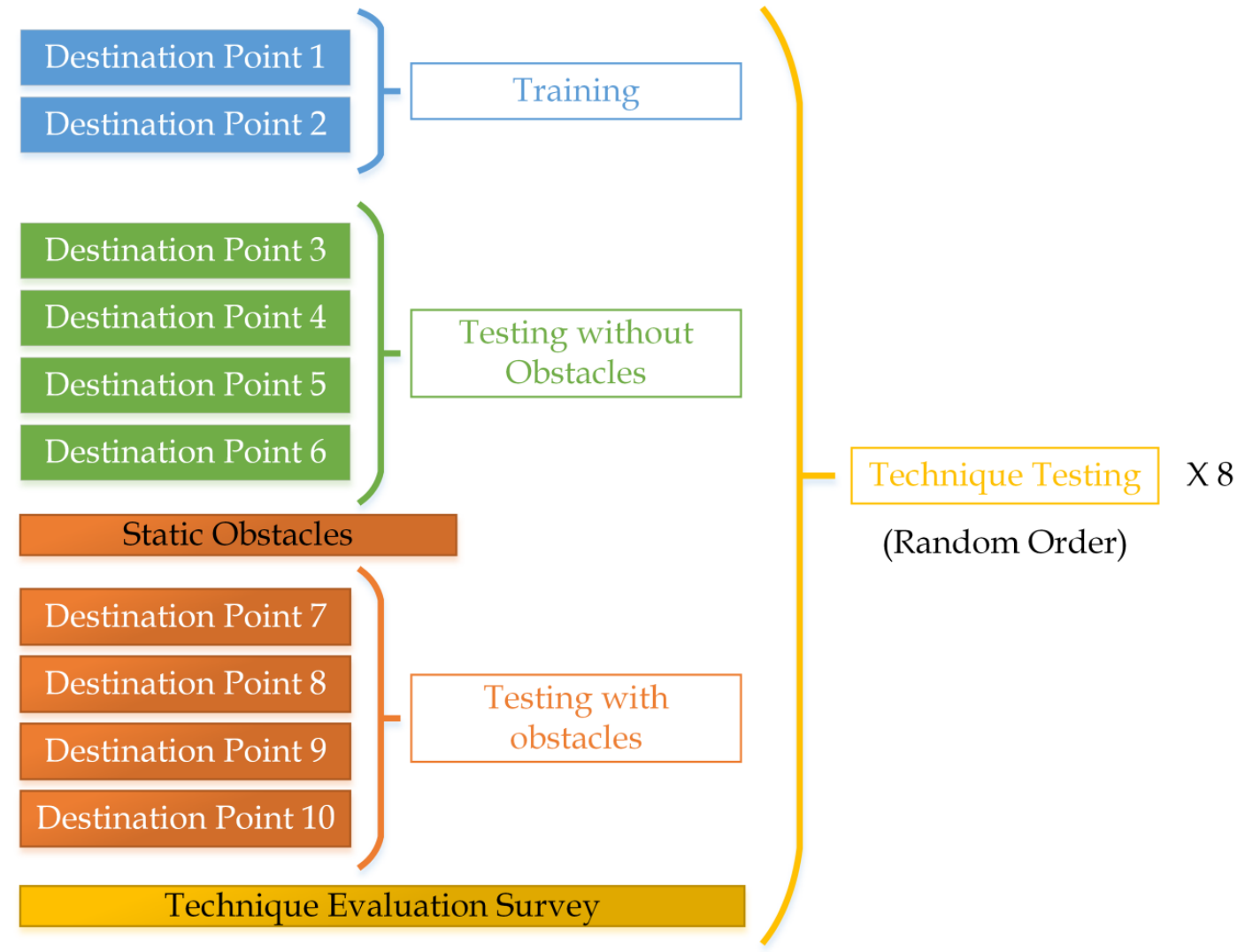

\section{Overall Preference Survey}

Figure 5-3: Block diagram for the experiment of the comparison of the eight locomotion techniques.

For the participants with ASD, a specially designed sorting table was used to rank the locomotion techniques. The techniques were represented by the descriptive icons that were mentioned in the previous chapter. The table was printed out on a large paper and photos were taken after the participant finished sorting to keep record. One example of a sorted preference of the locomotion techniques list can be seen in Figure 5-4. This ranking 
technique was utilized to decrease the cognitive effort required to rank the eight locomotion techniques, with the recommendation of the professional job trainers of individuals with ASD.

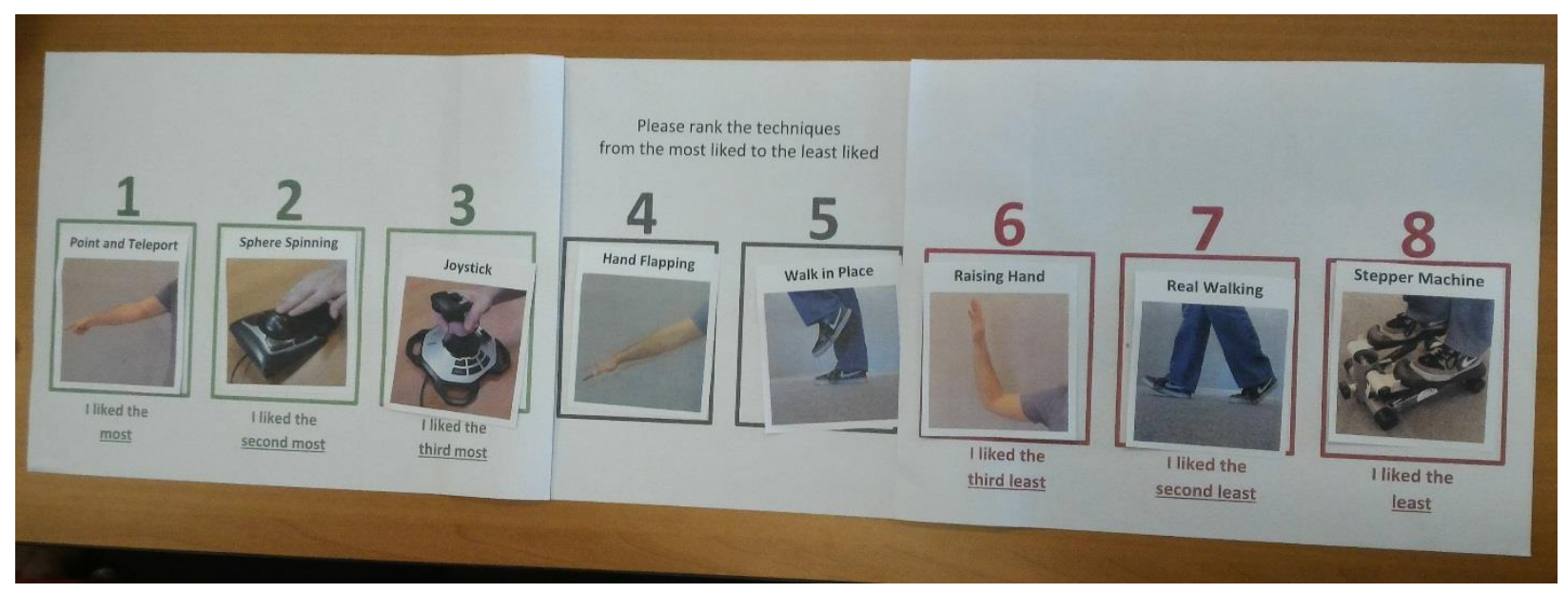

Figure 5-4: Sorting chart for the participants with ASD.

The eight locomotion techniques in the experiment were assigned to the users in random order. Counterbalancing was utilized to ensure an equal distribution among the possible ordering combinations. The experiment lasted around one hour per participant, with 3 minutes of VR exposure followed by 5 minute breaks to fill out the surveys for each locomotion technique. All sessions were video and audio recorded. The user study was performed under the IRB with the number Ame7_Pro00013008, the IRB approval letter can be seen in Appendix D.

\subsubsection{Data Collection}

During the user study sessions, timestamps for clearing each destination point and collision with obstacles, and virtual and real locomotion paths of each user was stored for each locomotion technique. After the completion of each technique trial, the users filled 
out a survey about the experience they had with that locomotion technique. The questions included the following aspects: ease of understanding, ease of operating, required effort, tiredness, being in control, enjoyment, being overwhelmed and frustrated; questions about motion sickness and presence. The survey was constructed as modified a version of Loewenthal's core elements of the gaming experience questionnaire [156], Pensacola Diagnostic Criteria survey on motion sickness [157] and Witmer and Singer's questionnaire on presence [158]. After completing the testing of all eight locomotion techniques, the users were asked to rank the eight techniques according to their preferences.

\subsection{Neurotypical Users}

This subsection presents neurotypical user demographics and the results for this population. Discussions, conclusions and future work directions were also included. The research question for this user study was: 'Which of the eight virtual locomotion techniques would provide the best user experience for neurotypical individuals in room scale tracked areas?' Based on this research question, the following null hypotheses were constructed: Hypothesis1,0: All locomotion techniques will result in similar performance in terms of average time to reach to the destination points, Hypothesis2,0: All locomotion techniques will result in similar ranking scores.

\subsubsection{Participants}

16 neurotypical individuals participated in the study. The gender distribution was 11 males and 5 females. The participants were aged between 21 and $33(\mu=25.8, \sigma=3.05)$. All participants were undergraduate or graduate university students from different 
majors. All participants' dominant side was right. All participants had none to minimal previous virtual reality experience. A $\$ 50$ gift card was given to the participants as an incentive. Due to a hardware malfunction during the testing session of one participant, their data was discarded and data analysis was performed for 15 neurotypical participants $(\mathrm{N}=15)$.

\subsubsection{Results}

This sub-subsection presents the user study results for neurotypical individuals under these categories: data results, survey results, participant comments, discussion, summary, conclusion and future work.

\subsubsection{Data Results}

The data of the time it took for the users to reach to the destination points was analyzed in two groups based on the presence of obstacles in the form of pillars: noobstacles and obstacles. The bar charts for the average times to reach the destination points can be seen in Figure 5-5. Error bars in all of the charts represent standard error of the mean.

One-way ANOVA with repeated measures with $\alpha=0.05$ resulted in significant difference for both the no-obstacles case $(F(7,7)=17.220, \mathrm{p}=0.000)$ and for the obstacles case $(F(7,7)=7.970, p=0.000)$. Mauchly's sphericity test failed and Greenhouse-Geisser correction was performed for both cases. Paired t-test analysis results that yielded the largest and the smallest statistically significant mean differences between two techniques are reported in Table 5-1. 


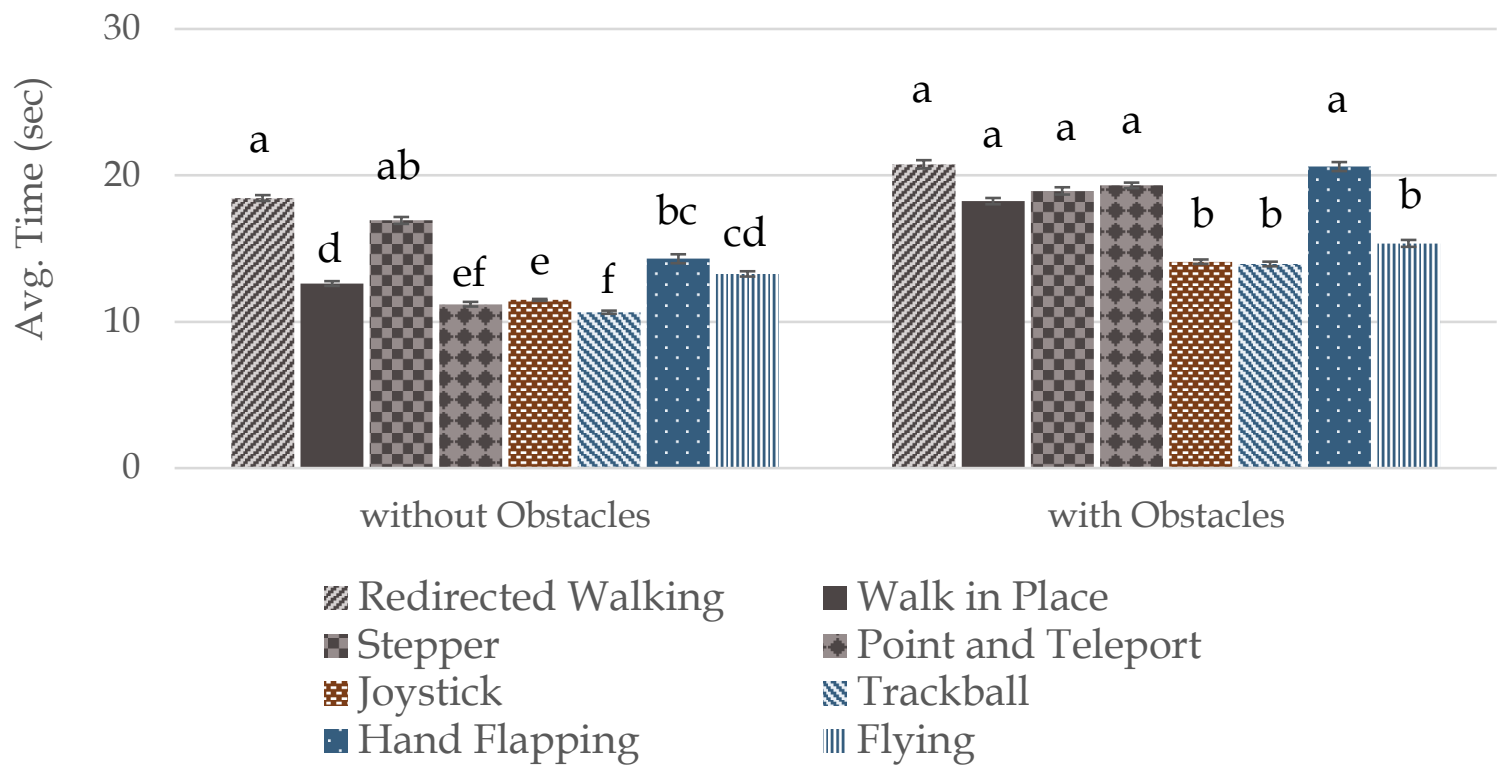

Figure 5-5: Average time to reach to the destination points without and with obstacles for the neurotypical users with the eight locomotion techniques.

Table 5-1: Paired t-test results for the largest and the smallest significant mean differences for average destination clearance times for the neurotypical users.

$\begin{array}{lcccc}\text { No-Obstacles } & & \boldsymbol{\mu} \text { Diff. } & \text { Std. E } & \mathbf{p} \\ \text { Redirected Walking } & \text { Trackball } & 7.798 & 0.878 & 0.000 \\ \text { Joystick } & \text { Trackball } & 0.839 & 0.346 & 0.029\end{array}$

\section{Obstacles}

$\begin{array}{lllll}\text { Hand Flapping } & \text { Trackball } & 6.655 & 1.946 & 0.004 \\ \text { Walk-in-Place } & \text { Flying } & 2.887 & 1.148 & 0.025\end{array}$

For the obstacles case, data for the number of collisions that were made with the static obstacles in the virtual environment were also analyzed. The bar charts for the 
average number of collisions can be seen in Figure 5-6. Collisions that were made with the dynamic objects (the pop-up walls in the redirected walking) were excluded from this analysis for comparable results among the locomotion techniques. One-way ANOVA with repeated measures with $a=0.05$ resulted in significant difference $F(7,7)=8.099, p$ $=0.000$. Mauchly's sphericity test was failed and Greenhouse-Geisser correction was performed. In the paired t-tests, the largest significant mean difference was between redirected walking and point $\&$ teleport $(\mu$ difference $=3.800, p=0.001$ ), and the smallest significant mean difference was between walk-in-place and point \& teleport ( $\mu$ difference $=0.867, \mathrm{p}=0.010)$. Detailed statistical analysis can be seen in Appendix G.

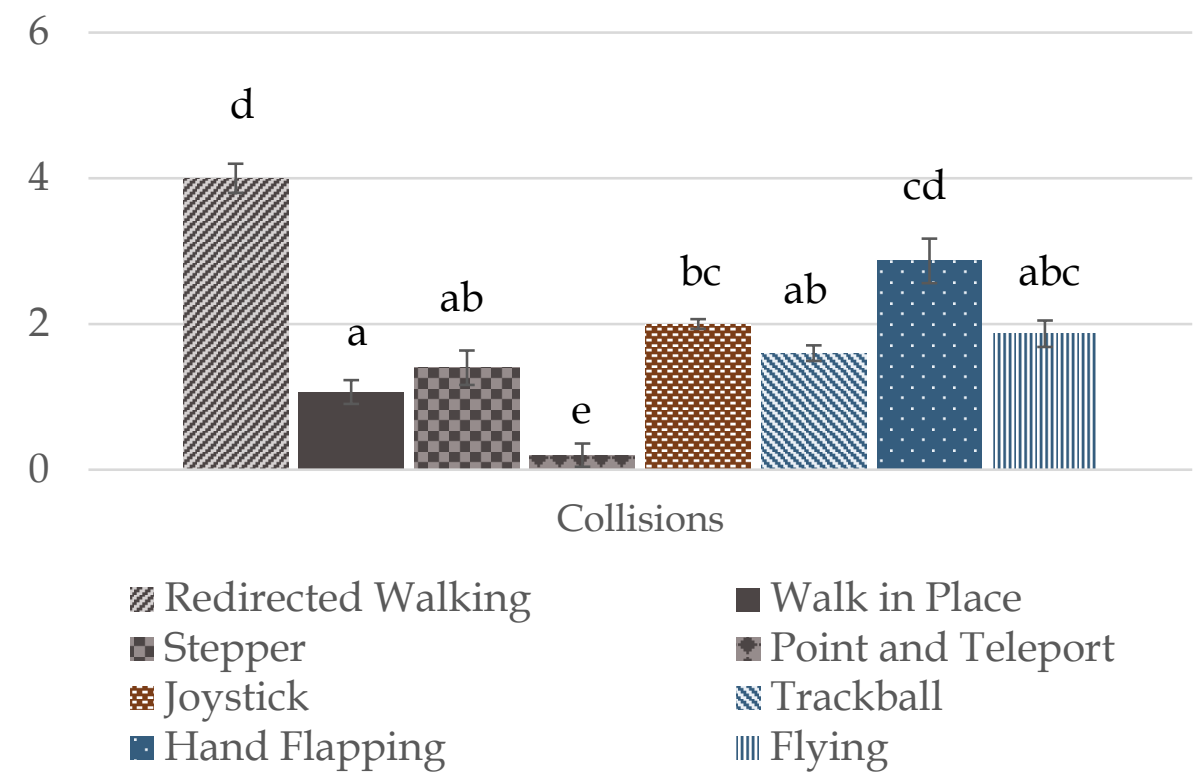

Figure 5-6: Average number of collisions with static obstacles for the neurotypical users.

\subsubsection{Survey Results}

The user experience survey included questions on eight categories: difficulty in understanding the locomotion method, difficulty in operating the locomotion method, 
feeling of being in control while using the locomotion method, required effort to use the locomotion method, feeling of tiredness the locomotion method caused, feeling of enjoyment the locomotion method caused, feeling of being overwhelmed the locomotion method caused and feeling of frustration the locomotion method caused. The answers were based on a 5 point Likert scale (1: not at all, 5: very much). Bar charts for these eight categories are presented in Figure 5-7. Results for one-way ANOVA with repeated measures with $a=0.05$ are reported in Table 5-2. Results for the paired t-tests which resulted in the largest and the smallest statistically significant mean differences are reported in Table 5-3.

Table 5-2: One-way ANOVA results for the user experience survey for the neurotypical users.

\begin{tabular}{|c|c|c|c|c|c|}
\hline & Correction & df & df Err. & $\mathbf{F}$ & $\mathbf{p}$ \\
\hline $\begin{array}{l}\text { Difficulty in } \\
\text { Understanding }\end{array}$ & $\begin{array}{l}\text { Greenhouse- } \\
\text { Geisser }\end{array}$ & 3.383 & 47.369 & 7.850 & 0.000 \\
\hline Difficulty in Operating & $\begin{array}{l}\text { Greenhouse- } \\
\text { Geisser }\end{array}$ & 3.663 & 51.286 & 10.243 & 0.000 \\
\hline In Control & $\begin{array}{l}\text { Sphericity } \\
\text { Assumed }\end{array}$ & 7 & 98 & 8.569 & 0.000 \\
\hline Enjoyment & $\begin{array}{l}\text { Sphericity } \\
\text { Assumed }\end{array}$ & 7 & 98 & 5.651 & 0.000 \\
\hline Required Effort & $\begin{array}{l}\text { Sphericity } \\
\text { Assumed }\end{array}$ & 7 & 98 & 8.660 & 0.000 \\
\hline Tiredness & $\begin{array}{l}\text { Sphericity } \\
\text { Assumed }\end{array}$ & 7 & 98 & 5.941 & 0.000 \\
\hline Overwhelmedness & $\begin{array}{l}\text { Greenhouse- } \\
\text { Geisser }\end{array}$ & 3.345 & 46.829 & 2.330 & 0.080 \\
\hline Frustration & $\begin{array}{l}\text { Greenhouse- } \\
\text { Geisser }\end{array}$ & 3.928 & 54.987 & 6.492 & 0.000 \\
\hline
\end{tabular}


Motion sickness and presence questions were based on a 4 point Likert answer scale (0: none, 3: major). The bar charts for the results of the motion sickness and presence are presented in Figure 5-8. One-way ANOVA with repeated measures with $\alpha=0.05$ resulted in no significant difference between the eight locomotion techniques for motion sickness $(F(7,7)=1.680, p=0.199)$ but resulted in significant difference for presence $(F(7$, 7) $=1.329, p=0.020)$. Mauchly's sphericity test was failed and Greenhouse-Geisser correction was performed for both motion sickness and presence data (Table 5-4).
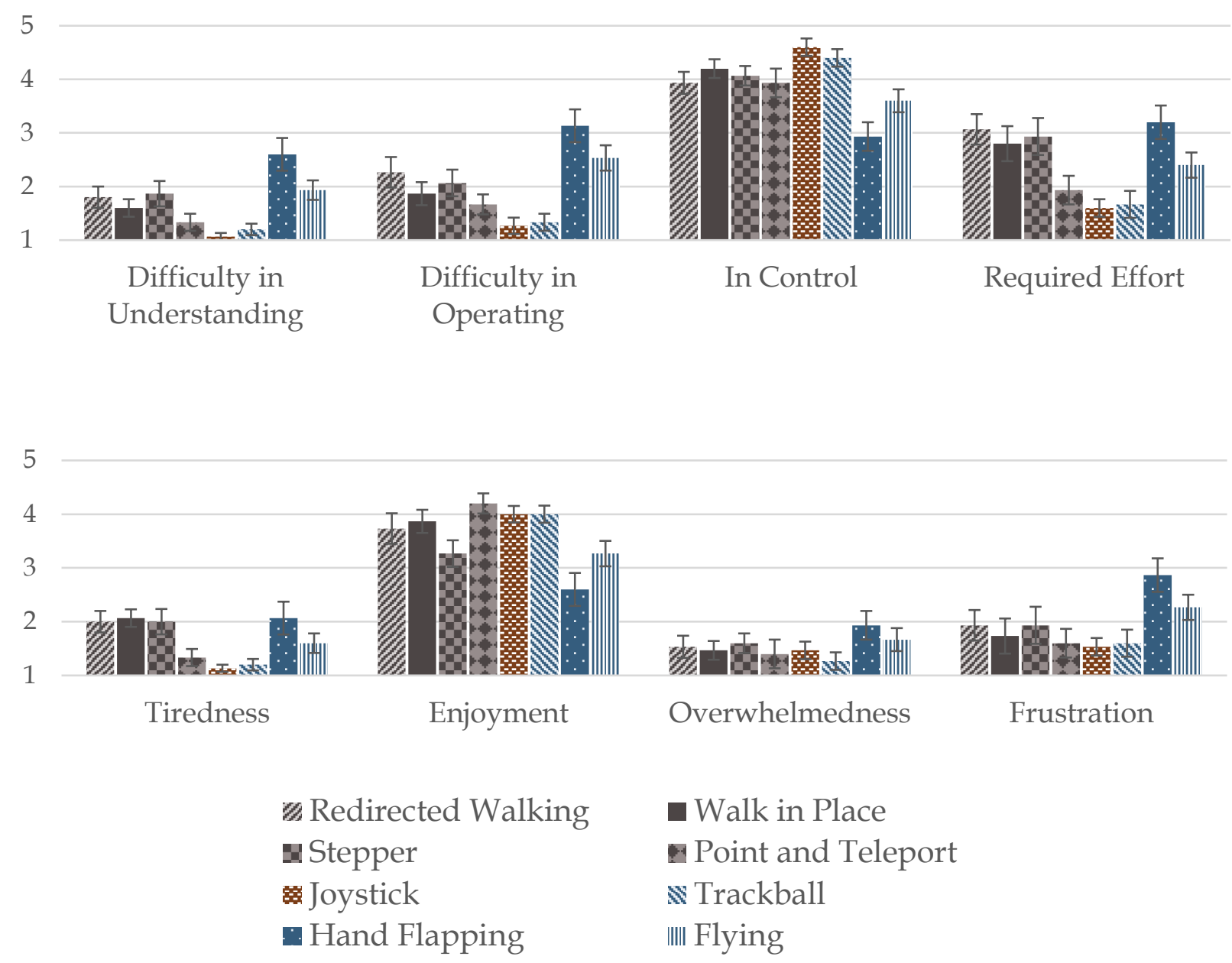

Figure 5-7: Survey results for the eight locomotion techniques for the neurotypical users. 
Table 5-3: Paired t-test results for the largest and the smallest significant mean differences for the user experience survey for the neurotypical users.

Difficulty in Understanding $\quad \mu$ Diff. $\quad$ Std. E $\quad p$

$\begin{array}{lllll}\text { Hand Flapping } & \text { Joystick } & 1.533 & 0.307 & 0.000\end{array}$

$\begin{array}{lllll}\text { Walk in Place } & \text { Trackball } & 0.400 & 0.163 & 0.028\end{array}$

Difficulty in Operating

$\begin{array}{lllll}\text { Hand Flapping } & \text { Joystick } & 1.867 & 0.291 & 0.000\end{array}$

$\begin{array}{lllll}\text { Walk in Place } & \text { Trackball } & 0.533 & 0.215 & 0.027\end{array}$

In Control

Joystick

Hand Flapping

1.667

$0.252 \quad 0.000$

Joystick

Stepper

0.533

$0.236 \quad 0.041$

Enjoyment

$\begin{array}{lllll}\text { Point \& Teleport } & \text { Hand Flapping } & 1.600 & 0.273 & 0.000\end{array}$

$\begin{array}{lllll}\text { Redirected Walking } & \text { Stepper } & 0.467 & 0.215 & 0.048\end{array}$

Required Effort

$\begin{array}{lllll}\text { Hand Flapping } & \text { Joystick } & 1.600 & 0.289 & 0.000 \\ \text { Flying } & \text { Trackball } & 0.733 & 0.316 & 0.036\end{array}$

Tiredness

$\begin{array}{lllll}\text { Walk in Place } & \text { Joystick } & 0.933 & 0.284 & 0.005\end{array}$

$\begin{array}{lllll}\text { Hand Flapping } & \text { Joystick } & 0.933 & 0.284 & 0.005\end{array}$

$\begin{array}{lllll}\text { Stepper } & \text { Flying } & 0.400 & 0.163 & 0.028\end{array}$

Frustration

$\begin{array}{lllll}\text { Hand Flapping } & \text { Joystick } & 1.333 & 0.232 & 0.000 \\ \text { Flying } & \text { Joystick } & 0.733 & 0.267 & 0.016\end{array}$ 
Table 5-4: Paired t-test results for the largest and the smallest significant mean differences for the presence results for the neurotypical users.

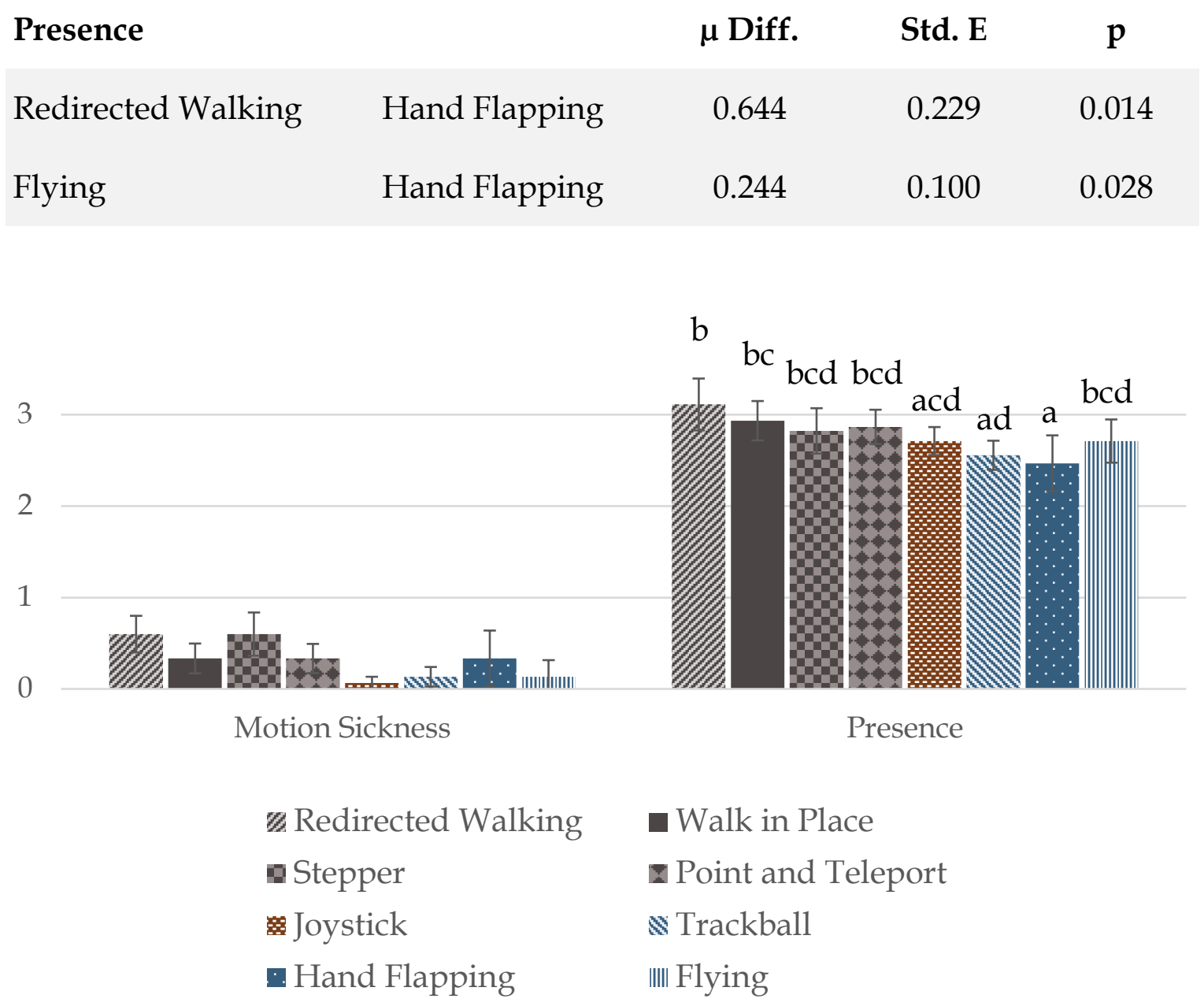

Figure 5-8: Average motion sickness and presence scores for the neurotypical users.

After they finished testing all of the eight techniques, the participants were requested to rank the eight locomotion techniques according to their preference. Results for the preference ranking were then transformed such that a score of 1 meant the least preferred and a score of 8 meant the most preferred. The bar charts for the average preference results are presented in Figure 5-9. One-way ANOVA with repeated measures with $a=0.05$ resulted in significant difference for the preference results $(F(7,7)=5.606$, 
$p=0.000)$. Mauchly's sphericity test was succeeded. As paired t-tests were performed, the largest significant mean difference was between the point \& teleport and hand flapping $(\mu$ difference $=4.133, \mathrm{p}=0.000)$, and the smallest significant mean difference was between the joystick and flying $(\mu$ difference $=1.667, \mathrm{p}=0.047)$.

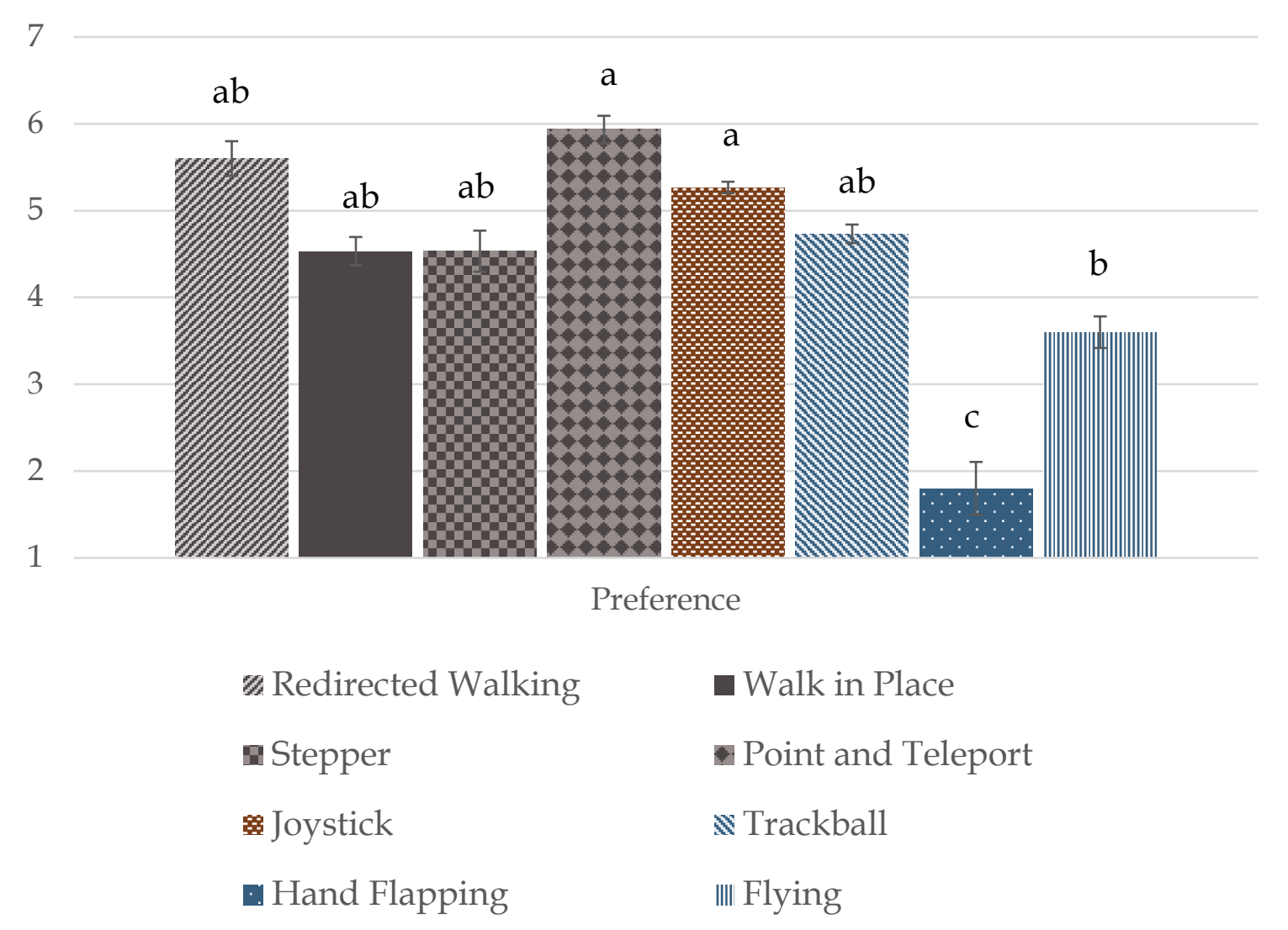

Figure 5-9: Weighted averages of the preference ranking scores for the neurotypical users.

Trajectories for the movements of the users in the virtual world are presented in the following figures: redirected walking (Figure 5-10), walk-in-place (Figure 5-11), stepper machine (Figure 5-12), point \& teleport (Figure 5-13), joystick (Figure 5-14), trackball (Figure 5-15), hand flapping (Figure 5-16), and flying (Figure 5-17). The virtual 
and real path trajectories for the locomotion techniques with and without obstacles can be seen in the figures.

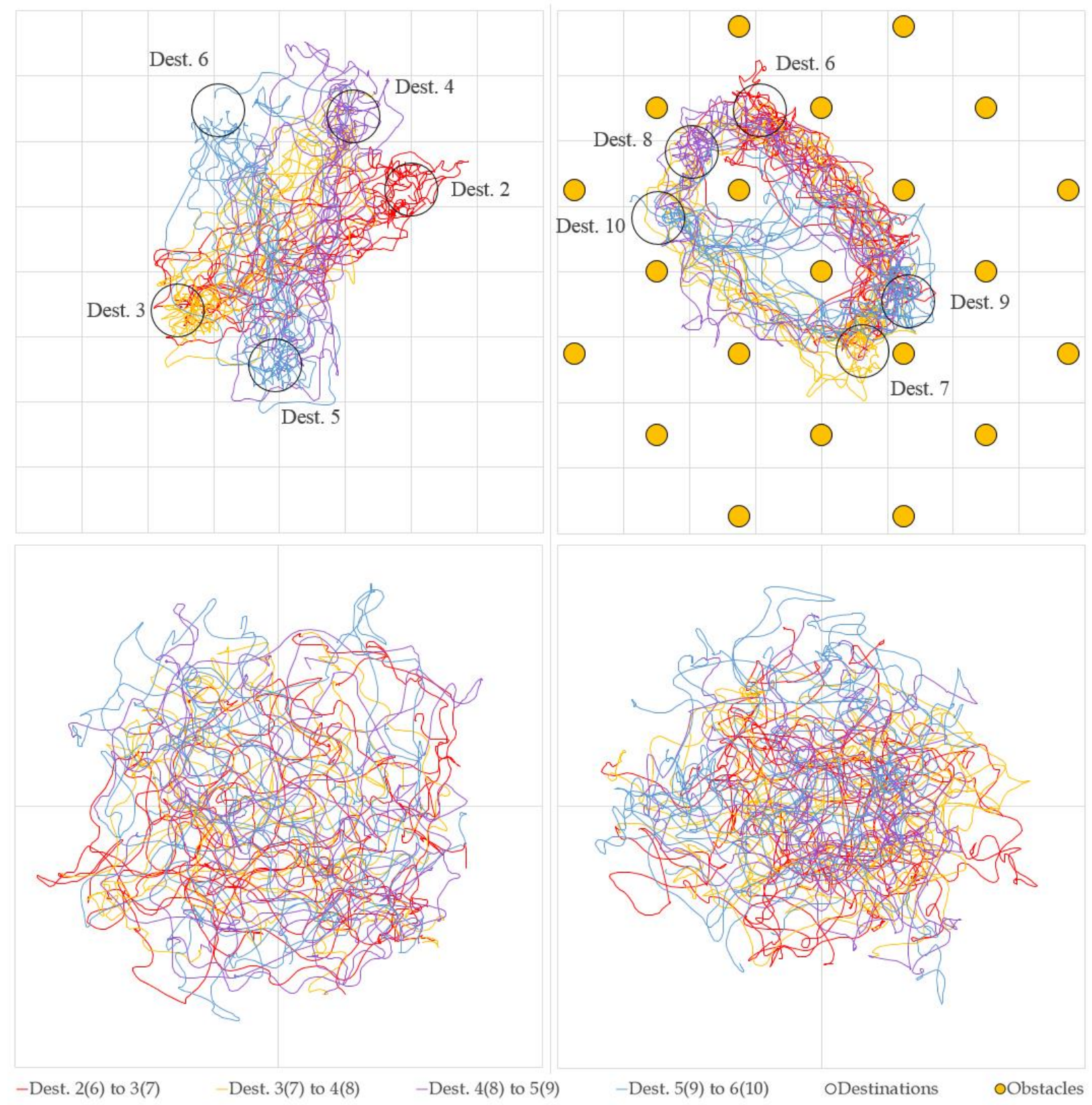

Figure 5-10: Redirected walking locomotion technique projected paths for the neurotypical users. Left top: Virtual projected paths in virtual environment between destination points 2 and 6 . Left bottom: Real projected paths in real tracking area between destination points 2 and 6. Right top: Virtual projected paths in virtual environment between destination points 9 and 10 with obstacles. Right bottom: Real projected paths in real tracking area between destination points 6 and 10. 


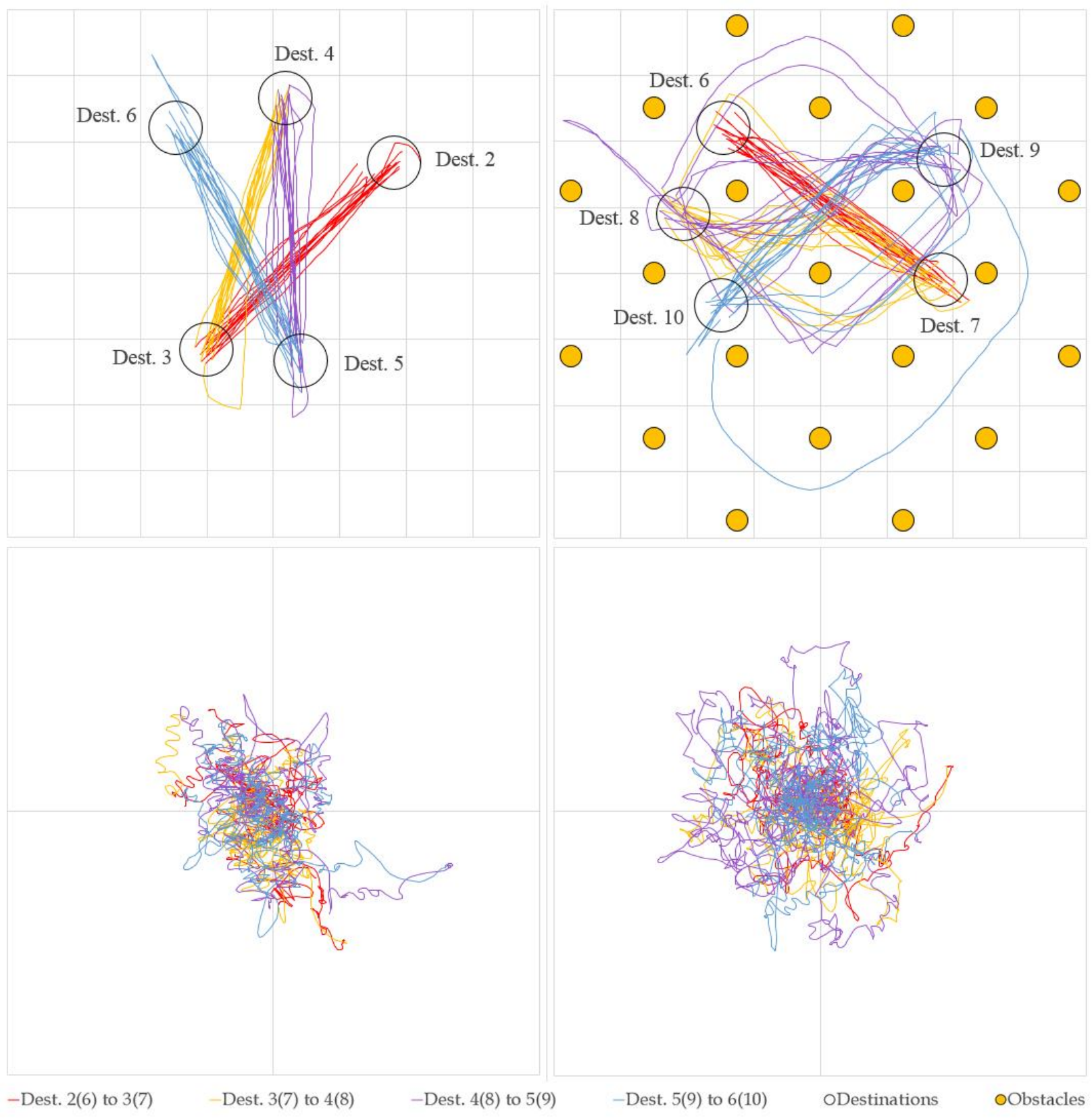

Figure 5-11: Walk-in-place locomotion technique projected paths for the neurotypical users. Left top: Virtual projected paths in virtual environment between destination points 2 and 6. Left bottom: Real projected paths in real tracking area between destination points 2 and 6. Right top: Virtual projected paths in virtual environment between destination points 9 and 10 with obstacles. Right bottom: Real projected paths in real tracking area between destination points 6 and 10 . 


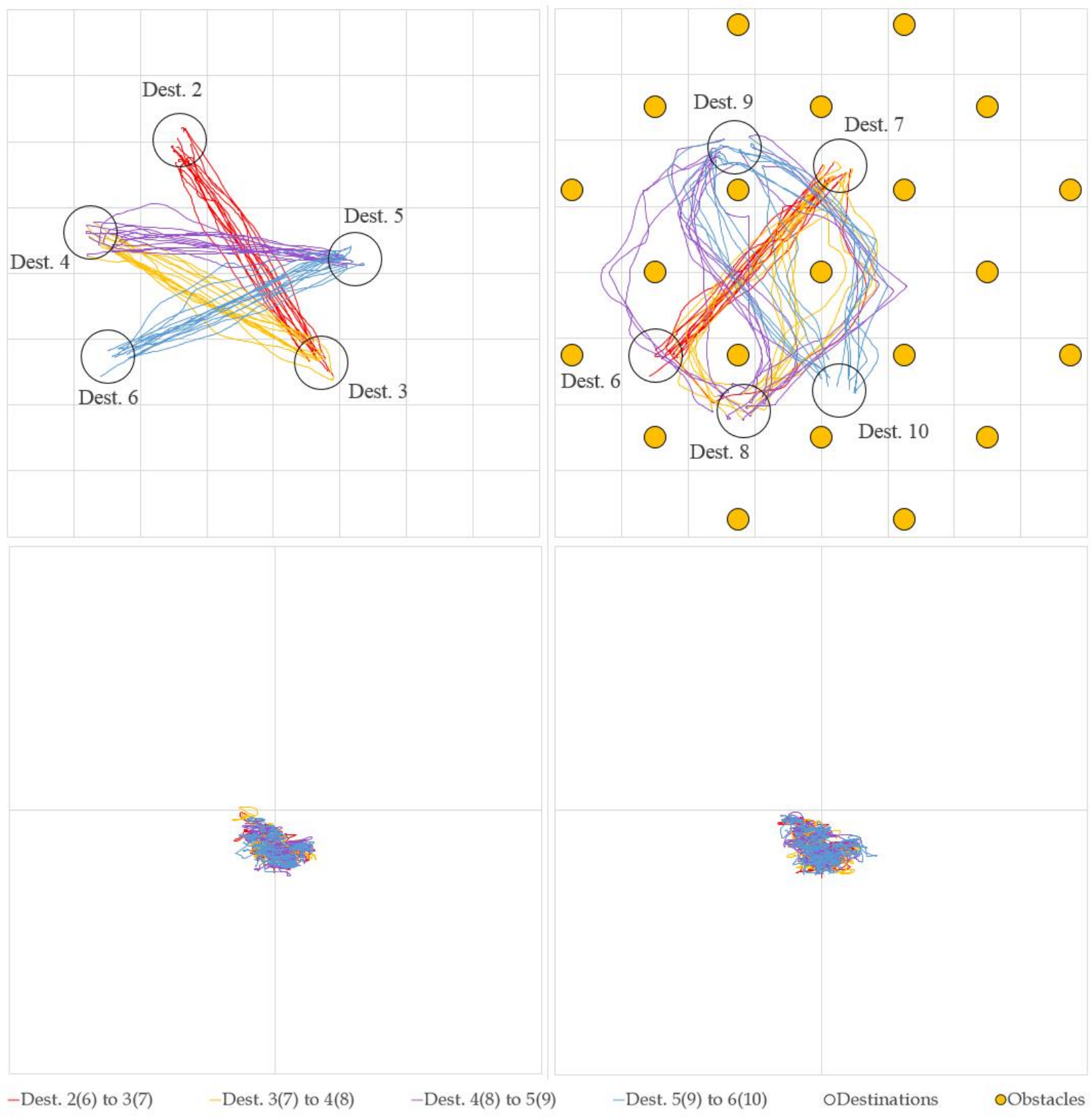

Figure 5-12: Stepper machine locomotion technique projected paths for the neurotypical users. Left top: Virtual projected paths in virtual environment between destination points 2 and 6 . Left bottom: Real projected paths in real tracking area between destination points 2 and 6. Right top: Virtual projected paths in virtual environment between destination points 9 and 10 with obstacles. Right bottom: Real projected paths in real tracking area between destination points 6 and 10 . 


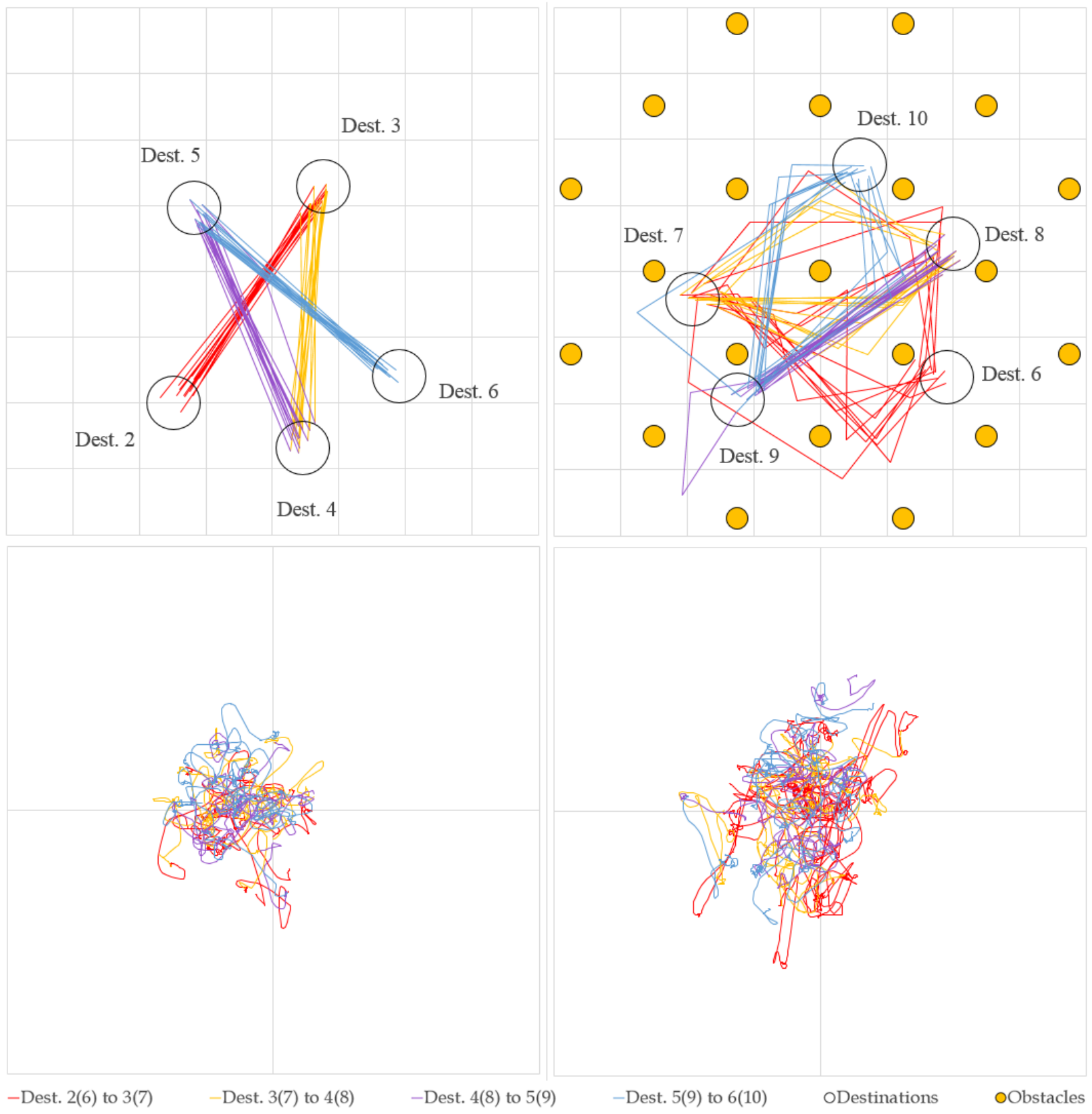

Figure 5-13: Point \& teleport locomotion technique projected paths for the neurotypical users. Left top: Virtual projected paths in virtual environment between destination points 2 and 6. Left bottom: Real projected paths in real tracking area between destination points 2 and 6. Right top: Virtual projected paths in virtual environment between destination points 9 and 10 with obstacles. Right bottom: Real projected paths in real tracking area between destination points 6 and 10 . 


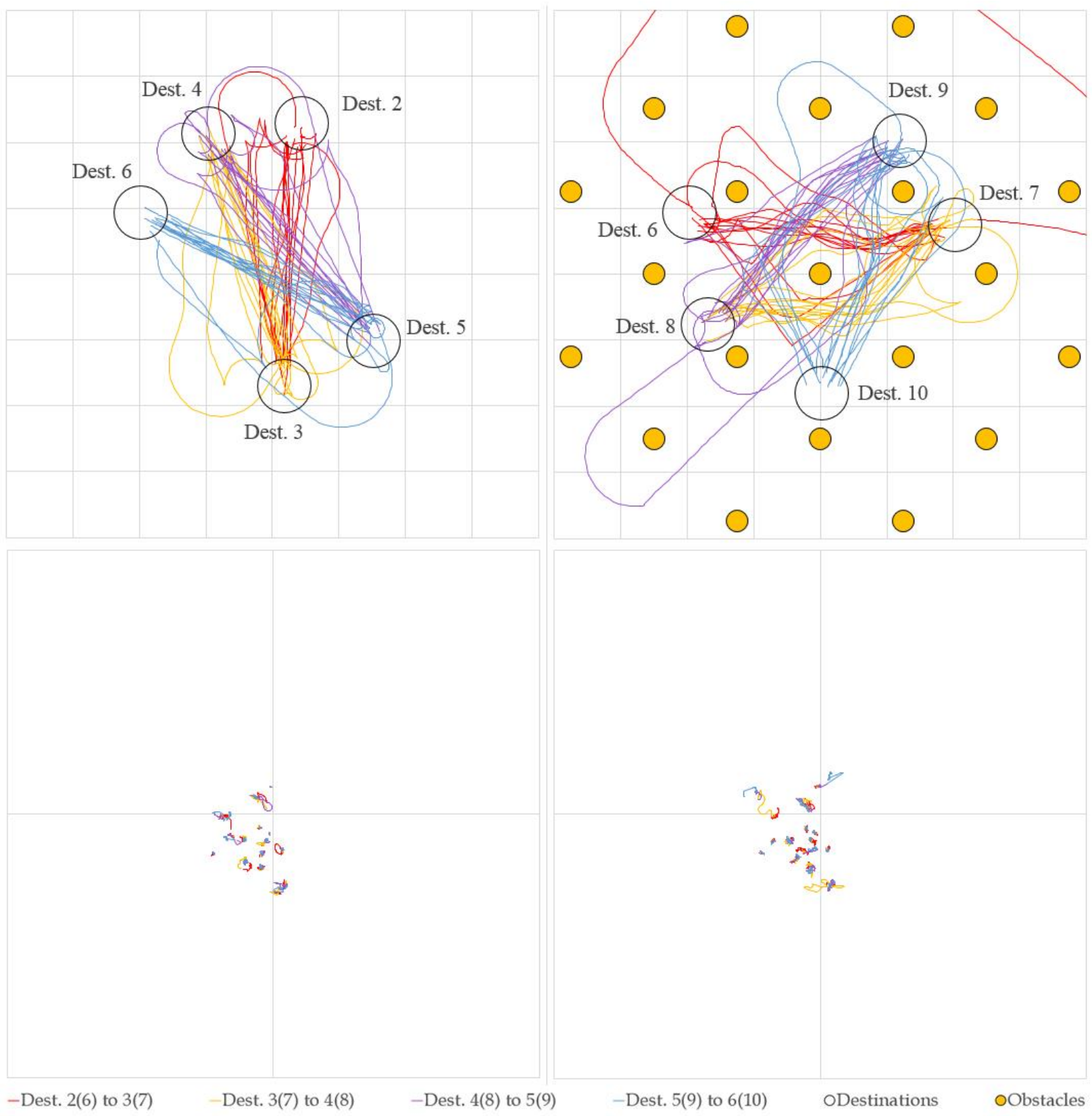

Figure 5-14: Joystick locomotion technique projected paths for the neurotypical users. Left top: Virtual projected paths in virtual environment between destination points 2 and 6. Left bottom: Real projected paths in real tracking area between destination points 2 and 6. Right top: Virtual projected paths in virtual environment between destination points 9 and 10 with obstacles. Right bottom: Real projected paths in real tracking area between destination points 6 and 10 . 


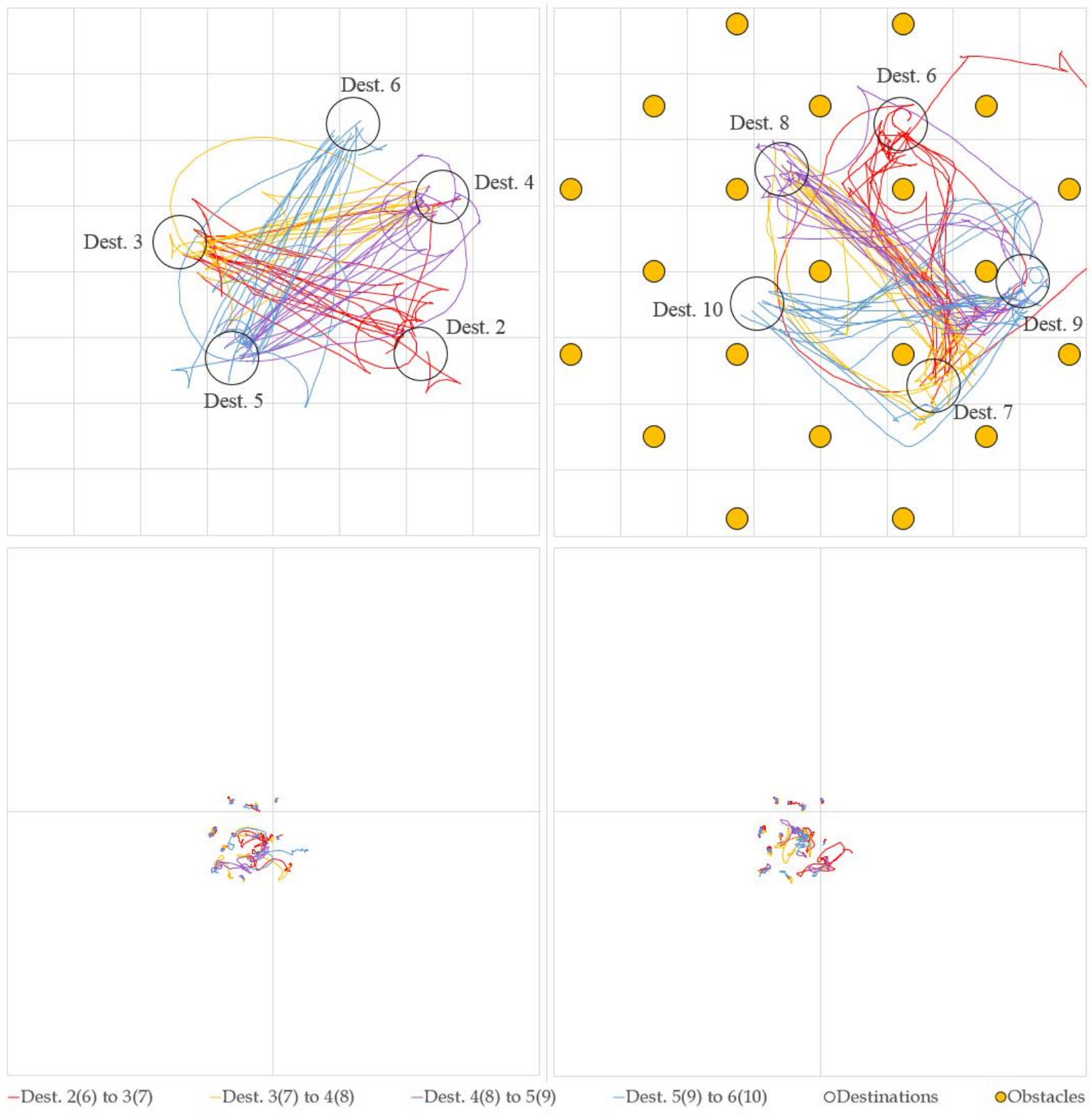

Figure 5-15: Trackball locomotion technique projected paths for the neurotypical users. Left top: Virtual projected paths in virtual environment between destination points 2 and 6. Left bottom: Real projected paths in real tracking area between destination points 2 and 6. Right top: Virtual projected paths in virtual environment between destination points 9 and 10 with obstacles. Right bottom: Real projected paths in real tracking area between destination points 6 and 10 . 


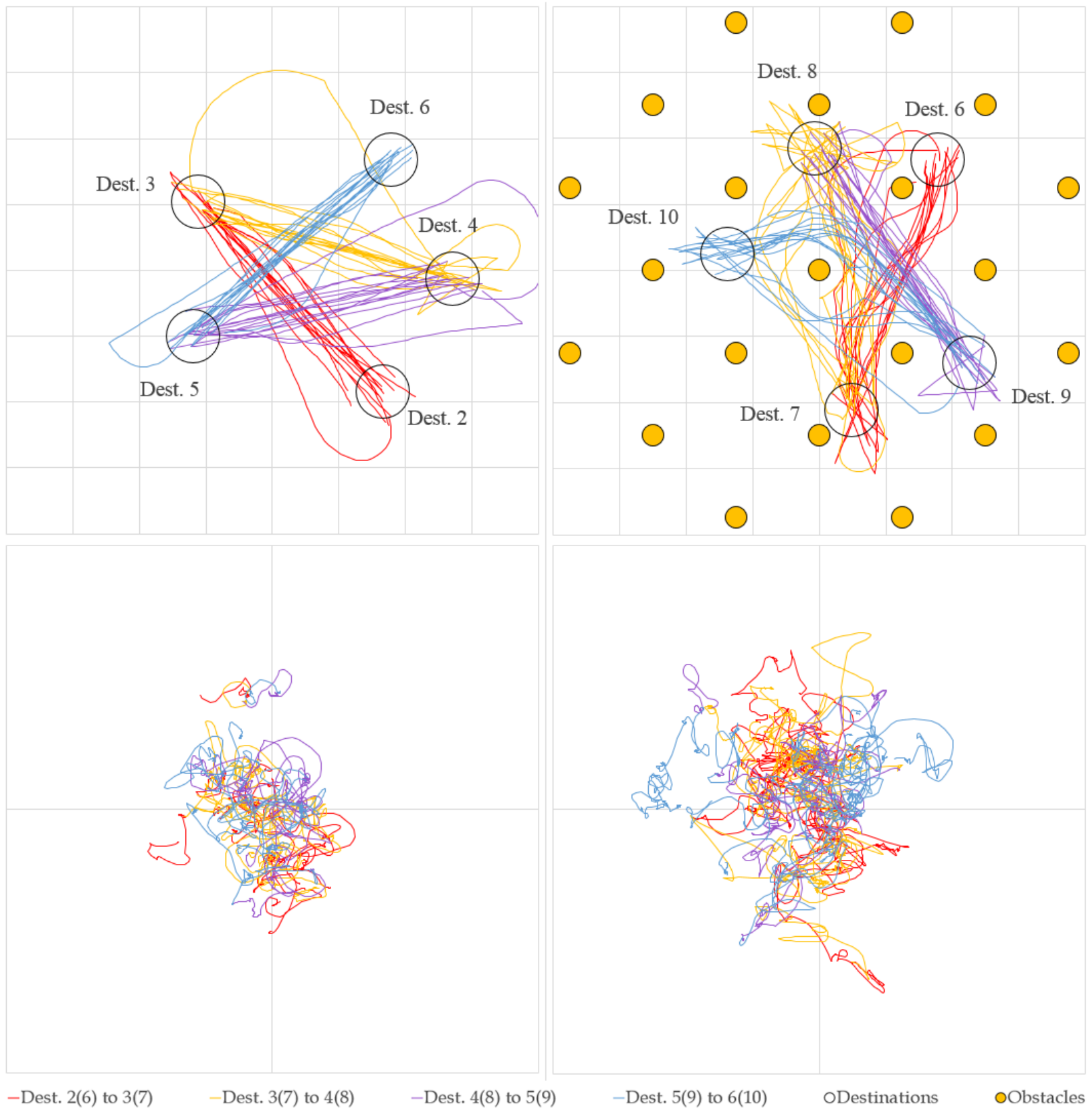

Figure 5-16: Hand flapping locomotion technique projected paths for the neurotypical users. Left top: Virtual projected paths in virtual environment between destination points 2 and 6. Left bottom: Real projected paths in real tracking area between destination points 2 and 6. Right top: Virtual projected paths in virtual environment between destination points 9 and 10 with obstacles. Right bottom: Real projected paths in real tracking area between destination points 6 and 10 . 


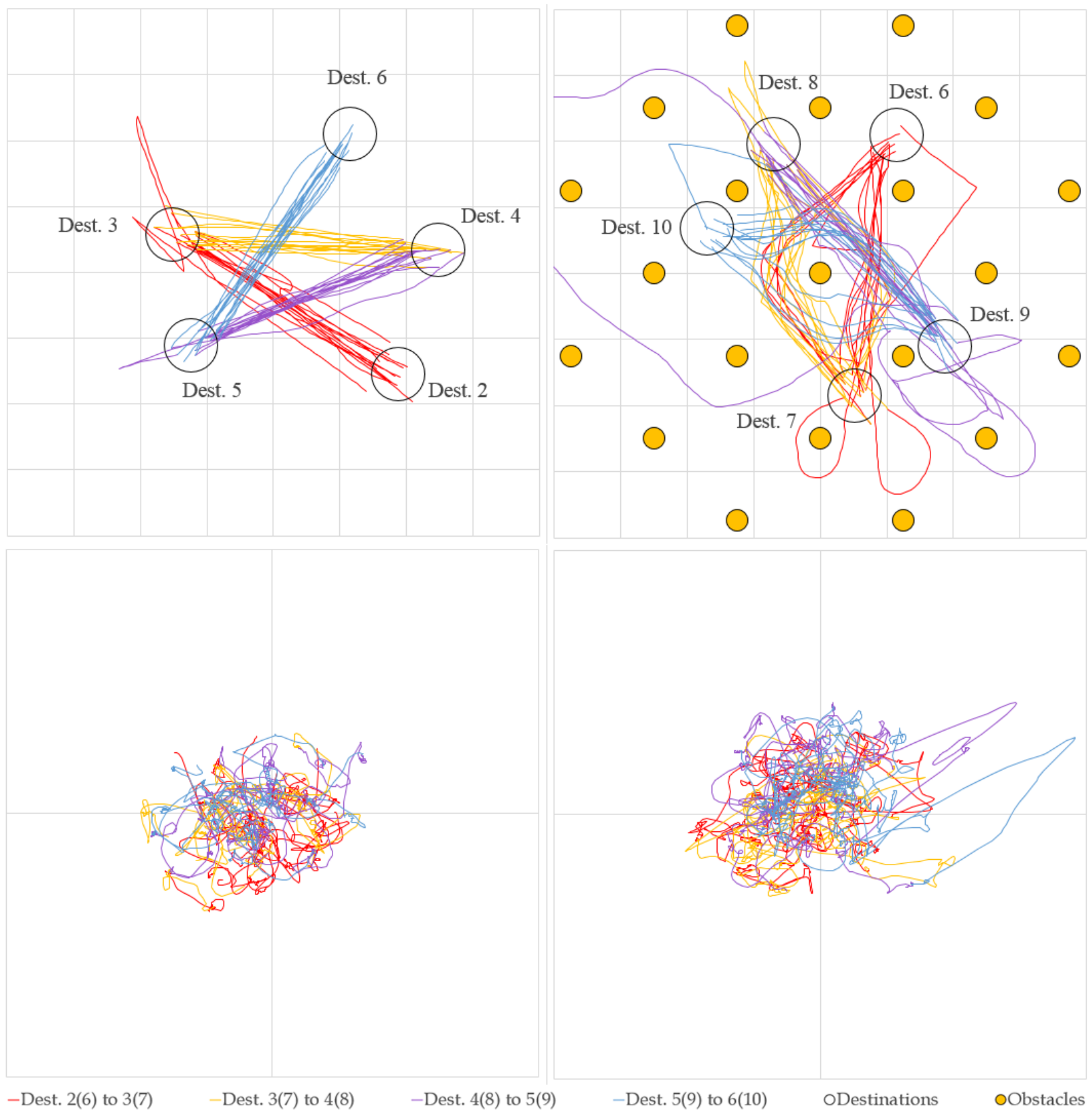

Figure 5-17: Flying locomotion technique projected paths for the neurotypical users. Left top: Virtual projected paths in virtual environment between destination points 2 and 6. Left bottom: Real projected paths in real tracking area between destination points 2 and 6. Right top: Virtual projected paths in virtual environment between destination points 9 and 10 with obstacles. Right bottom: Real projected paths in real tracking area between destination points 6 and 10 . 


\subsubsection{Participant Comments}

The comments that were made by the participants in the surveys were presented below under the relevant locomotion technique categories.

- Redirected Walking: (User 1) "Pretty straightforward method as long as you walked slowly." (User 9) "Real walking was very simple. I think it is a good method." (User 11) "I felt like at times when it would appear that I would just need to walk in a straight line, I found myself going off to the side rather than straight forward." (User 12) "Much like real world and easier to navigate." (User 13) "Controlling the speed was challenging but after a few walks easy." (User 17) “Nice. Close to reality." (User 18) "Felt really real, actually walking." (User 20) "While I did feel limited in motion and mildly frustrated, this was the most realistic method."

- Walk-in-Place: (User 6) “Actually it is intuitive but I cannot walk sideways." (User 9) "I think it is simple and also have real walk. I think it is good." (User 12) “Needs more effort than normal walking." (User 13) “I really liked this method." (User 17) “Good walking technique.” (User 20) “While not as easy as joystick, it did feel more immersive."

- Stepper: (User 11) "I like how there was no guesswork in the walking, when you stopped on the pedals, you stopped in the virtual world." (User 13) "It was fun stepping without walking." (User 15) "This method was the most difficult of all in terms of physical exertion." (User 18) "It didn't feel as real as walk in place using the stepper machine." (User 20) "This method was 
far more restrictive in turning and required more effort than walking in place."

- Point \& Teleport: (User 4) “Getting around obstacles was a lot of time. But good for longer, unobstructed distances." (User 5) “Easy to move and avoid obstacles." (User 6) "Pointing is very easy and interesting." (User 9) "It is a very simple method, very easy to apply." (User 11) "I enjoyed the accuracy in the whole 'point and you will teleport to that spot' because it took the guesswork out." (User 15) "This felt more like a video game because I wasn't actually walking to move in VR." (User 18) "Really cool method. Would be fun for video games." (User 20) "This method was very easy to use."

- Joystick: (User 6) “Love this method. No thinking, no effort. Know for sure. Accurate and safe.” (User 9) “This method is very easy to apply.” (User 11) "I really like how smooth it was moving in the virtual world. Movement was continuous, not stop and go like some other methods." (User 13) "It was easier to control the walking but completely conscious that I was controlling from outside." (User 17) “Complete control of motion. Perfect stoppage parts."

- Trackball: (User 1) “I liked this method. Easy to use." (User 4) “Slow, jerky motion. Difficult to get from place to place efficiently." (User 9) “It is simple, but about the operating feeling, I think the joystick is much better." (User 
13) "It was easy to control but completely aware of surroundings." (User

18) "Didn't feel real using the walking device."

- Hand Flapping: (User 1) “This method was a little difficult. Once I figured it out, it was better. Particularly difficult to navigate the pillars." (User 9) "It is not very easy to avoid the obstacles." (User 13) "The walking with the hand is not that easy to control." (User 18) "Not a big fan of this method. Think it's a little hard to get the hang at first."

- Flying: (User 1) “Difficult. Sometimes I forgot to put my hand down.” (User 3) "Felt like I was on a skateboard but there was no momentum." (User 4) "Hard to signal on then off for short distances." (User 9) "Sometimes, it is easy to walk over the position." (User 11) "I wasn't able to tell how close I was to the columns (i.e. if my shoulder would've brushed against the column)." (User 18) "Annoying to operate this way."

\subsubsection{Discussion}

The following implications were compiled for virtual reality applications for room scale tracked areas in the light of the analysis results and the comments from the participants. We recommend using point \& teleport, joystick like controllers and redirected walking depending on the scope of intended VR application. On the other hand, we recommend avoiding from using hand flapping and flying.

\subsubsection{Point \& Teleport}

As this technique was explained to the participants, many of them immediately made positive verbal comments stating that the technique seemed fun to try. They stated 
that the technique reminded them immediately of video games and made the experiment seem more like a video game as compared to the other locomotion techniques. Point \& teleport received good scores for many aspects and was ranked as the first preference of the neurotypical users among the eight locomotion techniques. Hence, evidence suggests that point \& teleport is a good candidate for locomotion in room scale VR. However, in VR applications that contain a lot of obstacles which the user needs to move around, point \& teleport might not be suitable. Such environments may need many teleportations which may be annoying for the users. The technique is suitable for vast virtual environments in which the user needs to travel long distances such as exploration games and applications.

\subsubsection{Joystick}

The joystick provided high level of control and simplicity in usage. The familiarity from video games might be a contributing factor for the preference for the joystick locomotion technique. The scores for many aspects were good for joystick, especially for the feeling of being in control, and it was the third choice in the preference ranking. These were in alignment with $[115,116]$ and contradiction with [117]. As a drawback, we observed some drifting in the turning around motions made with the joystick method. The reason behind this was that the users turned around by pushing the joystick diagonally (both forward and sideways), instead of stopping and turning back. Although it is a sign of a continuous movement that can be considered as positive, it may cause more time to reach to the desired position. This effect should be taken into account for applications requiring large instances of turning around. We suggest using joystick like 
controllers in VR applications that are fast paced and that needs accurate controlling of the user such as making fast moves to all sides in small increments or dodging.

\subsubsection{Redirected Walking}

Although redirected walking was implemented with limits that were beyond the recommended range in the literature and in a tracked area that was smaller than the recommended size, it received good scores in many aspects. This aligned with $[33,119$, 130] but contradicted with [117]. Redirected walking was the second choice of the participants in the preference ranking among the eight techniques. It did not induce motion sickness and it provided the highest level of presence. Many users mentioned how natural and realistic using this technique felt. We recommend using redirected walking in room scale VR applications that aims for high levels of presence. However, it should be noted that the exaggeration of the movements resulted in loss of control. This effect was observed in the survey results and the collision results for this technique. Hence, in room scale VR applications in which precision is important, we recommend avoiding redirected walking.

\subsubsection{Walk-in-Place}

Walk-in-place received middle range scores for many aspects. However, the users stated that locomotion with walk-in-place gave them proprioceptive feedback and felt realistic. It helped the users to stay in the same place and it had an aspect of physical effort as well. As an advantage over redirected walking, walk-in-place provided more control since the movements were transformed into the virtual world without alteration. On the other hand, it provided less presence than redirected walking and it was the fifth 
in the preference ranking. Hence, we suggest using walk-in-place as a second resort to redirected walking in room scale VR applications that require accurate locomotion and have the aim of making the users exert some physical effort.

\subsubsection{The Other Techniques}

Although stepper machine also gave the users proprioceptive feedback, it received poorer scores than the walk-in-place in several aspects. The users stated that they found the walk-in-place and stepper machine similar in general. However, some users stated that the stepper machine was not as natural as the walk-in-place and it gave them the feeling of controlling from the outside instead of being in the virtual world. We did not find any advantages of using stepper machine over walk-in-place. Hence, we recommend utilizing walk-in-place instead of stepper machine.

Trackball also did not provide any advantages over joystick. Some users stated that it was annoying to spin the ball many times for locomotion. The trajectories show that the strides were abrupt with the trackball locomotion technique instead of indicating a continuous movement. The trackball was behind the joystick in the preference ranking. Hence, we could not find any advantage of the trackball over the joystick and recommend choosing the joystick over trackball.

Flying received poor scores for many aspects and was the second least in the preference ranking among the eight techniques. The automatic movement created a loss of control and the users mentioned that it was difficult for them to estimate their distance in the virtual environment with this method. They stated that it was difficult for them to decide when to stop and most of the users made early stops. We interpret that the users 
might have expected some form of decelerated stop with this technique (such as stopping a moving car in the real life) and thus triggered the stopping a bit earlier than their desired point of destination. However, the flying technique was implemented such that it started and stopped moving immediately when the user made the triggering gesture.

Hand flapping received the poorest scores in many aspects and it was the last ranked technique among the eight, in terms of user preference. The users stated that it was awkward and tiring to continuously flap the hand for locomotion and they mentioned a loss of control with this technique, in alignment with the survey results. We interpret that the unintentional movements of the hand may have resulted in unintentional movements in the virtual world. The translation of the foot motion to the hand with the hand flapping was not found comfortable by the users. In lights of the negative results and user comments, we recommend avoiding hand flapping for locomotion in VR.

\subsubsection{Time to Reach the Destination Points and Number of Collisions}

For the no-obstacles case, the least time to reach to the destination points was with the trackball, joystick and point \& teleport locomotion techniques, rejecting Hypothesis $1,0$. These results are in alignment with [114] for joystick-like controllers. For the obstacles case, trackball and joystick still resulted in the least times whereas the point \& teleport became the technique with the third most time to reach to the destination points. We interpret that the multiple teleportations that were needed in the presence of the obstacles was the reason behind this. The most time to reach the to the destination points was with the redirected walking for both the no-obstacles and the obstacles cases. The reason 
behind this should be the altered movement of the users and the pop-up walls that appeared at times to redirect the users towards the center of the tracked area as they approached to the edges.

Point \& teleport resulted in significantly lower number of collisions with the pillars. It was followed by the walk-in-place and stepper machine. On the contrary, redirected walking resulted in the most number of collisions with the pillars. We interpret that the gains that were applied in redirected walking should have caused exaggerated movements in the virtual world, contributing to the more number of collisions with pillars.

\subsubsection{Survey Metrics}

Hand flapping resulted in the most difficulty in understanding whereas joystick, trackball and point \& teleport resulting in the least difficulty. These results are in alignment with [130]. Hand flapping and flying resulted in the most difficulty in operating whereas joystick and trackball were the least. Joystick and trackball provided the feeling of most being in control whereas hand flapping and flying provided the least. Hand flapping, redirected walking, stepper machine and walk-in-place were the techniques that required the most effort to operate respectively. On the contrary, joystick, trackball and point \& teleport required the least effort to operate. Similarly, hand flapping, walk-in-place, redirected walking and stepper machine caused the most tiredness in the users whereas joystick, trackball and point \& teleport caused the least. Point \& teleport provided the highest level of enjoyment followed by joystick and trackball. Hand flapping, stepper machine and flying provided the least level of 
enjoyment. There wasn't any significant difference in overwhelmedness for the eight techniques. Hand flapping caused the most level of frustration followed by flying. The least level of frustration was caused by joystick, trackball and point \& teleport. The poor results for the flying technique were in alignment with [33]. There wasn't any significant difference between the eight locomotion techniques in terms of motion sickness, aligning with the results in $[33,129]$. The highest level of presence was offered by redirected walking followed by walk-in-place. The lowest level of presence was with hand flapping and trackball.

For the user preference ranking scores, the point \& teleport was the highest, followed by the redirected walking and the joystick. The lowest scores were for the hand flapping and flying, respectively. Thus, Hypothesis 1,0 was rejected.

\subsubsection{Summary, Conclusion and Future Work}

This user study aimed at investigating eight VR locomotion techniques in room scale tracked areas. An immersive VR experiment was implemented and 16 neurotypical participants took part in the evaluation of the following locomotion techniques: redirected walking, walk-in-place, stepper machine, point \& teleport, joystick, trackball, hand flapping and flying. In light of the study results, point \& teleport, joystick and redirected walking was found to be suitable locomotion techniques for VR applications in room scale tracked areas whereas hand flapping and flying were not found to be suitable.

In summary, for locomotion in VR applications in room scale tracked areas targeting neurotypical individuals, we recommend using the point \& teleport for virtual 
environments that do not contain a lot of obstacles to be overcome; using joystick-like controllers for fast paced virtual environments in which accuracy of the movement is important; using the redirected walking for applications that aim for high levels of presence and physical motion; and using the walk-in-place as a second resort to redirected walking. We recommend avoiding using the hand flapping and flying locomotion techniques.

Future research areas may consist of evaluating modifications of the locomotion techniques that were found to be suitable for room scale VR in this user study, such as point \& teleport with controller triggering, and wireless hand held joystick or game pad controller. Evaluating the locomotion techniques in virtual environments with different levels of complexity would be another area for exploration.

\subsection{Individuals with ASD}

This subsection presents the demographics information of individuals with ASD and the user study results for this population. Discussions, summary, conclusions and future work directions were also included in the subsection. The research question for this user study was: 'Which of the eight virtual locomotion techniques would provide the best user experience for individuals with ASD in room scale tracked areas?' In the light of this research question, the following null hypotheses were constructed: Hypothesis 1,0 : All locomotion techniques will result in similar performance in terms of average time to reach to the destination points, Hypothesis2,0: All locomotion techniques will result in similar ranking scores. 


\subsubsection{Participants}

15 high functioning individuals with ASD (11 male, 4 female) participated in the user study. All participants were older than 18 years old, ages ranging from 18 to 41 , mean age $23.73(\mathrm{SD}=5.37) .11$ participants' dominant side was right and 4 participants' dominant side was left. Most of the participants (14 out of 15) had no prior VR experience while only 1 participant had minimal prior VR experience. A \$50 gift card was given to the participants as an incentive to participate in the user study.

\subsubsection{Results}

This sub-subsection presents the user study results for individuals with ASD under the following categories: data results, survey results, and the participant comments. One participant with ASD state that they felt nauseous during trying their first assigned locomotion technique, hence did not want to continue taking part in the experiment. Thus, the data of the 14 participants with ASD were used in the analysis in this sub-subsection. Two participants with ASD did not complete the flying technique trial due to feeling nauseous and being overwhelmed.

\subsubsection{Data Results}

We analyzed the time data to reach to the destination points based on two groups according to the presence of obstacles in the virtual world. The results are presented in Figure 5-18.

As one-way ANOVA with repeated measures analysis was performed, significant difference was found in the time to reach to the destination points for both of these cases: $F(7,6)=8.894, p=0.001$ for without obstacles case, and $F(7,6)=8.493, p=0.000$ for with 
obstacles case. Mauchly's sphericity test failed and Greenhouse-Geisser correction was done for both cases. As paired t-tests were performed, for without obstacles case, the largest significant mean difference was between the redirected walking and point \& teleport $(\mu$ difference $=9.549, \mathrm{p}=0.001)$, and the smallest significant mean difference was between the walk-in-place and joystick ( $\mu$ difference $=1.198, p=0.036$ ). For with obstacles case, the largest significant mean difference was between the redirected walking and joystick $(\mu$ difference $=12.774, p=0.000)$, and the smallest significant mean difference was between the walk-in-place and flying ( $\mu$ difference $=3.106, p=0.015)$.

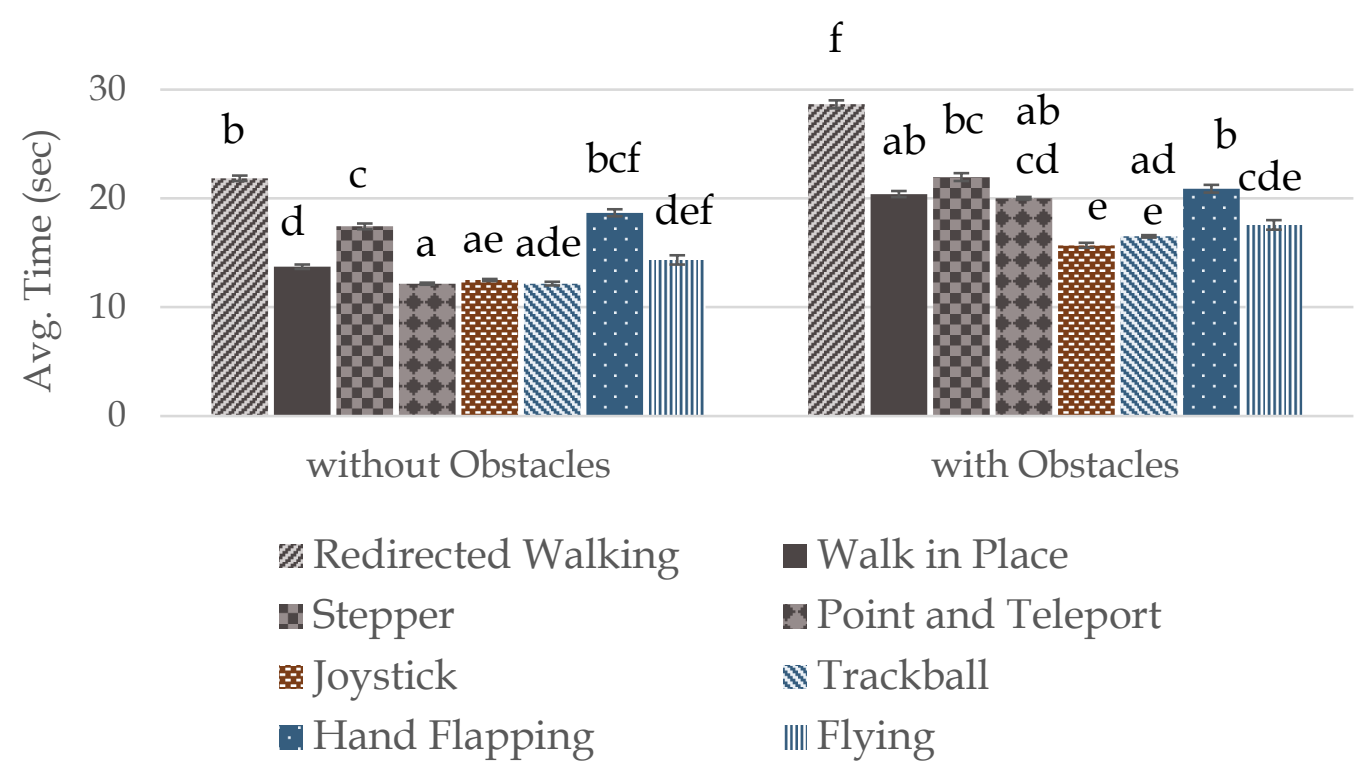

Figure 5-18: Average time to reach to the destination points without and with obstacles for the users with ASD with the eight locomotion techniques.

Number of collisions made with the static obstacles in the virtual environment were also analyzed (see Figure 5-19). Additional dynamic obstacles of the redirected walking technique in the form of pop-up walls aiming to keep the users inside the tracked 
area were not included in this analysis to obtain comparable results between the different locomotion techniques. One-way ANOVA with repeated measures yielded statistically significant difference between the techniques: $F(7,6)=11.296, p=0.000$, Mauchly's sphericity test failed and Greenhouse-Geisser correction was done. The largest significant mean difference in the paired t-tests was between the redirected walking and point \& teleport $(\mu$ difference $=5.833, p=0.000)$, and the smallest significant mean difference was between the walk-in-place and point $\&$ teleport $(\mu$ difference $=1.083, \mathrm{p}=0.02)$. Detailed statistical analysis can be seen in Appendix G.

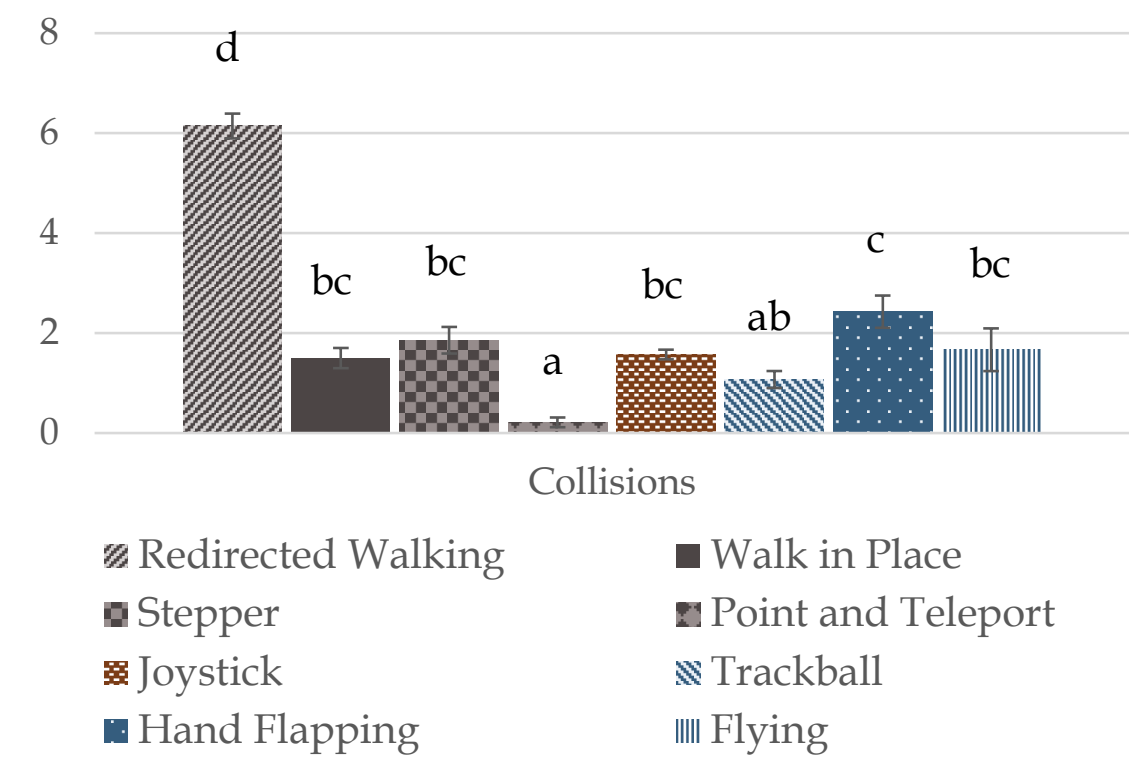

Figure 5-19: Average number of collisions with static obstacles for the users with ASD.

\subsubsection{Survey Results}

Usability part of the survey included questions on eight categories on user experience: difficulty in understanding the locomotion method, difficulty in operating the method, feeling of being in control while using the method, required effort to use the 
method, feeling of tiredness the method caused, feeling of enjoyment the method caused, feeling of being overwhelmed the method caused and feeling of frustration the method caused. The questions had answers on a 5 point Likert scale (1: not at all, 5: very much). Average results of these categories are presented in Figure 5-20. One-way ANOVA with repeated measures analysis was performed for each category yielding results reported in Table 5-5. All eight categories resulted in statistically significant difference. Results of the paired t-tests that yielded the largest and the smallest significant mean differences are presented in Table 5-6.

Table 5-5: One-way ANOVA results the user experience survey for the users with ASD.

\begin{tabular}{|c|c|c|c|c|c|}
\hline & Correction & df & df Err. & F & Sig. \\
\hline $\begin{array}{l}\text { Difficulty in } \\
\text { Understanding }\end{array}$ & Greenhouse-Geisser & 3.667 & 47.676 & 3.633 & 0.014 \\
\hline $\begin{array}{l}\text { Difficulty in } \\
\text { Operating }\end{array}$ & Sphericity Assumed & 7.000 & 91.000 & 7.519 & 0.000 \\
\hline In Control & Greenhouse-Geisser & 3.277 & 42.602 & 6.479 & 0.001 \\
\hline Enjoyment & Sphericity Assumed & 7.000 & 91.000 & 5.984 & 0.000 \\
\hline Required Effort & Sphericity Assumed & 7.000 & 91.000 & 6.249 & 0.000 \\
\hline Tiredness & Greenhouse-Geisser & 3.142 & 40.840 & 2.945 & 0.042 \\
\hline Overwhelmedness & Greenhouse-Geisser & 2.890 & 37.574 & 3.314 & 0.032 \\
\hline Frustration & Greenhouse-Geisser & 3.655 & 47.520 & 4.453 & 0.005 \\
\hline
\end{tabular}


There were also questions in the survey on motion sickness and presence. These questions had answers on a 4 point Likert scale (0: none, 3: major). Results for the motion sickness and presence are presented in Figure 5-21. One-way ANOVA with repeated measures analysis indicated no significant difference between the locomotion techniques for motion sickness $(F(7,6)=1.175, p=0.332)$ and presence $(F(7,6)=1.156, p=0.341)$. Mauchly's sphericity test failed and Greenhouse-Geisser correction was done for both the motion sickness and presence data.
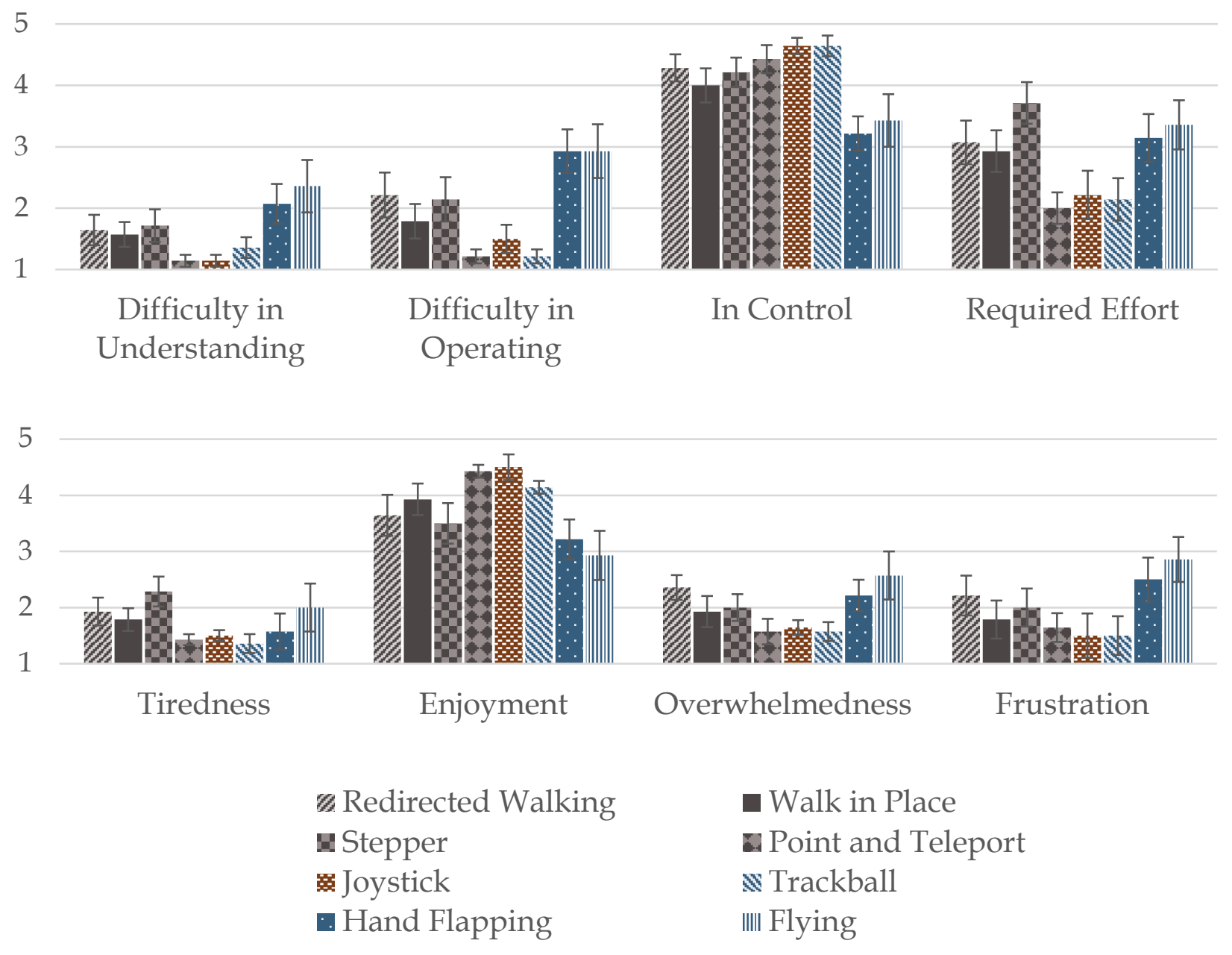

Figure 5-20: Survey results for the eight locomotion techniques for the users with ASD. 
Table 5-6: Paired t-test results for the largest and the smallest significant mean differences for the user experience survey for the users with ASD.

$\mu$ Diff. $\quad$ Std. Err. $\quad$ Sig.

\section{Difficulty in Understanding}

Flying

Point \& Teleport

1.214

0.447

0.018

Flying

Joystick

1.214

0.422

0.013

Hand Flapping

Point \& Teleport

0.929

0.286

0.006

Hand Flapping

Joystick

0.929

0.305

0.009

\section{Difficulty in Operating}

Hand Flapping

Point \& Teleport

1.714

0.304

0.000

Hand Flapping

Trackball

1.714

0.398

0.001

Flying

Point \& Teleport

1.714

0.425

0.001

Flying

Trackball

1.714

0.474

0.003

Stepper

Joystick

0.643

0.269

0.033

\section{In Control}

Joystick

Hand Flapping

1.429

0.291

0.000

Trackball

Hand Flapping

1.429

0.291

0.000

Walk in Place

Hand Flapping

0.786

0.155

0.000

\section{Enjoyment}

Joystick

Flying

1.571

0.416

0.002

Trackball

Stepper

0.643

0.269

0.033

Required Effort

Stepper

Point \& Teleport

1.714

0.304

0.000

Walk in Place

Joystick

0.714

0.244

0.012

\section{Tiredness}

Stepper

Trackball

0.929

0.339

0.017

Stepper

Walk in Place

0.500

0.228

0.047 
Table 5-6 (Continued)

Overwhelmedness

Flying

Flying

Stepper

Stepper

Frustration

Flying

Flying

Stepper

3

2

1

0

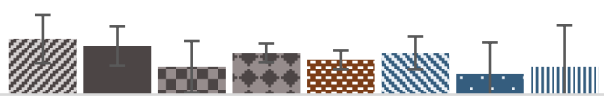

Motion Sickness

\# Redirected Walking

Stepper

罗 Joystick

Hand Flapping $\mu$ Diff. $\quad$ Std. Err. $\quad$ Sig.

Point \& Teleport

1.000

0.348

0.013

1.000

0.378

0.020

Point \& Teleport

0.429

0.173

0.028

Trackball

0.429

0.173

0.028
Joystick

Trackball

Joystick

0.500

0.203

0.029

Figure 5-21: Average motion sickness and presence scores for the users with ASD.

After the testing, the participants were requested to rank the locomotion techniques according to their preference. Results for the preference ranking are presented in Figure 5-22. One-way ANOVA with repeated measures analysis resulted in significant difference for the preference results $(F(7,6)=7.06, p=0.000$, Mauchly's sphericity test 
succeeded and sphericity assumed). Then, paired t-tests were conducted for finding out any differences in combinations of technique pairs. The largest significant mean difference was found to be between point $\&$ teleport and flying $(\mu$ difference $=3.667, \mathrm{p}=$ $0.000)$ and joystick and flying $(\mu$ difference $=3.667, \mathrm{p}=0.000)$ and the smallest significant mean difference was found to be between joystick and trackball $(\mu$ difference $=1.133, \mathrm{p}=$ $0.021)$.

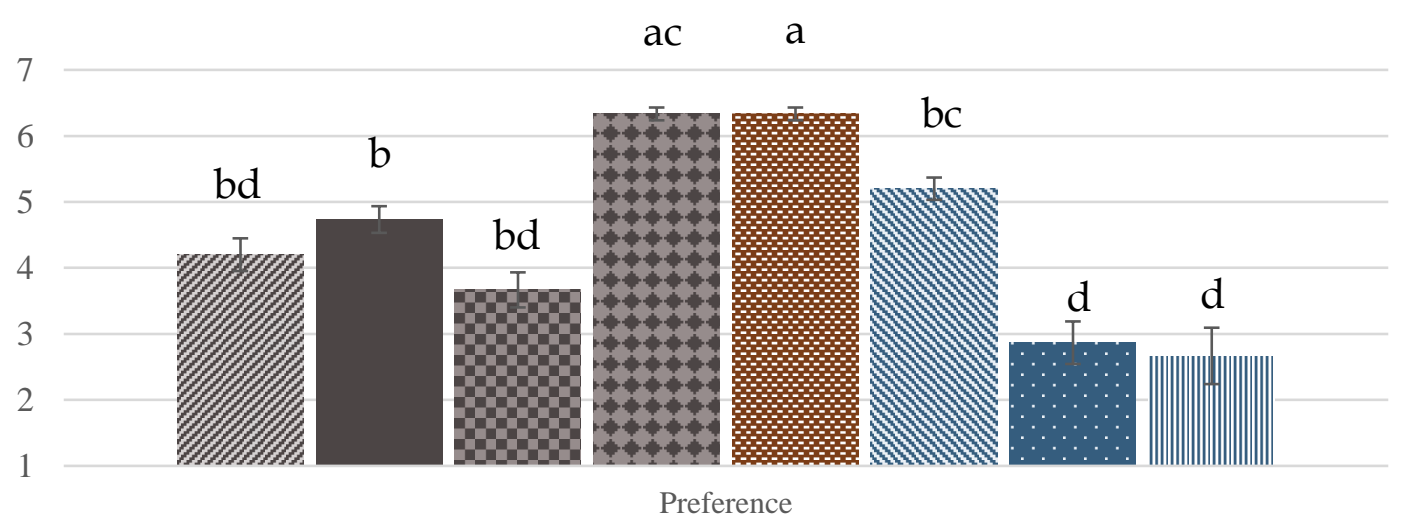

\begin{tabular}{|c|c|}
\hline $\mathbb{Z}$ Redirected Walking & - Walk in Place \\
\hline Stepper & Point and Teleport \\
\hline Joy & \& Trackball \\
\hline Hand Flapping & \|\|$\| \mid$ Flying \\
\hline
\end{tabular}

Figure 5-22: Weighted averages of the preference ranking data for all locomotion techniques.

Trajectories for the movements of the users with ASD in the virtual world are presented in the following figures: redirected walking (Figure 5-23), walk-in-place (Figure 5-24), stepper machine (Figure 5-25), point \& teleport (Figure 5-26), joystick (Figure 5-27), trackball (Figure 5-28), hand flapping (Figure 5-29), and flying (Figure 5-30). Virtual and real path trajectories for the eight locomotion techniques with and without 
obstacles can be seen in the figures. Users' movements between all eight destination points were plotted.

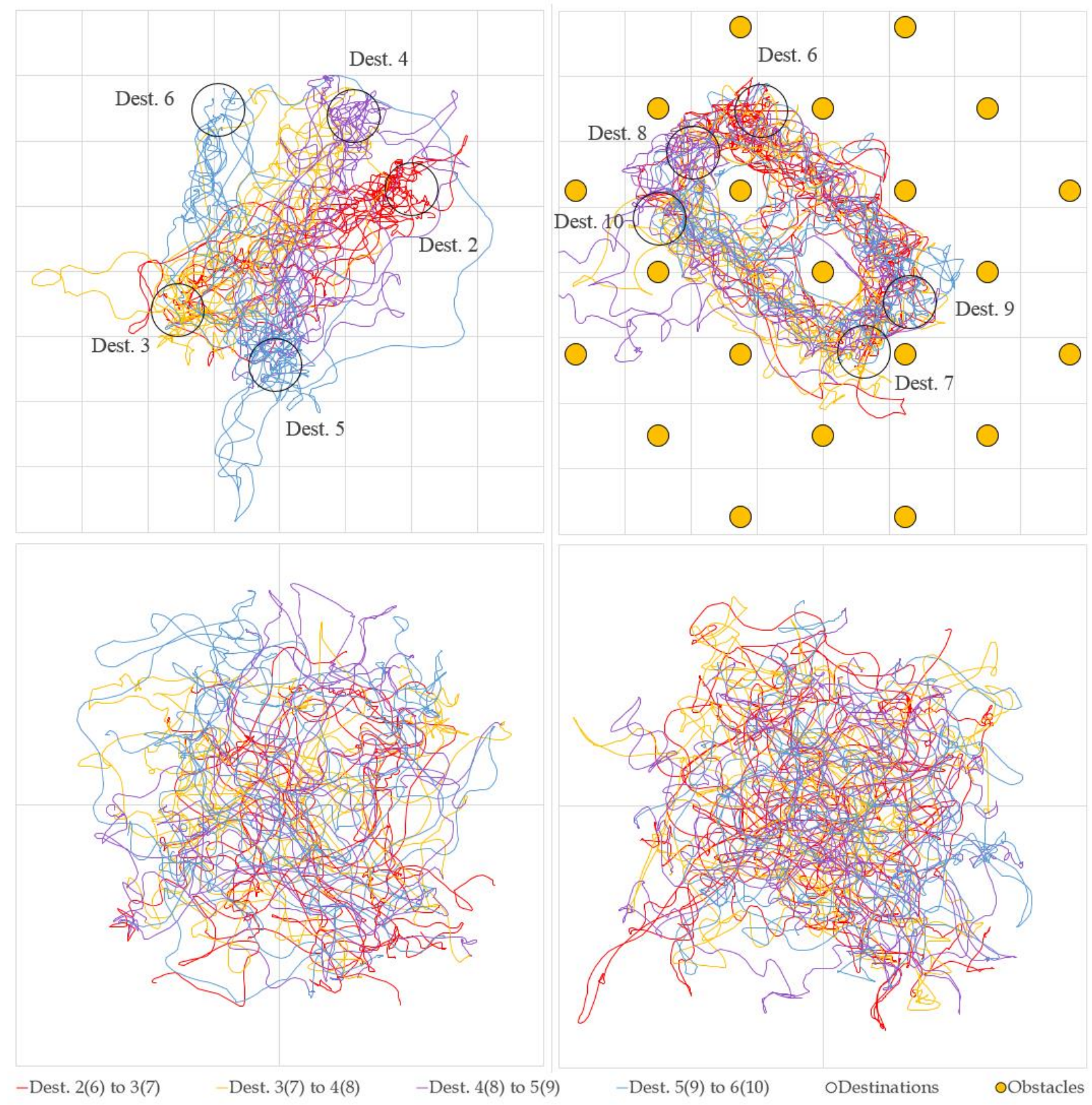

Figure 5-23: Redirected walking locomotion technique projected paths for the users with ASD. Left top: Virtual projected paths in virtual environment between destination points 2 and 6. Left bottom: Real projected paths in real tracking area between destination points 2 and 6 . Right top: Virtual projected paths in virtual environment between destination points 9 and 10 with obstacles. Right bottom: Real projected paths in real tracking area between destination points 6 and 10 . 


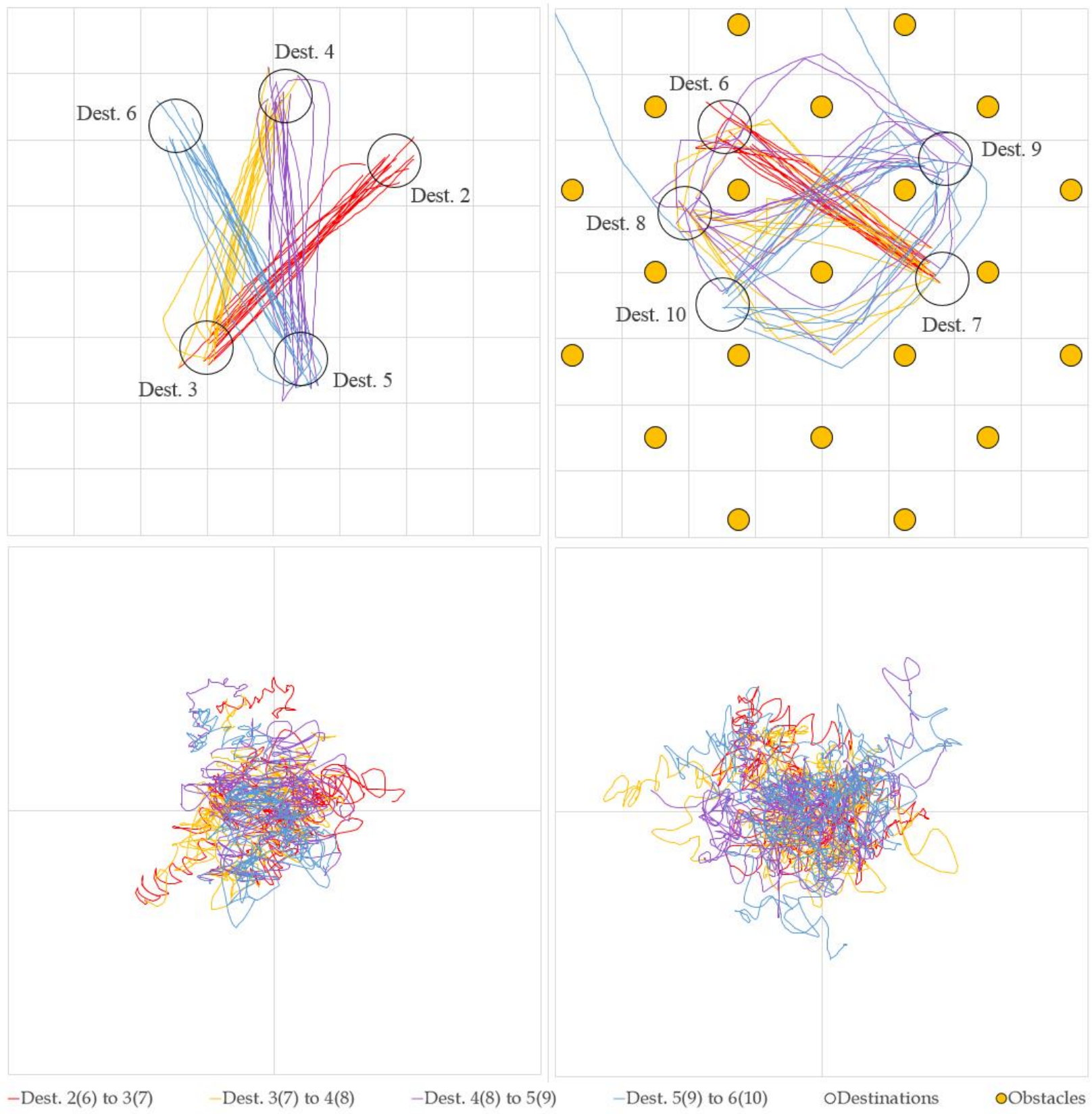

Figure 5-24: Walk-in-place locomotion technique projected paths for the users with ASD. Left top: Virtual projected paths in virtual environment between destination points 2 and 6. Left bottom: Real projected paths in real tracking area between destination points 2 and 6. Right top: Virtual projected paths in virtual environment between destination points 9 and 10 with obstacles. Right bottom: Real projected paths in real tracking area between destination points 6 and 10 . 


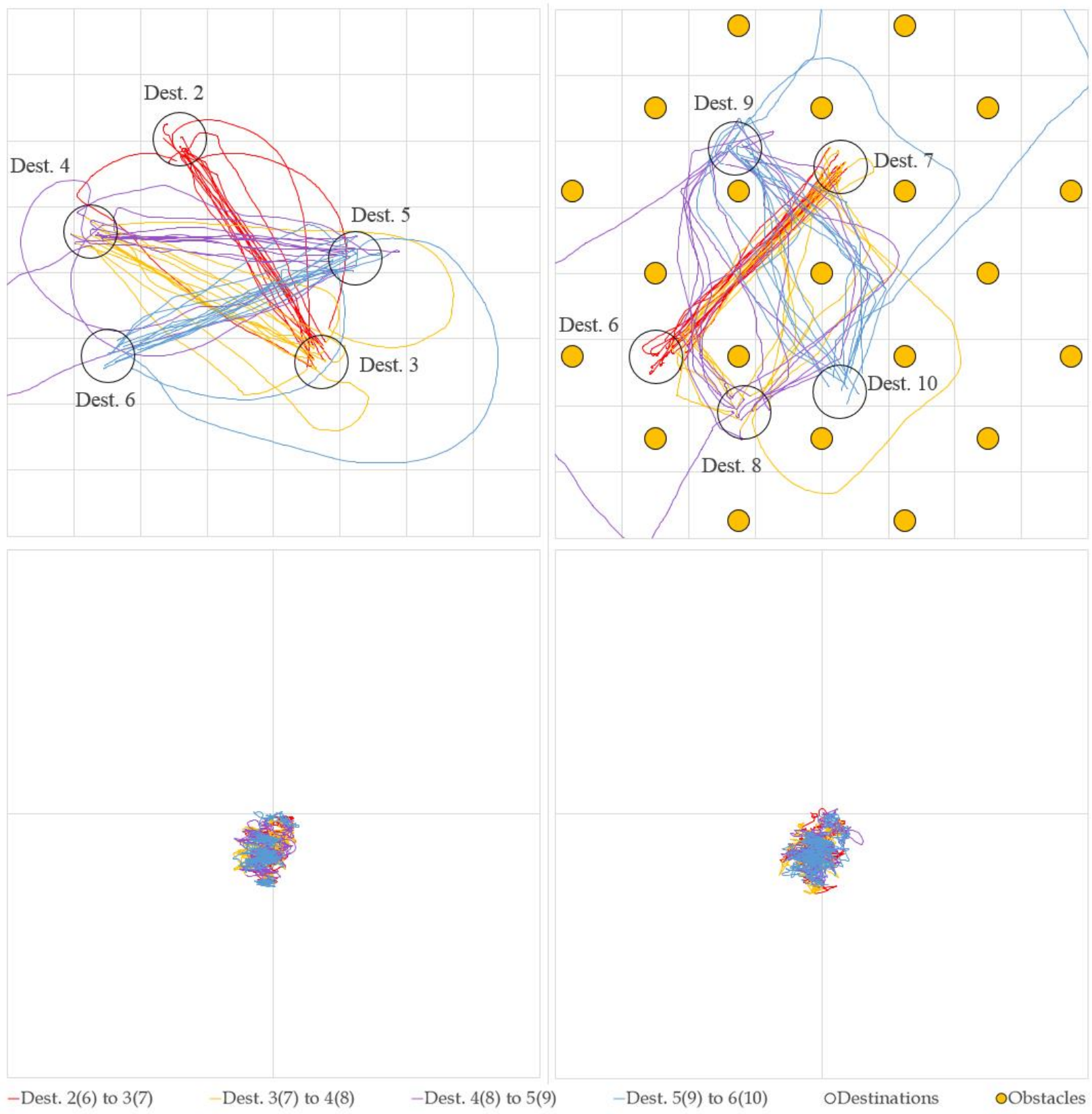

Figure 5-25: Stepper machine locomotion technique projected paths for the users with ASD. Left top: Virtual projected paths in virtual environment between destination points 2 and 6. Left bottom: Real projected paths in real tracking area between destination points 2 and 6 . Right top: Virtual projected paths in virtual environment between destination points 9 and 10 with obstacles. Right bottom: Real projected paths in real tracking area between destination points 6 and 10 . 


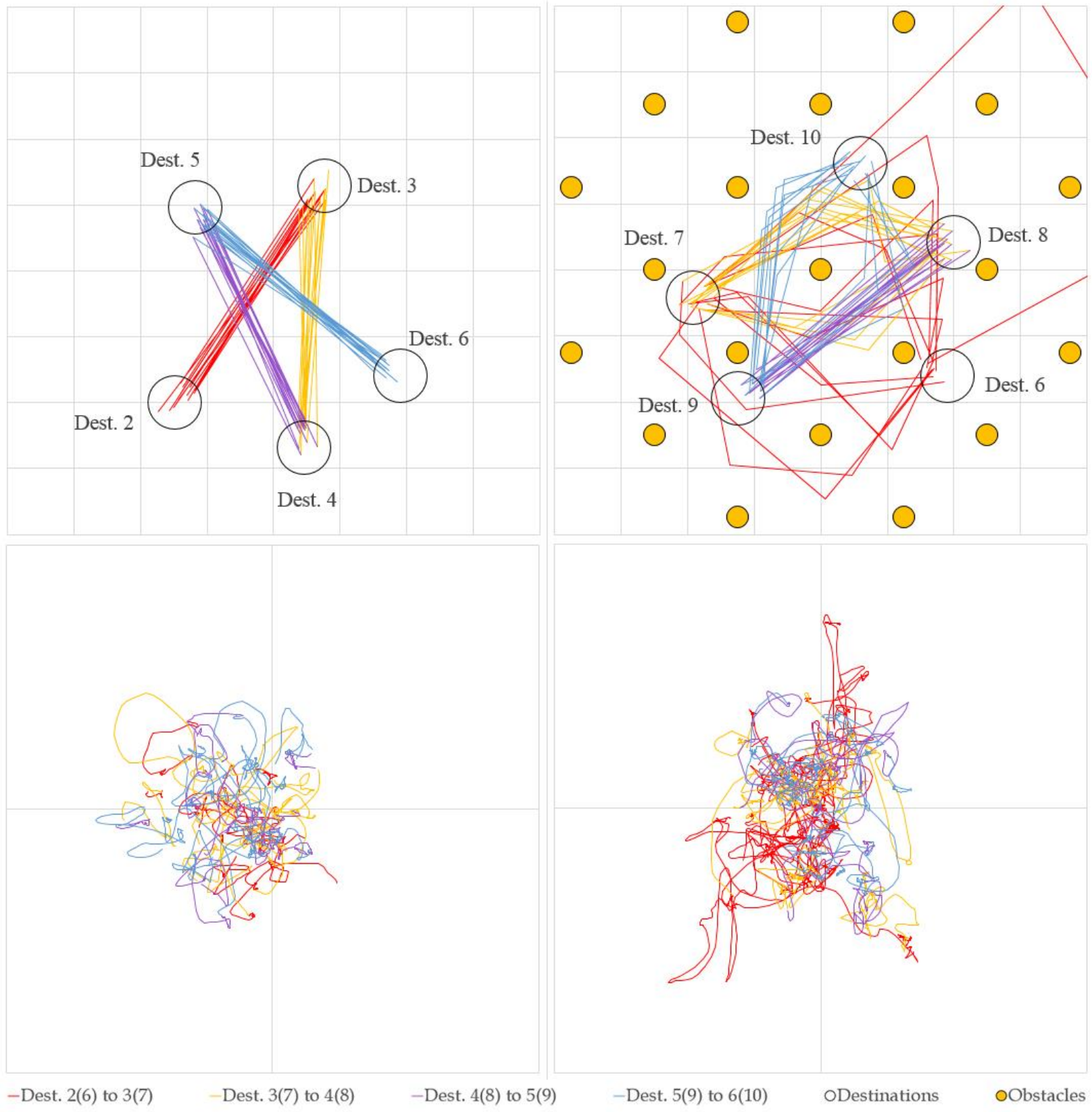

Figure 5-26: Point \& teleport locomotion technique projected paths for the users with ASD. Left top: Virtual projected paths in virtual environment between destination points 2 and 6. Left bottom: Real projected paths in real tracking area between destination points 2 and 6. Right top: Virtual projected paths in virtual environment between destination points 9 and 10 with obstacles. Right bottom: Real projected paths in real tracking area between destination points 6 and 10. 


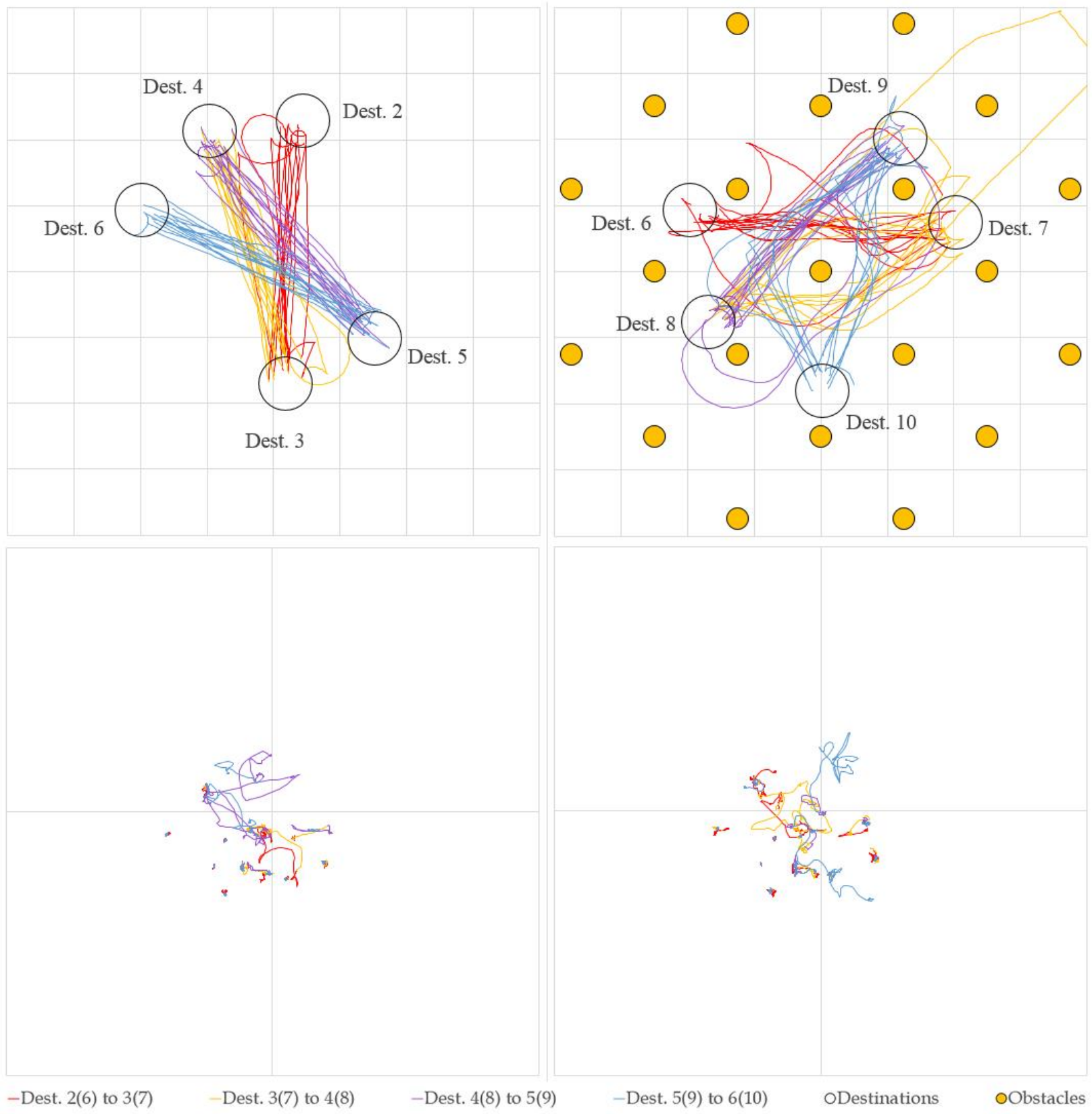

Figure 5-27: Joystick locomotion technique projected paths for the users with ASD. Left top: Virtual projected paths in virtual environment between destination points 2 and 6 . Left bottom: Real projected paths in real tracking area between destination points 2 and 6. Right top: Virtual projected paths in virtual environment between destination points 9 and 10 with obstacles. Right bottom: Real projected paths in real tracking area between destination points 6 and 10 . 


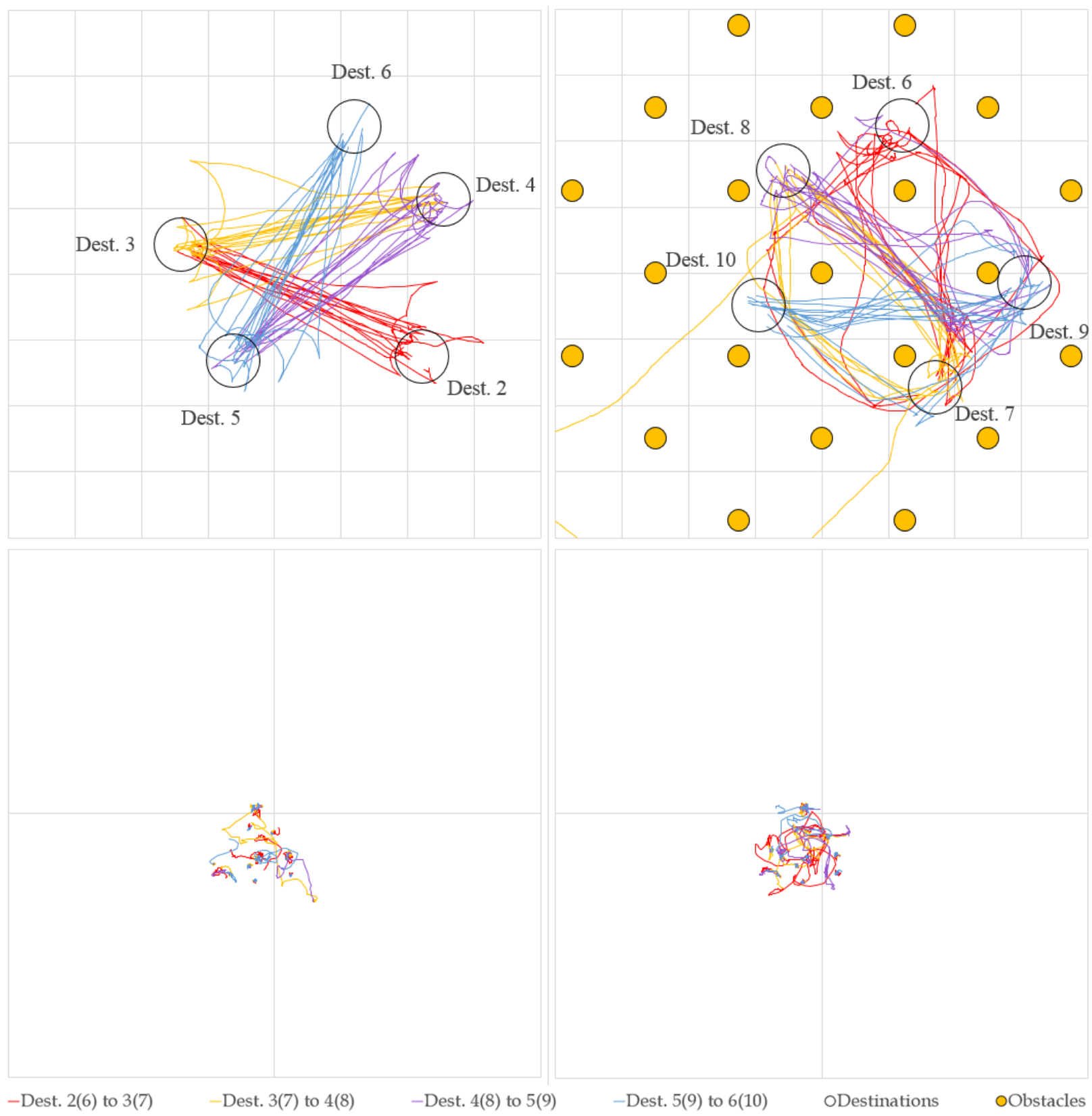

Figure 5-28: Trackball locomotion technique projected paths for the users with ASD. Left top: Virtual projected paths in virtual environment between destination points 2 and 6. Left bottom: Real projected paths in real tracking area between destination points 2 and 6. Right top: Virtual projected paths in virtual environment between destination points 9 and 10 with obstacles. Right bottom: Real projected paths in real tracking area between destination points 6 and 10 . 


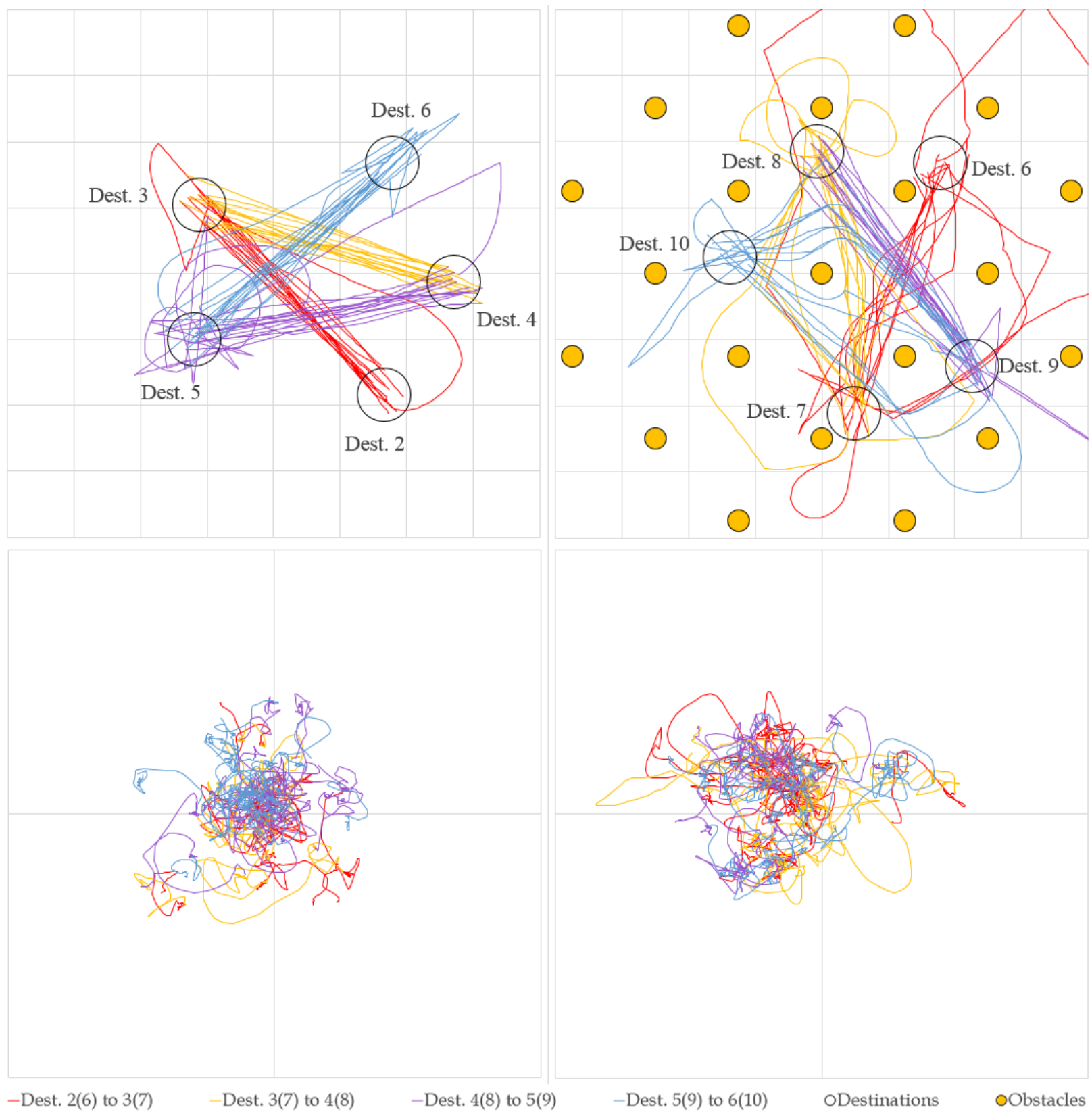

Figure 5-29: Hand flapping locomotion technique projected paths for the users with ASD. Left top: Virtual projected paths in virtual environment between destination points 2 and 6. Left bottom: Real projected paths in real tracking area between destination points 2 and 6. Right top: Virtual projected paths in virtual environment between destination points 9 and 10 with obstacles. Right bottom: Real projected paths in real tracking area between destination points 6 and 10 . 


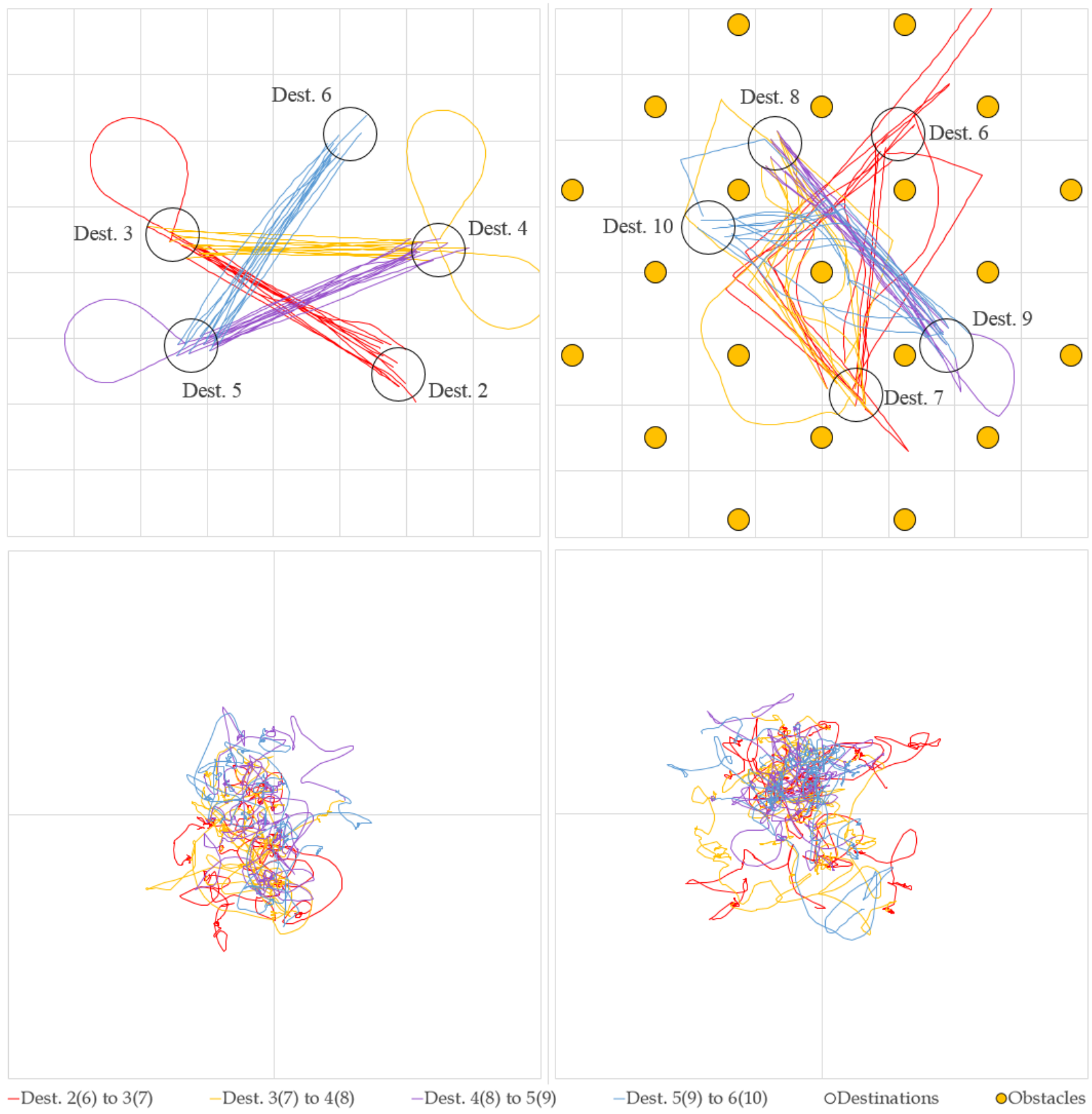

Figure 5-30: Flying locomotion technique projected paths for the users with ASD. Left top: Virtual projected paths in virtual environment between destination points 2 and 6 . Left bottom: Real projected paths in real tracking area between destination points 2 and 6. Right top: Virtual projected paths in virtual environment between destination points 9 and 10 with obstacles. Right bottom: Real projected paths in real tracking area between destination points 6 and 10 . 


\subsubsection{Participant Comments}

Participants were encouraged to share their comments, suggestions, likes and dislikes about any aspect of the experiment on the surveys. Some of these comments are shared following, under the categories based on the locomotion techniques.

Joystick received many positive comments from the participants with ASD: User 22: "Joystick is what my favorite tech is and it's perfect for walking in virtual world." User 25: “Awesome!” Many participants made positive comments for point \& teleport as well: User 7: "I do enjoy the technology used." User 22: "Teleport is really the way to walk." User 25: “I wanna buy this game.” User 26: “It's teleporting! It was really cool!" Trackball received mostly positive comments: User 7: “I liked that it was super easy. I want to play this one again." User 8: "It was very fun. Rolling it was like moving around on an office chair." User 22: "Very interesting indeed." User 26: "I liked that when you're trying to walk and turn all you had to do is use your hand." One user on the other hand, made a negative statement about trackball: User 24: "It was a bit difficult to move the ball to keep walking all the time."

Redirected walking received mixed comments from the users with ASD: User 8: "I liked it a lot. It was fast changing and challenging in a positive way." User 22: "I liked walking in virtual world, it was so interesting." User 23: "It was good to really walk." User 26: "I liked it since it felt like I was really in the game. User 22: "I walk fast in real world yet I can't control my speed in virtual world." User 25: "It was close to the edge and a bit confusing." Stepper machine also received mixed comments from the participants: User 16: "I liked it the most because it made me to exert the most effort." 
User 25: "I wanna do it again!" User 22: “It wasn't so easy to do stepping machine in virtual world." User 26: "I liked that one but it felt a bit tiring at the back of my legs." Walk-in-place also got mixed comments from the users: User 24: "It was realistic." User 25: It helped to keep me in the center and I liked it." User 28: "Really interesting concept." User 26: "I didn't like this method because it was like real walking but not so."

Hand flapping received mostly negative comments from the users with ASD: User 22: "Hand flapping for walking is harder than I thought." User 23: "It was a bit hard to use flapping." User 24: It was a little hard to control." User 26: "It was not so realistic to use hand flapping for walking." User 25: It was comfortable." Flying also received mostly negative comments from the participants: User 14: "It was completely frustrating." User 22: "It's hard to control it." User 25: "I didn't like this one."

\subsubsection{Discussion}

Analysis of the results, the observations that were made throughout the user study sessions and the comments from the participants with ASD shaped our interpretation on the implication of the user study results. For virtual reality applications targeting high functioning individuals with ASD, we recommend using the joystick, point \& teleport, redirected walking or walk-in-place as the locomotion techniques under different circumstances.

\subsubsection{Joystick}

The joystick shared the first ranking in the user preference along with the point \& teleport. We interpret that the users liked the feeling of being in control, the simplicity of use and the translation of movement into the virtual world with the joystick technique 
(pushing forward to go forward). Its reminding of video games and the familiarity may be other positive factors for the joystick, considering that individuals with ASD feel more comfortable using familiar objects. Although most of our participants with ASD didn't have any prior VR experience, more than half of them mentioned that they frequently played video games in real life. We recommend that in VR applications where the accuracy of the control is important and instant movements in small increments would be useful, the joystick can be used for locomotion.

\subsubsection{Point E Teleport}

This technique created excitement in our participants with ASD. Many users made positive statements such as 'Wow' and 'Cool' when they only heard its name or when we explained them how the technique worked. The uses stated that the technique resembled science fiction. We interpret that affinity of individuals with ASD to technology may have contributed to the preference for this locomotion technique. Since teleportation is a term reminding of technology, users may have felt sympathy for this technique. Beyond this, we observed that the users were comfortable in using this technique and embraced it very quickly as well. Pointing to where they wanted to be in the virtual world provided a simple form of representation. However, point \& teleport may not be applicable in virtual environments that contain many elements that the user needs to move around. In virtual reality applications with vast environments that require long travel distances, we recommend using point \& teleport locomotion technique for high functioning individuals with ASD. 


\subsubsection{Redirected Walking}

It may result in long times to reach to the destination points for individuals with ASD when redirected walking is implemented on smaller tracked areas. The reason behind this may be that in smaller tracked areas, there will be more frequent appearances of the dynamic obstacles that are used for directing users towards the center of the tracked area. These additional obstacles will yield longer travel times since the users need to overcome them by walking around. In addition, the alteration of the virtual view would create instant changes to which individuals with ASD may have difficulties in adjusting. Redirected walking resulted in low preference scores. Hence, in small tracked areas, we do not recommend using redirected walking as a virtual reality locomotion technique for individuals with ASD.

\subsubsection{Walk-in-Place}

For individuals with ASD the walk-in-place technique turned out to be a good alternative to redirected walking. Since it urged the users to stay in the same place, the size of the tracked area did not impose a limitation. The users were quick to grasp the concept. Hence, we recommend using walk-in-place in applications that encourage exercising for high functioning individuals with ASD in small tracked areas. The stepper machine gave the users a similar experience with walk-in-place. However, it did not provide additional comfort or ease of use. Hence, we recommend selecting walk-in-place over the stepper machine for high functioning individuals with ASD. 


\subsubsection{The Other Techniques}

In this user study, locomotion that required continuous input from the hands or feet did not provide convenient use for the participants with ASD. For flying, which required the users to release their hands after triggering the movement by raising it up, it was difficult for users with ASD to put their hands down in the idle pose. They tended to keep their hands close to their chests while waiting, which resulted in unintentional movements and problems in deactivation of the technique. Hence, we suggest that incorporating relaxed hand pose into gesture controlling for individuals with ASD may not work well. With the flying technique, two participants wanted to stop taking part in the experiment. They stated that they felt overwhelmed and nauseous. The main reason was stated to be the feeling of not being in control of the starting and stopping the locomotion technique. The participants did not wish to completely stop taking part in the user study and wished to try the remaining locomotion techniques. This emphasizes the importance of giving the control of the locomotion technique to the individuals with ASD with an easy to use interface for them in virtual reality experiences.

Another hand gesture controlled locomotion, hand flapping also didn't provide comfortable use. We observed that some users had difficulty in keeping their hands still while waiting for the destination points to disappear, which caused unintentional movements. Some users with ASD did the hand flapping motion with their hands around their chest level but tried to touch their bodies with their hands after stopping doing the flapping motion. This made the virtual viewpoint move more since moving the hand back to touch the body for the idle pose elongated the flapping motion, causing 
overshoots. Thus, we recommend that the gesture design for locomotion should give individuals with ASD concrete poses (such as putting their hands on their body or making a specific gesture). More abstract concepts such as releasing the hands and stopping making the flapping motion did not work well in our study for this population group.

\subsubsection{Time to Reach the Destination Points and Number of Collisions}

It took less time to reach to the destination points with the joystick and trackball independent of the presence of obstacles in the virtual world, in alignment with the results in [113]. The point \& teleport provided short times without the presence of obstacles whereas providing long times with the presence of obstacles, hence Hypothesis $_{1,0}$ was rejected. We interpret the reason behind this as the multiple teleportations needed to move around the obstacles requiring waiting times for the activation of the teleportation. Redirected walking yielded the longest times to reach to the destination points for both with obstacles and without obstacles cases. We interpret the reason behind this as the additional time it took for the participants to overcome the dynamic obstacles that appeared when the users got close to the edges of the tracked area and the time it took for the adjustment to the altered view.

Point \& teleport, trackball and joystick resulted in the least amount of collisions with the static obstacles whereas redirected walking resulting in the most. We interpret this as the point \& teleport, trackball and joystick providing more control to the users whereas redirected walking providing the least. Gains that were applied in redirected 
walking may have caused exaggerated movements resulting in unintentional hits to obstacles.

\subsubsection{Survey Metrics}

Flying resulted in the most difficulty in understanding whereas joystick, point \& teleport and trackball provided the least difficulty, in alignment with the results in [129]. Hand flapping and flying were the most difficult to operate whereas trackball, point \& teleport and joystick were the least difficult to operate. Joystick and trackball shared the feeling of most being in control whereas flying and hand flapping provided the least feeling of being in control. Joystick, point \& teleport and trackball provided high level of enjoyment whereas flying and hand flapping provided significantly lower levels of enjoyment. Stepper machine resulted in the most required effort whereas point \& teleport and trackball resulted in the least. Tiredness and overwhelmedness wasn't significantly different for all of the techniques. Flying, hand flapping and redirected walking caused the most frustration whereas joystick and trackball and causing the least. Low results for flying was in alignment with [33]. There wasn't any significant difference between the locomotion techniques in terms of motion sickness and presence, in alignment with the results in $[33,128]$.

The participants with ASD preferred joystick and point \& teleport the most, that was followed by walk-in-place and trackball. Flying and hand flapping were preferred the least. The Hypothesis 2,0 was also rejected. 


\subsubsection{Summary, Conclusion and Future Work}

In this study, eight VR locomotion techniques were implemented and evaluated with high functioning individuals with ASD. These techniques were: redirected walking, walk-in-place, joystick, stepper machine, point \& teleport, flying, hand flapping, and trackball controller. The locomotion techniques were implemented in an immersive VR environment and a user study was performed with 15 high functioning individuals with ASD. Results showed that for individuals with high functioning autism; joystick, point \& teleport and walk-in-place are suitable VR locomotion alternatives for small tracked areas whereas continuous hand gesture based (such as hand flapping) and automatic movement based locomotion techniques (such as flying) are not convenient for them.

Possible future work areas consist of evaluating different versions of the locomotion techniques that resulted in high preference scores in this user study, such as point \& teleport with controller, wireless hand held joystick and alterations of the walkin-place. The aim of these evaluations will be providing more comfortable VR experiences for individuals with ASD. Evaluating these VR locomotion techniques with low and medium functioning individuals with ASD would also be important areas for future work.

\subsection{Comparison of Neurotypical Individuals and Individuals with ASD}

This subsection presents the comparison of neurotypical individuals and individuals with high functioning ASD in virtual reality locomotion. Although this comparison was not the main focus of the study, it is still added with the motivation of providing insight into the developers for this specific population by clarifying the 
similarities and differences in these two populations regarding virtual reality locomotion. This subsection includes general differences in the scores, preferences and behavior of neurotypical individuals and individuals with ASD. Detailed statistical analysis outputs from the IBM SPSS Statistics Software for the comparison between the two population groups are presented in Appendix H.

As the completion time was considered, independent samples t-tests resulted in statistical significance only for the following: $\mathrm{t}(27)=-1.015, \mathrm{p}=0.023$ for joystick without obstacles; $\mathrm{t}(27)=-7.898, \mathrm{p}=0.002$ for redirected walking with obstacles (equal variances assumed by the Levene's test for equality of variances). The average time to clear the destination points was significantly higher for individuals with ASD (12.50) than neurotypical individuals (11.49) for the joystick technique when there were no obstacles in the scene. The average time to clear the destination points was again significantly higher for individuals with ASD (28.65) than neurotypical individuals (20.75) for the redirected walking technique when there were obstacles in the scene.

There wasn't any statistically significant difference for the number of collisions, difficulty in operation, difficulty in understanding, enjoyment, frustration, feeling of being in control, required effort, presence, motion sickness, and tiredness scores between neurotypical individuals and individuals with ASD.

For the feeling of being overwhelmed, there was significant difference: $t(27)=-$ $0.824, p=0.038$ for redirected walking (equal variances not assumed by the Levene's test for equality of variances). For individuals with ASD, the score for the feeling of being overwhelmed was significantly higher (2.36) than the neurotypical individuals (1.53) 
with the redirected walking. Although not statistically significant, required effort resulted in a small $\mathrm{p}$ value for the flying technique: $\mathrm{t}(27)=-0.957, \mathrm{p}=0.052$ (equal variances not assumed by the Levene's test for equality of variances). For individuals with ASD, the score for the required effort was significantly higher (3.36) than neurotypical individuals (2.4) for flying.

For the preference ranking scores, point \& teleport was the first choice in both populations (shared the first ranking with the joystick for individuals with ASD). Redirected walking was the second choice of neurotypical individuals whereas the fifth choice of individuals with ASD. Joystick was the third choice of neurotypical individuals whereas the trackball was the third choice of individuals with ASD.

We can interpret that the redirected walking was more overwhelming for the users with ASD, most probably because of the alteration of the virtual world based on the user's movement. Addition of the static obstacles in the scene made it more difficult for individuals with ASD to use the redirected walking method, whereas no similar effect was observed with the neurotypical users. We interpret the underlying reason as the additional rotations imposed by the static obstacles.

Another major difference was in the use of the flying technique. Individuals with ASD had more difficulties in using this technique than the neurotypical individuals. We interpret the underlying reason as the selected neutral pose gesture. Individuals with ASD tended to keep their hands at their chest level, which made it difficult for them to start and stop the locomotion with this technique. Two individuals with ASD wanted to stop trying the flying technique due to being overwhelmed and nauseous whereas all 
neurotypical participants completed testing the flying technique without reporting similar complaints.

The final behavioral difference that was observed while using the locomotion techniques was for the hand flapping. Users with ASD tended to keep their hands pressed to their bodies as they wanted to stop the flapping gesture. Neurotypical individuals did not make such an addition to the gesture. This addition made it more difficult for the users with ASD to use this technique. We interpret that individuals with ASD need more concrete starting and stopping poses that do not involve neutral or relaxed postures for triggering the locomotion in virtual reality.

Table 5-7: Locomotion technique recommendations for neurotypical individuals and individuals with ASD.

$\begin{array}{ccc}\begin{array}{c}\text { Virtual Environment } \\ \text { Property/Aim }\end{array} & \text { Neurotypical Individuals } & \text { Individuals with ASD } \\ \text { Cognitively demanding } & \text { Point and teleport/Joystick } & \text { Joystick } \\ \text { Fast paced } & \text { Joystick } & \text { Joystick } \\ \text { A few virtual obstacles } & \text { Point and teleport } & \text { Point and teleport } \\ \text { High levels of presence } & \text { Redirected walking } & \text { Joystick } \\ \text { Physical motion } & \text { Redirected walking } & \text { Walk-in-place }\end{array}$

Based on the results of all user studies that were performed in scope of this dissertation, we compiled a table that includes the implications for virtual environments 
of different properties and aims, regarding neurotypical individuals and high functioning individuals with ASD (Table 5-7).

\subsection{Limitations}

This study was performed in a tracked area of $2 \mathrm{~m}$ by $2 \mathrm{~m}$. The findings of the study may not be transferrable to larger tracked areas. The task in the experiment was intentionally designed as simple and there were no distractions in the virtual environment for letting the users focus on their experience with the locomotion techniques. It remains questionable that the findings would transfer to more challenging virtual environments and more complex task designs. Finally, we would like to note that the gesture design of the locomotion techniques (such as triggering point \& teleport by hand pointing and triggering flying by hand raising) is expected to have a direct effect on the results. Hence, the findings may not be transferrable to the same locomotion techniques that are implemented with different gestures or control mechanisms.

It should also be noted that this study focused on the high functioning individuals with ASD. Since Autism is a spectrum based disorder, individuals on the different sides of the spectrum may have different needs and characteristics. Hence, the results may not be generalizable to the medium and low functioning populations with ASD. Redirected walking locomotion technique was implemented on a $2 \mathrm{~m} \times 2 \mathrm{~m}$ tracked area in this study, which is considered smaller as compared to the large tracked areas recommended in the previous studies on redirected walking [19]. The main concern of the previous studies while recommending large tracked areas was the motion sickness that would be induced 
by the large gains that are needed to be applied in smaller areas. In our study, although there wasn't any significant difference between the locomotion techniques in terms of motion sickness, in overall redirected walking did not provide a comfortable locomotion experience for the users with ASD. We would like to emphasize that the results for the redirected walking should not be generalized to all sizes of tracked areas. 


\section{CHAPTER 6: DIRECTION SPECIFICATION MODIFICATION OF THE POINT \& TELEPORT LOCOMOTION TECHNIQUE}

In this chapter, a modification of the point \& teleport locomotion technique with direction specification was evaluated. Although teleportation is a simple yet powerful possible alternative to the commonly used locomotion techniques, which has been recently started to be used in commercial virtual reality video games, it has not been quite explored in the literature yet. This chapter presents an experiment that aims to compare point \& teleport with a modified version of itself: point \& teleport with direction specification. In this modified version, the users could specify the direction they would be facing when teleported by rotating their hands in the rolling axis before the teleportation. In this chapter, the experiment design, results of the user study and limitations are discussed.

\subsection{Note to Reader}

Portions of this chapter were published in CHI PLAY 2016 (Bozgeyikli, E., Raij, A., Katkoori, S., and Dubey, R. Point \& Teleport Locomotion Technique for Virtual Reality. In Proceedings of the 2016 Annual Symposium on Computer-Human Interaction in Play (CHI PLAY '16). ACM, New York, NY, USA.) Permission is included in Appendix C. 


\subsection{Experiment Design}

A within subject experiment was designed. Each participant tried both the point \& teleport (P\&T) and the point \& teleport with direction specification (P\&T w/DS) techniques with a random order. Similar to the experiment in Chapter 5, the first two destination points for each technique were considered as training and discarded, and the remaining four destination points were taken into consideration for evaluation. After each technique, the participants filled out a survey about the technique they tried. After completing both trials, the participants were asked to state their preferences between the two techniques on an additional survey question.

The research question for this user study was: 'Would there be an improvement in the point \& teleport locomotion technique with the addition of the direction specification feature?' In the light of this research question, the following hypotheses were built for this experiment: Hypothesis1: The P\&T w/DS will give lower average time to reach the destination points than the P\&T. Hypothesis: The P\&T w/DS will result in better ranking scores than the P\&T. The same 16 neurotypical participants who attended the experiment in Chapter 5 attended this experiment. Due to the hardware malfunction in the testing session of one user, their data was not included in the analysis $(\mathrm{N}=15)$.

\subsubsection{Virtual Environment}

In this experiment, the virtual world was designed as a simple maze (Figure 6-1) to measure effects of the additional direction specification component more effectively. The maze was designed not to be challenging, with $14 \mathrm{~m}$ length and $14 \mathrm{~m}$ width. No gaps were placed on the exterior walls so the user could not go outside the virtual maze. The 
corridors had $2 \mathrm{~m}$ length for easy navigation. The longest dead-end corridor was $2 \mathrm{~m}$ long, so the users did not waste too much time with the wayfinding if they made wrong path choices. The height of the maze walls was $1.5 \mathrm{~m}$, which made it possible for the users to see the destination points from anywhere in the maze. Since the environment was designed as a maze and the maze walls inherently were obstacles, no additional obstacles in the form of pillars were used in this experiment. That's why this experiment had 6 destination points in total.

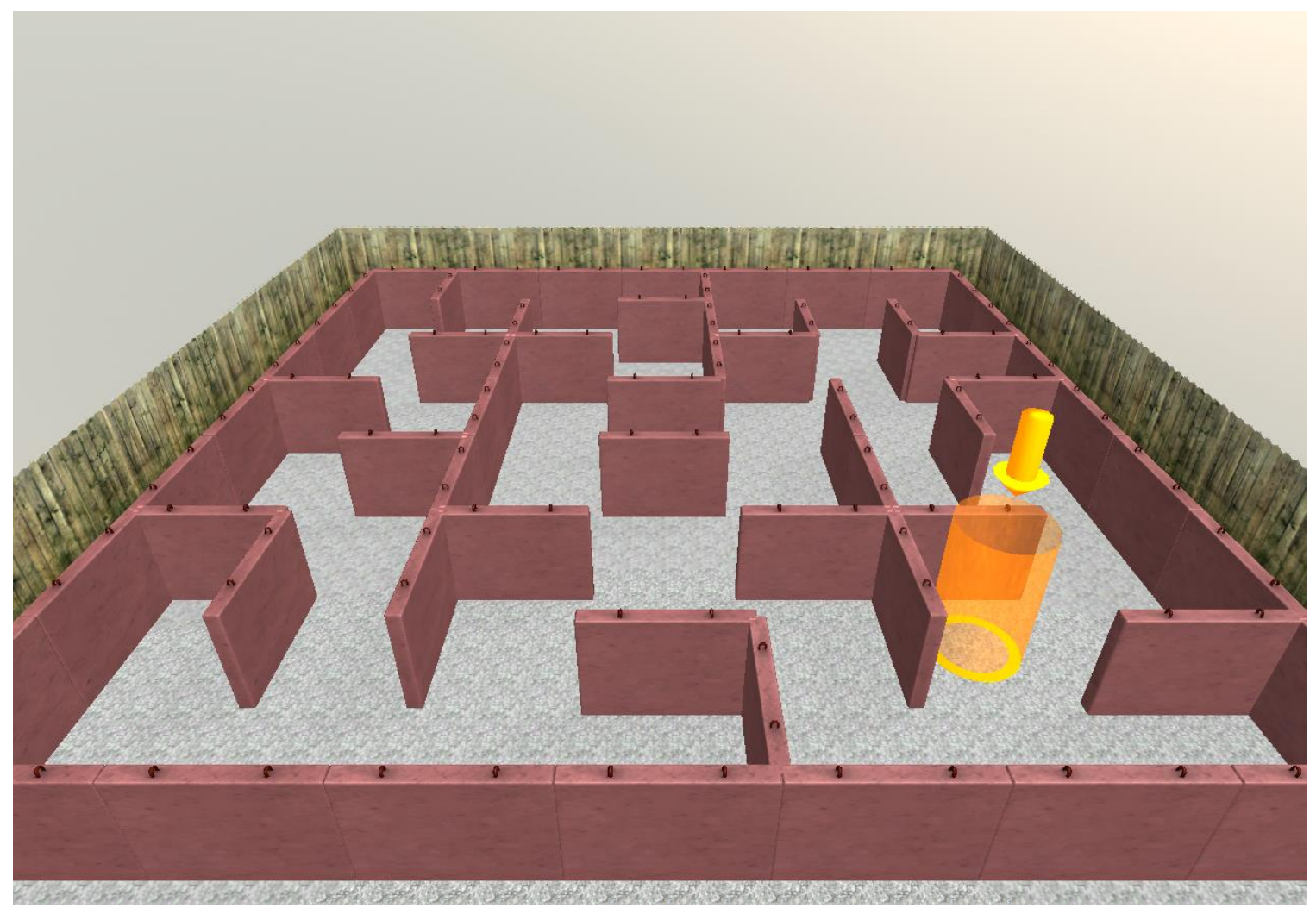

Figure 6-1: The virtual maze environment.

\subsubsection{Objective}

This experiment's objective was similar to the experiment in Chapter 5. The participants were asked to go to the destination points and wait inside until another 
destination point appeared somewhere else in the virtual maze. The same destination point marker objects as in the experiment in Chapter 5 were used to designate the target positions. Users completed two trials, each with 6 destination points. One trial included P\&T and the other trial included P\&T w/DS. The order of the trials was decided randomly with counterbalancing. The destination points in the two trials were different to eliminate any possible learning effect. The users started the testing at the center of the virtual maze. Each destination point was $8 \mathrm{~m}$ away from the previous destination point. The experiment block design can be seen in Figure 6-2.

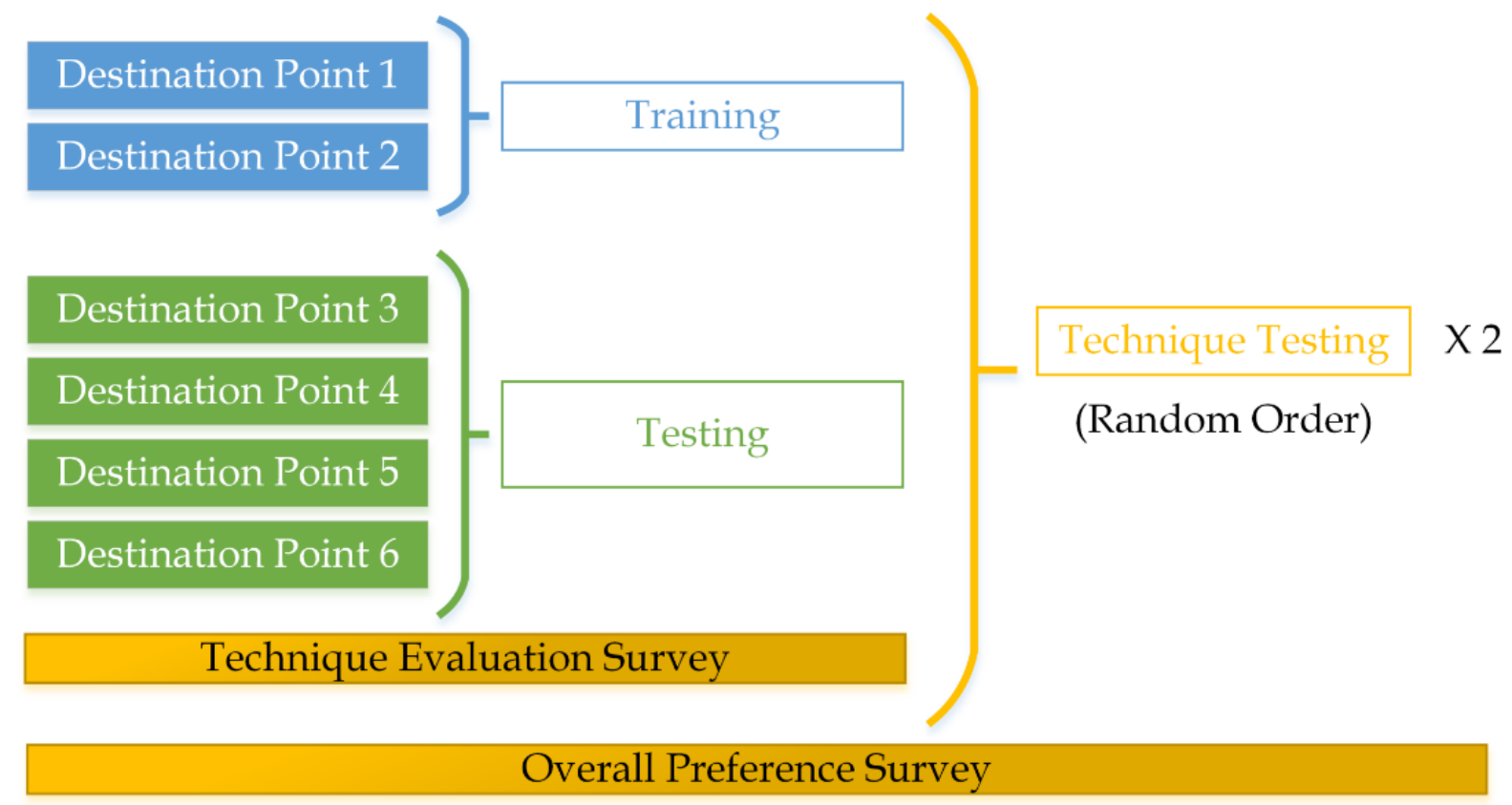

Figure 6-2: Block design of the direction specification modification of the point \& teleport experiment.

Photos taken during testing sessions with the point \& teleport and point \& teleport with direction specification can be seen in Figure 6-3 and Figure 6-4 respectively. 


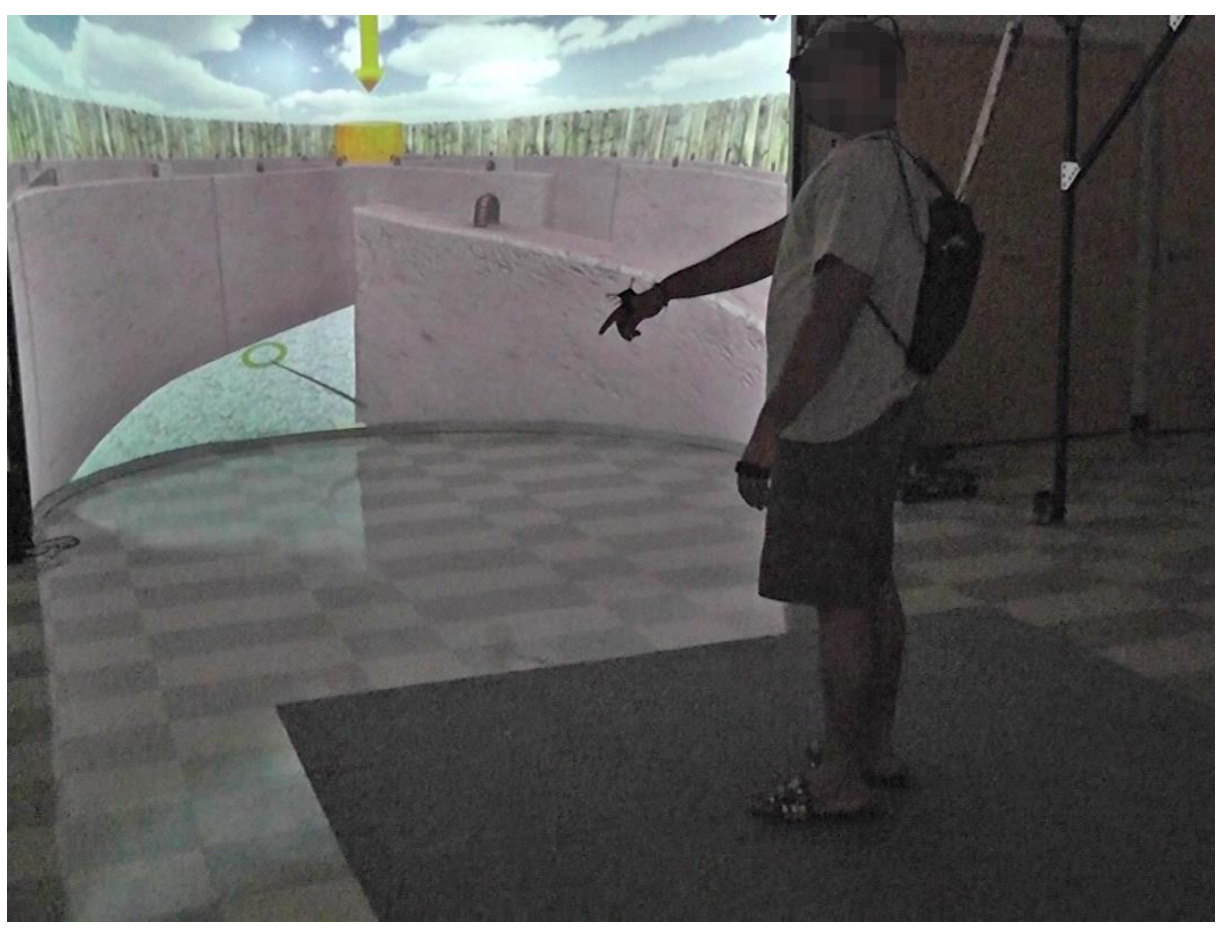

Figure 6-3: Photo of a testing session with the point \& teleport locomotion technique in the virtual maze.

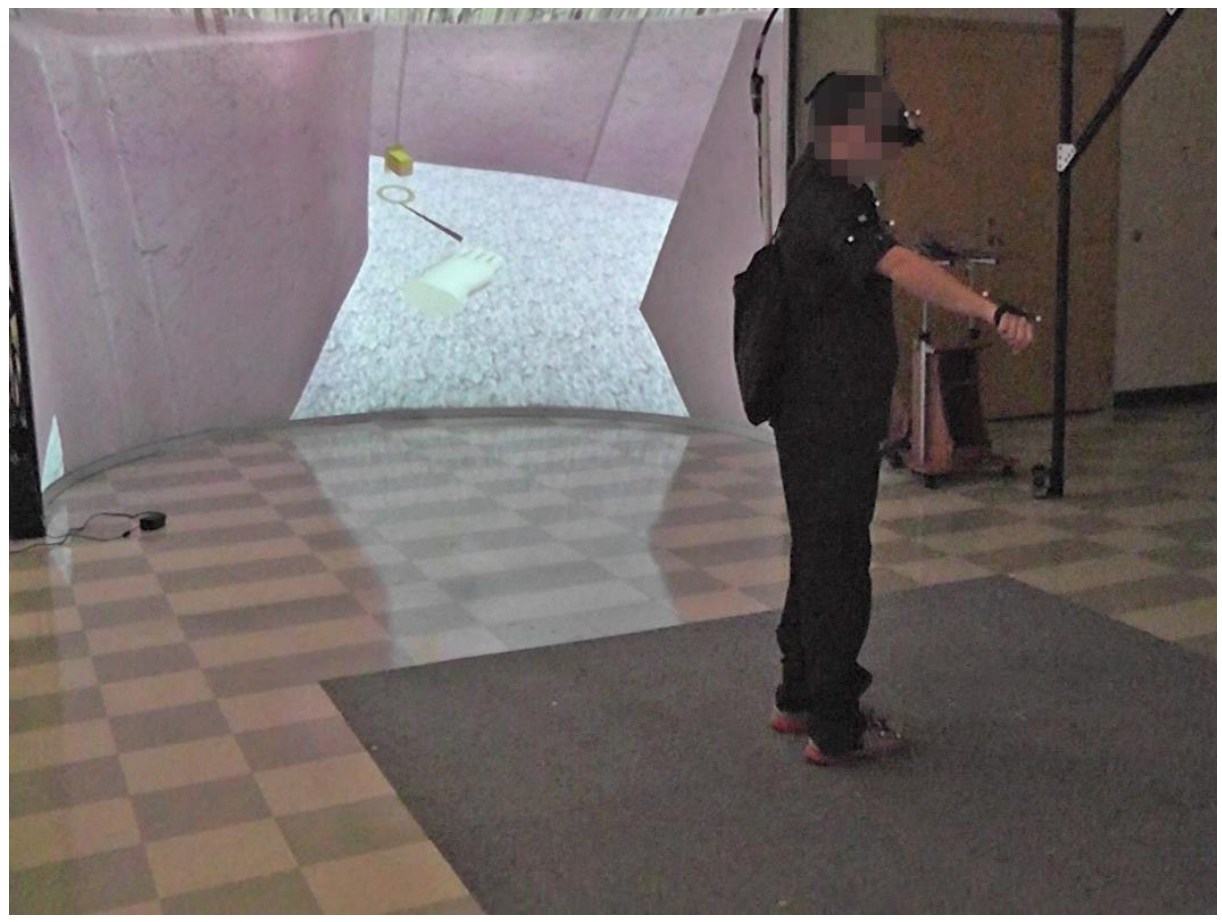

Figure 6-4: Photo of a testing session with the point \& teleport with direction specification locomotion technique in the virtual maze. 


\subsection{Results}

This subsection presents the results of the user study under the sub-subsections of data results, survey results and user comments.

\subsubsection{Data Results}

The average time to reach the destination points are presented in Figure 6-5. Two sample $\mathrm{t}$-tests resulted in no significant difference $(\mathrm{t}(14)=-1.289, \mathrm{p}=0.200$, Cohen's $\mathrm{d}=$ 0.235) in the time it took to reach the destination points with the point \& teleport and the point \& teleport with direction specification.

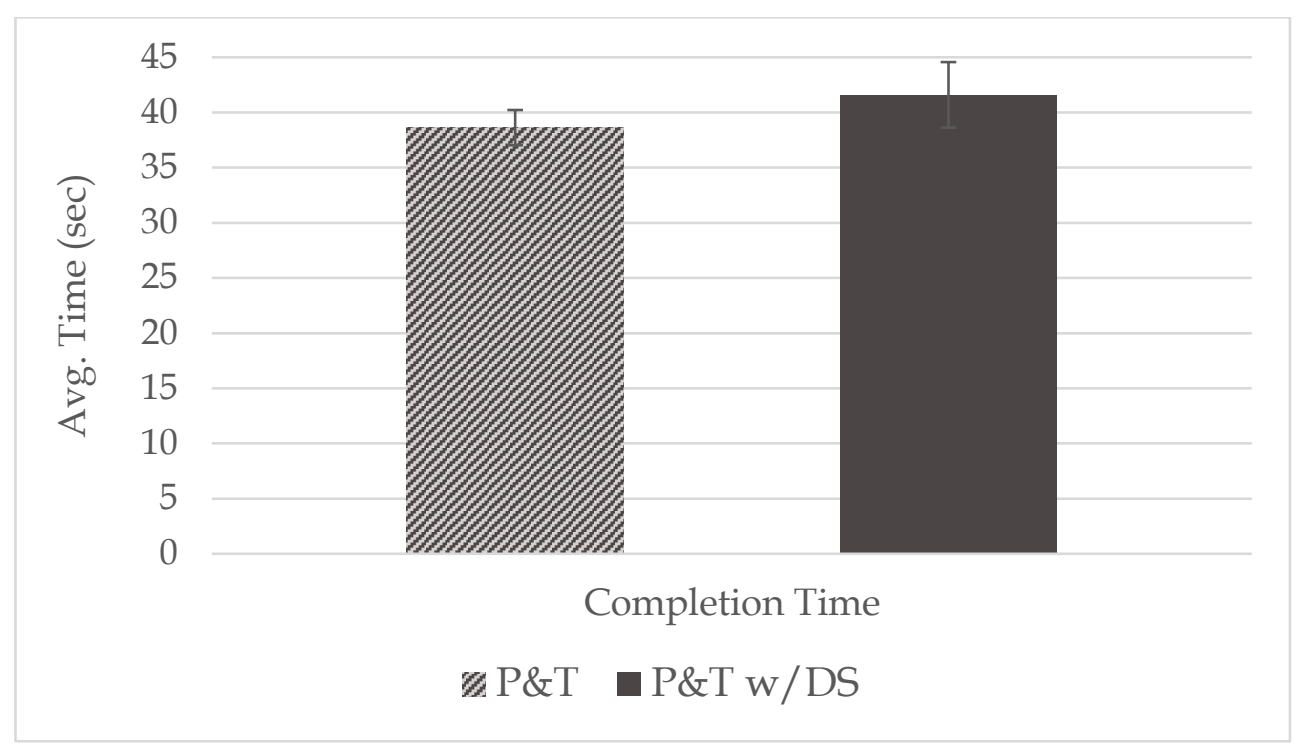

Figure 6-5: Average time to reach the destination points in the direction specification modification of the point \& teleport experiment.

In terms of the number of collisions made by the users with the maze walls, two sample t-tests resulted in no significant difference between the two locomotion techniques $(t(14)=-1.339, p=0.191$, Cohen's $d=0.489)$. Data can be seen in Figure 6-6. Detailed statistical analysis can be seen in Appendix I. 


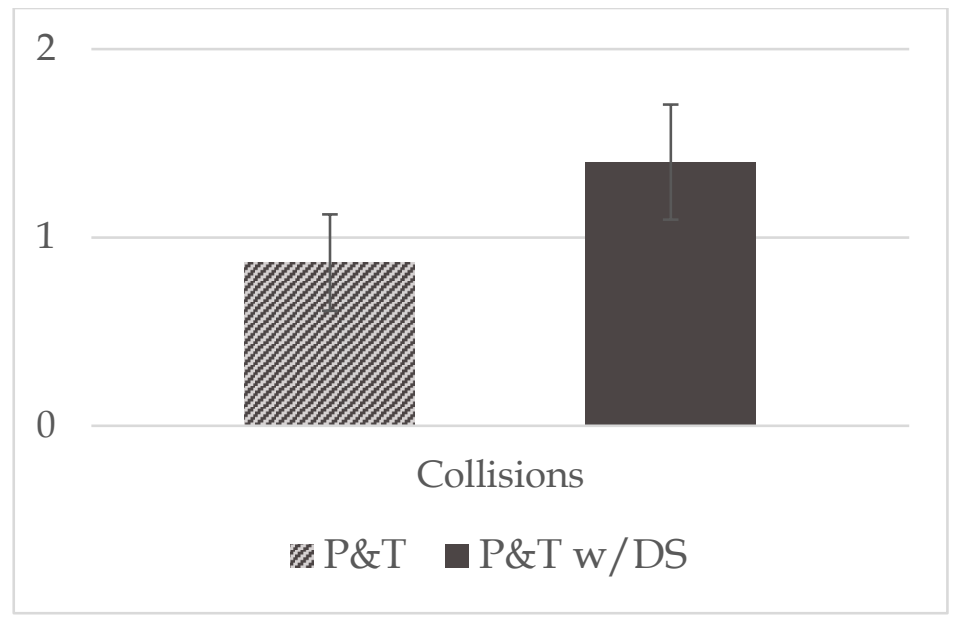

Figure 6-6: Average number of collisions with maze walls in the direction specification modification of the point \& teleport experiment.
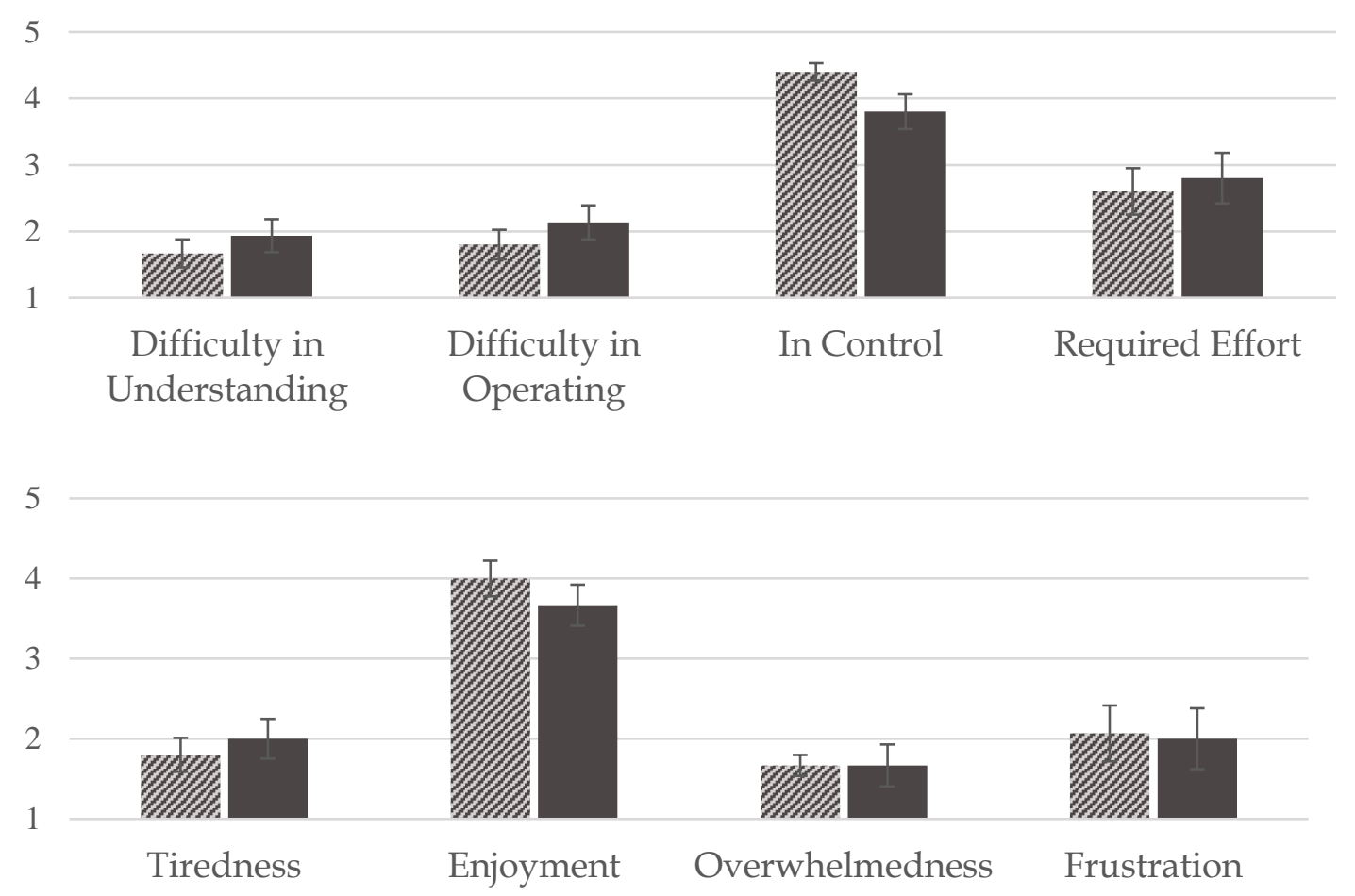

$$
\text { ZP\&T ロ P\&T w/DS }
$$

Figure 6-7: Survey results for point \& teleport and point \& teleport with direction specification locomotion techniques. 
Table 6-1: T-test results for the survey data of the direction specification modification of the point \& teleport experiment.

\begin{tabular}{lcccc}
\hline & $\mathbf{t}$ & $\mathbf{d f}$ & $\mathbf{p}$ & Cohen's d \\
\hline Diff. Understand & -0.819 & 14 & 0.420 & 0.299 \\
Diff. Operate & -0.983 & 14 & 0.334 & 0.359 \\
In Control & 2.049 & 14 & 0.050 & 0.748 \\
Required Effort & -0.387 & 14 & 0.701 & 0.141 \\
Tiredness & -0.456 & 14 & 0.652 & 0.167 \\
Enjoyment & 0.924 & 14 & 0.363 & 0.337 \\
Overwhelm & 0.000 & 14 & 1.000 & 0.000 \\
Frustration & 0.159 & 14 & 0.875 & 0.058 \\
Motion Sickness & -0.638 & 14 & 0.529 & 0.233 \\
Presence & 0.838 & 14 & 0.409 & 0.306 \\
\hline
\end{tabular}

\subsubsection{Survey Results}

To analyze the usability aspects of the two versions of the point \& teleport, we used the same eight sub categories with the experiment in Chapter 5: difficulty in understanding, difficulty in operating, feeling of being in control, required effort to move, feeling of tiredness, enjoyment, being overwhelmed and frustration. Each question had answers on a 5 point Likert scale (1: not at all, 5: very much). Results of these eight categories are presented in Figure 6-7.

As two sample t-tests were conducted, the only significant difference between the two locomotion techniques was in the feeling of being in control. The two sample t-tests results are reported in Table 6-1. No statistically significant difference was evident in 
terms of motion sickness although P\&T w/DS got higher scores $(M=0.47, \mathrm{SD}=1.06)$ than P\&T $(M=0.27, S D=0.59)$. The presence results weren't significantly different as well although P\&T got slightly higher scores $(\mathrm{M}=3.04, \mathrm{SD}=0.52)$ than $\mathrm{P} \& \mathrm{~T} w / \mathrm{DS}(\mathrm{M}=$ $2.89, \mathrm{SD}=0.50)$.

To analyze the user preference data (see Figure 6-8), the Friedman test was conducted and no statistically significant difference was found $\left(\chi^{2}(1, N=15)=0.067, p=\right.$ 0.796).

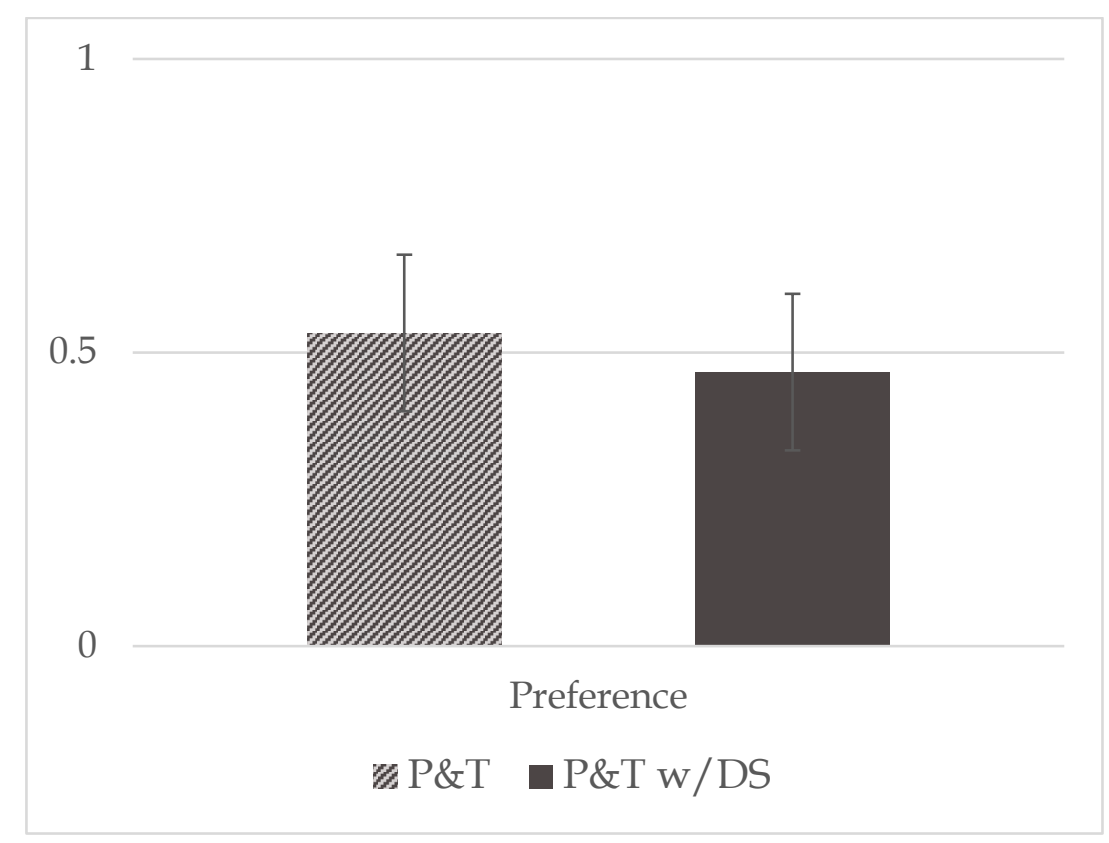

Figure 6-8: Average user preference score results for point \& teleport and point \& teleport with direction specification locomotion techniques.

The trajectories for the movements of the participants in the virtual maze and the real tracking area for the point \& teleport and the point \& teleport with direction specification locomotion techniques are presented in Figure 6-9 and Figure 6-10 respectively. 


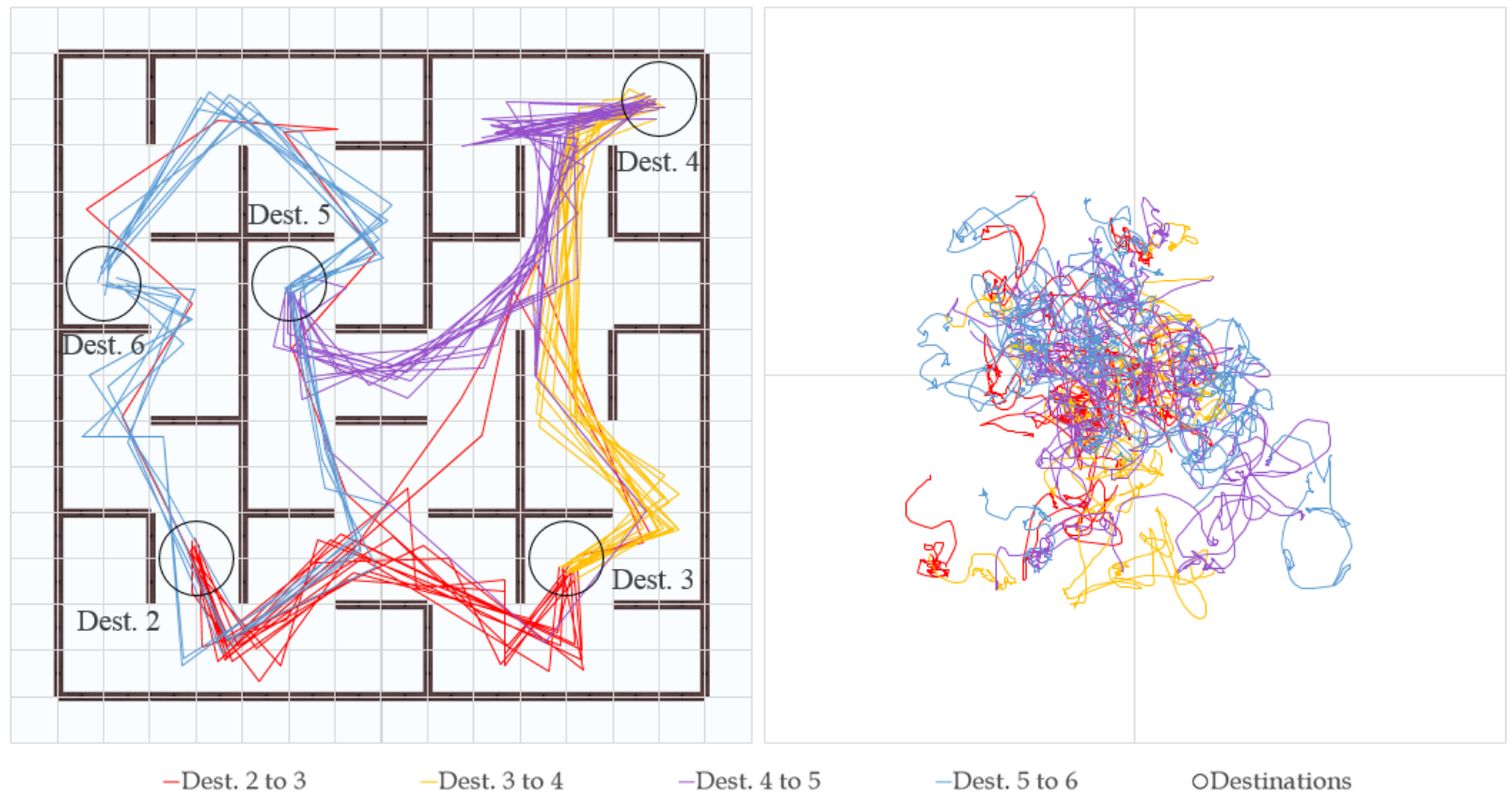

Figure 6-9: Point \& teleport technique's projected virtual (left) and real (right) user paths for the direction specification modification of the point \& teleport experiment.

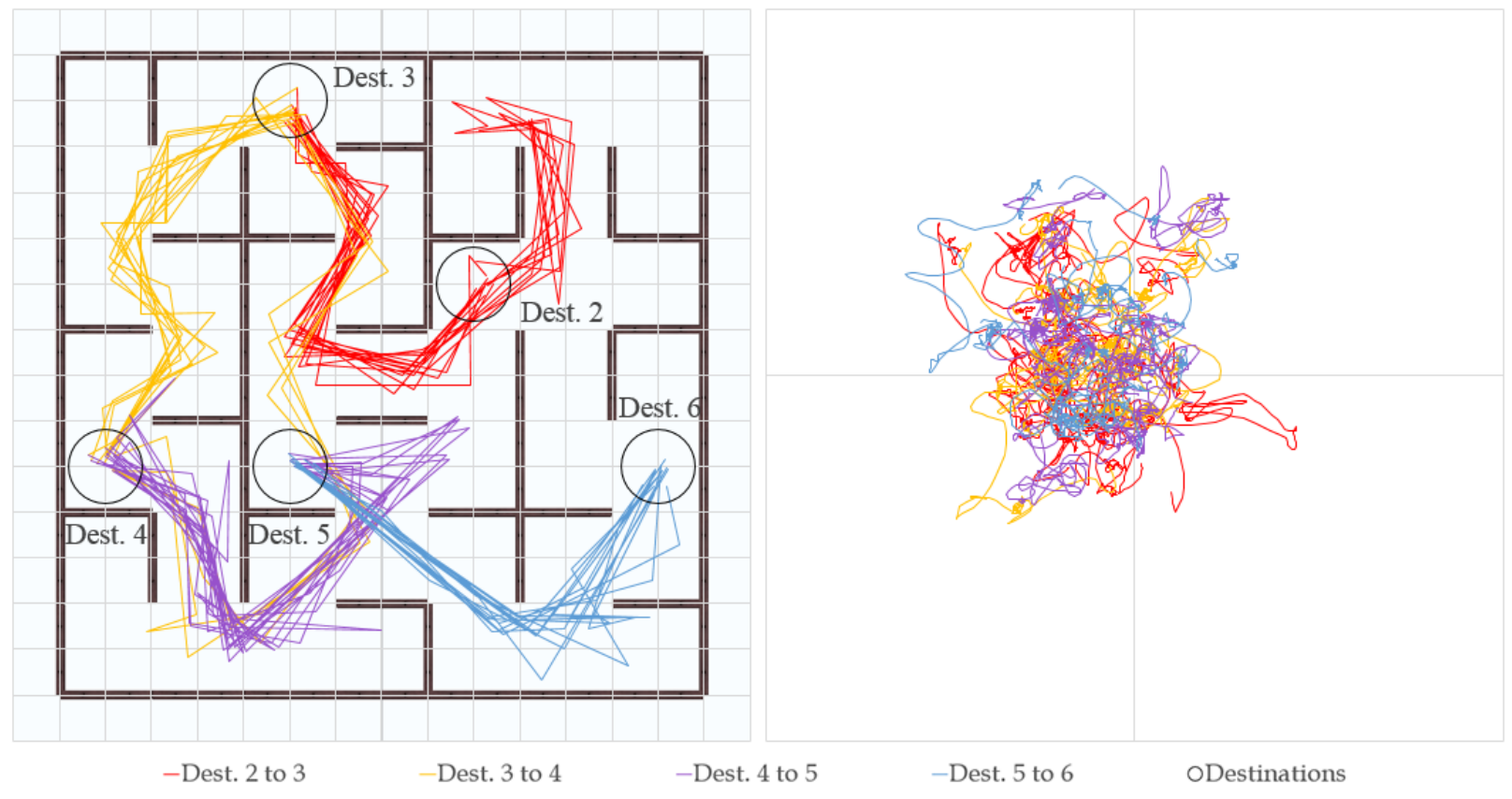

Figure 6-10: Point \& teleport with direction specification technique projected virtual (left) and real (right) user paths for the direction specification modification of the point \& teleport experiment. 


\subsubsection{Participant Comments}

Many participants stated preference for P\&T over P\&T w/DS in their comments "I liked this method [P\&T]. It made navigating the maze relatively easy." "I think it is [P\&T] better than the first one [P\&T w/DS]. I am not very dizzy." "I like pointing and then I look for directions. I think I do it faster this way." "It was easier overall to reach my destination in this method [P\&T]." Only a few users made comments stating preference over P\&T w/DS such as "I liked the added control of choosing the direction. It made the method feel more efficient." A lot of users complained about feeling dizzy or disoriented with P\&T w/DS “Might be [P\&T w/DS] more confusing than before [P\&T]. Difficult to fully realize own orientation after teleporting." "A little bit dizziness for me [with P\&T w/DS]." "While I really liked this method [P\&T w/DS] over regular teleporting $[\mathrm{P} \& \mathrm{~T}]$, the directional controls were a bit touchy. Accordingly, I sometimes felt a little disoriented."

\subsection{Discussion}

Average time to reach the destination points was similar for the two techniques, rejecting Hypothesis1. In fact, it took more time to reach the destination points with the P\&T w/DS as opposed to what we expected. No difference was observed in the required effort to use the two techniques, although the P\&T w/DS required slightly more effort than the P\&T. The P\&T w/DS resulted in slightly higher motion sickness results. User preference results were similar between the two techniques, rejecting Hypothesis2. The P\&T w/DS was more difficult to operate and understand. We interpret this as the effect caused from merging the two components of moving and rotating into one in this 
modified version. In the original P\&T, users controlled the locomotion sequentially; first they moved to the desired location, then they turned to face wherever they wanted. The P\&T w/DS merged these components and induced more cognitive load in users.

There were a lot of complaints about the feeling of being disoriented and dizziness that the P\&T w/DS caused whereas there was none for the P\&T. Since the P\&T w/DS changed the environment's orientation instantly, this may have caused disorientation in the users. Hence, we do not recommend using the additional direction specification feature and we recommend keeping the P\&T in its simple form. As possible solutions to disorientation that would be caused by the instant change of the direction of the users, we recommend using in-game mini maps or making the user's previous position marked for a while after they teleported to somewhere else to help them maintain their sense of orientation. To sum up, although the additional direction specification feature was expected to enhance the P\&T locomotion technique, the experiment results indicated the opposite.

\subsection{Limitations}

The gesture design for the direction specification and the teleportation can be considered as a limitation for this user study. Since the users performed the direction specification by the rolling gesture and performed the teleportation by pointing with their hands, this may have affected the results. Other controlling mechanisms such as gamepads might have led to different results. 


\subsection{Summary, Conclusion and Future Work}

In this chapter, a modification of the point \& teleport locomotion technique was described and evaluated. This modification included the direction specification. A virtual reality experiment that took place in a maze environment was designed. A user study was performed with 16 neurotypical individuals to compare the point \& teleport with its modified version of point \& teleport with direction specification. The results of the user study indicated that the additional direction specification component degraded the user experience. Thus, point \& teleport is recommended to be kept in its simple form in which the teleported orientation of the users would match what they saw before the teleportation.

Areas for future work may include evaluating the different versions of the point and teleport locomotion technique such as triggering with controller or other gestures. Evaluating the usability of the point \& teleport for multiplayer virtual reality experiences would be another area for exploration. 


\section{CHAPTER 7: CONCLUSIONS AND FUTURE WORK}

In this dissertation, we presented an extensive evaluation of locomotion techniques for room scale tracked virtual reality. Three immersive virtual reality experiments were developed and user studies were performed with neurotypical individuals and individuals with high functioning ASD. Results of these user studies were reported and discussed with the motivation of providing insight into the future virtual reality studies targeting neurotypical individuals and individuals with ASD.

The first experiment consisted of the evaluation of an advanced VR system for vocational rehabilitation of individuals with disabilities by 10 neurotypical individuals and 9 individuals with high functioning ASD in terms of locomotion, interaction, and display methods. For neurotypical individuals, results indicated that the real walking was better than the walk-in-place; the tangible interaction was better than the haptic device, touch \& snap and touch screen; and the head mounted display was better than the curtain screen. For individuals with high functioning ASD, the real walking was found out to be better than the walk-in-place; the touch screen was found out to be better than the haptic device, tangible interaction and touch \& snap; and the curtain screen was found to be better than the head mounted display.

In the second experiment eight locomotion techniques were evaluated in an immersive virtual reality experiment for a room scale tracked area $(2 \mathrm{~m}$ by $2 \mathrm{~m})$ : redirected 
walking, walk-in-place, stepper machine, point \& teleport, joystick, trackball, hand flapping and flying. A user study was performed with 16 neurotypical participants and 15 participants with high functioning ASD. For the neurotypical users, the results of the user study indicated that the point \& teleport, joystick and redirected walking were suitable virtual reality locomotion techniques for room scale tracked areas whereas the hand flapping and flying were not found to be suitable locomotion techniques. For individuals with high functioning ASD the results of the user study indicated that the point \& teleport, joystick and walk-in-place turned out to be suitable virtual reality locomotion techniques for room scale tracked areas. On the contrary, the hand flapping and flying turned out to be not suitable locomotion techniques for individuals with high functioning ASD.

The third experiment included the evaluation of the point \& teleport technique's direction specification modification. A user study was performed with 16 neurotypical individuals. The results indicated that the additional direction specification feature did not improve the user experience, thus found to be unnecessary.

Future work directions consist of evaluating the modifications of the virtual reality locomotion techniques that were found to be working well for room scale tracked areas in this dissertation, such as the modifications of the point \& teleport, the controllers, and the redirected walking. Evaluating the virtual reality locomotion techniques with task designs of different complexities may be another area for exploration. Finally, the locomotion techniques may be evaluated with more population groups, i.e. children, 
elderly, individuals with low functioning ASD and individuals with medium functioning ASD. 


\section{REFERENCES}

1. First, M.B., Diagnostic and statistical manual of mental disorders. DSM IV-4th edition. APA, 1994: p. 1994.

2. Janzen, J.E. and Zenko, C.B., Understanding the nature of autism: A guide to the autism spectrum disorders. 2003: Therapy Skill Builders San Antonio, TX.

3. Burdea, G.C. and Coiffet, P., Virtual Reality Technology. 2003: John Wiley \& Sons, Inc. 444 .

4. Sutherland, I.E., A head-mounted three dimensional display, in Proceedings of the December 9-11, 1968, fall joint computer conference, part I. 1968, ACM: San Francisco, California. p. 757-764.

5. Oculus. Rift. [cited 6/13/2016]; Available from: https://www.oculus.com/.

6. Microsoft. Kinect. 6/13/2016]; Available from: https:// developer.microsoft.com/en-us/windows/kinect.

7. Witmer, B.G., et al., Virtual spaces and real world places: transfer of route knowledge. International Journal of Human-Computer Studies, 1996. 45(4): p. 413-428.

8. Finkelstein, S., et al. Evaluation of the exertion and motivation factors of a virtual reality exercise game for children with autism. in Virtual and Augmented Assistive Technology (VAAT), 2013 1st Workshop on. 2013.

9. Lorenzo, G., Pomares, J., and Lledó, A., Inclusion of immersive virtual learning environments and visual control systems to support the learning of students with Asperger syndrome. Computers \& Education, 2013. 62: p. 88-101.

10. Bernardes, M., et al. A serious game with virtual reality for travel training with Autism Spectrum Disorder. in Virtual Rehabilitation Proceedings (ICVR), 2015 International Conference on. 2015.

11. Kandalaft, M.R., et al., Virtual Reality Social Cognition Training for Young Adults with High-Functioning Autism. Journal of Autism and Developmental Disorders, 2012. 43(1): p. 34-44. 
12. Chen, S.S.A. and Bernard-Opitz, V., Comparison of personal and computer-assisted instruction for children with autism. Mental retardation, 1993. 31(6): p. 368.

13. Putnam, C. and Chong, L. Software and technologies designed for people with autism: what do users want? in Proceedings of the 10th international ACM SIGACCESS conference on Computers and accessibility. 2008. ACM.

14. Bowman, D.A., et al., 3D User Interfaces: Theory and Practice. 2004: Addison Wesley Longman Publishing Co., Inc.

15. Hale, K.S. and Stanney, K.M., Handbook of virtual environments: Design, implementation, and applications. 2014: CRC Press.

16. Council, N.R., Virtual Reality: Scientific and Technological Challenges, ed. I.D. Nathaniel and S.M. Anne. 1994: National Academy Press. 542.

17. Matthies, D.J.C., InEar BioFeedController: a headset for hands-free and eyes-free interaction with mobile devices, in CHI '13 Extended Abstracts on Human Factors in Computing Systems. 2013, ACM: Paris, France. p. 1293-1298.

18. Goldsmith, T.R. and LeBlanc, L.A., Use of technology in interventions for children with autism. Journal of Early and Intensive Behavior Intervention, 2004. 1(2): p. 166.

19. Razzaque, S., Kohn, Z., and Whitton, M.C., Redirected Walking. 2001, University of North Carolina at Chapel Hill.

20. Feasel, J., Whitton, M.C., and Wendt, J.D. LLCM-WIP: Low-Latency, ContinuousMotion Walking-in-Place. in 3D User Interfaces, 2008. 3DUI 2008. IEEE Symposium on. 2008.

21. Hourcade, J.P., et al. Evaluation of tablet apps to encourage social interaction in children with autism spectrum disorders. in Proceedings of the SIGCHI Conference on Human Factors in Computing Systems. 2013. ACM.

22. Madsen, M., et al. Lessons from participatory design with adolescents on the autism spectrum. in CHI'09 Extended Abstracts on Human Factors in Computing Systems. 2009. ACM.

23. Harrold, N., Tan, C.T., and Rosser, D. Towards an expression recognition game to assist the emotional development of children with autism spectrum disorders. in Proceedings of the Workshop at SIGGRAPH Asia. 2012. ACM. 
24. Kientz, J.A., et al., Pervasive computing and autism: Assisting caregivers of children with special needs. IEEE Pervasive Computing, 2007. 6(1): p. 28-35.

25. Bartoli, L., et al. Exploring motion-based touchless games for autistic children's learning. in Proceedings of the 12th International Conference on Interaction Design and Children. 2013. ACM.

26. Garzotto, F., et al. Motion-based touchless interaction for ASD children: a case study. in Proceedings of the 2014 International Working Conference on Advanced Visual Interfaces. 2014. ACM.

27. Parés, N., et al., Starting research in interaction design with visuals for low-functioning children in the autistic spectrum: A protocol. Cyberpsychology \& Behavior, 2006. 9(2): p. 218-223.

28. Josman, N., et al., Effectiveness of virtual reality for teaching street-crossing skills to children and adolescents with autism. International Journal on Disability and Human Development, 2008. 7(1): p. 49-56.

29. Wade, J., et al. A virtual reality driving environment for training safe gaze patterns: Application in individuals with ASD. in International Conference on Universal Access in Human-Computer Interaction. 2015. Springer.

30. Bauminger-Zviely, N., et al., Increasing social engagement in children with highfunctioning autism spectrum disorder using collaborative technologies in the school environment. Autism, 2013. 17(3): p. 317-339.

31. Sitdhisanguan, K., et al., Using tangible user interfaces in computer-based training systems for low-functioning autistic children. Personal and Ubiquitous Computing, 2012. 16(2): p. 143-155.

32. Mei, C., Mason, L., and Quarles, J. Usability issues with 3D user interfaces for adolescents with high functioning autism. in Proceedings of the 16th international ACM SIGACCESS conference on Computers \& accessibility. 2014. ACM.

33. Usoh, M., et al., Walking > walking-in-place > flying, in virtual environments, in Proceedings of the 26th annual conference on Computer graphics and interactive techniques. 1999, ACM Press/ Addison-Wesley Publishing Co. p. 359-364.

34. Ruddle, R.A. and Lessels, S., The benefits of using a walking interface to navigate virtual environments. ACM Trans. Comput.-Hum. Interact., 2009. 16(1): p. 1-18. 
35. Ruddle, R.A., et al., Walking improves your cognitive map in environments that are large-scale and large in extent. ACM Trans. Comput.-Hum. Interact., 2011. 18(2): p. $1-20$.

36. Marsh, W., et al., Cognitive Demands of Semi-Natural Virtual Locomotion. Presence, 2013. 22(3): p. 216-234.

37. Whitton, M.C., et al. Comparing VE locomotion interfaces. in Virtual Reality, 2005. Proceedings. VR 2005. IEEE. 2005.

38. Lappe, M., Bremmer, F., and van den Berg, A.V., Perception of self-motion from visual flow. Trends in Cognitive Sciences, 1999. 3(9): p. 329-336.

39. Interrante, V., Ries, B., and Anderson, L. Distance Perception in Immersive Virtual Environments, Revisited. in Virtual Reality Conference, 2006. 2006.

40. Steinicke, F., et al., Transitional environments enhance distance perception in immersive virtual reality systems, in Proceedings of the 6th Symposium on Applied Perception in Graphics and Visualization. 2009, ACM: Chania, Crete, Greece. p. 1926.

41. Frenz, H., et al., Estimation of travel distance from visual motion in virtual environments. ACM Trans. Appl. Percept., 2007. 4(1): p. 3.

42. Banton, T., et al., The perception of walking speed in a virtual environment. Presence: Teleoper. Virtual Environ., 2005. 14(4): p. 394-406.

43. Steinicke, F., et al., Estimation of Detection Thresholds for Redirected Walking Techniques. IEEE Transactions on Visualization and Computer Graphics, 2010. 16(1): p. 17-27.

44. Suma, E.A., et al. A taxonomy for deploying redirection techniques in immersive virtual environments. in Virtual Reality Short Papers and Posters (VRW), 2012 IEEE. 2012.

45. Steinicke, F., et al., Real Walking through Virtual Environments by Redirection Techniques. JVRB - Journal of Virtual Reality and Broadcasting, 2009. 6(2009)(2).

46. Steinicke, F., et al., Analyses of human sensitivity to redirected walking, in Proceedings of the 2008 ACM symposium on Virtual reality software and technology. 2008, ACM: Bordeaux, France. p. 149-156. 
47. Bruder, G., et al., Reorientation during body turns, in Proceedings of the 15th Joint virtual reality Eurographics conference on Virtual Environments. 2009, Eurographics Association: Lyon, France. p. 145-152.

48. Jerald, J., et al., Sensitivity to scene motion for phases of head yaws, in Proceedings of the 5th symposium on Applied perception in graphics and visualization. 2008, ACM: Los Angeles, California. p. 155-162.

49. Bruder, G., Lubas, P., and Steinicke, F., Cognitive Resource Demands of Redirected Walking. Visualization and Computer Graphics, IEEE Transactions on, 2015. 21(4): p. 539-544.

50. Neth, C.T., et al. Velocity-dependent dynamic curvature gain for redirected walking. in Virtual Reality Conference (VR), 2011 IEEE. 2011.

51. Zhang, R. and Kuhl, S.A., Human sensitivity to dynamic rotation gains in headmounted displays, in Proceedings of the ACM Symposium on Applied Perception. 2013, ACM: Dublin, Ireland. p. 71-74.

52. Interrante, V., Ries, B., and Anderson, L. Seven League Boots: A New Metaphor for Augmented Locomotion through Moderately Large Scale Immersive Virtual Environments. in 3D User Interfaces, 2007. 3DUI '07. IEEE Symposium on. 2007.

53. Azmandian, M., et al. An enhanced steering algorithm for redirected walking in virtual environments. in Virtual Reality (VR), 2014 iEEE. 2014.

54. Hodgson, E., Bachmann, E., and Waller, D., Redirected walking to explore virtual environments: Assessing the potential for spatial interference. ACM Trans. Appl. Percept., 2008. 8(4): p. 1-22.

55. Bruder, G., et al., Redirecting Walking and Driving for Natural Navigation in Immersive Virtual Environments. IEEE Transactions on Visualization and Computer Graphics, 2012. 18(4): p. 538-545.

56. Williams, B., et al., Exploring large virtual environments with an HMD when physical space is limited, in Proceedings of the 4th symposium on Applied perception in graphics and visualization. 2007, ACM: Tubingen, Germany. p. 41-48.

57. Peck, T.C., Whitton, M.C., and Fuchs, H. Evaluation of Reorientation Techniques for Walking in Large Virtual Environments. in Virtual Reality Conference, 2008. VR '08. IEEE. 2008. 
58. Kohli, L., et al., Combining passive haptics with redirected walking, in Proceedings of the 2005 international conference on Augmented tele-existence. 2005, ACM: Christchurch, New Zealand. p. 253-254.

59. Peck, T.C., Fuchs, H., and Whitton, M.C. Improved Redirection with Distractors: A large-scale-real-walking locomotion interface and its effect on navigation in virtual environments. in Virtual Reality Conference (VR), 2010 IEEE. 2010.

60. Grechkin, T., et al. Towards context-sensitive reorientation for real walking in virtual reality. in Virtual Reality (VR), 2015 IEEE. 2015.

61. Steinicke, F., et al. Taxonomy and Implementation of Redirection Techniques for Ubiquitous Passive Haptic Feedback. in Cyberworlds, 2008 International Conference on. 2008.

62. Insko, B.E., Passive haptics significantly enhances virtual environments. 2001, The University of North Carolina at Chapel Hill. p. 100.

63. Bruder, G., Steinicke, F., and Hinrichs, K.H. Arch-Explore: A natural user interface for immersive architectural walkthroughs. in 3D User Interfaces, 2009. 3DUI 2009. IEEE Symposium on. 2009.

64. Suma, E.A., et al. Leveraging change blindness for redirection in virtual environments. in Virtual Reality Conference (VR), 2011 IEEE. 2011.

65. Suma, E.A., et al., Impossible Spaces: Maximizing Natural Walking in Virtual Environments with Self-Overlapping Architecture. Visualization and Computer Graphics, IEEE Transactions on, 2012. 18(4): p. 555-564.

66. Vasylevska, K., et al. Flexible spaces: Dynamic layout generation for infinite walking in virtual environments. in 3D User Interfaces (3DUI), 2013 IEEE Symposium on. 2013.

67. Freitag, S., Rausch, D., and Kuhlen, T. Reorientation in virtual environments using interactive portals. in 3D User Interfaces (3DUI), 2014 IEEE Symposium on. 2014.

68. Steinicke, F., et al., Human Walking in Virtual Environments: Perception, Technology, and Applications. 2013: Springer Publishing Company, Incorporated. 460.

69. Slater, M., Usoh, M., and Steed, A., Taking steps: the influence of a walking technique on presence in virtual reality. ACM Trans. Comput.-Hum. Interact., 1995. 2(3): p. 201-219. 
70. Slater, M., Usoh, M., and Steed, A., Steps and ladders in virtual reality, in Proceedings of the conference on Virtual reality software and technology. 1994, World Scientific Publishing Co., Inc.: Singapore, Singapore. p. 45-54.

71. Slater, M., Steed, A., and Usoh, M., The virtual treadmill: a naturalistic metaphor for navigation in immersive virtual environments, in Selected papers of the Eurographics workshops on Virtual environments '95. 1995, Springer-Verlag: Barcelona, Spain. p. 135-148.

72. Whitton, M.C. and Peck, T.C., Stepping-Driven Locomotion Interfaces, in Human Walking in Virtual Environments: Perception, Technology, and Applications, F. Steinicke, et al., Editors. 2013, Springer New York: New York, NY. p. 241-262.

73. Durgin, F.H., Gigone, K., and Scott, R., Perception of Visual Speed While Moving. Journal of Experimental Psychology: Human Perception and Performance, 2005. 31(2): p. 339-353.

74. Durgin, F.H., When Walking Makes Perception Better. Current Directions in Psychological Science, 2009. 18(1): p. 43-47.

75. Nilsson, N.C., Serafin, S., and Nordahl, R., Establishing the Range of Perceptually Natural Visual Walking Speeds for Virtual Walking-In-Place Locomotion. Visualization and Computer Graphics, IEEE Transactions on, 2014. 20(4): p. 569578.

76. Wendt, J.D., Whitton, M.C., and Brooks, F.P., Jr. GUD WIP: Gait-UnderstandingDriven Walking-In-Place. in Virtual Reality Conference (VR), 2010 IEEE. 2010.

77. Kim, J., Gracanin, D., and Quek, F. Sensor-fusion walking-in-place interaction technique using mobile devices. in Virtual Reality Short Papers and Posters (VRW), 2012 IEEE. 2012.

78. Nilsson, N.C., et al. Tapping-In-Place: Increasing the naturalness of immersive walking-in-place locomotion through novel gestural input. in 3D User Interfaces (3DUI), 2013 IEEE Symposium on. 2013.

79. Nilsson, N.C., Serafin, S., and Nordahl, R., The Perceived Naturalness of Virtual Locomotion Methods Devoid of Explicit Leg Movements, in Proceedings of Motion on Games. 2013, ACM: Dublin 2, Ireland. p. 155-164.

80. Templeman, J., Denbrook, P., and Sibert, L., Virtual Locomotion: Walking in Place through Virtual Environments. Presence, 1999. 8(6): p. 598-617. 
81. Williams, B., et al., Evaluation of walking in place on a Wii balance board to explore a virtual environment. ACM Trans. Appl. Percept., 2011. 8(3): p. 1-14.

82. Zielinski, D.J., McMahan, R.P., and Brady, R.B. Shadow walking: An unencumbered locomotion technique for systems with under-floor projection. in Virtual Reality Conference (VR), 2011 IEEE. 2011.

83. Razzaque, S., et al., Redirected walking in place, in Proceedings of the workshop on Virtual environments 2002. 2002, Eurographics Association: Barcelona, Spain. p. 123-130.

84. Terziman, L., et al., Shake-your-head: revisiting walking-in-place for desktop virtual reality, in Proceedings of the 17th ACM Symposium on Virtual Reality Software and Technology. 2010, ACM: Hong Kong. p. 27-34.

85. Ware, C. and Osborne, S., Exploration and virtual camera control in virtual three dimensional environments. SIGGRAPH Comput. Graph., 1990. 24(2): p. 175-183.

86. Robinett, W. and Holloway, R., Implementation of flying, scaling and grabbing in virtual worlds, in Proceedings of the 1992 symposium on Interactive 3D graphics. 1992, ACM: Cambridge, Massachusetts, USA. p. 189-192.

87. Bowman, D.A., Koller, D., and Hodges, L.F. Travel in immersive virtual environments: an evaluation of viewpoint motion control techniques. in Virtual Reality Annual International Symposium, 1997., IEEE 1997. 1997.

88. Valkov, D., et al. A multi-touch enabled human-transporter metaphor for virtual 3D traveling. in 3D User Interfaces (3DUI), 2010 IEEE Symposium on. 2010.

89. Harris, A., et al., Human joystick: Wii-leaning to translate in large virtual environments, in Proceedings of the 13th ACM SIGGRAPH International Conference on Virtual-Reality Continuum and its Applications in Industry. 2014, ACM: Shenzhen, China. p. 231-234.

90. Cirio, G., et al., The magic barrier tape: a novel metaphor for infinite navigation in virtual worlds with a restricted walking workspace, in Proceedings of the 16th ACM Symposium on Virtual Reality Software and Technology. 2009, ACM: Kyoto, Japan. p. 155-162.

91. Guy, E., et al. LazyNav: 3D ground navigation with non-critical body parts. in 3D User Interfaces (3DUI), 2015 IEEE Symposium on. 2015. 
92. Bolte, B., Bruder, G., and Steinicke, F., The Jumper Metaphor: An Effective Navigation Technique for Immersive Display Setups, in Virtual Reality International Conference (VRIC). 2011. p. 1-7.

93. Frederick P. Brooks, J., Walkthrough-a dynamic graphics system for simulating virtual buildings, in Proceedings of the 1986 workshop on Interactive 3D graphics. 1987, ACM: Chapel Hill, North Carolina, USA. p. 9-21.

94. Darken, R.P., Cockayne, W.R., and Carmein, D., The omni-directional treadmill: a locomotion device for virtual worlds, in Proceedings of the 10th annual ACM symposium on User interface software and technology. 1997, ACM: Banff, Alberta, Canada. p. 213-221.

95. Iwata, H. Walking about virtual environments on an infinite floor. in Virtual Reality, 1999. Proceedings., IEEE. 1999.

96. Iwata, H., The Torus Treadmill: realizing locomotion in VEs. Computer Graphics and Applications, IEEE, 1999. 19(6): p. 30-35.

97. Schwaiger, M., Thummel, T., and Ulbrich, H. Cyberwalk: An advanced prototype of a belt array platform. in Haptic, Audio and Visual Environments and Games, 2007. HAVE 2007. IEEE International Workshop on. 2007.

98. Souman, J.L., et al., CyberWalk: Enabling unconstrained omnidirectional walking through virtual environments. ACM Trans. Appl. Percept., 2008. 8(4): p. 1-22.

99. Jiung-Yao, H., An omnidirectional stroll-based virtual reality interface and its application on overhead crane training. Multimedia, IEEE Transactions on, 2003. 5(1): p. 39-51.

100. Suryajaya, M., Lambert, T., and Fowler, C., Camera-based OBDP locomotion system, in Proceedings of the 16th ACM Symposium on Virtual Reality Software and Technology. 2009, ACM: Kyoto, Japan. p. 31-34.

101. De Luca, A., et al., Motion Control of the CyberCarpet Platform. Control Systems Technology, IEEE Transactions on, 2013. 21(2): p. 410-427.

102. Swapp, D., Williams, J., and Steed, A. The implementation of a novel walking interface within an immersive display. in 3D User Interfaces (3DUI), 2010 IEEE Symposium on. 2010. 
103. Cakmak, T. and Hager, H., Cyberith virtualizer: a locomotion device for virtual reality, in ACM SIGGRAPH 2014 Emerging Technologies. 2014, ACM: Vancouver, Canada. p. 1-1.

104. Marchal, M., Pettre, J., and Lecuyer, A. Joyman: A human-scale joystick for navigating in virtual worlds. in 3D User Interfaces (3DUI), 2011 IEEE Symposium on. 2011.

105. Bouguila, L., et al., Walking-pad: a step-in-place locomotion interface for virtual environments, in Proceedings of the 6th international conference on Multimodal interfaces. 2004, ACM: State College, PA, USA. p. 77-81.

106. Mi, F., et al. Haptic ChairIO: A system to study the effect of wind and floor vibration feedback on spatial orientation in VEs. in 3D User Interfaces (3DUI), 2015 IEEE Symposium on. 2015.

107. Matthies, D.J.C., et al., VR-Stepper: A Do-It-Yourself Game Interface For Locomotion In Virtual Environments. CoRR, 2014. abs/1407.3948.

108. Nilsson, N.C., Serafin, S., and Nordahl, R., Comparisons of Two Commercial and Two Low-cost Interfaces for Virtual Skiing. PsychNology Journal, 2015. 13(1): p. 5774.

109. Medina, E., Fruland, R., and Weghorst, S., Virtusphere: Walking in a Human Size $V R$ "Hamster Ball". Proceedings of the Human Factors and Ergonomics Society Annual Meeting, 2008. 52(27): p. 2102-2106.

110. Iwata, H., Yano, H., and Tomiyoshi, M., String walker, in ACM SIGGRAPH 2007 emerging technologies. 2007, ACM: San Diego, California. p. 20.

111. Darken, R.P. and Sibert, J.L., Navigating large virtual spaces. International Journal of Human-Computer Interaction, 1996. 8(1): p. 49-71.

112. Zhixin, Y. and Lindeman, R.W. A multi-touch finger gesture based low-fatigue VR travel framework. in 3D User Interfaces (3DUI), 2015 IEEE Symposium on. 2015.

113. Cirio, G., et al., Kinematic Evaluation of Virtual Walking Trajectories. Visualization and Computer Graphics, IEEE Transactions on, 2013. 19(4): p. 671-680.

114. Grechkin, T.Y., Plumert, J.M., and Kearney, J.K., Dynamic Affordances in Embodied Interactive Systems: The Role of Display and Mode of Locomotion. Visualization and Computer Graphics, IEEE Transactions on, 2014. 20(4): p. 596-605. 
115. Peck, T.C., Fuchs, H., and Whitton, M.C. An evaluation of navigational ability comparing Redirected Free Exploration with Distractors to Walking-in-Place and joystick locomotio interfaces. in Virtual Reality Conference (VR), 2011 IEEE. 2011.

116. Peck, T.C., Fuchs, H., and Whitton, M.C., The Design and Evaluation of a LargeScale Real-Walking Locomotion Interface. IEEE Transactions on Visualization and Computer Graphics, 2012. 18(7): p. 1053-1067.

117. Nabiyouni, M., et al. Comparing the performance of natural, semi-natural, and nonnatural locomotion techniques in virtual reality. in 3D User Interfaces (3DUI), 2015 IEEE Symposium on. 2015.

118. Riecke, B.E., et al., Do We Need to Walk for Effective Virtual Reality Navigation? Physical Rotations Alone May Suffice, in Spatial Cognition VII: International Conference, Spatial Cognition 2010, Mt. Hood/Portland, OR, USA, August 15-19, 2010. Proceedings, C. Hölscher, et al., Editors. 2010, Springer Berlin Heidelberg: Berlin, Heidelberg. p. 234-247.

119. Ruddle, R.A. and Lessels, S., For Efficient Navigational Search, Humans Require Full Physical Movement, but Not a Rich Visual Scene. Psychological Science, 2006. 17(6): p. $460-465$.

120. Pakkanen, T. and Raisamo, R., Appropriateness of foot interaction for non-accurate spatial tasks, in CHI '04 Extended Abstracts on Human Factors in Computing Systems. 2004, ACM: Vienna, Austria. p. 1123-1126.

121. Insook, C. and Ricci, C. Foot-mounted gesture detection and its application in virtual environments. in Systems, Man, and Cybernetics, 1997. Computational Cybernetics and Simulation., 1997 IEEE International Conference on. 1997.

122. Barrera, S., et al. Real time detection interface for walking on CAVE. in Computer Graphics International, 2003. Proceedings. 2003.

123. Barrera, S., Takahashi, H., and Nakajima, M. Hands-free navigation methods for moving through a virtual landscape walking interface virtual reality input devices. in Computer Graphics International, 2004. Proceedings. 2004.

124. Matthies, D.J.C., et al., ShoeSoleSense: proof of concept for a wearable foot interface for virtual and real environments, in Proceedings of the 19th ACM Symposium on Virtual Reality Software and Technology. 2013, ACM: Singapore. p. 93-96. 
125. Scott, J., et al., Sensing foot gestures from the pocket, in Proceedings of the 23nd annual ACM symposium on User interface software and technology. 2010, ACM: New York, New York, USA. p. 199-208.

126. Higuchi, H. and Nojima, T., Shoe-shaped i/o interface, in Adjunct proceedings of the 23nd annual ACM symposium on User interface software and technology. 2010, ACM: New York, New York, USA. p. 423-424.

127. Iwata, H., Yano, H., and Tomioka, H., Powered shoes, in ACM SIGGRAPH 2006 Emerging technologies. 2006, ACM: Boston, Massachusetts. p. 28.

128. Iwata, H., et al., CirculaFloor [locomotion interface]. Computer Graphics and Applications, IEEE, 2005. 25(1): p. 64-67.

129. Suma, E.A., et al., Evaluation of the Cognitive Effects of Travel Technique in Complex Real and Virtual Environments. Visualization and Computer Graphics, IEEE Transactions on, 2010. 16(4): p. 690-702.

130. Zanbaka, C., et al. Effects of travel technique on cognition in virtual environments. in Virtual Reality, 2004. Proceedings. IEEE. 2004.

131. Zanbaka, C.A., et al., Comparison of Path Visualizations and Cognitive Measures Relative to Travel Technique in a Virtual Environment. IEEE Transactions on Visualization and Computer Graphics, 2005. 11(6): p. 694-705.

132. Finkelstein, S., et al., Astrojumper: motivating children with autism to exercise using a $V R$ game, in CHI '10 Extended Abstracts on Human Factors in Computing Systems. 2010, ACM: Atlanta, Georgia, USA. p. 4189-4194.

133. Yiyu, C., et al., Design and Development of a Virtual Dolphinarium for Children With Autism. Neural Systems and Rehabilitation Engineering, IEEE Transactions on, 2013. 21(2): p. 208-217.

134. Self, T., et al., A virtual approach to teaching safety skills to children with autism spectrum disorder. Topics in Language Disorders, 2007. 27(3): p. 242-253.

135. Strickland, D.C., et al., An evolution of virtual reality training designs for children with autism and fetal alcohol spectrum disorders. Topics in language disorders, 2007. 27(3): p. 226.

136. Mitchell, P., Parsons, S., and Leonard, A., Using virtual environments for teaching social understanding to 6 adolescents with autistic spectrum disorders. Journal of autism and developmental disorders, 2007. 37(3): p. 589-600. 
137. Ward, K., Teaching Students with Autism Spectrum Disorders. Vol. Book 9 in the Programming for Students with Special Needs series. 2003: Alberta Learning.

138. Developmental, D.M.N.S.Y. and Investigators, P., Prevalence of autism spectrum disorder among children aged 8 years-autism and developmental disabilities monitoring network, 11 sites, United States, 2010. Morbidity and mortality weekly report. Surveillance summaries (Washington, DC: 2002), 2014. 63(2): p. 1.

139. Boyle, C.A., et al., Trends in the prevalence of developmental disabilities in US children, 1997-2008. Pediatrics, 2011. 127(6): p. 1034-1042.

140. Hendricks, D., Employment and adults with autism spectrum disorders: Challenges and strategies for success. Journal of Vocational Rehabilitation, 2010. 32(2): p. 125-134.

141. Barry Issenberg, S., et al., Features and uses of high-fidelity medical simulations that lead to effective learning: a BEME systematic review. Medical teacher, 2005. 27(1): p. 10-28.

142. Hubal, R.C. and Frank, G.A., Interactive training applications using responsive virtual human technology. children, 2001. 21: p. 25.

143. Fleming, M., et al., Virtual reality skills training for health care professionals in alcohol screening and brief intervention. The Journal of the American Board of Family Medicine, 2009. 22(4): p. 387-398.

144. Smith, M.J., et al., Virtual reality job interview training in adults with autism spectrum disorder. Journal of autism and developmental disorders, 2014. 44(10): p. 24502463.

145. Unity. Game Engine. 6/13/2016]; Available from: https://unity3d.com/.

146. MiddleVR. Middleware. 6/13/2016]; Available from: http://www.middlevr.com/.

147. OptiTrack. Motion capture systems. 6/13/2016]; Available from: http://optitrack.com/.

148. Rosenberg, M. and Vance, J.M. Virtual Hand Representations to Support Natural Interaction in Immersive Environments. in ASME 2013 International Design Engineering Technical Conferences and Computers and Information in Engineering Conference. 2013. American Society of Mechanical Engineers. 
149. Sensable. Phantom Omni. 6/13/2016]; Available from: http://www.dentsable.com/haptic-phantom-omni.htm.

150. Likert, R., A technique for the measurement of attitudes. Archives of psychology, 1932.

151. Logitech. Joystick. 6/13/2016]; Available from: http://gaming.logitech.com/enus/product/extreme-3d-pro-joystick.

152. Dodd, S., Understanding autism. 2005, Marrickville, N.S.W: Elsevier Australia.

153. Kensington. Trackball. 6/13/2016]; Available from: http://www.kensington.com/us/us/4493/k64325/expert-mousespanclassregsymspan-wired-trackball.

154. Lessels, S. and Ruddle, R.A., Movement around real and virtual cluttered environments. Presence: Teleoperators and Virtual Environments, 2005. 14(5): p. 580-596.

155. Loewenthal, K.M., An introduction to psychological tests and scales. 2001: Psychology Press.

156. Lawson, B.D., et al., Signs and symptoms of human syndromes associated with synthetic experiences. Handbook of virtual environments: Design, implementation, and applications, 2002: p. 589-618.

157. Witmer, B.G. and Singer, M.J., Measuring Presence in Virtual Environments: A Presence Questionnaire. Presence: Teleoper. Virtual Environ., 1998. 7(3): p. 225-240. 


\section{APPENDIX A: SUMMARY OF THE STATE-OF-THE-ART PREVIOUS STUDIES}

Table A-1: A summary of the key studies with generalized properties.

\begin{tabular}{|c|c|c|c|c|c|c|c|c|c|c|c|c|c|}
\hline $\begin{array}{l}\text { Taxonomy } \\
\text { Group }\end{array}$ & $\begin{array}{c}\text { Specific } \\
\text { Tool }\end{array}$ & $\begin{array}{l}\text { Motion } \\
\text { Tracking }\end{array}$ & $\begin{array}{l}\text { Part of } \\
\text { Body }\end{array}$ & $\begin{array}{l}\text { Motion } \\
\text { Type }\end{array}$ & Posture & $\begin{array}{l}\text { Rotation } \\
\text { Technique }\end{array}$ & $\begin{array}{l}\text { Locomotion } \\
\text { Direction }\end{array}$ & Speed & $\begin{array}{l}\text { Backward } \\
\text { Walking }\end{array}$ & $\begin{array}{l}\text { Proprio- } \\
\text { ceptive }\end{array}$ & Limitations & $\begin{array}{c}\text { Learning } \\
\text { Require- } \\
\text { ment }\end{array}$ & $\begin{array}{l}\text { Key } \\
\text { Works }\end{array}$ \\
\hline $\begin{array}{l}\text { Redirected } \\
\text { Walking }\end{array}$ & No & Yes & $\begin{array}{l}\text { Full } \\
\text { body }\end{array}$ & $\begin{array}{c}\text { Real } \\
\text { walking }\end{array}$ & Standing & Body & Walking & $\begin{array}{c}\text { User } \\
\text { defined }\end{array}$ & Yes & Yes & Tracking area & Low & $\begin{array}{c}\text { [19], [56], } \\
\text { [59] }\end{array}$ \\
\hline $\begin{array}{c}\text { Environment } \\
\text { Change }\end{array}$ & No & Yes & $\begin{array}{l}\text { Full } \\
\text { body }\end{array}$ & $\begin{array}{c}\text { Real } \\
\text { walking }\end{array}$ & Standing & Body & Walking & $\begin{array}{c}\text { User } \\
\text { defined }\end{array}$ & Yes & Yes & $\begin{array}{l}\text { Tracking area, } \\
\text { usually indoor }\end{array}$ & Low & $\begin{array}{c}\text { [63], [64], } \\
{[65]}\end{array}$ \\
\hline $\begin{array}{l}\text { Walking } \\
\text { in Place }\end{array}$ & No & Yes & $\begin{array}{l}\text { Full } \\
\text { body }\end{array}$ & $\begin{array}{l}\text { Walking } \\
\text { in place }\end{array}$ & Standing & Body & Gaze/Torso & $\begin{array}{c}\text { User } \\
\text { defined }\end{array}$ & No & Limited & $\begin{array}{l}\text { Latency, } \\
\text { computational } \\
\text { cost }\end{array}$ & Medium & $\begin{array}{c}\text { [20], [71], } \\
{[78]}\end{array}$ \\
\hline Flying & Optional & $\begin{array}{l}\text { Optional } \\
\text { (Head } \\
\text { tracking) }\end{array}$ & $\begin{array}{l}\text { Hand/ } \\
\text { Finger }\end{array}$ & Triggering & $\begin{array}{c}\text { Standing/ } \\
\text { Sitting }\end{array}$ & $\begin{array}{l}\text { Head/ } \\
\text { Body }\end{array}$ & Gaze/Point & Constant & No & No & $\begin{array}{l}\text { Lack of realism } \\
\text { and presence }\end{array}$ & Low & [85] \\
\hline Leaning & No & No & $\begin{array}{l}\text { Full } \\
\text { body }\end{array}$ & Leaning & Standing & $\begin{array}{l}\text { Head/ } \\
\text { Body }\end{array}$ & Torso & $\begin{array}{c}\text { User } \\
\text { defined } \\
\text { (limited) }\end{array}$ & No & No & $\begin{array}{l}\text { Lack of realism } \\
\text { and presence }\end{array}$ & Medium & [89] \\
\hline Walker & Yes & Partial & $\begin{array}{l}\text { Full } \\
\text { body }\end{array}$ & Walking & Standing & Body & Walking & $\begin{array}{c}\text { User } \\
\text { defined }\end{array}$ & Optional & Yes & $\begin{array}{l}\text { Difficult to build, } \\
\text { expensive, speed }\end{array}$ & Low & $\begin{array}{c}\text { [94], [99], } \\
\text { [108] }\end{array}$ \\
\hline
\end{tabular}


Table A-1 (Continued)

\begin{tabular}{|c|c|c|c|c|c|c|c|c|c|c|c|c|c|}
\hline $\begin{array}{l}\text { Taxonomy } \\
\text { Group }\end{array}$ & $\begin{array}{c}\text { Specific } \\
\text { Tool }\end{array}$ & $\begin{array}{c}\text { Motion } \\
\text { Tracking }\end{array}$ & $\begin{array}{l}\text { Part of } \\
\text { Body }\end{array}$ & $\begin{array}{c}\text { Motion } \\
\text { Type }\end{array}$ & Posture & $\begin{array}{l}\text { Rotation } \\
\text { Technique }\end{array}$ & $\begin{array}{l}\text { Locomotion } \\
\text { Direction }\end{array}$ & Speed & $\begin{array}{c}\text { Backward } \\
\text { Walking }\end{array}$ & $\begin{array}{l}\text { Proprio- } \\
\text { ceptive }\end{array}$ & Limitations & $\begin{array}{c}\text { Learning } \\
\text { Require- } \\
\text { ment }\end{array}$ & $\begin{array}{l}\text { Key } \\
\text { Works }\end{array}$ \\
\hline $\begin{array}{l}\text { Standard } \\
\text { Controller }\end{array}$ & Yes & No & $\begin{array}{l}\text { Hand/ } \\
\text { Finger }\end{array}$ & $\begin{array}{c}\text { Triggering } \\
\text { Pushing/ } \\
\text { Pulling }\end{array}$ & $\begin{array}{l}\text { Standing/ } \\
\text { Sitting }\end{array}$ & Controller & Controller & Constant & Yes & No & $\begin{array}{l}\text { Lack of realism } \\
\text { and presence }\end{array}$ & Low & [114], [116] \\
\hline Wearable & Yes & Partial & $\begin{array}{l}\text { Full } \\
\text { body }\end{array}$ & $\begin{array}{c}\text { Real } \\
\text { walking }\end{array}$ & Standing & Body & Walking & $\begin{array}{c}\text { User } \\
\text { defined }\end{array}$ & Optional & Yes & Speed, weight & Medium & $\begin{array}{c}{[120],[123]} \\
{[126]}\end{array}$ \\
\hline Robot & Yes & Partial & $\begin{array}{l}\text { Full } \\
\text { body }\end{array}$ & $\begin{array}{c}\text { Real } \\
\text { walking }\end{array}$ & Standing & Body & Walking & $\begin{array}{c}\text { User } \\
\text { defined } \\
\text { (limited) }\end{array}$ & Optional & Yes & Speed & High & [127] \\
\hline
\end{tabular}




\section{APPENDIX B: SUMMARY OF THE VIRTUAL REALITY APPLICATIONS FOR INDIVIDUALS WITH AUTISM}

Table B-1: A summary of virtual reality applications for individuals with autism, with properties regarding locomotion.

\begin{tabular}{|c|c|c|c|c|c|c|}
\hline Reference & Locomotion Related Task & $\begin{array}{c}\text { Navigation } \\
\text { Requirement }\end{array}$ & $\begin{array}{l}\text { Locomotion } \\
\text { Technique }\end{array}$ & $\begin{array}{l}\text { Locomotion } \\
\text { Tool }\end{array}$ & $\begin{array}{l}\text { Display } \\
\text { Device }\end{array}$ & Number of Participants \\
\hline$[131],[8]$ & Avoiding moving objects & Low & Limited real walking & $\begin{array}{c}\text { Motion tracking } \\
\text { (electromagnetic markers) }\end{array}$ & CAVE & 10 ASD (8-20 years old) \\
\hline [132] & $\begin{array}{l}\text { Performing hand gestures to } \\
\text { communicate with dolphins }\end{array}$ & Low & Limited real walking & $\begin{array}{c}\text { Motion tracking } \\
\text { (electromagnetic markers) }\end{array}$ & CAVE & 15 ASD (6-17 years old) \\
\hline [25] & $\begin{array}{l}\text { Avoiding moving balls, hitting } \\
\text { balls, swinging arms and legs to } \\
\text { throw virtual balls, creating body } \\
\text { postures }\end{array}$ & Low & Limited real walking & $\begin{array}{l}\text { Motion tracking } \\
\text { (Kinect) }\end{array}$ & TV & 5 ASD (10-12 years old) \\
\hline [26] & Creating body postures & Low & Limited real walking & $\begin{array}{l}\text { Motion tracking } \\
\text { (Kinect) }\end{array}$ & TV & 5 ASD (6-8 years old) \\
\hline [27] & $\begin{array}{l}\text { Interacting with particles using } \\
\text { body movements }\end{array}$ & Low & Limited real walking & Motion tracking & Projection & $90 \mathrm{ASD}$ \\
\hline [9] & $\begin{array}{l}\text { Fulfilling several tasks such as } \\
\text { picking up items and conversation }\end{array}$ & Low & Limited real walking & $\begin{array}{c}\text { Motion tracking } \\
\text { (Optical cameras \& Kinect) }\end{array}$ & CAVE & 20 ASD (children) \\
\hline [10] & Moving to reach destination points & Middle & Standard controller & Gamepad & $\begin{array}{c}\text { Head } \\
\text { Mounted } \\
\text { Display } \\
\end{array}$ & $\begin{array}{c}5 \text { ASD (32.2 years old mean), } \\
5 \text { typical ( } 30.7 \text { years old } \\
\text { mean) }\end{array}$ \\
\hline$[133]$ & $\begin{array}{l}\text { Navigating through a virtual } \\
\text { building to find the exit }\end{array}$ & High & Standard controller & Mouse & Monitor & 8 ASD (6-12 years old) \\
\hline [134] & Crossing a street & Middle & Standard controller & Joystick & $\begin{array}{c}\text { Head } \\
\text { Mounted } \\
\text { Display }\end{array}$ & 2 ASD (children) \\
\hline
\end{tabular}


Table B-1 (Continued)

\begin{tabular}{|c|c|c|c|c|c|c|}
\hline Reference & Locomotion Related Task & $\begin{array}{l}\text { Navigation } \\
\text { Requirement }\end{array}$ & $\begin{array}{c}\text { Locomotion } \\
\text { Technique }\end{array}$ & $\begin{array}{l}\text { Locomotion } \\
\text { Tool }\end{array}$ & $\begin{array}{c}\text { Display } \\
\text { Device }\end{array}$ & Number of Participants \\
\hline$[134]$ & $\begin{array}{l}\text { Navigating through a virtual } \\
\text { building to find the exit }\end{array}$ & High & Standard controller & Mouse and keyboard & Monitor & 14 ASD (children) \\
\hline$[28]$ & Crossing a street & Middle & Standard controller & Keyboard & Monitor & $\begin{array}{l}6 \text { ASD (children), } \\
6 \text { typical (children) }\end{array}$ \\
\hline [135] & $\begin{array}{l}\text { Moving in the virtual environment } \\
\text { and interacting with virtual objects }\end{array}$ & Middle & Standard controller & Joystick & Monitor & 7 ASD (14-15 years old) \\
\hline$[11]$ & $\begin{array}{l}\text { Moving in the virtual environment } \\
\text { and interacting with virtual } \\
\text { characters }\end{array}$ & Middle & Standard controller & Keyboard & Monitor & 8 ASD (18-26 years old) \\
\hline
\end{tabular}




\title{
APPENDIX C: COPYRIGHT PERMISSIONS
}

\section{Below is the permission for use of material in Chapter 3.}

\section{Consent to Publish \\ Lecture Notes in Computer Science \\ 照 Springer}

\author{
Title of the Book or Conference Name: . HCl. International 2016 \\ Volume Editor(s): Will be provided during publication. \\ Title of the Contribution: virtual Reality interaction Techniques for Individuals with Autism Spectrum Disorder. Design Considerations and Preliminary Results . .

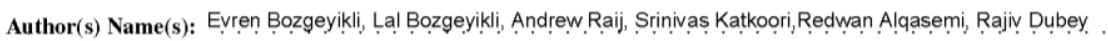 \\ Corresponding Author's Name, Address, Affiliation and Email: Evren. Bozgeyikli.

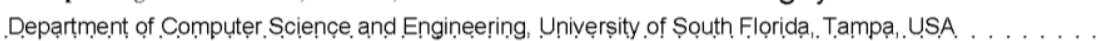 \\ .Research Assistant, .evren@mail.usf.edu
}

When Author is more than one person the expression "Author" as used in this agreement will apply collectively unless otherwise indicated.

\section{\$1 Rights Granted}

Author hereby grants and assigns to Springer International Publishing AG, Cham (hereinafter called Springer) the exclusive, sole, permanent, world-wide, transferable, sub-licensable and unlimited right to reproduce, publish, distribute, transmit, make available or otherwise communicate to the public, translate, publicly perform, archive, store, lease or lend and sell the Contribution or parts thereof individually or together with other works in any language, in all revisions and versions (including soft cover, book club and collected editions, anthologies, advance printing, reprints or print to order, microfilm editions, audiograms and videograms), in all forms and media of expression including in electronic form (including offline and online use, push or pull technologies, use in databases and networks for display, print and storing on any and all stationary or portable end-user devices, e.g. text readers, audio, video or interactive devices, and for use in multimedia or interactive versions as well as for the display or transmission of the Contribution or parts thereof in data networks or seach engines), in whole, in part or in abridged form, in each case as now known or developed in the future, including the right to grant further time-limited or permanent rights. For the purposes of use in electronic forms, Springer may adjust the Contribution to the respective form of use and include links or otherwise combine it with other works. For the avoidance of doubt, Springer has the right to permit others to use individual illustrations and may use the Contribution for advertising purposes.

$$
\text { (10 }
$$

The copyright of the Contribution will be held in the name of Springer. Springer may take, either in its own name or in that of copyright holder, any necessary steps to protect these rights against infringement by third parties. It will have the copyright notice inserted into all editions of the Contribution according to the provisions of the Universal Copyright Convention (UCC) and dutifully take care of all formalities in this connection in the name of the copyright holder.

\section{$\$ 2$ Regulations for Authors under Special Copyright Law}

The parties acknowledge that there may be no basis for claim of copyright in the United States to a Contribution prepared by an officer or employee of the United States government as part of that person's official duties. If the Contribution was performed under a United States government contract, but Author is not a United States government employee, Springer grants the United States government royalty-free permission to reproduce all or part of the Contribution and to authorize others to do so for United States government purposes.

If the Contribution was prepared or published by or under the direction or control of Her Majesty (i.e., the constitutional monarch of the Commonwealth realm) or any Crown government department, the copyright in the Contribution shall, subject to any agreement with Author, belong to Her Majesty.

If the Contribution was created by an employee of the European Union or the European Atomic Energy Community (EU/Euratom) in the performance of their duties, the regulation 31/EEC, 11/EAEC (Staff Regulations) applies, and copyright in the Contribution shall, subject to the Publication Framework Agreement (EC Plug), belong to the European Union or the European Atomic Energy Community.

If Author is an officer or employee of the United States government, of the Crown, or of EU/Euratom, reference will be made to this status on the signature page.

\section{\$3 Rights Retained by Author}

Author retains, in addition to uses permitted by law, the right to communicate the content of the Contribution to other scientists, to share the Contribution with them in manuscript form, to perform or present the Contribution or to use the content for non-commercial internal and educational purposes, provided the Springer publication is mentioned as the 


\begin{abstract}
original source of publication in any printed or electronic materials. Author retains the right to republish the Contribution in any collection consisting solely of Author's own works without charge subject to ensuring that the publication by Springer is properly credited and that the relevant copyright notice is repeated verbatim.

Author may self-atchive an author-created ver sion of hisher Contribution on hisher own website andror the repository of Author's department or faculty. Author may also deposit this version on his/her funder's or funder's designated repository at the funder's request or as a result of a legal obligation. He/she may not use the publisher's PDF version, which is posted on SpringerLink and other Springer websites, for the purpose of self-archiving or deposit. Furthermore, Author may only post hisher own version, provided acknowledgment. is given to the original source of publication and a link is inserted to the published article on Springer's website. The link must be provided by inserting the DOI mumber of the article in the following sentence: "The final publication is available at Springer via http//dx.doi.org/[insert DOI". The DOI (Digital Object Identifier' can be found at the bottom of the first page of the publi shed paper.

Frior versions of the Contribution published on non-commercial pre-print servers like ArXiv/CORR and HAL can remain on these servers andior can be updated with Author's accepted version. The final published version (in pdf or html/xml format) cannot be used for this purpose. Acknowledgment needs to be given to the final publication and a link must be inserted to the published Contribution on Springer's website, by inserting the DOI number of the article in the following sentence: "The final publication is available at Springer via http://dx.doi.org/[insert DOI".

Author retains the right to use hisher Contribution for hisher further scientific career by including the final published paper in hisher dissertation or doctoral thesis provided ackmowledgment is given to the original source of publication. Author also retains the right to use, without having to pay a fee and without having to inform the publisher, parts of the Contribution (e.g, illustrations) for inclusion in future work, and to publish a substantially revised version (at least 30\% new content) elsewhere, prowided that the original Springer Contribution is properly cited.
\end{abstract}

\title{
$\$ 4$ Warranties
}

Author warrants that the Contribution is original except for such excerpts from copyrighted works (including illustrations, tables, animations and text quotations) as may be included with the permission of the copyright holder thereof, in which case(s) Author is required to obtain written permission to the extent necessary and to indicate the precise sources of the excerpts in the manuscript. Author is also requested to store the signed permission forms and to make them available to Springer if required.

Author warrants that he/she is entitled to grant the rights in aocordance with Clause 1 "Rights Granted", that he/she has not assigned such rights to third parties, that the Contribution has not heretofore been published in whole or in part, that the Contribution contains no libelous statements and does not infringe on any copyright, trademark, patent, statutory right or proprietary right of others, including rights obtained through licenses; and that Author will indermify Springer against, any costs, expenses or damages for which Springer may become liable as a result of any breach of this warr anty.

\section{Delivery of the Work and Publication}

Author agrees to deliver to the responsible Volume Editor (for conferences, usually one of the Frogram Chairs), on a date to be agreed upon, the manuscript created according to the Springer Instructions for Authors. Springer will undertake the reproduction and distribution of the Contribution at its own expense and risk. After submission of the Consent to Publish form Signed by the Corresponding Author, changes of author ship, or in the order of the authors listed, will not be accepted by Springer.

\section{Author's Discount}

Author is entitled to purchase for hisher personal use (directly from Springer) the Work or other books published by Springer at a discount of $331 / 3 \%$ off the list price as long as there is a contractual arrangement between Author and Springer and subject to applicable book price regulation Resale of such copies or of free copies is not permitted.

\section{Governing Law and Jurisdiction}

This agrement shall be governed by, and shall be construed in accordance with, the laws of Switzerland. The courts of Zug, Switzerland shall have the exclusive jurisdiction.

Corresponding Author signs for and accepts responsibility for releasing this material on behalf of any and all Co-authors.

\section{Signature of Corresponding Author:}

Date:

\begin{tabular}{|l} 
I'm an employe of the US Government and transfer the rights to the extent transferable \\
(Title 17 § 105 U.S.C. applies) \\
I'm an employe of the Crown and copyright on the Contribution belongs to Her Majesty \\
I'm an employe of the EU or Euratom and copyright on the Contribution belongs to EU or Euratom
\end{tabular}




\section{Below is the permission for use of material in Chapter 5.}

\section{ACM Copyright and Audio/Video Release}

Title of the Work: Point \& Teleport Locomotion Technique for Virtual Reality

Submission ID:fp218

Author/Presenter(s): Evren Bozgeyikli (Univ. of South Florida); Andrew Raij (Institute for Simulation and Training); Srinivas Katkoori (Department of Computer Science and Engineering); Rajiv Dubey (Department of Mechanical Engineering)

Type of material:Paper

Publication and/or Conference Name: CHI PLAY '16: The Annual Symposium on Computer-Human Interaction in Play Proceedings

I. Copyright Transfer, Reserved Rights and Permitted Uses

* Your Copyright Transfer is conditional upon you agreeing to the terms set out below.

Copyright to the Work and to any supplemental files integral to the Work which are submitted with it for review and publication such as an extended proof, a PowerPoint outline, or appendices that may exceed a printed page limit, (including without limitation, the right to publish the Work in whole or in part in any and all forms of media, now or hereafter known) is hereby transferred to the ACM (for Government work, to the extent transferable) effective as of the date of this agreement, on the understanding that the Work has been accepted for publication by ACM.

Reserved Rights and Permitted Uses

(a) All rights and permissions the author has not granted to $\mathrm{ACM}$ are reserved to the Owner, including all other proprietary rights such as patent or trademark rights.

(b) Furthermore, notwithstanding the exclusive rights the Owner has granted to ACM, Owner shall have the right to do the following:

(i) Reuse any portion of the Work, without fee, in any future works written or edited by the Author, including books, lectures and presentations in any and all media.

(ii) Create a "Major Revision" which is wholly owned by the author

(iii) Post the Accepted Version of the Work on (1) the Author's home page, (2) the Owner's institutional repository, (3) any repository legally mandated by an agency funding the research on which the Work is based, and (4) any non-commercial repository or aggregation that does not duplicate ACM tables of contents, i.e., whose patterns of links do not substantially duplicate an ACM-copyrighted volume or issue. Non-commercial repositories are here understood as repositories owned by non-profit organizations that do not charge a fee for accessing deposited articles and that do not sell advertising or otherwise profit from serving articles.

(iv) Post an "Author-Izer" link enabling free downloads of the Version of Record in the ACM Digital Library on (1) the Author's home page or (2) the Owner's institutional repository;

(v) Prior to commencement of the ACM peer review process, post the version of the Work as submitted to ACM ("Submitted Version" or any earlier versions) to non-peer reviewed servers;

(vi) Make free distributions of the final published Version of Record internally to the Owner's employees, if applicable;

(vii) Make free distributions of the published Version of Record for Classroom and Personal Use; 
(viii) Bundle the Work in any of Owner's software distributions; and

(ix) Use any Auxiliary Material independent from the Work.

When preparing your paper for submission using the ACM TeX templates, the rights and permissions information and the bibliographic strip must appear on the lower left hand portion of the first page.

The new Authorized ACM TeX template cls version 2.8, automatically creates and positions these text blocks for you based on the code snippet which is system-generated based on your rights management choice and this particular conference.

Please copy and paste the following code snippet into your TeX file between Ibegin\{document\} and Imaketitle, either after or before CCS codes.

CopyrightYear $\{2016\}$

setcopyright $\{$ acm copyright $\}$

lconferenceinfo \{CHI PLAY'16 CD-ROM, \}\{October 16-19, 2016, Austin, TX, USA

$\backslash$ isbn $\{978-1-4503-4456-2 / 16 / 10\} \backslash$ acm Price $\{\backslash \$ 15.00\}$

doi\{http://dx.doi.org/10.1145/2967934.2968105\}

If you are using the ACM Microsoft Word template, or still using an older version of the ACM TeX template, or the current versions of the ACM SIGCHI, SIGGRAPH, or SIGPLAN TeX templates, you must copy and paste the following text block into your document as per the instructions provided with the templates you are using:

Permission to make digital or hard copies of all or part of this work for personal or classroom use is granted without fee provided that copies are not made or distributed for profit or commercial advantage and that copies bear this notice and the full citation on the first page. Copyrights for components of this work owned by others than ACM must be honored. Abstracting with credit is permitted. To copy otherwise, or republish, to post on servers or to redistribute to lists, requires prior specific permission and/or a fee. Request permissions from Permissions@acm.org.

CHI PLAY'16 CD-ROM, October 16-19, 2016, Austin, TX, USA

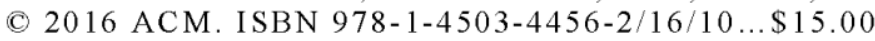

DOI: http://dx.doi.org/10.1145/2967934.2968105

NOTE: Make sure to include your article's DOI as part of the bibstrip data; DOIs will be registered and become active shortly after publication in the ACM Digital Library

A. Assent to Assignment. I hereby represent and warrant that I am the sole owner (or authorized agent of the copyright owner(s)), with the exception of third party materials detailed in section III below. I have obtained permission for any third-party material included in the Work.

B. Declaration for Government Work. I am an employee of the National Government of 
my country and my Government claims rights to this work, or it is not copyrightable (Government work is classified as Public Domain in U.S. only)

Are any of the co-authors, employees or contractors of a National Government? $\bigcirc$ Yes $\mathbb{N o}$ Country:

\section{PERMISSION FOR CONFERENCE TAPING AND DISTRIBUTION}

\section{Audio/Video Release}

* Your Audio/Video Release is conditional upon you agreeing to the terms set out below.

I hereby grant permission for ACM to include my name, likeness, presentation and comments in any and all forms, for the Conference and/or Publication.

I further grant permission for $\mathrm{ACM}$ to record and/or transcribe and reproduce my presentation as part of the ACM Digital Library, and to distribute the same for sale in complete or partial form as part of an ACM product on CD-ROM, DVD, webcast, USB device, streaming video or any other media format now or hereafter known.

I understand that my presentation will not be sold separately as a stand-alone product without my direct consent. Accordingly, I give ACM the right to use my image, voice, pronouncements, likeness, and my name, and any biographical material submitted by me, in connection with the Conference and/or Publication, whether used in excerpts or in full, for distribution described above and for any associated advertising or exhibition.

Do you agree to the above Audio/Video Release? 9 Yes $\square$ No

\section{Auxiliary Material}

Do you have any Auxiliary Materials? Yes 0 No

\section{Third Party Materials}

In the event that any materials used in my presentation or Auxiliary Materials contain the work of third-party individuals or organizations (including copyrighted music or movie excerpts or anything not owned by me), I understand that it is my responsibility to secure any necessary permissions and/or licenses for print and/or digital publication, and cite or attach them below.

We/I have not used third-party material.

$0 \mathrm{We} / \mathrm{I}$ have used third-party materials and have necessary permissions.

\section{Artistic Images}

If your paper includes images that were created for any purpose other than this paper and to which you or your employer claim copyright, you must complete Part V and be sure to include a notice of copyright with each such image in the paper.

(Q) We/I do not have any artistic images.

$\bigcirc \mathrm{We} / \mathrm{I}$ have any artistic images.

\section{Representations, Warranties and Covenants}


The undersigned hereby represents, warrants and covenants as follows:

(a) Owner is the sole owner or authorized agent of Owner(s) of the Work;

(b) The undersigned is authorized to enter into this Agreement and grant the rights included in this license to ACM;

(c) The Work is original and does not infringe the rights of any third party; all permissions for use of third-party materials consistent in scope and duration with the rights granted to ACM have been obtained, copies of such permissions have been provided to ACM, and the Work as submitted to ACM clearly and accurately indicates the credit to the proprietors of any such third-party materials (including any applicable copyright notice), or will be revised to indicate such credit;

(d) The Work has not been published except for informal postings on non-peer reviewed servers, and Owner covenants to use best efforts to place ACM DOI pointers on any such prior postings;

(e) The Auxiliary Materials, if any, contain no malicious code, virus, trojan horse or other software routines or hardware components designed to permit unauthorized access or to disable, erase or otherwise harm any computer systems or software; and

(f) The Artistic Images, if any, are clearly and accurately noted as such (including any applicable copyright notice) in the Submitted Version.

I agree to the Representations, Warranties and Covenants

DATE: 07/16/2016 sent to evren@mail.usf.edu at 00:07:20 


\section{Below is the permission for use of material in Chapter 6.}

\section{ACM Copyright and Audio/Video Release}

Title of the Work: Locomotion in Virtual Reality for Individuals with Autism Spectrum Disorder Submission ID:suifp200

Author/Presenter(s): Evren Bozgeyikli (Univ. of South Florida); Andrew Raij (Univ. of Central Florida); Srinivas Katkoori (Univ. of South Florida); Rajiv Dubey (Univ. of South Florida)

Type of material:Full Paper

Publication and/or Conference Name: SUI '16: 2016 Symposium on Spatial User Interaction Proceedings

I. Copyright Transfer, Reserved Rights and Permitted Uses

* Your Copyright Transfer is conditional upon you agreeing to the terms set out below.

Copyright to the Work and to any supplemental files integral to the Work which are submitted with it for review and publication such as an extended proof, a PowerPoint outline, or appendices that may exceed a printed page limit, (including without limitation, the right to publish the Work in whole or in part in any and all forms of media, now or hereafter known) is hereby transferred to the ACM (for Government work, to the extent transferable) effective as of the date of this agreement, on the understanding that the Work has been accepted for publication by ACM.

Reserved Rights and Permitted Uses

(a) All rights and permissions the author has not granted to ACM are reserved to the Owner, including all other proprietary rights such as patent or trademark rights.

(b) Furthermore, notwithstanding the exclusive rights the Owner has granted to ACM, Owner shall have the right to do the following:

(i) Reuse any portion of the Work, without fee, in any future works written or edited by the Author, including books, lectures and presentations in any and all media.

(ii) Create a "Major Revision" which is wholly owned by the author

(iii) Post the Accepted Version of the Work on (1) the Author's home page, (2) the Owner's institutional repository, (3) any repository legally mandated by an agency funding the research on which the Work is based, and (4) any non-commercial repository or aggregation that does not duplicate ACM tables of contents, i.e., whose patterns of links do not substantially duplicate an ACM-copyrighted volume or issue. Non-commercial repositories are here understood as repositories owned by non-profit organizations that do not charge a fee for accessing deposited articles and that do not sell advertising or otherwise profit from serving articles.

(iv) Post an "Author-Izer" link enabling free downloads of the Version of Record in the ACM Digital Library on (1) the Author's home page or (2) the Owner's institutional repository;

(v) Prior to commencement of the ACM peer review process, post the version of the Work as submitted to ACM ("Submitted Version" or any earlier versions) to non-peer reviewed servers;

(vi) Make free distributions of the final published Version of Record internally to the Owner's employees, if applicable;

(vii) Make free distributions of the published Version of Record for Classroom and Personal Use;

(viii) Bundle the Work in any of Owner's software distributions; and 
(ix) Use any Auxiliary Material independent from the Work.

When preparing your paper for submission using the ACM TeX templates, the rights and permissions information and the bibliographic strip must appear on the lower left hand portion of the first page.

The new Authorized ACM TeX template cls version 2.8, automatically creates and positions these text blocks for you based on the code snippet which is system-generated based on your rights management choice and this particular conference.

Please copy and paste the following code snippet into your TeX file between Ibegin\{document\} and Imaketitle, either after or before CCS codes.

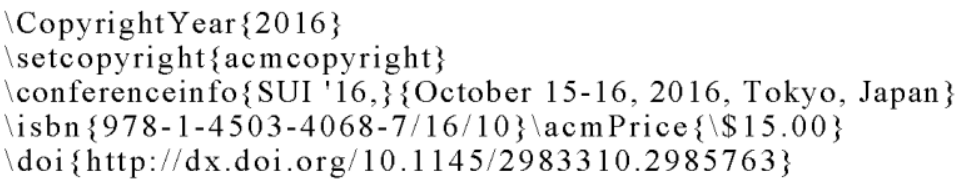

If you are using the ACM Microsoft Word template, or still using an older version of the ACM TeX template, or the current versions of the ACM SIGCHI, SIGGRAPH, or SIGPLAN TeX templates, you must copy and paste the following text block into your document as per the instructions provided with the templates you are using:

Permission to make digital or hard copies of all or part of this work for personal or classroom use is granted without fee provided that copies are not made or distributed for profit or commercial advantage and that copies bear this notice and the full citation on the first page. Copyrights for components of this work owned by others than ACM must be honored. Abstracting with credit is permitted. To copy otherwise, or republish, to post on servers or to redistribute to lists, requires prior specific permission and/or a fee. Request permissions from Permissions@acm.org.

SUI'16, October 15-16, 2016, Tokyo, Japan

(C) 2016 ACM. ISBN 978-1-4503-4068-7/16/10 $\$ 15.00$

DOI: http://dx.doi.org/10.1145/2983310.2985763

NOTE: Make sure to include your article's DOI as part of the bibstrip data; DOIs will be registered and become active shortly after publication in the ACM Digital Library

A. Assent to Assignment. I hereby represent and warrant that I am the sole owner (or authorized agent of the copyright owner(s)), with the exception of third party materials detailed in section III below. I have obtained permission for any third-party material included in the Work.

B. Declaration for Government Work. I am an employee of the National Government of my country and my Government claims rights to this work, or it is not copyrightable (Government work is classified as Public Domain in U.S. only)

Are any of the co-authors, employees or contractors of a National Government? $\bigcirc$ Yes No 
Country:

\title{
II. PERMISSION FOR CONFERENCE TAPING AND DISTRIBUTION
}

\author{
Audio/Video Release \\ * Your Audio/Video Release is conditional upon you agreeing to the terms set out below.
}

I hereby grant permission for ACM to include my name, likeness, presentation and comments in any and all forms, for the Conference and/or Publication.

I further grant permission for $\mathrm{ACM}$ to record and/or transcribe and reproduce my presentation as part of the ACM Digital Library, and to distribute the same for sale in complete or partial form as part of an ACM product on CD-ROM, DVD, webcast, USB device, streaming video or any other media format now or hereafter known.

I understand that my presentation will not be sold separately as a stand-alone product without my direct consent. Accordingly, I give ACM the right to use my image, voice, pronouncements, likeness, and my name, and any biographical material submitted by me, in connection with the Conference and/or Publication, whether used in excerpts or in full, for distribution described above and for any associated advertising or exhibition.

Do you agree to the above Audio/Video Release? $O$ Yes $\bigcirc$ No

\section{Auxiliary Material}

Do you have any Auxiliary Materials? $\bigcirc$ Yes 0 No

\section{Third Party Materials}

In the event that any materials used in my presentation or Auxiliary Materials contain the work of third-party individuals or organizations (including copyrighted music or movie excerpts or anything not owned by me), I understand that it is my responsibility to secure any necessary permissions and/or licenses for print and/or digital publication, and cite or attach them below.

We/I have not used third-party material.

$\mathrm{We} / \mathrm{I}$ have used third-party materials and have necessary permissions.

\section{Artistic Images}

If your paper includes images that were created for any purpose other than this paper and to which you or your employer claim copyright, you must complete Part V and be sure to include a notice of copyright with each such image in the paper.

(O) We/I do not have any artistic images.

$0 \mathrm{We} / \mathrm{I}$ have any artistic images.

\section{Representations, Warranties and Covenants}

The undersigned hereby represents, warrants and covenants as follows:

(a) Owner is the sole owner or authorized agent of Owner(s) of the Work; 
(b) The undersigned is authorized to enter into this Agreement and grant the rights included in this license to ACM;

(c) The Work is original and does not infringe the rights of any third party; all permissions for use of third-party materials consistent in scope and duration with the rights granted to ACM have been obtained, copies of such permissions have been provided to ACM, and the Work as submitted to ACM clearly and accurately indicates the credit to the proprietors of any such third-party materials (including any applicable copyright notice), or will be revised to indicate such credit;

(d) The Work has not been published except for informal postings on non-peer reviewed servers, and Owner covenants to use best efforts to place ACM DOI pointers on any such prior postings;

(e) The Auxiliary Materials, if any, contain no malicious code, virus, trojan horse or other software routines or hardware components designed to permit unauthorized access or to disable, erase or otherwise harm any computer systems or software; and

(f) The Artistic Images, if any, are clearly and accurately noted as such (including any applicable copyright notice) in the Submitted Version.

I agree to the Representations, Warranties and Covenants

DATE: 08/14/2016 sent to evren@mail.usf.edu at 18:08:19 


\section{APPENDIX D: IRB APPROVAL DOCUMENT}

\section{IRB approval document for the user studies that were performed in this}

\section{dissertation is below.}

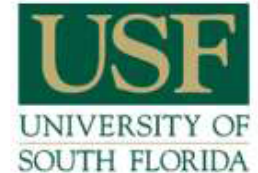

$6 / 6 / 2016$

Rajiv Dubey, $\mathrm{PhD}$

Mechanical Engineering

4202 East Fowler Ave

Tampa, FL 33620

RE: Expedited Approval of Am endm ent

IRB\#: Ame7_Pro00013008

Title: Using Virtual Reality and Robotics Technologies for Vocational Evaluation, Training and Placement

Dear Dr. Dubey:

On 6/6/2016, the Institutional Review Board (RB) reviewed and APPROVED your

Amendment. The submitted request and all documents contained within have been approved, including those outlined below.

We are removing the gift card incentive from the study. We are also adding a consent form for an online survey and the online survey itself. We are also adding Daniel Ashley and Kyle Ashley to the study staff, and removing Samia Dutra from the study staff

Revised Protocol, version 8, dated 05/20/2016

Revised Disability Consent Form, version 9, dated 05/20/2016

Revised Heal thy Consent From, version 7, dated 05/20/2016

\section{Approved Item(s):}

Protocol Document(s):

VR4VRProtocol 052016 Clean.docx

Consent Document(s) ${ }^{*}$ :

VR4VRDisabilityConsentForm 052016 Clean.docx.pdf VR4VRHealthyConsentForm 05.20.2016 Clean.docx.pdf

VR4VROnlineSurveyConsentForm $06 \quad 02 \quad 16$.docx

*Please use only the official IRB stamped informed consent/assent document(s) found under the "Attachments" tab on the main study's workspace. Please note, these consent/assent document(s) 
are only valid during the approval period indicated at the top of the form(s) and replace previously approved versions. Online consent forms are not stamped.

The $\mathbb{R B}$ does not require that subjects be reconsented.

The online survey portion of your study qualifies for a waiver of the requirements for the documentation of informed consent as outlined in the federal regulations at 45 CFR 46.117(c) which states that an $\mathbb{R B}$ may waive the requirement for the investigator to obtain a signed consent form for some or all subjects if it finds either: (1) That the only record linking the subject and the research would be the consent document and the principal risk would be potential harm resulting from a breach of confidentiality. Each subject will be asked whether the subject wants documentation linking the subject with the research, and the subject's wishes will govern; or (2) That the research presents no more than minimal risk of harm to subjects and involves no procedures for which written consent is norm ally required outside of the research context

As the principal investigator of this study, it is your responsibility to conduct this study in accordance with USF HRPP policies and procedures and as approved by the USF IRB. Any changes to the approved research must be submitted to the $R B$ for review and approval via an amendment. Additionally, all unanticipated problems must be reported to the USF IRB within five (5) calendar days.

We appreciate your dedication to the ethical conduct of hum an subject research at the University of South Florida and your continued commitment to human research protections. If you have any questions regarding this matter, please call 813-974-5638.

Sincerely,

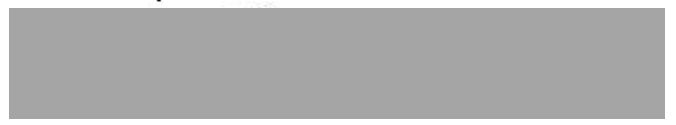

John Schinka, Ph.D., Chairperson

USF Institutional Review Board 


\section{APPENDIX E: DATA ANALYSIS OF THE VR4VR EXPERIMENT}

\begin{tabular}{|c|c|c|c|c|c|c|c|c|c|}
\hline \multicolumn{10}{|c|}{ Paired Samples Test } \\
\hline & & \multicolumn{5}{|c|}{ Paired Differences } & \multirow[b]{3}{*}{$\mathrm{t}$} & \multirow[b]{3}{*}{ df } & \multirow[b]{3}{*}{ Sig. (2-tailed) } \\
\hline & & \multirow[b]{2}{*}{ Mean } & \multirow[b]{2}{*}{ Std. Deviation } & \multirow{2}{*}{$\begin{array}{l}\text { Std. Error } \\
\text { Mean }\end{array}$} & \multicolumn{2}{|c|}{$\begin{array}{l}95 \% \text { Confidence Interval of the } \\
\text { Difference }\end{array}$} & & & \\
\hline & & & & & Lower & Upper & & & \\
\hline Pair 1 & $\begin{array}{l}\text { EaseOflnt_RW- } \\
\text { EaseOflnt_WiP }\end{array}$ & 1.11111 & .70711 & .23570 & .56758 & 1.65464 & 4.714 & 8 & .002 \\
\hline Pair 2 & Enjoy_RW - Enjoy_WiP & .77778 & 1.23603 & .41201 & -.17232 & 1.72788 & 1.888 & 8 & .096 \\
\hline Pair 3 & Frust_RW - Frust_WiP & -.85185 & .85165 & .28388 & -1.50649 & -.19722 & -3.001 & 8 & .017 \\
\hline Pair 4 & Tired_RW-Tired_WiP & -.22222 & .57735 & .19245 & -.66601 & .22157 & -1.155 & 8 & .282 \\
\hline Pair 5 & Immer_RW - Immer_WiP & .29630 & .77180 & .25727 & -.29696 & .88956 & 1.152 & 8 & .283 \\
\hline Pair 6 & Score_RW-Score_WiP & 6.18519 & 11.27172 & 3.75724 & -2.47903 & 14.84940 & 1.646 & 8 & .138 \\
\hline
\end{tabular}

Figure E-1: Paired samples t-test results of VR4VR locomotion techniques for neurotypical individuals.

\begin{tabular}{|c|c|c|c|c|c|c|c|c|c|}
\hline \multicolumn{10}{|c|}{ Paired Samples Test } \\
\hline & & \multicolumn{5}{|c|}{ Paired Differences } & \multirow[b]{3}{*}{$t$} & \multirow[b]{3}{*}{ df } & \multirow[b]{3}{*}{ Sig. (2-tailed) } \\
\hline & & \multirow[b]{2}{*}{ Mean } & \multirow[b]{2}{*}{ Std. Deviation } & \multirow{2}{*}{$\begin{array}{l}\text { Std. Error } \\
\text { Mean }\end{array}$} & \multicolumn{2}{|c|}{$\begin{array}{l}\text { 95\% Confidence Interval of the } \\
\text { Difference }\end{array}$} & & & \\
\hline & & & & & Lower & Upper & & & \\
\hline Pair 1 & $\begin{array}{l}\text { EaseOflnt_RW- } \\
\text { EaseOflnt_WiP }\end{array}$ & .33333 & 1.24722 & .55777 & -1.21529 & 1.88196 & .598 & 4 & .582 \\
\hline Pair 2 & Enjoy_RW - Enjoy_WiP & .26667 & .43461 & .19437 & -.27298 & .80631 & 1.372 & 4 & .242 \\
\hline Pair 3 & Frust_RW - Frust_WiP & -.86667 & 1.59164 & .71181 & -2.84295 & 1.10962 & -1.218 & 4 & .290 \\
\hline Pair 4 & Tired_RW - Tired_WiP & .06667 & .82999 & .37118 & -.96391 & 1.09724 & .180 & 4 & .866 \\
\hline Pair 5 & Immer_RW-Immer_WiP & .13333 & .18257 & .08165 & -.09336 & .36003 & 1.633 & 4 & .178 \\
\hline Pair 6 & Score_RW-Score_WiP & .45455 & 1.44039 & .64416 & -1.33394 & 2.24303 & .706 & 4 & .519 \\
\hline
\end{tabular}

Figure E-2: Paired samples t-test results of VR4VR locomotion techniques for individuals with ASD.

\begin{tabular}{|l|l|}
\hline Interaction & \multicolumn{1}{|c|}{ Dependent Variable } \\
\hline 1 & Haptic Device \\
2 & Tangible Interaction \\
3 & Touch and Snap \\
4 & Touch Screen \\
\hline
\end{tabular}

Figure E-3: Pairwise comparison factors for the data analysis of selection and manipulation techniques. 


\section{Mauchly's Test of Sphericity}

Measure: Easeoflnteraction

\begin{tabular}{|c|c|c|c|c|c|c|c|}
\hline \multirow[b]{2}{*}{ Within Subjects Effect } & \multirow[b]{2}{*}{ Mauchly's W } & \multirow[b]{2}{*}{$\begin{array}{l}\text { Approx. Chi- } \\
\text { Square }\end{array}$} & \multirow[b]{2}{*}{ df } & \multirow[b]{2}{*}{ Sig. } & \multicolumn{3}{|c|}{ Epsilon } \\
\hline & & & & & $\begin{array}{c}\text { Greenhouse- } \\
\text { Geisser }\end{array}$ & Huynh-Feldt & Lower-bound \\
\hline Interaction & 252 & 9.267 & 5 & .102 & .556 & .685 & .333 \\
\hline
\end{tabular}

Tests the null hypothesis that the error covariance matrix of the orthonormalized transformed dependent variables is proportional to an identity matrix.

Tests of Within-Subjects Effects

Measure: Easeoflnteraction

\begin{tabular}{|c|c|c|c|c|c|c|c|c|c|}
\hline Source & & $\begin{array}{c}\text { Type III Sum } \\
\text { of Squares }\end{array}$ & df & Mean Square & $\mathrm{F}$ & Sig. & $\begin{array}{l}\text { Partial Eta } \\
\text { Squared }\end{array}$ & $\begin{array}{l}\text { Noncent. } \\
\text { Parameter }\end{array}$ & $\begin{array}{l}\text { Observed } \\
\text { Power }^{\mathrm{a}}\end{array}$ \\
\hline \multirow[t]{4}{*}{ Interaction } & Sphericity Assumed & 13.188 & 3 & 4.396 & 8.115 & .001 & .504 & 24.346 & .979 \\
\hline & Greenhouse-Geisser & 13.188 & 1.668 & 7.907 & 8.115 & .007 & .504 & 13.535 & .870 \\
\hline & Huynh-Feldt & 13.188 & 2.055 & 6.419 & 8.115 & .003 & .504 & 16.673 & .921 \\
\hline & Lower-bound & 13.188 & 1.000 & 13.188 & 8.115 & .022 & .504 & 8.115 & .705 \\
\hline \multirow[t]{4}{*}{ Error(Interaction) } & Sphericity Assumed & 13.000 & 24 & .542 & & & & & \\
\hline & Greenhouse-Geisser & 13.000 & 13.342 & .974 & & & & & \\
\hline & Huynh-Feldt & 13.000 & 16.436 & .791 & & & & & \\
\hline & Lower-bound & 13.000 & 8.000 & 1.625 & & & & & \\
\hline
\end{tabular}

a. Computed using alpha $=.05$

\section{Pairwise Comparisons}

Measure: EaseOflnteraction

\begin{tabular}{|c|c|c|c|c|c|c|}
\hline \multirow[b]{2}{*}{ (l) Interaction } & \multirow[b]{2}{*}{ (J) Interaction } & \multirow{2}{*}{$\begin{array}{c}\text { Mean } \\
\text { Difference (I- } \\
\mathrm{J})\end{array}$} & \multirow[b]{2}{*}{ Std. Error } & \multirow[b]{2}{*}{ Sig. ${ }^{b}$} & \multicolumn{2}{|c|}{$\begin{array}{l}95 \% \text { Confidence Interval for } \\
\text { Difference }\end{array}$} \\
\hline & & & & & Lower Bound & Upper Bound \\
\hline \multirow[t]{3}{*}{1} & 2 & .389 & .389 & .347 & -.508 & 1.286 \\
\hline & 3 & 1.000 & .500 & .081 & -.153 & 2.153 \\
\hline & 4 & -.667 & .333 & .081 & -1.435 & .102 \\
\hline \multirow[t]{3}{*}{2} & 1 & -.389 & .389 & .347 & -1.286 & .508 \\
\hline & 3 & $.611^{*}$ & .200 & .016 & .149 & 1.073 \\
\hline & 4 & $-1.056^{\pi}$ & .242 & .002 & -1.614 & -.497 \\
\hline \multirow[t]{3}{*}{3} & 1 & -1.000 & .500 & .081 & -2.153 & .153 \\
\hline & 2 & $-.611^{\star}$ & .200 & .016 & -1.073 & -.149 \\
\hline & 4 & $-1.667^{\star}$ & .333 & .001 & -2.435 & -.898 \\
\hline \multirow[t]{3}{*}{4} & 1 & .667 & .333 & .081 & -.102 & 1.435 \\
\hline & 2 & $1.056^{*}$ & .242 & .002 & .497 & 1.614 \\
\hline & 3 & $1.667^{*}$ & .333 & .001 & .898 & 2.435 \\
\hline
\end{tabular}

Based on estimated marginal means

${ }^{\star}$. The mean difference is significant at the .05 level.

b. Adjustment for multiple comparisons: Least Significant Difference (equivalent to no adjustments).

Figure E-4: Ease of interaction detailed data analysis for VR4VR's object selection and manipulation techniques for neurotypical individuals. 
Mauchly's Test of Sphericity

Measure: Easeoflnteraction

\begin{tabular}{|c|c|c|c|c|c|c|c|}
\hline \multirow[b]{2}{*}{ Within Subjects Effect } & \multirow[b]{2}{*}{ Mauchly's W } & \multirow[b]{2}{*}{$\begin{array}{l}\text { Approx. Chi- } \\
\text { Square }\end{array}$} & \multirow[b]{2}{*}{ df } & \multirow[b]{2}{*}{ Sig. } & \multicolumn{3}{|c|}{ Epsilon } \\
\hline & & & & & $\begin{array}{l}\text { Greenhouse- } \\
\text { Geisser }\end{array}$ & Huynh-Feldt & Lower-bound \\
\hline Interaction & .222 & 4.098 & 5 & .558 & .678 & 1.000 & .333 \\
\hline
\end{tabular}

Tests the null hypothesis that the error covariance matrix of the orthonormalized transformed dependent variables is proportional to an identity matrix.

Tests of Within-Subjects Effects

\begin{tabular}{|c|c|c|c|c|c|c|c|c|c|}
\hline Source & & $\begin{array}{l}\text { Type III Sum } \\
\text { of Squares }\end{array}$ & df & Mean Square & $\mathrm{F}$ & Sig. & $\begin{array}{l}\text { Partial Eta } \\
\text { Squared }\end{array}$ & $\begin{array}{l}\text { Noncent. } \\
\text { Parameter }\end{array}$ & $\begin{array}{l}\text { Observed } \\
\text { Power }^{\mathrm{a}}\end{array}$ \\
\hline \multirow[t]{4}{*}{ Interaction } & Sphericity Assumed & 7.600 & 3 & 2.533 & 4.406 & .026 & .524 & 13.217 & .738 \\
\hline & Greenhouse-Geisser & 7.600 & 2.034 & 3.737 & 4.406 & .050 & .524 & 8.960 & .589 \\
\hline & Huynh-Feldt & 7.600 & 3.000 & 2.533 & 4.406 & .026 & .524 & 13.217 & .738 \\
\hline & Lower-bound & 7.600 & 1.000 & 7.600 & 4.406 & .104 & .524 & 4.406 & .363 \\
\hline \multirow[t]{4}{*}{ Error(Interaction) } & Sphericity Assumed & 6.900 & 12 & .575 & & & & & \\
\hline & Greenhouse-Geisser & 6.900 & 8.135 & .848 & & & & & \\
\hline & Huynh-Feldt & 6.900 & 12.000 & .575 & & & & & \\
\hline & Lower-bound & 6.900 & 4.000 & 1.725 & & & & & \\
\hline
\end{tabular}

a. Computed using alpha $=.05$

\section{Pairwise Comparisons}

Measure: Easeoflnteraction

\begin{tabular}{|c|c|c|c|c|c|c|}
\hline \multirow[b]{2}{*}{ (I) Interaction } & \multirow[b]{2}{*}{ (J) Interaction } & \multirow{2}{*}{$\begin{array}{c}\text { Mean } \\
\text { Difference (I- } \\
\mathrm{J})\end{array}$} & \multirow[b]{2}{*}{ Std. Error } & \multirow[b]{2}{*}{ Sig. $^{b}$} & \multicolumn{2}{|c|}{$\begin{array}{l}95 \% \text { Confidence Interval for } \\
\text { Difference }^{\mathrm{b}}\end{array}$} \\
\hline & & & & & Lower Bound & Upper Bound \\
\hline \multirow[t]{3}{*}{1} & 2 & -1.000 & .632 & .189 & -2.756 & .756 \\
\hline & 3 & -1.400 & .510 & .052 & -2.816 & .016 \\
\hline & 4 & $-1.600^{*}$ & .510 & .035 & -3.016 & -.184 \\
\hline \multirow[t]{3}{*}{2} & 1 & 1.000 & .632 & .189 & -.756 & 2.756 \\
\hline & 3 & -.400 & .510 & .477 & -1.816 & 1.016 \\
\hline & 4 & -.600 & .400 & .208 & -1.711 & .511 \\
\hline \multirow[t]{3}{*}{3} & 1 & 1.400 & .510 & .052 & -.016 & 2.816 \\
\hline & 2 & .400 & .510 & .477 & -1.016 & 1.816 \\
\hline & 4 & -.200 & .200 & .374 & -.755 & .355 \\
\hline \multirow[t]{3}{*}{4} & 1 & $1.600^{\pi}$ & .510 & .035 & .184 & 3.016 \\
\hline & 2 & .600 & .400 & .208 & -.511 & 1.711 \\
\hline & 3 & .200 & .200 & .374 & -.355 & .755 \\
\hline
\end{tabular}

Based on estimated marginal means

*. The mean difference is significant at the .05 level.

b. Adjustment for multiple comparisons: Least Significant Difference (equivalent to no adjustments).

Figure E-5: Ease of interaction detailed data analysis for VR4VR's object selection and manipulation techniques for individuals with ASD. 
Mauchly's Test of Sphericity

Measure: Enjoyment

\begin{tabular}{|c|c|c|c|c|c|c|c|}
\hline \multirow[b]{2}{*}{ Within Subjects Effect } & \multirow[b]{2}{*}{ Mauchly's W } & \multirow[b]{2}{*}{$\begin{array}{l}\text { Approx. Chi- } \\
\text { Square }\end{array}$} & \multirow[b]{2}{*}{ df } & \multirow[b]{2}{*}{ Sig. } & \multicolumn{3}{|c|}{ Epsilon } \\
\hline & & & & & $\begin{array}{c}\text { Greenhouse- } \\
\text { Geisser }\end{array}$ & Huynh-Feldt & Lower-bound \\
\hline Interaction & .662 & 2.775 & 5 & .737 & .832 & 1.000 & .333 \\
\hline
\end{tabular}

Tests the null hypothesis that the error covariance matrix of the orthonormalized transformed dependent variables is proportional to an identity matrix.

Tests of Within-Subjects Effects

\begin{tabular}{|c|c|c|c|c|c|c|c|c|c|}
\hline Source & & $\begin{array}{c}\text { Type III Sum } \\
\text { of Squares }\end{array}$ & $d f$ & Mean Square & $\mathrm{F}$ & Sig. & $\begin{array}{l}\text { Partial Eta } \\
\text { Squared }\end{array}$ & $\begin{array}{l}\text { Noncent. } \\
\text { Parameter }\end{array}$ & $\begin{array}{l}\text { Observed } \\
\text { Power }^{\text {a }}\end{array}$ \\
\hline \multirow[t]{4}{*}{ Interaction } & Sphericity Assumed & 4.667 & 3 & 1.556 & 2.881 & .057 & .265 & 8.643 & .613 \\
\hline & Greenhouse-Geisser & 4.667 & 2.497 & 1.869 & 2.881 & .070 & .265 & 7.194 & .552 \\
\hline & Huynh-Feldt & 4.667 & 3.000 & 1.556 & 2.881 & .057 & .265 & 8.643 & .613 \\
\hline & Lower-bound & 4.667 & 1.000 & 4.667 & 2.881 & .128 & .265 & 2.881 & .322 \\
\hline \multirow[t]{4}{*}{ Error(Interaction) } & Sphericity Assumed & 12.958 & 24 & .540 & & & & & \\
\hline & Greenhouse-Geisser & 12.958 & 19.975 & .649 & & & & & \\
\hline & Huynh-Feldt & 12.958 & 24.000 & .540 & & & & & \\
\hline & Lower-bound & 12.958 & 8.000 & 1.620 & & & & & \\
\hline
\end{tabular}

a. Computed using alpha $=.05$

\section{Pairwise Comparisons}

Measure: Enjoyment

\begin{tabular}{|c|c|c|c|c|c|c|}
\hline \multirow[b]{2}{*}{ (1) Interaction } & \multirow[b]{2}{*}{ (J) Interaction } & \multirow{2}{*}{$\begin{array}{c}\text { Mean } \\
\text { Difference (I- } \\
\mathrm{J})\end{array}$} & \multirow[b]{2}{*}{ Std. Error } & \multirow[b]{2}{*}{ Sig. ${ }^{b}$} & \multicolumn{2}{|c|}{$\begin{array}{l}95 \% \text { Confidence Interval for } \\
\text { Difference }{ }^{b}\end{array}$} \\
\hline & & & & & Lower Bound & Upper Bound \\
\hline \multirow[t]{3}{*}{1} & 2 & .111 & .415 & .796 & -.845 & 1.068 \\
\hline & 3 & .444 & .338 & .225 & -.335 & 1.224 \\
\hline & 4 & -.556 & .294 & .095 & -1.233 & .122 \\
\hline \multirow[t]{3}{*}{2} & 1 & -.111 & .415 & .796 & -1.068 & .845 \\
\hline & 3 & .333 & .323 & .332 & -.411 & 1.078 \\
\hline & 4 & -.667 & .323 & .073 & -1.411 & .078 \\
\hline \multirow[t]{3}{*}{3} & 1 & -.444 & .338 & .225 & -1.224 & .335 \\
\hline & 2 & -.333 & .323 & .332 & -1.078 & .411 \\
\hline & 4 & $-1.000^{*}$ & .373 & .028 & -1.859 & -.141 \\
\hline \multirow[t]{3}{*}{4} & 1 & .556 & .294 & .095 & -.122 & 1.233 \\
\hline & 2 & .667 & .323 & .073 & -.078 & 1.411 \\
\hline & 3 & $1.000^{*}$ & .373 & .028 & .141 & 1.859 \\
\hline
\end{tabular}

Based on estimated marginal means

*. The mean difference is significant at the .05 level.

b. Adjustment for multiple comparisons: Least Significant Difference (equivalent to no adjustments).

Figure E-6: Enjoyment detailed data analysis for VR4VR's object selection and manipulation techniques for neurotypical individuals. 
Mauchly's Test of Sphericity

Measure: Enjoyment

\begin{tabular}{|c|c|c|c|c|c|c|c|}
\hline \multirow[b]{2}{*}{ Within Subjects Effect } & \multirow[b]{2}{*}{ Mauchly's W } & \multirow[b]{2}{*}{$\begin{array}{l}\text { Approx. Chi- } \\
\text { Square }\end{array}$} & \multirow[b]{2}{*}{ df } & \multirow[b]{2}{*}{ Sig. } & \multicolumn{3}{|c|}{ Epsilon } \\
\hline & & & & & $\begin{array}{l}\text { Greenhouse- } \\
\text { Geisser }\end{array}$ & Huynh-Feldt & Lower-bound \\
\hline Interaction & .000 & & 5 & & 399 & 475 & .333 \\
\hline
\end{tabular}

Tests the null hypothesis that the error covariance matrix of the orthonormalized transformed dependent variables is proportional to an identity matrix.

Tests of Within-Subjects Effects

\begin{tabular}{|c|c|c|c|c|c|c|c|c|c|}
\hline Source & & $\begin{array}{c}\text { Type III Sum } \\
\text { of Squares }\end{array}$ & df & Mean Square & $\mathrm{F}$ & Sig. & $\begin{array}{c}\text { Partial Eta } \\
\text { Squared }\end{array}$ & $\begin{array}{l}\text { Noncent. } \\
\text { Parameter }\end{array}$ & $\begin{array}{l}\text { Observed } \\
\text { Power }^{\text {a }}\end{array}$ \\
\hline \multirow[t]{4}{*}{ Interaction } & Sphericity Assumed & .738 & 3 & .246 & 1.341 & .307 & .251 & 4.023 & .270 \\
\hline & Greenhouse-Geisser & .738 & 1.198 & .616 & 1.341 & .314 & .251 & 1.606 & .161 \\
\hline & Huynh-Feldt & .738 & 1.424 & .518 & 1.341 & .315 & .251 & 1.909 & .176 \\
\hline & Lower-bound & .738 & 1.000 & .738 & 1.341 & .311 & .251 & 1.341 & .147 \\
\hline \multirow[t]{4}{*}{ Error(Interaction) } & Sphericity Assumed & 2.200 & 12 & .183 & & & & & \\
\hline & Greenhouse-Geisser & 2.200 & 4.792 & .459 & & & & & \\
\hline & Huynh-Feldt & 2.200 & 5.696 & .386 & & & & & \\
\hline & Lower-bound & 2.200 & 4.000 & .550 & & & & & \\
\hline
\end{tabular}

a. Computed using alpha $=.05$

\section{Pairwise Comparisons}

Measure: Enjoyment

\begin{tabular}{|c|c|c|c|c|c|c|}
\hline \multirow[b]{2}{*}{ (I) Interaction } & \multirow[b]{2}{*}{ (J) Interaction } & \multirow{2}{*}{$\begin{array}{c}\text { Mean } \\
\text { Difference (I- } \\
\text { J) }\end{array}$} & \multirow[b]{2}{*}{ Std. Error } & \multirow[b]{2}{*}{ Sig. ${ }^{a}$} & \multicolumn{2}{|c|}{$\begin{array}{l}\text { 95\% Confidence Interval for } \\
\text { Difference }\end{array}$} \\
\hline & & & & & Lower Bound & Upper Bound \\
\hline \multirow[t]{3}{*}{1} & 2 & .100 & .245 & .704 & -.580 & .780 \\
\hline & 3 & .000 & .000 & & .000 & .000 \\
\hline & 4 & -.400 & .245 & .178 & -1.080 & .280 \\
\hline \multirow[t]{3}{*}{2} & 1 & -.100 & .245 & .704 & -.780 & .580 \\
\hline & 3 & -.100 & .245 & .704 & -.780 & .580 \\
\hline & 4 & -.500 & .447 & .326 & -1.742 & .742 \\
\hline \multirow[t]{3}{*}{3} & 1 & .000 & .000 & & .000 & .000 \\
\hline & 2 & .100 & .245 & .704 & -.580 & .780 \\
\hline & 4 & -.400 & .245 & .178 & -1.080 & .280 \\
\hline \multirow[t]{3}{*}{4} & 1 & .400 & .245 & .178 & -.280 & 1.080 \\
\hline & 2 & .500 & .447 & .326 & -.742 & 1.742 \\
\hline & 3 & .400 & .245 & .178 & -.280 & 1.080 \\
\hline
\end{tabular}

Based on estimated marginal means

a. Adjustment for multiple comparisons: Least Significant Difference (equivalent to no adjustments).

Figure E-7: Enjoyment detailed data analysis for VR4VR's object selection and manipulation techniques for individuals with ASD. 
Mauchly's Test of Sphericity

Measure: Frustration

\begin{tabular}{|c|c|c|c|c|c|c|c|}
\hline \multirow[b]{2}{*}{ Within Subjects Effect } & \multirow[b]{2}{*}{ Mauchly's W } & \multirow[b]{2}{*}{$\begin{array}{l}\text { Approx. Chi- } \\
\text { Square }\end{array}$} & \multirow[b]{2}{*}{ df } & \multirow[b]{2}{*}{ Sig. } & \multicolumn{3}{|c|}{ Epsilon } \\
\hline & & & & & $\begin{array}{l}\text { Greenhouse- } \\
\text { Geisser }\end{array}$ & Huynh-Feldt & Lower-bound \\
\hline Interaction & .296 & 8.186 & 5 & .150 & 663 & .882 & .333 \\
\hline
\end{tabular}

Tests the null hypothesis that the error covariance matrix of the orthonormalized transformed dependent variables is proportional to an identity matrix.

Tests of Within-Subjects Effects

\begin{tabular}{|c|c|c|c|c|c|c|c|c|c|}
\hline Source & & $\begin{array}{c}\text { Type III Sum } \\
\text { of Squares }\end{array}$ & $\mathrm{df}$ & Mean Square & $\mathrm{F}$ & Sig. & $\begin{array}{l}\text { Partial Eta } \\
\text { Squared }\end{array}$ & $\begin{array}{l}\text { Noncent. } \\
\text { Parameter }\end{array}$ & $\begin{array}{l}\text { Observed } \\
\text { Power }^{\text {a }}\end{array}$ \\
\hline \multirow[t]{4}{*}{ Interaction } & Sphericity Assumed & 10.889 & 3 & 3.630 & 6.066 & .003 & .431 & 18.197 & .925 \\
\hline & Greenhouse-Geisser & 10.889 & 1.990 & 5.473 & 6.066 & .011 & .431 & 12.069 & .814 \\
\hline & Huynh-Feldt & 10.889 & 2.647 & 4.114 & 6.066 & .005 & .431 & 16.053 & .896 \\
\hline & Lower-bound & 10.889 & 1.000 & 10.889 & 6.066 & .039 & .431 & 6.066 & .581 \\
\hline \multirow[t]{4}{*}{ Error(Interaction) } & Sphericity Assumed & 14.361 & 24 & .598 & & & & & \\
\hline & Greenhouse-Geisser & 14.361 & 15.917 & .902 & & & & & \\
\hline & Huynh-Feldt & 14.361 & 21.172 & .678 & & & & & \\
\hline & Lower-bound & 14.361 & 8.000 & 1.795 & & & & & \\
\hline
\end{tabular}

a. Computed using alpha $=.05$

\section{Pairwise Comparisons}

Measure: Frustration

\begin{tabular}{|c|c|c|c|c|c|c|}
\hline \multirow[b]{2}{*}{ (l) Interaction } & \multirow[b]{2}{*}{ (J) Interaction } & \multirow{2}{*}{$\begin{array}{c}\text { Mean } \\
\text { Difference (1- } \\
\mathrm{J}) \\
\end{array}$} & \multirow[b]{2}{*}{ Std. Error } & \multirow[b]{2}{*}{ Sig. ${ }^{\text {b }}$} & \multicolumn{2}{|c|}{$\begin{array}{l}\text { 95\% Confidence Interval for } \\
\text { Difference }^{\mathrm{b}}\end{array}$} \\
\hline & & & & & Lower Bound & Upper Bound \\
\hline \multirow[t]{3}{*}{1} & 2 & .556 & .412 & .214 & -.395 & 1.506 \\
\hline & 3 & .222 & .364 & .559 & -.618 & 1.062 \\
\hline & 4 & $1.444^{\pi}$ & .294 & .001 & .767 & 2.122 \\
\hline \multirow[t]{3}{*}{2} & 1 & -.556 & .412 & .214 & -1.506 & .395 \\
\hline & 3 & -.333 & .500 & .524 & -1.486 & .820 \\
\hline & 4 & $.889^{*}$ & .286 & .014 & .229 & 1.548 \\
\hline \multirow[t]{3}{*}{3} & 1 & -.222 & .364 & .559 & -1.062 & .618 \\
\hline & 2 & .333 & .500 & .524 & -.820 & 1.486 \\
\hline & 4 & $1.222^{\star}$ & .278 & .002 & .582 & 1.863 \\
\hline \multirow[t]{3}{*}{4} & 1 & $-1.444^{\pi}$ & .294 & .001 & -2.122 & -.767 \\
\hline & 2 & $-.889^{\star}$ & .286 & .014 & -1.548 & -.229 \\
\hline & 3 & $-1.222^{*}$ & .278 & .002 & -1.863 & -.582 \\
\hline
\end{tabular}

Based on estimated marginal means

*. The mean difference is significant at the .05 level.

b. Adjustment for multiple comparisons: Least Significant Difference (equivalent to no adjustments).

Figure E-8: Frustration detailed data analysis for VR4VR's object selection and manipulation techniques for neurotypical individuals. 
Mauchly's Test of Sphericity

Measure: Frustration

\begin{tabular}{|c|c|c|c|c|c|c|c|}
\hline \multirow[b]{2}{*}{ Within Subjects Effect } & \multirow[b]{2}{*}{ Mauchly's W } & \multirow[b]{2}{*}{$\begin{array}{c}\text { Approx. Chi- } \\
\text { Square }\end{array}$} & \multirow[b]{2}{*}{ df } & \multirow[b]{2}{*}{ Sig. } & \multicolumn{3}{|c|}{ Epsilon } \\
\hline & & & & & $\begin{array}{c}\text { Greenhouse- } \\
\text { Geisser }\end{array}$ & Huynh-Feldt & Lower-bound \\
\hline Interaction & .031 & 9.479 & 5 & .110 & .391 & .455 & .333 \\
\hline
\end{tabular}

Tests the null hypothesis that the error covariance matrix of the orthonormalized transformed dependent variables is proportional to an identity matrix.

Tests of Within-Subjects Effects

\begin{tabular}{|c|c|c|c|c|c|c|c|c|c|}
\hline Source & & $\begin{array}{c}\text { Type III Sum } \\
\text { of Squares }\end{array}$ & df & Mean Square & $\mathrm{F}$ & Sig. & $\begin{array}{c}\text { Partial Eta } \\
\text { Squared }\end{array}$ & $\begin{array}{l}\text { Noncent. } \\
\text { Parameter }\end{array}$ & $\begin{array}{l}\text { Observed } \\
\text { Power }^{\text {a }}\end{array}$ \\
\hline \multirow[t]{4}{*}{ Interaction } & Sphericity Assumed & 1.138 & 3 & .379 & 1.625 & .236 & .289 & 4.875 & .322 \\
\hline & Greenhouse-Geisser & 1.138 & 1.172 & .971 & 1.625 & .270 & .289 & 1.904 & .183 \\
\hline & Huynh-Feldt & 1.138 & 1.365 & .834 & 1.625 & .267 & .289 & 2.218 & .199 \\
\hline & Lower-bound & 1.138 & 1.000 & 1.138 & 1.625 & .271 & .289 & 1.625 & .168 \\
\hline \multirow[t]{4}{*}{ Error(Interaction) } & Sphericity Assumed & 2.800 & 12 & .233 & & & & & \\
\hline & Greenhouse-Geisser & 2.800 & 4.688 & .597 & & & & & \\
\hline & Huynh-Feldt & 2.800 & 5.459 & .513 & & & & & \\
\hline & Lower-bound & 2.800 & 4.000 & .700 & & & & & \\
\hline
\end{tabular}

a. Computed using alpha $=.05$

\section{Pairwise Comparisons}

Measure: Frustration

\begin{tabular}{|c|c|c|c|c|c|c|}
\hline \multirow[b]{2}{*}{ (l) Interaction } & \multirow[b]{2}{*}{ (J) Interaction } & \multirow{2}{*}{$\begin{array}{c}\text { Mean } \\
\text { Difference (I- } \\
\text { J) }\end{array}$} & \multirow[b]{2}{*}{ Std. Error } & \multirow[b]{2}{*}{ Sig. ${ }^{a}$} & \multicolumn{2}{|c|}{$\begin{array}{l}\text { 95\% Confidence Interval for } \\
\text { Difference }\end{array}$} \\
\hline & & & & & Lower Bound & Upper Bound \\
\hline \multirow[t]{3}{*}{1} & 2 & .300 & .300 & .374 & -.533 & 1.133 \\
\hline & 3 & -.200 & .200 & .374 & -.755 & .355 \\
\hline & 4 & .400 & .245 & .178 & -.280 & 1.080 \\
\hline \multirow[t]{3}{*}{2} & 1 & -.300 & .300 & .374 & -1.133 & .533 \\
\hline & 3 & -.500 & .447 & .326 & -1.742 & .742 \\
\hline & 4 & .100 & .100 & .374 & -.178 & .378 \\
\hline \multirow[t]{3}{*}{3} & 1 & .200 & .200 & .374 & -.355 & .755 \\
\hline & 2 & .500 & .447 & .326 & -.742 & 1.742 \\
\hline & 4 & .600 & .400 & .208 & -.511 & 1.711 \\
\hline \multirow[t]{3}{*}{4} & 1 & -.400 & .245 & .178 & -1.080 & .280 \\
\hline & 2 & -.100 & .100 & .374 & -.378 & .178 \\
\hline & 3 & -.600 & .400 & .208 & -1.711 & .511 \\
\hline
\end{tabular}

Based on estimated marginal means

a. Adjustment for multiple comparisons: Least Significant Difference (equivalent to no adjustments).

Figure E-9: Frustration detailed data analysis for VR4VR's object selection and manipulation techniques for individuals with ASD. 
Mauchly's Test of Sphericity

Measure: Tiredness

\begin{tabular}{|c|c|c|c|c|c|c|c|}
\hline \multirow[b]{2}{*}{ Within Subjects Effect } & \multirow[b]{2}{*}{ Mauchly's W } & \multirow[b]{2}{*}{$\begin{array}{l}\text { Approx. Chi- } \\
\text { Square }\end{array}$} & \multirow[b]{2}{*}{ df } & \multirow[b]{2}{*}{ Sig. } & \multicolumn{3}{|c|}{ Epsilon } \\
\hline & & & & & $\begin{array}{l}\text { Greenhouse- } \\
\text { Geisser }\end{array}$ & Huynh-Feldt & Lower-bound \\
\hline Interaction & .244 & 9.479 & 5 & .095 & .686 & .927 & .333 \\
\hline
\end{tabular}

Tests the null hypothesis that the error covariance matrix of the orthonormalized transformed dependent variables is proportional to an identity matrix.

Tests of Within-Subjects Effects

\begin{tabular}{|c|c|c|c|c|c|c|c|c|c|}
\hline Source & & $\begin{array}{c}\text { Type III Sum } \\
\text { of Squares }\end{array}$ & df & Mean Square & $\mathrm{F}$ & Sig. & $\begin{array}{c}\text { Partial Eta } \\
\text { Squared }\end{array}$ & $\begin{array}{l}\text { Noncent. } \\
\text { Parameter }\end{array}$ & $\begin{array}{l}\text { Observed } \\
\text { Power }^{\text {a }}\end{array}$ \\
\hline \multirow[t]{4}{*}{ Interaction } & Sphericity Assumed & 1.639 & 3 & .546 & 3.006 & .050 & .273 & 9.019 & .633 \\
\hline & Greenhouse-Geisser & 1.639 & 2.058 & .796 & 3.006 & .076 & .273 & 6.187 & .510 \\
\hline & Huynh-Feldt & 1.639 & 2.781 & .589 & 3.006 & .055 & .273 & 8.360 & .607 \\
\hline & Lower-bound & 1.639 & 1.000 & 1.639 & 3.006 & .121 & .273 & 3.006 & .333 \\
\hline \multirow[t]{4}{*}{ Error(Interaction) } & Sphericity Assumed & 4.361 & 24 & .182 & & & & & \\
\hline & Greenhouse-Geisser & 4.361 & 16.464 & .265 & & & & & \\
\hline & Huynh-Feldt & 4.361 & 22.245 & .196 & & & & & \\
\hline & Lower-bound & 4.361 & 8.000 & .545 & & & & & \\
\hline
\end{tabular}

a. Computed using alpha $=.05$

\section{Pairwise Comparisons}

Measure: Tiredness

\begin{tabular}{|c|c|c|c|c|c|c|}
\hline \multirow[b]{2}{*}{ (l) Interaction } & \multirow[b]{2}{*}{ (J) Interaction } & \multirow{2}{*}{$\begin{array}{c}\text { Mean } \\
\text { Difference (I- } \\
\mathrm{J}) \\
\end{array}$} & \multirow[b]{2}{*}{ Std. Error } & \multirow[b]{2}{*}{ Sig. ${ }^{\text {b }}$} & \multicolumn{2}{|c|}{$\begin{array}{l}\text { 95\% Confidence Interval for } \\
\text { Difference }\end{array}$} \\
\hline & & & & & Lower Bound & Upper Bound \\
\hline \multirow[t]{3}{*}{1} & 2 & -.444 & .212 & .069 & -.932 & .043 \\
\hline & 3 & -.222 & .222 & .347 & -.735 & .290 \\
\hline & 4 & .111 & .261 & .681 & -.490 & .712 \\
\hline \multirow[t]{3}{*}{2} & 1 & .444 & .212 & .069 & -.043 & .932 \\
\hline & 3 & $.222^{\star}$ & .088 & .035 & .020 & .425 \\
\hline & 4 & $.556^{*}$ & .212 & .030 & .068 & 1.043 \\
\hline \multirow[t]{3}{*}{3} & 1 & .222 & .222 & .347 & -.290 & .735 \\
\hline & 2 & $-.222^{x}$ & .088 & .035 & -.425 & -.020 \\
\hline & 4 & .333 & .167 & .081 & -.051 & .718 \\
\hline \multirow[t]{3}{*}{4} & 1 & -.111 & .261 & .681 & -.712 & .490 \\
\hline & 2 & $-.556^{\star}$ & .212 & .030 & -1.043 & -.068 \\
\hline & 3 & -.333 & .167 & .081 & -.718 & .051 \\
\hline
\end{tabular}

Based on estimated marginal means

*. The mean difference is significant at the .05 level.

b. Adjustment for multiple comparisons: Least Significant Difference (equivalent to no adjustments).

Figure E-10: Tiredness detailed data analysis for VR4VR's object selection and manipulation techniques for neurotypical individuals. 
Mauchly's Test of Sphericity

Measure: Tiredness

\begin{tabular}{|c|c|c|c|c|c|c|c|}
\hline \multirow[b]{2}{*}{ Within Subjects Effect } & \multirow[b]{2}{*}{ Mauchly's W } & \multirow[b]{2}{*}{$\begin{array}{l}\text { Approx. Chi- } \\
\text { Square }\end{array}$} & \multirow[b]{2}{*}{ df } & \multirow[b]{2}{*}{ Sig. } & \multicolumn{3}{|c|}{ Epsilon } \\
\hline & & & & & $\begin{array}{l}\text { Greenhouse- } \\
\text { Geisser }\end{array}$ & Huynh-Feldt & Lower-bound \\
\hline Interaction & .000 & & 5 & & .480 & .678 & .333 \\
\hline
\end{tabular}

Tests the null hypothesis that the error covariance matrix of the orthonormalized transformed dependent variables is proportional to an identity matrix.

Tests of Within-Subjects Effects

\begin{tabular}{|c|c|c|c|c|c|c|c|c|c|}
\hline Source & & $\begin{array}{c}\text { Type III Sum } \\
\text { of Squares }\end{array}$ & df & Mean Square & $\mathrm{F}$ & Sig. & $\begin{array}{c}\text { Partial Eta } \\
\text { Squared }\end{array}$ & $\begin{array}{l}\text { Noncent. } \\
\text { Parameter }\end{array}$ & $\begin{array}{l}\text { Observed } \\
\text { Power }^{\text {a }}\end{array}$ \\
\hline \multirow[t]{4}{*}{ Interaction } & Sphericity Assumed & 1.350 & 3 & .450 & 2.139 & .149 & .348 & 6.416 & .414 \\
\hline & Greenhouse-Geisser & 1.350 & 1.441 & .937 & 2.139 & .201 & .348 & 3.081 & .256 \\
\hline & Huynh-Feldt & 1.350 & 2.033 & .664 & 2.139 & .179 & .348 & 4.348 & .320 \\
\hline & Lower-bound & 1.350 & 1.000 & 1.350 & 2.139 & .217 & .348 & 2.139 & .205 \\
\hline \multirow[t]{4}{*}{ Error(Interaction) } & Sphericity Assumed & 2.525 & 12 & .210 & & & & & \\
\hline & Greenhouse-Geisser & 2.525 & 5.762 & .438 & & & & & \\
\hline & Huynh-Feldt & 2.525 & 8.132 & .311 & & & & & \\
\hline & Lower-bound & 2.525 & 4.000 & .631 & & & & & \\
\hline
\end{tabular}

a. Computed using alpha $=.05$

\section{Pairwise Comparisons}

Measure: Tiredness

\begin{tabular}{|c|c|c|c|c|c|c|}
\hline \multirow[b]{2}{*}{ (l) Interaction } & \multirow[b]{2}{*}{ (J) Interaction } & \multirow{2}{*}{$\begin{array}{c}\text { Mean } \\
\text { Difference (I- } \\
\text { J) }\end{array}$} & \multirow[b]{2}{*}{ Std. Error } & \multirow[b]{2}{*}{ Sig. ${ }^{a}$} & \multicolumn{2}{|c|}{$\begin{array}{l}\text { 95\% Confidence Interval for } \\
\text { Difference }\end{array}$} \\
\hline & & & & & Lower Bound & Upper Bound \\
\hline \multirow[t]{3}{*}{1} & 2 & -.400 & .292 & .242 & -1.209 & .409 \\
\hline & 3 & -.400 & .245 & .178 & -1.080 & .280 \\
\hline & 4 & .200 & .200 & .374 & -.355 & .755 \\
\hline \multirow[t]{3}{*}{2} & 1 & .400 & .292 & .242 & -.409 & 1.209 \\
\hline & 3 & .000 & .158 & 1.000 & -.439 & .439 \\
\hline & 4 & .600 & .367 & .178 & -.420 & 1.620 \\
\hline \multirow[t]{3}{*}{3} & 1 & .400 & .245 & .178 & -.280 & 1.080 \\
\hline & 2 & .000 & .158 & 1.000 & -.439 & .439 \\
\hline & 4 & .600 & .400 & .208 & -.511 & 1.711 \\
\hline \multirow[t]{3}{*}{4} & 1 & -.200 & .200 & .374 & -.755 & .355 \\
\hline & 2 & -.600 & .367 & .178 & -1.620 & .420 \\
\hline & 3 & -.600 & .400 & .208 & -1.711 & .511 \\
\hline
\end{tabular}

Based on estimated marginal means

a. Adjustment for multiple comparisons: Least Significant Difference (equivalent to no adjustments).

Figure E-11: Tiredness detailed data analysis for VR4VR's object selection and manipulation techniques for individuals with ASD. 
Mauchly's Test of Sphericity

Measure: Score

\begin{tabular}{|c|c|c|c|c|c|c|c|}
\hline \multirow[b]{2}{*}{ Within Subjects Effect } & \multirow[b]{2}{*}{ Mauchly's W } & \multirow[b]{2}{*}{$\begin{array}{c}\text { Approx. Chi- } \\
\text { Square }\end{array}$} & \multirow[b]{2}{*}{ df } & \multirow[b]{2}{*}{ Sig. } & \multicolumn{3}{|c|}{ Epsilon } \\
\hline & & & & & $\begin{array}{c}\text { Greenhouse- } \\
\text { Geisser }\end{array}$ & Huynh-Feldt & Lower-bound \\
\hline Interaction & .476 & 4.985 & 5 & .422 & .727 & 1.000 & .333 \\
\hline
\end{tabular}

Tests the null hypothesis that the error covariance matrix of the orthonormalized transformed dependent variables is proportional to an identity matrix.

\begin{tabular}{|c|c|c|c|c|c|c|c|c|c|}
\hline \multicolumn{10}{|c|}{ Tests of Within-Subjects Effects } \\
\hline Measure: Score & & & & & & & & & \\
\hline \multicolumn{2}{|l|}{ Source } & $\begin{array}{l}\text { Type III Sum } \\
\text { of Squares }\end{array}$ & df & Mean Square & $\mathrm{F}$ & Sig. & $\begin{array}{c}\text { Partial Eta } \\
\text { Squared }\end{array}$ & $\begin{array}{l}\text { Noncent. } \\
\text { Parameter }\end{array}$ & $\begin{array}{l}\text { Observed } \\
\text { Power }^{\text {a }}\end{array}$ \\
\hline \multirow[t]{4}{*}{ Interaction } & Sphericity Assumed & 7847.938 & 3 & 2615.979 & 27.559 & .000 & .775 & 82.676 & 1.000 \\
\hline & Greenhouse-Geisser & 7847.938 & 2.182 & 3596.799 & 27.559 & .000 & .775 & 60.131 & 1.000 \\
\hline & Huynh-Feldt & 7847.938 & 3.000 & 2615.979 & 27.559 & .000 & .775 & 82.676 & 1.000 \\
\hline & Lower-bound & 7847.938 & 1.000 & 7847.938 & 27.559 & .001 & .775 & 27.559 & .995 \\
\hline \multirow[t]{4}{*}{ Error(Interaction) } & Sphericity Assumed & 2278.173 & 24 & 94.924 & & & & & \\
\hline & Greenhouse-Geisser & 2278.173 & 17.455 & 130.514 & & & & & \\
\hline & Huynh-Feldt & 2278.173 & 24.000 & 94.924 & & & & & \\
\hline & Lower-bound & 2278.173 & 8.000 & 284.772 & & & & & \\
\hline
\end{tabular}

a. Computed using alpha $=.05$

\section{Pairwise Comparisons}

Measure: Score

\begin{tabular}{|c|c|c|c|c|c|c|}
\hline \multirow[b]{2}{*}{ (I) Interaction } & \multirow[b]{2}{*}{ (J) Interaction } & \multirow{2}{*}{$\begin{array}{c}\text { Mean } \\
\text { Difference (I- } \\
\text { J) }\end{array}$} & \multirow[b]{2}{*}{ Std. Error } & \multirow[b]{2}{*}{ Sig. ${ }^{b}$} & \multicolumn{2}{|c|}{$\begin{array}{l}\text { 95\% Confidence Interval for } \\
\text { Difference }\end{array}$} \\
\hline & & & & & Lower Bound & Upper Bound \\
\hline \multirow[t]{3}{*}{1} & 2 & $-34.000^{\pi}$ & 4.861 & .000 & -45.210 & -22.790 \\
\hline & 3 & $-35.185^{\star}$ & 5.849 & .000 & -48.674 & -21.696 \\
\hline & 4 & $-32.963^{x}$ & 5.342 & .000 & -45.281 & -20.645 \\
\hline \multirow[t]{3}{*}{2} & 1 & $34.000^{*}$ & 4.861 & .000 & 22.790 & 45.210 \\
\hline & 3 & -1.185 & 3.016 & .705 & -8.141 & 5.771 \\
\hline & 4 & 1.037 & 3.285 & .760 & -6.538 & 8.612 \\
\hline \multirow[t]{3}{*}{3} & 1 & $35.185^{\star}$ & 5.849 & .000 & 21.696 & 48.674 \\
\hline & 2 & 1.185 & 3.016 & .705 & -5.771 & 8.141 \\
\hline & 4 & 2.222 & 4.505 & .635 & -8.166 & 12.610 \\
\hline \multirow[t]{3}{*}{4} & 1 & $32.963^{*}$ & 5.342 & .000 & 20.645 & 45.281 \\
\hline & 2 & -1.037 & 3.285 & .760 & -8.612 & 6.538 \\
\hline & 3 & -2.222 & 4.505 & .635 & -12.610 & 8.166 \\
\hline
\end{tabular}

Based on estimated marginal means

*. The mean difference is significant at the .05 level.

b. Adjustment for multiple comparisons: Least Significant Difference (equivalent to no adjustments).

Figure E-12: Score detailed data analysis for VR4VR's object selection and manipulation techniques for neurotypical individuals. 
Mauchly's Test of Sphericity

Measure: Score

\begin{tabular}{|c|c|c|c|c|c|c|c|}
\hline \multirow[b]{2}{*}{ Within Subjects Effect } & \multirow[b]{2}{*}{ Mauchly's W } & \multirow[b]{2}{*}{$\begin{array}{l}\text { Approx. Chi- } \\
\text { Square }\end{array}$} & \multirow[b]{2}{*}{ df } & \multirow[b]{2}{*}{ Sig. } & \multicolumn{3}{|c|}{ Epsilon } \\
\hline & & & & & $\begin{array}{l}\text { Greenhouse- } \\
\text { Geisser }\end{array}$ & Huynh-Feldt & Lower-bound \\
\hline Interaction & .038 & 8.935 & 5 & .132 & 481 & 680 & .333 \\
\hline
\end{tabular}

Tests the null hypothesis that the error covariance matrix of the orthonormalized transformed dependent variables is proportional to an identity matrix.

\begin{tabular}{|c|c|c|c|c|c|c|c|c|c|}
\hline \multicolumn{10}{|c|}{ Tests of Within-Subjects Effects } \\
\hline \multicolumn{10}{|l|}{ Measure: Score } \\
\hline Source & & $\begin{array}{c}\text { Type III Sum } \\
\text { of Squares }\end{array}$ & df & Mean Square & $\mathrm{F}$ & Sig. & $\begin{array}{c}\text { Partial Eta } \\
\text { Squared }\end{array}$ & $\begin{array}{l}\text { Noncent. } \\
\text { Parameter }\end{array}$ & $\begin{array}{l}\text { Observed } \\
\text { Power }^{\text {a }}\end{array}$ \\
\hline \multirow[t]{4}{*}{ Interaction } & Sphericity Assumed & 10055.067 & 3 & 3351.689 & 7.325 & .005 & .647 & 21.975 & .929 \\
\hline & Greenhouse-Geisser & 10055.067 & 1.443 & 6966.127 & 7.325 & .031 & .647 & 10.573 & .681 \\
\hline & Huynh-Feldt & 10055.067 & 2.041 & 4927.351 & 7.325 & .015 & .647 & 14.948 & .815 \\
\hline & Lower-bound & 10055.067 & 1.000 & 10055.067 & 7.325 & .054 & .647 & 7.325 & .537 \\
\hline \multirow[t]{4}{*}{ Error(Interaction) } & Sphericity Assumed & 5490.933 & 12 & 457.578 & & & & & \\
\hline & Greenhouse-Geisser & 5490.933 & 5.774 & 951.026 & & & & & \\
\hline & Huynh-Feldt & 5490.933 & 8.163 & 672.690 & & & & & \\
\hline & Lower-bound & 5490.933 & 4.000 & 1372.733 & & & & & \\
\hline
\end{tabular}

a. Computed using alpha $=.05$

\section{Pairwise Comparisons}

Measure: Score

\begin{tabular}{|c|c|c|c|c|c|c|}
\hline \multirow[b]{2}{*}{ (l) Interaction } & \multirow[b]{2}{*}{ (J) Interaction } & \multirow{2}{*}{$\begin{array}{c}\text { Mean } \\
\text { Difference (I- } \\
\mathrm{J})\end{array}$} & \multirow[b]{2}{*}{ Std. Error } & \multirow[b]{2}{*}{ Sig. $^{\text {b }}$} & \multicolumn{2}{|c|}{$\begin{array}{l}\text { 95\% Confidence Interval for } \\
\text { Difference }^{\mathrm{b}}\end{array}$} \\
\hline & & & & & Lower Bound & Upper Bound \\
\hline \multirow[t]{3}{*}{1} & 2 & -47.200 & 18.492 & .063 & -98.541 & 4.141 \\
\hline & 3 & $-59.000^{\star}$ & 18.148 & .031 & -109.386 & -8.614 \\
\hline & 4 & -26.333 & 15.197 & .158 & -68.527 & 15.860 \\
\hline \multirow[t]{3}{*}{2} & 1 & 47.200 & 18.492 & .063 & -4.141 & 98.541 \\
\hline & 3 & $-11.800^{\star}$ & 2.518 & .009 & -18.791 & -4.809 \\
\hline & 4 & 20.867 & 9.444 & .092 & -5.353 & 47.087 \\
\hline \multirow[t]{3}{*}{3} & 1 & $59.000^{x}$ & 18.148 & .031 & 8.614 & 109.386 \\
\hline & 2 & $11.800^{*}$ & 2.518 & .009 & 4.809 & 18.791 \\
\hline & 4 & $32.667^{*}$ & 10.022 & .031 & 4.841 & 60.493 \\
\hline \multirow[t]{3}{*}{4} & 1 & 26.333 & 15.197 & .158 & -15.860 & 68.527 \\
\hline & 2 & -20.867 & 9.444 & .092 & -47.087 & 5.353 \\
\hline & 3 & $-32.667^{\star}$ & 10.022 & .031 & -60.493 & -4.841 \\
\hline
\end{tabular}

Based on estimated marginal means

*. The mean difference is significant at the .05 level.

b. Adjustment for multiple comparisons: Least Significant Difference (equivalent to no adjustments).

Figure E-13: Score detailed data analysis for VR4VR's object selection and manipulation techniques for individuals with ASD. 
Mauchly's Test of Sphericity

Measure: Immersion

\begin{tabular}{|c|c|c|c|c|c|c|c|}
\hline \multirow[b]{2}{*}{ Within Subjects Effect } & \multirow[b]{2}{*}{ Mauchly's W } & \multirow[b]{2}{*}{$\begin{array}{l}\text { Approx. Chi- } \\
\text { Square }\end{array}$} & \multirow[b]{2}{*}{ df } & \multirow[b]{2}{*}{ Sig. } & \multicolumn{3}{|c|}{ Epsilon } \\
\hline & & & & & $\begin{array}{l}\text { Greenhouse- } \\
\text { Geisser }\end{array}$ & Huynh-Feldt & Lower-bound \\
\hline Interaction & .243 & 9.520 & 5 & .093 & .567 & .705 & .333 \\
\hline
\end{tabular}

Tests the null hypothesis that the error covariance matrix of the orthonormalized transformed dependent variables is proportional to an identity matrix.

Tests of Within-Subjects Effects

\begin{tabular}{|c|c|c|c|c|c|c|c|c|c|}
\hline Source & & $\begin{array}{c}\text { Type III Sum } \\
\text { of Squares }\end{array}$ & df & Mean Square & $\mathrm{F}$ & Sig. & $\begin{array}{c}\text { Partial Eta } \\
\text { Squared }\end{array}$ & $\begin{array}{l}\text { Noncent. } \\
\text { Parameter }\end{array}$ & $\begin{array}{l}\text { Observed } \\
\text { Power }^{\text {a }}\end{array}$ \\
\hline \multirow[t]{4}{*}{ Interaction } & Sphericity Assumed & 2.354 & 3 & .785 & 2.404 & .092 & .231 & 7.213 & .528 \\
\hline & Greenhouse-Geisser & 2.354 & 1.702 & 1.383 & 2.404 & .133 & .231 & 4.092 & .376 \\
\hline & Huynh-Feldt & 2.354 & 2.114 & 1.113 & 2.404 & .118 & .231 & 5.083 & .428 \\
\hline & Lower-bound & 2.354 & 1.000 & 2.354 & 2.404 & .160 & .231 & 2.404 & .277 \\
\hline \multirow[t]{4}{*}{ Error(Interaction) } & Sphericity Assumed & 7.833 & 24 & .326 & & & & & \\
\hline & Greenhouse-Geisser & 7.833 & 13.615 & .575 & & & & & \\
\hline & Huynh-Feldt & 7.833 & 16.915 & .463 & & & & & \\
\hline & Lower-bound & 7.833 & 8.000 & .979 & & & & & \\
\hline
\end{tabular}

a. Computed using alpha $=.05$

\section{Pairwise Comparisons}

Measure: Immersion

\begin{tabular}{|c|c|c|c|c|c|c|}
\hline \multirow[b]{2}{*}{ (l) Interaction } & \multirow[b]{2}{*}{ (J) Interaction } & \multirow{2}{*}{$\begin{array}{c}\text { Mean } \\
\text { Difference (I- } \\
\mathrm{J}) \\
\end{array}$} & \multirow[b]{2}{*}{ Std. Error } & \multirow[b]{2}{*}{ Sig. ${ }^{b}$} & \multicolumn{2}{|c|}{$\begin{array}{l}\text { 95\% Confidence Interval for } \\
\text { Difference }\end{array}$} \\
\hline & & & & & Lower Bound & Upper Bound \\
\hline \multirow[t]{3}{*}{1} & 2 & -.167 & .264 & .545 & -.774 & .441 \\
\hline & 3 & -.111 & .389 & .782 & -1.008 & .786 \\
\hline & 4 & $-.667^{\star}$ & .167 & .004 & -1.051 & -.282 \\
\hline \multirow[t]{3}{*}{2} & 1 & .167 & .264 & .545 & -.441 & .774 \\
\hline & 3 & .056 & .269 & .842 & -.565 & .677 \\
\hline & 4 & $-.500^{*}$ & .167 & .017 & -.884 & -.116 \\
\hline \multirow[t]{3}{*}{3} & 1 & .111 & .389 & .782 & -.786 & 1.008 \\
\hline & 2 & -.056 & .269 & .842 & -.677 & .565 \\
\hline & 4 & -.556 & .294 & .095 & -1.233 & .122 \\
\hline \multirow[t]{3}{*}{4} & 1 & $.667^{*}$ & .167 & .004 & .282 & 1.051 \\
\hline & 2 & $.500^{\star}$ & .167 & .017 & .116 & .884 \\
\hline & 3 & .556 & .294 & .095 & -.122 & 1.233 \\
\hline
\end{tabular}

Based on estimated marginal means

*. The mean difference is significant at the .05 level.

b. Adjustment for multiple comparisons: Least Significant Difference (equivalent to no adjustments).

Figure E-14: Presence detailed data analysis for VR4VR's object selection and manipulation techniques for neurotypical individuals. 
Mauchly's Test of Sphericity

Measure: Immersion

\begin{tabular}{|c|c|c|c|c|c|c|c|}
\hline \multirow[b]{2}{*}{ Within Subjects Effect } & \multirow[b]{2}{*}{ Mauchly's W } & \multirow[b]{2}{*}{$\begin{array}{l}\text { Approx. Chi- } \\
\text { Square }\end{array}$} & \multirow[b]{2}{*}{ df } & \multirow[b]{2}{*}{ Sig. } & \multicolumn{3}{|c|}{ Epsilon } \\
\hline & & & & & $\begin{array}{c}\text { Greenhouse- } \\
\text { Geisser }\end{array}$ & Huynh-Feldt & Lower-bound \\
\hline Interaction & .000 & & 5 & & 372 & .414 & .333 \\
\hline
\end{tabular}

Tests the null hypothesis that the error covariance matrix of the orthonormalized transformed dependent variables is proportional to an identity matrix.

Tests of Within-Subjects Effects

\begin{tabular}{|c|c|c|c|c|c|c|c|c|c|}
\hline Source & & $\begin{array}{c}\text { Type III Sum } \\
\text { of Squares }\end{array}$ & df & Mean Square & $\mathrm{F}$ & Sig. & $\begin{array}{l}\text { Partial Eta } \\
\text { Squared }\end{array}$ & $\begin{array}{l}\text { Noncent. } \\
\text { Parameter }\end{array}$ & $\begin{array}{l}\text { Observed } \\
\text { Power }^{\text {a }}\end{array}$ \\
\hline \multirow[t]{4}{*}{ Interaction } & Sphericity Assumed & .738 & 3 & .246 & 2.458 & .113 & .381 & 7.375 & .469 \\
\hline & Greenhouse-Geisser & .738 & 1.116 & .661 & 2.458 & .186 & .381 & 2.744 & .244 \\
\hline & Huynh-Feldt & .738 & 1.242 & .594 & 2.458 & .180 & .381 & 3.053 & .262 \\
\hline & Lower-bound & .738 & 1.000 & .738 & 2.458 & .192 & .381 & 2.458 & .228 \\
\hline \multirow[t]{4}{*}{ Error(Interaction) } & Sphericity Assumed & 1.200 & 12 & .100 & & & & & \\
\hline & Greenhouse-Geisser & 1.200 & 4.465 & .269 & & & & & \\
\hline & Huynh-Feldt & 1.200 & 4.968 & .242 & & & & & \\
\hline & Lower-bound & 1.200 & 4.000 & .300 & & & & & \\
\hline
\end{tabular}

a. Computed using alpha $=.05$

\section{Pairwise Comparisons}

Measure: Immersion

\begin{tabular}{|c|c|c|c|c|c|c|}
\hline \multirow[b]{2}{*}{ (l) Interaction } & \multirow[b]{2}{*}{ (J) Interaction } & \multirow{2}{*}{$\begin{array}{c}\text { Mean } \\
\text { Difference (I- } \\
\text { J) }\end{array}$} & \multirow[b]{2}{*}{ Std. Error } & \multirow[b]{2}{*}{ Sig. ${ }^{a}$} & \multicolumn{2}{|c|}{$\begin{array}{l}\text { 95\% Confidence Interval for } \\
\text { Difference }\end{array}$} \\
\hline & & & & & Lower Bound & Upper Bound \\
\hline \multirow[t]{3}{*}{1} & 2 & .100 & .100 & .374 & -.178 & .378 \\
\hline & 3 & .000 & .000 & & .000 & .000 \\
\hline & 4 & -.400 & .245 & .178 & -1.080 & .280 \\
\hline \multirow[t]{3}{*}{2} & 1 & -.100 & .100 & .374 & -.378 & .178 \\
\hline & 3 & -.100 & .100 & .374 & -.378 & .178 \\
\hline & 4 & -.500 & .316 & .189 & -1.378 & .378 \\
\hline \multirow[t]{3}{*}{3} & 1 & .000 & .000 & & .000 & .000 \\
\hline & 2 & .100 & .100 & .374 & -.178 & .378 \\
\hline & 4 & -.400 & .245 & .178 & -1.080 & .280 \\
\hline \multirow[t]{3}{*}{4} & 1 & .400 & .245 & .178 & -.280 & 1.080 \\
\hline & 2 & .500 & .316 & .189 & -.378 & 1.378 \\
\hline & 3 & .400 & .245 & .178 & -.280 & 1.080 \\
\hline
\end{tabular}

Based on estimated marginal means

a. Adjustment for multiple comparisons: Least Significant Difference (equivalent to no adjustments).

Figure E-15: Presence detailed data analysis for VR4VR's object selection and manipulation techniques for individuals with ASD. 
Paired Samples Test

\begin{tabular}{|c|c|c|c|c|c|c|c|c|c|}
\hline & & \multicolumn{5}{|c|}{ Paired Differences } & \multirow[b]{3}{*}{$t$} & \multirow[b]{3}{*}{ df } & \multirow[b]{3}{*}{ Sig. (2-tailed) } \\
\hline & & \multirow[b]{2}{*}{ Mean } & \multirow[b]{2}{*}{ Std. Deviation } & \multirow{2}{*}{$\begin{array}{l}\text { Std. Error } \\
\text { Mean }\end{array}$} & \multicolumn{2}{|c|}{$\begin{array}{l}\text { 95\% Confidence Interval of the } \\
\text { Difference }\end{array}$} & & & \\
\hline & & & & & Lower & Upper & & & \\
\hline Pair 1 & $\begin{array}{l}\text { EaseOfint_Curtain - } \\
\text { EaseOfInt_HMD }\end{array}$ & .81667 & .82757 & .26170 & .22466 & 1.40868 & 3.121 & 9 & .012 \\
\hline Pair 2 & $\begin{array}{l}\text { Enjoy_Curtain - } \\
\text { Enjoy_HMD }\end{array}$ & .69167 & .87405 & .27640 & .06641 & 1.31693 & 2.502 & 9 & .034 \\
\hline Pair 3 & $\begin{array}{l}\text { Frust_Curtain - } \\
\text { Frust_HMD }\end{array}$ & -.57500 & .61545 & .19462 & -1.01527 & -.13473 & -2.954 & 9 & .016 \\
\hline Pair 4 & $\begin{array}{l}\text { Tired_Curtain - } \\
\text { Tired_HMD }\end{array}$ & -.75000 & .41759 & .13205 & -1.04873 & -.45127 & -5.679 & 9 & .000 \\
\hline Pair 5 & $\begin{array}{l}\text { Immer_Curtain - } \\
\text { Immer_HMD }\end{array}$ & .26667 & .58505 & .18501 & -.15185 & .68519 & 1.441 & 9 & .183 \\
\hline Pair 6 & $\begin{array}{l}\text { Score_Curtain - } \\
\text { Score_HMD }\end{array}$ & -2.75327 & 6.01981 & 1.90363 & -7.05958 & 1.55305 & -1.446 & 9 & .182 \\
\hline
\end{tabular}

Figure E-16: Detailed data analysis of VR4VR's display methods for neurotypical individuals.

Paired Samples Test

\begin{tabular}{|c|c|c|c|c|c|c|c|c|c|}
\hline & & \multicolumn{5}{|c|}{ Paired Differences } & \multirow[b]{3}{*}{$t$} & \multirow[b]{3}{*}{ df } & \multirow[b]{3}{*}{ Sig. (2-tailed) } \\
\hline & & \multirow[b]{2}{*}{ Mean } & \multirow[b]{2}{*}{ Std. Deviation } & \multirow{2}{*}{$\begin{array}{l}\text { Std. Error } \\
\text { Mean }\end{array}$} & \multicolumn{2}{|c|}{$\begin{array}{l}\text { 95\% Confidence Interval of the } \\
\text { Difference }\end{array}$} & & & \\
\hline & & & & & Lower & Upper & & & \\
\hline Pair 1 & $\begin{array}{l}\text { EaseOflnt_Curtain - } \\
\text { EaseOflnt_HMD }\end{array}$ & .58333 & 1.04194 & .39382 & -.38030 & 1.54697 & 1.481 & 6 & .189 \\
\hline Pair 2 & $\begin{array}{l}\text { Enjoy_Curtain - } \\
\text { Enjoy_HMD }\end{array}$ & .83333 & .95864 & .36233 & -.05326 & 1.71992 & 2.300 & 6 & .061 \\
\hline Pair 3 & $\begin{array}{l}\text { Frust_Curtain - } \\
\text { Frust_HMD }\end{array}$ & -.17857 & .86392 & .32653 & -.97757 & .62042 & -.547 & 6 & .604 \\
\hline Pair 4 & $\begin{array}{l}\text { Tired_Curtain - } \\
\text { Tired_HMD }\end{array}$ & -.72619 & .97029 & .36674 & -1.62356 & .17118 & -1.980 & 6 & .095 \\
\hline Pair 5 & $\begin{array}{l}\text { Immer_Curtain - } \\
\text { Immer_HMD }\end{array}$ & .45238 & .66988 & .25319 & -.16716 & 1.07192 & 1.787 & 6 & .124 \\
\hline Pair 6 & $\begin{array}{l}\text { Score_Curtain - } \\
\text { Score_HMD }\end{array}$ & -5.50420 & 22.37367 & 8.45645 & -26.19640 & 15.18800 & -.651 & 6 & .539 \\
\hline
\end{tabular}

Figure E-17: Detailed data analysis of VR4VR's display methods for individuals with ASD. 


\begin{tabular}{|c|c|c|c|c|c|c|c|c|c|c|}
\hline \multicolumn{11}{|c|}{ Independent Samples Test } \\
\hline & & \multicolumn{2}{|c|}{$\begin{array}{l}\text { Levene's Test for Equality of } \\
\text { Variances }\end{array}$} & \multicolumn{7}{|c|}{ t-test for Equality of Means } \\
\hline & & \multirow[b]{2}{*}{$\mathrm{F}$} & \multirow[b]{2}{*}{ Sig. } & \multirow[b]{2}{*}{$t$} & \multirow[b]{2}{*}{ df } & \multirow[b]{2}{*}{ Sig. (2-tailed) } & \multirow{2}{*}{$\begin{array}{c}\text { Mean } \\
\text { Difference }\end{array}$} & \multirow{2}{*}{$\begin{array}{l}\text { Std. Error } \\
\text { Difference }\end{array}$} & \multicolumn{2}{|c|}{$\begin{array}{l}\text { 95\% Confidence Interval of the } \\
\text { Difference }\end{array}$} \\
\hline & & & & & & & & & Lower & Upper \\
\hline \multirow[t]{2}{*}{ Haptic } & $\begin{array}{l}\text { Equal variances } \\
\text { assumed }\end{array}$ & \multirow[t]{2}{*}{26.286} & \multirow[t]{2}{*}{.000} & 1.485 & 14 & .160 & 20.83333 & 14.03039 & -9.25886 & 50.92553 \\
\hline & $\begin{array}{l}\text { Equal variances not } \\
\text { assumed }\end{array}$ & & & 1.198 & 5.762 & .278 & 20.83333 & 17.38498 & -22.13634 & 63.80301 \\
\hline \multirow[t]{2}{*}{ Tangible } & $\begin{array}{l}\text { Equal variances } \\
\text { assumed }\end{array}$ & \multirow[t]{2}{*}{6.397} & \multirow[t]{2}{*}{.024} & 2.333 & 14 & .035 & 8.93333 & 3.82905 & .72085 & 17.14582 \\
\hline & $\begin{array}{l}\text { Equal variances not } \\
\text { assumed }\end{array}$ & & & 2.004 & 6.739 & .087 & 8.93333 & 4.45812 & -1.69179 & 19.55846 \\
\hline \multirow[t]{2}{*}{ T_Snap } & $\begin{array}{l}\text { Equal variances } \\
\text { assumed }\end{array}$ & \multirow[t]{2}{*}{.070} & \multirow[t]{2}{*}{.795} & -.137 & 13 & .893 & -.55556 & 4.06208 & -9.33114 & 8.22003 \\
\hline & $\begin{array}{l}\text { Equal variances not } \\
\text { assumed }\end{array}$ & & & -.133 & 9.750 & .897 & -.55556 & 4.18699 & -9.91726 & 8.80615 \\
\hline \multirow[t]{2}{*}{ T_Screen } & $\begin{array}{l}\text { Equal variances } \\
\text { assumed }\end{array}$ & \multirow[t]{2}{*}{5.999} & \multirow[t]{2}{*}{.026} & 2.521 & 16 & .023 & 22.08333 & 8.75892 & 3.51524 & 40.65142 \\
\hline & $\begin{array}{l}\text { Equal variances not } \\
\text { assumed }\end{array}$ & & & 2.283 & 8.016 & .052 & 22.08333 & 9.67412 & -.21725 & 44.38391 \\
\hline \multirow[t]{2}{*}{ RealW } & $\begin{array}{l}\text { Equal variances } \\
\text { assumed }\end{array}$ & \multirow[t]{2}{*}{2.303} & \multirow[t]{2}{*}{.153} & 2.466 & 13 & .028 & 13.23333 & 5.36659 & 1.63953 & 24.82714 \\
\hline & $\begin{array}{l}\text { Equal variances not } \\
\text { assumed }\end{array}$ & & & 2.113 & 5.685 & .082 & 13.23333 & 6.26346 & -2.30127 & 28.76793 \\
\hline \multirow[t]{2}{*}{ W_inplace } & $\begin{array}{l}\text { Equal variances } \\
\text { assumed }\end{array}$ & \multirow[t]{2}{*}{6.873} & \multirow[t]{2}{*}{.020} & 1.276 & 14 & .223 & 5.64214 & 4.42132 & -3.84065 & 15.12492 \\
\hline & $\begin{array}{l}\text { Equal variances not } \\
\text { assumed }\end{array}$ & & & 1.158 & 7.533 & .282 & 5.64214 & 4.87254 & -5.71623 & 17.00050 \\
\hline \multirow[t]{2}{*}{ Curtain } & $\begin{array}{l}\text { Equal variances } \\
\text { assumed }\end{array}$ & \multirow[t]{2}{*}{25.557} & \multirow[t]{2}{*}{.000} & 1.453 & 17 & .164 & 11.56481 & 7.95775 & -5.22457 & 28.35420 \\
\hline & $\begin{array}{l}\text { Equal variances not } \\
\text { assumed }\end{array}$ & & & 1.383 & 8.904 & .200 & 11.56481 & 8.36004 & -7.37792 & 30.50755 \\
\hline \multirow[t]{2}{*}{ HMD } & $\begin{array}{l}\text { Equal variances } \\
\text { assumed }\end{array}$ & \multirow[t]{2}{*}{12.586} & .003 & 1.889 & 15 & .078 & 7.53081 & 3.98614 & -.96545 & 16.02707 \\
\hline & $\begin{array}{l}\text { Equal variances not } \\
\text { assumed }\end{array}$ & & & 1.598 & 6.573 & .157 & 7.53081 & 4.71352 & -3.76350 & 18.82512 \\
\hline
\end{tabular}

Figure E-18: Detailed score analysis between neurotypical individuals and individuals with ASD 


\section{APPENDIX F: SURVEYS THAT WERE USED IN THE USER STUDIES}

Please answer the following questions

How easy was it to understand this method?

$\square$ Completely easy $\square$ Easy

How easy was it to operate this method?

$\square$ Completely easy $\square$ Easy

How much effort was it to walk with this method?
$\square$ Alot of effort
$\square$ some effort
$\square$ Neutral
Minimal effort
No effort

Did you feel tired when you tried this walking method?
$\square$ Completely tired
$\square$ Tired
$\square$ Neutral
Not tired
Not tired at al

Did you feel like you were in control of the walking?

$\square$ Completely in

$\square$ In control

$\square$ Neutral

Not in control

Not in control at all

Did you enjoy using this walking method?

Did you feel overwhelmed while training?

$\square$ Completely

overwhelmed

Overwhelmed

$\square$ Neutral

Not overwhelmed

Not overwhelmed at all

Did this walking method make you frustrated?
$\square \begin{aligned} & \text { Completely } \\ & \text { frustrated }\end{aligned}$
$\square$ Frustrated
$\square$ Neutral
Not frustrated
$\square$ Not frustrated at all

How are you feeling now? Please select one for each.

\begin{tabular}{|c|l|l|l|l|}
\hline & None & Minimal & Moderate & Major \\
\hline Nauseous & & & & \\
\hline Cold sweating & & & & \\
\hline Drowsiness & & & & \\
\hline Headache & & & & \\
\hline Flushing/warmth & & & & \\
\hline Dizziness & & & & \\
\hline
\end{tabular}

Figure F-1: A sample survey that was used in the user studies about a locomotion technique for neurotypical individuals. 
Did you have the sense of being in the virtual world?

$\square$ Completely in the virtual world

$\square$ Mostly in the virtual world

$\square$ Somewhat in the virtual world

$\square$ Not in the virtual world at all

Did you feel like the virtual world was real?

$\square$ Completely real

$\square$ Mostly real

$\square$ Somewhat real

$\square$ Not real at all

Did you feel like you were visiting the virtual world or viewing a scene?

$\square$ Completely visiting the virtual world

$\square$ Mostly visiting the virtual world

$\square$ Mostly viewing a scene

$\square$ Completely viewing a scene

\section{Additional Comments}

Is there anything you liked/disliked/suggest about this walking method? If so, please describe.

Figure F-1 (Continued) 
Please answer the following questions for the

Stepper Machine technique you tried.

\section{Straightforwardness}

How easy was it to understand this method?

$\square$ Completely easy

$\square$ Easy

$\square$ Neutral

$\square$ Not easy

$\square$ Not easy at all

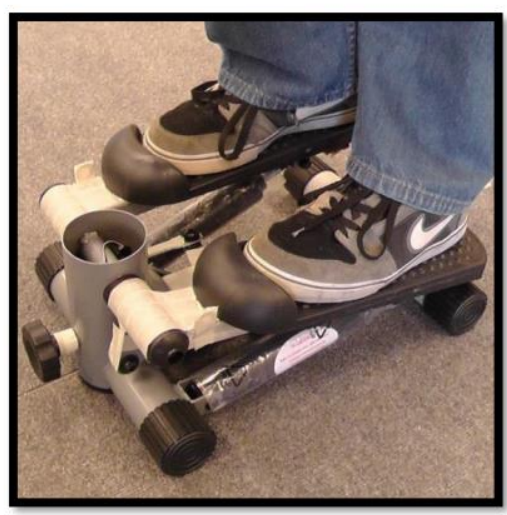

How easy was it to operate this method?

$\square$ Completely easy

$\square$ Easy

$\square$ Neutral

$\square$ Not easy

$\square$ Not easy at all

\section{Effort}

How much effort was it to walk with this method?
$\square$ A lot of effort
$\square$ Some effort
$\square$ Neutral
$\square$ Minimal effort
$\square$ No effort

\section{Tiredness}

Did you feel tired when you tried this walking method?

$\square$ Completely tired

$\square$ Tired

$\square$ Neutral

$\square$ Not tired

$\square$ Not tired at all

Figure F-2: A sample survey that was used in the user studies about the stepper machine locomotion technique for individuals with ASD. 


\section{Control}

Did you feel like you were in control of the walking?

$\square$ Completely in control

$\square$ In control

$\square$ Neutral

$\square$ Not in control

$\square$ Not in control at all

\section{Enjoyment}

Did you enjoy using this walking method?

$\square$ Completely enjoyed

$\square$ Enjoyed

$\square$ Neutral

$\square$ Did not enjoy

$\square$ Did not enjoy at all

\section{Overwhelmedness}

Did you feel overwhelmed while training?

$\square$ Completely overwhelmed

$\square$ Overwhelmed

$\square$ Neutral

$\square$ Not overwhelmed

$\square$ Not overwhelmed at all

\section{Frustration}

Did this walking method make you frustrated?

$\square$ Completely frustrated

$\square$ Frustrated

$\square$ Neutral

$\square$ Not frustrated

$\square$ Not frustrated at all

Figure F-2 (Continued) 


\section{Nauseousness}

Are you feeling nauseous?

$\square$ None

$\square$ Minimal

$\square$ Moderate

$\square$ Major

\section{Cold sweating}

Are you cold sweating?

$\square$ None

$\square$ Minimal

$\square$ Moderate

$\square$ Major

\section{Drowsiness}

Do you feel drowsy?

$\square$ None

$\square$ Minimal

$\square$ Moderate

$\square$ Major

Headache

Do you feel headache?

$\square$ None

$\square$ Minimal

$\square$ Moderate

$\square$ Major

\section{Flushing/warmth}

Do you feel flushing/warmth?

$\square$ None

$\square$ Minimal

$\square$ Moderate

$\square$ Major

Figure F-2 (Continued) 


\section{Dizziness}

Do you feel dizzy?

$\square$ None

$\square$ Minimal

$\square$ Moderate

$\square$ Major

\section{Sense of being}

Did you have the sense of being in the virtual world?

$\square$ Completely in the virtual world

$\square$ Mostly in the virtual world

$\square$ Somewhat in the virtual world

$\square$ Not in the virtual world at all

\section{Reality}

Did you feel like the virtual world was real?

$\square$ Completely real

$\square$ Mostly real

$\square$ Somewhat real

$\square$ Not real at all

\section{Visiting vs viewing}

Did you feel like you were visiting the virtual world or viewing a scene?

$\square$ Completely visiting the virtual world

$\square$ Mostly visiting the virtual world

$\square$ Mostly viewing a scene

$\square$ Completely viewing a scene

\section{Additional Comments}

Is there anything you liked/disliked/suggest about this walking method? If so, please describe.

Figure F-2 (Continued) 


\section{APPENDIX G: DATA ANALYSIS OF THE LOCOMOTION TECHNIQUES}

\section{EXPERIMENT FOR NEUROTYPICAL INDIVIDUALS AND INDIVIDUALS WITH}

\section{ASD}

\begin{tabular}{|l|l|}
\hline Technique & Dependent Variable \\
\hline 1 & RedirectedWalking \\
2 & WalkinPlace \\
3 & Stepper \\
4 & PointandTeleport \\
5 & Joystick \\
6 & Trackball \\
7 & HandFlapping \\
8 & Flying \\
\hline
\end{tabular}

Figure G-1: Pairwise comparison factors for the data analysis of the eight virtual reality locomotion techniques. 


\begin{tabular}{|c|c|c|c|c|c|c|}
\hline \multicolumn{7}{|c|}{ Pairwise Comparisons } \\
\hline & & \multirow{3}{*}{$\begin{array}{c}\text { Mean } \\
\text { Difference (I- } \\
\mathrm{J})\end{array}$} & & & & \\
\hline \multirow[b]{2}{*}{ (I) Technique } & \multirow[b]{2}{*}{ (J) Technique } & & \multirow[b]{2}{*}{ Std. Error } & \multirow[b]{2}{*}{ Sig. } & \multicolumn{2}{|c|}{$\begin{array}{l}95 \% \text { Confidence Interval for } \\
\text { Difference }\end{array}$} \\
\hline & & & & & Lower Bound & Upper Bound \\
\hline \multirow[t]{7}{*}{1} & 2 & 5.842 & .755 & .000 & 4.223 & 7.461 \\
\hline & 3 & 1.530 & 1.737 & .393 & -2.195 & 5.255 \\
\hline & 4 & 7.253 & .978 & .000 & 5.156 & 9.350 \\
\hline & 5 & 6.959 & .877 & .000 & 5.079 & 8.839 \\
\hline & 6 & 7.798 & .878 & .000 & 5.914 & 9.682 \\
\hline & 7 & 4.146 & 1.049 & .001 & 1.896 & 6.397 \\
\hline & 8 & 5.183 & 1.250 & .001 & 2.502 & 7.864 \\
\hline \multirow[t]{7}{*}{2} & 1 & -5.842 & .755 & .000 & -7.461 & -4.223 \\
\hline & 3 & -4.312 & 1.441 & .010 & -7.402 & -1.221 \\
\hline & 4 & 1.411 & .477 & .010 & .388 & 2.435 \\
\hline & 5 & 1.118 & .419 & .018 & .220 & 2.015 \\
\hline & 6 & 1.956 & .552 & .003 & .773 & 3.139 \\
\hline & 7 & -1.695 & .610 & .015 & -3.004 & -.387 \\
\hline & 8 & -.659 & .914 & .483 & -2.620 & 1.303 \\
\hline \multirow[t]{7}{*}{3} & 1 & -1.530 & 1.737 & .393 & -5.255 & 2.195 \\
\hline & 2 & 4.312 & 1.441 & .010 & 1.221 & 7.402 \\
\hline & 4 & 5.723 & 1.411 & .001 & 2.697 & 8.748 \\
\hline & 5 & 5.429 & 1.235 & .001 & 2.780 & 8.079 \\
\hline & 6 & 6.268 & 1.282 & .000 & 3.519 & 9.017 \\
\hline & 7 & 2.616 & 1.416 & .086 & -.421 & 5.653 \\
\hline & 8 & 3.653 & 1.057 & .004 & 1.385 & 5.921 \\
\hline \multirow[t]{7}{*}{4} & 1 & -7.253 & .978 & .000 & -9.350 & -5.156 \\
\hline & 2 & -1.411 & .477 & .010 & -2.435 & -.388 \\
\hline & 3 & -5.723 & 1.411 & .001 & -8.748 & -2.697 \\
\hline & 5 & -.293 & .556 & .606 & -1.487 & .900 \\
\hline & 6 & .545 & .657 & .421 & -.864 & 1.955 \\
\hline & 7 & -3.106 & .845 & .002 & -4.918 & -1.295 \\
\hline & 8 & -2.070 & .848 & .029 & -3.888 & -.252 \\
\hline \multirow[t]{7}{*}{5} & 1 & -6.959 & .877 & .000 & -8.839 & -5.079 \\
\hline & 2 & -1.118 & .419 & .018 & -2.015 & -.220 \\
\hline & 3 & -5.429 & 1.235 & .001 & -8.079 & -2.780 \\
\hline & 4 & .293 & .556 & .606 & -.900 & 1.487 \\
\hline & 6 & .839 & .346 & .029 & .097 & 1.581 \\
\hline & 7 & -2.813 & .602 & .000 & -4.105 & -1.521 \\
\hline & 8 & -1.776 & .624 & .013 & -3.114 & -.439 \\
\hline \multirow[t]{7}{*}{6} & 1 & -7.798 & .878 & .000 & -9.682 & -5.914 \\
\hline & 2 & -1.956 & .552 & .003 & -3.139 & -.773 \\
\hline & 3 & -6.268 & 1.282 & .000 & -9.017 & -3.519 \\
\hline & 4 & -.545 & .657 & .421 & -1.955 & .864 \\
\hline & 5 & -.839 & .346 & .029 & -1.581 & -.097 \\
\hline & 7 & -3.652 & .598 & .000 & -4.934 & -2.369 \\
\hline & 8 & -2.615 & .694 & .002 & -4.103 & -1.127 \\
\hline \multirow[t]{7}{*}{7} & 1 & -4.146 & 1.049 & .001 & -6.397 & -1.896 \\
\hline & 2 & 1.695 & .610 & .015 & .387 & 3.004 \\
\hline & 3 & -2.616 & 1.416 & .086 & -5.653 & .421 \\
\hline & 4 & 3.106 & .845 & .002 & 1.295 & 4.918 \\
\hline & 5 & 2.813 & .602 & .000 & 1.521 & 4.105 \\
\hline & 6 & 3.652 & .598 & .000 & 2.369 & 4.934 \\
\hline & 8 & 1.037 & .820 & .227 & -.721 & 2.795 \\
\hline \multirow[t]{7}{*}{8} & 1 & -5.183 & 1.250 & .001 & -7.864 & -2.502 \\
\hline & 2 & .659 & .914 & .483 & -1.303 & 2.620 \\
\hline & 3 & -3.653 & 1.057 & .004 & -5.921 & -1.385 \\
\hline & 4 & 2.070 & .848 & .029 & .252 & 3.888 \\
\hline & 5 & 1.776 & .624 & .013 & .439 & 3.114 \\
\hline & 6 & 2.615 & .694 & .002 & 1.127 & 4.103 \\
\hline & 7 & -1.037 & .820 & .227 & -2.795 & .721 \\
\hline
\end{tabular}

Figure G-2: Detailed data analysis for the completion time without obstacles for neurotypical individuals. 


\section{Mauchly's Test of Sphericity ${ }^{a}$}

Measure: CompletionTime

\begin{tabular}{|l|r|r|r|r|r|r|r|}
\hline & & & & \multicolumn{3}{|c|}{ Epsilon $^{\text {b }}$} \\
\cline { 6 - 9 } Within Subjects Effect & Mauchly's W & $\begin{array}{c}\text { Approx. Chi- } \\
\text { Square }\end{array}$ & df & Sig. & $\begin{array}{c}\text { Greenhouse- } \\
\text { Geisser }\end{array}$ & Huynh-Feldt & Lower-bound \\
\hline Technique & .003 & 67.944 & 27 & .000 & .381 & .478 & .143 \\
\hline
\end{tabular}

Tests the null hypothesis that the error covariance matrix of the orthonormalized transformed dependent variables is proportional to an identity matrix.

a. Design: Intercept

Within Subjects Design: Technique

b. May be used to adjust the degrees of freedom for the averaged tests of significance. Corrected tests are displayed in the Tests of Within-Subjects Effects table.

Tests of Within-Subjects Effects

Measure: CompletionTime

\begin{tabular}{|c|c|c|c|c|c|c|c|c|c|}
\hline Source & & $\begin{array}{c}\text { Type III Sum } \\
\text { of Squares }\end{array}$ & df & Mean Square & $\mathrm{F}$ & Sig. & $\begin{array}{l}\text { Partial Eta } \\
\text { Squared }\end{array}$ & $\begin{array}{l}\text { Noncent. } \\
\text { Parameter }\end{array}$ & $\begin{array}{l}\text { Observed } \\
\text { Power }^{\text {a }}\end{array}$ \\
\hline \multirow[t]{4}{*}{ Technique } & Sphericity Assumed & 825.704 & 7 & 117.958 & 17.220 & .000 & .552 & 120.538 & 1.000 \\
\hline & Greenhouse-Geisser & 825.704 & 2.665 & 309.891 & 17.220 & .000 & .552 & 45.882 & 1.000 \\
\hline & Huynh-Feldt & 825.704 & 3.349 & 246.520 & 17.220 & .000 & .552 & 57.676 & 1.000 \\
\hline & Lower-bound & 825.704 & 1.000 & 825.704 & 17.220 & .001 & .552 & 17.220 & .971 \\
\hline \multirow[t]{4}{*}{ Error(Technique) } & Sphericity Assumed & 671.318 & 98 & 6.850 & & & & & \\
\hline & Greenhouse-Geisser & 671.318 & 37.303 & 17.996 & & & & & \\
\hline & Huynh-Feldt & 671.318 & 46.892 & 14.316 & & & & & \\
\hline & Lower-bound & 671.318 & 14.000 & 47.951 & & & & & \\
\hline
\end{tabular}

a. Computed using alpha $=.05$

Figure G-2 (Continued) 


\begin{tabular}{|c|c|c|c|c|c|c|}
\hline \multicolumn{7}{|c|}{ Pairwise Comparisons } \\
\hline & & & & & & \\
\hline \multirow[b]{2}{*}{ (I) Technique } & \multirow[b]{2}{*}{ (J) Technique } & \multirow{2}{*}{$\begin{array}{c}\text { Mean } \\
\text { Difference (I- } \\
\mathrm{J}) \\
\end{array}$} & \multirow[b]{2}{*}{ Std. Error } & \multirow[b]{2}{*}{ Sig. } & \multicolumn{2}{|c|}{$\begin{array}{l}95 \% \text { Confidence Interval for } \\
\text { Difference }\end{array}$} \\
\hline & & & & & Lower Bound & Upper Bound \\
\hline \multirow[t]{7}{*}{1} & 2 & 7.947 & 2.132 & .003 & 3.256 & 12.639 \\
\hline & 3 & 4.808 & 2.145 & .047 & .087 & 9.529 \\
\hline & 4 & 9.549 & 2.225 & .001 & 4.652 & 14.446 \\
\hline & 5 & 9.146 & 2.328 & .002 & 4.021 & 14.271 \\
\hline & 6 & 9.256 & 2.234 & .002 & 4.339 & 14.173 \\
\hline & 7 & 2.201 & 3.175 & .503 & -4.787 & 9.190 \\
\hline & 8 & 7.469 & 2.064 & .004 & 2.926 & 12.011 \\
\hline \multirow[t]{7}{*}{2} & 1 & -7.947 & 2.132 & .003 & -12.639 & -3.256 \\
\hline & 3 & -3.140 & .630 & .000 & -4.526 & -1.753 \\
\hline & 4 & 1.601 & .641 & .030 & .191 & 3.012 \\
\hline & 5 & 1.198 & .502 & .036 & .093 & 2.303 \\
\hline & 6 & 1.309 & .837 & .146 & -.533 & 3.150 \\
\hline & 7 & -5.746 & 2.574 & .047 & -11.411 & -.081 \\
\hline & 8 & -.479 & .826 & .574 & -2.297 & 1.340 \\
\hline \multirow[t]{7}{*}{3} & 1 & -4.808 & 2.145 & .047 & -9.529 & -.087 \\
\hline & 2 & 3.140 & .630 & .000 & 1.753 & 4.526 \\
\hline & 4 & 4.741 & .442 & .000 & 3.769 & 5.713 \\
\hline & 5 & 4.338 & .464 & .000 & 3.316 & 5.360 \\
\hline & 6 & 4.448 & .646 & .000 & 3.026 & 5.870 \\
\hline & 7 & -2.607 & 2.288 & .279 & -7.643 & 2.430 \\
\hline & 8 & 2.661 & .932 & .016 & .609 & 4.713 \\
\hline \multirow[t]{7}{*}{4} & 1 & -9.549 & 2.225 & .001 & -14.446 & -4.652 \\
\hline & 2 & -1.601 & .641 & .030 & -3.012 & -.191 \\
\hline & 3 & -4.741 & .442 & .000 & -5.713 & -3.769 \\
\hline & 5 & -.403 & .380 & .312 & -1.240 & .433 \\
\hline & 6 & -.293 & .614 & .643 & -1.644 & 1.058 \\
\hline & 7 & -7.348 & 2.265 & .008 & -12.333 & -2.363 \\
\hline & 8 & -2.080 & .781 & .022 & -3.799 & -.362 \\
\hline \multirow[t]{7}{*}{5} & 1 & -9.146 & 2.328 & .002 & -14.271 & -4.021 \\
\hline & 2 & -1.198 & .502 & .036 & -2.303 & -.093 \\
\hline & 3 & -4.338 & .464 & .000 & -5.360 & -3.316 \\
\hline & 4 & .403 & .380 & .312 & -.433 & 1.240 \\
\hline & 6 & .110 & .741 & .884 & -1.520 & 1.740 \\
\hline & 7 & -6.945 & 2.503 & .018 & -12.453 & -1.436 \\
\hline & 8 & -1.677 & .912 & .093 & -3.685 & .331 \\
\hline \multirow[t]{7}{*}{6} & 1 & -9.256 & 2.234 & .002 & -14.173 & -4.339 \\
\hline & 2 & -1.309 & .837 & .146 & -3.150 & .533 \\
\hline & 3 & -4.448 & .646 & .000 & -5.870 & -3.026 \\
\hline & 4 & .293 & .614 & .643 & -1.058 & 1.644 \\
\hline & 5 & -.110 & .741 & .884 & -1.740 & 1.520 \\
\hline & 7 & -7.055 & 2.135 & .007 & -11.754 & -2.356 \\
\hline & 8 & -1.787 & 1.049 & .116 & -4.096 & .521 \\
\hline \multirow[t]{7}{*}{7} & 1 & -2.201 & 3.175 & .503 & -9.190 & 4.787 \\
\hline & 2 & 5.746 & 2.574 & .047 & .081 & 11.411 \\
\hline & 3 & 2.607 & 2.288 & .279 & -2.430 & 7.643 \\
\hline & 4 & 7.348 & 2.265 & .008 & 2.363 & 12.333 \\
\hline & 5 & 6.945 & 2.503 & .018 & 1.436 & 12.453 \\
\hline & 6 & 7.055 & 2.135 & .007 & 2.356 & 11.754 \\
\hline & 8 & 5.268 & 2.591 & .067 & -.435 & 10.970 \\
\hline \multirow[t]{7}{*}{8} & 1 & -7.469 & 2.064 & .004 & -12.011 & -2.926 \\
\hline & 2 & .479 & .826 & .574 & -1.340 & 2.297 \\
\hline & 3 & -2.661 & .932 & .016 & -4.713 & -.609 \\
\hline & 4 & 2.080 & .781 & .022 & .362 & 3.799 \\
\hline & 5 & 1.677 & .912 & .093 & -.331 & 3.685 \\
\hline & 6 & 1.787 & 1.049 & .116 & -.521 & 4.096 \\
\hline & 7 & -5.268 & 2.591 & .067 & -10.970 & .435 \\
\hline
\end{tabular}

Figure G-3: Detailed data analysis for the completion time without obstacles for individuals with ASD. 


\section{Mauchly's Test of Sphericity ${ }^{a}$}

Measure: CompletionTime

\begin{tabular}{|l|r|r|r|r|r|r|r|}
\hline & & & & \multicolumn{3}{|c|}{ Epsilon $^{\text {b }}$} \\
\cline { 6 - 9 } Within Subjects Effect & Mauchly's W & $\begin{array}{c}\text { Approx. Chi- } \\
\text { Square }\end{array}$ & df & Sig. & $\begin{array}{c}\text { Greenhouse- } \\
\text { Geisser }\end{array}$ & Huynh-Feldt & Lower-bound \\
\hline Technique & .000 & 85.029 & 27 & .000 & .341 & .442 & .143 \\
\hline
\end{tabular}

Tests the null hypothesis that the error covariance matrix of the orthonormalized transformed dependent variables is proportional to an identity matrix.

a. Design: Intercept

Within Subjects Design: Technique

b. May be used to adjust the degrees of freedom for the averaged tests of significance. Corrected tests are displayed in the Tests of Within-Subjects Effects table.

Tests of Within-Subjects Effects

Measure: CompletionTime

\begin{tabular}{|c|c|c|c|c|c|c|c|c|c|}
\hline Source & & $\begin{array}{l}\text { Type III Sum } \\
\text { of Squares }\end{array}$ & df & Mean Square & $\mathrm{F}$ & Sig. & $\begin{array}{l}\text { Partial Eta } \\
\text { Squared }\end{array}$ & $\begin{array}{l}\text { Noncent. } \\
\text { Parameter }\end{array}$ & $\begin{array}{l}\text { Observed } \\
\text { Power }^{\mathrm{a}}\end{array}$ \\
\hline \multirow[t]{4}{*}{ Technique } & Sphericity Assumed & 1082.267 & 7 & 154.610 & 8.894 & .000 & .447 & 62.255 & 1.000 \\
\hline & Greenhouse-Geisser & 1082.267 & 2.386 & 453.522 & 8.894 & .001 & .447 & 21.223 & .973 \\
\hline & Huynh-Feldt & 1082.267 & 3.092 & 349.983 & 8.894 & .000 & .447 & 27.502 & .992 \\
\hline & Lower-bound & 1082.267 & 1.000 & 1082.267 & 8.894 & .012 & .447 & 8.894 & .775 \\
\hline \multirow[t]{4}{*}{ Error(Technique) } & Sphericity Assumed & 1338.594 & 77 & 17.384 & & & & & \\
\hline & Greenhouse-Geisser & 1338.594 & 26.250 & 50.994 & & & & & \\
\hline & Huynh-Feldt & 1338.594 & 34.016 & 39.352 & & & & & \\
\hline & Lower-bound & 1338.594 & 11.000 & 121.690 & & & & & \\
\hline
\end{tabular}

a. Computed using alpha $=.05$

Figure G-3 (Continued) 


\begin{tabular}{|c|c|c|c|c|c|c|}
\hline \multicolumn{7}{|c|}{ Pairwise Comparisons } \\
\hline & & & & & & \\
\hline \multirow[b]{2}{*}{ (I) Technique } & \multirow[b]{2}{*}{ (J) Technique } & \multirow{2}{*}{$\begin{array}{c}\text { Mean } \\
\text { Difference (I- } \\
\text { J) }\end{array}$} & \multirow[b]{2}{*}{ Std. Error } & \multirow[b]{2}{*}{ Sig. } & \multicolumn{2}{|c|}{$\begin{array}{l}95 \% \text { Confidence Interval for } \\
\text { Difference }\end{array}$} \\
\hline & & & & & Lower Bound & Upper Bound \\
\hline \multirow[t]{7}{*}{1} & 2 & 2.513 & 1.472 & .110 & -.645 & 5.671 \\
\hline & 3 & 1.815 & 1.426 & .224 & -1.243 & 4.873 \\
\hline & 4 & 1.435 & 1.307 & .291 & -1.368 & 4.239 \\
\hline & 5 & 6.652 & .848 & .000 & 4.835 & 8.470 \\
\hline & 6 & 6.808 & 1.429 & .000 & 3.743 & 9.872 \\
\hline & 7 & .153 & 2.239 & .947 & -4.649 & 4.954 \\
\hline & 8 & 5.400 & .893 & .000 & 3.485 & 7.315 \\
\hline \multirow[t]{7}{*}{2} & 1 & -2.513 & 1.472 & .110 & -5.671 & .645 \\
\hline & 3 & -.698 & 1.122 & .544 & -3.105 & 1.709 \\
\hline & 4 & -1.077 & 1.580 & .506 & -4.466 & 2.312 \\
\hline & 5 & 4.140 & 1.285 & .006 & 1.384 & 6.895 \\
\hline & 6 & 4.295 & 1.407 & .009 & 1.278 & 7.312 \\
\hline & 7 & -2.360 & 1.366 & .106 & -5.290 & .570 \\
\hline & 8 & 2.887 & 1.148 & .025 & .425 & 5.349 \\
\hline \multirow[t]{7}{*}{3} & 1 & -1.815 & 1.426 & .224 & -4.873 & 1.243 \\
\hline & 2 & .698 & 1.122 & .544 & -1.709 & 3.105 \\
\hline & 4 & -.379 & 1.503 & .804 & -3.604 & 2.845 \\
\hline & 5 & 4.838 & .972 & .000 & 2.754 & 6.921 \\
\hline & 6 & 4.993 & .806 & .000 & 3.263 & 6.723 \\
\hline & 7 & -1.662 & 1.511 & .290 & -4.902 & 1.578 \\
\hline & 8 & 3.585 & .907 & .001 & 1.639 & 5.531 \\
\hline \multirow[t]{7}{*}{4} & 1 & -1.435 & 1.307 & .291 & -4.239 & 1.368 \\
\hline & 2 & 1.077 & 1.580 & .506 & -2.312 & 4.466 \\
\hline & 3 & .379 & 1.503 & .804 & -2.845 & 3.604 \\
\hline & 5 & 5.217 & 1.134 & .000 & 2.784 & 7.650 \\
\hline & 6 & 5.372 & 1.668 & .006 & 1.796 & 8.949 \\
\hline & 7 & -1.283 & 2.069 & .545 & -5.720 & 3.154 \\
\hline & 8 & 3.964 & 1.308 & .009 & 1.159 & 6.770 \\
\hline \multirow[t]{7}{*}{5} & 1 & -6.652 & .848 & .000 & -8.470 & -4.835 \\
\hline & 2 & -4.140 & 1.285 & .006 & -6.895 & -1.384 \\
\hline & 3 & -4.838 & .972 & .000 & -6.921 & -2.754 \\
\hline & 4 & -5.217 & 1.134 & .000 & -7.650 & -2.784 \\
\hline & 6 & .155 & .999 & .879 & -1.987 & 2.298 \\
\hline & 7 & -6.500 & 1.945 & .005 & -10.671 & -2.329 \\
\hline & 8 & -1.253 & .649 & .074 & -2.643 & .138 \\
\hline \multirow[t]{7}{*}{6} & 1 & -6.808 & 1.429 & .000 & -9.872 & -3.743 \\
\hline & 2 & -4.295 & 1.407 & .009 & -7.312 & -1.278 \\
\hline & 3 & -4.993 & .806 & .000 & -6.723 & -3.263 \\
\hline & 4 & -5.372 & 1.668 & .006 & -8.949 & -1.796 \\
\hline & 5 & -.155 & .999 & .879 & -2.298 & 1.987 \\
\hline & 7 & -6.655 & 1.946 & .004 & -10.829 & -2.482 \\
\hline & 8 & -1.408 & .780 & .093 & -3.080 & .265 \\
\hline \multirow[t]{7}{*}{7} & 1 & -.153 & 2.239 & .947 & -4.954 & 4.649 \\
\hline & 2 & 2.360 & 1.366 & .106 & -.570 & 5.290 \\
\hline & 3 & 1.662 & 1.511 & .290 & -1.578 & 4.902 \\
\hline & 4 & 1.283 & 2.069 & .545 & -3.154 & 5.720 \\
\hline & 5 & 6.500 & 1.945 & .005 & 2.329 & 10.671 \\
\hline & 6 & 6.655 & 1.946 & .004 & 2.482 & 10.829 \\
\hline & 8 & 5.247 & 1.919 & .016 & 1.131 & 9.364 \\
\hline \multirow[t]{7}{*}{8} & 1 & -5.400 & .893 & .000 & -7.315 & -3.485 \\
\hline & 2 & -2.887 & 1.148 & .025 & -5.349 & -.425 \\
\hline & 3 & -3.585 & .907 & .001 & -5.531 & -1.639 \\
\hline & 4 & -3.964 & 1.308 & .009 & -6.770 & -1.159 \\
\hline & 5 & 1.253 & .649 & .074 & -.138 & 2.643 \\
\hline & 6 & 1.408 & .780 & .093 & -.265 & 3.080 \\
\hline & 7 & -5.247 & 1.919 & .016 & -9.364 & -1.131 \\
\hline
\end{tabular}

Figure G-4: Detailed data analysis for the completion time with obstacles for neurotypical individuals. 


\section{Mauchly's Test of Sphericity ${ }^{a}$}

Measure: CompletionTime

\begin{tabular}{|l|r|r|r|r|r|r|r|}
\hline & & & & \multicolumn{3}{|c|}{ Epsilon $^{\text {b }}$} \\
\cline { 6 - 9 } Within Subjects Effect & Mauchly's W & $\begin{array}{c}\text { Approx. Chi- } \\
\text { Square }\end{array}$ & df & Sig. & $\begin{array}{c}\text { Greenhouse- } \\
\text { Geisser }\end{array}$ & Huynh-Feldt & Lower-bound \\
\hline Technique & .011 & 51.499 & 27 & .004 & .445 & .587 & .143 \\
\hline
\end{tabular}

Tests the null hypothesis that the error covariance matrix of the orthonormalized transformed dependent variables is proportional to an identity matrix.

a. Design: Intercept

Within Subjects Design: Technique

b. May be used to adjust the degrees of freedom for the averaged tests of significance. Corrected tests are displayed in the Tests of Within-Subjects Effects table.

Tests of Within-Subjects Effects

Measure: CompletionTime

\begin{tabular}{|c|c|c|c|c|c|c|c|c|c|}
\hline Source & & $\begin{array}{l}\text { Type III Sum } \\
\text { of Squares }\end{array}$ & $d f$ & Mean Square & $\mathrm{F}$ & Sig. & $\begin{array}{c}\text { Partial Eta } \\
\text { Squared }\end{array}$ & $\begin{array}{l}\text { Noncent. } \\
\text { Parameter }\end{array}$ & $\begin{array}{l}\text { Observed } \\
\text { Power }^{\mathrm{a}}\end{array}$ \\
\hline \multirow[t]{4}{*}{ Technique } & Sphericity Assumed & 820.829 & 7 & 117.261 & 7.910 & .000 & .361 & 55.371 & 1.000 \\
\hline & Greenhouse-Geisser & 820.829 & 3.114 & 263.598 & 7.910 & .000 & .361 & 24.632 & .986 \\
\hline & Huynh-Feldt & 820.829 & 4.107 & 199.861 & 7.910 & .000 & .361 & 32.487 & .997 \\
\hline & Lower-bound & 820.829 & 1.000 & 820.829 & 7.910 & .014 & .361 & 7.910 & .744 \\
\hline \multirow[t]{4}{*}{ Error(Technique) } & Sphericity Assumed & 1452.762 & 98 & 14.824 & & & & & \\
\hline & Greenhouse-Geisser & 1452.762 & 43.595 & 33.324 & & & & & \\
\hline & Huynh-Feldt & 1452.762 & 57.498 & 25.266 & & & & & \\
\hline & Lower-bound & 1452.762 & 14.000 & 103.769 & & & & & \\
\hline
\end{tabular}

a. Computed using alpha $=.05$

Figure G-4 (Continued) 


\begin{tabular}{|c|c|c|c|c|c|c|}
\hline \multicolumn{7}{|c|}{ Pairwise Comparisons } \\
\hline & & & & & & \\
\hline \multirow[b]{2}{*}{ (I) Technique } & \multirow[b]{2}{*}{ (J) Technique } & \multirow{2}{*}{$\begin{array}{c}\text { Mean } \\
\text { Difference (I- } \\
\mathrm{J})\end{array}$} & \multirow[b]{2}{*}{ Std. Error } & \multirow[b]{2}{*}{ Sig. } & \multicolumn{2}{|c|}{$\begin{array}{l}95 \% \text { Confidence Interval for } \\
\text { Difference }\end{array}$} \\
\hline & & & & & Lower Bound & Upper Bound \\
\hline \multirow[t]{7}{*}{1} & 2 & 7.986 & 2.062 & .003 & 3.447 & 12.525 \\
\hline & 3 & 7.769 & 3.272 & .037 & .567 & 14.972 \\
\hline & 4 & 9.586 & 2.457 & .002 & 4.178 & 14.994 \\
\hline & 5 & 12.774 & 2.252 & .000 & 7.817 & 17.731 \\
\hline & 6 & 11.531 & 3.120 & .004 & 4.665 & 18.398 \\
\hline & 7 & 7.222 & 2.346 & .011 & 2.058 & 12.386 \\
\hline & 8 & 11.092 & 1.948 & .000 & 6.803 & 15.380 \\
\hline \multirow[t]{7}{*}{2} & 1 & -7.986 & 2.062 & .003 & -12.525 & -3.447 \\
\hline & 3 & -.216 & 2.311 & .927 & -5.302 & 4.869 \\
\hline & 4 & 1.600 & 1.737 & .377 & -2.223 & 5.424 \\
\hline & 5 & 4.788 & 1.388 & .005 & 1.732 & 7.844 \\
\hline & 6 & 3.546 & 2.283 & .149 & -1.480 & 8.571 \\
\hline & 7 & -.764 & 1.841 & .686 & -4.817 & 3.289 \\
\hline & 8 & 3.106 & 1.074 & .015 & .742 & 5.470 \\
\hline \multirow[t]{7}{*}{3} & 1 & -7.769 & 3.272 & .037 & -14.972 & -.567 \\
\hline & 2 & .216 & 2.311 & .927 & -4.869 & 5.302 \\
\hline & 4 & 1.817 & 1.864 & .351 & -2.286 & 5.919 \\
\hline & 5 & 5.005 & 1.852 & .021 & .929 & 9.080 \\
\hline & 6 & 3.762 & 1.474 & .027 & .519 & 7.005 \\
\hline & 7 & -.547 & 2.084 & .798 & -5.135 & 4.040 \\
\hline & 8 & 3.322 & 1.754 & .085 & -.538 & 7.183 \\
\hline \multirow[t]{7}{*}{4} & 1 & -9.586 & 2.457 & .002 & -14.994 & -4.178 \\
\hline & 2 & -1.600 & 1.737 & .377 & -5.424 & 2.223 \\
\hline & 3 & -1.817 & 1.864 & .351 & -5.919 & 2.286 \\
\hline & 5 & 3.188 & 1.409 & .045 & .086 & 6.290 \\
\hline & 6 & 1.945 & 2.207 & .397 & -2.912 & 6.803 \\
\hline & 7 & -2.364 & 2.015 & .266 & -6.800 & 2.072 \\
\hline & 8 & 1.506 & 1.297 & .270 & -1.349 & 4.360 \\
\hline \multirow[t]{7}{*}{5} & 1 & -12.774 & 2.252 & .000 & -17.731 & -7.817 \\
\hline & 2 & -4.788 & 1.388 & .005 & -7.844 & -1.732 \\
\hline & 3 & -5.005 & 1.852 & .021 & -9.080 & -.929 \\
\hline & 4 & -3.188 & 1.409 & .045 & -6.290 & -.086 \\
\hline & 6 & -1.242 & 1.265 & .347 & -4.027 & 1.542 \\
\hline & 7 & -5.552 & .880 & .000 & -7.489 & -3.615 \\
\hline & 8 & -1.682 & .837 & .070 & -3.523 & .159 \\
\hline \multirow[t]{7}{*}{6} & 1 & -11.531 & 3.120 & .004 & -18.398 & -4.665 \\
\hline & 2 & -3.546 & 2.283 & .149 & -8.571 & 1.480 \\
\hline & 3 & -3.762 & 1.474 & .027 & -7.005 & -.519 \\
\hline & 4 & -1.945 & 2.207 & .397 & -6.803 & 2.912 \\
\hline & 5 & 1.242 & 1.265 & .347 & -1.542 & 4.027 \\
\hline & 7 & -4.309 & 1.238 & .005 & -7.035 & -1.584 \\
\hline & 8 & -.440 & 1.743 & .806 & -4.277 & 3.397 \\
\hline \multirow[t]{7}{*}{7} & 1 & -7.222 & 2.346 & .011 & -12.386 & -2.058 \\
\hline & 2 & .764 & 1.841 & .686 & -3.289 & 4.817 \\
\hline & 3 & .547 & 2.084 & .798 & -4.040 & 5.135 \\
\hline & 4 & 2.364 & 2.015 & .266 & -2.072 & 6.800 \\
\hline & 5 & 5.552 & .880 & .000 & 3.615 & 7.489 \\
\hline & 6 & 4.309 & 1.238 & .005 & 1.584 & 7.035 \\
\hline & 8 & 3.870 & 1.326 & .014 & .951 & 6.789 \\
\hline \multirow[t]{7}{*}{8} & 1 & -11.092 & 1.948 & .000 & -15.380 & -6.803 \\
\hline & 2 & -3.106 & 1.074 & .015 & -5.470 & -.742 \\
\hline & 3 & -3.322 & 1.754 & .085 & -7.183 & .538 \\
\hline & 4 & -1.506 & 1.297 & .270 & -4.360 & 1.349 \\
\hline & 5 & 1.682 & .837 & .070 & -.159 & 3.523 \\
\hline & 6 & .440 & 1.743 & .806 & -3.397 & 4.277 \\
\hline & 7 & -3.870 & 1.326 & .014 & -6.789 & -.951 \\
\hline
\end{tabular}

Figure G-5: Detailed data analysis for the completion time with obstacles for individuals with ASD. 


\section{Mauchly's Test of Sphericity ${ }^{a}$}

Measure: CompletionTime

\begin{tabular}{|l|r|r|r|r|r|r|r|}
\hline & & & & \multicolumn{3}{|c|}{ Epsilon $^{\text {b }}$} \\
\cline { 6 - 9 } Within Subjects Effect & Mauchly's W & $\begin{array}{c}\text { Approx. Chi- } \\
\text { Square }\end{array}$ & df & Sig. & $\begin{array}{c}\text { Greenhouse- } \\
\text { Geisser }\end{array}$ & Huynh-Feldt & Lower-bound \\
\hline Technique & .001 & 63.502 & 27 & .000 & .403 & .556 & .143 \\
\hline
\end{tabular}

Tests the null hypothesis that the error covariance matrix of the orthonormalized transformed dependent variables is proportional to an identity matrix.

a. Design: Intercept

Within Subjects Design: Technique

b. May be used to adjust the degrees of freedom for the averaged tests of significance. Corrected tests are displayed in the Tests of Within-Subjects Effects table.

Tests of Within-Subjects Effects

Measure: CompletionTime

\begin{tabular}{|c|c|c|c|c|c|c|c|c|c|}
\hline Source & & $\begin{array}{l}\text { Type III Sum } \\
\text { of Squares }\end{array}$ & df & Mean Square & $\mathrm{F}$ & Sig. & $\begin{array}{c}\text { Partial Eta } \\
\text { Squared }\end{array}$ & $\begin{array}{l}\text { Noncent. } \\
\text { Parameter }\end{array}$ & $\begin{array}{l}\text { Observed } \\
\text { Power }^{\text {a }}\end{array}$ \\
\hline \multirow[t]{4}{*}{ Technique } & Sphericity Assumed & 1320.418 & 7 & 188.631 & 8.493 & .000 & 436 & 59.449 & 1.000 \\
\hline & Greenhouse-Geisser & 1320.418 & 2.818 & 468.552 & 8.493 & .000 & .436 & 23.933 & .983 \\
\hline & Huynh-Feldt & 1320.418 & 3.889 & 339.553 & 8.493 & .000 & .436 & 33.026 & .997 \\
\hline & Lower-bound & 1320.418 & 1.000 & 1320.418 & 8.493 & .014 & .436 & 8.493 & .756 \\
\hline \multirow[t]{4}{*}{ Error(Technique) } & Sphericity Assumed & 1710.235 & 77 & 22.211 & & & & & \\
\hline & Greenhouse-Geisser & 1710.235 & 30.999 & 55.171 & & & & & \\
\hline & Huynh-Feldt & 1710.235 & 42.776 & 39.982 & & & & & \\
\hline & Lower-bound & 1710.235 & 11.000 & 155.476 & & & & & \\
\hline
\end{tabular}

a. Computed using alpha $=.05$

Figure G-5 (Continued) 


\begin{tabular}{|c|c|c|c|c|c|c|}
\hline \multicolumn{7}{|c|}{ Pairwise Comparisons } \\
\hline & & & \multirow[b]{3}{*}{ Std. Error } & & & \\
\hline \multirow[b]{2}{*}{ (l) Technique } & \multirow[b]{2}{*}{ (J) Technique } & \multirow{2}{*}{$\begin{array}{c}\text { Mean } \\
\text { Difference (I- } \\
\mathrm{J})\end{array}$} & & \multirow[b]{2}{*}{ Sig. } & \multicolumn{2}{|c|}{$\begin{array}{l}95 \% \text { Confidence Interval for } \\
\text { Difference }\end{array}$} \\
\hline & & & & & Lower Bound & Upper Bound \\
\hline \multirow[t]{7}{*}{1} & 2 & 2.933 & .848 & .004 & 1.115 & 4.752 \\
\hline & 3 & 2.600 & .914 & .013 & .639 & 4.561 \\
\hline & 4 & 3.800 & .890 & .001 & 1.891 & 5.709 \\
\hline & 5 & 2.000 & .834 & .031 & .212 & 3.788 \\
\hline & 6 & 2.400 & .838 & .013 & .602 & 4.198 \\
\hline & 7 & 1.133 & .894 & .225 & -.783 & 3.050 \\
\hline & 8 & 2.133 & .742 & .012 & .541 & 3.726 \\
\hline \multirow[t]{7}{*}{2} & 1 & -2.933 & .848 & .004 & -4.752 & -1.115 \\
\hline & 3 & -.333 & .475 & .494 & -1.352 & .685 \\
\hline & 4 & .867 & .291 & .010 & .243 & 1.490 \\
\hline & 5 & -.933 & .431 & .048 & -1.857 & -.010 \\
\hline & 6 & -.533 & .363 & .164 & -1.313 & .246 \\
\hline & 7 & -1.800 & .571 & .007 & -3.024 & -.576 \\
\hline & 8 & -.800 & .509 & .138 & -1.892 & .292 \\
\hline \multirow[t]{7}{*}{3} & 1 & -2.600 & .914 & .013 & -4.561 & -.639 \\
\hline & 2 & .333 & .475 & .494 & -.685 & 1.352 \\
\hline & 4 & 1.200 & .355 & .004 & .440 & 1.960 \\
\hline & 5 & -.600 & .466 & .219 & -1.599 & .399 \\
\hline & 6 & -.200 & .368 & .595 & -.989 & .589 \\
\hline & 7 & -1.467 & .593 & .027 & -2.738 & -.196 \\
\hline & 8 & -.467 & .487 & .354 & -1.510 & .577 \\
\hline \multirow[t]{7}{*}{4} & 1 & -3.800 & .890 & .001 & -5.709 & -1.891 \\
\hline & 2 & -.867 & .291 & .010 & -1.490 & -.243 \\
\hline & 3 & -1.200 & .355 & .004 & -1.960 & -.440 \\
\hline & 5 & -1.800 & .380 & .000 & -2.616 & -.984 \\
\hline & 6 & -1.400 & .289 & .000 & -2.021 & -.779 \\
\hline & 7 & -2.667 & .513 & .000 & -3.768 & -1.566 \\
\hline & 8 & -1.667 & .485 & .004 & -2.706 & -.627 \\
\hline \multirow[t]{7}{*}{5} & 1 & -2.000 & .834 & .031 & -3.788 & -.212 \\
\hline & 2 & .933 & .431 & .048 & .010 & 1.857 \\
\hline & 3 & .600 & .466 & .219 & -.399 & 1.599 \\
\hline & 4 & 1.800 & .380 & .000 & .984 & 2.616 \\
\hline & 6 & .400 & .363 & .288 & -.378 & 1.178 \\
\hline & 7 & -.867 & .467 & .084 & -1.868 & .134 \\
\hline & 8 & .133 & .456 & .774 & -.845 & 1.112 \\
\hline \multirow[t]{7}{*}{6} & 1 & -2.400 & .838 & .013 & -4.198 & -.602 \\
\hline & 2 & .533 & .363 & .164 & -.246 & 1.313 \\
\hline & 3 & .200 & .368 & .595 & -.589 & .989 \\
\hline & 4 & 1.400 & .289 & .000 & .779 & 2.021 \\
\hline & 5 & -.400 & .363 & .288 & -1.178 & .378 \\
\hline & 7 & -1.267 & .384 & .005 & -2.090 & -.443 \\
\hline & 8 & -.267 & .284 & .364 & -.876 & .342 \\
\hline \multirow[t]{7}{*}{7} & 1 & -1.133 & .894 & .225 & -3.050 & .783 \\
\hline & 2 & 1.800 & .571 & .007 & .576 & 3.024 \\
\hline & 3 & 1.467 & .593 & .027 & .196 & 2.738 \\
\hline & 4 & 2.667 & .513 & .000 & 1.566 & 3.768 \\
\hline & 5 & .867 & .467 & .084 & -.134 & 1.868 \\
\hline & 6 & 1.267 & .384 & .005 & .443 & 2.090 \\
\hline & 8 & 1.000 & .526 & .078 & -.127 & 2.127 \\
\hline \multirow[t]{7}{*}{8} & 1 & -2.133 & .742 & .012 & -3.726 & -.541 \\
\hline & 2 & .800 & .509 & .138 & -.292 & 1.892 \\
\hline & 3 & .467 & .487 & .354 & -.577 & 1.510 \\
\hline & 4 & 1.667 & .485 & .004 & .627 & 2.706 \\
\hline & 5 & -.133 & .456 & .774 & -1.112 & .845 \\
\hline & 6 & .267 & .284 & .364 & -.342 & .876 \\
\hline & 7 & -1.000 & .526 & .078 & -2.127 & .127 \\
\hline
\end{tabular}

Figure G-6: Detailed data analysis for the number of collisions for neurotypical individuals. 


\section{Mauchly's Test of Sphericity ${ }^{a}$}

Measure: StaticObstacleCollision

\begin{tabular}{|l|r|r|r|r|r|r|r|}
\hline & & & & \multicolumn{3}{|c|}{ Epsilon $^{\text {b }}$} \\
\cline { 6 - 9 } Within Subjects Effect & Mauchly's W & $\begin{array}{c}\text { Approx. Chi- } \\
\text { Square }\end{array}$ & df & Sig. & $\begin{array}{c}\text { Greenhouse- } \\
\text { Geisser }\end{array}$ & Huynh-Feldt & Lower-bound \\
\hline Technique & .009 & 54.335 & 27 & .002 & .439 & .576 & .143 \\
\hline
\end{tabular}

Tests the null hypothesis that the error covariance matrix of the orthonormalized transformed dependent variables is proportional to an identity matrix.

a. Design: Intercept

Within Subjects Design: Technique

b. May be used to adjust the degrees of freedom for the averaged tests of significance. Corrected tests are displayed in the Tests of Within-Subjects Effects table.

Tests of Within-Subjects Effects

Measure: StaticObstacleCollision

\begin{tabular}{|c|c|c|c|c|c|c|c|c|c|}
\hline Source & & $\begin{array}{l}\text { Type III Sum } \\
\text { of Squares }\end{array}$ & df & Mean Square & $\mathrm{F}$ & Sig. & $\begin{array}{l}\text { Partial Eta } \\
\text { Squared }\end{array}$ & $\begin{array}{l}\text { Noncent. } \\
\text { Parameter }\end{array}$ & $\begin{array}{l}\text { Observed } \\
\text { Power }^{\text {a }}\end{array}$ \\
\hline \multirow[t]{4}{*}{ Technique } & Sphericity Assumed & 139.125 & 7 & 19.875 & 8.099 & .000 & .366 & 56.691 & 1.000 \\
\hline & Greenhouse-Geisser & 139.125 & 3.071 & 45.309 & 8.099 & .000 & .366 & 24.868 & .987 \\
\hline & Huynh-Feldt & 139.125 & 4.031 & 34.512 & 8.099 & .000 & .366 & 32.648 & .997 \\
\hline & Lower-bound & 139.125 & 1.000 & 139.125 & 8.099 & .013 & .366 & 8.099 & .754 \\
\hline \multirow[t]{4}{*}{ Error(Technique) } & Sphericity Assumed & 240.500 & 98 & 2.454 & & & & & \\
\hline & Greenhouse-Geisser & 240.500 & 42.988 & 5.595 & & & & & \\
\hline & Huynh-Feldt & 240.500 & 56.437 & 4.261 & & & & & \\
\hline & Lower-bound & 240.500 & 14.000 & 17.179 & & & & & \\
\hline
\end{tabular}

a. Computed using alpha $=.05$

Figure G-6 (Continued) 


\begin{tabular}{|c|c|c|c|c|c|c|}
\hline \multicolumn{7}{|c|}{ Pairwise Comparisons } \\
\hline & & & \multirow[b]{3}{*}{ Std. Error } & & & \\
\hline \multirow[b]{2}{*}{ (l) Technique } & \multirow[b]{2}{*}{ (J) Technique } & \multirow{2}{*}{$\begin{array}{c}\text { Mean } \\
\text { Difference (I- } \\
\mathrm{J})\end{array}$} & & \multirow[b]{2}{*}{ Sig. } & \multicolumn{2}{|c|}{$\begin{array}{l}95 \% \text { Confidence Interval for } \\
\text { Difference }\end{array}$} \\
\hline & & & & & Lower Bound & Upper Bound \\
\hline \multirow[t]{7}{*}{1} & 2 & 4.750 & 1.088 & .001 & 2.355 & 7.145 \\
\hline & 3 & 4.500 & 1.171 & .003 & 1.923 & 7.077 \\
\hline & 4 & 5.833 & 1.065 & .000 & 3.490 & 8.177 \\
\hline & 5 & 4.333 & 1.293 & .006 & 1.488 & 7.179 \\
\hline & 6 & 5.333 & 1.103 & .001 & 2.905 & 7.762 \\
\hline & 7 & 3.583 & 1.158 & .010 & 1.035 & 6.131 \\
\hline & 8 & 4.417 & 1.203 & .004 & 1.770 & 7.064 \\
\hline \multirow[t]{7}{*}{2} & 1 & -4.750 & 1.088 & .001 & -7.145 & -2.355 \\
\hline & 3 & -.250 & .372 & .515 & -1.068 & .568 \\
\hline & 4 & 1.083 & .398 & .020 & .207 & 1.959 \\
\hline & 5 & -.417 & .529 & .447 & -1.581 & .747 \\
\hline & 6 & .583 & .417 & .189 & -.334 & 1.500 \\
\hline & 7 & -1.167 & .777 & .161 & -2.877 & .543 \\
\hline & 8 & -.333 & .644 & .615 & -1.750 & 1.083 \\
\hline \multirow[t]{7}{*}{3} & 1 & -4.500 & 1.171 & .003 & -7.077 & -1.923 \\
\hline & 2 & .250 & .372 & .515 & -.568 & 1.068 \\
\hline & 4 & 1.333 & .333 & .002 & .600 & 2.067 \\
\hline & 5 & -.167 & .490 & .740 & -1.245 & .911 \\
\hline & 6 & .833 & .490 & .117 & -.245 & 1.911 \\
\hline & 7 & -.917 & .743 & .243 & -2.553 & .719 \\
\hline & 8 & -.083 & .417 & .845 & -1.000 & .834 \\
\hline \multirow[t]{7}{*}{4} & 1 & -5.833 & 1.065 & .000 & -8.177 & -3.490 \\
\hline & 2 & -1.083 & .398 & .020 & -1.959 & -.207 \\
\hline & 3 & -1.333 & .333 & .002 & -2.067 & -.600 \\
\hline & 5 & -1.500 & .435 & .005 & -2.458 & -.542 \\
\hline & 6 & -.500 & .337 & .166 & -1.242 & .242 \\
\hline & 7 & -2.250 & .719 & .010 & -3.833 & -.667 \\
\hline & 8 & -1.417 & .452 & .009 & -2.411 & -.423 \\
\hline \multirow[t]{7}{*}{5} & 1 & -4.333 & 1.293 & .006 & -7.179 & -1.488 \\
\hline & 2 & .417 & .529 & .447 & -.747 & 1.581 \\
\hline & 3 & .167 & .490 & .740 & -.911 & 1.245 \\
\hline & 4 & 1.500 & .435 & .005 & .542 & 2.458 \\
\hline & 6 & 1.000 & .461 & .053 & -.014 & 2.014 \\
\hline & 7 & -.750 & .799 & .368 & -2.508 & 1.008 \\
\hline & 8 & .083 & .570 & .886 & -1.172 & 1.338 \\
\hline \multirow[t]{7}{*}{6} & 1 & -5.333 & 1.103 & .001 & -7.762 & -2.905 \\
\hline & 2 & -.583 & .417 & .189 & -1.500 & .334 \\
\hline & 3 & -.833 & .490 & .117 & -1.911 & .245 \\
\hline & 4 & .500 & .337 & .166 & -.242 & 1.242 \\
\hline & 5 & -1.000 & .461 & .053 & -2.014 & .014 \\
\hline & 7 & -1.750 & .770 & .044 & -3.445 & -.055 \\
\hline & 8 & -.917 & .529 & .111 & -2.081 & .247 \\
\hline \multirow[t]{7}{*}{7} & 1 & -3.583 & 1.158 & .010 & -6.131 & -1.035 \\
\hline & 2 & 1.167 & .777 & .161 & -.543 & 2.877 \\
\hline & 3 & .917 & .743 & .243 & -.719 & 2.553 \\
\hline & 4 & 2.250 & .719 & .010 & .667 & 3.833 \\
\hline & 5 & .750 & .799 & .368 & -1.008 & 2.508 \\
\hline & 6 & 1.750 & .770 & .044 & .055 & 3.445 \\
\hline & 8 & .833 & .548 & .157 & -.373 & 2.040 \\
\hline \multirow[t]{7}{*}{8} & 1 & -4.417 & 1.203 & .004 & -7.064 & -1.770 \\
\hline & 2 & .333 & .644 & .615 & -1.083 & 1.750 \\
\hline & 3 & .083 & .417 & .845 & -.834 & 1.000 \\
\hline & 4 & 1.417 & .452 & .009 & .423 & 2.411 \\
\hline & 5 & -.083 & .570 & .886 & -1.338 & 1.172 \\
\hline & 6 & .917 & .529 & .111 & -.247 & 2.081 \\
\hline & 7 & -.833 & .548 & .157 & -2.040 & .373 \\
\hline
\end{tabular}

Figure G-7: Detailed data analysis for the number of collisions for individuals with ASD. 


\section{Mauchly's Test of Sphericity ${ }^{a}$}

Measure: StaticObstacleCollision

\begin{tabular}{|l|r|r|r|r|r|r|r|}
\hline & & & & \multicolumn{3}{|c|}{ Epsilon $^{\text {b }}$} \\
\cline { 6 - 9 } Within Subjects Effect & Mauchly's W & $\begin{array}{c}\text { Approx. Chi- } \\
\text { Square }\end{array}$ & df & Sig. & $\begin{array}{c}\text { Greenhouse- } \\
\text { Geisser }\end{array}$ & Huynh-Feldt & Lower-bound \\
\hline Technique & .001 & 56.401 & 27 & .001 & .378 & .509 & .143 \\
\hline
\end{tabular}

Tests the null hypothesis that the error covariance matrix of the orthonormalized transformed dependent variables is proportional to an identity matrix.

a. Design: Intercept

Within Subjects Design: Technique

b. May be used to adjust the degrees of freedom for the averaged tests of significance. Corrected tests are displayed in the Tests of Within-Subjects Effects table.

Tests of Within-Subjects Effects

Measure: StaticObstacleCollision

\begin{tabular}{|c|c|c|c|c|c|c|c|c|c|}
\hline Source & & $\begin{array}{l}\text { Type III Sum } \\
\text { of Squares }\end{array}$ & df & Mean Square & $\mathrm{F}$ & Sig. & $\begin{array}{l}\text { Partial Eta } \\
\text { Squared }\end{array}$ & $\begin{array}{l}\text { Noncent. } \\
\text { Parameter }\end{array}$ & $\begin{array}{l}\text { Observed } \\
\text { Power }^{\text {a }}\end{array}$ \\
\hline \multirow[t]{4}{*}{ Technique } & Sphericity Assumed & 268.073 & 7 & 38.296 & 11.296 & .000 & .507 & 79.071 & 1.000 \\
\hline & Greenhouse-Geisser & 268.073 & 2.645 & 101.346 & 11.296 & .000 & .507 & 29.879 & .996 \\
\hline & Huynh-Feldt & 268.073 & 3.560 & 75.306 & 11.296 & .000 & .507 & 40.211 & 1.000 \\
\hline & Lower-bound & 268.073 & 1.000 & 268.073 & 11.296 & .006 & .507 & 11.296 & .864 \\
\hline \multirow[t]{4}{*}{ Error(Technique) } & Sphericity Assumed & 261.052 & 77 & 3.390 & & & & & \\
\hline & Greenhouse-Geisser & 261.052 & 29.096 & 8.972 & & & & & \\
\hline & Huynh-Feldt & 261.052 & 39.158 & 6.667 & & & & & \\
\hline & Lower-bound & 261.052 & 11.000 & 23.732 & & & & & \\
\hline
\end{tabular}

a. Computed using alpha $=.05$

Figure G-7 (Continued) 


\begin{tabular}{|c|c|c|c|c|c|c|}
\hline \multicolumn{7}{|c|}{ Pairwise Comparisons } \\
\hline & & & \multirow[b]{3}{*}{ Std. Error } & & & \\
\hline \multirow[b]{2}{*}{ (I) Technique } & \multirow[b]{2}{*}{ (J) Technique } & \multirow{2}{*}{$\begin{array}{c}\text { Mean } \\
\text { Difference (I- } \\
\mathrm{J})\end{array}$} & & \multirow[b]{2}{*}{ Sig. } & \multicolumn{2}{|c|}{$\begin{array}{l}\text { 95\% Confidence Interval for } \\
\text { Difference }\end{array}$} \\
\hline & & & & & Lower Bound & Upper Bound \\
\hline \multirow[t]{7}{*}{1} & 2 & .200 & .223 & .384 & -.277 & .677 \\
\hline & 3 & -.067 & .182 & .719 & -.456 & .323 \\
\hline & 4 & .467 & .236 & .068 & -.040 & .974 \\
\hline & 5 & .733 & .206 & .003 & .291 & 1.176 \\
\hline & 6 & .600 & .214 & .014 & .141 & 1.059 \\
\hline & 7 & -.800 & .393 & .061 & -1.642 & .042 \\
\hline & 8 & -.133 & .307 & .670 & -.791 & .524 \\
\hline \multirow[t]{7}{*}{2} & 1 & -.200 & .223 & .384 & -.677 & .277 \\
\hline & 3 & -.267 & .267 & .334 & -.839 & .305 \\
\hline & 4 & .267 & .153 & .104 & -.062 & .595 \\
\hline & 5 & .533 & .165 & .006 & .179 & .888 \\
\hline & 6 & .400 & .163 & .028 & .050 & .750 \\
\hline & 7 & -1.000 & .276 & .003 & -1.592 & -.408 \\
\hline & 8 & -.333 & .252 & .207 & -.874 & .207 \\
\hline \multirow[t]{7}{*}{3} & 1 & .067 & .182 & .719 & -.323 & .456 \\
\hline & 2 & .267 & .267 & .334 & -.305 & .839 \\
\hline & 4 & .533 & .291 & .088 & -.090 & 1.157 \\
\hline & 5 & .800 & .243 & .005 & .279 & 1.321 \\
\hline & 6 & .667 & .252 & .019 & .126 & 1.207 \\
\hline & 7 & -.733 & .358 & .060 & -1.501 & .035 \\
\hline & 8 & -.067 & .284 & .818 & -.676 & .542 \\
\hline \multirow[t]{7}{*}{4} & 1 & -.467 & .236 & .068 & -.974 & .040 \\
\hline & 2 & -.267 & .153 & .104 & -.595 & .062 \\
\hline & 3 & -.533 & .291 & .088 & -1.157 & .090 \\
\hline & 5 & .267 & .153 & .104 & -.062 & .595 \\
\hline & 6 & .133 & .165 & .433 & -.221 & .488 \\
\hline & 7 & -1.267 & .284 & .001 & -1.876 & -.658 \\
\hline & 8 & -.600 & .273 & .045 & -1.185 & -.015 \\
\hline \multirow[t]{7}{*}{5} & 1 & -.733 & .206 & .003 & -1.176 & -.291 \\
\hline & 2 & -.533 & .165 & .006 & -.888 & -.179 \\
\hline & 3 & -.800 & .243 & .005 & -1.321 & -.279 \\
\hline & 4 & -.267 & .153 & .104 & -.595 & .062 \\
\hline & 6 & -.133 & .091 & .164 & -.328 & .062 \\
\hline & 7 & -1.533 & .307 & .000 & -2.191 & -.876 \\
\hline & 8 & -.867 & .192 & .000 & -1.278 & -.455 \\
\hline \multirow[t]{7}{*}{6} & 1 & -.600 & .214 & .014 & -1.059 & -.141 \\
\hline & 2 & -.400 & .163 & .028 & -.750 & -.050 \\
\hline & 3 & -.667 & .252 & .019 & -1.207 & -.126 \\
\hline & 4 & -.133 & .165 & .433 & -.488 & .221 \\
\hline & 5 & .133 & .091 & .164 & -.062 & .328 \\
\hline & 7 & -1.400 & .306 & .000 & -2.055 & -.745 \\
\hline & 8 & -.733 & .206 & .003 & -1.176 & -.291 \\
\hline \multirow[t]{7}{*}{7} & 1 & .800 & .393 & .061 & -.042 & 1.642 \\
\hline & 2 & 1.000 & .276 & .003 & .408 & 1.592 \\
\hline & 3 & .733 & .358 & .060 & -.035 & 1.501 \\
\hline & 4 & 1.267 & .284 & .001 & .658 & 1.876 \\
\hline & 5 & 1.533 & .307 & .000 & .876 & 2.191 \\
\hline & 6 & 1.400 & .306 & .000 & .745 & 2.055 \\
\hline & 8 & .667 & .252 & .019 & .126 & 1.207 \\
\hline \multirow[t]{7}{*}{8} & 1 & .133 & .307 & .670 & -.524 & .791 \\
\hline & 2 & .333 & .252 & .207 & -.207 & .874 \\
\hline & 3 & .067 & .284 & .818 & -.542 & .676 \\
\hline & 4 & .600 & .273 & .045 & .015 & 1.185 \\
\hline & 5 & .867 & .192 & .000 & .455 & 1.278 \\
\hline & 6 & .733 & .206 & .003 & .291 & 1.176 \\
\hline & 7 & -.667 & .252 & .019 & -1.207 & -.126 \\
\hline
\end{tabular}

Figure G-8: Detailed data analysis for the difficulty in understanding for neurotypical individuals. 


\section{Mauchly's Test of Sphericity ${ }^{a}$}

Measure: DifficultylnUnderstanding

\begin{tabular}{|l|r|r|r|r|r|r|r|}
\hline & & & & \multicolumn{3}{|c|}{ Epsilon $^{\text {b }}$} \\
\cline { 6 - 9 } Within Subjects Effect & Mauchly's W & $\begin{array}{c}\text { Approx. Chi- } \\
\text { Square }\end{array}$ & df & Sig. & $\begin{array}{c}\text { Greenhouse- } \\
\text { Geisser }\end{array}$ & Huynh-Feldt & Lower-bound \\
\hline Technique & .014 & 48.919 & 27 & .008 & .483 & .656 & .143 \\
\hline
\end{tabular}

Tests the null hypothesis that the error covariance matrix of the orthonormalized transformed dependent variables is proportional to an identity matrix.

a. Design: Intercept

Within Subjects Design: Technique

b. May be used to adjust the degrees of freedom for the averaged tests of significance. Corrected tests are displayed in the Tests of Within-Subjects Effects table.

Tests of Within-Subjects Effects

Measure: DifficultyInUnderstanding

\begin{tabular}{|c|c|c|c|c|c|c|c|c|c|}
\hline Source & & $\begin{array}{l}\text { Type III Sum } \\
\text { of Squares }\end{array}$ & df & Mean Square & $\mathrm{F}$ & Sig. & $\begin{array}{l}\text { Partial Eta } \\
\text { Squared }\end{array}$ & $\begin{array}{l}\text { Noncent. } \\
\text { Parameter }\end{array}$ & $\begin{array}{l}\text { Observed } \\
\text { Power }^{\mathrm{a}}\end{array}$ \\
\hline \multirow[t]{4}{*}{ Technique } & Sphericity Assumed & 25.392 & 7 & 3.627 & 7.859 & .000 & .360 & 55.012 & 1.000 \\
\hline & Greenhouse-Geisser & 25.392 & 3.383 & 7.505 & 7.859 & .000 & .360 & 26.590 & .990 \\
\hline & Huynh-Feldt & 25.392 & 4.592 & 5.529 & 7.859 & .000 & .360 & 36.089 & .998 \\
\hline & Lower-bound & 25.392 & 1.000 & 25.392 & 7.859 & .014 & .360 & 7.859 & .741 \\
\hline \multirow[t]{4}{*}{ Error(Technique) } & Sphericity Assumed & 45.233 & 98 & .462 & & & & & \\
\hline & Greenhouse-Geisser & 45.233 & 47.369 & .955 & & & & & \\
\hline & Huynh-Feldt & 45.233 & 64.289 & .704 & & & & & \\
\hline & Lower-bound & 45.233 & 14.000 & 3.231 & & & & & \\
\hline
\end{tabular}

a. Computed using alpha $=.05$

Figure G-8 (Continued) 


\begin{tabular}{|c|c|c|c|c|c|c|}
\hline \multicolumn{7}{|c|}{ Pairwise Comparisons } \\
\hline & & & & & & \\
\hline \multirow[b]{2}{*}{ (I) Technique } & \multirow[b]{2}{*}{ (J) Technique } & \multirow{2}{*}{$\begin{array}{c}\text { Mean } \\
\text { Difference (I- } \\
\text { J) }\end{array}$} & \multirow[b]{2}{*}{ Std. Error } & \multirow[b]{2}{*}{ Sig. } & \multicolumn{2}{|c|}{$\begin{array}{l}95 \% \text { Confidence Interval for } \\
\text { Difference }\end{array}$} \\
\hline & & & & & Lower Bound & Upper Bound \\
\hline \multirow[t]{7}{*}{1} & 2 & .071 & .245 & .775 & -.458 & .601 \\
\hline & 3 & -.071 & .127 & .583 & -.345 & .203 \\
\hline & 4 & .500 & .251 & .068 & -.043 & 1.043 \\
\hline & 5 & .500 & .251 & .068 & -.043 & 1.043 \\
\hline & 6 & .286 & .244 & .263 & -.242 & .813 \\
\hline & 7 & -.429 & .374 & .272 & -1.236 & .379 \\
\hline & 8 & -.714 & .398 & .096 & -1.575 & .146 \\
\hline \multirow[t]{7}{*}{2} & 1 & -.071 & .245 & .775 & -.601 & .458 \\
\hline & 3 & -.143 & .254 & .583 & -.691 & .405 \\
\hline & 4 & .429 & .228 & .082 & -.063 & .920 \\
\hline & 5 & .429 & .251 & .111 & -.113 & .970 \\
\hline & 6 & .214 & .261 & .426 & -.349 & .777 \\
\hline & 7 & -.500 & .374 & .205 & -1.309 & .309 \\
\hline & 8 & -.786 & .447 & .102 & -1.751 & .180 \\
\hline \multirow[t]{7}{*}{3} & 1 & .071 & .127 & .583 & -.203 & .345 \\
\hline & 2 & .143 & .254 & .583 & -.405 & .691 \\
\hline & 4 & .571 & .272 & .055 & -.015 & 1.158 \\
\hline & 5 & .571 & .272 & .055 & -.015 & 1.158 \\
\hline & 6 & .357 & .248 & .174 & -.179 & .893 \\
\hline & 7 & -.357 & .401 & .389 & -1.223 & .508 \\
\hline & 8 & -.643 & .427 & .156 & -1.566 & .280 \\
\hline \multirow[t]{7}{*}{4} & 1 & -.500 & .251 & .068 & -1.043 & .043 \\
\hline & 2 & -.429 & .228 & .082 & -.920 & .063 \\
\hline & 3 & -.571 & .272 & .055 & -1.158 & .015 \\
\hline & 5 & .000 & .148 & 1.000 & -.320 & .320 \\
\hline & 6 & -.214 & .214 & .336 & -.677 & .249 \\
\hline & 7 & -.929 & .286 & .006 & -1.547 & -.310 \\
\hline & 8 & -1.214 & .447 & .018 & -2.180 & -.249 \\
\hline \multirow[t]{7}{*}{5} & 1 & -.500 & .251 & .068 & -1.043 & .043 \\
\hline & 2 & -.429 & .251 & .111 & -.970 & .113 \\
\hline & 3 & -.571 & .272 & .055 & -1.158 & .015 \\
\hline & 4 & .000 & .148 & 1.000 & -.320 & .320 \\
\hline & 6 & -.214 & .155 & .189 & -.549 & .120 \\
\hline & 7 & -.929 & .305 & .009 & -1.587 & -.270 \\
\hline & 8 & -1.214 & .422 & .013 & -2.125 & -.303 \\
\hline \multirow[t]{7}{*}{6} & 1 & -.286 & .244 & .263 & -.813 & .242 \\
\hline & 2 & -.214 & .261 & .426 & -.777 & .349 \\
\hline & 3 & -.357 & .248 & .174 & -.893 & .179 \\
\hline & 4 & .214 & .214 & .336 & -.249 & .677 \\
\hline & 5 & .214 & .155 & .189 & -.120 & .549 \\
\hline & 7 & -.714 & .339 & .055 & -1.446 & .017 \\
\hline & 8 & -1.000 & .363 & .016 & -1.785 & -.215 \\
\hline \multirow[t]{7}{*}{7} & 1 & .429 & .374 & .272 & -.379 & 1.236 \\
\hline & 2 & .500 & .374 & .205 & -.309 & 1.309 \\
\hline & 3 & .357 & .401 & .389 & -.508 & 1.223 \\
\hline & 4 & .929 & .286 & .006 & .310 & 1.547 \\
\hline & 5 & .929 & .305 & .009 & .270 & 1.587 \\
\hline & 6 & .714 & .339 & .055 & -.017 & 1.446 \\
\hline & 8 & -.286 & .485 & .566 & -1.334 & .763 \\
\hline \multirow[t]{7}{*}{8} & 1 & .714 & .398 & .096 & -.146 & 1.575 \\
\hline & 2 & .786 & .447 & .102 & -.180 & 1.751 \\
\hline & 3 & .643 & .427 & .156 & -.280 & 1.566 \\
\hline & 4 & 1.214 & .447 & .018 & .249 & 2.180 \\
\hline & 5 & 1.214 & .422 & .013 & .303 & 2.125 \\
\hline & 6 & 1.000 & .363 & .016 & .215 & 1.785 \\
\hline & 7 & .286 & .485 & .566 & -.763 & 1.334 \\
\hline
\end{tabular}

Figure G-9: Detailed data analysis for the difficulty in understanding for individuals with ASD. 


\section{Mauchly's Test of Sphericity ${ }^{a}$}

Measure: DifficultylnUnderstanding

\begin{tabular}{|l|r|r|r|r|r|r|r|}
\hline & & & & \multicolumn{3}{|c|}{ Epsilon $^{\text {b }}$} \\
\cline { 6 - 9 } Within Subjects Effect & Mauchly's W & $\begin{array}{c}\text { Approx. Chi- } \\
\text { Square }\end{array}$ & df & Sig. & $\begin{array}{c}\text { Greenhouse- } \\
\text { Geisser }\end{array}$ & Huynh-Feldt & Lower-bound \\
\hline Technique & .019 & 41.498 & 27 & .048 & .524 & .755 & .143 \\
\hline
\end{tabular}

Tests the null hypothesis that the error covariance matrix of the orthonormalized transformed dependent variables is proportional to an identity matrix.

a. Design: Intercept

Within Subjects Design: Technique

b. May be used to adjust the degrees of freedom for the averaged tests of significance. Corrected tests are displayed in the Tests of Within-Subjects Effects table.

Tests of Within-Subjects Effects

Measure: DifficultyInUnderstanding

\begin{tabular}{|c|c|c|c|c|c|c|c|c|c|}
\hline Source & & $\begin{array}{l}\text { Type III Sum } \\
\text { of Squares }\end{array}$ & df & Mean Square & $\mathrm{F}$ & Sig. & $\begin{array}{l}\text { Partial Eta } \\
\text { Squared }\end{array}$ & $\begin{array}{l}\text { Noncent. } \\
\text { Parameter }\end{array}$ & $\begin{array}{l}\text { Observed } \\
\text { Power }^{\text {a }}\end{array}$ \\
\hline \multirow[t]{4}{*}{ Technique } & Sphericity Assumed & 17.964 & 7 & 2.566 & 3.633 & .002 & .218 & 25.429 & .965 \\
\hline & Greenhouse-Geisser & 17.964 & 3.667 & 4.898 & 3.633 & .014 & .218 & 13.323 & .819 \\
\hline & Huynh-Feldt & 17.964 & 5.287 & 3.398 & 3.633 & .005 & .218 & 19.207 & .917 \\
\hline & Lower-bound & 17.964 & 1.000 & 17.964 & 3.633 & .079 & .218 & 3.633 & .423 \\
\hline \multirow[t]{4}{*}{ Error(Technique) } & Sphericity Assumed & 64.286 & 91 & .706 & & & & & \\
\hline & Greenhouse-Geisser & 64.286 & 47.676 & 1.348 & & & & & \\
\hline & Huynh-Feldt & 64.286 & 68.734 & .935 & & & & & \\
\hline & Lower-bound & 64.286 & 13.000 & 4.945 & & & & & \\
\hline
\end{tabular}

a. Computed using alpha $=.05$

Figure G-9 (Continued) 


\begin{tabular}{|c|c|c|c|c|c|c|}
\hline \multicolumn{7}{|c|}{ Pairwise Comparisons } \\
\hline & & & & & & \\
\hline \multirow[b]{2}{*}{ (I) Technique } & \multirow[b]{2}{*}{ (J) Technique } & \multirow{2}{*}{$\begin{array}{c}\text { Mean } \\
\text { Difference (I- } \\
\mathrm{J})\end{array}$} & \multirow[b]{2}{*}{ Std. Error } & \multirow[b]{2}{*}{ Sig. } & \multicolumn{2}{|c|}{$\begin{array}{l}\text { 95\% Confidence Interval for } \\
\text { Difference }\end{array}$} \\
\hline & & & & & Lower Bound & Upper Bound \\
\hline \multirow[t]{7}{*}{1} & 2 & .400 & .254 & .138 & -.146 & .946 \\
\hline & 3 & .200 & .279 & .486 & -.399 & .799 \\
\hline & 4 & .600 & .321 & .082 & -.088 & 1.288 \\
\hline & 5 & 1.000 & .309 & .006 & .338 & 1.662 \\
\hline & 6 & .933 & .267 & .004 & .361 & 1.505 \\
\hline & 7 & -.867 & .413 & .054 & -1.751 & .018 \\
\hline & 8 & -.267 & .431 & .546 & -1.190 & .657 \\
\hline \multirow[t]{7}{*}{2} & 1 & -.400 & .254 & .138 & -.946 & .146 \\
\hline & 3 & -.200 & .279 & .486 & -.799 & .399 \\
\hline & 4 & .200 & .175 & .271 & -.174 & .574 \\
\hline & 5 & .600 & .235 & .023 & .096 & 1.104 \\
\hline & 6 & .533 & .215 & .027 & .072 & .995 \\
\hline & 7 & -1.267 & .248 & .000 & -1.799 & -.734 \\
\hline & 8 & -.667 & .287 & .036 & -1.283 & -.050 \\
\hline \multirow[t]{7}{*}{3} & 1 & -.200 & .279 & .486 & -.799 & .399 \\
\hline & 2 & .200 & .279 & .486 & -.399 & .799 \\
\hline & 4 & .400 & .289 & .189 & -.221 & 1.021 \\
\hline & 5 & .800 & .279 & .013 & .201 & 1.399 \\
\hline & 6 & .733 & .248 & .010 & .201 & 1.266 \\
\hline & 7 & -1.067 & .345 & .008 & -1.806 & -.328 \\
\hline & 8 & -.467 & .336 & .187 & -1.188 & .254 \\
\hline \multirow[t]{7}{*}{4} & 1 & -.600 & .321 & .082 & -1.288 & .088 \\
\hline & 2 & -.200 & .175 & .271 & -.574 & .174 \\
\hline & 3 & -.400 & .289 & .189 & -1.021 & .221 \\
\hline & 5 & .400 & .214 & .082 & -.059 & .859 \\
\hline & 6 & .333 & .211 & .136 & -.119 & .785 \\
\hline & 7 & -1.467 & .256 & .000 & -2.015 & -.918 \\
\hline & 8 & -.867 & .256 & .004 & -1.415 & -.318 \\
\hline \multirow[t]{7}{*}{5} & 1 & -1.000 & .309 & .006 & -1.662 & -.338 \\
\hline & 2 & -.600 & .235 & .023 & -1.104 & -.096 \\
\hline & 3 & -.800 & .279 & .013 & -1.399 & -.201 \\
\hline & 4 & -.400 & .214 & .082 & -.859 & .059 \\
\hline & 6 & -.067 & .118 & .582 & -.320 & .187 \\
\hline & 7 & -1.867 & .291 & .000 & -2.490 & -1.243 \\
\hline & 8 & -1.267 & .182 & .000 & -1.656 & -.877 \\
\hline \multirow[t]{7}{*}{6} & 1 & -.933 & .267 & .004 & -1.505 & -.361 \\
\hline & 2 & -.533 & .215 & .027 & -.995 & -.072 \\
\hline & 3 & -.733 & .248 & .010 & -1.266 & -.201 \\
\hline & 4 & -.333 & .211 & .136 & -.785 & .119 \\
\hline & 5 & .067 & .118 & .582 & -.187 & .320 \\
\hline & 7 & -1.800 & .279 & .000 & -2.399 & -1.201 \\
\hline & 8 & -1.200 & .243 & .000 & -1.721 & -.679 \\
\hline \multirow[t]{7}{*}{7} & 1 & .867 & .413 & .054 & -.018 & 1.751 \\
\hline & 2 & 1.267 & .248 & .000 & .734 & 1.799 \\
\hline & 3 & 1.067 & .345 & .008 & .328 & 1.806 \\
\hline & 4 & 1.467 & .256 & .000 & .918 & 2.015 \\
\hline & 5 & 1.867 & .291 & .000 & 1.243 & 2.490 \\
\hline & 6 & 1.800 & .279 & .000 & 1.201 & 2.399 \\
\hline & 8 & .600 & .289 & .057 & -.021 & 1.221 \\
\hline \multirow[t]{7}{*}{8} & 1 & .267 & .431 & .546 & -.657 & 1.190 \\
\hline & 2 & .667 & .287 & .036 & .050 & 1.283 \\
\hline & 3 & .467 & .336 & .187 & -.254 & 1.188 \\
\hline & 4 & .867 & .256 & .004 & .318 & 1.415 \\
\hline & 5 & 1.267 & .182 & .000 & .877 & 1.656 \\
\hline & 6 & 1.200 & .243 & .000 & .679 & 1.721 \\
\hline & 7 & -.600 & .289 & .057 & -1.221 & .021 \\
\hline
\end{tabular}

Figure G-10: Detailed data analysis for the difficulty in operating for neurotypical individuals. 


\section{Mauchly's Test of Sphericity ${ }^{a}$}

Measure: Difficultylnoperating

\begin{tabular}{|l|r|r|r|r|r|r|r|}
\hline & & & & \multicolumn{3}{|c|}{ Epsilon $^{\text {b }}$} \\
\cline { 6 - 9 } Within Subjects Effect & Mauchly's W & $\begin{array}{c}\text { Approx. Chi- } \\
\text { Square }\end{array}$ & df & Sig. & $\begin{array}{c}\text { Greenhouse- } \\
\text { Geisser }\end{array}$ & Huynh-Feldt & Lower-bound \\
\hline Technique & .022 & 43.704 & 27 & .028 & .523 & .732 & .143 \\
\hline
\end{tabular}

Tests the null hypothesis that the error covariance matrix of the orthonormalized transformed dependent variables is proportional to an identity matrix.

a. Design: Intercept

Within Subjects Design: Technique

b. May be used to adjust the degrees of freedom for the averaged tests of significance. Corrected tests are displayed in the Tests of Within-Subjects Effects table.

Tests of Within-Subjects Effects

Measure: DifficultylnOperating

\begin{tabular}{|c|c|c|c|c|c|c|c|c|c|}
\hline Source & & $\begin{array}{l}\text { Type III Sum } \\
\text { of Squares }\end{array}$ & df & Mean Square & $\mathrm{F}$ & Sig. & $\begin{array}{c}\text { Partial Eta } \\
\text { Squared }\end{array}$ & $\begin{array}{l}\text { Noncent. } \\
\text { Parameter }\end{array}$ & $\begin{array}{l}\text { Observed } \\
\text { Power }^{\text {a }}\end{array}$ \\
\hline \multirow[t]{4}{*}{ Technique } & Sphericity Assumed & 41.300 & 7 & 5.900 & 10.243 & .000 & .423 & 71.699 & 1.000 \\
\hline & Greenhouse-Geisser & 41.300 & 3.663 & 11.274 & 10.243 & .000 & .423 & 37.522 & .999 \\
\hline & Huynh-Feldt & 41.300 & 5.123 & 8.062 & 10.243 & .000 & .423 & 52.468 & 1.000 \\
\hline & Lower-bound & 41.300 & 1.000 & 41.300 & 10.243 & .006 & .423 & 10.243 & .845 \\
\hline \multirow[t]{4}{*}{ Error(Technique) } & Sphericity Assumed & 56.450 & 98 & .576 & & & & & \\
\hline & Greenhouse-Geisser & 56.450 & 51.286 & 1.101 & & & & & \\
\hline & Huynh-Feldt & 56.450 & 71.715 & .787 & & & & & \\
\hline & Lower-bound & 56.450 & 14.000 & 4.032 & & & & & \\
\hline
\end{tabular}

a. Computed using alpha $=.05$

Figure G-10 (Continued) 


\begin{tabular}{|c|c|c|c|c|c|c|}
\hline \multicolumn{7}{|c|}{ Pairwise Comparisons } \\
\hline sure. & & & & & & \\
\hline \multirow[b]{2}{*}{ (I) Technique } & \multirow[b]{2}{*}{ (J) Technique } & \multirow{2}{*}{$\begin{array}{c}\text { Mean } \\
\text { Difference (I- } \\
\mathrm{J})\end{array}$} & \multirow[b]{2}{*}{ Std. Error } & \multirow[b]{2}{*}{ Sig. } & \multicolumn{2}{|c|}{$\begin{array}{l}95 \% \text { Confidence Interval for } \\
\text { Difference }\end{array}$} \\
\hline & & & & & Lower Bound & Upper Bound \\
\hline \multirow[t]{7}{*}{1} & 2 & .429 & .327 & .212 & -.277 & 1.134 \\
\hline & 3 & .071 & .221 & .752 & -.407 & .550 \\
\hline & 4 & 1.000 & .392 & .024 & .153 & 1.847 \\
\hline & 5 & .714 & .286 & .027 & .097 & 1.332 \\
\hline & 6 & 1.000 & .392 & .024 & .153 & 1.847 \\
\hline & 7 & -.714 & .425 & .117 & -1.632 & .204 \\
\hline & 8 & -.714 & .450 & .136 & -1.686 & .258 \\
\hline \multirow[t]{7}{*}{2} & 1 & -.429 & .327 & .212 & -1.134 & .277 \\
\hline & 3 & -.357 & .289 & .239 & -.982 & .267 \\
\hline & 4 & .571 & .272 & .055 & -.015 & 1.158 \\
\hline & 5 & .286 & .163 & .104 & -.067 & .639 \\
\hline & 6 & .571 & .291 & .071 & -.058 & 1.200 \\
\hline & 7 & -1.143 & .390 & .012 & -1.986 & -.300 \\
\hline & 8 & -1.143 & .430 & .020 & -2.073 & -.213 \\
\hline \multirow[t]{7}{*}{3} & 1 & -.071 & .221 & .752 & -.550 & .407 \\
\hline & 2 & .357 & .289 & .239 & -.267 & .982 \\
\hline & 4 & .929 & .370 & .026 & .129 & 1.728 \\
\hline & 5 & .643 & .269 & .033 & .061 & 1.225 \\
\hline & 6 & .929 & .385 & .031 & .098 & 1.760 \\
\hline & 7 & -.786 & .422 & .085 & -1.697 & .125 \\
\hline & 8 & -.786 & .447 & .102 & -1.751 & .180 \\
\hline \multirow[t]{7}{*}{4} & 1 & -1.000 & .392 & .024 & -1.847 & -.153 \\
\hline & 2 & -.571 & .272 & .055 & -1.158 & .015 \\
\hline & 3 & -.929 & .370 & .026 & -1.728 & -.129 \\
\hline & 5 & -.286 & .194 & .165 & -.705 & .134 \\
\hline & 6 & .000 & .182 & 1.000 & -.392 & .392 \\
\hline & 7 & -1.714 & .304 & .000 & -2.372 & -1.057 \\
\hline & 8 & -1.714 & .425 & .001 & -2.632 & -.796 \\
\hline \multirow[t]{7}{*}{5} & 1 & -.714 & .286 & .027 & -1.332 & -.097 \\
\hline & 2 & -.286 & .163 & .104 & -.639 & .067 \\
\hline & 3 & -.643 & .269 & .033 & -1.225 & -.061 \\
\hline & 4 & .286 & .194 & .165 & -.134 & .705 \\
\hline & 6 & .286 & .266 & .302 & -.288 & .860 \\
\hline & 7 & -1.429 & .309 & .000 & -2.097 & -.760 \\
\hline & 8 & -1.429 & .374 & .002 & -2.236 & -.621 \\
\hline \multirow[t]{7}{*}{6} & 1 & -1.000 & .392 & .024 & -1.847 & -.153 \\
\hline & 2 & -.571 & .291 & .071 & -1.200 & .058 \\
\hline & 3 & -.929 & .385 & .031 & -1.760 & -.098 \\
\hline & 4 & .000 & .182 & 1.000 & -.392 & .392 \\
\hline & 5 & -.286 & .266 & .302 & -.860 & .288 \\
\hline & 7 & -1.714 & .398 & .001 & -2.575 & -.854 \\
\hline & 8 & -1.714 & .474 & .003 & -2.738 & -.691 \\
\hline \multirow[t]{7}{*}{7} & 1 & .714 & .425 & .117 & -.204 & 1.632 \\
\hline & 2 & 1.143 & .390 & .012 & .300 & 1.986 \\
\hline & 3 & .786 & .422 & .085 & -.125 & 1.697 \\
\hline & 4 & 1.714 & .304 & .000 & 1.057 & 2.372 \\
\hline & 5 & 1.429 & .309 & .000 & .760 & 2.097 \\
\hline & 6 & 1.714 & .398 & .001 & .854 & 2.575 \\
\hline & 8 & .000 & .480 & 1.000 & -1.038 & 1.038 \\
\hline \multirow[t]{7}{*}{8} & 1 & .714 & .450 & .136 & -.258 & 1.686 \\
\hline & 2 & 1.143 & .430 & .020 & .213 & 2.073 \\
\hline & 3 & .786 & .447 & .102 & -.180 & 1.751 \\
\hline & 4 & 1.714 & .425 & .001 & .796 & 2.632 \\
\hline & 5 & 1.429 & .374 & .002 & .621 & 2.236 \\
\hline & 6 & 1.714 & .474 & .003 & .691 & 2.738 \\
\hline & 7 & .000 & .480 & 1.000 & -1.038 & 1.038 \\
\hline
\end{tabular}

Figure G-11: Detailed data analysis for the difficulty in operating for individuals with ASD. 


\section{Mauchly's Test of Sphericity ${ }^{a}$}

Measure: Difficultylnoperating

\begin{tabular}{|l|r|r|r|r|r|r|r|}
\hline & & & & \multicolumn{3}{|c|}{ Epsilon $^{\text {b }}$} \\
\cline { 6 - 9 } Within Subjects Effect & Mauchly's W & $\begin{array}{c}\text { Approx. Chi- } \\
\text { Square }\end{array}$ & df & Sig. & $\begin{array}{c}\text { Greenhouse- } \\
\text { Geisser }\end{array}$ & Huynh-Feldt & Lower-bound \\
\hline Technique & .019 & 41.234 & 27 & .050 & .588 & .894 & .143 \\
\hline
\end{tabular}

Tests the null hypothesis that the error covariance matrix of the orthonormalized transformed dependent variables is proportional to an identity matrix.

a. Design: Intercept

Within Subjects Design: Technique

b. May be used to adjust the degrees of freedom for the averaged tests of significance. Corrected tests are displayed in the Tests of Within-Subjects Effects table.

Tests of Within-Subjects Effects

Measure: DifficultylnOperating

\begin{tabular}{|c|c|c|c|c|c|c|c|c|c|}
\hline Source & & $\begin{array}{l}\text { Type III Sum } \\
\text { of Squares }\end{array}$ & df & Mean Square & $\mathrm{F}$ & Sig. & $\begin{array}{l}\text { Partial Eta } \\
\text { Squared }\end{array}$ & $\begin{array}{l}\text { Noncent. } \\
\text { Parameter }\end{array}$ & $\begin{array}{l}\text { Observed } \\
\text { Power }^{\mathrm{a}}\end{array}$ \\
\hline \multirow[t]{4}{*}{ Technique } & Sphericity Assumed & 46.491 & 7 & 6.642 & 7.519 & .000 & .366 & 52.631 & 1.000 \\
\hline & Greenhouse-Geisser & 46.491 & 4.113 & 11.303 & 7.519 & .000 & .366 & 30.926 & .995 \\
\hline & Huynh-Feldt & 46.491 & 6.255 & 7.433 & 7.519 & .000 & .366 & 47.028 & 1.000 \\
\hline & Lower-bound & 46.491 & 1.000 & 46.491 & 7.519 & .017 & .366 & 7.519 & .718 \\
\hline \multirow[t]{4}{*}{ Error(Technique) } & Sphericity Assumed & 80.384 & 91 & .883 & & & & & \\
\hline & Greenhouse-Geisser & 80.384 & 53.472 & 1.503 & & & & & \\
\hline & Huynh-Feldt & 80.384 & 81.312 & .989 & & & & & \\
\hline & Lower-bound & 80.384 & 13.000 & 6.183 & & & & & \\
\hline
\end{tabular}

a. Computed using alpha $=.05$

Figure G-11 (Continued) 


\begin{tabular}{|c|c|c|c|c|c|c|}
\hline \multicolumn{7}{|c|}{ Pairwise Comparisons } \\
\hline Measure: In & ontrol & & & & & \\
\hline \multirow[b]{2}{*}{ (l) Technique } & \multirow[b]{2}{*}{ (J) Technique } & \multirow{2}{*}{$\begin{array}{c}\text { Mean } \\
\text { Difference (I- } \\
\mathrm{J})\end{array}$} & \multirow[b]{2}{*}{ Std. Error } & \multirow[b]{2}{*}{ Sig. } & \multicolumn{2}{|c|}{$\begin{array}{l}\text { 95\% Confidence Interval for } \\
\text { Difference }\end{array}$} \\
\hline & & & & & Lower Bound & Upper Bound \\
\hline \multirow[t]{7}{*}{1} & 2 & -.267 & .228 & .262 & -.756 & .223 \\
\hline & 3 & -.133 & .256 & .610 & -.682 & .415 \\
\hline & 4 & .000 & .324 & 1.000 & -.694 & .694 \\
\hline & 5 & -.667 & .232 & .012 & -1.165 & -.168 \\
\hline & 6 & -.467 & .274 & .110 & -1.054 & .120 \\
\hline & 7 & 1.000 & .293 & .004 & .372 & 1.628 \\
\hline & 8 & .333 & .319 & .313 & -.350 & 1.017 \\
\hline \multirow[t]{7}{*}{2} & 1 & .267 & .228 & .262 & -.223 & .756 \\
\hline & 3 & .133 & .236 & .582 & -.374 & .640 \\
\hline & 4 & .267 & .228 & .262 & -.223 & .756 \\
\hline & 5 & -.400 & .214 & .082 & -.859 & .059 \\
\hline & 6 & -.200 & .243 & .424 & -.721 & .321 \\
\hline & 7 & 1.267 & .206 & .000 & .824 & 1.709 \\
\hline & 8 & .600 & .273 & .045 & .015 & 1.185 \\
\hline \multirow[t]{7}{*}{3} & 1 & .133 & .256 & .610 & -.415 & .682 \\
\hline & 2 & -.133 & .236 & .582 & -.640 & .374 \\
\hline & 4 & .133 & .274 & .634 & -.454 & .720 \\
\hline & 5 & -.533 & .236 & .041 & -1.040 & -.026 \\
\hline & 6 & -.333 & .211 & .136 & -.785 & .119 \\
\hline & 7 & 1.133 & .307 & .002 & .476 & 1.791 \\
\hline & 8 & .467 & .236 & .068 & -.040 & .974 \\
\hline \multirow[t]{7}{*}{4} & 1 & .000 & .324 & 1.000 & -.694 & .694 \\
\hline & 2 & -.267 & .228 & .262 & -.756 & .223 \\
\hline & 3 & -.133 & .274 & .634 & -.720 & .454 \\
\hline & 5 & -.667 & .252 & .019 & -1.207 & -.126 \\
\hline & 6 & -.467 & .291 & .131 & -1.090 & .157 \\
\hline & 7 & 1.000 & .218 & .000 & .532 & 1.468 \\
\hline & 8 & .333 & .211 & .136 & -.119 & .785 \\
\hline \multirow[t]{7}{*}{5} & 1 & .667 & .232 & .012 & .168 & 1.165 \\
\hline & 2 & .400 & .214 & .082 & -.059 & .859 \\
\hline & 3 & .533 & .236 & .041 & .026 & 1.040 \\
\hline & 4 & .667 & .252 & .019 & .126 & 1.207 \\
\hline & 6 & .200 & .145 & .189 & -.110 & .510 \\
\hline & 7 & 1.667 & .252 & .000 & 1.126 & 2.207 \\
\hline & 8 & 1.000 & .169 & .000 & .637 & 1.363 \\
\hline \multirow[t]{7}{*}{6} & 1 & .467 & .274 & .110 & -.120 & 1.054 \\
\hline & 2 & .200 & .243 & .424 & -.321 & .721 \\
\hline & 3 & .333 & .211 & .136 & -.119 & .785 \\
\hline & 4 & .467 & .291 & .131 & -.157 & 1.090 \\
\hline & 5 & -.200 & .145 & .189 & -.510 & .110 \\
\hline & 7 & 1.467 & .274 & .000 & .880 & 2.054 \\
\hline & 8 & .800 & .200 & .001 & .371 & 1.229 \\
\hline \multirow[t]{7}{*}{7} & 1 & -1.000 & .293 & .004 & -1.628 & -.372 \\
\hline & 2 & -1.267 & .206 & .000 & -1.709 & -.824 \\
\hline & 3 & -1.133 & .307 & .002 & -1.791 & -.476 \\
\hline & 4 & -1.000 & .218 & .000 & -1.468 & -.532 \\
\hline & 5 & -1.667 & .252 & .000 & -2.207 & -1.126 \\
\hline & 6 & -1.467 & .274 & .000 & -2.054 & -.880 \\
\hline & 8 & -.667 & .270 & .027 & -1.246 & -.087 \\
\hline \multirow[t]{7}{*}{8} & 1 & -.333 & .319 & .313 & -1.017 & .350 \\
\hline & 2 & -.600 & .273 & .045 & -1.185 & -.015 \\
\hline & 3 & -.467 & .236 & .068 & -.974 & .040 \\
\hline & 4 & -.333 & .211 & .136 & -.785 & .119 \\
\hline & 5 & -1.000 & .169 & .000 & -1.363 & -.637 \\
\hline & 6 & -.800 & .200 & .001 & -1.229 & -.371 \\
\hline & 7 & .667 & .270 & .027 & .087 & 1.246 \\
\hline
\end{tabular}

Figure G-12: Detailed data analysis for the feeling of being in control for neurotypical individuals. 


\section{Mauchly's Test of Sphericity ${ }^{a}$}

Measure: InControl

\begin{tabular}{|l|r|r|r|r|r|r|r|}
\hline & & & & \multicolumn{3}{|c|}{ Epsilon $^{\text {b }}$} \\
\cline { 6 - 9 } Within Subjects Effect & Mauchly's W & $\begin{array}{c}\text { Approx. Chi- } \\
\text { Square }\end{array}$ & df & Sig. & $\begin{array}{c}\text { Greenhouse- } \\
\text { Geisser }\end{array}$ & Huynh-Feldt & Lower-bound \\
\hline Technique & .070 & 30.477 & 27 & .322 & .634 & .965 & .143 \\
\hline
\end{tabular}

Tests the null hypothesis that the error covariance matrix of the orthonormalized transformed dependent variables is proportional to an identity matrix.

a. Design: Intercept

Within Subjects Design: Technique

b. May be used to adjust the degrees of freedom for the averaged tests of significance. Corrected tests are displayed in the Tests of Within-Subjects Effects table.

Tests of Within-Subjects Effects

Measure: InControl

\begin{tabular}{|c|c|c|c|c|c|c|c|c|c|}
\hline Source & & $\begin{array}{l}\text { Type III Sum } \\
\text { of Squares }\end{array}$ & df & Mean Square & $\mathrm{F}$ & Sig. & $\begin{array}{l}\text { Partial Eta } \\
\text { Squared }\end{array}$ & $\begin{array}{l}\text { Noncent. } \\
\text { Parameter }\end{array}$ & $\begin{array}{l}\text { Observed } \\
\text { Power }^{\mathrm{a}}\end{array}$ \\
\hline \multirow[t]{4}{*}{ Technique } & Sphericity Assumed & 27.858 & 7 & 3.980 & 8.569 & .000 & .380 & 59.981 & 1.000 \\
\hline & Greenhouse-Geisser & 27.858 & 4.440 & 6.275 & 8.569 & .000 & .380 & 38.043 & .999 \\
\hline & Huynh-Feldt & 27.858 & 6.757 & 4.123 & 8.569 & .000 & .380 & 57.898 & 1.000 \\
\hline & Lower-bound & 27.858 & 1.000 & 27.858 & 8.569 & .011 & .380 & 8.569 & .777 \\
\hline \multirow[t]{4}{*}{ Error(Technique) } & Sphericity Assumed & 45.517 & 98 & .464 & & & & & \\
\hline & Greenhouse-Geisser & 45.517 & 62.157 & .732 & & & & & \\
\hline & Huynh-Feldt & 45.517 & 94.597 & .481 & & & & & \\
\hline & Lower-bound & 45.517 & 14.000 & 3.251 & & & & & \\
\hline
\end{tabular}

a. Computed using alpha $=.05$

Figure G-12 (Continued) 


\begin{tabular}{|c|c|c|c|c|c|c|}
\hline \multicolumn{7}{|c|}{ Pairwise Comparisons } \\
\hline Measure: In & ontrol & & & & & \\
\hline \multirow[b]{2}{*}{ (l) Technique } & \multirow[b]{2}{*}{ (J) Technique } & \multirow{2}{*}{$\begin{array}{c}\text { Mean } \\
\text { Difference (I- } \\
\mathrm{J})\end{array}$} & \multirow[b]{2}{*}{ Std. Error } & \multirow[b]{2}{*}{ Sig. } & \multicolumn{2}{|c|}{$\begin{array}{l}\text { 95\% Confidence Interval for } \\
\text { Difference }\end{array}$} \\
\hline & & & & & Lower Bound & Upper Bound \\
\hline \multirow[t]{7}{*}{1} & 2 & .286 & .163 & .104 & -.067 & .639 \\
\hline & 3 & .071 & .165 & .671 & -.284 & .427 \\
\hline & 4 & -.143 & .177 & .435 & -.526 & .240 \\
\hline & 5 & -.357 & .269 & .208 & -.939 & .225 \\
\hline & 6 & -.357 & .289 & .239 & -.982 & .267 \\
\hline & 7 & 1.071 & .221 & .000 & .593 & 1.550 \\
\hline & 8 & .857 & .443 & .075 & -.100 & 1.814 \\
\hline \multirow[t]{7}{*}{2} & 1 & -.286 & .163 & .104 & -.639 & .067 \\
\hline & 3 & -.214 & .187 & .272 & -.618 & .189 \\
\hline & 4 & -.429 & .202 & .054 & -.865 & .008 \\
\hline & 5 & -.643 & .325 & .069 & -1.345 & .059 \\
\hline & 6 & -.643 & .308 & .057 & -1.307 & .022 \\
\hline & 7 & .786 & .155 & .000 & .451 & 1.120 \\
\hline & 8 & .571 & .374 & .150 & -.236 & 1.379 \\
\hline \multirow[t]{7}{*}{3} & 1 & -.071 & .165 & .671 & -.427 & .284 \\
\hline & 2 & .214 & .187 & .272 & -.189 & .618 \\
\hline & 4 & -.214 & .187 & .272 & -.618 & .189 \\
\hline & 5 & -.429 & .251 & .111 & -.970 & .113 \\
\hline & 6 & -.429 & .272 & .139 & -1.015 & .158 \\
\hline & 7 & 1.000 & .210 & .000 & .547 & 1.453 \\
\hline & 8 & .786 & .381 & .059 & -.036 & 1.608 \\
\hline \multirow[t]{7}{*}{4} & 1 & .143 & .177 & .435 & -.240 & .526 \\
\hline & 2 & .429 & .202 & .054 & -.008 & .865 \\
\hline & 3 & .214 & .187 & .272 & -189 & .618 \\
\hline & 5 & -.214 & .300 & .487 & -.862 & .433 \\
\hline & 6 & -.214 & .318 & .512 & -.900 & .472 \\
\hline & 7 & 1.214 & .300 & .001 & .567 & 1.862 \\
\hline & 8 & 1.000 & .378 & .020 & .183 & 1.817 \\
\hline \multirow[t]{7}{*}{5} & 1 & .357 & .269 & .208 & -.225 & .939 \\
\hline & 2 & .643 & .325 & .069 & -.059 & 1.345 \\
\hline & 3 & .429 & .251 & .111 & -.113 & .970 \\
\hline & 4 & .214 & .300 & .487 & -.433 & .862 \\
\hline & 6 & .000 & .148 & 1.000 & -.320 & .320 \\
\hline & 7 & 1.429 & .291 & .000 & .800 & 2.058 \\
\hline & 8 & 1.214 & .447 & .018 & .249 & 2.180 \\
\hline \multirow[t]{7}{*}{6} & 1 & .357 & .289 & .239 & -.267 & .982 \\
\hline & 2 & .643 & .308 & .057 & -.022 & 1.307 \\
\hline & 3 & .429 & .272 & .139 & -.158 & 1.015 \\
\hline & 4 & .214 & .318 & .512 & -.472 & .900 \\
\hline & 5 & .000 & .148 & 1.000 & -.320 & .320 \\
\hline & 7 & 1.429 & .291 & .000 & .800 & 2.058 \\
\hline & 8 & 1.214 & .434 & .015 & .276 & 2.153 \\
\hline \multirow[t]{7}{*}{7} & 1 & -1.071 & .221 & .000 & -1.550 & -.593 \\
\hline & 2 & -.786 & .155 & .000 & -1.120 & -.451 \\
\hline & 3 & -1.000 & .210 & .000 & -1.453 & -.547 \\
\hline & 4 & -1.214 & .300 & .001 & -1.862 & -.567 \\
\hline & 5 & -1.429 & .291 & .000 & -2.058 & -.800 \\
\hline & 6 & -1.429 & .291 & .000 & -2.058 & -.800 \\
\hline & 8 & -.214 & .408 & .609 & -1.097 & .668 \\
\hline \multirow[t]{7}{*}{8} & 1 & -.857 & .443 & .075 & -1.814 & .100 \\
\hline & 2 & -.571 & .374 & .150 & -1.379 & .236 \\
\hline & 3 & -.786 & .381 & .059 & -1.608 & .036 \\
\hline & 4 & -1.000 & .378 & .020 & -1.817 & -.183 \\
\hline & 5 & -1.214 & .447 & .018 & -2.180 & -.249 \\
\hline & 6 & -1.214 & .434 & .015 & -2.153 & -.276 \\
\hline & 7 & .214 & .408 & .609 & -.668 & 1.097 \\
\hline
\end{tabular}

Figure G-13: Detailed data analysis for the feeling of being in control for individuals with ASD. 


\section{Mauchly's Test of Sphericity ${ }^{a}$}

Measure: InControl

\begin{tabular}{|l|r|r|r|r|r|r|r|}
\hline & & & & \multicolumn{3}{|c|}{ Epsilon $^{\text {b }}$} \\
\cline { 6 - 9 } Within Subjects Effect & Mauchly's W & $\begin{array}{c}\text { Approx. Chi- } \\
\text { Square }\end{array}$ & df & Sig. & $\begin{array}{c}\text { Greenhouse- } \\
\text { Geisser }\end{array}$ & Huynh-Feldt & Lower-bound \\
\hline Technique & .003 & 60.732 & 27 & .000 & .468 & .645 & .143 \\
\hline
\end{tabular}

Tests the null hypothesis that the error covariance matrix of the orthonormalized transformed dependent variables is proportional to an identity matrix.

a. Design: Intercept

Within Subjects Design: Technique

b. May be used to adjust the degrees of freedom for the averaged tests of significance. Corrected tests are displayed in the Tests of Within-Subjects Effects table.

Tests of Within-Subjects Effects

Measure: InControl

\begin{tabular}{|c|c|c|c|c|c|c|c|c|c|}
\hline Source & & $\begin{array}{l}\text { Type III Sum } \\
\text { of Squares }\end{array}$ & df & Mean Square & $\mathrm{F}$ & Sig. & $\begin{array}{l}\text { Partial Eta } \\
\text { Squared }\end{array}$ & $\begin{array}{l}\text { Noncent. } \\
\text { Parameter }\end{array}$ & $\begin{array}{l}\text { Observed } \\
\text { Power }^{\mathrm{a}}\end{array}$ \\
\hline \multirow[t]{4}{*}{ Technique } & Sphericity Assumed & 27.857 & 7 & 3.980 & 6.479 & .000 & .333 & 45.355 & 1.000 \\
\hline & Greenhouse-Geisser & 27.857 & 3.277 & 8.501 & 6.479 & .001 & .333 & 21.233 & .966 \\
\hline & Huynh-Feldt & 27.857 & 4.513 & 6.173 & 6.479 & .000 & .333 & 29.240 & .992 \\
\hline & Lower-bound & 27.857 & 1.000 & 27.857 & 6.479 & .024 & .333 & 6.479 & .653 \\
\hline \multirow[t]{4}{*}{ Error(Technique) } & Sphericity Assumed & 55.893 & 91 & .614 & & & & & \\
\hline & Greenhouse-Geisser & 55.893 & 42.602 & 1.312 & & & & & \\
\hline & Huynh-Feldt & 55.893 & 58.668 & .953 & & & & & \\
\hline & Lower-bound & 55.893 & 13.000 & 4.299 & & & & & \\
\hline
\end{tabular}

a. Computed using alpha $=.05$

Figure G-13 (Continued) 


\begin{tabular}{|c|c|c|c|c|c|c|}
\hline \multicolumn{7}{|c|}{ Pairwise Comparisons } \\
\hline : En & & & & & & \\
\hline \multirow[b]{2}{*}{ (l) Technique } & \multirow[b]{2}{*}{ (J) Technique } & \multirow{2}{*}{$\begin{array}{c}\text { Mean } \\
\text { Difference (I- } \\
\mathrm{J})\end{array}$} & \multirow[b]{2}{*}{ Std. Error } & \multirow[b]{2}{*}{ Sig. } & \multicolumn{2}{|c|}{$\begin{array}{l}95 \% \text { Confidence Interval for } \\
\text { Difference }\end{array}$} \\
\hline & & & & & Lower Bound & Upper Bound \\
\hline \multirow[t]{7}{*}{1} & 2 & -.133 & .291 & .653 & -.757 & .490 \\
\hline & 3 & .467 & .215 & .048 & .005 & .928 \\
\hline & 4 & -.467 & .322 & .169 & -1.157 & .223 \\
\hline & 5 & -.267 & .396 & .512 & -1.116 & .583 \\
\hline & 6 & -.267 & .371 & .484 & -1.063 & .529 \\
\hline & 7 & 1.133 & .350 & .006 & .383 & 1.884 \\
\hline & 8 & .467 & .435 & .301 & -.466 & 1.400 \\
\hline \multirow[t]{7}{*}{2} & 1 & .133 & .291 & .653 & -.490 & .757 \\
\hline & 3 & .600 & .254 & .033 & .054 & 1.146 \\
\hline & 4 & -.333 & .187 & .096 & -.734 & .067 \\
\hline & 5 & -.133 & .336 & .698 & -.854 & .588 \\
\hline & 6 & -.133 & .291 & .653 & -.757 & .490 \\
\hline & 7 & 1.267 & .206 & .000 & .824 & 1.709 \\
\hline & 8 & .600 & .375 & .132 & -.205 & 1.405 \\
\hline \multirow[t]{7}{*}{3} & 1 & -.467 & .215 & .048 & -.928 & -.005 \\
\hline & 2 & -.600 & .254 & .033 & -1.146 & -.054 \\
\hline & 4 & -.933 & .267 & .004 & -1.505 & -.361 \\
\hline & 5 & -.733 & .419 & .102 & -1.633 & .166 \\
\hline & 6 & -.733 & .345 & .052 & -1.472 & .006 \\
\hline & 7 & .667 & .319 & .055 & -.017 & 1.350 \\
\hline & 8 & .000 & .378 & 1.000 & -.811 & .811 \\
\hline \multirow[t]{7}{*}{4} & 1 & .467 & .322 & .169 & -.223 & 1.157 \\
\hline & 2 & .333 & .187 & .096 & -.067 & .734 \\
\hline & 3 & .933 & .267 & .004 & .361 & 1.505 \\
\hline & 5 & .200 & .341 & .567 & -.531 & .931 \\
\hline & 6 & .200 & .312 & .531 & -.468 & .868 \\
\hline & 7 & 1.600 & .273 & .000 & 1.015 & 2.185 \\
\hline & 8 & .933 & .345 & .017 & .194 & 1.672 \\
\hline \multirow[t]{7}{*}{5} & 1 & .267 & .396 & .512 & -.583 & 1.116 \\
\hline & 2 & .133 & .336 & .698 & -.588 & .854 \\
\hline & 3 & .733 & .419 & .102 & -.166 & 1.633 \\
\hline & 4 & -.200 & .341 & .567 & -.931 & .531 \\
\hline & 6 & .000 & .258 & 1.000 & -.554 & .554 \\
\hline & 7 & 1.400 & .273 & .000 & .815 & 1.985 \\
\hline & 8 & .733 & .267 & .016 & .161 & 1.305 \\
\hline \multirow[t]{7}{*}{6} & 1 & .267 & .371 & .484 & -.529 & 1.063 \\
\hline & 2 & .133 & .291 & .653 & -.490 & .757 \\
\hline & 3 & .733 & .345 & .052 & -.006 & 1.472 \\
\hline & 4 & -.200 & .312 & .531 & -.868 & .468 \\
\hline & 5 & .000 & .258 & 1.000 & -.554 & .554 \\
\hline & 7 & 1.400 & .273 & .000 & .815 & 1.985 \\
\hline & 8 & .733 & .316 & .036 & .056 & 1.410 \\
\hline \multirow[t]{7}{*}{7} & 1 & -1.133 & .350 & .006 & -1.884 & -.383 \\
\hline & 2 & -1.267 & .206 & .000 & -1.709 & -.824 \\
\hline & 3 & -.667 & .319 & .055 & -1.350 & .017 \\
\hline & 4 & -1.600 & .273 & .000 & -2.185 & -1.015 \\
\hline & 5 & -1.400 & .273 & .000 & -1.985 & -.815 \\
\hline & 6 & -1.400 & .273 & .000 & -1.985 & -.815 \\
\hline & 8 & -.667 & .303 & .045 & -1.317 & -.016 \\
\hline \multirow[t]{7}{*}{8} & 1 & -.467 & .435 & .301 & -1.400 & .466 \\
\hline & 2 & -.600 & .375 & .132 & -1.405 & .205 \\
\hline & 3 & .000 & .378 & 1.000 & -.811 & .811 \\
\hline & 4 & -.933 & .345 & .017 & -1.672 & -.194 \\
\hline & 5 & -.733 & .267 & .016 & -1.305 & -.161 \\
\hline & 6 & -.733 & .316 & .036 & -1.410 & -.056 \\
\hline & 7 & .667 & .303 & .045 & .016 & 1.317 \\
\hline
\end{tabular}

Figure G-14: Detailed data analysis for the enjoyment for neurotypical individuals. 


\section{Mauchly's Test of Sphericity ${ }^{a}$}

Measure: Enjoyment

\begin{tabular}{|l|r|r|r|r|r|r|r|}
\hline & & & & \multicolumn{3}{|c|}{ Epsilon $^{\text {b }}$} \\
\cline { 6 - 9 } Within Subjects Effect & Mauchly's W & $\begin{array}{c}\text { Approx. Chi- } \\
\text { Square }\end{array}$ & df & Sig. & $\begin{array}{c}\text { Greenhouse- } \\
\text { Geisser }\end{array}$ & Huynh-Feldt & Lower-bound \\
\hline Technique & .049 & 34.586 & 27 & .172 & .547 & .778 & .143 \\
\hline
\end{tabular}

Tests the null hypothesis that the error covariance matrix of the orthonormalized transformed dependent variables is proportional to an identity matrix.

a. Design: Intercept

Within Subjects Design: Technique

b. May be used to adjust the degrees of freedom for the averaged tests of significance. Corrected tests are displayed in the Tests of Within-Subjects Effects table.

Tests of Within-Subjects Effects

Measure: Enjoyment

\begin{tabular}{|c|c|c|c|c|c|c|c|c|c|}
\hline Source & & $\begin{array}{l}\text { Type III Sum } \\
\text { of Squares }\end{array}$ & df & Mean Square & $\mathrm{F}$ & Sig. & $\begin{array}{l}\text { Partial Eta } \\
\text { Squared }\end{array}$ & $\begin{array}{l}\text { Noncent. } \\
\text { Parameter }\end{array}$ & $\begin{array}{l}\text { Observed } \\
\text { Power }^{\text {a }}\end{array}$ \\
\hline \multirow[t]{4}{*}{ Technique } & Sphericity Assumed & 29.833 & 7 & 4.262 & 5.651 & .000 & .288 & 39.554 & .998 \\
\hline & Greenhouse-Geisser & 29.833 & 3.827 & 7.796 & 5.651 & .001 & .288 & 21.623 & .965 \\
\hline & Huynh-Feldt & 29.833 & 5.446 & 5.478 & 5.651 & .000 & .288 & 30.771 & .992 \\
\hline & Lower-bound & 29.833 & 1.000 & 29.833 & 5.651 & .032 & .288 & 5.651 & .600 \\
\hline \multirow[t]{4}{*}{ Error(Technique) } & Sphericity Assumed & 73.917 & 98 & .754 & & & & & \\
\hline & Greenhouse-Geisser & 73.917 & 53.574 & 1.380 & & & & & \\
\hline & Huynh-Feldt & 73.917 & 76.240 & .970 & & & & & \\
\hline & Lower-bound & 73.917 & 14.000 & 5.280 & & & & & \\
\hline
\end{tabular}

a. Computed using alpha $=.05$

Figure G-14 (Continued) 


\begin{tabular}{|c|c|c|c|c|c|c|}
\hline \multicolumn{7}{|c|}{ Pairwise Comparisons } \\
\hline e: En & & & & & & \\
\hline \multirow[b]{2}{*}{ (I) Technique } & \multirow[b]{2}{*}{ (J) Technique } & \multirow{2}{*}{$\begin{array}{c}\text { Mean } \\
\text { Difference (I- } \\
\mathrm{J})\end{array}$} & \multirow[b]{2}{*}{ Std. Error } & \multirow[b]{2}{*}{ Sig. } & \multicolumn{2}{|c|}{$\begin{array}{l}95 \% \text { Confidence Interval for } \\
\text { Difference }\end{array}$} \\
\hline & & & & & Lower Bound & Upper Bound \\
\hline \multirow[t]{7}{*}{1} & 2 & -.286 & .286 & .336 & -.903 & .332 \\
\hline & 3 & .143 & .177 & .435 & -.240 & .526 \\
\hline & 4 & -.786 & .261 & .010 & -1.349 & -.223 \\
\hline & 5 & -.857 & .294 & .012 & -1.492 & -.222 \\
\hline & 6 & -.500 & .251 & .068 & -1.043 & .043 \\
\hline & 7 & .429 & .359 & .254 & -.347 & 1.204 \\
\hline & 8 & .714 & .398 & .096 & -.146 & 1.575 \\
\hline \multirow[t]{7}{*}{2} & 1 & .286 & .286 & .336 & -.332 & .903 \\
\hline & 3 & .429 & .251 & .111 & -.113 & .970 \\
\hline & 4 & -.500 & .251 & .068 & -1.043 & .043 \\
\hline & 5 & -.571 & .309 & .088 & -1.240 & .097 \\
\hline & 6 & -.214 & .366 & .568 & -1.005 & .576 \\
\hline & 7 & .714 & .266 & .019 & .140 & 1.288 \\
\hline & 8 & 1.000 & .348 & .013 & .249 & 1.751 \\
\hline \multirow[t]{7}{*}{3} & 1 & -.143 & .177 & .435 & -.526 & .240 \\
\hline & 2 & -.429 & .251 & .111 & -.970 & .113 \\
\hline & 4 & -.929 & .245 & .002 & -1.458 & -.399 \\
\hline & 5 & -1.000 & .277 & .003 & -1.599 & -.401 \\
\hline & 6 & -.643 & .269 & .033 & -1.225 & -.061 \\
\hline & 7 & .286 & .384 & .470 & -.544 & 1.116 \\
\hline & 8 & .571 & .402 & .179 & -.297 & 1.440 \\
\hline \multirow[t]{7}{*}{4} & 1 & .786 & .261 & .010 & .223 & 1.349 \\
\hline & 2 & .500 & .251 & .068 & -.043 & 1.043 \\
\hline & 3 & .929 & .245 & .002 & .399 & 1.458 \\
\hline & 5 & -.071 & .165 & .671 & -.427 & .284 \\
\hline & 6 & .286 & .266 & .302 & -.288 & .860 \\
\hline & 7 & 1.214 & .281 & .001 & .608 & 1.821 \\
\hline & 8 & 1.500 & .416 & .003 & .601 & 2.399 \\
\hline \multirow[t]{7}{*}{5} & 1 & .857 & .294 & .012 & .222 & 1.492 \\
\hline & 2 & .571 & .309 & .088 & -.097 & 1.240 \\
\hline & 3 & 1.000 & .277 & .003 & .401 & 1.599 \\
\hline & 4 & .071 & .165 & .671 & -.284 & .427 \\
\hline & 6 & .357 & .225 & .136 & -.129 & .843 \\
\hline & 7 & 1.286 & .384 & .005 & .456 & 2.116 \\
\hline & 8 & 1.571 & .416 & .002 & .674 & 2.469 \\
\hline \multirow[t]{7}{*}{6} & 1 & .500 & .251 & .068 & -.043 & 1.043 \\
\hline & 2 & .214 & .366 & .568 & -.576 & 1.005 \\
\hline & 3 & .643 & .269 & .033 & .061 & 1.225 \\
\hline & 4 & -.286 & .266 & .302 & -.860 & .288 \\
\hline & 5 & -.357 & .225 & .136 & -.843 & .129 \\
\hline & 7 & .929 & .438 & .054 & -.018 & 1.875 \\
\hline & 8 & 1.214 & .434 & .015 & .276 & 2.153 \\
\hline \multirow[t]{7}{*}{7} & 1 & -.429 & .359 & .254 & -1.204 & .347 \\
\hline & 2 & -.714 & .266 & .019 & -1.288 & -.140 \\
\hline & 3 & -.286 & .384 & .470 & -1.116 & .544 \\
\hline & 4 & -1.214 & .281 & .001 & -1.821 & -.608 \\
\hline & 5 & -1.286 & .384 & .005 & -2.116 & -.456 \\
\hline & 6 & -.929 & .438 & .054 & -1.875 & .018 \\
\hline & 8 & .286 & .450 & .537 & -.686 & 1.258 \\
\hline \multirow[t]{7}{*}{8} & 1 & -.714 & .398 & .096 & -1.575 & .146 \\
\hline & 2 & -1.000 & .348 & .013 & -1.751 & -.249 \\
\hline & 3 & -.571 & .402 & .179 & -1.440 & .297 \\
\hline & 4 & -1.500 & .416 & .003 & -2.399 & -.601 \\
\hline & 5 & -1.571 & .416 & .002 & -2.469 & -.674 \\
\hline & 6 & -1.214 & .434 & .015 & -2.153 & -.276 \\
\hline & 7 & -.286 & .450 & .537 & -1.258 & .686 \\
\hline
\end{tabular}

Figure G-15: Detailed data analysis for the enjoyment for individuals with ASD. 


\section{Mauchly's Test of Sphericity ${ }^{a}$}

Measure: Enjoyment

\begin{tabular}{|l|r|r|r|r|r|r|r|}
\hline & & & & \multicolumn{3}{|c|}{ Epsilon $^{\text {b }}$} \\
\cline { 6 - 9 } Within Subjects Effect & Mauchly's W & $\begin{array}{c}\text { Approx. Chi- } \\
\text { Square }\end{array}$ & df & Sig. & $\begin{array}{c}\text { Greenhouse- } \\
\text { Geisser }\end{array}$ & Huynh-Feldt & Lower-bound \\
\hline Technique & .024 & 39.168 & 27 & .077 & .557 & .825 & .143 \\
\hline
\end{tabular}

Tests the null hypothesis that the error covariance matrix of the orthonormalized transformed dependent variables is proportional to an identity matrix.

a. Design: Intercept

Within Subjects Design: Technique

b. May be used to adjust the degrees of freedom for the averaged tests of significance. Corrected tests are displayed in the Tests of Within-Subjects Effects table.

Tests of Within-Subjects Effects

Measure: Enjoyment

\begin{tabular}{|c|c|c|c|c|c|c|c|c|c|}
\hline Source & & $\begin{array}{l}\text { Type III Sum } \\
\text { of Squares }\end{array}$ & df & Mean Square & $\mathrm{F}$ & Sig. & $\begin{array}{l}\text { Partial Eta } \\
\text { Squared }\end{array}$ & $\begin{array}{l}\text { Noncent. } \\
\text { Parameter }\end{array}$ & $\begin{array}{l}\text { Observed } \\
\text { Power }^{\text {a }}\end{array}$ \\
\hline \multirow[t]{4}{*}{ Technique } & Sphericity Assumed & 31.286 & 7 & 4.469 & 5.984 & .000 & .315 & 41.890 & .999 \\
\hline & Greenhouse-Geisser & 31.286 & 3.897 & 8.029 & 5.984 & .001 & .315 & 23.318 & .974 \\
\hline & Huynh-Feldt & 31.286 & 5.773 & 5.419 & 5.984 & .000 & .315 & 34.546 & .996 \\
\hline & Lower-bound & 31.286 & 1.000 & 31.286 & 5.984 & .029 & .315 & 5.984 & .619 \\
\hline \multirow[t]{4}{*}{ Error(Technique) } & Sphericity Assumed & 67.964 & 91 & .747 & & & & & \\
\hline & Greenhouse-Geisser & 67.964 & 50.656 & 1.342 & & & & & \\
\hline & Huynh-Feldt & 67.964 & 75.047 & .906 & & & & & \\
\hline & Lower-bound & 67.964 & 13.000 & 5.228 & & & & & \\
\hline
\end{tabular}

a. Computed using alpha $=.05$

Figure G-15 (Continued) 


\begin{tabular}{|c|c|c|c|c|c|c|}
\hline \multicolumn{7}{|c|}{ Pairwise Comparisons } \\
\hline & & \multirow{3}{*}{$\begin{array}{c}\text { Mean } \\
\text { Difference (I- } \\
\mathrm{J})\end{array}$} & & & & \\
\hline \multirow[b]{2}{*}{ (l) Technique } & \multirow[b]{2}{*}{ (J) Technique } & & \multirow[b]{2}{*}{ Std. Error } & \multirow[b]{2}{*}{ Sig. } & \multicolumn{2}{|c|}{$\begin{array}{l}95 \% \text { Confidence Interval for } \\
\text { Difference }\end{array}$} \\
\hline & & & & & Lower Bound & Upper Bound \\
\hline \multirow[t]{7}{*}{1} & 2 & .267 & .358 & .469 & -.501 & 1.035 \\
\hline & 3 & .133 & .322 & .685 & -.557 & .823 \\
\hline & 4 & 1.133 & .363 & .008 & .354 & 1.913 \\
\hline & 5 & 1.467 & .291 & .000 & .843 & 2.090 \\
\hline & 6 & 1.400 & .321 & .001 & .712 & 2.088 \\
\hline & 7 & -.133 & .307 & .670 & -.791 & .524 \\
\hline & 8 & .667 & .319 & .055 & -.017 & 1.350 \\
\hline \multirow[t]{7}{*}{2} & 1 & -.267 & .358 & .469 & -1.035 & .501 \\
\hline & 3 & -.133 & .350 & .709 & -.884 & .617 \\
\hline & 4 & .867 & .256 & .004 & .318 & 1.415 \\
\hline & 5 & 1.200 & .312 & .002 & .532 & 1.868 \\
\hline & 6 & 1.133 & .307 & .002 & .476 & 1.791 \\
\hline & 7 & -.400 & .254 & .138 & -.946 & .146 \\
\hline & 8 & .400 & .335 & .253 & -.319 & 1.119 \\
\hline \multirow[t]{7}{*}{3} & 1 & -.133 & .322 & .685 & -.823 & .557 \\
\hline & 2 & .133 & .350 & .709 & -.617 & .884 \\
\hline & 4 & 1.000 & .352 & .013 & .245 & 1.755 \\
\hline & 5 & 1.333 & .270 & .000 & .754 & 1.913 \\
\hline & 6 & 1.267 & .284 & .001 & .658 & 1.876 \\
\hline & 7 & -.267 & .396 & .512 & -1.116 & .583 \\
\hline & 8 & .533 & .350 & .150 & -.217 & 1.284 \\
\hline \multirow[t]{7}{*}{4} & 1 & -1.133 & .363 & .008 & -1.913 & -.354 \\
\hline & 2 & -.867 & .256 & .004 & -1.415 & -.318 \\
\hline & 3 & -1.000 & .352 & .013 & -1.755 & -.245 \\
\hline & 5 & .333 & .287 & .265 & -.283 & .950 \\
\hline & 6 & .267 & .316 & .413 & -.410 & .944 \\
\hline & 7 & -1.267 & .316 & .001 & -1.944 & -.590 \\
\hline & 8 & -.467 & .307 & .150 & -1.124 & .191 \\
\hline \multirow[t]{7}{*}{5} & 1 & -1.467 & .291 & .000 & -2.090 & -.843 \\
\hline & 2 & -1.200 & .312 & .002 & -1.868 & -.532 \\
\hline & 3 & -1.333 & .270 & .000 & -1.913 & -.754 \\
\hline & 4 & -.333 & .287 & .265 & -.950 & .283 \\
\hline & 6 & -.067 & .228 & .774 & -.556 & .423 \\
\hline & 7 & -1.600 & .289 & .000 & -2.221 & -.979 \\
\hline & 8 & -.800 & .175 & .000 & -1.174 & -.426 \\
\hline \multirow[t]{7}{*}{6} & 1 & -1.400 & .321 & .001 & -2.088 & -.712 \\
\hline & 2 & -1.133 & .307 & .002 & -1.791 & -.476 \\
\hline & 3 & -1.267 & .284 & .001 & -1.876 & -.658 \\
\hline & 4 & -.267 & .316 & .413 & -.944 & .410 \\
\hline & 5 & .067 & .228 & .774 & -.423 & .556 \\
\hline & 7 & -1.533 & .307 & .000 & -2.191 & -.876 \\
\hline & 8 & -.733 & .316 & .036 & -1.410 & -.056 \\
\hline \multirow[t]{7}{*}{7} & 1 & .133 & .307 & .670 & -.524 & .791 \\
\hline & 2 & .400 & .254 & .138 & -.146 & .946 \\
\hline & 3 & .267 & .396 & .512 & -.583 & 1.116 \\
\hline & 4 & 1.267 & .316 & .001 & .590 & 1.944 \\
\hline & 5 & 1.600 & .289 & .000 & .979 & 2.221 \\
\hline & 6 & 1.533 & .307 & .000 & .876 & 2.191 \\
\hline & 8 & .800 & .296 & .017 & .165 & 1.435 \\
\hline \multirow[t]{7}{*}{8} & 1 & -.667 & .319 & .055 & -1.350 & .017 \\
\hline & 2 & -.400 & .335 & .253 & -1.119 & .319 \\
\hline & 3 & -.533 & .350 & .150 & -1.284 & .217 \\
\hline & 4 & .467 & .307 & .150 & -.191 & 1.124 \\
\hline & 5 & .800 & .175 & .000 & .426 & 1.174 \\
\hline & 6 & .733 & .316 & .036 & .056 & 1.410 \\
\hline & 7 & -.800 & .296 & .017 & -1.435 & -.165 \\
\hline
\end{tabular}

Figure G-16: Detailed data analysis for the required effort for neurotypical individuals. 


\section{Mauchly's Test of Sphericity ${ }^{a}$}

Measure: RequiredEffort

\begin{tabular}{|l|r|r|r|r|r|r|r|}
\hline & & & & \multicolumn{3}{|c|}{ Epsilon $^{\text {b }}$} \\
\cline { 6 - 9 } Within Subjects Effect & Mauchly's W & $\begin{array}{c}\text { Approx. Chi- } \\
\text { Square }\end{array}$ & df & Sig. & $\begin{array}{c}\text { Greenhouse- } \\
\text { Geisser }\end{array}$ & Huynh-Feldt & Lower-bound \\
\hline Technique & .169 & 20.352 & 27 & .832 & .731 & 1.000 & .143 \\
\hline
\end{tabular}

Tests the null hypothesis that the error covariance matrix of the orthonormalized transformed dependent variables is proportional to an identity matrix.

a. Design: Intercept

Within Subjects Design: Technique

b. May be used to adjust the degrees of freedom for the averaged tests of significance. Corrected tests are displayed in the Tests of Within-Subjects Effects table.

Tests of Within-Subjects Effects

Measure: RequiredEffort

\begin{tabular}{|c|c|c|c|c|c|c|c|c|c|}
\hline Source & & $\begin{array}{l}\text { Type III Sum } \\
\text { of Squares }\end{array}$ & df & Mean Square & $\mathrm{F}$ & Sig. & $\begin{array}{c}\text { Partial Eta } \\
\text { Squared }\end{array}$ & $\begin{array}{l}\text { Noncent. } \\
\text { Parameter }\end{array}$ & $\begin{array}{l}\text { Observed } \\
\text { Power }^{\text {a }}\end{array}$ \\
\hline \multirow[t]{4}{*}{ Technique } & Sphericity Assumed & 43.567 & 7 & 6.224 & 8.660 & .000 & .382 & 60.618 & 1.000 \\
\hline & Greenhouse-Geisser & 43.567 & 5.114 & 8.520 & 8.660 & .000 & .382 & 44.283 & 1.000 \\
\hline & Huynh-Feldt & 43.567 & 7.000 & 6.224 & 8.660 & .000 & .382 & 60.618 & 1.000 \\
\hline & Lower-bound & 43.567 & 1.000 & 43.567 & 8.660 & .011 & .382 & 8.660 & .781 \\
\hline \multirow[t]{4}{*}{ Error(Technique) } & Sphericity Assumed & 70.433 & 98 & .719 & & & & & \\
\hline & Greenhouse-Geisser & 70.433 & 71.591 & .984 & & & & & \\
\hline & Huynh-Feldt & 70.433 & 98.000 & .719 & & & & & \\
\hline & Lower-bound & 70.433 & 14.000 & 5.031 & & & & & \\
\hline
\end{tabular}

a. Computed using alpha $=.05$

Figure G-16 (Continued) 


\begin{tabular}{|c|c|c|c|c|c|c|}
\hline \multicolumn{7}{|c|}{ Pairwise Comparisons } \\
\hline Ire: & & & & & & \\
\hline \multirow[b]{2}{*}{ (I) Technique } & \multirow[b]{2}{*}{ (J) Technique } & \multirow{2}{*}{$\begin{array}{c}\text { Mean } \\
\text { Difference (I- } \\
\text { J) }\end{array}$} & \multirow[b]{2}{*}{ Std. Error } & \multirow[b]{2}{*}{ Sig. } & \multicolumn{2}{|c|}{$\begin{array}{l}95 \% \text { Confidence Interval for } \\
\text { Difference }\end{array}$} \\
\hline & & & & & Lower Bound & Upper Bound \\
\hline \multirow[t]{7}{*}{1} & 2 & .143 & .345 & .686 & -.603 & .889 \\
\hline & 3 & -.643 & .308 & .057 & -1.307 & .022 \\
\hline & 4 & 1.071 & .305 & .004 & .413 & 1.730 \\
\hline & 5 & .857 & .345 & .028 & .111 & 1.603 \\
\hline & 6 & .929 & .399 & .037 & .067 & 1.790 \\
\hline & 7 & -.071 & .370 & .850 & -.871 & .728 \\
\hline & 8 & -.286 & .384 & .470 & -1.116 & .544 \\
\hline \multirow[t]{7}{*}{2} & 1 & -.143 & .345 & .686 & -.889 & .603 \\
\hline & 3 & -.786 & .318 & .028 & -1.472 & -.100 \\
\hline & 4 & .929 & .267 & .004 & .353 & 1.504 \\
\hline & 5 & .714 & .244 & .012 & .187 & 1.242 \\
\hline & 6 & .786 & .350 & .043 & .029 & 1.543 \\
\hline & 7 & -.214 & .261 & .426 & -.777 & .349 \\
\hline & 8 & -.429 & .374 & .272 & -1.236 & .379 \\
\hline \multirow[t]{7}{*}{3} & 1 & .643 & .308 & .057 & -.022 & 1.307 \\
\hline & 2 & .786 & .318 & .028 & .100 & 1.472 \\
\hline & 4 & 1.714 & .304 & .000 & 1.057 & 2.372 \\
\hline & 5 & 1.500 & .442 & .005 & .546 & 2.454 \\
\hline & 6 & 1.571 & .453 & .004 & .592 & 2.551 \\
\hline & 7 & .571 & .429 & .205 & -.354 & 1.497 \\
\hline & 8 & .357 & .341 & .315 & -.380 & 1.095 \\
\hline \multirow[t]{7}{*}{4} & 1 & -1.071 & .305 & .004 & -1.730 & -.413 \\
\hline & 2 & -.929 & .267 & .004 & -1.504 & -.353 \\
\hline & 3 & -1.714 & .304 & .000 & -2.372 & -1.057 \\
\hline & 5 & -.214 & .350 & .551 & -.971 & .543 \\
\hline & 6 & -.143 & .294 & .635 & -.778 & .492 \\
\hline & 7 & -1.143 & .361 & .007 & -1.923 & -.363 \\
\hline & 8 & -1.357 & .325 & .001 & -2.059 & -.655 \\
\hline \multirow[t]{7}{*}{5} & 1 & -.857 & .345 & .028 & -1.603 & -.111 \\
\hline & 2 & -.714 & .244 & .012 & -1.242 & -.187 \\
\hline & 3 & -1.500 & .442 & .005 & -2.454 & -.546 \\
\hline & 4 & .214 & .350 & .551 & -.543 & .971 \\
\hline & 6 & .071 & .339 & .836 & -.661 & .804 \\
\hline & 7 & -.929 & .286 & .006 & -1.547 & -.310 \\
\hline & 8 & -1.143 & .361 & .007 & -1.923 & -.363 \\
\hline \multirow[t]{7}{*}{6} & 1 & -.929 & .399 & .037 & -1.790 & -.067 \\
\hline & 2 & -.786 & .350 & .043 & -1.543 & -.029 \\
\hline & 3 & -1.571 & .453 & .004 & -2.551 & -.592 \\
\hline & 4 & .143 & .294 & .635 & -.492 & .778 \\
\hline & 5 & -.071 & .339 & .836 & -.804 & .661 \\
\hline & 7 & -1.000 & .406 & .029 & -1.877 & -.123 \\
\hline & 8 & -1.214 & .459 & .020 & -2.206 & -.223 \\
\hline \multirow[t]{7}{*}{7} & 1 & .071 & .370 & .850 & -.728 & .871 \\
\hline & 2 & .214 & .261 & .426 & -.349 & .777 \\
\hline & 3 & -.571 & .429 & .205 & -1.497 & .354 \\
\hline & 4 & 1.143 & .361 & .007 & .363 & 1.923 \\
\hline & 5 & .929 & .286 & .006 & .310 & 1.547 \\
\hline & 6 & 1.000 & .406 & .029 & .123 & 1.877 \\
\hline & 8 & -.214 & .408 & .609 & -1.097 & .668 \\
\hline \multirow[t]{7}{*}{8} & 1 & .286 & .384 & .470 & -.544 & 1.116 \\
\hline & 2 & .429 & .374 & .272 & -.379 & 1.236 \\
\hline & 3 & -.357 & .341 & .315 & -1.095 & .380 \\
\hline & 4 & 1.357 & .325 & .001 & .655 & 2.059 \\
\hline & 5 & 1.143 & .361 & .007 & .363 & 1.923 \\
\hline & 6 & 1.214 & .459 & .020 & .223 & 2.206 \\
\hline & 7 & .214 & .408 & .609 & -.668 & 1.097 \\
\hline
\end{tabular}

Figure G-17: Detailed data analysis for the required effort for individuals with ASD. 


\section{Mauchly's Test of Sphericity ${ }^{a}$}

Measure: RequiredEffort

\begin{tabular}{|l|r|r|r|r|r|r|r|}
\hline & & & & \multicolumn{3}{|c|}{ Epsilon $^{\text {b }}$} \\
\cline { 6 - 9 } Within Subjects Effect & Mauchly's W & $\begin{array}{c}\text { Approx. Chi- } \\
\text { Square }\end{array}$ & df & Sig. & $\begin{array}{c}\text { Greenhouse- } \\
\text { Geisser }\end{array}$ & Huynh-Feldt & Lower-bound \\
\hline Technique & .056 & 30.087 & 27 & .346 & .668 & 1.000 & .143 \\
\hline
\end{tabular}

Tests the null hypothesis that the error covariance matrix of the orthonormalized transformed dependent variables is proportional to an identity matrix.

a. Design: Intercept

Within Subjects Design: Technique

b. May be used to adjust the degrees of freedom for the averaged tests of significance. Corrected tests are displayed in the Tests of Within-Subjects Effects table.

Tests of Within-Subjects Effects

Measure: RequiredEffort

\begin{tabular}{|c|c|c|c|c|c|c|c|c|c|}
\hline Source & & $\begin{array}{l}\text { Type III Sum } \\
\text { of Squares }\end{array}$ & df & Mean Square & $\mathrm{F}$ & Sig. & $\begin{array}{l}\text { Partial Eta } \\
\text { Squared }\end{array}$ & $\begin{array}{l}\text { Noncent. } \\
\text { Parameter }\end{array}$ & $\begin{array}{l}\text { Observed } \\
\text { Power }^{\mathrm{a}}\end{array}$ \\
\hline \multirow[t]{4}{*}{ Technique } & Sphericity Assumed & 38.714 & 7 & 5.531 & 6.249 & .000 & .325 & 43.745 & .999 \\
\hline & Greenhouse-Geisser & 38.714 & 4.675 & 8.282 & 6.249 & .000 & .325 & 29.213 & .991 \\
\hline & Huynh-Feldt & 38.714 & 7.000 & 5.531 & 6.249 & .000 & .325 & 43.745 & .999 \\
\hline & Lower-bound & 38.714 & 1.000 & 38.714 & 6.249 & .027 & .325 & 6.249 & .638 \\
\hline \multirow[t]{4}{*}{ Error(Technique) } & Sphericity Assumed & 80.536 & 91 & .885 & & & & & \\
\hline & Greenhouse-Geisser & 80.536 & 60.770 & 1.325 & & & & & \\
\hline & Huynh-Feldt & 80.536 & 91.000 & .885 & & & & & \\
\hline & Lower-bound & 80.536 & 13.000 & 6.195 & & & & & \\
\hline
\end{tabular}

a. Computed using alpha $=.05$

Figure G-17 (Continued) 


\begin{tabular}{|c|c|c|c|c|c|c|}
\hline \multicolumn{7}{|c|}{ Pairwise Comparisons } \\
\hline & & & & & & \\
\hline \multirow[b]{2}{*}{ (l) Technique } & \multirow[b]{2}{*}{ (J) Technique } & \multirow{2}{*}{$\begin{array}{c}\text { Mean } \\
\text { Difference (I- } \\
\mathrm{J})\end{array}$} & \multirow[b]{2}{*}{ Std. Error } & \multirow[b]{2}{*}{ Sig. } & \multicolumn{2}{|c|}{$\begin{array}{l}\text { 95\% Confidence Interval for } \\
\text { Difference }\end{array}$} \\
\hline & & & & & Lower Bound & Upper Bound \\
\hline \multirow[t]{7}{*}{1} & 2 & -.067 & .267 & .806 & -.639 & .505 \\
\hline & 3 & .000 & .258 & 1.000 & -.554 & .554 \\
\hline & 4 & .667 & .303 & .045 & .016 & 1.317 \\
\hline & 5 & .867 & .291 & .010 & .243 & 1.490 \\
\hline & 6 & .800 & .279 & .013 & .201 & 1.399 \\
\hline & 7 & -.067 & .267 & .806 & -.639 & .505 \\
\hline & 8 & .400 & .214 & .082 & -.059 & .859 \\
\hline \multirow[t]{7}{*}{2} & 1 & .067 & .267 & .806 & -.505 & .639 \\
\hline & 3 & .067 & .206 & .751 & -.376 & .509 \\
\hline & 4 & .733 & .248 & .010 & .201 & 1.266 \\
\hline & 5 & .933 & .284 & .005 & .324 & 1.542 \\
\hline & 6 & .867 & .256 & .004 & .318 & 1.415 \\
\hline & 7 & .000 & .169 & 1.000 & -.363 & .363 \\
\hline & 8 & .467 & .236 & .068 & -.040 & .974 \\
\hline \multirow[t]{7}{*}{3} & 1 & .000 & .258 & 1.000 & -.554 & .554 \\
\hline & 2 & -.067 & .206 & .751 & -.509 & .376 \\
\hline & 4 & .667 & .252 & .019 & .126 & 1.207 \\
\hline & 5 & .867 & .165 & .000 & .512 & 1.221 \\
\hline & 6 & .800 & .175 & .000 & .426 & 1.174 \\
\hline & 7 & -.067 & .182 & .719 & -.456 & .323 \\
\hline & 8 & .400 & .163 & .028 & .050 & .750 \\
\hline \multirow[t]{7}{*}{4} & 1 & -.667 & .303 & .045 & -1.317 & -.016 \\
\hline & 2 & -.733 & .248 & .010 & -1.266 & -.201 \\
\hline & 3 & -.667 & .252 & .019 & -1.207 & -.126 \\
\hline & 5 & .200 & .243 & .424 & -.321 & .721 \\
\hline & 6 & .133 & .236 & .582 & -.374 & .640 \\
\hline & 7 & -.733 & .284 & .022 & -1.342 & -.124 \\
\hline & 8 & -.267 & .182 & .164 & -.656 & .123 \\
\hline \multirow[t]{7}{*}{5} & 1 & -.867 & .291 & .010 & -1.490 & -.243 \\
\hline & 2 & -.933 & .284 & .005 & -1.542 & -.324 \\
\hline & 3 & -.867 & .165 & .000 & -1.221 & -.512 \\
\hline & 4 & -.200 & .243 & .424 & -.721 & .321 \\
\hline & 6 & -.067 & .153 & .670 & -.395 & .262 \\
\hline & 7 & -.933 & .284 & .005 & -1.542 & -.324 \\
\hline & 8 & -.467 & .215 & .048 & -.928 & -.005 \\
\hline \multirow[t]{7}{*}{6} & 1 & -.800 & .279 & .013 & -1.399 & -.201 \\
\hline & 2 & -.867 & .256 & .004 & -1.415 & -.318 \\
\hline & 3 & -.800 & .175 & .000 & -1.174 & -.426 \\
\hline & 4 & -.133 & .236 & .582 & -.640 & .374 \\
\hline & 5 & .067 & .153 & .670 & -.262 & .395 \\
\hline & 7 & -.867 & .274 & .007 & -1.454 & -.280 \\
\hline & 8 & -.400 & .214 & .082 & -.859 & .059 \\
\hline \multirow[t]{7}{*}{7} & 1 & .067 & .267 & .806 & -.505 & .639 \\
\hline & 2 & .000 & .169 & 1.000 & -.363 & .363 \\
\hline & 3 & .067 & .182 & .719 & -.323 & .456 \\
\hline & 4 & .733 & .284 & .022 & .124 & 1.342 \\
\hline & 5 & .933 & .284 & .005 & .324 & 1.542 \\
\hline & 6 & .867 & .274 & .007 & .280 & 1.454 \\
\hline & 8 & .467 & .192 & .029 & .055 & .878 \\
\hline \multirow[t]{7}{*}{8} & 1 & -.400 & .214 & .082 & -.859 & .059 \\
\hline & 2 & -.467 & .236 & .068 & -.974 & .040 \\
\hline & 3 & -.400 & .163 & .028 & -.750 & -.050 \\
\hline & 4 & .267 & .182 & .164 & -.123 & .656 \\
\hline & 5 & .467 & .215 & .048 & .005 & .928 \\
\hline & 6 & .400 & .214 & .082 & -.059 & .859 \\
\hline & 7 & -.467 & .192 & .029 & -.878 & -.055 \\
\hline
\end{tabular}

Figure G-18: Detailed data analysis for the tiredness for neurotypical individuals. 


\section{Mauchly's Test of Sphericity ${ }^{a}$}

Measure: Tiredness

\begin{tabular}{|l|r|r|r|r|r|r|r|}
\hline & & & & \multicolumn{3}{|c|}{ Epsilon $^{\text {b }}$} \\
\cline { 6 - 9 } Within Subjects Effect & Mauchly's W & $\begin{array}{c}\text { Approx. Chi- } \\
\text { Square }\end{array}$ & df & Sig. & $\begin{array}{c}\text { Greenhouse- } \\
\text { Geisser }\end{array}$ & Huynh-Feldt & Lower-bound \\
\hline Technique & .031 & 39.633 & 27 & .067 & .615 & .922 & .143 \\
\hline
\end{tabular}

Tests the null hypothesis that the error covariance matrix of the orthonormalized transformed dependent variables is proportional to an identity matrix.

a. Design: Intercept

Within Subjects Design: Technique

b. May be used to adjust the degrees of freedom for the averaged tests of significance. Corrected tests are displayed in the Tests of Within-Subjects Effects table.

Tests of Within-Subjects Effects

Measure: Tiredness

\begin{tabular}{|c|c|c|c|c|c|c|c|c|c|}
\hline Source & & $\begin{array}{l}\text { Type III Sum } \\
\text { of Squares }\end{array}$ & df & Mean Square & $\mathrm{F}$ & Sig. & $\begin{array}{l}\text { Partial Eta } \\
\text { Squared }\end{array}$ & $\begin{array}{l}\text { Noncent. } \\
\text { Parameter }\end{array}$ & $\begin{array}{l}\text { Observed } \\
\text { Power }^{\mathrm{a}}\end{array}$ \\
\hline \multirow[t]{4}{*}{ Technique } & Sphericity Assumed & 17.392 & 7 & 2.485 & 5.941 & .000 & .298 & 41.587 & .999 \\
\hline & Greenhouse-Geisser & 17.392 & 4.304 & 4.041 & 5.941 & .000 & .298 & 25.569 & .983 \\
\hline & Huynh-Feldt & 17.392 & 6.452 & 2.696 & 5.941 & .000 & .298 & 38.330 & .998 \\
\hline & Lower-bound & 17.392 & 1.000 & 17.392 & 5.941 & .029 & .298 & 5.941 & .621 \\
\hline \multirow[t]{4}{*}{ Error(Technique) } & Sphericity Assumed & 40.983 & 98 & .418 & & & & & \\
\hline & Greenhouse-Geisser & 40.983 & 60.253 & .680 & & & & & \\
\hline & Huynh-Feldt & 40.983 & 90.324 & .454 & & & & & \\
\hline & Lower-bound & 40.983 & 14.000 & 2.927 & & & & & \\
\hline
\end{tabular}

a. Computed using alpha $=.05$

Figure G-18 (Continued) 


\begin{tabular}{|c|c|c|c|c|c|c|}
\hline \multicolumn{7}{|c|}{ Pairwise Comparisons } \\
\hline & & \multirow{3}{*}{$\begin{array}{c}\text { Mean } \\
\text { Difference (I- } \\
\mathrm{J})\end{array}$} & \multirow[b]{3}{*}{ Std. Error } & & & \\
\hline \multirow[b]{2}{*}{ (I) Technique } & \multirow[b]{2}{*}{ (J) Technique } & & & \multirow[b]{2}{*}{ Sig. } & \multicolumn{2}{|c|}{$\begin{array}{l}95 \% \text { Confidence Interval for } \\
\text { Difference }\end{array}$} \\
\hline & & & & & Lower Bound & Upper Bound \\
\hline \multirow[t]{7}{*}{1} & 2 & .143 & .143 & .336 & -.166 & .451 \\
\hline & 3 & -.357 & .225 & .136 & -.843 & .129 \\
\hline & 4 & .500 & .272 & .089 & -.088 & 1.088 \\
\hline & 5 & .429 & .202 & .054 & -.008 & .865 \\
\hline & 6 & .571 & .327 & .104 & -.134 & 1.277 \\
\hline & 7 & .357 & .199 & .096 & -.073 & .787 \\
\hline & 8 & -.071 & .355 & .844 & -.838 & .695 \\
\hline \multirow[t]{7}{*}{2} & 1 & -.143 & .143 & .336 & -.451 & .166 \\
\hline & 3 & -.500 & .228 & .047 & -.994 & -.006 \\
\hline & 4 & .357 & .248 & .174 & -.179 & .893 \\
\hline & 5 & .286 & .194 & .165 & -.134 & .705 \\
\hline & 6 & .429 & .272 & .139 & -.158 & 1.015 \\
\hline & 7 & .214 & .187 & .272 & -189 & .618 \\
\hline & 8 & -.214 & .366 & .568 & -1.005 & .576 \\
\hline \multirow[t]{7}{*}{3} & 1 & .357 & .225 & .136 & -.129 & .843 \\
\hline & 2 & .500 & .228 & .047 & .006 & .994 \\
\hline & 4 & .857 & .329 & .022 & .146 & 1.568 \\
\hline & 5 & .786 & .281 & .015 & .179 & 1.392 \\
\hline & 6 & .929 & .339 & .017 & .196 & 1.661 \\
\hline & 7 & .714 & .286 & .027 & .097 & 1.332 \\
\hline & 8 & .286 & .412 & .500 & -.604 & 1.175 \\
\hline \multirow[t]{7}{*}{4} & 1 & -.500 & .272 & .089 & -1.088 & .088 \\
\hline & 2 & -.357 & .248 & .174 & -.893 & .179 \\
\hline & 3 & -.857 & .329 & .022 & -1.568 & -.146 \\
\hline & 5 & -.071 & .127 & .583 & -.345 & .203 \\
\hline & 6 & .071 & .165 & .671 & -.284 & .427 \\
\hline & 7 & -.143 & .143 & .336 & -.451 & .166 \\
\hline & 8 & -.571 & .291 & .071 & -1.200 & .058 \\
\hline \multirow[t]{7}{*}{5} & 1 & -.429 & .202 & .054 & -.865 & .008 \\
\hline & 2 & -.286 & .194 & .165 & -.705 & .134 \\
\hline & 3 & -.786 & .281 & .015 & -1.392 & -.179 \\
\hline & 4 & .071 & .127 & .583 & -.203 & .345 \\
\hline & 6 & .143 & .177 & .435 & -.240 & .526 \\
\hline & 7 & -.071 & .071 & .336 & -.226 & .083 \\
\hline & 8 & -.500 & .310 & .131 & -1.170 & .170 \\
\hline \multirow[t]{7}{*}{6} & 1 & -.571 & .327 & .104 & -1.277 & .134 \\
\hline & 2 & -.429 & .272 & .139 & -1.015 & .158 \\
\hline & 3 & -.929 & .339 & .017 & -1.661 & -.196 \\
\hline & 4 & -.071 & .165 & .671 & -.427 & .284 \\
\hline & 5 & -.143 & .177 & .435 & -.526 & .240 \\
\hline & 7 & -.214 & .187 & .272 & -.618 & .189 \\
\hline & 8 & -.643 & .401 & .133 & -1.508 & .223 \\
\hline \multirow[t]{7}{*}{7} & 1 & -.357 & .199 & .096 & -.787 & .073 \\
\hline & 2 & -.214 & .187 & .272 & -.618 & .189 \\
\hline & 3 & -.714 & .286 & .027 & -1.332 & -.097 \\
\hline & 4 & .143 & .143 & .336 & -.166 & .451 \\
\hline & 5 & .071 & .071 & .336 & -.083 & .226 \\
\hline & 6 & .214 & .187 & .272 & -.189 & .618 \\
\hline & 8 & -.429 & .327 & .212 & -1.134 & .277 \\
\hline \multirow[t]{7}{*}{8} & 1 & .071 & .355 & .844 & -.695 & .838 \\
\hline & 2 & .214 & .366 & .568 & -.576 & 1.005 \\
\hline & 3 & -.286 & .412 & .500 & -1.175 & .604 \\
\hline & 4 & .571 & .291 & .071 & -.058 & 1.200 \\
\hline & 5 & .500 & .310 & .131 & -.170 & 1.170 \\
\hline & 6 & .643 & .401 & .133 & -.223 & 1.508 \\
\hline & 7 & .429 & .327 & .212 & -.277 & 1.134 \\
\hline
\end{tabular}

Figure G-19: Detailed data analysis for the tiredness for individuals with ASD. 


\section{Mauchly's Test of Sphericity ${ }^{a}$}

Measure: Tiredness

\begin{tabular}{|l|r|r|r|r|r|r|r|}
\hline & & & & \multicolumn{3}{|c|}{ Epsilon $^{\text {b }}$} \\
\cline { 6 - 9 } Within Subjects Effect & Mauchly's W & $\begin{array}{c}\text { Approx. Chi- } \\
\text { Square }\end{array}$ & df & Sig. & $\begin{array}{c}\text { Greenhouse- } \\
\text { Geisser }\end{array}$ & Huynh-Feldt & Lower-bound \\
\hline Technique & .003 & 61.102 & 27 & .000 & .449 & .608 & .143 \\
\hline
\end{tabular}

Tests the null hypothesis that the error covariance matrix of the orthonormalized transformed dependent variables is proportional to an identity matrix.

a. Design: Intercept

Within Subjects Design: Technique

b. May be used to adjust the degrees of freedom for the averaged tests of significance. Corrected tests are displayed in the Tests of Within-Subjects Effects table.

Tests of Within-Subjects Effects

Measure: Tiredness

\begin{tabular}{|c|c|c|c|c|c|c|c|c|c|}
\hline Source & & $\begin{array}{l}\text { Type III Sum } \\
\text { of Squares }\end{array}$ & df & Mean Square & $\mathrm{F}$ & Sig. & $\begin{array}{l}\text { Partial Eta } \\
\text { Squared }\end{array}$ & $\begin{array}{l}\text { Noncent. } \\
\text { Parameter }\end{array}$ & $\begin{array}{l}\text { Observed } \\
\text { Power }^{\mathrm{a}}\end{array}$ \\
\hline \multirow[t]{4}{*}{ Technique } & Sphericity Assumed & 10.250 & 7 & 1.464 & 2.945 & .008 & .185 & 20.613 & .914 \\
\hline & Greenhouse-Geisser & 10.250 & 3.142 & 3.263 & 2.945 & .042 & .185 & 9.251 & .669 \\
\hline & Huynh-Feldt & 10.250 & 4.258 & 2.407 & 2.945 & .026 & .185 & 12.540 & .772 \\
\hline & Lower-bound & 10.250 & 1.000 & 10.250 & 2.945 & .110 & .185 & 2.945 & .356 \\
\hline \multirow[t]{4}{*}{ Error(Technique) } & Sphericity Assumed & 45.250 & 91 & .497 & & & & & \\
\hline & Greenhouse-Geisser & 45.250 & 40.840 & 1.108 & & & & & \\
\hline & Huynh-Feldt & 45.250 & 55.359 & .817 & & & & & \\
\hline & Lower-bound & 45.250 & 13.000 & 3.481 & & & & & \\
\hline
\end{tabular}

a. Computed using alpha $=.05$

Figure G-19 (Continued) 


\begin{tabular}{|c|c|c|c|c|c|c|}
\hline \multicolumn{7}{|c|}{ Pairwise Comparisons } \\
\hline Measure: Ov & rwhelm & & & & & \\
\hline \multirow[b]{2}{*}{ (I) Technique } & \multirow[b]{2}{*}{ (J) Technique } & \multirow{2}{*}{$\begin{array}{c}\text { Mean } \\
\text { Difference (I- } \\
\mathrm{J})\end{array}$} & \multirow[b]{2}{*}{ Std. Error } & \multirow[b]{2}{*}{ Sig. } & \multicolumn{2}{|c|}{$\begin{array}{l}\text { 95\% Confidence Interval for } \\
\text { Difference }\end{array}$} \\
\hline & & & & & Lower Bound & Upper Bound \\
\hline \multirow[t]{7}{*}{1} & 2 & .067 & .118 & .582 & -.187 & .320 \\
\hline & 3 & -.067 & .153 & .670 & -.395 & .262 \\
\hline & 4 & .133 & .165 & .433 & -.221 & .488 \\
\hline & 5 & .067 & .153 & .670 & -.262 & .395 \\
\hline & 6 & .267 & .153 & .104 & -.062 & .595 \\
\hline & 7 & -.400 & .235 & .111 & -.904 & .104 \\
\hline & 8 & -.133 & .215 & .546 & -.595 & .328 \\
\hline \multirow[t]{7}{*}{2} & 1 & -.067 & .118 & .582 & -.320 & .187 \\
\hline & 3 & -.133 & .165 & .433 & -.488 & .221 \\
\hline & 4 & .067 & .118 & .582 & -.187 & .320 \\
\hline & 5 & .000 & .138 & 1.000 & -.296 & .296 \\
\hline & 6 & .200 & .145 & .189 & -.110 & .510 \\
\hline & 7 & -.467 & .192 & .029 & -.878 & -.055 \\
\hline & 8 & -.200 & .175 & .271 & -.574 & .174 \\
\hline \multirow[t]{7}{*}{3} & 1 & .067 & .153 & .670 & -.262 & .395 \\
\hline & 2 & .133 & .165 & .433 & -.221 & .488 \\
\hline & 4 & .200 & .200 & .334 & -.229 & .629 \\
\hline & 5 & .133 & .215 & .546 & -.328 & .595 \\
\hline & 6 & .333 & .211 & .136 & -.119 & .785 \\
\hline & 7 & -.333 & .232 & .173 & -.832 & .165 \\
\hline & 8 & -.067 & .228 & .774 & -.556 & .423 \\
\hline \multirow[t]{7}{*}{4} & 1 & -.133 & .165 & .433 & -.488 & .221 \\
\hline & 2 & -.067 & .118 & .582 & -.320 & .187 \\
\hline & 3 & -.200 & .200 & .334 & -.629 & .229 \\
\hline & 5 & -.067 & .182 & .719 & -.456 & .323 \\
\hline & 6 & .133 & .133 & .334 & -.153 & .419 \\
\hline & 7 & -.533 & .236 & .041 & -1.040 & -.026 \\
\hline & 8 & -.267 & .206 & .217 & -.709 & .176 \\
\hline \multirow[t]{7}{*}{5} & 1 & -.067 & .153 & .670 & -.395 & .262 \\
\hline & 2 & .000 & .138 & 1.000 & -.296 & .296 \\
\hline & 3 & -.133 & .215 & .546 & -.595 & .328 \\
\hline & 4 & .067 & .182 & .719 & -.323 & .456 \\
\hline & 6 & .200 & .107 & .082 & -.029 & .429 \\
\hline & 7 & -.467 & .215 & .048 & -.928 & -.005 \\
\hline & 8 & -.200 & .145 & .189 & -.510 & .110 \\
\hline \multirow[t]{7}{*}{6} & 1 & -.267 & .153 & .104 & -.595 & .062 \\
\hline & 2 & -.200 & .145 & .189 & -.510 & .110 \\
\hline & 3 & -.333 & .211 & .136 & -.785 & .119 \\
\hline & 4 & -.133 & .133 & .334 & -.419 & .153 \\
\hline & 5 & -.200 & .107 & .082 & -.429 & .029 \\
\hline & 7 & -.667 & .270 & .027 & -1.246 & -.087 \\
\hline & 8 & -.400 & .214 & .082 & -.859 & .059 \\
\hline \multirow[t]{7}{*}{7} & 1 & .400 & .235 & .111 & -.104 & .904 \\
\hline & 2 & .467 & .192 & .029 & .055 & .878 \\
\hline & 3 & .333 & .232 & .173 & -.165 & .832 \\
\hline & 4 & .533 & .236 & .041 & .026 & 1.040 \\
\hline & 5 & .467 & .215 & .048 & .005 & .928 \\
\hline & 6 & .667 & .270 & .027 & .087 & 1.246 \\
\hline & 8 & .267 & .118 & .041 & .013 & .520 \\
\hline \multirow[t]{7}{*}{8} & 1 & .133 & .215 & .546 & -.328 & .595 \\
\hline & 2 & .200 & .175 & .271 & -.174 & .574 \\
\hline & 3 & .067 & .228 & .774 & -.423 & .556 \\
\hline & 4 & .267 & .206 & .217 & -.176 & .709 \\
\hline & 5 & .200 & .145 & .189 & -.110 & .510 \\
\hline & 6 & .400 & .214 & .082 & -.059 & .859 \\
\hline & 7 & -.267 & .118 & .041 & -.520 & -.013 \\
\hline
\end{tabular}

Figure G-20: Detailed data analysis for the overwhelmedness for neurotypical individuals. 


\section{Mauchly's Test of Sphericity ${ }^{a}$}

Measure: Overwhelm

\begin{tabular}{|l|r|r|r|r|r|r|r|}
\hline & & & & \multicolumn{3}{|c|}{ Epsilon $^{\text {b }}$} \\
\cline { 6 - 9 } Within Subjects Effect & Mauchly's W & $\begin{array}{c}\text { Approx. Chi- } \\
\text { Square }\end{array}$ & df & Sig. & $\begin{array}{c}\text { Greenhouse- } \\
\text { Geisser }\end{array}$ & Huynh-Feldt & Lower-bound \\
\hline Technique & .002 & 69.127 & 27 & .000 & .478 & .646 & .143 \\
\hline
\end{tabular}

Tests the null hypothesis that the error covariance matrix of the orthonormalized transformed dependent variables is proportional to an identity matrix.

a. Design: Intercept

Within Subjects Design: Technique

b. May be used to adjust the degrees of freedom for the averaged tests of significance. Corrected tests are displayed in the Tests of Within-Subjects Effects table.

Tests of Within-Subjects Effects

Measure: Overwhelm

\begin{tabular}{|c|c|c|c|c|c|c|c|c|c|}
\hline Source & & $\begin{array}{l}\text { Type III Sum } \\
\text { of Squares }\end{array}$ & df & Mean Square & $\mathrm{F}$ & Sig. & $\begin{array}{c}\text { Partial Eta } \\
\text { Squared }\end{array}$ & $\begin{array}{l}\text { Noncent. } \\
\text { Parameter }\end{array}$ & $\begin{array}{c}\text { Observed } \\
\text { Power }^{\text {a }}\end{array}$ \\
\hline \multirow[t]{4}{*}{ Technique } & Sphericity Assumed & 4.192 & 7 & .599 & 2.330 & .030 & .143 & 16.312 & .826 \\
\hline & Greenhouse-Geisser & 4.192 & 3.345 & 1.253 & 2.330 & .080 & .143 & 7.795 & .580 \\
\hline & Huynh-Feldt & 4.192 & 4.521 & .927 & 2.330 & .058 & .143 & 10.536 & .681 \\
\hline & Lower-bound & 4.192 & 1.000 & 4.192 & 2.330 & .149 & .143 & 2.330 & .296 \\
\hline \multirow[t]{4}{*}{ Error(Technique) } & Sphericity Assumed & 25.183 & 98 & .257 & & & & & \\
\hline & Greenhouse-Geisser & 25.183 & 46.829 & .538 & & & & & \\
\hline & Huynh-Feldt & 25.183 & 63.297 & .398 & & & & & \\
\hline & Lower-bound & 25.183 & 14.000 & 1.799 & & & & & \\
\hline
\end{tabular}

a. Computed using alpha $=.05$

Figure G-20 (Continued) 


\begin{tabular}{|c|c|c|c|c|c|c|}
\hline \multicolumn{7}{|c|}{ Pairwise Comparisons } \\
\hline & & \multirow{3}{*}{$\begin{array}{c}\text { Mean } \\
\text { Difference (I- } \\
\mathrm{J})\end{array}$} & \multirow[b]{3}{*}{ Std. Error } & & & \\
\hline \multirow[b]{2}{*}{ (I) Technique } & \multirow[b]{2}{*}{ (J) Technique } & & & \multirow[b]{2}{*}{ Sig. } & \multicolumn{2}{|c|}{$\begin{array}{l}95 \% \text { Confidence Interval for } \\
\text { Difference }\end{array}$} \\
\hline & & & & & Lower Bound & Upper Bound \\
\hline \multirow[t]{7}{*}{1} & 2 & .429 & .309 & .189 & -.240 & 1.097 \\
\hline & 3 & .357 & .289 & .239 & -.267 & .982 \\
\hline & 4 & .786 & .300 & .021 & .138 & 1.433 \\
\hline & 5 & .714 & .266 & .019 & .140 & 1.288 \\
\hline & 6 & .786 & .239 & .006 & .270 & 1.301 \\
\hline & 7 & .143 & .206 & .500 & -.302 & .588 \\
\hline & 8 & -.214 & .505 & .678 & -1.305 & .876 \\
\hline \multirow[t]{7}{*}{2} & 1 & -.429 & .309 & .189 & -1.097 & .240 \\
\hline & 3 & -.071 & .221 & .752 & -.550 & .407 \\
\hline & 4 & .357 & .199 & .096 & -.073 & .787 \\
\hline & 5 & .286 & .244 & .263 & -.242 & .813 \\
\hline & 6 & .357 & .225 & .136 & -.129 & .843 \\
\hline & 7 & -.286 & .354 & .435 & -1.051 & .480 \\
\hline & 8 & -.643 & .401 & .133 & -1.508 & .223 \\
\hline \multirow[t]{7}{*}{3} & 1 & -.357 & .289 & .239 & -.982 & .267 \\
\hline & 2 & .071 & .221 & .752 & -.407 & .550 \\
\hline & 4 & .429 & .173 & .028 & .055 & .802 \\
\hline & 5 & .357 & .199 & .096 & -.073 & .787 \\
\hline & 6 & .429 & .173 & .028 & .055 & .802 \\
\hline & 7 & -.214 & .261 & .426 & -.777 & .349 \\
\hline & 8 & -.571 & .374 & .150 & -1.379 & .236 \\
\hline \multirow[t]{7}{*}{4} & 1 & -.786 & .300 & .021 & -1.433 & -.138 \\
\hline & 2 & -.357 & .199 & .096 & -.787 & .073 \\
\hline & 3 & -.429 & .173 & .028 & -.802 & -.055 \\
\hline & 5 & -.071 & .221 & .752 & -.550 & .407 \\
\hline & 6 & .000 & .148 & 1.000 & -.320 & .320 \\
\hline & 7 & -.643 & .289 & .045 & -1.267 & -.018 \\
\hline & 8 & -1.000 & .348 & .013 & -1.751 & -.249 \\
\hline \multirow[t]{7}{*}{5} & 1 & -.714 & .266 & .019 & -1.288 & -.140 \\
\hline & 2 & -.286 & .244 & .263 & -.813 & .242 \\
\hline & 3 & -.357 & .199 & .096 & -.787 & .073 \\
\hline & 4 & .071 & .221 & .752 & -.407 & .550 \\
\hline & 6 & .071 & .127 & .583 & -.203 & .345 \\
\hline & 7 & -.571 & .202 & .014 & -1.008 & -.135 \\
\hline & 8 & -.929 & .425 & .048 & -1.847 & -.010 \\
\hline \multirow[t]{7}{*}{6} & 1 & -.786 & .239 & .006 & -1.301 & -.270 \\
\hline & 2 & -.357 & .225 & .136 & -.843 & .129 \\
\hline & 3 & -.429 & .173 & .028 & -.802 & -.055 \\
\hline & 4 & .000 & .148 & 1.000 & -.320 & .320 \\
\hline & 5 & -.071 & .127 & .583 & -.345 & .203 \\
\hline & 7 & -.643 & .199 & .007 & -1.073 & -.213 \\
\hline & 8 & -1.000 & .378 & .020 & -1.817 & -.183 \\
\hline \multirow[t]{7}{*}{7} & 1 & -.143 & .206 & .500 & -.588 & .302 \\
\hline & 2 & .286 & .354 & .435 & -.480 & 1.051 \\
\hline & 3 & .214 & .261 & .426 & -.349 & .777 \\
\hline & 4 & .643 & .289 & .045 & .018 & 1.267 \\
\hline & 5 & .571 & .202 & .014 & .135 & 1.008 \\
\hline & 6 & .643 & .199 & .007 & .213 & 1.073 \\
\hline & 8 & -.357 & .476 & .466 & -1.385 & .671 \\
\hline \multirow[t]{7}{*}{8} & 1 & .214 & .505 & .678 & -.876 & 1.305 \\
\hline & 2 & .643 & .401 & .133 & -.223 & 1.508 \\
\hline & 3 & .571 & .374 & .150 & -.236 & 1.379 \\
\hline & 4 & 1.000 & .348 & .013 & .249 & 1.751 \\
\hline & 5 & .929 & .425 & .048 & .010 & 1.847 \\
\hline & 6 & 1.000 & .378 & .020 & .183 & 1.817 \\
\hline & 7 & .357 & .476 & .466 & -.671 & 1.385 \\
\hline
\end{tabular}

Figure G-21: Detailed data analysis for the overwhelmedness for individuals with ASD. 


\section{Mauchly's Test of Sphericity ${ }^{a}$}

Measure: Overwhelm

\begin{tabular}{|l|r|r|r|r|r|r|r|}
\hline & & & & \multicolumn{3}{|c|}{ Epsilon $^{\text {b }}$} \\
\cline { 6 - 9 } Within Subjects Effect & Mauchly's W & $\begin{array}{c}\text { Approx. Chi- } \\
\text { Square }\end{array}$ & df & Sig. & $\begin{array}{c}\text { Greenhouse- } \\
\text { Geisser }\end{array}$ & Huynh-Feldt & Lower-bound \\
\hline Technique & .008 & 50.922 & 27 & .005 & .413 & .544 & .143 \\
\hline
\end{tabular}

Tests the null hypothesis that the error covariance matrix of the orthonormalized transformed dependent variables is proportional to an identity matrix.

a. Design: Intercept

Within Subjects Design: Technique

b. May be used to adjust the degrees of freedom for the averaged tests of significance. Corrected tests are displayed in the Tests of Within-Subjects Effects table.

Tests of Within-Subjects Effects

Measure: Overwhelm

\begin{tabular}{|c|c|c|c|c|c|c|c|c|c|}
\hline Source & & $\begin{array}{l}\text { Type III Sum } \\
\text { of Squares }\end{array}$ & df & Mean Square & $\mathrm{F}$ & Sig. & $\begin{array}{l}\text { Partial Eta } \\
\text { Squared }\end{array}$ & $\begin{array}{l}\text { Noncent. } \\
\text { Parameter }\end{array}$ & $\begin{array}{l}\text { Observed } \\
\text { Power }^{\mathrm{a}}\end{array}$ \\
\hline \multirow[t]{4}{*}{ Technique } & Sphericity Assumed & 13.964 & 7 & 1.995 & 3.314 & .003 & .203 & 23.195 & .946 \\
\hline & Greenhouse-Geisser & 13.964 & 2.890 & 4.831 & 3.314 & .032 & .203 & 9.577 & .698 \\
\hline & Huynh-Feldt & 13.964 & 3.805 & 3.670 & 3.314 & .019 & .203 & 12.607 & .790 \\
\hline & Lower-bound & 13.964 & 1.000 & 13.964 & 3.314 & .092 & .203 & 3.314 & .392 \\
\hline \multirow[t]{4}{*}{ Error(Technique) } & Sphericity Assumed & 54.786 & 91 & .602 & & & & & \\
\hline & Greenhouse-Geisser & 54.786 & 37.574 & 1.458 & & & & & \\
\hline & Huynh-Feldt & 54.786 & 49.460 & 1.108 & & & & & \\
\hline & Lower-bound & 54.786 & 13.000 & 4.214 & & & & & \\
\hline
\end{tabular}

a. Computed using alpha $=.05$

Figure G-21 (Continued) 


\begin{tabular}{|c|c|c|c|c|c|c|}
\hline \multicolumn{7}{|c|}{ Pairwise Comparisons } \\
\hline & & & \multirow[b]{3}{*}{ Std. Error } & & & \\
\hline \multirow[b]{2}{*}{ (I) Technique } & \multirow[b]{2}{*}{ (J) Technique } & \multirow{2}{*}{$\begin{array}{c}\text { Mean } \\
\text { Difference (I- } \\
\mathrm{J})\end{array}$} & & \multirow[b]{2}{*}{ Sig. } & \multicolumn{2}{|c|}{$\begin{array}{l}95 \% \text { Confidence Interval for } \\
\text { Difference }\end{array}$} \\
\hline & & & & & Lower Bound & Upper Bound \\
\hline \multirow[t]{7}{*}{1} & 2 & .200 & .175 & .271 & -.174 & .574 \\
\hline & 3 & .000 & .195 & 1.000 & -.419 & .419 \\
\hline & 4 & .333 & .252 & .207 & -.207 & .874 \\
\hline & 5 & .400 & .190 & .054 & -.008 & .808 \\
\hline & 6 & .333 & .187 & .096 & -.067 & .734 \\
\hline & 7 & -.933 & .300 & .008 & -1.577 & -.289 \\
\hline & 8 & -.333 & .374 & .388 & -1.135 & .468 \\
\hline \multirow[t]{7}{*}{2} & 1 & -.200 & .175 & .271 & -.574 & .174 \\
\hline & 3 & -.200 & .175 & .271 & -.574 & .174 \\
\hline & 4 & .133 & .215 & .546 & -.328 & .595 \\
\hline & 5 & .200 & .145 & .189 & -.110 & .510 \\
\hline & 6 & .133 & .091 & .164 & -.062 & .328 \\
\hline & 7 & -1.133 & .215 & .000 & -1.595 & -.672 \\
\hline & 8 & -.533 & .322 & .120 & -1.223 & .157 \\
\hline \multirow[t]{7}{*}{3} & 1 & .000 & .195 & 1.000 & -.419 & .419 \\
\hline & 2 & .200 & .175 & .271 & -.174 & .574 \\
\hline & 4 & .333 & .270 & .238 & -.246 & .913 \\
\hline & 5 & .400 & .214 & .082 & -.059 & .859 \\
\hline & 6 & .333 & .211 & .136 & -.119 & .785 \\
\hline & 7 & -.933 & .284 & .005 & -1.542 & -.324 \\
\hline & 8 & -.333 & .287 & .265 & -.950 & .283 \\
\hline \multirow[t]{7}{*}{4} & 1 & -.333 & .252 & .207 & -.874 & .207 \\
\hline & 2 & -.133 & .215 & .546 & -.595 & .328 \\
\hline & 3 & -.333 & .270 & .238 & -.913 & .246 \\
\hline & 5 & .067 & .228 & .774 & -.423 & .556 \\
\hline & 6 & .000 & .239 & 1.000 & -.513 & .513 \\
\hline & 7 & -1.267 & .316 & .001 & -1.944 & -.590 \\
\hline & 8 & -.667 & .333 & .065 & -1.382 & .048 \\
\hline \multirow[t]{7}{*}{5} & 1 & -.400 & .190 & .054 & -.808 & .008 \\
\hline & 2 & -.200 & .145 & .189 & -.510 & .110 \\
\hline & 3 & -.400 & .214 & .082 & -.859 & .059 \\
\hline & 4 & -.067 & .228 & .774 & -.556 & .423 \\
\hline & 6 & -.067 & .153 & .670 & -.395 & .262 \\
\hline & 7 & -1.333 & .232 & .000 & -1.832 & -.835 \\
\hline & 8 & -.733 & .267 & .016 & -1.305 & -.161 \\
\hline \multirow[t]{7}{*}{6} & 1 & -.333 & .187 & .096 & -.734 & .067 \\
\hline & 2 & -.133 & .091 & .164 & -.328 & .062 \\
\hline & 3 & -.333 & .211 & .136 & -.785 & .119 \\
\hline & 4 & .000 & .239 & 1.000 & -.513 & .513 \\
\hline & 5 & .067 & .153 & .670 & -.262 & .395 \\
\hline & 7 & -1.267 & .248 & .000 & -1.799 & -.734 \\
\hline & 8 & -.667 & .319 & .055 & -1.350 & .017 \\
\hline \multirow[t]{7}{*}{7} & 1 & .933 & .300 & .008 & .289 & 1.577 \\
\hline & 2 & 1.133 & .215 & .000 & .672 & 1.595 \\
\hline & 3 & .933 & .284 & .005 & .324 & 1.542 \\
\hline & 4 & 1.267 & .316 & .001 & .590 & 1.944 \\
\hline & 5 & 1.333 & .232 & .000 & .835 & 1.832 \\
\hline & 6 & 1.267 & .248 & .000 & .734 & 1.799 \\
\hline & 8 & .600 & .289 & .057 & -.021 & 1.221 \\
\hline \multirow[t]{7}{*}{8} & 1 & .333 & .374 & .388 & -.468 & 1.135 \\
\hline & 2 & .533 & .322 & .120 & -.157 & 1.223 \\
\hline & 3 & .333 & .287 & .265 & -.283 & .950 \\
\hline & 4 & .667 & .333 & .065 & -.048 & 1.382 \\
\hline & 5 & .733 & .267 & .016 & .161 & 1.305 \\
\hline & 6 & .667 & .319 & .055 & -.017 & 1.350 \\
\hline & 7 & -.600 & .289 & .057 & -1.221 & .021 \\
\hline
\end{tabular}

Figure G-22: Detailed data analysis for the frustration for neurotypical individuals. 


\section{Mauchly's Test of Sphericity ${ }^{a}$}

Measure: Frustrated

\begin{tabular}{|l|r|r|r|r|r|r|r|}
\hline & & & & \multicolumn{3}{|c|}{ Epsilon $^{\text {b }}$} \\
\cline { 6 - 9 } Within Subjects Effect & Mauchly's W & $\begin{array}{c}\text { Approx. Chi- } \\
\text { Square }\end{array}$ & df & Sig. & $\begin{array}{c}\text { Greenhouse- } \\
\text { Geisser }\end{array}$ & Huynh-Feldt & Lower-bound \\
\hline Technique & .010 & 53.055 & 27 & .003 & .561 & .807 & .143 \\
\hline
\end{tabular}

Tests the null hypothesis that the error covariance matrix of the orthonormalized transformed dependent variables is proportional to an identity matrix.

a. Design: Intercept

Within Subjects Design: Technique

b. May be used to adjust the degrees of freedom for the averaged tests of significance. Corrected tests are displayed in the Tests of Within-Subjects Effects table.

Tests of Within-Subjects Effects

Measure: Frustrated

\begin{tabular}{|c|c|c|c|c|c|c|c|c|c|}
\hline Source & & $\begin{array}{l}\text { Type III Sum } \\
\text { of Squares }\end{array}$ & df & Mean Square & $\mathrm{F}$ & Sig. & $\begin{array}{c}\text { Partial Eta } \\
\text { Squared }\end{array}$ & $\begin{array}{l}\text { Noncent. } \\
\text { Parameter }\end{array}$ & $\begin{array}{c}\text { Observed } \\
\text { Power }^{\text {a }}\end{array}$ \\
\hline \multirow[t]{4}{*}{ Technique } & Sphericity Assumed & 21.067 & 7 & 3.010 & 6.492 & .000 & .317 & 45.441 & 1.000 \\
\hline & Greenhouse-Geisser & 21.067 & 3.928 & 5.364 & 6.492 & .000 & .317 & 25.497 & .985 \\
\hline & Huynh-Feldt & 21.067 & 5.651 & 3.728 & 6.492 & .000 & .317 & 36.681 & .998 \\
\hline & Lower-bound & 21.067 & 1.000 & 21.067 & 6.492 & .023 & .317 & 6.492 & .659 \\
\hline \multirow[t]{4}{*}{ Error(Technique) } & Sphericity Assumed & 45.433 & 98 & .464 & & & & & \\
\hline & Greenhouse-Geisser & 45.433 & 54.987 & .826 & & & & & \\
\hline & Huynh-Feldt & 45.433 & 79.108 & .574 & & & & & \\
\hline & Lower-bound & 45.433 & 14.000 & 3.245 & & & & & \\
\hline
\end{tabular}

a. Computed using alpha $=.05$

Figure G-22 (Continued) 


\begin{tabular}{|c|c|c|c|c|c|c|}
\hline \multicolumn{7}{|c|}{ Pairwise Comparisons } \\
\hline Measure: $\mathrm{Fr}$ & strated & & & & & \\
\hline \multirow[b]{2}{*}{ (l) Technique } & \multirow[b]{2}{*}{ (J) Technique } & \multirow{2}{*}{$\begin{array}{c}\text { Mean } \\
\text { Difference (I- } \\
\mathrm{J})\end{array}$} & \multirow[b]{2}{*}{ Std. Error } & \multirow[b]{2}{*}{ Sig. } & \multicolumn{2}{|c|}{$\begin{array}{l}\text { 95\% Confidence Interval for } \\
\text { Difference }\end{array}$} \\
\hline & & & & & Lower Bound & Upper Bound \\
\hline \multirow[t]{7}{*}{1} & 2 & .429 & .359 & .254 & -.347 & 1.204 \\
\hline & 3 & .214 & .422 & .620 & -.697 & 1.125 \\
\hline & 4 & .571 & .510 & .283 & -.531 & 1.674 \\
\hline & 5 & .714 & .339 & .055 & -.017 & 1.446 \\
\hline & 6 & .714 & .304 & .035 & .057 & 1.372 \\
\hline & 7 & -.286 & .450 & .537 & -1.258 & .686 \\
\hline & 8 & -.643 & .452 & .179 & -1.620 & .334 \\
\hline \multirow[t]{7}{*}{2} & 1 & -.429 & .359 & .254 & -1.204 & .347 \\
\hline & 3 & -.214 & .214 & .336 & -.677 & .249 \\
\hline & 4 & .143 & .312 & .655 & -.531 & .817 \\
\hline & 5 & .286 & .163 & .104 & -.067 & .639 \\
\hline & 6 & .286 & .221 & .218 & -.191 & .762 \\
\hline & 7 & -.714 & .339 & .055 & -1.446 & .017 \\
\hline & 8 & -1.071 & .286 & .002 & -1.690 & -.453 \\
\hline \multirow[t]{7}{*}{3} & 1 & -.214 & .422 & .620 & -1.125 & .697 \\
\hline & 2 & .214 & .214 & .336 & -.249 & .677 \\
\hline & 4 & .357 & .269 & .208 & -.225 & .939 \\
\hline & 5 & .500 & .203 & .029 & .061 & .939 \\
\hline & 6 & .500 & .251 & .068 & -.043 & 1.043 \\
\hline & 7 & -.500 & .327 & .151 & -1.207 & .207 \\
\hline & 8 & -.857 & .275 & .008 & -1.450 & -.264 \\
\hline \multirow[t]{7}{*}{4} & 1 & -.571 & .510 & .283 & -1.674 & .531 \\
\hline & 2 & -.143 & .312 & .655 & -.817 & .531 \\
\hline & 3 & -.357 & .269 & .208 & -.939 & .225 \\
\hline & 5 & .143 & .312 & .655 & -.531 & .817 \\
\hline & 6 & .143 & .345 & .686 & -.603 & .889 \\
\hline & 7 & -.857 & .275 & .008 & -1.450 & -.264 \\
\hline & 8 & -1.214 & .395 & .009 & -2.067 & -.362 \\
\hline \multirow[t]{7}{*}{5} & 1 & -.714 & .339 & .055 & -1.446 & .017 \\
\hline & 2 & -.286 & .163 & .104 & -.639 & .067 \\
\hline & 3 & -.500 & .203 & .029 & -.939 & -.061 \\
\hline & 4 & -.143 & .312 & .655 & -.817 & .531 \\
\hline & 6 & .000 & .105 & 1.000 & -.226 & .226 \\
\hline & 7 & -1.000 & .331 & .010 & -1.716 & -.284 \\
\hline & 8 & -1.357 & .357 & .002 & -2.129 & -.586 \\
\hline \multirow[t]{7}{*}{6} & 1 & -.714 & .304 & .035 & -1.372 & -.057 \\
\hline & 2 & -.286 & .221 & .218 & -.762 & .191 \\
\hline & 3 & -.500 & .251 & .068 & -1.043 & .043 \\
\hline & 4 & -.143 & .345 & .686 & -.889 & .603 \\
\hline & 5 & .000 & .105 & 1.000 & -.226 & .226 \\
\hline & 7 & -1.000 & .348 & .013 & -1.751 & -.249 \\
\hline & 8 & -1.357 & .387 & .004 & -2.193 & -.522 \\
\hline \multirow[t]{7}{*}{7} & 1 & .286 & .450 & .537 & -.686 & 1.258 \\
\hline & 2 & .714 & .339 & .055 & -.017 & 1.446 \\
\hline & 3 & .500 & .327 & .151 & -.207 & 1.207 \\
\hline & 4 & .857 & .275 & .008 & .264 & 1.450 \\
\hline & 5 & 1.000 & .331 & .010 & .284 & 1.716 \\
\hline & 6 & 1.000 & .348 & .013 & .249 & 1.751 \\
\hline & 8 & -.357 & .372 & .355 & -1.161 & .447 \\
\hline \multirow[t]{7}{*}{8} & 1 & .643 & .452 & .179 & -.334 & 1.620 \\
\hline & 2 & 1.071 & .286 & .002 & .453 & 1.690 \\
\hline & 3 & .857 & .275 & .008 & .264 & 1.450 \\
\hline & 4 & 1.214 & .395 & .009 & .362 & 2.067 \\
\hline & 5 & 1.357 & .357 & .002 & .586 & 2.129 \\
\hline & 6 & 1.357 & .387 & .004 & .522 & 2.193 \\
\hline & 7 & .357 & .372 & .355 & -.447 & 1.161 \\
\hline
\end{tabular}

Figure G-23: Detailed data analysis for the frustration for individuals with ASD. 


\section{Mauchly's Test of Sphericity ${ }^{a}$}

Measure: Frustrated

\begin{tabular}{|l|r|r|r|r|r|r|r|}
\hline & & & & \multicolumn{3}{|c|}{ Epsilon $^{\text {b }}$} \\
\cline { 6 - 9 } Within Subjects Effect & Mauchly's W & $\begin{array}{c}\text { Approx. Chi- } \\
\text { Square }\end{array}$ & df & Sig. & $\begin{array}{c}\text { Greenhouse- } \\
\text { Geisser }\end{array}$ & Huynh-Feldt & Lower-bound \\
\hline Technique & .010 & 48.350 & 27 & .010 & .522 & .752 & .143 \\
\hline
\end{tabular}

Tests the null hypothesis that the error covariance matrix of the orthonormalized transformed dependent variables is proportional to an identity matrix.

a. Design: Intercept

Within Subjects Design: Technique

b. May be used to adjust the degrees of freedom for the averaged tests of significance. Corrected tests are displayed in the Tests of Within-Subjects Effects table.

Tests of Within-Subjects Effects

Measure: Frustrated

\begin{tabular}{|c|c|c|c|c|c|c|c|c|c|}
\hline Source & & $\begin{array}{l}\text { Type III Sum } \\
\text { of Squares }\end{array}$ & df & Mean Square & $\mathrm{F}$ & Sig. & $\begin{array}{l}\text { Partial Eta } \\
\text { Squared }\end{array}$ & $\begin{array}{l}\text { Noncent. } \\
\text { Parameter }\end{array}$ & $\begin{array}{l}\text { Observed } \\
\text { Power }^{\mathrm{a}}\end{array}$ \\
\hline \multirow[t]{4}{*}{ Technique } & Sphericity Assumed & 23.857 & 7 & 3.408 & 4.453 & .000 & .255 & 31.173 & .989 \\
\hline & Greenhouse-Geisser & 23.857 & 3.655 & 6.527 & 4.453 & .005 & .255 & 16.279 & .896 \\
\hline & Huynh-Feldt & 23.857 & 5.262 & 4.533 & 4.453 & .001 & .255 & 23.435 & .964 \\
\hline & Lower-bound & 23.857 & 1.000 & 23.857 & 4.453 & .055 & .255 & 4.453 & .498 \\
\hline \multirow[t]{4}{*}{ Error(Technique) } & Sphericity Assumed & 69.643 & 91 & .765 & & & & & \\
\hline & Greenhouse-Geisser & 69.643 & 47.520 & 1.466 & & & & & \\
\hline & Huynh-Feldt & 69.643 & 68.412 & 1.018 & & & & & \\
\hline & Lower-bound & 69.643 & 13.000 & 5.357 & & & & & \\
\hline
\end{tabular}

a. Computed using alpha $=.05$

Figure G-23 (Continued) 


\begin{tabular}{|c|c|c|c|c|c|c|}
\hline \multicolumn{7}{|c|}{ Pairwise Comparisons } \\
\hline & & & & & & \\
\hline \multirow[b]{2}{*}{ (l) Technique } & \multirow[b]{2}{*}{ (J) Technique } & \multirow{2}{*}{$\begin{array}{c}\text { Mean } \\
\text { Difference (I- } \\
\mathrm{J})\end{array}$} & \multirow[b]{2}{*}{ Std. Error } & \multirow[b]{2}{*}{ Sig. } & \multicolumn{2}{|c|}{$\begin{array}{l}\text { 95\% Confidence Interval for } \\
\text { Difference }\end{array}$} \\
\hline & & & & & Lower Bound & Upper Bound \\
\hline \multirow[t]{7}{*}{1} & 2 & .267 & .153 & .104 & -.062 & .595 \\
\hline & 3 & .000 & .258 & 1.000 & -.554 & .554 \\
\hline & 4 & .267 & .182 & .164 & -.123 & .656 \\
\hline & 5 & .533 & .291 & .088 & -.090 & 1.157 \\
\hline & 6 & .467 & .291 & .131 & -.157 & 1.090 \\
\hline & 7 & .267 & .153 & .104 & -.062 & .595 \\
\hline & 8 & .467 & .256 & .089 & -.082 & 1.015 \\
\hline \multirow[t]{7}{*}{2} & 1 & -.267 & .153 & .104 & -.595 & .062 \\
\hline & 3 & -.267 & .267 & .334 & -.839 & .305 \\
\hline & 4 & .000 & .169 & 1.000 & -.363 & .363 \\
\hline & 5 & .267 & .153 & .104 & -.062 & .595 \\
\hline & 6 & .200 & .145 & .189 & -.110 & .510 \\
\hline & 7 & .000 & .000 & & .000 & .000 \\
\hline & 8 & .200 & .107 & .082 & -.029 & .429 \\
\hline \multirow[t]{7}{*}{3} & 1 & .000 & .258 & 1.000 & -.554 & .554 \\
\hline & 2 & .267 & .267 & .334 & -.305 & .839 \\
\hline & 4 & .267 & .371 & .484 & -.529 & 1.063 \\
\hline & 5 & .533 & .291 & .088 & -.090 & 1.157 \\
\hline & 6 & .467 & .350 & .204 & -.284 & 1.217 \\
\hline & 7 & .267 & .267 & .334 & -.305 & .839 \\
\hline & 8 & .467 & .336 & .187 & -.254 & 1.188 \\
\hline \multirow[t]{7}{*}{4} & 1 & -.267 & .182 & .164 & -.656 & .123 \\
\hline & 2 & .000 & .169 & 1.000 & -.363 & .363 \\
\hline & 3 & -.267 & .371 & .484 & -1.063 & .529 \\
\hline & 5 & .267 & .284 & .364 & -.342 & .876 \\
\hline & 6 & .200 & .279 & .486 & -.399 & .799 \\
\hline & 7 & .000 & .169 & 1.000 & -.363 & .363 \\
\hline & 8 & .200 & .200 & .334 & -.229 & .629 \\
\hline \multirow[t]{7}{*}{5} & 1 & -.533 & .291 & .088 & -1.157 & .090 \\
\hline & 2 & -.267 & .153 & .104 & -.595 & .062 \\
\hline & 3 & -.533 & .291 & .088 & -1.157 & .090 \\
\hline & 4 & -.267 & .284 & .364 & -.876 & .342 \\
\hline & 6 & -.067 & .118 & .582 & -.320 & .187 \\
\hline & 7 & -.267 & .153 & .104 & -.595 & .062 \\
\hline & 8 & -.067 & .118 & .582 & -.320 & .187 \\
\hline \multirow[t]{7}{*}{6} & 1 & -.467 & .291 & .131 & -1.090 & .157 \\
\hline & 2 & -.200 & .145 & .189 & -.510 & .110 \\
\hline & 3 & -.467 & .350 & .204 & -1.217 & .284 \\
\hline & 4 & -.200 & .279 & .486 & -.799 & .399 \\
\hline & 5 & .067 & .118 & .582 & -.187 & .320 \\
\hline & 7 & -.200 & .145 & .189 & -.510 & .110 \\
\hline & 8 & .000 & .098 & 1.000 & -.209 & .209 \\
\hline \multirow[t]{7}{*}{7} & 1 & -.267 & .153 & .104 & -.595 & .062 \\
\hline & 2 & .000 & .000 & . & .000 & .000 \\
\hline & 3 & -.267 & .267 & .334 & -.839 & .305 \\
\hline & 4 & .000 & .169 & 1.000 & -.363 & .363 \\
\hline & 5 & .267 & .153 & .104 & -.062 & .595 \\
\hline & 6 & .200 & .145 & .189 & -.110 & .510 \\
\hline & 8 & .200 & .107 & .082 & -.029 & .429 \\
\hline \multirow[t]{7}{*}{8} & 1 & -.467 & .256 & .089 & -1.015 & .082 \\
\hline & 2 & -.200 & .107 & .082 & -.429 & .029 \\
\hline & 3 & -.467 & .336 & .187 & -1.188 & .254 \\
\hline & 4 & -.200 & .200 & .334 & -.629 & .229 \\
\hline & 5 & .067 & .118 & .582 & -.187 & .320 \\
\hline & 6 & .000 & .098 & 1.000 & -.209 & .209 \\
\hline & 7 & -.200 & .107 & .082 & -.429 & .029 \\
\hline
\end{tabular}

Figure G-24: Detailed data analysis for the motion sickness for neurotypical individuals. 
Mauchly's Test of Sphericity ${ }^{2}$

Measure: MotionSickness

\begin{tabular}{|l|r|r|r|r|r|r|r|}
\hline & & & & \multicolumn{3}{|c|}{ Epsilon $^{\text {b }}$} \\
\cline { 6 - 9 } Within Subjects Effect & Mauchly's W & $\begin{array}{c}\text { Approx. Chi- } \\
\text { Square }\end{array}$ & df & Sig. & $\begin{array}{c}\text { Greenhouse- } \\
\text { Geisser }\end{array}$ & Huynh-Feldt & Lower-bound \\
\hline Technique & .000 & & 27 & & .329 & .398 & .143 \\
\hline
\end{tabular}

Tests the null hypothesis that the error covariance matrix of the orthonormalized transformed dependent variables is proportional to an identity matrix.

a. Design: Intercept

Within Subjects Design: Technique

b. May be used to adjust the degrees of freedom for the averaged tests of significance. Corrected tests are displayed in the Tests of Within-Subjects Effects table.

Tests of Within-Subjects Effects

Measure: MotionSickness

\begin{tabular}{|c|c|c|c|c|c|c|c|c|c|}
\hline Source & & $\begin{array}{l}\text { Type III Sum } \\
\text { of Squares }\end{array}$ & df & Mean Square & $\mathrm{F}$ & Sig. & $\begin{array}{l}\text { Partial Eta } \\
\text { Squared }\end{array}$ & $\begin{array}{l}\text { Noncent. } \\
\text { Parameter }\end{array}$ & $\begin{array}{l}\text { Observed } \\
\text { Power }^{\text {a }}\end{array}$ \\
\hline \multirow[t]{4}{*}{ Technique } & Sphericity Assumed & 4.367 & 7 & .624 & 1.680 & .123 & .107 & 11.762 & .663 \\
\hline & Greenhouse-Geisser & 4.367 & 2.305 & 1.894 & 1.680 & .199 & .107 & 3.873 & .350 \\
\hline & Huynh-Feldt & 4.367 & 2.786 & 1.568 & 1.680 & .190 & .107 & 4.681 & .391 \\
\hline & Lower-bound & 4.367 & 1.000 & 4.367 & 1.680 & .216 & .107 & 1.680 & .227 \\
\hline \multirow[t]{4}{*}{ Error(Technique) } & Sphericity Assumed & 36.383 & 98 & .371 & & & & & \\
\hline & Greenhouse-Geisser & 36.383 & 32.273 & 1.127 & & & & & \\
\hline & Huynh-Feldt & 36.383 & 38.999 & .933 & & & & & \\
\hline & Lower-bound & 36.383 & 14.000 & 2.599 & & & & & \\
\hline
\end{tabular}

a. Computed using alpha $=.05$

Figure G-24 (Continued) 


\begin{tabular}{|c|c|c|c|c|c|c|}
\hline \multicolumn{7}{|c|}{ Pairwise Comparisons } \\
\hline Measure: Mc & onSickness & & & & & \\
\hline \multirow[b]{2}{*}{ (l) Technique } & \multirow[b]{2}{*}{ (J) Technique } & \multirow{2}{*}{$\begin{array}{c}\text { Mean } \\
\text { Difference (I- } \\
\mathrm{J})\end{array}$} & \multirow[b]{2}{*}{ Std. Error } & \multirow[b]{2}{*}{ Sig. } & \multicolumn{2}{|c|}{$\begin{array}{l}\text { 95\% Confidence Interval for } \\
\text { Difference }\end{array}$} \\
\hline & & & & & Lower Bound & Upper Bound \\
\hline \multirow[t]{7}{*}{1} & 2 & .071 & .195 & .720 & -.350 & .493 \\
\hline & 3 & .286 & .221 & .218 & -.191 & .762 \\
\hline & 4 & .143 & .177 & .435 & -.240 & .526 \\
\hline & 5 & .214 & .187 & .272 & -.189 & .618 \\
\hline & 6 & .143 & .206 & .500 & -.302 & .588 \\
\hline & 7 & .357 & .199 & .096 & -.073 & .787 \\
\hline & 8 & .286 & .163 & .104 & -.067 & .639 \\
\hline \multirow[t]{7}{*}{2} & 1 & -.071 & .195 & .720 & -.493 & .350 \\
\hline & 3 & .214 & .155 & .189 & -.120 & .549 \\
\hline & 4 & .071 & .245 & .775 & -.458 & .601 \\
\hline & 5 & .143 & .177 & .435 & -.240 & .526 \\
\hline & 6 & .071 & .127 & .583 & -.203 & .345 \\
\hline & 7 & .286 & .163 & .104 & -.067 & .639 \\
\hline & 8 & .214 & .187 & .272 & -.189 & .618 \\
\hline \multirow[t]{7}{*}{3} & 1 & -.286 & .221 & .218 & -.762 & .191 \\
\hline & 2 & -.214 & .155 & .189 & -.549 & .120 \\
\hline & 4 & -.143 & .177 & .435 & -.526 & .240 \\
\hline & 5 & -.071 & .127 & .583 & -.345 & .203 \\
\hline & 6 & -.143 & .143 & .336 & -.451 & .166 \\
\hline & 7 & .071 & .071 & .336 & -.083 & .226 \\
\hline & 8 & .000 & .105 & 1.000 & -.226 & .226 \\
\hline \multirow[t]{7}{*}{4} & 1 & -.143 & .177 & .435 & -.526 & .240 \\
\hline & 2 & -.071 & .245 & .775 & -.601 & .458 \\
\hline & 3 & .143 & .177 & .435 & -.240 & .526 \\
\hline & 5 & .071 & .127 & .583 & -.203 & .345 \\
\hline & 6 & .000 & .182 & 1.000 & -.392 & .392 \\
\hline & 7 & .214 & .155 & .189 & -.120 & .549 \\
\hline & 8 & .143 & .097 & .165 & -.067 & .353 \\
\hline \multirow[t]{7}{*}{5} & 1 & -.214 & .187 & .272 & -.618 & .189 \\
\hline & 2 & -.143 & .177 & .435 & -.526 & .240 \\
\hline & 3 & .071 & .127 & .583 & -.203 & .345 \\
\hline & 4 & -.071 & .127 & .583 & -.345 & .203 \\
\hline & 6 & -.071 & .127 & .583 & -.345 & .203 \\
\hline & 7 & .143 & .097 & .165 & -.067 & .353 \\
\hline & 8 & .071 & .071 & .336 & -.083 & .226 \\
\hline \multirow[t]{7}{*}{6} & 1 & -.143 & .206 & .500 & -.588 & .302 \\
\hline & 2 & -.071 & .127 & .583 & -.345 & .203 \\
\hline & 3 & .143 & .143 & .336 & -.166 & .451 \\
\hline & 4 & .000 & .182 & 1.000 & -.392 & .392 \\
\hline & 5 & .071 & .127 & .583 & -.203 & .345 \\
\hline & 7 & .214 & .114 & .082 & -.032 & .460 \\
\hline & 8 & .143 & .143 & .336 & -.166 & .451 \\
\hline \multirow[t]{7}{*}{7} & 1 & -.357 & .199 & .096 & -.787 & .073 \\
\hline & 2 & -.286 & .163 & .104 & -.639 & .067 \\
\hline & 3 & -.071 & .071 & .336 & -.226 & .083 \\
\hline & 4 & -.214 & .155 & .189 & -.549 & .120 \\
\hline & 5 & -.143 & .097 & .165 & -.353 & .067 \\
\hline & 6 & -.214 & .114 & .082 & -.460 & .032 \\
\hline & 8 & -.071 & .071 & .336 & -.226 & .083 \\
\hline \multirow[t]{7}{*}{8} & 1 & -.286 & .163 & .104 & -.639 & .067 \\
\hline & 2 & -.214 & .187 & .272 & -.618 & .189 \\
\hline & 3 & .000 & .105 & 1.000 & -.226 & .226 \\
\hline & 4 & -.143 & .097 & .165 & -.353 & .067 \\
\hline & 5 & -.071 & .071 & .336 & -.226 & .083 \\
\hline & 6 & -.143 & .143 & .336 & -.451 & .166 \\
\hline & 7 & .071 & .071 & .336 & -.083 & .226 \\
\hline
\end{tabular}

Figure G-25: Detailed data analysis for the motion sickness for individuals with ASD. 
Mauchly's Test of Sphericity ${ }^{2}$

Measure: MotionSickness

\begin{tabular}{|l|r|r|r|r|r|r|r|}
\hline & & & & \multicolumn{3}{|c|}{ Epsilon $^{\text {b }}$} \\
\cline { 6 - 9 } Within Subjects Effect & Mauchly's W & $\begin{array}{c}\text { Approx. Chi- } \\
\text { Square }\end{array}$ & df & Sig. & $\begin{array}{c}\text { Greenhouse- } \\
\text { Geisser }\end{array}$ & Huynh-Feldt & Lower-bound \\
\hline Technique & .000 & & 27 & & .446 & .604 & .143 \\
\hline
\end{tabular}

Tests the null hypothesis that the error covariance matrix of the orthonormalized transformed dependent variables is proportional to an identity matrix.

a. Design: Intercept

Within Subjects Design: Technique

b. May be used to adjust the degrees of freedom for the averaged tests of significance. Corrected tests are displayed in the Tests of Within-Subjects Effects table.

Tests of Within-Subjects Effects

Measure: MotionSickness

\begin{tabular}{|c|c|c|c|c|c|c|c|c|c|}
\hline Source & & $\begin{array}{l}\text { Type III Sum } \\
\text { of Squares }\end{array}$ & df & Mean Square & $\mathrm{F}$ & Sig. & $\begin{array}{l}\text { Partial Eta } \\
\text { Squared }\end{array}$ & $\begin{array}{l}\text { Noncent. } \\
\text { Parameter }\end{array}$ & $\begin{array}{l}\text { Observed } \\
\text { Power }^{\text {a }}\end{array}$ \\
\hline \multirow[t]{4}{*}{ Technique } & Sphericity Assumed & 1.420 & 7 & .203 & 1.175 & .325 & .083 & 8.226 & .479 \\
\hline & Greenhouse-Geisser & 1.420 & 3.124 & .454 & 1.175 & .332 & .083 & 3.672 & .298 \\
\hline & Huynh-Feldt & 1.420 & 4.227 & .336 & 1.175 & .332 & .083 & 4.967 & .354 \\
\hline & Lower-bound & 1.420 & 1.000 & 1.420 & 1.175 & .298 & .083 & 1.175 & .171 \\
\hline \multirow[t]{4}{*}{ Error(Technique) } & Sphericity Assumed & 15.705 & 91 & .173 & & & & & \\
\hline & Greenhouse-Geisser & 15.705 & 40.618 & .387 & & & & & \\
\hline & Huynh-Feldt & 15.705 & 54.949 & .286 & & & & & \\
\hline & Lower-bound & 15.705 & 13.000 & 1.208 & & & & & \\
\hline
\end{tabular}

a. Computed using alpha $=.05$

Figure G-25 (Continued) 


\begin{tabular}{|c|c|c|c|c|c|c|}
\hline \multicolumn{7}{|c|}{ Pairwise Comparisons } \\
\hline Measure: $\mathrm{Pr}$ & sence & & & & & \\
\hline \multirow[b]{2}{*}{ (I) Technique } & \multirow[b]{2}{*}{ (J) Technique } & \multirow{2}{*}{$\begin{array}{c}\text { Mean } \\
\text { Difference (I- } \\
\mathrm{J})\end{array}$} & \multirow[b]{2}{*}{ Std. Error } & \multirow[b]{2}{*}{ Sig. } & \multicolumn{2}{|c|}{$\begin{array}{l}\text { 95\% Confidence Interval for } \\
\text { Difference }\end{array}$} \\
\hline & & & & & Lower Bound & Upper Bound \\
\hline \multirow[t]{7}{*}{1} & 2 & .178 & .141 & .229 & -.125 & .481 \\
\hline & 3 & .289 & .138 & .054 & -.006 & .584 \\
\hline & 4 & .244 & .202 & .246 & -.188 & .677 \\
\hline & 5 & .400 & .153 & .021 & .071 & .729 \\
\hline & 6 & .556 & .189 & .011 & .151 & .960 \\
\hline & 7 & .644 & .229 & .014 & .154 & 1.135 \\
\hline & 8 & .400 & .230 & .105 & -.094 & .894 \\
\hline \multirow[t]{7}{*}{2} & 1 & -.178 & .141 & .229 & -.481 & .125 \\
\hline & 3 & .111 & .096 & .265 & -.094 & .317 \\
\hline & 4 & .067 & .201 & .745 & -.365 & .498 \\
\hline & 5 & .222 & .106 & .055 & -.006 & .450 \\
\hline & 6 & .378 & .125 & .009 & .109 & .647 \\
\hline & 7 & .467 & .172 & .017 & .099 & .835 \\
\hline & 8 & .222 & .144 & .146 & -.087 & .532 \\
\hline \multirow[t]{7}{*}{3} & 1 & -.289 & .138 & .054 & -.584 & .006 \\
\hline & 2 & -.111 & .096 & .265 & -.317 & .094 \\
\hline & 4 & -.044 & .134 & .744 & -.331 & .242 \\
\hline & 5 & .111 & .120 & .371 & -.147 & .369 \\
\hline & 6 & .267 & .157 & .111 & -.069 & .603 \\
\hline & 7 & .356 & .132 & .017 & .072 & .639 \\
\hline & 8 & .111 & .137 & .430 & -.182 & .404 \\
\hline \multirow[t]{7}{*}{4} & 1 & -.244 & .202 & .246 & -.677 & .188 \\
\hline & 2 & -.067 & .201 & .745 & -.498 & .365 \\
\hline & 3 & .044 & .134 & .744 & -.242 & .331 \\
\hline & 5 & .156 & .165 & .363 & -.199 & .510 \\
\hline & 6 & .311 & .212 & .164 & -.144 & .766 \\
\hline & 7 & .400 & .143 & .014 & .094 & .706 \\
\hline & 8 & .156 & .165 & .363 & -.199 & .510 \\
\hline \multirow[t]{7}{*}{5} & 1 & -.400 & .153 & .021 & -.729 & -.071 \\
\hline & 2 & -.222 & .106 & .055 & -.450 & .006 \\
\hline & 3 & -.111 & .120 & .371 & -.369 & .147 \\
\hline & 4 & -.156 & .165 & .363 & -.510 & .199 \\
\hline & 6 & .156 & .107 & .169 & -.074 & .386 \\
\hline & 7 & .244 & .140 & .102 & -.055 & .544 \\
\hline & 8 & $-6.667 \mathrm{E}-11$ & .145 & 1.000 & -.312 & .312 \\
\hline \multirow[t]{7}{*}{6} & 1 & -.556 & .189 & .011 & -.960 & -.151 \\
\hline & 2 & -.378 & .125 & .009 & -.647 & -.109 \\
\hline & 3 & -.267 & .157 & .111 & -.603 & .069 \\
\hline & 4 & -.311 & .212 & .164 & -.766 & .144 \\
\hline & 5 & -.156 & .107 & .169 & -.386 & .074 \\
\hline & 7 & .089 & .161 & .589 & -.256 & .434 \\
\hline & 8 & -.156 & .162 & .354 & -.503 & .192 \\
\hline \multirow[t]{7}{*}{7} & 1 & -.644 & .229 & .014 & -1.135 & -.154 \\
\hline & 2 & -.467 & .172 & .017 & -.835 & -.099 \\
\hline & 3 & -.356 & .132 & .017 & -.639 & -.072 \\
\hline & 4 & -.400 & .143 & .014 & -.706 & -.094 \\
\hline & 5 & -.244 & .140 & .102 & -.544 & .055 \\
\hline & 6 & -.089 & .161 & .589 & -.434 & .256 \\
\hline & 8 & -.244 & .100 & .028 & -.459 & -.030 \\
\hline \multirow[t]{7}{*}{8} & 1 & -.400 & .230 & .105 & -.894 & .094 \\
\hline & 2 & -.222 & .144 & .146 & -.532 & .087 \\
\hline & 3 & -.111 & .137 & .430 & -.404 & .182 \\
\hline & 4 & -.156 & .165 & .363 & -.510 & .199 \\
\hline & 5 & $6.667 \mathrm{E}-11$ & .145 & 1.000 & -.312 & .312 \\
\hline & 6 & .156 & .162 & .354 & -.192 & .503 \\
\hline & 7 & .244 & .100 & .028 & .030 & .459 \\
\hline
\end{tabular}

Figure G-26: Detailed data analysis for the presence for neurotypical individuals. 


\section{Mauchly's Test of Sphericity ${ }^{a}$}

Measure: Presence

\begin{tabular}{|l|r|r|r|r|r|r|r|}
\hline & & & & \multicolumn{3}{|c|}{ Epsilon $^{\text {b }}$} \\
\cline { 6 - 9 } Within Subjects Effect & Mauchly's W & $\begin{array}{c}\text { Approx. Chi- } \\
\text { Square }\end{array}$ & df & Sig. & $\begin{array}{c}\text { Greenhouse- } \\
\text { Geisser }\end{array}$ & Huynh-Feldt & Lower-bound \\
\hline Technique & .009 & 54.395 & 27 & .002 & .484 & .657 & .143 \\
\hline
\end{tabular}

Tests the null hypothesis that the error covariance matrix of the orthonormalized transformed dependent variables is proportional to an identity matrix.

a. Design: Intercept

Within Subjects Design: Technique

b. May be used to adjust the degrees of freedom for the averaged tests of significance. Corrected tests are displayed in the Tests of Within-Subjects Effects table.

Tests of Within-Subjects Effects

Measure: Presence

\begin{tabular}{|c|c|c|c|c|c|c|c|c|c|}
\hline Source & & $\begin{array}{l}\text { Type III Sum } \\
\text { of Squares }\end{array}$ & df & Mean Square & $\mathrm{F}$ & Sig. & $\begin{array}{l}\text { Partial Eta } \\
\text { Squared }\end{array}$ & $\begin{array}{l}\text { Noncent. } \\
\text { Parameter }\end{array}$ & $\begin{array}{l}\text { Observed } \\
\text { Power }^{\mathrm{a}}\end{array}$ \\
\hline \multirow[t]{4}{*}{ Technique } & Sphericity Assumed & 4.500 & 7 & .643 & 3.436 & .002 & .197 & 24.055 & .955 \\
\hline & Greenhouse-Geisser & 4.500 & 3.385 & 1.329 & 3.436 & .020 & .197 & 11.634 & .772 \\
\hline & Huynh-Feldt & 4.500 & 4.596 & .979 & 3.436 & .010 & .197 & 15.793 & .865 \\
\hline & Lower-bound & 4.500 & 1.000 & 4.500 & 3.436 & .085 & .197 & 3.436 & .408 \\
\hline \multirow[t]{4}{*}{ Error(Technique) } & Sphericity Assumed & 18.333 & 98 & .187 & & & & & \\
\hline & Greenhouse-Geisser & 18.333 & 47.396 & .387 & & & & & \\
\hline & Huynh-Feldt & 18.333 & 64.340 & .285 & & & & & \\
\hline & Lower-bound & 18.333 & 14.000 & 1.310 & & & & & \\
\hline
\end{tabular}

a. Computed using alpha $=.05$

Figure G-26 (Continued) 


\begin{tabular}{|c|c|c|c|c|c|c|}
\hline \multicolumn{7}{|c|}{ Pairwise Comparisons } \\
\hline $\mathrm{Pr}$ & & & & & & \\
\hline \multirow[b]{2}{*}{ (I) Technique } & \multirow[b]{2}{*}{ (J) Technique } & \multirow{2}{*}{$\begin{array}{c}\text { Mean } \\
\text { Difference (I- } \\
\mathrm{J})\end{array}$} & \multirow[b]{2}{*}{ Std. Error } & \multirow[b]{2}{*}{ Sig. } & \multicolumn{2}{|c|}{$\begin{array}{l}95 \% \text { Confidence Interval for } \\
\text { Difference }\end{array}$} \\
\hline & & & & & Lower Bound & Upper Bound \\
\hline \multirow[t]{7}{*}{1} & 2 & .095 & .150 & .537 & -.229 & .419 \\
\hline & 3 & .000 & .105 & 1.000 & -.226 & .226 \\
\hline & 4 & .048 & .130 & .720 & -.233 & .329 \\
\hline & 5 & .214 & .124 & .108 & -.054 & .482 \\
\hline & 6 & .214 & .103 & .057 & -.007 & .436 \\
\hline & 7 & .143 & .103 & .189 & -.080 & .366 \\
\hline & 8 & .167 & .115 & .169 & -.081 & .414 \\
\hline \multirow[t]{7}{*}{2} & 1 & -.095 & .150 & .537 & -.419 & .229 \\
\hline & 3 & -.095 & .158 & .557 & -.436 & .246 \\
\hline & 4 & -.048 & .110 & .671 & -.285 & .189 \\
\hline & 5 & .119 & .159 & .466 & -.224 & .462 \\
\hline & 6 & .119 & .147 & .431 & -.198 & .436 \\
\hline & 7 & .048 & .115 & .686 & -.201 & .296 \\
\hline & 8 & .071 & .106 & .512 & -.157 & .300 \\
\hline \multirow[t]{7}{*}{3} & 1 & .000 & .105 & 1.000 & -.226 & .226 \\
\hline & 2 & .095 & .158 & .557 & -.246 & .436 \\
\hline & 4 & .048 & .143 & .745 & -.262 & .358 \\
\hline & 5 & .214 & .159 & .200 & -.128 & .557 \\
\hline & 6 & .214 & .124 & .108 & -.054 & .482 \\
\hline & 7 & .143 & .091 & .139 & -.053 & .338 \\
\hline & 8 & .167 & .134 & .236 & -.123 & .457 \\
\hline \multirow[t]{7}{*}{4} & 1 & -.048 & .130 & .720 & -.329 & .233 \\
\hline & 2 & .048 & .110 & .671 & -.189 & .285 \\
\hline & 3 & -.048 & .143 & .745 & -.358 & .262 \\
\hline & 5 & .167 & .120 & .187 & -.092 & .425 \\
\hline & 6 & .167 & .109 & .151 & -.069 & .402 \\
\hline & 7 & .095 & .065 & .165 & -.045 & .235 \\
\hline & 8 & .119 & .083 & .174 & -.060 & .298 \\
\hline \multirow[t]{7}{*}{5} & 1 & -.214 & .124 & .108 & -.482 & .054 \\
\hline & 2 & -.119 & .159 & .466 & -.462 & .224 \\
\hline & 3 & -.214 & .159 & .200 & -.557 & .128 \\
\hline & 4 & -.167 & .120 & .187 & -.425 & .092 \\
\hline & 6 & $-1.429 \mathrm{E}-10$ & .070 & 1.000 & -.151 & .151 \\
\hline & 7 & -.071 & .117 & .551 & -.324 & .181 \\
\hline & 8 & -.048 & .092 & .612 & -.245 & .150 \\
\hline \multirow[t]{7}{*}{6} & 1 & -.214 & .103 & .057 & -.436 & .007 \\
\hline & 2 & -.119 & .147 & .431 & -.436 & .198 \\
\hline & 3 & -.214 & .124 & .108 & -.482 & .054 \\
\hline & 4 & -.167 & .109 & .151 & -.402 & .069 \\
\hline & 5 & $1.429 \mathrm{E}-10$ & .070 & 1.000 & -.151 & .151 \\
\hline & 7 & -.071 & .087 & .426 & -.259 & .116 \\
\hline & 8 & -.048 & .059 & .435 & -.175 & .080 \\
\hline \multirow[t]{7}{*}{7} & 1 & $\begin{array}{l}-143 \\
\end{array}$ & .103 & .189 & -.366 & .080 \\
\hline & 2 & -.048 & .115 & .686 & -.296 & .201 \\
\hline & 3 & -.143 & .091 & .139 & -.338 & .053 \\
\hline & 4 & -.095 & .065 & .165 & -.235 & .045 \\
\hline & 5 & .071 & .117 & .551 & -.181 & .324 \\
\hline & 6 & .071 & .087 & .426 & -.116 & .259 \\
\hline & 8 & .024 & .074 & .752 & -.136 & .183 \\
\hline \multirow[t]{7}{*}{8} & 1 & $\begin{array}{l}-167 \\
\end{array}$ & .115 & .169 & -.414 & .081 \\
\hline & 2 & -.071 & .106 & .512 & -.300 & .157 \\
\hline & 3 & -.167 & .134 & .236 & -.457 & .123 \\
\hline & 4 & -.119 & .083 & .174 & -.298 & .060 \\
\hline & 5 & .048 & .092 & .612 & -.150 & .245 \\
\hline & 6 & .048 & .059 & .435 & -.080 & .175 \\
\hline & 7 & -.024 & .074 & .752 & -.183 & .136 \\
\hline
\end{tabular}

Figure G-27: Detailed data analysis for the presence for individuals with ASD. 


\section{Mauchly's Test of Sphericity ${ }^{a}$}

Measure: Presence

\begin{tabular}{|l|r|r|r|r|r|r|r|}
\hline & & & & \multicolumn{3}{|c|}{ Epsilon $^{\text {b }}$} \\
\cline { 6 - 9 } Within Subjects Effect & Mauchly's W & $\begin{array}{c}\text { Approx. Chi- } \\
\text { Square }\end{array}$ & df & Sig. & $\begin{array}{c}\text { Greenhouse- } \\
\text { Geisser }\end{array}$ & Huynh-Feldt & Lower-bound \\
\hline Technique & .006 & 53.271 & 27 & .003 & .528 & .763 & .143 \\
\hline
\end{tabular}

Tests the null hypothesis that the error covariance matrix of the orthonormalized transformed dependent variables is proportional to an identity matrix.

a. Design: Intercept

Within Subjects Design: Technique

b. May be used to adjust the degrees of freedom for the averaged tests of significance. Corrected tests are displayed in the Tests of Within-Subjects Effects table.

Tests of Within-Subjects Effects

Measure: Presence

\begin{tabular}{|c|c|c|c|c|c|c|c|c|c|}
\hline Source & & $\begin{array}{l}\text { Type III Sum } \\
\text { of Squares }\end{array}$ & df & Mean Square & $\mathrm{F}$ & Sig. & $\begin{array}{l}\text { Partial Eta } \\
\text { Squared }\end{array}$ & $\begin{array}{l}\text { Noncent. } \\
\text { Parameter }\end{array}$ & $\begin{array}{l}\text { Observed } \\
\text { Power }^{\mathrm{a}}\end{array}$ \\
\hline \multirow[t]{4}{*}{ Technique } & Sphericity Assumed & .761 & 7 & .109 & 1.156 & .336 & .082 & 8.091 & .472 \\
\hline & Greenhouse-Geisser & .761 & 3.693 & .206 & 1.156 & .341 & .082 & 4.269 & .322 \\
\hline & Huynh-Feldt & .761 & 5.341 & .142 & 1.156 & .340 & .082 & 6.173 & .401 \\
\hline & Lower-bound & .761 & 1.000 & .761 & 1.156 & .302 & .082 & 1.156 & .169 \\
\hline \multirow[t]{4}{*}{ Error(Technique) } & Sphericity Assumed & 8.559 & 91 & .094 & & & & & \\
\hline & Greenhouse-Geisser & 8.559 & 48.015 & .178 & & & & & \\
\hline & Huynh-Feldt & 8.559 & 69.437 & .123 & & & & & \\
\hline & Lower-bound & 8.559 & 13.000 & .658 & & & & & \\
\hline
\end{tabular}

a. Computed using alpha $=.05$

Figure G-27 (Continued) 


\begin{tabular}{|c|c|c|c|c|c|c|}
\hline \multicolumn{7}{|c|}{ Pairwise Comparisons } \\
\hline Measure: $\mathrm{Pr}$ & erence & & & & & \\
\hline \multirow[b]{2}{*}{ (I) Technique } & \multirow[b]{2}{*}{ (J) Technique } & \multirow{2}{*}{$\begin{array}{c}\text { Mean } \\
\text { Difference (I- } \\
\mathrm{J})\end{array}$} & \multirow[b]{2}{*}{ Std. Error } & \multirow[b]{2}{*}{ Sig. } & \multicolumn{2}{|c|}{$\begin{array}{l}\text { 95\% Confidence Interval for } \\
\text { Difference }\end{array}$} \\
\hline & & & & & Lower Bound & Upper Bound \\
\hline \multirow[t]{7}{*}{1} & 2 & 1.067 & .628 & .112 & -.281 & 2.415 \\
\hline & 3 & 1.067 & .790 & .198 & -.627 & 2.760 \\
\hline & 4 & -.333 & .969 & .736 & -2.412 & 1.746 \\
\hline & 5 & .333 & .898 & .716 & -1.593 & 2.259 \\
\hline & 6 & .867 & .899 & .351 & -1.062 & 2.795 \\
\hline & 7 & 3.800 & .794 & .000 & 2.097 & 5.503 \\
\hline & 8 & 2.000 & 1.095 & .089 & -.349 & 4.349 \\
\hline \multirow[t]{7}{*}{2} & 1 & -1.067 & .628 & .112 & -2.415 & .281 \\
\hline & 3 & .000 & .655 & 1.000 & -1.404 & 1.404 \\
\hline & 4 & -1.400 & .767 & .089 & -3.045 & .245 \\
\hline & 5 & -.733 & .765 & .354 & -2.374 & .908 \\
\hline & 6 & -.200 & .906 & .828 & -2.143 & 1.743 \\
\hline & 7 & 2.733 & .621 & .001 & 1.402 & 4.065 \\
\hline & 8 & .933 & .870 & .301 & -.933 & 2.799 \\
\hline \multirow[t]{7}{*}{3} & 1 & -1.067 & .790 & .198 & -2.760 & .627 \\
\hline & 2 & .000 & .655 & 1.000 & -1.404 & 1.404 \\
\hline & 4 & -1.400 & .925 & .152 & -3.383 & .583 \\
\hline & 5 & -.733 & .802 & .376 & -2.453 & .986 \\
\hline & 6 & -.200 & .890 & .825 & -2.109 & 1.709 \\
\hline & 7 & 2.733 & .720 & .002 & 1.189 & 4.278 \\
\hline & 8 & .933 & .886 & .310 & -.967 & 2.834 \\
\hline \multirow[t]{7}{*}{4} & 1 & .333 & .969 & .736 & -1.746 & 2.412 \\
\hline & 2 & 1.400 & .767 & .089 & -.245 & 3.045 \\
\hline & 3 & 1.400 & .925 & .152 & -.583 & 3.383 \\
\hline & 5 & .667 & .760 & .395 & -.964 & 2.297 \\
\hline & 6 & 1.200 & .738 & .126 & -.383 & 2.783 \\
\hline & 7 & 4.133 & .616 & .000 & 2.812 & 5.455 \\
\hline & 8 & 2.333 & .652 & .003 & .934 & 3.732 \\
\hline \multirow[t]{7}{*}{5} & 1 & -.333 & .898 & .716 & -2.259 & 1.593 \\
\hline & 2 & .733 & .765 & .354 & -.908 & 2.374 \\
\hline & 3 & .733 & .802 & .376 & -.986 & 2.453 \\
\hline & 4 & -.667 & .760 & .395 & -2.297 & .964 \\
\hline & 6 & .533 & .616 & .401 & -.788 & 1.855 \\
\hline & 7 & 3.467 & .524 & .000 & 2.342 & 4.591 \\
\hline & 8 & 1.667 & .766 & .047 & .023 & 3.310 \\
\hline \multirow[t]{7}{*}{6} & 1 & -.867 & .899 & .351 & -2.795 & 1.062 \\
\hline & 2 & .200 & .906 & .828 & -1.743 & 2.143 \\
\hline & 3 & .200 & .890 & .825 & -1.709 & 2.109 \\
\hline & 4 & -1.200 & .738 & .126 & -2.783 & .383 \\
\hline & 5 & -.533 & .616 & .401 & -1.855 & .788 \\
\hline & 7 & 2.933 & .539 & .000 & 1.778 & 4.089 \\
\hline & 8 & 1.133 & .822 & .189 & -.629 & 2.895 \\
\hline \multirow[t]{7}{*}{7} & 1 & -3.800 & .794 & .000 & -5.503 & -2.097 \\
\hline & 2 & -2.733 & .621 & .001 & -4.065 & -1.402 \\
\hline & 3 & -2.733 & .720 & .002 & -4.278 & -1.189 \\
\hline & 4 & -4.133 & .616 & .000 & -5.455 & -2.812 \\
\hline & 5 & -3.467 & .524 & .000 & -4.591 & -2.342 \\
\hline & 6 & -2.933 & .539 & .000 & -4.089 & -1.778 \\
\hline & 8 & -1.800 & .663 & .017 & -3.223 & -.377 \\
\hline \multirow[t]{7}{*}{8} & 1 & -2.000 & 1.095 & .089 & -4.349 & .349 \\
\hline & 2 & -.933 & .870 & .301 & -2.799 & .933 \\
\hline & 3 & -.933 & .886 & .310 & -2.834 & .967 \\
\hline & 4 & -2.333 & .652 & .003 & -3.732 & -.934 \\
\hline & 5 & -1.667 & .766 & .047 & -3.310 & -.023 \\
\hline & 6 & -1.133 & .822 & .189 & -2.895 & .629 \\
\hline & 7 & 1.800 & .663 & .017 & .377 & 3.223 \\
\hline
\end{tabular}

Figure G-28: Detailed data analysis for the preference ranking scores for neurotypical individuals. 


\section{Mauchly's Test of Sphericity ${ }^{a}$}

Measure: Preference

\begin{tabular}{|l|r|r|r|r|r|r|r|}
\hline & & & & \multicolumn{3}{|c|}{ Epsilon $^{\text {b }}$} \\
\cline { 6 - 9 } Within Subjects Effect & Mauchly's W & $\begin{array}{c}\text { Approx. Chi- } \\
\text { Square }\end{array}$ & df & Sig. & $\begin{array}{c}\text { Greenhouse- } \\
\text { Geisser }\end{array}$ & Huynh-Feldt & Lower-bound \\
\hline Technique & .134 & 23.038 & 27 & .707 & .629 & .953 & .143 \\
\hline
\end{tabular}

Tests the null hypothesis that the error covariance matrix of the orthonormalized transformed dependent variables is proportional to an identity matrix.

a. Design: Intercept

Within Subjects Design: Technique

b. May be used to adjust the degrees of freedom for the averaged tests of significance. Corrected tests are displayed in the Tests of Within-Subjects Effects table.

Tests of Within-Subjects Effects

Measure: Preference

\begin{tabular}{|c|c|c|c|c|c|c|c|c|c|}
\hline Source & & $\begin{array}{l}\text { Type III Sum } \\
\text { of Squares }\end{array}$ & df & Mean Square & $\mathrm{F}$ & Sig. & $\begin{array}{c}\text { Partial Eta } \\
\text { Squared }\end{array}$ & $\begin{array}{l}\text { Noncent. } \\
\text { Parameter }\end{array}$ & $\begin{array}{c}\text { Observed } \\
\text { Power }^{\text {a }}\end{array}$ \\
\hline \multirow[t]{4}{*}{ Technique } & Sphericity Assumed & 180.133 & 7 & 25.733 & 5.606 & .000 & .286 & 39.241 & .998 \\
\hline & Greenhouse-Geisser & 180.133 & 4.402 & 40.925 & 5.606 & .000 & .286 & 24.674 & .978 \\
\hline & Huynh-Feldt & 180.133 & 6.670 & 27.006 & 5.606 & .000 & .286 & 37.391 & .998 \\
\hline & Lower-bound & 180.133 & 1.000 & 180.133 & 5.606 & .033 & .286 & 5.606 & .596 \\
\hline \multirow[t]{4}{*}{ Error(Technique) } & Sphericity Assumed & 449.867 & 98 & 4.590 & & & & & \\
\hline & Greenhouse-Geisser & 449.867 & 61.621 & 7.301 & & & & & \\
\hline & Huynh-Feldt & 449.867 & 93.381 & 4.818 & & & & & \\
\hline & Lower-bound & 449.867 & 14.000 & 32.133 & & & & & \\
\hline
\end{tabular}

a. Computed using alpha $=.05$

Figure G-28 (Continued) 


\begin{tabular}{|c|c|c|c|c|c|c|}
\hline \multicolumn{7}{|c|}{ Pairwise Comparisons } \\
\hline sure: & & & & & & \\
\hline \multirow[b]{2}{*}{ (I) Technique } & \multirow[b]{2}{*}{ (J) Technique } & \multirow{2}{*}{$\begin{array}{c}\text { Mean } \\
\text { Difference (I- } \\
\text { J) }\end{array}$} & \multirow[b]{2}{*}{ Std. Error } & \multirow[b]{2}{*}{ Sig. } & \multicolumn{2}{|c|}{$\begin{array}{l}95 \% \text { Confidence Interval for } \\
\text { Difference }\end{array}$} \\
\hline & & & & & Lower Bound & Upper Bound \\
\hline \multirow[t]{7}{*}{1} & 2 & -.533 & .786 & .508 & -2.219 & 1.152 \\
\hline & 3 & .533 & .940 & .580 & -1.484 & 2.550 \\
\hline & 4 & -2.133 & .883 & .030 & -4.027 & -.239 \\
\hline & 5 & -2.133 & .639 & .005 & -3.504 & -.763 \\
\hline & 6 & -1.000 & .840 & .253 & -2.801 & .801 \\
\hline & 7 & 1.333 & 1.022 & .213 & -.859 & 3.525 \\
\hline & 8 & 1.533 & .736 & .056 & -.045 & 3.112 \\
\hline \multirow[t]{7}{*}{2} & 1 & .533 & .786 & .508 & -1.152 & 2.219 \\
\hline & 3 & 1.067 & .686 & .142 & -.405 & 2.539 \\
\hline & 4 & -1.600 & .646 & .027 & -2.985 & -.215 \\
\hline & 5 & -1.600 & .638 & .025 & -2.969 & -.231 \\
\hline & 6 & -.467 & .822 & .579 & -2.229 & 1.295 \\
\hline & 7 & 1.867 & .696 & .018 & .374 & 3.359 \\
\hline & 8 & 2.067 & .636 & .006 & .703 & 3.431 \\
\hline \multirow[t]{7}{*}{3} & 1 & -.533 & .940 & .580 & -2.550 & 1.484 \\
\hline & 2 & -1.067 & .686 & .142 & -2.539 & .405 \\
\hline & 4 & -2.667 & .919 & .012 & -4.638 & -.696 \\
\hline & 5 & -2.667 & .797 & .005 & -4.376 & -.958 \\
\hline & 6 & -1.533 & .975 & .138 & -3.625 & .558 \\
\hline & 7 & .800 & 1.038 & .454 & -1.427 & 3.027 \\
\hline & 8 & 1.000 & .828 & .247 & -.776 & 2.776 \\
\hline \multirow[t]{7}{*}{4} & 1 & 2.133 & .883 & .030 & .239 & 4.027 \\
\hline & 2 & 1.600 & .646 & .027 & .215 & 2.985 \\
\hline & 3 & 2.667 & .919 & .012 & .696 & 4.638 \\
\hline & 5 & .000 & .569 & 1.000 & -1.220 & 1.220 \\
\hline & 6 & 1.133 & .601 & .080 & -.155 & 2.421 \\
\hline & 7 & 3.467 & .639 & .000 & 2.096 & 4.837 \\
\hline & 8 & 3.667 & .454 & .000 & 2.692 & 4.641 \\
\hline \multirow[t]{7}{*}{5} & 1 & 2.133 & .639 & .005 & .763 & 3.504 \\
\hline & 2 & 1.600 & .638 & .025 & .231 & 2.969 \\
\hline & 3 & 2.667 & .797 & .005 & .958 & 4.376 \\
\hline & 4 & .000 & .569 & 1.000 & -1.220 & 1.220 \\
\hline & 6 & 1.133 & .435 & .021 & .200 & 2.066 \\
\hline & 7 & 3.467 & .736 & .000 & 1.888 & 5.045 \\
\hline & 8 & 3.667 & .475 & .000 & 2.648 & 4.685 \\
\hline \multirow[t]{7}{*}{6} & 1 & 1.000 & .840 & .253 & -.801 & 2.801 \\
\hline & 2 & .467 & .822 & .579 & -1.295 & 2.229 \\
\hline & 3 & 1.533 & .975 & .138 & -.558 & 3.625 \\
\hline & 4 & -1.133 & .601 & .080 & -2.421 & .155 \\
\hline & 5 & -1.133 & .435 & .021 & -2.066 & -.200 \\
\hline & 7 & 2.333 & .919 & .024 & .362 & 4.304 \\
\hline & 8 & 2.533 & .584 & .001 & 1.280 & 3.787 \\
\hline \multirow[t]{7}{*}{7} & 1 & -1.333 & 1.022 & .213 & -3.525 & .859 \\
\hline & 2 & -1.867 & .696 & .018 & -3.359 & -.374 \\
\hline & 3 & -.800 & 1.038 & .454 & -3.027 & 1.427 \\
\hline & 4 & -3.467 & .639 & .000 & -4.837 & -2.096 \\
\hline & 5 & -3.467 & .736 & .000 & -5.045 & -1.888 \\
\hline & 6 & -2.333 & .919 & .024 & -4.304 & -.362 \\
\hline & 8 & .200 & .691 & .777 & -1.283 & 1.683 \\
\hline \multirow[t]{7}{*}{8} & 1 & -1.533 & .736 & .056 & -3.112 & .045 \\
\hline & 2 & -2.067 & .636 & .006 & -3.431 & -.703 \\
\hline & 3 & -1.000 & .828 & .247 & -2.776 & .776 \\
\hline & 4 & -3.667 & .454 & .000 & -4.641 & -2.692 \\
\hline & 5 & -3.667 & .475 & .000 & -4.685 & -2.648 \\
\hline & 6 & -2.533 & .584 & .001 & -3.787 & -1.280 \\
\hline & 7 & -.200 & .691 & .777 & -1.683 & 1.283 \\
\hline
\end{tabular}

Figure G-29: Detailed data analysis for the preference ranking scores for individuals with ASD. 


\section{Mauchly's Test of Sphericity ${ }^{a}$}

Measure: Preference

\begin{tabular}{|l|r|r|r|r|r|r|r|}
\hline & & & & \multicolumn{3}{|c|}{ Epsilon $^{\text {b }}$} \\
\cline { 6 - 9 } Within Subjects Effect & Mauchly's W & $\begin{array}{c}\text { Approx. Chi- } \\
\text { Square }\end{array}$ & df & Sig. & $\begin{array}{c}\text { Greenhouse- } \\
\text { Geisser }\end{array}$ & Huynh-Feldt & Lower-bound \\
\hline Technique & .062 & 31.865 & 27 & .264 & .613 & .918 & .143 \\
\hline
\end{tabular}

Tests the null hypothesis that the error covariance matrix of the orthonormalized transformed dependent variables is proportional to an identity matrix.

a. Design: Intercept

Within Subjects Design: Technique

b. May be used to adjust the degrees of freedom for the averaged tests of significance. Corrected tests are displayed in the Tests of Within-Subjects Effects table.

Tests of Within-Subjects Effects

Measure: Preference

\begin{tabular}{|c|c|c|c|c|c|c|c|c|c|}
\hline Source & & $\begin{array}{l}\text { Type III Sum } \\
\text { of Squares }\end{array}$ & df & Mean Square & $\mathrm{F}$ & Sig. & $\begin{array}{l}\text { Partial Eta } \\
\text { Squared }\end{array}$ & $\begin{array}{l}\text { Noncent. } \\
\text { Parameter }\end{array}$ & $\begin{array}{l}\text { Observed } \\
\text { Power }^{\mathrm{a}}\end{array}$ \\
\hline \multirow[t]{4}{*}{ Technique } & Sphericity Assumed & 211.200 & 7 & 30.171 & 7.060 & .000 & .335 & 49.421 & 1.000 \\
\hline & Greenhouse-Geisser & 211.200 & 4.292 & 49.205 & 7.060 & .000 & .335 & 30.304 & .994 \\
\hline & Huynh-Feldt & 211.200 & 6.426 & 32.866 & 7.060 & .000 & .335 & 45.370 & 1.000 \\
\hline & Lower-bound & 211.200 & 1.000 & 211.200 & 7.060 & .019 & .335 & 7.060 & 696 \\
\hline \multirow[t]{4}{*}{ Error(Technique) } & Sphericity Assumed & 418.800 & 98 & 4.273 & & & & & \\
\hline & Greenhouse-Geisser & 418.800 & 60.091 & 6.969 & & & & & \\
\hline & Huynh-Feldt & 418.800 & 89.966 & 4.655 & & & & & \\
\hline & Lower-bound & 418.800 & 14.000 & 29.914 & & & & & \\
\hline
\end{tabular}

a. Computed using alpha $=.05$

Figure G-29 (Continued) 
APPENDIX H: DETAILED COMPARISON ANALYSIS BETWEEN

NEUROTYPICAL INDIVIDUALS AND INDIVIDUALS WITH ASD FOR THE LOCOMOTION TECHNIQUES EXPERIMENT 
Independent Samples Test

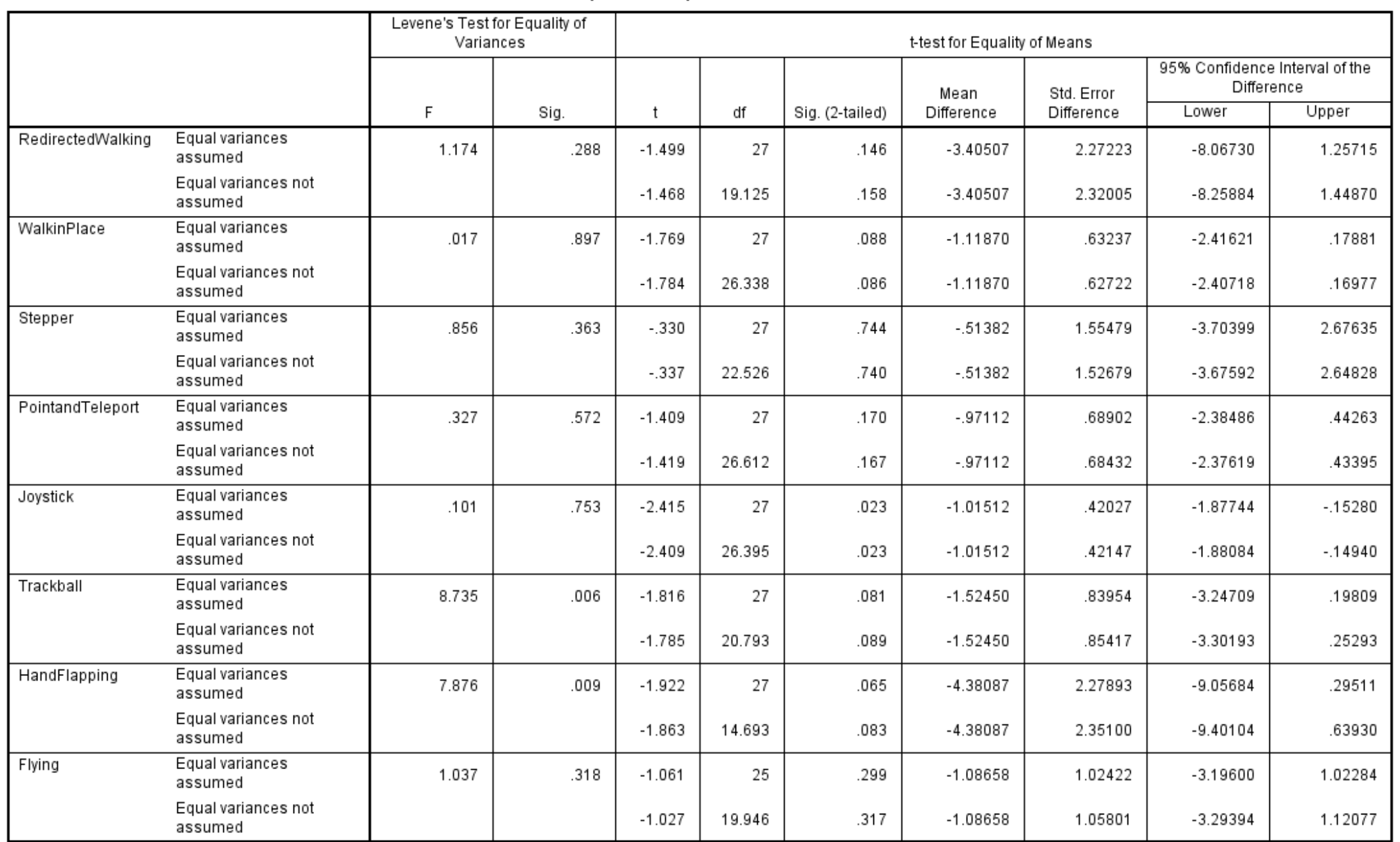

Figure H-1: Detailed comparison analysis for the completion time without obstacles for neurotypical individuals and individuals with ASD. 
Independent Samples Test

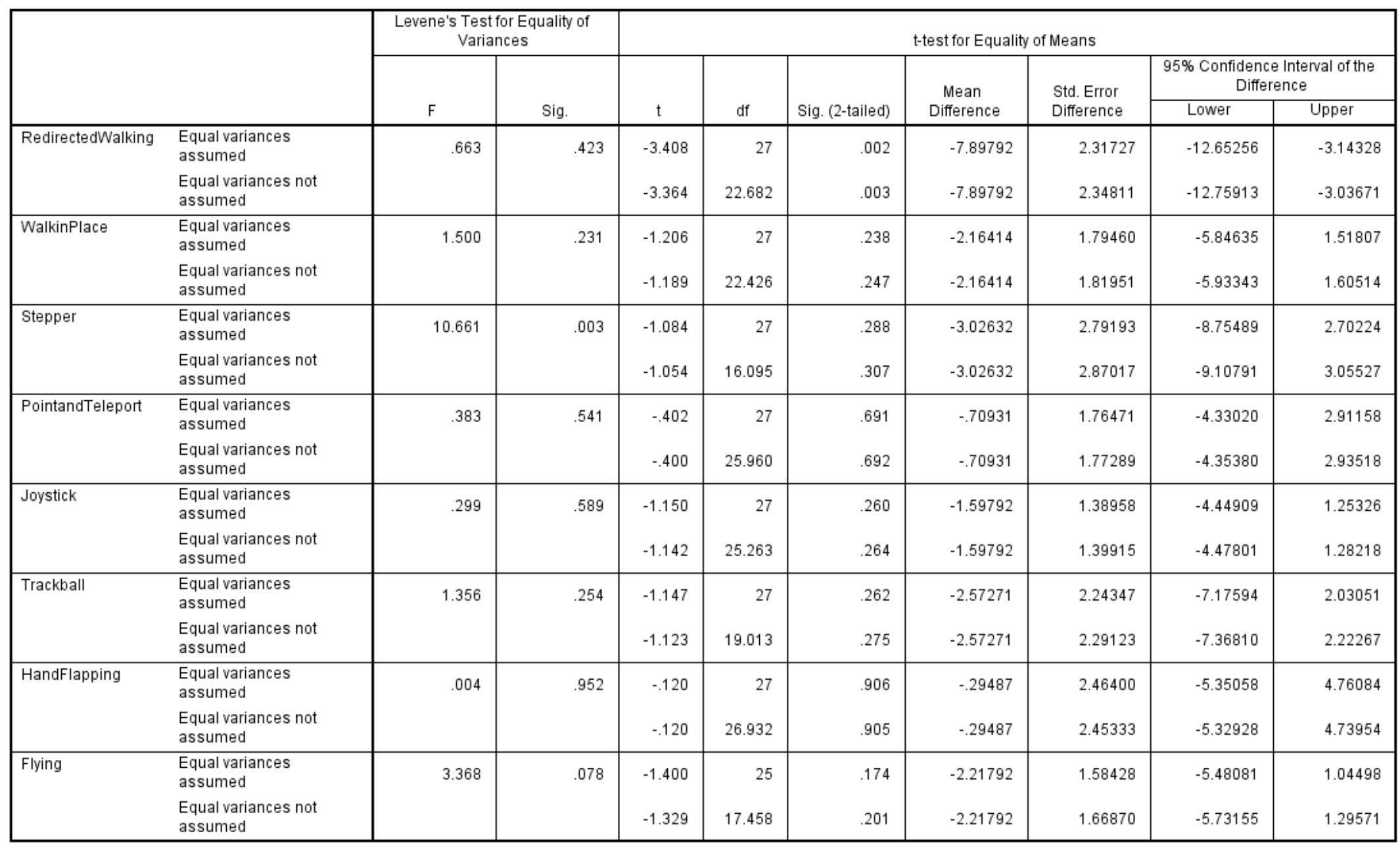

Figure H-2: Detailed comparison analysis for the completion time with obstacles for neurotypical individuals and individuals with ASD. 
Independent Samples Test

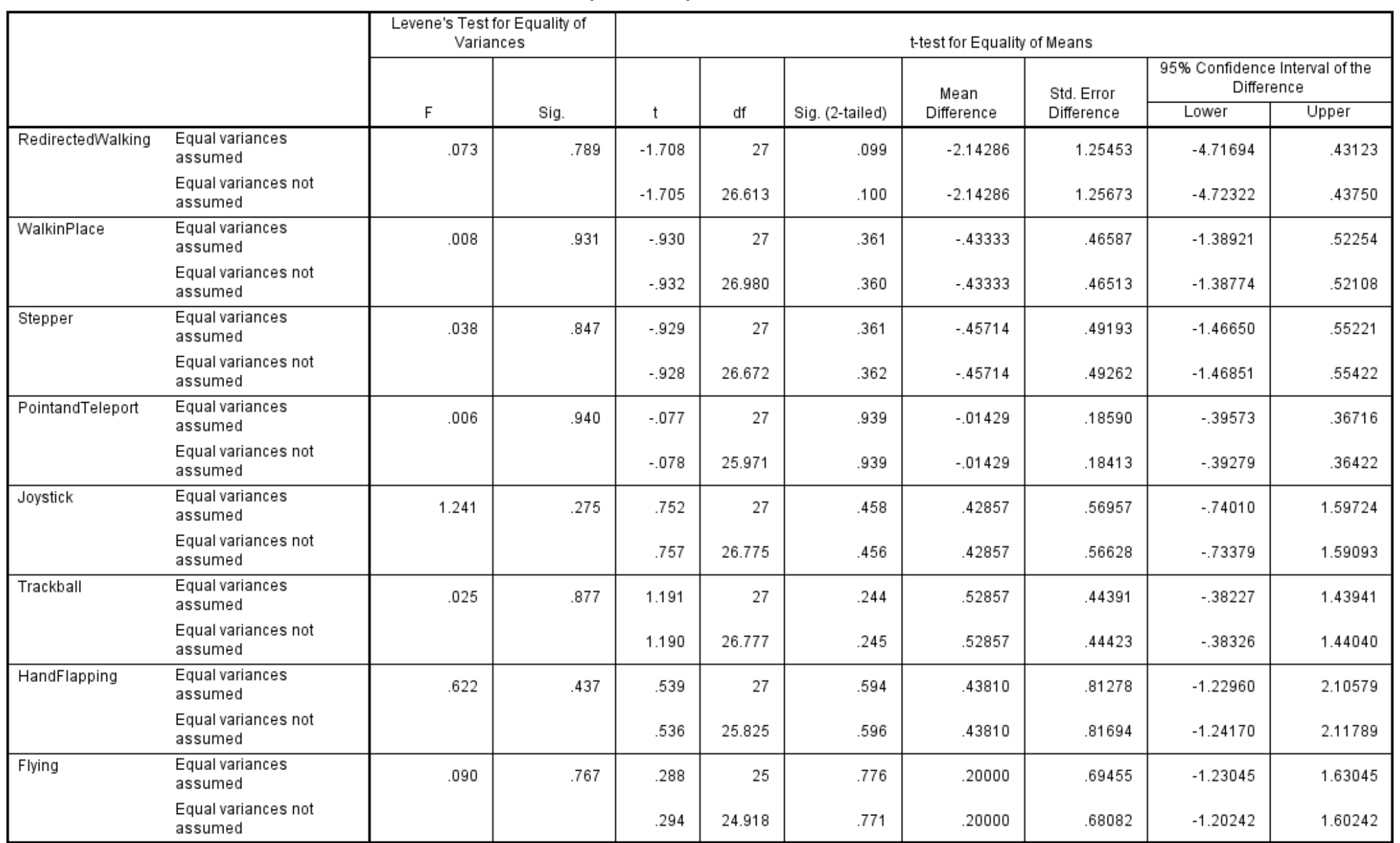

Figure H-3: Detailed comparison analysis for the number of collisions for neurotypical individuals and individuals with ASD. 
Independent Samples Test

\begin{tabular}{|c|c|c|c|c|c|c|c|c|c|c|}
\hline & & \multicolumn{2}{|c|}{$\begin{array}{l}\text { Levene's Test for Equality of } \\
\text { Variances }\end{array}$} & \multicolumn{7}{|c|}{ t-test for Equality of Means } \\
\hline & & \multirow[b]{2}{*}{$\mathrm{F}$} & \multirow[b]{2}{*}{ Sig. } & \multirow[b]{2}{*}{$t$} & \multirow[b]{2}{*}{ df } & \multirow[b]{2}{*}{ Sig. (2-tailed) } & \multirow{2}{*}{$\begin{array}{c}\text { Mean } \\
\text { Difference }\end{array}$} & \multirow{2}{*}{$\begin{array}{l}\text { Std. Error } \\
\text { Difference }\end{array}$} & \multicolumn{2}{|c|}{$\begin{array}{l}95 \% \text { Confidence Interval of the } \\
\text { Difference }\end{array}$} \\
\hline & & & & & & & & & Lower & Upper \\
\hline \multirow[t]{2}{*}{ RedirectedWalking } & $\begin{array}{l}\text { Equal variances } \\
\text { assumed }\end{array}$ & \multirow[t]{2}{*}{1.018} & \multirow[t]{2}{*}{.322} & \multirow[t]{2}{*}{.496} & \multirow{2}{*}{$\begin{array}{r}27 \\
25.413\end{array}$} & \multirow{2}{*}{$\begin{array}{r}.624 \\
.626\end{array}$} & \multirow{2}{*}{$\begin{array}{l}.15714 \\
.15714\end{array}$} & \multirow{2}{*}{$\begin{array}{l}.31673 \\
.31877\end{array}$} & \multirow{2}{*}{$\begin{array}{l}-.49274 \\
-.49884\end{array}$} & \multirow{2}{*}{$\begin{array}{l}.80703 \\
.81313\end{array}$} \\
\hline & $\begin{array}{l}\text { Equal variances not } \\
\text { assumed }\end{array}$ & & & & & & & & & \\
\hline \multirow[t]{2}{*}{ WalkinPlace } & $\begin{array}{l}\text { Equal variances } \\
\text { assumed }\end{array}$ & \multirow[t]{2}{*}{.719} & \multirow[t]{2}{*}{.404} & \multirow{2}{*}{$\begin{array}{l}.111 \\
.110\end{array}$} & \multirow{2}{*}{$\begin{array}{r}27 \\
25.449\end{array}$} & \multirow{2}{*}{$\begin{array}{l}.913 \\
.913\end{array}$} & \multirow{2}{*}{$\begin{array}{l}.02857 \\
.02857\end{array}$} & \multirow{2}{*}{$\begin{array}{l}.25814 \\
.25977\end{array}$} & \multirow{2}{*}{$\begin{array}{l}-.50109 \\
-.50597\end{array}$} & \multirow{2}{*}{$\begin{array}{l}.55823 \\
.56311\end{array}$} \\
\hline & $\begin{array}{l}\text { Equal variances not } \\
\text { assumed }\end{array}$ & & & & & & & & & \\
\hline \multirow[t]{2}{*}{ Stepper } & $\begin{array}{l}\text { Equal variances } \\
\text { assumed }\end{array}$ & \multirow[t]{2}{*}{.367} & \multirow[t]{2}{*}{.549} & \multirow{2}{*}{$\begin{array}{l}.430 \\
.428\end{array}$} & 27 & .671 & .15238 & .35464 & -.57529 & .88005 \\
\hline & $\begin{array}{l}\text { Equal variances not } \\
\text { assumed }\end{array}$ & & & & 26.375 & .672 & .15238 & .35569 & -.57825 & .88301 \\
\hline PointandTeleport & $\begin{array}{l}\text { Equal variances } \\
\text { assumed }\end{array}$ & 4.438 & .045 & 1.003 & 27 & .325 & .19048 & .18986 & -.19908 & .58003 \\
\hline & $\begin{array}{l}\text { Equal variances not } \\
\text { assumed }\end{array}$ & & & 1.021 & 22.916 & .318 & .19048 & .18659 & -.19559 & .57655 \\
\hline Joystick & $\begin{array}{l}\text { Equal variances } \\
\text { assumed }\end{array}$ & 1.800 & .191 & -.655 & 27 & .518 & -.07619 & .11637 & -.31496 & .16258 \\
\hline & $\begin{array}{l}\text { Equal variances not } \\
\text { assumed }\end{array}$ & & & -.647 & 23.338 & .524 & -.07619 & .11774 & -.31957 & .16719 \\
\hline Trackball & $\begin{array}{l}\text { Equal variances } \\
\text { assumed }\end{array}$ & 2.906 & .100 & -.796 & 27 & .433 & -.15714 & .19734 & -.56206 & .24777 \\
\hline & $\begin{array}{l}\text { Equal variances not } \\
\text { assumed }\end{array}$ & & & -.785 & 22.165 & .441 & -.15714 & .20020 & -.57215 & .25786 \\
\hline HandFlapping & $\begin{array}{l}\text { Equal variances } \\
\text { assumed }\end{array}$ & .013 & .909 & 1.191 & 27 & .244 & .52857 & .44391 & -.38227 & 1.43941 \\
\hline & $\begin{array}{l}\text { Equal variances not } \\
\text { assumed }\end{array}$ & & & 1.190 & 26.777 & .245 & .52857 & .44423 & -.38326 & 1.44040 \\
\hline Flying & $\begin{array}{l}\text { Equal variances } \\
\text { assumed }\end{array}$ & 18.690 & .000 & -.935 & 27 & .358 & -.42381 & .45315 & -1.35359 & .50597 \\
\hline & $\begin{array}{l}\text { Equal variances not } \\
\text { assumed }\end{array}$ & & & -.913 & 17.594 & .374 & -.42381 & .46423 & -1.40074 & .55312 \\
\hline
\end{tabular}

Figure H-4: Detailed comparison analysis for the difficulty in understanding for neurotypical individuals and individuals with ASD. 
Independent Samples Test

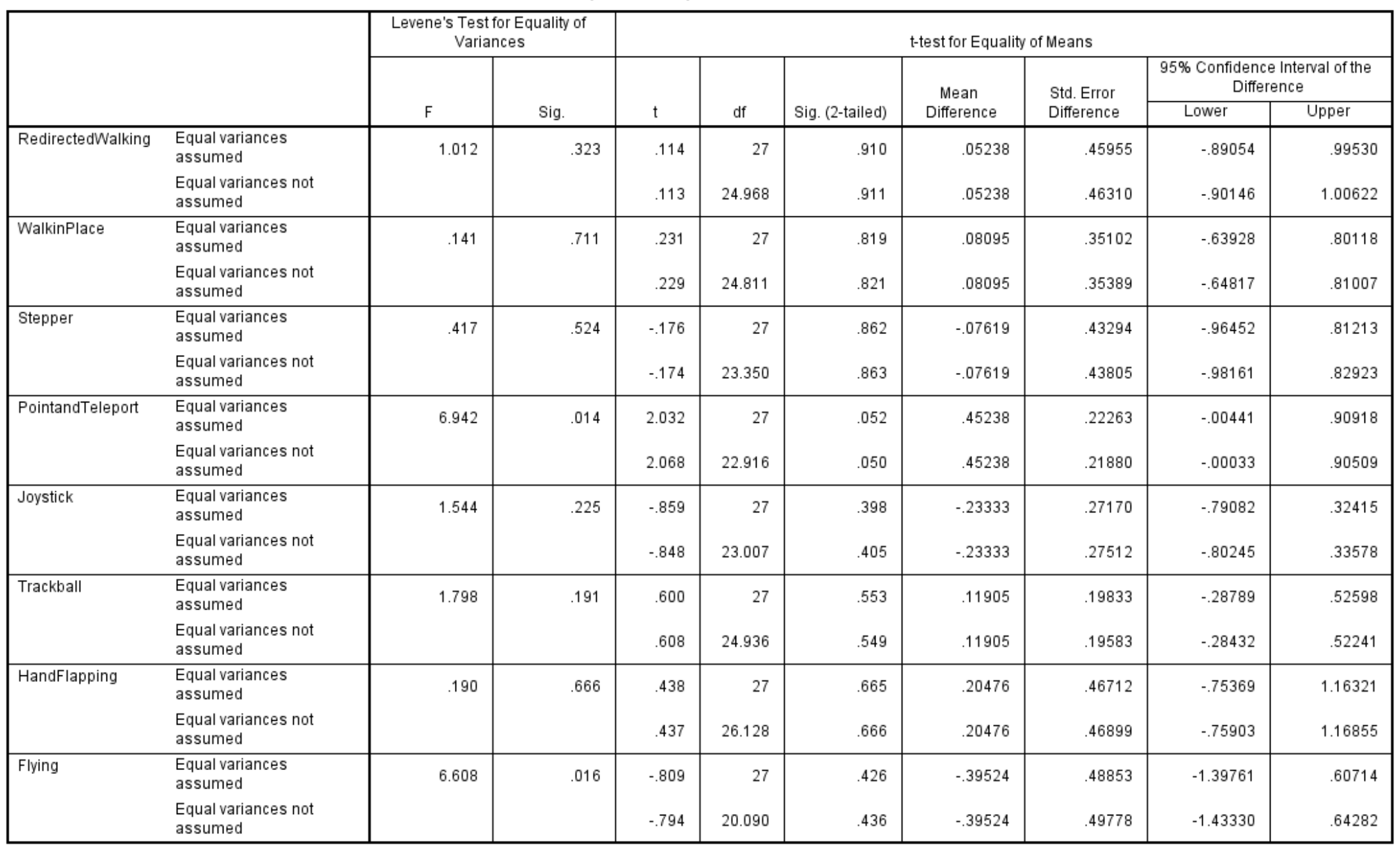

Figure H-5: Detailed comparison analysis for the difficulty in operating for neurotypical individuals and individuals with ASD. 
Independent Samples Test

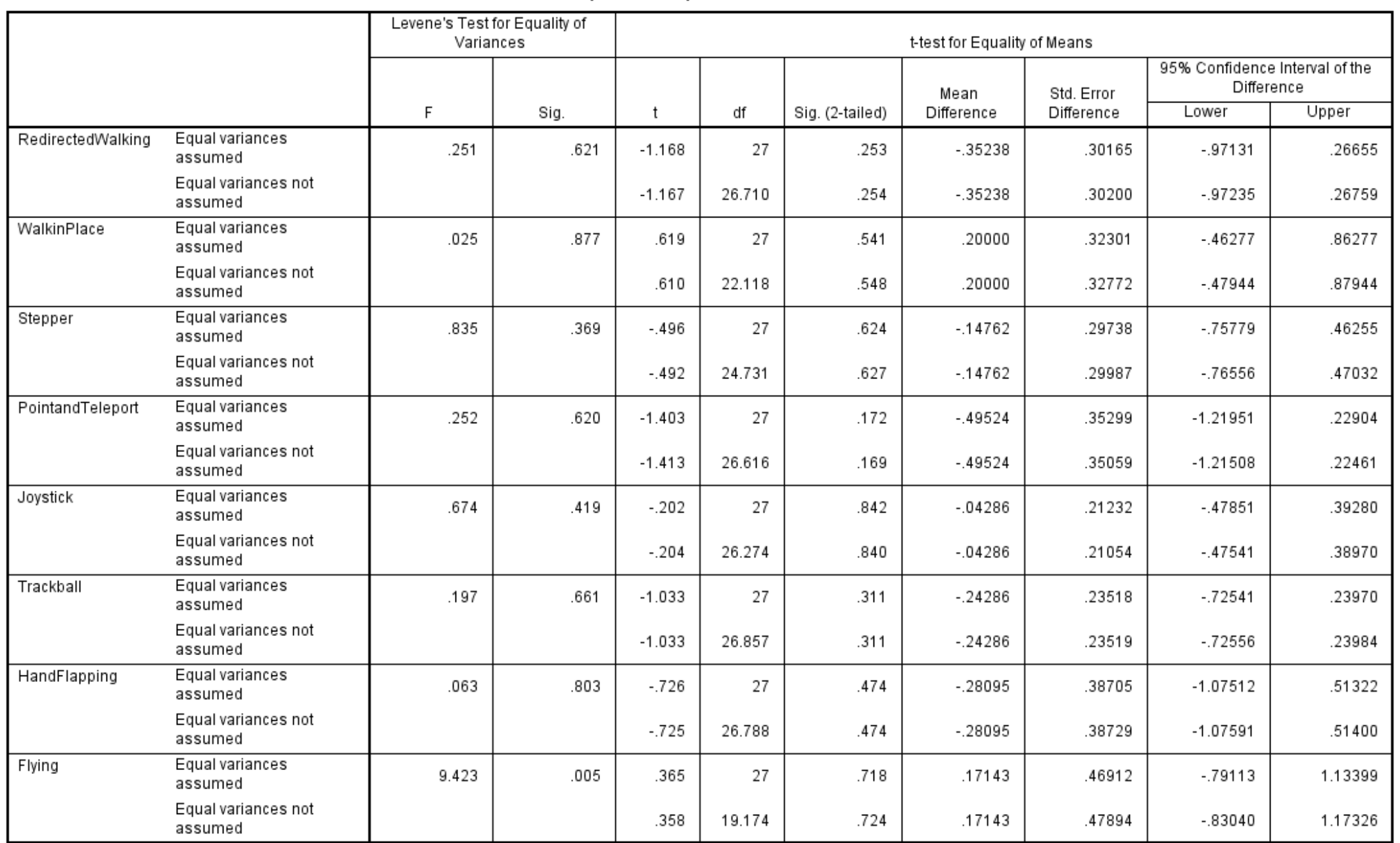

Figure H-6: Detailed comparison analysis for the feeling of being in control for neurotypical individuals and individuals with ASD. 
Independent Samples Test

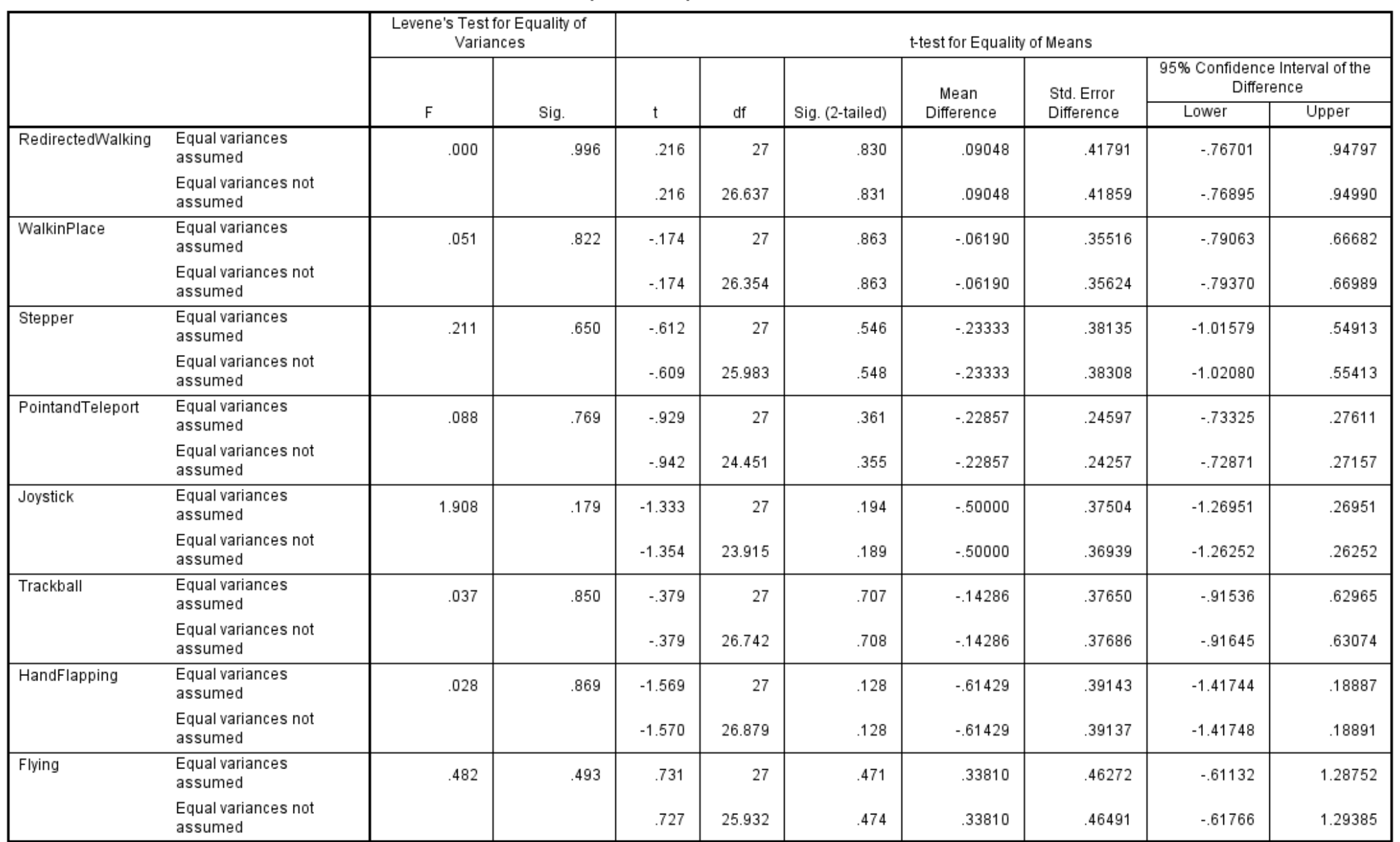

Figure H-7: Detailed comparison analysis for the enjoyment for neurotypical individuals and individuals with ASD. 
Independent Samples Test

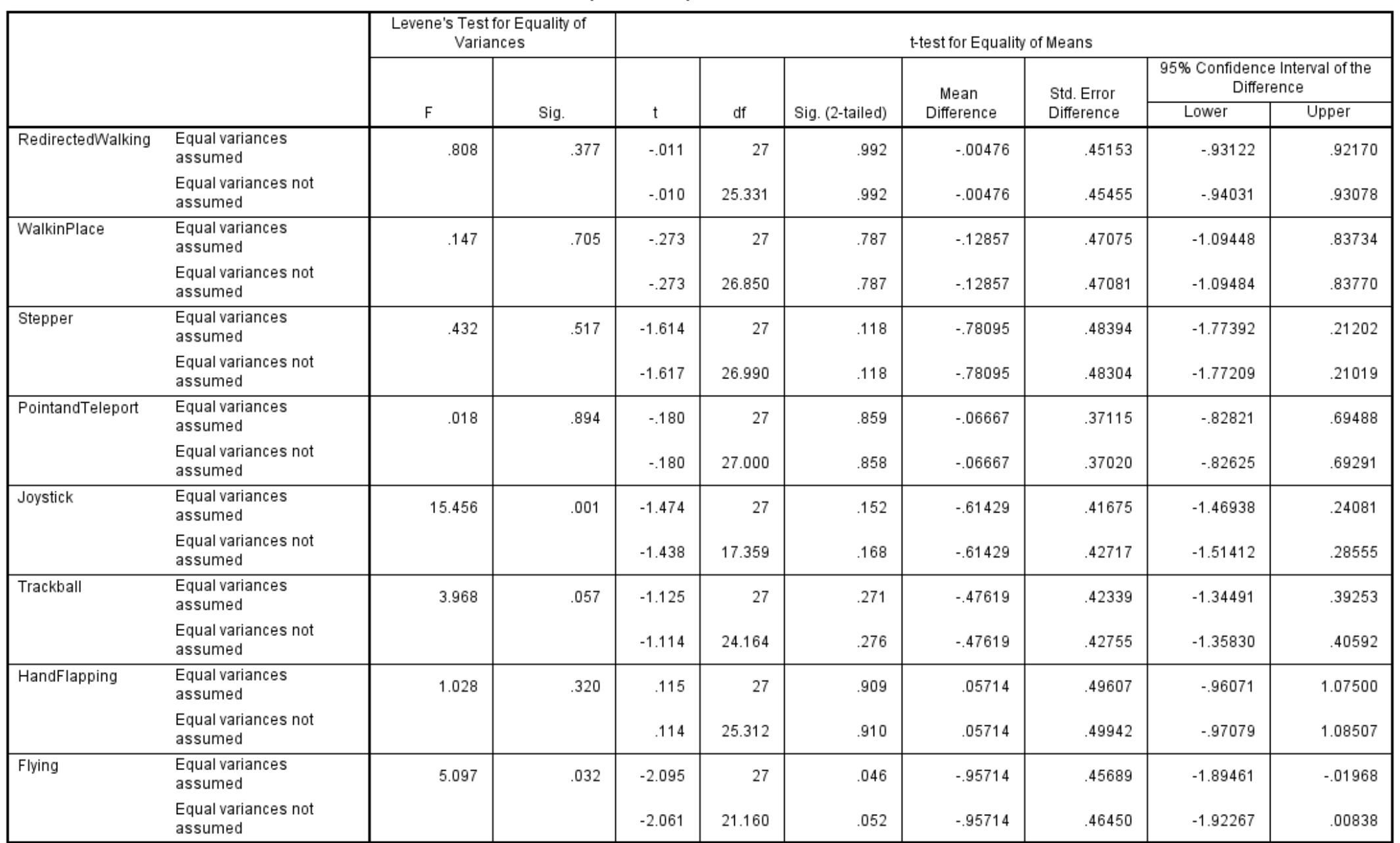

Figure H-8: Detailed comparison analysis for the required effort for neurotypical individuals and individuals with ASD. 
Independent Samples Test

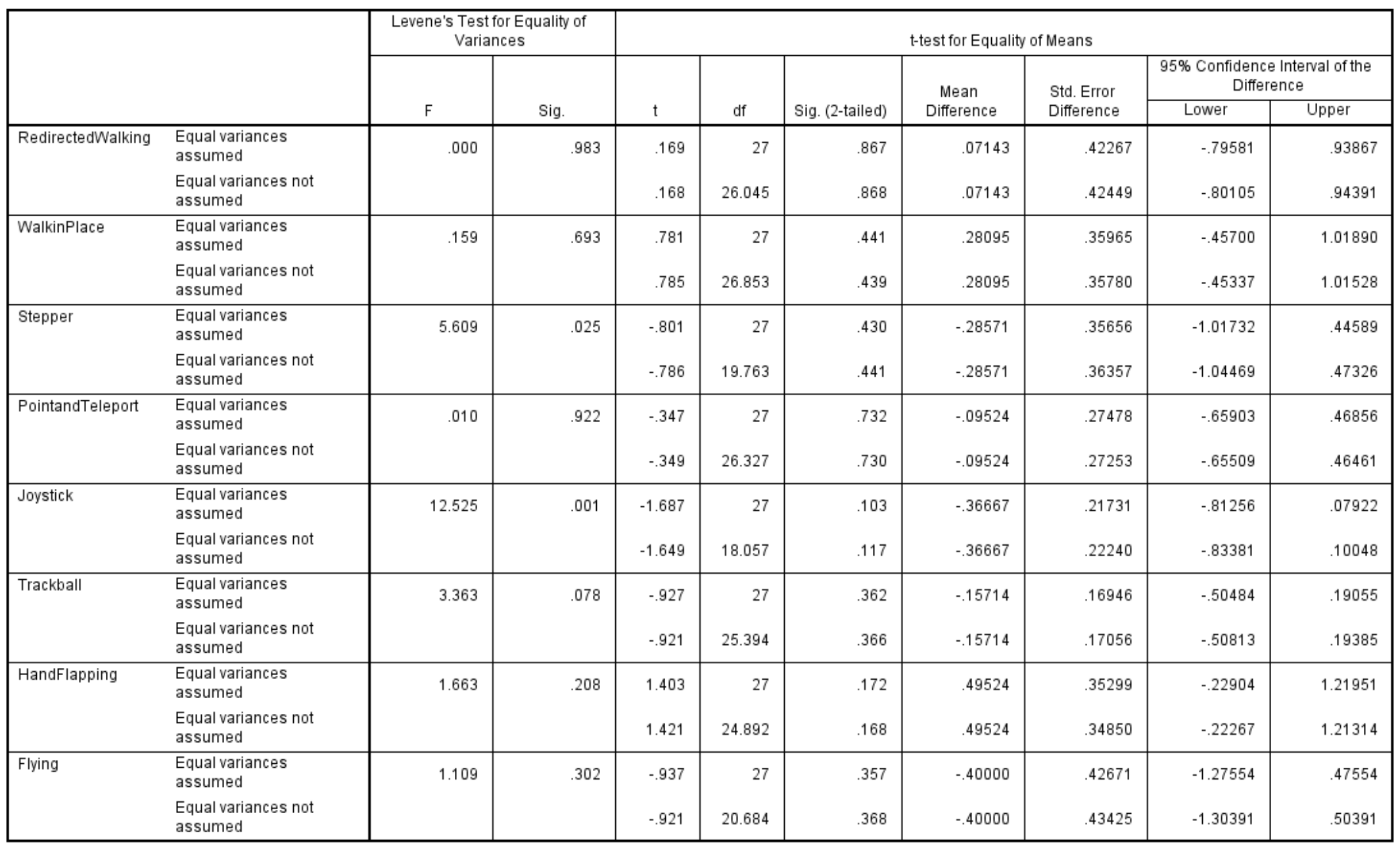

Figure H-9: Detailed comparison analysis for the tiredness for neurotypical individuals and individuals with ASD. 
Independent Samples Test

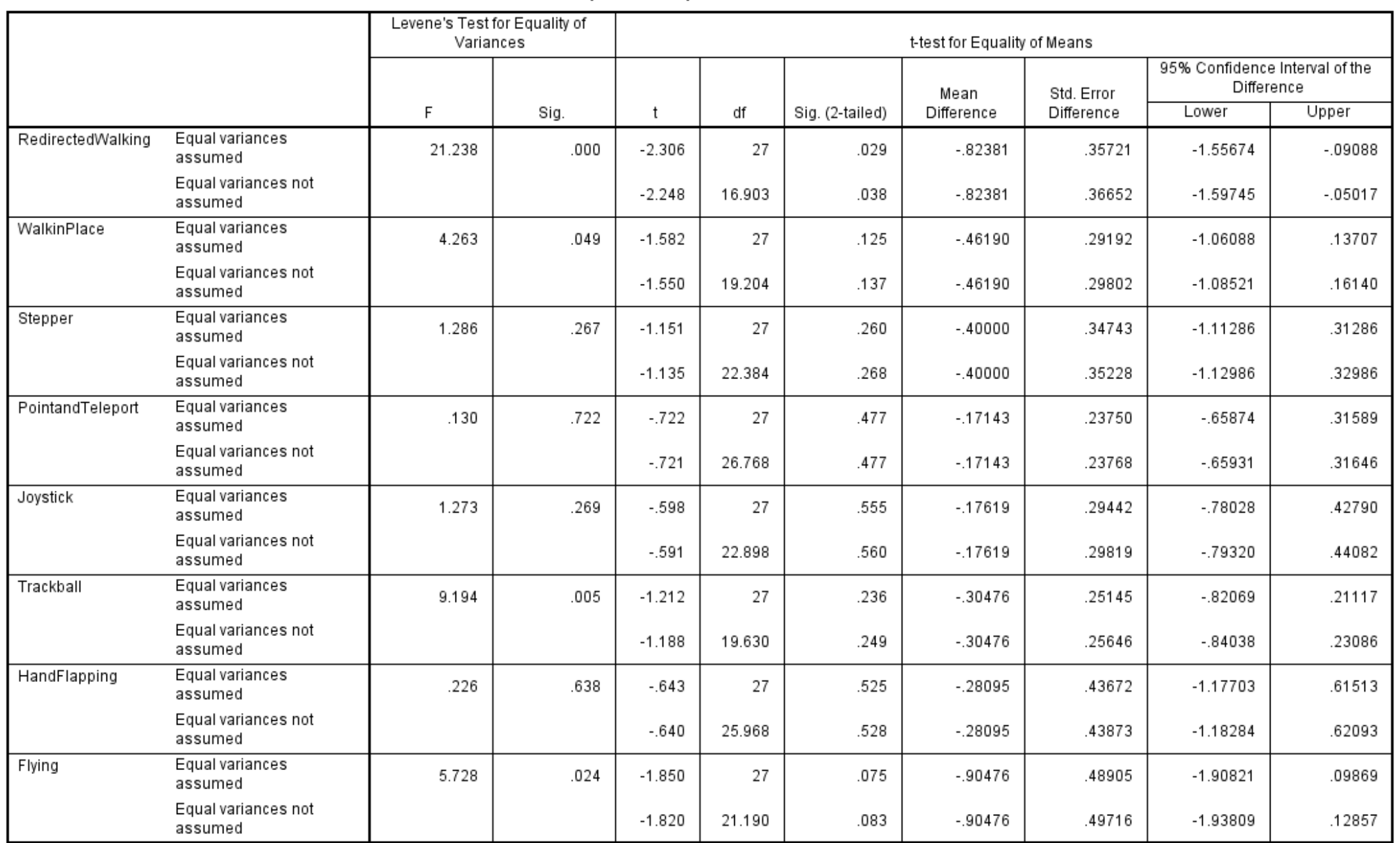

Figure H-10: Detailed comparison analysis for the overwhelmedness for neurotypical individuals and individuals with ASD. 
Independent Samples Test

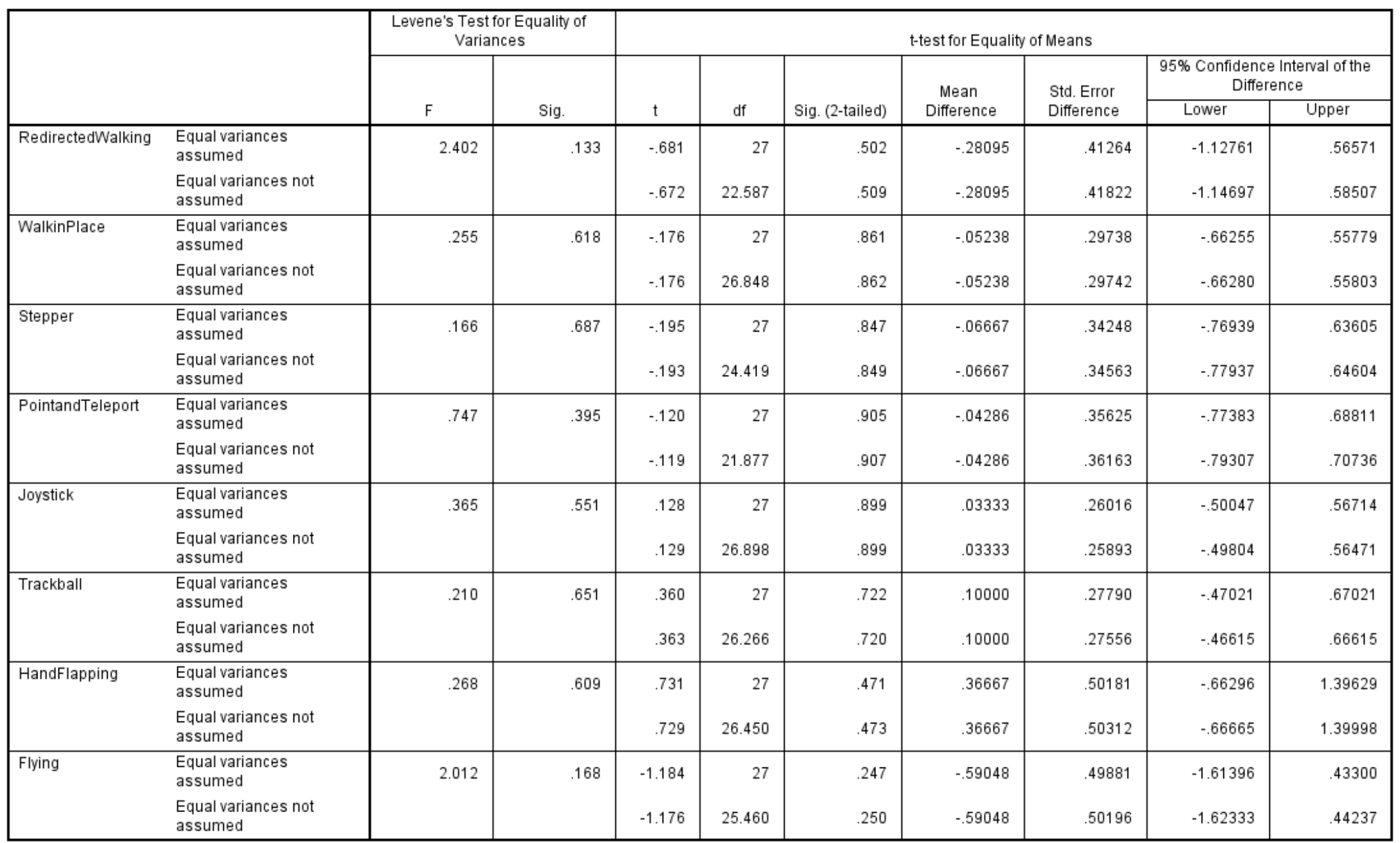

Figure H-11: Detailed comparison analysis for the frustration for neurotypical individuals and individuals with ASD. 
Independent Samples Test

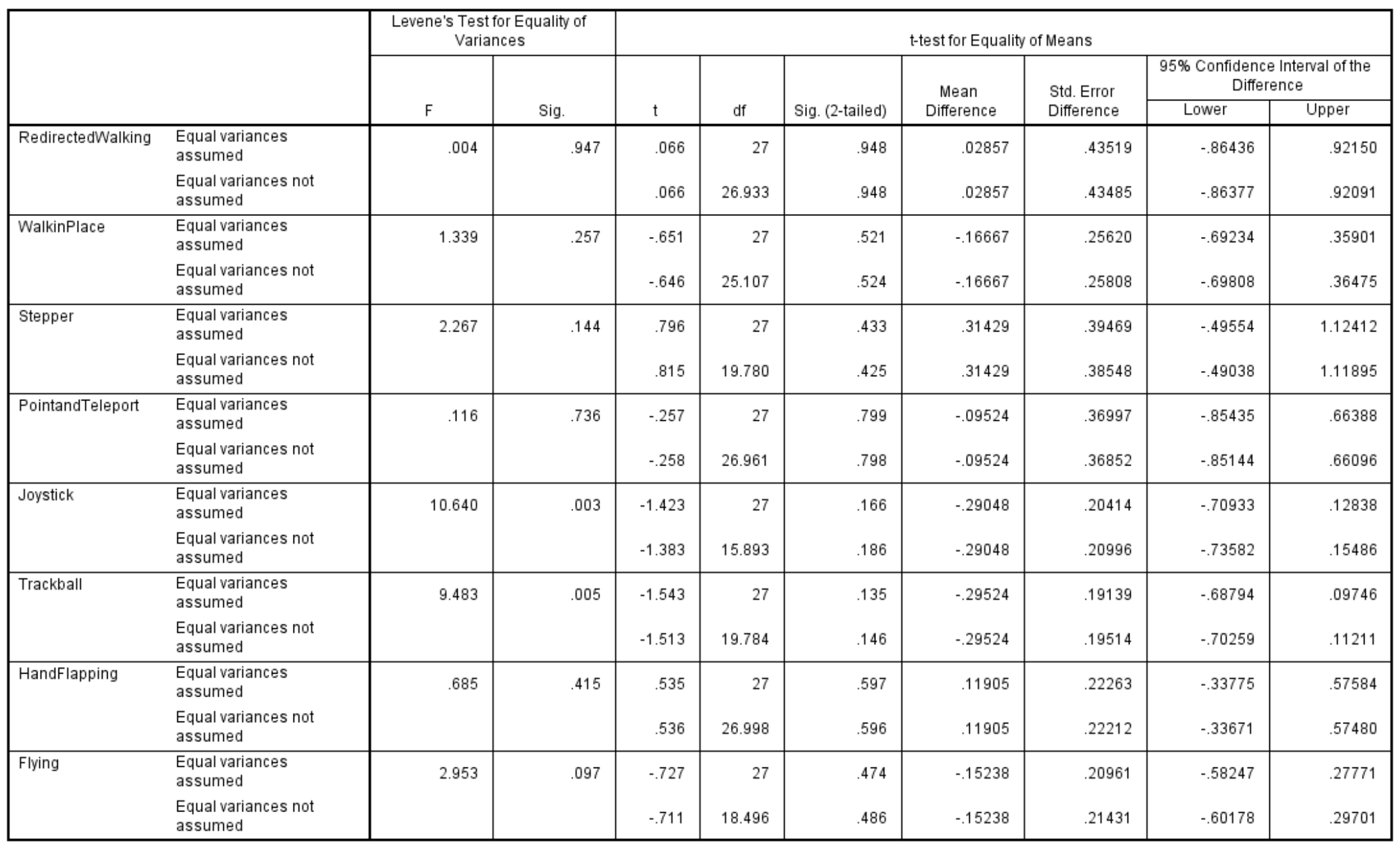

Figure H-12: Detailed comparison analysis for the motion sickness for neurotypical individuals and individuals with ASD. 
Independent Samples Test

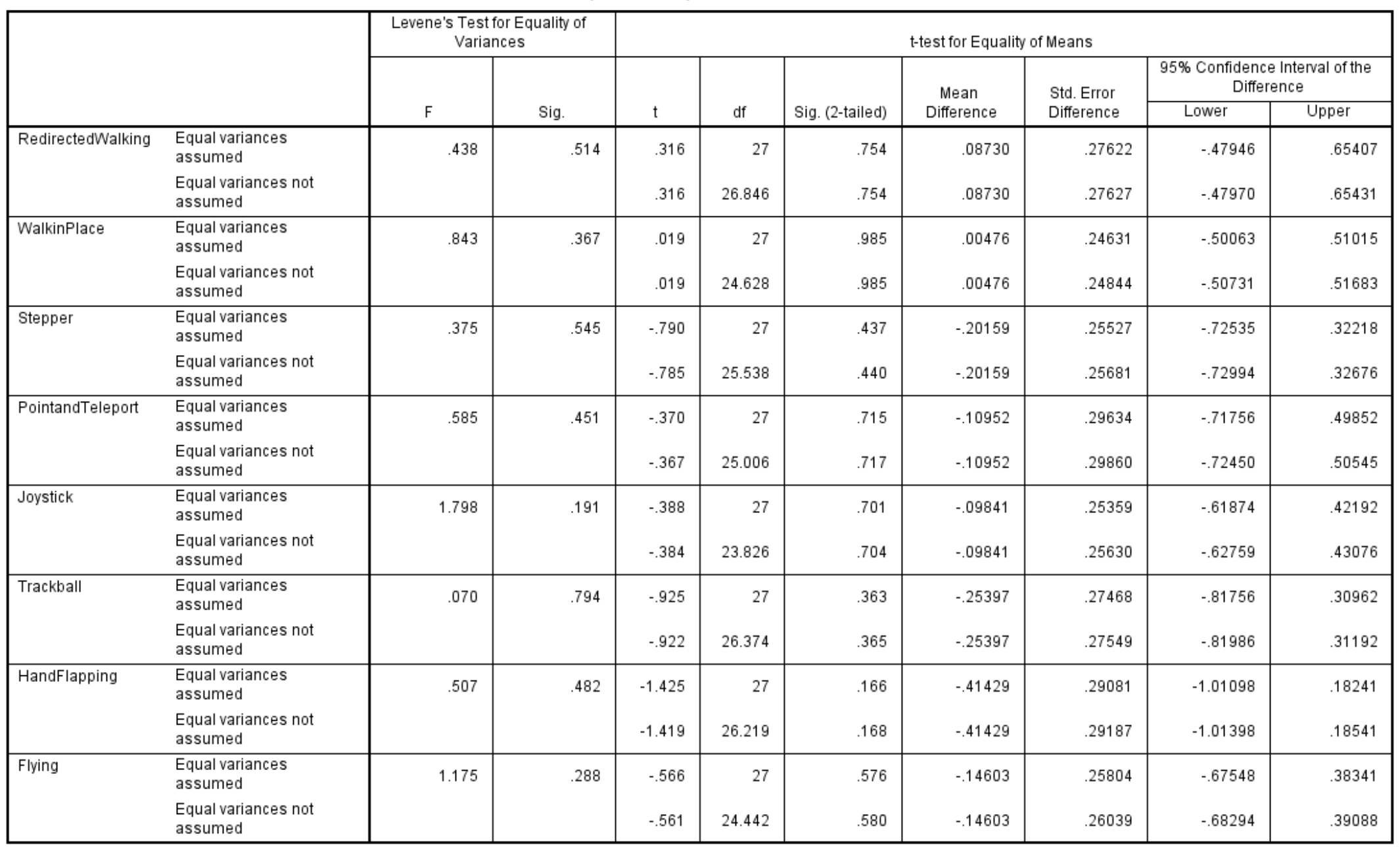

Figure H-13: Detailed comparison analysis for the presence for neurotypical individuals and individuals with ASD. 
Independent Samples Test

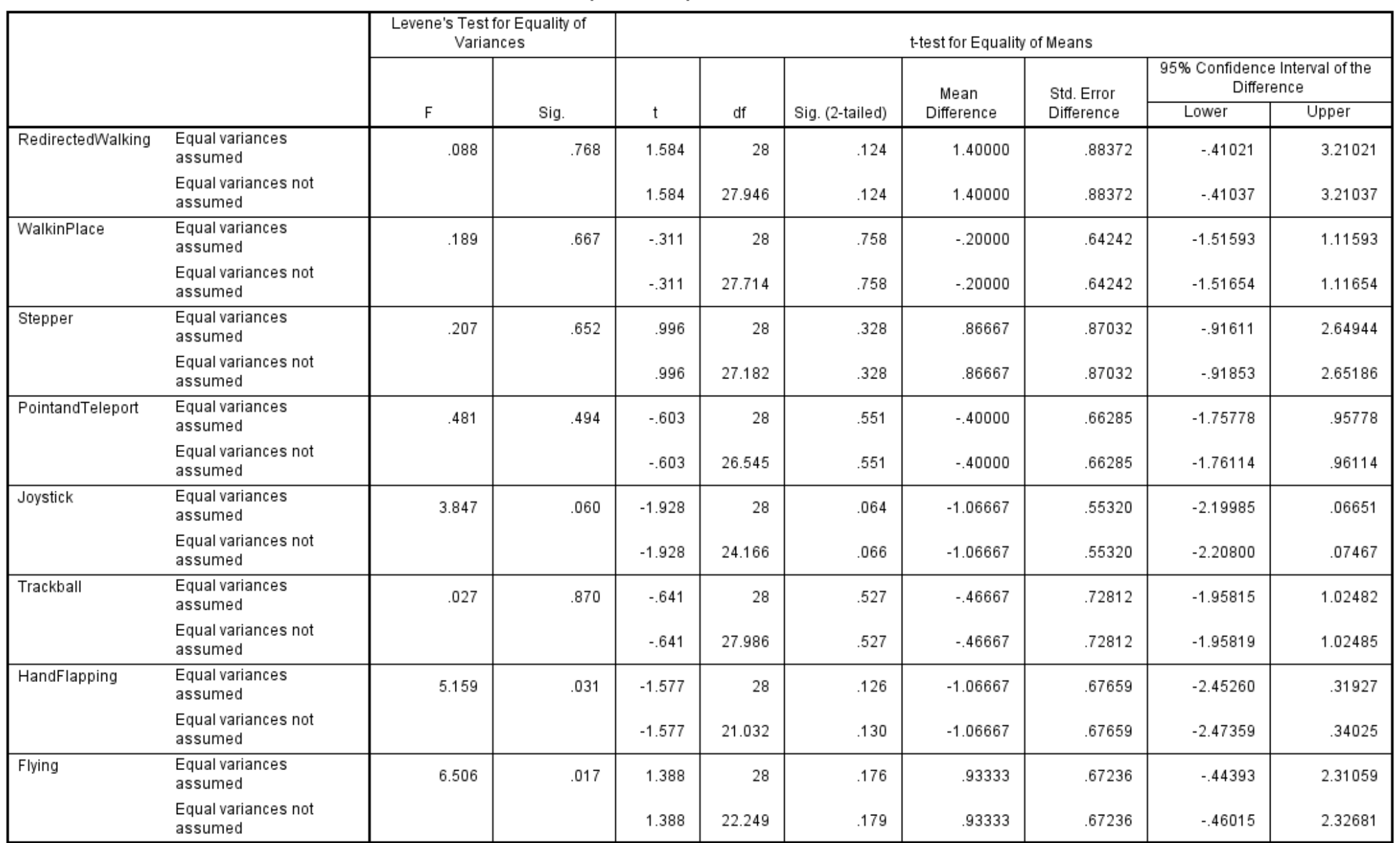

Figure H-14: Detailed comparison analysis for the preference ranking scores for neurotypical individuals and individuals with ASD. 


\section{APPENDIX I: DATA ANALYSIS OF THE POINT \& TELEPORT DIRECTION SPECIFICATION EXPERIMENT}

\begin{tabular}{|c|c|c|c|c|c|c|c|c|c|}
\hline \multicolumn{10}{|c|}{ Paired Samples Test } \\
\hline & & \multicolumn{5}{|c|}{ Paired Differences } & \multirow[b]{3}{*}{$t$} & \multirow[b]{3}{*}{ df } & \multirow[b]{3}{*}{ Sig. (2-tailed) } \\
\hline & & \multirow[b]{2}{*}{ Mean } & \multirow[b]{2}{*}{ Std. Deviation } & \multirow{2}{*}{$\begin{array}{l}\text { Std. Error } \\
\text { Mean }\end{array}$} & \multicolumn{2}{|c|}{$\begin{array}{l}\text { 95\% Confidence Interval of the } \\
\text { Difference }\end{array}$} & & & \\
\hline & & & & & Lower & Upper & & & \\
\hline Pair 1 & Maze - MazeWithDirection & -2.96733 & 8.72920 & 2.25387 & -7.80140 & 1.86674 & -1.317 & 14 & .209 \\
\hline
\end{tabular}

Figure I-1: Paired samples t-test for the completion time for neurotypical individuals for the point \& teleport direction specification experiment.

\begin{tabular}{|c|c|c|c|c|c|c|c|c|c|}
\hline \multicolumn{10}{|c|}{ Paired Samples Test } \\
\hline & & \multicolumn{5}{|c|}{ Paired Differences } & \multirow[b]{3}{*}{$t$} & \multirow[b]{3}{*}{ df } & \multirow[b]{3}{*}{ Sig. (2-tailed) } \\
\hline & & \multirow[b]{2}{*}{ Mean } & \multirow[b]{2}{*}{ Std. Deviation } & \multirow{2}{*}{$\begin{array}{l}\text { Std. Error } \\
\text { Mean }\end{array}$} & \multicolumn{2}{|c|}{$\begin{array}{l}\text { 95\% Confidence Interval of the } \\
\text { Difference }\end{array}$} & & & \\
\hline & & & & & Lower & Upper & & & \\
\hline Pair 1 & Maze - MazeWithDirection & -.53333 & 1.45733 & .37628 & -1.34038 & .27371 & -1.417 & 14 & .178 \\
\hline
\end{tabular}

Figure I-2: Paired samples t-test for the number of collision with maze walls for neurotypical individuals for the point \& teleport direction specification experiment.

\begin{tabular}{|c|c|c|c|c|c|c|c|c|c|}
\hline \multicolumn{10}{|c|}{ Paired Samples Test } \\
\hline & & \multicolumn{5}{|c|}{ Paired Differences } & \multirow[b]{3}{*}{$\mathrm{t}$} & \multirow[b]{3}{*}{ df } & \multirow[b]{3}{*}{ Sig. (2-tailed) } \\
\hline & & \multirow[b]{2}{*}{ Mean } & \multirow[b]{2}{*}{ Std. Deviation } & \multirow{2}{*}{$\begin{array}{l}\text { Std. Error } \\
\text { Mean }\end{array}$} & \multicolumn{2}{|c|}{$\begin{array}{l}95 \% \text { Confidence Interval of the } \\
\text { Difference }\end{array}$} & & & \\
\hline & & & & & Lower & Upper & & & \\
\hline Pair 1 & Maze - MazeWithDirection & -.26667 & .45774 & .11819 & -.52015 & -.01318 & -2.256 & 14 & .041 \\
\hline
\end{tabular}

Figure I-3: Paired samples t-test for the difficulty in understanding for neurotypical individuals for the point \& teleport direction specification experiment. 
Paired Samples Test

\begin{tabular}{|c|c|c|c|c|c|c|c|c|c|}
\hline & & \multicolumn{5}{|c|}{ Paired Differences } & \multirow[b]{3}{*}{$t$} & \multirow[b]{3}{*}{ df } & \multirow[b]{3}{*}{ Sig. (2-tailed) } \\
\hline & & \multirow[b]{2}{*}{ Mean } & \multirow[b]{2}{*}{ Std. Deviation } & \multirow{2}{*}{$\begin{array}{l}\text { Std. Error } \\
\text { Mean }\end{array}$} & \multicolumn{2}{|c|}{$\begin{array}{l}95 \% \text { Confidence Interval of the } \\
\text { Difference }\end{array}$} & & & \\
\hline & & & & & Lower & Upper & & & \\
\hline Pair 1 & Maze - MazeWithDirection & -.33333 & .48795 & .12599 & -.60355 & -.06312 & -2.646 & 14 & .019 \\
\hline
\end{tabular}

Figure I-4: Paired samples t-test for the difficulty in operating for neurotypical individuals for the point \& teleport direction specification experiment.

\begin{tabular}{|c|c|c|c|c|c|c|c|c|c|}
\hline \multicolumn{10}{|c|}{ Paired Samples Test } \\
\hline & & \multicolumn{5}{|c|}{ Paired Differences } & \multirow[b]{3}{*}{$t$} & \multirow[b]{3}{*}{ df } & \multirow[b]{3}{*}{ Sig. (2-tailed) } \\
\hline & & \multirow[b]{2}{*}{ Mean } & \multirow[b]{2}{*}{ Std. Deviation } & \multirow{2}{*}{$\begin{array}{l}\text { Std. Error } \\
\text { Mean }\end{array}$} & \multicolumn{2}{|c|}{$\begin{array}{l}95 \% \text { Confidence Interval of the } \\
\text { Difference }\end{array}$} & & & \\
\hline & & & & & Lower & Upper & & & \\
\hline Pair 1 & Maze - MazeWithDirection & .60000 & .82808 & .21381 & .14143 & 1.05857 & 2.806 & 14 & .014 \\
\hline
\end{tabular}

Figure I-5: Paired samples t-test for the feeling of being in control for neurotypical individuals for the point \& teleport direction specification experiment.

\begin{tabular}{|c|c|c|c|c|c|c|c|c|c|}
\hline \multicolumn{10}{|c|}{ Paired Samples Test } \\
\hline & & \multicolumn{5}{|c|}{ Paired Differences } & \multirow[b]{3}{*}{$t$} & \multirow[b]{3}{*}{ df } & \multirow[b]{3}{*}{ Sig. (2-tailed) } \\
\hline & & \multirow[b]{2}{*}{ Mean } & \multirow[b]{2}{*}{ Std. Deviation } & \multirow{2}{*}{$\begin{array}{l}\text { Std. Error } \\
\text { Mean }\end{array}$} & \multicolumn{2}{|c|}{$\begin{array}{l}95 \% \text { Confidence Interval of the } \\
\text { Difference }\end{array}$} & & & \\
\hline & & & & & Lower & Upper & & & \\
\hline Pair 1 & Maze - MazeWithDirection & .33333 & .89974 & .23231 & -.16492 & .83159 & 1.435 & 14 & .173 \\
\hline
\end{tabular}

Figure I-6: Paired samples t-test for the enjoyment for neurotypical individuals for the point \& teleport direction specification experiment.

Paired Samples Test

\begin{tabular}{|c|c|c|c|c|c|c|c|c|c|}
\hline & & \multicolumn{5}{|c|}{ Paired Differences } & \multirow[b]{3}{*}{$\mathrm{t}$} & \multirow[b]{3}{*}{ df } & \multirow[b]{3}{*}{ Sig. (2-tailed) } \\
\hline & & \multirow[b]{2}{*}{ Mean } & \multirow[b]{2}{*}{ Std. Deviation } & \multirow{2}{*}{$\begin{array}{l}\text { Std. Error } \\
\text { Mean }\end{array}$} & \multicolumn{2}{|c|}{$\begin{array}{l}\text { 95\% Confidence Interval of the } \\
\text { Difference }\end{array}$} & & & \\
\hline & & & & & Lower & Upper & & & \\
\hline Pair 1 & Maze - MazeWithDirection & -.20000 & .77460 & .20000 & -.62896 & .22896 & -1.000 & 14 & .334 \\
\hline
\end{tabular}

Figure I-7: Paired samples t-test for the required effort for neurotypical individuals for the point \& teleport direction specification experiment. 
Paired Samples Test

\begin{tabular}{|c|c|c|c|c|c|c|c|c|c|}
\hline & & \multicolumn{5}{|c|}{ Paired Differences } & \multirow[b]{3}{*}{$t$} & \multirow[b]{3}{*}{ df } & \multirow[b]{3}{*}{ Sig. (2-tailed) } \\
\hline & & \multirow[b]{2}{*}{ Mean } & \multirow[b]{2}{*}{ Std. Deviation } & \multirow{2}{*}{$\begin{array}{l}\text { Std. Error } \\
\text { Mean }\end{array}$} & \multicolumn{2}{|c|}{$\begin{array}{l}95 \% \text { Confidence Interval of the } \\
\text { Difference }\end{array}$} & & & \\
\hline & & & & & Lower & Upper & & & \\
\hline Pair 1 & Maze - MazeWithDirection & -.20000 & .41404 & .10690 & -.42929 & .02929 & -1.871 & 14 & .082 \\
\hline
\end{tabular}

Figure I-8: Paired samples t-test for the tiredness for neurotypical individuals for the point \& teleport direction specification experiment.

\begin{tabular}{|c|c|c|c|c|c|c|c|c|c|}
\hline \multicolumn{10}{|c|}{ Paired Samples Test } \\
\hline & & \multicolumn{5}{|c|}{ Paired Differences } & \multirow[b]{3}{*}{$t$} & \multirow[b]{3}{*}{ df } & \multirow[b]{3}{*}{ Sig. (2-tailed) } \\
\hline & & \multirow[b]{2}{*}{ Mean } & \multirow[b]{2}{*}{ Std. Deviation } & \multirow{2}{*}{$\begin{array}{l}\text { Std. Error } \\
\text { Mean }\end{array}$} & \multicolumn{2}{|c|}{$\begin{array}{l}\text { 95\% Confidence Interval of the } \\
\text { Difference }\end{array}$} & & & \\
\hline & & & & & Lower & Upper & & & \\
\hline Pair 1 & Maze - MazeWithDirection & .00000 & .37796 & .09759 & -.20931 & .20931 & .000 & 14 & 1.000 \\
\hline
\end{tabular}

Figure I-9: Paired samples t-test for the overwhelmedness for neurotypical individuals for the point \& teleport direction specification experiment.

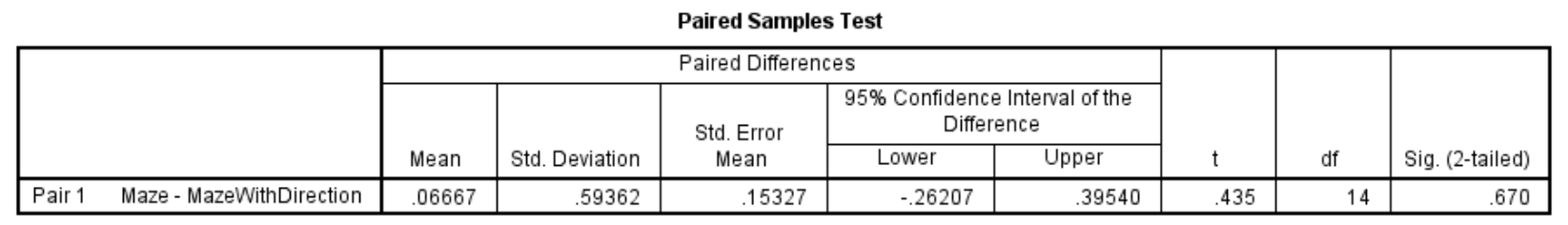

Figure I-10: Paired samples t-test for the frustration for neurotypical individuals for the point \& teleport direction specification experiment.

Paired Samples Test

\begin{tabular}{|c|c|c|c|c|c|c|c|c|c|}
\hline & & \multicolumn{5}{|c|}{ Paired Differences } & \multirow[b]{3}{*}{$\mathrm{t}$} & \multirow[b]{3}{*}{$\mathrm{df}$} & \multirow[b]{3}{*}{ Sig. (2-tailed) } \\
\hline & & \multirow[b]{2}{*}{ Mean } & \multirow[b]{2}{*}{ Std. Deviation } & \multirow{2}{*}{$\begin{array}{l}\text { Std. Error } \\
\text { Mean }\end{array}$} & \multicolumn{2}{|c|}{$\begin{array}{l}95 \% \text { Confidence Interval of the } \\
\text { Difference }\end{array}$} & & & \\
\hline & & & & & Lower & Upper & & & \\
\hline Pair 1 & Maze - MazeWithDirection & -.20000 & .56061 & .14475 & -.51046 & .11046 & -1.382 & 14 & .189 \\
\hline
\end{tabular}

Figure I-11: Paired samples t-test for the motion sickness for neurotypical individuals for the point \& teleport direction specification experiment. 
Paired Samples Test

\begin{tabular}{|c|c|c|c|c|c|c|c|c|c|}
\hline & \multicolumn{5}{|c|}{ Paired Differences } & \multirow[b]{3}{*}{$t$} & \multirow[b]{3}{*}{ df } & \multirow[b]{3}{*}{ Sig. (2-tailed) } \\
\hline & & \multirow[b]{2}{*}{ Mean } & \multirow[b]{2}{*}{ Std. Deviation } & \multirow{2}{*}{$\begin{array}{l}\text { Std. Error } \\
\text { Mean }\end{array}$} & \multicolumn{2}{|c|}{$\begin{array}{l}\text { 95\% Confidence Interval of the } \\
\text { Difference }\end{array}$} & & & \\
\hline & & & & & Lower & Upper & & & \\
\hline Pair 1 & Maze - MazeWithDirection & .15556 & .30516 & .07879 & -.01344 & .32455 & 1.974 & 14 & .068 \\
\hline
\end{tabular}

Figure I-12: Paired samples t-test for the presence for neurotypical individuals for the point \& teleport direction specification experiment.

Pairwise Comparisons

Measure: MazePreference

\begin{tabular}{|c|c|c|c|c|c|c|}
\hline \multirow[b]{2}{*}{ (l) Techniques } & \multirow[b]{2}{*}{ (J) Techniques } & \multirow{2}{*}{$\begin{array}{c}\text { Mean } \\
\text { Difference (I- } \\
\mathrm{J}) \\
\end{array}$} & \multirow[b]{2}{*}{ Std. Error } & \multirow[b]{2}{*}{ Sig. } & \multicolumn{2}{|c|}{$\begin{array}{l}\text { 95\% Confidence Interval for } \\
\text { Difference }\end{array}$} \\
\hline & & & & & Lower Bound & Upper Bound \\
\hline Maze & MazeWithDirection & .067 & .267 & .806 & -.505 & .639 \\
\hline
\end{tabular}

Figure I-13: Paired samples t-test for the preference ranking scores for neurotypical individuals for the point \& teleport direction specification experiment. 